MÉMOIRES DE LA SMF 96

\title{
UNE CONJECTURE DE LUSZTIG POUR LES GROUPES CLASSIQUES
}

\author{
Jean-Loup Waldspurger
}

Société Mathématique de France 2004

Publié avec le concours du Centre National de la Recherche Scientifique 


\section{J.-L. Waldspurger}

CNRS, Institut mathématique de Jussieu, 175, rue du Chevaleret, 75013 Paris.

E-mail : waldspur@math.jussieu.fr

Classification mathématique par sujets (2000). - 11E57, 20C33.

Mots clefs. - Groupes classiques, représentations, faisceaux-caractères, symboles, conjecture de Lusztig. 


\title{
UNE CONJECTURE DE LUSZTIG POUR LES GROUPES CLASSIQUES
}

\author{
Jean-Loup Waldspurger
}

Résumé. - Pour un groupe classique défini sur un corps fini de caractéristique assez grande, on prouve une conjecture de Lusztig reliant les caractères des représentations irréductibles aux fonctions traces des faisceaux-caractères. La preuve inclut une normalisation précise de ces dernières fonctions. Cela généralise des résultats de Shoji à tous les groupes classiques, en particulier au groupe orthogonal pair qui n'est ni connexe, ni à centre connexe.

Abstract (A conjecture of Lusztig for classical groups). - For a classical group defined over a finite field of sufficiently large characteristic, we prove a conjecture of Lusztig connecting characters of irreducible representations with characteristic functions of character-sheaves. Those functions are precisely normalized in the proof. Our result generalizes Shoji's results to all classical groups. We consider in particular the even orthogonal group, that is neither connected nor with connected center. 



\section{TABLE DES MATIÈRES}

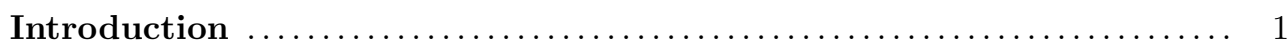

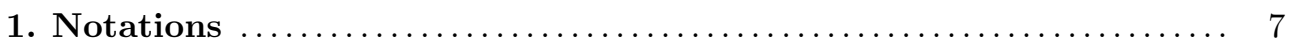

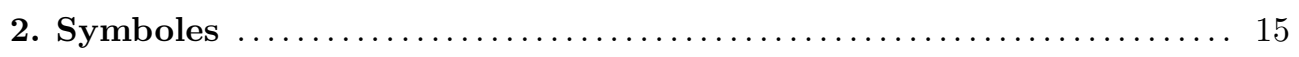

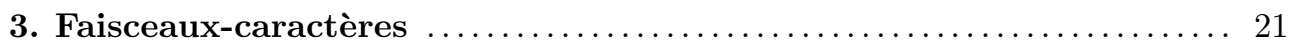

4. Représentations quadratiques-unipotentes $\ldots \ldots \ldots \ldots \ldots \ldots \ldots \ldots \ldots$

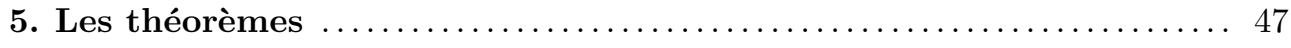

6. Détermination des faisceaux-caractères quadratiques-unipotents . . 49

7. Valeurs des fonctions traces de faisceaux-caractères $\ldots \ldots \ldots \ldots \ldots .57$

8. Formules d'induction $\ldots \ldots \ldots \ldots \ldots \ldots \ldots \ldots \ldots \ldots \ldots \ldots \ldots \ldots \ldots \ldots \ldots \ldots \ldots \ldots \ldots \ldots$

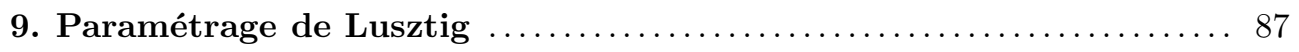

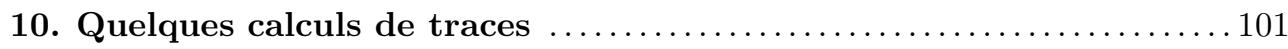

11. Commutation à l'induction de Deligne-Lusztig $\ldots \ldots \ldots \ldots \ldots \ldots \ldots$

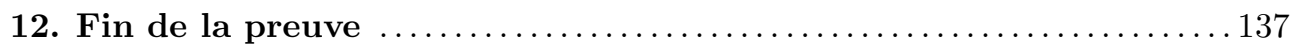

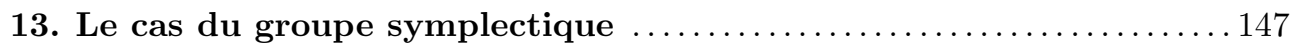

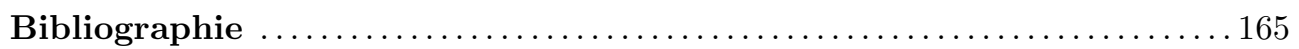





\section{INTRODUCTION}

Soit $\boldsymbol{G}$ un groupe réductif connexe défini sur un corps fini $\mathbb{F}_{q}$, notons $G=\boldsymbol{G}\left(\mathbb{F}_{q}\right)$ son groupe de points sur $\mathbb{F}_{q}$ et $\mathcal{C}(G)$ l'espace des fonctions sur $G$, à valeurs complexes, invariantes par conjugaison. Cet espace a pour base l'ensemble des caractères des représentations irréductibles de $G$. Lusztig a introduit en [L6] une autre base, formée des fonctions traces associées aux faisceaux-caractères sur $\boldsymbol{G}$ invariants par le Frobenius. Il a conjecturé la forme de la matrice de transition entre ces deux bases. Cette conjecture a été prouvée par Shoji, en $[\mathbf{S 1}]$, dans le cas où le centre de $\boldsymbol{G}$ est connexe. Une autre démonstration a été esquissée par Lusztig dans [L10]. Il faut préciser qu'un problème de normalisation se pose pour ces fonctions traces associées aux faisceaux-caractères. Elles ne sont bien définies qu'à un scalaire près. Le résultat de Shoji, comme la conjecture de Lusztig elle-même, consiste à dire que l'on peut normaliser ces fonctions de sorte que la matrice évoquée ci-dessus ait la forme prédite. Mais la normalisation n'est pas précisée en général. Elle l'a été ultérieurement par Shoji, en $[\mathbf{S 2}]$, dans le cas des représentations unipotentes des groupes classiques déployés à centre connexe.

Dans le présent article, nous considérons le cas des groupes classiques $\boldsymbol{G}=\boldsymbol{S} \boldsymbol{p}(2 n)$, $\boldsymbol{S} \boldsymbol{O}(2 n+1)$ et $\boldsymbol{O}(2 n)$. Ce dernier n'est pas connexe, mais il n'est pas difficile de généraliser dans son cas les différentes constructions utiles, en particulier celles des faisceauxcaractères. D'un point de vue combinatoire, le groupe $\boldsymbol{O}(2 n)$ est d'ailleurs plus simple que son sous-groupe $\boldsymbol{S} \boldsymbol{O}(2 n)$. Signalons que le cas des groupes non connexes a été abordé par différents auteurs, $c f$. [DM2], [E].

Nous nous limitons à un sous-espace $\mathbb{C}[\operatorname{Quad}(G)]$ de $\mathcal{C}(G)$. Expliquons sa définition. D'après Lusztig, on sait associer à toute représentation irréductible de $G$ une classe de conjugaison semi-simple dans le groupe dual. On dira qu'une représentation 
irréductible est quadratique-unipotente (cette terminologie s'inspire de $[\mathbf{M}]$ ) si les éléments de cette classe de conjugaison ont pour carré l'identité. C'est équivalent à dire que leurs valeurs propres dans la représentation naturelle du groupe dual sont \pm 1 , ou encore que le commutant d'un de ces éléments n'est pas inclus dans un groupe de Lévi propre du groupe dual (on appelle groupe de Lévi un sous-groupe de Lévi d'un sous-groupe parabolique). On note $\operatorname{Quad}(G)$ l'ensemble des représentations irréductibles quadratiques-unipotentes de $G$ et $\mathbb{C}[\operatorname{Quad}(G)]$ le sous-espace de $\mathcal{C}(G)$ engendré par leurs caractères. On peut caractériser les faisceaux-caractères dont les fonctions traces appartiennent à cet espace. Un faisceau-caractère apparaît toujours comme composante d'un complexe « induit » à partir d'un système local cuspidal $\mathcal{L}$ porté par une sous-variété d'un groupe de Lévi $\boldsymbol{M}$ de $\boldsymbol{G}$. Notons $\boldsymbol{Z}_{\boldsymbol{M}}^{0}$ la composante neutre du centre de $\boldsymbol{M}$. Il existe un système local $\mathcal{L}_{Z}$ sur $\boldsymbol{Z}_{M}^{0}$ qui joue le rôle de «caractère central » de $\mathcal{L}$. La trace du faisceau-caractère appartient à $\mathbb{C}[\operatorname{Quad}(G)]$ si et seulement si $\mathcal{L}_{Z}^{\otimes 2}$ est trivial. Se limiter à $\mathbb{C}[\operatorname{Quad}(G)]$ n'est pas une restriction essentielle car l'espace $\mathcal{C}(G)$ tout entier se reconstruit à l'aide d'espaces $R_{M}^{G} \mathbb{C}[\operatorname{Quad}(M) \otimes \tau]$ où $\boldsymbol{M}$ est un groupe de Lévi de $\boldsymbol{G}, \tau$ est un caractère de degré 1 de $M$ et $R_{M}^{G}$ est le foncteur d'induction de Deligne-Lusztig.

Nous imposons une hypothèse plus sérieusement restrictive, à savoir $q>2 n$ (et $q$ impair). Cela est nécessaire pour utiliser les résultats de [L8] comparant les foncteurs d'induction de Deligne-Lusztig à l'induction des faisceaux-caractères.

Sous cette hypothèse, on donne dans cet article :

- une classification des représentations irréductibles quadratiques-unipotentes de $G$ (le travail est presque entièrement fait en $[\mathbf{L 2}]$ );

- une construction et une classification des fonctions-traces de faisceaux-caractères qui appartiennent à $\mathbb{C}[\operatorname{Quad}(G)]$. Ces fonctions sont précisément normalisées;

- une preuve de la conjecture de Lusztig dans ce cadre, qui exprime la matrice de transition entre les deux bases ci-dessus de $\mathbb{C}[\operatorname{Quad}(G)]$.

Les constructions sont effectuées dans les paragraphes 3 et 4 . Le théorème principal est énoncé au paragraphe 5 . Nous n'en donnons une démonstration complète que dans le cas du groupe $\boldsymbol{O}(2 n)$ (paragraphes 6 à 12). Nous passons sous silence le cas du groupe $\boldsymbol{S} \boldsymbol{O}(2 n+1)$. Parce qu'il est connexe et adjoint, son cas relève beaucoup plus simplement des travaux de Lusztig et Shoji. Nous indiquons brièvement au paragraphe 13 les modifications à apporter à la preuve dans le cas du groupe $\boldsymbol{S} \boldsymbol{p}(2 n)$.

Expliquons la structure de la démonstration dans le cas du groupe orthogonal pair. Il est commode de regrouper les deux formes de ce groupe, la forme déployée $\boldsymbol{O}_{+}(2 n)$ et la forme non déployée $\boldsymbol{O}_{-}(2 n)$. On étudie donc l'espace $\mathbb{C}[\mathrm{Quad}(O(2 n))]=$ $\mathbb{C}\left[\operatorname{Quad}\left(O_{+}(2 n)\right)\right] \oplus \mathbb{C}\left[\operatorname{Quad}\left(O_{-}(2 n)\right)\right]$. Pour un entier $m \geqslant 0$, Lusztig a introduit en [L2] l'ensemble $\mathcal{S}_{m \text {,pair }}$ des symboles de rang $m$ et de défaut pair. Un tel symbole est une classe d'équivalence, pour une relation convenable, de paires $\left(\Lambda^{+}, \Lambda^{-}\right)$de sous-ensembles finis de $\mathbb{N}$, où l'on ne tient pas compte de l'ordre, c'est-à-dire que 
$\left(\Lambda^{+}, \Lambda^{-}\right) \equiv\left(\Lambda^{-}, \Lambda^{+}\right)$. On note $\widetilde{\mathcal{S}}_{m \text {,pair }}$ l'ensemble des symboles ordonnés de rang $m$ et de défaut pair : la définition est la même, sauf que l'on tient compte de l'ordre, c'està-dire que l'on n'identifie pas $\left(\Lambda^{+}, \Lambda^{-}\right)$à $\left(\Lambda^{-}, \Lambda^{+}\right)$. On note $\widetilde{\mathcal{S}} \widetilde{\mathcal{S}}_{n \text {,pair }}$ est la réunion

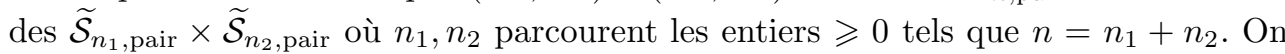
note $\mathbb{C}\left[\widetilde{\mathcal{S}} \widetilde{\mathcal{S}}_{n, \text { pair }}\right]$ l'espace vectoriel complexe de base $\widetilde{\mathcal{S}} \widetilde{\mathcal{S}}_{n, \text { pair }}$. On le munit du produit hermitien pour lequel cette base est orthonormée. À la suite de Lusztig, on définit une isométrie involutive $\mathcal{F}$ de cet espace. Lusztig l'appelle transformation de Fourier.

On construit explicitement une base de $\mathbb{C}[\mathrm{Quad}(O(2 n))]$ formée de fonctions traces de faisceaux-caractères, qui est naturellement paramétrée par $\widetilde{\mathcal{S}} \widetilde{\mathcal{S}}_{n \text {,pair }}$. Cela détermine une isométrie $k_{n}: \mathbb{C}\left[\widetilde{\mathcal{S}} \widetilde{\mathcal{S}}_{n, \text { pair }}\right] \rightarrow \mathbb{C}[\operatorname{Quad}(O(2 n))]$. On classifie ensuite les représentations irréductibles quadratiques-unipotentes de $O_{+}(2 n)$ et $O_{-}(2 n)$. La méthode consiste à classifier d'abord les cuspidales, grâce à [L2], puis à décomposer les représentations induites de cuspidales (cette méthode remonte à Harish-Chandra). Il s'avère que l'ensemble des représentations irréductibles quadratiques unipotentes est aussi paramétré par $\widetilde{\mathcal{S}} \widetilde{\mathcal{S}}_{n, \text { pair }}$. Cela détermine une autre isométrie $\pi_{n}: \mathbb{C}\left[\widetilde{\mathcal{S}} \widetilde{\mathcal{S}}_{n \text {,pair }}\right] \rightarrow$ $\mathbb{C}[\operatorname{Quad}(O(2 n))]$. Posons $i_{n}=\pi_{n}^{-1} \circ k_{n} \circ \mathcal{F}$. Le théorème est que $i_{n}$ est l'identité.

La première étape est de prouver que $i_{n}$ est l'identité sur un gros sous-espace de $\mathbb{C}\left[\widetilde{\mathcal{S}} \widetilde{\mathcal{S}}_{n, \text { pair }}\right]$. Soit $m$ un entier $<n$. On définit des homomorphismes d'induction $R: \mathbb{C}[\operatorname{Quad}(O(2 m))] \rightarrow \mathbb{C}[\operatorname{Quad}(O(2 n))]$ de la façon suivante. Soient $\eta, \varepsilon$ deux signes \pm ; soit $\boldsymbol{T}$ un tore tel que $\boldsymbol{M}=\boldsymbol{T} \times \boldsymbol{O}_{\eta}(2 m)$ soit un groupe de Lévi défini sur $\mathbb{F}_{q}$ de $\boldsymbol{G}=\boldsymbol{O}_{\eta \varepsilon}(2 n)$; soit $\chi$ un caractère quadratique-unipotent de $T$. On dispose du foncteur d'induction de Deligne-Lusztig $R_{M}^{G}$. Pour $f \in \mathbb{C}\left[\operatorname{Quad}\left(O_{\eta}(2 m)\right)\right]$, on pose $R(f)=R_{M}^{G}(\chi \times f)$. Comme on le voit, on dispose de plusieurs choix possibles pour $\boldsymbol{T}$ et $\chi$, d'où plusieurs homomorphismes $R$. Fixons-en un. Notons $R^{k}, R^{\pi}$ les applications linéaires qui rendent les diagrammes suivants commutatifs :
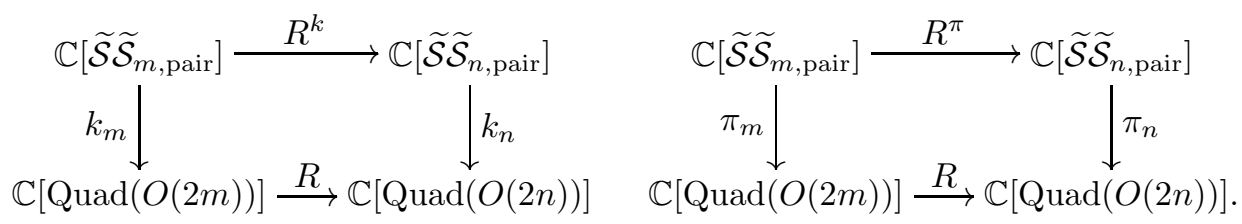

Lusztig a montré dans [L8] comment se comportaient les faisceaux-caractères pour l'induction de Deligne-Lusztig. On en déduit le calcul de $R^{k}$.

$\mathrm{Si}$, dans la construction ci-dessus, $\boldsymbol{M}$ est un sous-groupe de Lévi d'un sous-groupe parabolique défini sur $\mathbb{F}_{q}$, il est facile de calculer $R^{\pi}$. On voit alors que $\mathcal{F} \circ R^{\pi}=R^{k} \circ \mathcal{F}$ (le premier $\mathcal{F}$ agissant dans $\mathbb{C}\left[\widetilde{\mathcal{S}} \widetilde{\mathcal{S}}_{n, \text { pair }}\right]$, le second dans $\mathbb{C}\left[\widetilde{\mathcal{S}}_{m, \text { pair }}\right]$ ). En supposant par récurrence que $i_{m}$ est l'identité, on en déduit que $i_{n}$ est l'identité sur l'image de $R^{\pi}$.

Si $M$ n'est plus un sous-groupe de Lévi d'un sous-groupe parabolique défini sur $\mathbb{F}_{q}$, le calcul de $R^{\pi}$ devient difficile. Il est essentiellement dû̀ à Asai. Soit $\left(\Lambda_{1}, \Lambda_{2}\right) \in$ $\widetilde{\mathcal{S}} \widetilde{\mathcal{S}}_{m, \text { pair }}$. On connaît trois propriétés de $R^{\pi}\left(\Lambda_{1}, \Lambda_{2}\right)$ : 
- notons $\pi=\pi_{m}\left(\Lambda_{1}, \Lambda_{2}\right)$ la représentation irréductible associée à $\left(\Lambda_{1}, \Lambda_{2}\right)$. On sait que $R_{M}^{G}(\operatorname{trace} \pi)$ est la trace d'une représentation virtuelle de $G$, c'est-à-dire une combinaison linéaire à coefficients entiers relatifs de traces de représentations irréductibles. Donc, avec une notation évidente, $R^{\pi}\left(\Lambda_{1}, \Lambda_{2}\right) \in \mathbb{Z}\left[\widetilde{\mathcal{S}} \widetilde{\mathcal{S}}_{n, \text { pair }}\right]$;

- soit $\left(\Lambda_{1}^{\prime}, \Lambda_{2}^{\prime}\right) \in \widetilde{\mathcal{S}} \widetilde{\mathcal{S}}_{n \text {,pair }}$ un couple de symboles de défaut 0 . On sait calculer le produit scalaire $\left(R^{\pi}\left(\Lambda_{1}, \Lambda_{2}\right), \mathcal{F}\left(\Lambda_{1}^{\prime}, \Lambda_{2}^{\prime}\right)\right)$. Cela résulte de l'égalité $i_{n} \circ \mathcal{F}\left(\Lambda_{1}^{\prime}, \Lambda_{2}^{\prime}\right)=$ $\mathcal{F}\left(\Lambda_{1}^{\prime}, \Lambda_{2}^{\prime}\right)$. Celle-ci résulte elle-même de la comparaison entre notre paramétrage « à la Harish-Chandra » des représentations irréductibles et le paramétrage de Lusztig. Ce dernier classifie les représentations par le produit scalaire de leur trace contre les fonctions $k_{n}\left(\Lambda_{1}^{\prime}, \Lambda_{2}^{\prime}\right)$, pour $\left(\Lambda_{1}^{\prime}, \Lambda_{2}^{\prime}\right)$ comme ci-dessus ;

- si $R^{\prime}: \mathbb{C}\left[\operatorname{Quad}\left(O\left(2 m^{\prime}\right)\right)\right] \rightarrow \mathbb{C}[\operatorname{Quad}(O(2 n))]$ est un homomorphisme analogue à $R$, si $\left(\Lambda_{1}^{\prime}, \Lambda_{2}^{\prime}\right) \in \widetilde{\mathcal{S}} \widetilde{\mathcal{S}}_{m^{\prime}, \text { pair }}$, on peut calculer le produit scalaire $\left(R^{\pi}\left(\Lambda_{1}, \Lambda_{2}\right), R^{\prime \pi}\left(\Lambda_{1}^{\prime}, \Lambda_{2}^{\prime}\right)\right)$. En particulier, on connait la norme de $R^{\pi}\left(\Lambda_{1}, \Lambda_{2}\right)$.

À l'aide de ces trois propriétés, un peu de combinatoire permet de déterminer $R^{\pi}\left(\Lambda_{1}, \Lambda_{2}\right)$ (en fait, pas complètement, $c f$. ci-dessous). De nouveau, on constate que $\mathcal{F} \circ R^{\pi}=R^{k} \circ \mathcal{F}$. Alors $i_{n}$ est l'identité sur l'image de $R^{\pi}$.

On note $\mathbb{C}\left[\widetilde{\mathcal{S}} \widetilde{\mathcal{S}}_{n, \text { pair }}\right]_{I J}$ le sous-espace de $\mathbb{C}\left[\widetilde{\mathcal{S}} \widetilde{\mathcal{S}}_{n, \text { pair }}\right]$ engendré par les images des homomorphismes $R^{\pi}$ pour tous les $R$ possibles. À ce point, on a démontré que $i_{n}$ était l'identité sur ce sous-espace. Puisque $i_{n}$ est une isométrie, elle se restreint en une isométrie de l'orthogonal de ce sous-espace. Cet orthogonal a pour base la famille des $\mathcal{F}\left(\Lambda_{1}, \Lambda_{2}\right)$, quand $\left(\Lambda_{1}, \Lambda_{2}\right)$ décrit le sous-ensemble $H$ des éléments « cuspidaux » de $\widetilde{\mathcal{S}} \widetilde{\mathcal{S}}_{n \text {,pair }}$. On introduit la matrice carrée $C$ d'ordre $|H| \times|H|$ telle que $C_{\Lambda_{1}, \Lambda_{2} ; \Lambda_{1}^{\prime}, \Lambda_{2}^{\prime}}=\left(i_{n} \mathcal{F}\left(\Lambda_{1}, \Lambda_{2}\right), \mathcal{F}\left(\Lambda_{1}^{\prime}, \Lambda_{2}^{\prime}\right)\right)$ pour $\left(\Lambda_{1}, \Lambda_{2}\right),\left(\Lambda_{1}^{\prime}, \Lambda_{2}^{\prime}\right) \in H$. Pour achever de prouver le théorème, on doit montrer que $C$ est l'identité. Remarquons que $C_{\Lambda_{1}, \Lambda_{2} ; \Lambda_{1}^{\prime}, \Lambda_{2}^{\prime}}=\left(k_{n}\left(\Lambda_{1}, \Lambda_{2}\right), \pi_{n} \circ \mathcal{F}\left(\Lambda_{1}^{\prime}, \Lambda_{2}^{\prime}\right)\right)$. De cette égalité, on déduit sans peine que $C_{\Lambda_{1}, \Lambda_{2} ; \Lambda_{1}^{\prime}, \Lambda_{2}^{\prime}}$ est rationnel et même dans $2^{-N} \mathbb{Z}$ pour un entier $N$ assez grand. Soit $\left(\Lambda_{1}, \Lambda_{2}\right) \in \widetilde{\mathcal{S}} \widetilde{\mathcal{S}}_{n \text {,pair }}$. Le terme $\pi_{n}\left(\Lambda_{1}, \Lambda_{2}\right)-k_{n} \circ \mathcal{F}\left(\Lambda_{1}, \Lambda_{2}\right)$ se calcule en fonction des coefficients de la matrice $C$. En effet, on écrit $\left(\Lambda_{1}, \Lambda_{2}\right)=\mathcal{F} \circ \mathcal{F}\left(\Lambda_{1}, \Lambda_{2}\right)$; on décompose $\mathcal{F}\left(\Lambda_{1}, \Lambda_{2}\right)$ en $Z+Z^{\prime}$ où $Z \in \mathbb{C}\left[\widetilde{\mathcal{S}} \widetilde{\mathcal{S}}_{n, \text { pair }}\right]_{I J}$ et $Z^{\prime}$ appartient à l'orthogonal de ce sous-espace; on sait que $i_{n} \circ \mathcal{F}(Z)=\mathcal{F}(Z)$; donc

$$
\pi_{n}\left(\Lambda_{1}, \Lambda_{2}\right)-k_{n} \circ \mathcal{F}\left(\Lambda_{1}, \Lambda_{2}\right)=\pi_{n} \circ \mathcal{F}\left(Z^{\prime}\right)-k_{n}\left(Z^{\prime}\right)
$$

et ce dernier terme s'exprime à l'aide de la matrice $C$ par définition de cette matrice. Supposons la conjecture à démontrer vraie. Alors $k_{n} \circ \mathcal{F}\left(\Lambda_{1}, \Lambda_{2}\right)=\pi_{n}\left(\Lambda_{1}, \Lambda_{2}\right)$. Ce terme est la trace d'une représentation irréductible, en particulier il est à valeurs entières. On peut effectivement montrer que $k_{n} \circ \mathcal{F}\left(\Lambda_{1}, \Lambda_{2}\right)$, calculé en certains points particuliers, a pour valeur, sinon un entier, du moins un rationnel dont on peut minorer la valuation 2-adique. C'est l'idée de Shoji, que l'on reprend en y insérant quelques résultats combinatoires de $[\mathbf{W}]$. Il est curieux de constater à ce propos la similitude entre les problèmes combinatoires qui se posent ici et ceux qui se posaient en $[\mathbf{W}]$ concernant l'endoscopie pour les groupes $p$-adiques. Traduite en termes des coefficients 
de la matrice $C$, cette propriété entraîne des congruences mod $2 \mathbb{Z}_{2}$ de ces coefficients. On sait donc que $C$ est unitaire, que ses coefficients appartiennent à $2^{-N} \mathbb{Z}$, et vérifient ces congruences. Cela détermine entièrement $C$, qui est bien l'identité.

En fait, il y a quelques difficultés supplémentaires. D'abord, les congruences auxquelles on vient de faire allusion ne sont assez fortes que si $n \geqslant 5$. Pour $n \leqslant 4$, il faut ajouter des arguments ad hoc, utilisant des descriptions explicites de certaines représentations.

D'autre part, toutes nos classifications reposent sur des choix. Il ne semble pas y en avoir toujours de naturels et nous ne prétendons pas avoir fait les plus judicieux. Par exemple, l'ensemble $\operatorname{Quad}\left(O_{+}(2)\right)$ a 4 éléments : le caractère trivial, le caractère det et les deux caractères prolongeant l'unique caractère quadratique non trivial de $S_{+}(2)$. Les deux premiers caractères se distinguent aisément mais il n'y a pas de moyen naturel de distinguer chacun des deux autres. Pour identifier $\operatorname{Quad}\left(O_{+}(2)\right)$ à un sous-ensemble de $\widetilde{\mathcal{S}} \widetilde{\mathcal{S}}_{1 \text {,pair }}$, on doit nécessairement faire un choix. Plus généralement, on doit faire des choix à deux niveaux :

- celui du paramétrage des représentations cuspidales. On verra qu'à tout couple d'entiers $h_{1}, h_{2} \geqslant 0$ tels que $h_{1}^{2}+h_{2}^{2}=n$, on peut naturellement associer un ensemble de $|H|$ représentations irréductibles cuspidales de $O_{+}(2 n)$ ou $O_{-}(2 n)$ (selon la parité de $\left.h_{1}-h_{2}\right)$, où $H$ est l'ensemble des couples d'entiers relatifs de la forme $\left( \pm h_{1}, \pm h_{2}\right)$. On doit fixer une bijection entre $H$ et cet ensemble de représentations. En fait, nous ne précisons pas cette bijection. Notre résultat est qu'on peut la fixer de sorte que la conjecture de Lusztig soit vérifiée;

- si $\pi$ est une représentation irréductible cuspidale, disons de $O_{+}(2 n)$, que l'on prolonge en une représentation $\pi_{M}$ de $M=\mathbb{F}_{q}^{\times} \times O_{+}(2 n)$ par un caractère quadratique de $\mathbb{F}_{q}^{\times}$, il arrive que l'induite à la Harish-Chandra de $\pi_{M}$ à $O_{+}(2 n+2)$ se décompose en deux représentations de même dimension. Elles ne sont alors pas naturellement discernables. Nous les séparons en imposant les valeurs de leurs caractères sur certains éléments particuliers de $O_{+}(2 n+2)$ (les symétries orthogonales par rapport aux hyperplans, $c f$. paragraphe 10).

Évidemment, la nécessité de faire des choix se reflète au cours de la démonstration et explique la difficulté technique du paragraphe 11 : les méthodes purement combinatoires développées par Asai ne peuvent suffire à calculer les foncteurs $R_{M}^{G}$; il faut forcément montrer que les choix effectués pour classifier les représentations de $M$ sont compatibles à ceux effectués pour classifier celles de $G$.

Il est clair que notre article n'a rien d'original et n'apporte que des précisions minimes sur les travaux antérieurs. L'essentiel a bien sûr été fait par Lusztig. Le reste repose sur les idées d'Asai et Shoji. L'auteur n'est pas spécialiste de la théorie des groupes finis. La motivation de ce travail est l' article antérieur $[\mathbf{M W}]$ de C. Moeglin et l'auteur. Dans cet article, concernant les $L$-paquets de représentations de groupes $S O(2 n+1)$ sur un corps $p$-adique, on avait impérativement besoin de connaître la 
conjecture de Lusztig pour les représentations unipotentes d'un groupe $O(2 n)$. Cette conjecture avait été admise en $[\mathbf{M W}]$, rendant la preuve de cet article incomplète. La principale conséquence, à nos yeux, du présent article, est que, maintenant, la preuve du théorème de $[\mathbf{M W}]$ est complète.

Je remercie vivement C. Moeglin pour l'aide qu'elle a apportée pour mettre au point les preuves combinatoires du paragraphe 11 . 


\section{CHAPITRE 1}

\section{NOTATIONS}

On introduit des notations et on pose des définitions concernant les groupes algébriques, les foncteurs d'induction de Deligne-Lusztig, les groupes classiques et leurs éléments unipotents.

1.1. Soient $p$ un nombre premier $\neq 2, q$ une puissance de $p, \mathbb{F}_{q}$ le corps fini à $q$ éléments, $\overline{\mathbb{F}}_{q}$ une clôture algébrique de $\mathbb{F}_{q}$. Pour tout entier $n \geqslant 1$, on fixe un isomorphisme :

$$
\operatorname{Hom}\left(\mathbb{F}_{q^{n}}^{\times}, \mathbb{C}^{\times}\right) \simeq \mathbb{F}_{q^{n}}^{\times},
$$

de sorte que, si $m$ divise $n$, le diagramme suivant soit commutatif :

$$
\begin{array}{cc}
\operatorname{Hom}\left(\mathbb{F}_{q^{m}}^{\times}, \mathbb{C}^{\times}\right) & \simeq \mathbb{F}_{q^{m}}^{\times} \\
\downarrow & \downarrow \\
\operatorname{Hom}\left(\mathbb{F}_{q^{n}}^{\times}, \mathbb{C}^{\times}\right) & \simeq \mathbb{F}_{q^{n}}^{\times}
\end{array}
$$

(la flèche de droite est l'inclusion naturelle, celle de gauche se déduit de la norme).

En particulier, on note $\zeta$ le caractère de $\mathbb{F}_{q}^{\times}$correspondant à -1 par cet isomorphisme. C'est l'unique caractère d'ordre 2 de $\mathbb{F}_{q}^{\times}$.

1.2. Soient $X$ un ensemble et $A$ un anneau commutatif. On note $A[X]$ le $A$-module libre de base $X$. Supposons $X$ fini. On note $|X|$ son nombre d'éléments.

Supposons que $X$ soit un groupe fini. On note $\mathcal{C}(X)$ l'espace des fonctions sur $X$, à valeurs complexes, invariantes par conjugaison. On munit $\mathcal{C}(X)$ du produit hermitien :

$$
\left(f, f^{\prime}\right)=|X|^{-1} \sum_{x \in X} \bar{f}(x) f^{\prime}(x) .
$$

On appelle représentation de $X$ un couple $(\pi, E)$, où $E$ est un espace vectoriel complexe de dimension finie et $\pi: X \rightarrow G L(E)$ est un homomorphisme. On oubliera souvent l'un des termes du couple en parlant d'une représentation $\pi$ ou d'un $X$ module $E$. On identifiera souvent des représentations équivalentes. On note $\operatorname{Irr}(X)$ 
l'ensemble des classes d'équivalence de représentations irréductibles de $X$. L'application qui à $\pi \in \operatorname{Irr}(X)$ associe le caractère trace $(\pi)$ définit un isomorphisme de $\mathbb{C}[\operatorname{Irr}(X)] \operatorname{sur} \mathcal{C}(X)$. On identifie ainsi ces deux espaces. On note $Z_{X}$ le centre de $X$. Pour $x \in X$, resp. $Y \subset X$, et $X^{\prime}$ un sous-groupe de $X$, on note $Z_{X^{\prime}}(x)$, resp. $Z_{X^{\prime}}(Y)$, le centralisateur de $x$, resp. $Y$, dans $X^{\prime}$.

1.3. Soit $\boldsymbol{H}$ un groupe algébrique défini sur $\mathbb{F}_{q}$, réductif. On note $\boldsymbol{H}^{0}$ sa composante neutre et $H=\boldsymbol{H}\left(\mathbb{F}_{q}\right)$ son groupe de points sur $\mathbb{F}_{q}$. Fixons un sous-groupe de Borel $\boldsymbol{B}$ de $\boldsymbol{H}^{0}$ et un sous-tore maximal $\boldsymbol{T}$ de $\boldsymbol{B}$, tous deux définis sur $\mathbb{F}_{q}$. Ces groupes déterminent une donnée de racines $\mathcal{D}=\left(X^{*}, \Delta, X_{*}, \breve{\Delta}\right)$, où $X^{*}$, resp. $X_{*}$, est le groupes des caractères, resp. des sous-groupes à un paramètre, de $\boldsymbol{T}$, et $\Delta$, resp. $\check{\Delta}$, est l'ensemble de racines, resp. coracines, simples déterminé par $\boldsymbol{B}$. Notons $\operatorname{Aut}\left(\boldsymbol{H}^{0}\right)$, resp. $\operatorname{Aut}(\mathcal{D})$, le groupe des automorphismes de $\boldsymbol{H}^{0}$, resp. $\mathcal{D}$. Il y a un homomorphisme naturel $\boldsymbol{H} / \boldsymbol{H}^{0} \rightarrow \operatorname{Aut}(\mathcal{D})$. D'autre part, le choix d'un épinglage détermine un homomorphisme $\sigma: \operatorname{Aut}(\mathcal{D}) \rightarrow \operatorname{Aut}\left(\boldsymbol{H}^{0}\right)$. Ces homomorphismes sont équivariants pour les actions de Frobenius, pourvu que l'épinglage soit stable par cette action.

On note $\widehat{\boldsymbol{H}}^{0}$ le groupe dual de $\boldsymbol{H}^{0}$. C'est aussi un groupe algébrique défini sur $\mathbb{F}_{q}$ et réductif. On effectue pour ce groupe les mêmes constructions que ci-dessus : on fixe une paire $\widehat{\boldsymbol{B}}, \widehat{\boldsymbol{T}}$, on définit $\widehat{\mathcal{D}}$ et un homomorphisme équivariant pour l'action de Frobenius $\widehat{\sigma}: \operatorname{Aut}(\widehat{\mathcal{D}}) \rightarrow \operatorname{Aut}\left(\widehat{\boldsymbol{H}}^{0}\right)$. On a $\widehat{\mathcal{D}}=\left(X_{*}, \check{\Delta}, X^{*}, \Delta\right)$, $\operatorname{donc} \operatorname{Aut}(\mathcal{D}) \simeq \operatorname{Aut}(\widehat{\mathcal{D}})$. Cet isomorphisme n'est pas équivariant pour les actions de Frobenius : il entrelace l'action du Frobenius sur $\operatorname{Aut}(\mathcal{D})$ avec l'inverse de cette action sur $\operatorname{Aut}(\widehat{\mathcal{D}})$. On note $\left(\boldsymbol{H} / \boldsymbol{H}^{0}\right)$ le groupe $\boldsymbol{H} / \boldsymbol{H}^{0}$ dont on inverse l'action de Frobenius. Ce groupe agit sur $\widehat{\boldsymbol{H}}^{0}$ grâce à la suite d'homomorphismes :

$$
\left(\boldsymbol{H} / \boldsymbol{H}^{0}\right)=\boldsymbol{H} / \boldsymbol{H}^{0} \longrightarrow \operatorname{Aut}(\mathcal{D}) \simeq \operatorname{Aut}(\widehat{\mathcal{D}}) \stackrel{\widehat{\sigma}}{\longrightarrow} \operatorname{Aut}\left(\widehat{\boldsymbol{H}}^{0}\right) .
$$

On définit alors $\widehat{\boldsymbol{H}}$ comme le produit semi-direct de $\widehat{\boldsymbol{H}}^{0}$ et $\left(\boldsymbol{H} / \boldsymbol{H}^{0}\right)$.

Deligne et Lusztig ont défini une décomposition en union disjointe :

$$
\operatorname{Irr}\left(H^{0}\right)=\bigcup \operatorname{Irr}\left(H^{0}, s\right),
$$

où $s$ parcourt les éléments semi-simples de $\widehat{H}^{0}$, à conjugaison près par $\widehat{H}^{0}$. Soient $s$ un tel élément et $\pi \in \operatorname{Irr}(H)$. Pour une sous-représentation irréductible $\pi^{0}$ de la restriction de $\pi$ à $H^{0}$, considérons la condition : il existe $s^{\prime} \in \widehat{H}^{0}$, conjugué à $s$ par un élément de $\widehat{H}$, tel que $\pi^{0} \in \operatorname{Irr}\left(H^{0}, s^{\prime}\right)$. Alors toutes les sous-représentations irréductibles $\pi^{0}$ vérifient cette condition ou aucune ne la vérifie. On note $\operatorname{Irr}(H, s)$ l'ensemble des $\pi$ pour lesquelles la première éventualité se produit. On a une décomposition en union disjointe :

$$
\operatorname{Irr}(H)=\bigcup \operatorname{Irr}(H, s),
$$

où $s$ parcourt les éléments semi-simples de $\widehat{H}^{0}$, à conjugaison près par $\widehat{H}$. 
1.4. Soit $\boldsymbol{H}$ comme ci-dessus, supposons-le d'abord connexe. On appelle groupe de Lévi de $\boldsymbol{H}$ tout sous-groupe $\boldsymbol{M}$ qui est sous-groupe de Lévi d'un sous-groupe parabolique $\boldsymbol{P}$ de $\boldsymbol{H}$. On sait que l'on peut caractériser les groupes de Lévi de la façon suivante. Pour tout sous-groupe algébrique $\boldsymbol{M}$ de $\boldsymbol{H}$, notons $\boldsymbol{T}_{\boldsymbol{M}}$ le plus grand soustore central dans $\boldsymbol{M}$ et $\boldsymbol{A}_{\boldsymbol{M}}$ le plus grand sous-tore défini sur $\mathbb{F}_{q}$ et déployé de $\boldsymbol{T}_{\boldsymbol{M}}$. Alors $\boldsymbol{M}$ est un groupe de Lévi si et seulement si $\boldsymbol{M}=\boldsymbol{Z}_{\boldsymbol{H}}\left(\boldsymbol{T}_{\boldsymbol{M}}\right)$. Et $\boldsymbol{M}$ est un sous-groupe de Lévi défini sur $\mathbb{F}_{q}$ d'un sous-groupe parabolique $\boldsymbol{P}$ défini sur $\mathbb{F}_{q}$ si et seulement si $\boldsymbol{M}=\boldsymbol{Z}_{\boldsymbol{H}}\left(\boldsymbol{A}_{\boldsymbol{M}}\right)$.

Ne supposons plus $\boldsymbol{H}$ connexe. Pour tout sous-groupe algébrique $\boldsymbol{M}$ de $\boldsymbol{H}$, définissons $\boldsymbol{T}_{\boldsymbol{M}}$ et $\boldsymbol{A}_{\boldsymbol{M}}$ comme ci-dessus. Nous dirons que $\boldsymbol{M}$ est un groupe de Lévi si $\boldsymbol{M}=\boldsymbol{Z}_{\boldsymbol{H}}\left(\boldsymbol{T}_{\boldsymbol{M}}\right)$. Soit $\boldsymbol{M}$ un tel groupe. Alors $\boldsymbol{M}^{0}$ est un groupe de Lévi de $\boldsymbol{H}^{0}$. Soit $\boldsymbol{P}^{0}$ un sous-groupe parabolique de $\boldsymbol{H}^{0}$ de sous-groupe de Lévi $\boldsymbol{M}^{0}$, notons $\boldsymbol{U}$ son radical unipotent. Supposons que $\boldsymbol{U}$ soit stable par conjugaison par $\boldsymbol{M}$ (ce n'est pas toujours le cas, mais on peut choisir $\boldsymbol{P}^{0}$ de sorte qu'il en soit ainsi). Posons $\boldsymbol{P}=\boldsymbol{M U}$. Un tel sous-groupe sera appelé sous-groupe parabolique de $\boldsymbol{H}$. On dira que $\boldsymbol{M}$ est défini sur $\mathbb{F}_{q}$ s'il est stable par l'action de Frobenius. Par abus de terminologie, on dira que $\boldsymbol{P}$ est défini sur $\mathbb{F}_{q}$ s'il est stable par l'action de Frobenius et si, de plus, on a l'égalité $M=Z_{H}\left(A_{M}\right)$.

Soit $\boldsymbol{P}=\boldsymbol{M U}$ un sous-groupe parabolique de $\boldsymbol{H}$ (ici comme dans la suite, une telle phrase signifie implicitement que $\boldsymbol{M}$ est un sous-groupe de Lévi de $\boldsymbol{P}$ et $\boldsymbol{U}$ le radical unipotent de $\boldsymbol{P}$ ). Supposons $\boldsymbol{P}$ et $\boldsymbol{M}$ définis sur $\mathbb{F}_{q}$. Soit $A$ un sous-anneau de $\mathbb{C}$. On définit de façon usuelle l'induction d'Harish-Chandra :

$$
\operatorname{Ind}_{P}^{H}: A[\operatorname{Irr}(M)] \longrightarrow A[\operatorname{Irr}(H)] \text {. }
$$

Supposons seulement $\boldsymbol{M}$ défini sur $\mathbb{F}_{q}$. À la suite de Lusztig, on définit un homomorphisme :

$$
R_{M}^{H}: A[\operatorname{Irr}(M)] \longrightarrow A[\operatorname{Irr}(H)] .
$$

Notons $F$ le Frobenius de $\boldsymbol{H}$, introduisons la variété :

$$
\boldsymbol{X}=\left\{x(\boldsymbol{U} \cap F(\boldsymbol{U})) \in \boldsymbol{H} /(\boldsymbol{U} \cap F(\boldsymbol{U})) ; x^{-1} F(x) \in F(\boldsymbol{U})\right\} .
$$

Considérons ses espaces de cohomologie $H_{c}^{i}(\boldsymbol{X})$. Ce sont des $\overline{\mathbb{Q}}_{\ell}$-espaces vectoriels, où $\ell$ est un nombre premier fixé, $\neq p$. Ici comme dans la suite, on les considère comme des espaces sur $\mathbb{C}$, en fixant une fois pour toutes un isomorphisme $\overline{\mathbb{Q}}_{\ell} \simeq \mathbb{C}$. Le groupe $H \times M$ agit sur $\boldsymbol{X}$ par $(h, m) x(\boldsymbol{U} \cap F(\boldsymbol{U}))=h x m^{-1}(\boldsymbol{U} \cap F(\boldsymbol{U}))$. Il agit aussi sur les espaces $H_{c}^{i}(\boldsymbol{X})$. Pour un $M$-module $E$ et $i \in \mathbb{N}$, l'espace d'invariants $\left(H_{c}^{i}(\boldsymbol{X}) \otimes_{\mathbb{C}} E\right)^{M}$ est un $H$-module. On pose :

$$
R_{M}^{H}(E)=\sum_{i \in \mathbb{N}}(-1)^{i}\left(H_{c}^{i}(\boldsymbol{X}) \otimes_{\mathbb{C}} E\right)^{M} .
$$

Remarque. - On utilise la notation traditionnelle $R_{M}^{H}$. En fait, on ne sait pas, en général, que cet homomorphisme est indépendant du choix de $\boldsymbol{P}$. Ce n'est connu que si l'on suppose, comme nous le faisons, que $q$ est grand. 
Il y a des variantes à cette construction. Par exemple, on peut remplacer $\boldsymbol{M}$ par $\boldsymbol{M}^{0}$ et définir $R_{M^{0}}^{H}$. Dans le cas où $\boldsymbol{P}$ est défini sur $\mathbb{F}_{q}$, on a l'égalité $R_{M}^{H}=\operatorname{Ind}_{P}^{H}$.

Soient $\boldsymbol{P}=\boldsymbol{M U}, \boldsymbol{P}^{\prime}=\boldsymbol{M}^{\prime} \boldsymbol{U}^{\prime}$ deux sous-groupes paraboliques de $\boldsymbol{H}$. Supposons $\boldsymbol{P} \subseteq \boldsymbol{P}^{\prime}, \boldsymbol{M} \subseteq \boldsymbol{M}^{\prime}$ et $\boldsymbol{M}$ et $\boldsymbol{M}^{\prime}$ définis sur $\mathbb{F}_{q}$. On a la relation de transitivité :

$$
R_{M^{\prime}}^{H} \circ R_{M}^{M^{\prime}}=R_{M}^{H} \text {. }
$$

1.5. Soit $n \in \mathbb{N}$. On note $\mathcal{P}(n)$ l'ensemble des partitions de $n$. Pour $\underline{\lambda} \in$ $\mathcal{P}(n)$ et $i \in \mathbb{N}, i \geqslant 1$, on note mult $_{\underline{\lambda}}(i)$ la multiplicité de $i$ dans $\underline{\lambda}$. On pose $\operatorname{Jord}(\underline{\lambda})=\left\{i \geqslant 1 ; \operatorname{mult}_{\underline{\lambda}}(i) \geqslant 1\right\}$. On dit que $\underline{\lambda}$ est orthogonale, resp. symplectique, si mult $\underline{\lambda}_{\underline{\lambda}}(i)$ est paire pour tout $i$ pair, resp. impair. Quand $\underline{\lambda}$ est supposée orthogonale, resp. symplectique, on note $\operatorname{Jord}^{\mathrm{bp}}(\underline{\lambda})$ le sous-ensemble des éléments impairs, resp. pairs, de $\operatorname{Jord}(\underline{\lambda})$ ('exposant bp signifiant « de bonne parité »). On appelle partition un élément de $\bigcup_{n \in \mathbb{N}} \mathcal{P}(n)$.

1.6. Soit $V$ un espace vectoriel de dimension finie $d$ sur $\mathbb{F}_{q}$, muni d'une forme quadratique non dégénérée $Q$. On pose :

$$
\eta(V)=\eta(Q)=\zeta\left((-1)^{[d / 2]} \operatorname{det}(Q)\right),
$$

où $\operatorname{det}(Q)$ est le déterminant de $Q$ calculé dans une base quelconque de $V$. Par convention, $\eta(V)=1$ si $d=0$. On introduit les groupes usuels $\boldsymbol{O}(V), \boldsymbol{S O}(V), \boldsymbol{C O}(V)$ (groupe des similitudes). On note :

$$
\lambda: C O(V) \longrightarrow \mathbb{F}_{q}^{\times}
$$

l'homomorphisme tel que, pour tous $g \in C O(V), v, v^{\prime} \in V, Q\left(g v, g v^{\prime}\right)=\lambda(g) Q\left(v, v^{\prime}\right)$. On définit un homomorphisme «norme spinorielle»:

$$
\text { sp : } O(V) \longrightarrow\{ \pm 1\},
$$

par la propriété suivante. Soient $v \in V$ tel que $Q(v, v) \neq 0$ et $s_{v}$ la symétrie par rapport à l'hyperplan orthogonal à $v$; alors $\operatorname{sp}\left(s_{v}\right)=\zeta(Q(v, v))$. Cet homomorphisme dépend de $Q$. Par contre, sa restriction à $S O(V)$ en est indépendante (c'est-à-dire que l'on peut changer $Q$ en $z Q$, avec $z \in \mathbb{F}_{q}^{\times}$, sans changer cette restriction). Soit $s$ un élément de $O(V)$ dont toutes les valeurs propres sont \pm 1 . Notons $V^{-}$le sous-espace caractéristique associé à la valeur propre -1 . On calcule :

$$
\operatorname{sp}(s)=\eta\left(V^{-}\right) \zeta(-1)^{\left[\operatorname{dim}\left(V^{-}\right) / 2\right]} .
$$

Soit $u$ un élément unipotent de $O(V)$. Notons $\underline{\lambda}(u)$ la partition de $d$ formée des dimensions des blocs de Jordan de $u$. Elle est orthogonale. Posons $\operatorname{Jord}^{\text {bp }}(u)=$ $\operatorname{Jord}^{\mathrm{bp}}(\underline{\lambda}(u))$. Soit $i \in \operatorname{Jord}^{\mathrm{bp}}(u)$. Décomposons $V$ en une somme directe orthogonale $V=V_{i} \oplus V_{\neq i}$, de sorte que $V_{i}$ et $V_{\neq i}$ soient stables par $u$ et les blocs de Jordan de $u$ agissant dans $V_{i}$, resp. $V_{\neq i}$, soient tous de dimension $i$, resp. $\neq i$. On pose $\eta_{u}(i)=\eta\left(V_{i}\right)$. Cela définit une fonction :

$$
\eta_{u}: \operatorname{Jord}^{\mathrm{bp}}(u) \longrightarrow\{ \pm 1\} .
$$


On a l'égalité :

$$
\prod_{i \in \operatorname{Jord}^{\mathrm{bp}}(u)} \eta_{u}(i)=\eta(V) \zeta(-1)^{\left[\left|\operatorname{Jord}^{\mathrm{bp}}(u)\right| / 2\right]} .
$$

Les données $\left(\underline{\lambda}(u), \eta_{u}\right)$ caractérisent la classe de conjugaison de $u$ dans $O(V)$.

1.7. Soit $n \in \mathbb{N}$. On note $W_{n}$ le groupe des permutations $w$ de $\{ \pm 1, \ldots, \pm n\}$ telles que $w(-i)=-w(i)$ pour tout $i$. Pour $i \in\{1, \ldots, n-1\}$ on note $s_{i}$ l'élément de $W_{n}$ qui permute $i$ et $i+1$ et fixe $j$ pour tout $j \in\{1, \ldots, n\} \backslash\{i, i+1\}$. On note $s_{n}$ l'élément qui envoie $n$ sur $-n$ et fixe $j$ pour tout $j \in\{1, \ldots, n-1\}$. On note sgn le caractère signe usuel de $W_{n}$ et on définit le caractère :

$$
\begin{aligned}
\operatorname{sgn}_{C D}: W_{n} & \longrightarrow\{ \pm 1\} \\
w & \longmapsto(-1)^{|\{i \in\{1, \ldots, n\} ; w(i)<0\}|} .
\end{aligned}
$$

On note $W_{n}^{D}$ le noyau de $\operatorname{sgn}_{C D}$.

Soit $(V, Q)$ comme en 1.6, de dimension $d$. Si $d=2 n+1$, resp. $d=2 n$ et $\eta(Q)=1$, resp. $d=2 n$ et $\eta(Q)=-1$, il est bien connu que les classes de conjugaison de soustores maximaux définis sur $\mathbb{F}_{q}$ de $\boldsymbol{O}(V)$ sont paramétrées par les classes de conjugaison dans $W_{n}$, resp. les classes de $W_{n}$-conjugaison dans $W_{n}^{D}$, resp. les classes de $s_{n}$-conjugaison dans $W_{n}^{D}\left(w, w^{\prime} \in W_{n}^{D}\right.$ sont $s_{n}$-conjugués s'il existe $w^{\prime \prime} \in W_{n}$ tel que $\left.w=w^{\prime \prime} w^{\prime}\left(s_{n} w^{\prime \prime} s_{n}\right)^{-1}\right)$. Dans le dernier cas, par l'application $w \mapsto w s_{n}$, on remplace les classes de $s_{n}$-conjugaison dans $W_{n}^{D}$ par les classes de $W_{n}$-conjugaison dans l'ensemble $\left\{w \in W_{n} ; \operatorname{sgn}_{C D}(w)=-1\right\}$.

1.8. Soit $V$ un espace vectoriel de dimension finie $d$ paire sur $\mathbb{F}_{q}$, muni d'une forme symplectique $Q$, non dégénérée. On introduit les groupes $\boldsymbol{S} \boldsymbol{p}(V), \boldsymbol{C S} \boldsymbol{p}(V)$ et l'homomorphisme $\lambda: C S p(V) \rightarrow \mathbb{F}_{q}^{\times}$.

Soit $u$ un élément unipotent de $S p(V)$. On définit $\underline{\lambda}(u)$ et $\operatorname{Jord}^{\mathrm{bp}}(u)$ comme en 1.6, la partition $\underline{\lambda}(u)$ étant cette fois symplectique. Soit $i \in \operatorname{Jord}^{\mathrm{bp}}(u)$. On décompose encore $V$ en $V_{i} \oplus V_{\neq i}$. L'espace $V_{i} /(u-1)\left(V_{i}\right)$ est muni d'une forme quadratique non dégénérée $q_{i}$ définie par :

$$
q_{i}\left(v, v^{\prime}\right)=(-1)^{i / 2-1} Q\left((u-1)^{i-1}(v), v^{\prime}\right) .
$$

On pose $\eta_{u}(i)=\eta\left(q_{i}\right)$. Cela définit une fonction :

$$
\eta_{u}: \operatorname{Jord}^{\mathrm{bp}}(u) \longrightarrow\{ \pm 1\} .
$$

Les données $\left(\underline{\lambda}(u), \eta_{u}\right)$ caractérisent la classe de conjugaison de $u$ dans $S p(V)$.

Les classes de conjugaison de sous-tores maximaux définis sur $\mathbb{F}_{q}$ de $\boldsymbol{S} \boldsymbol{p}(V)$ sont paramétrées par les classes de conjugaison dans $W_{d / 2}$. 
1.9. Soit $V$ un espace vectoriel sur $\mathbb{F}_{q}$, de dimension finie $d$, muni d'une forme bilinéaire non dégénérée $Q$. On suppose vérifiée l'une des hypothèses :

(i) $d$ est pair, $Q$ est symétrique;

(ii) $d$ est impair, $Q$ est symétrique;

(iii) $d$ est pair, $Q$ est symplectique.

On pose respectivement $\boldsymbol{H}=\boldsymbol{O}(V), \boldsymbol{S} \boldsymbol{O}(V), \boldsymbol{S} \boldsymbol{p}(V)$. On peut fixer un espace $\widehat{V}$ de dimension finie sur $\mathbb{F}_{q}$, muni d'une forme bilinéaire non dégénérée $\widehat{Q}$, de sorte que, respectivement :

(i) $\widehat{V}$ est de dimension $d$, $\widehat{Q}$ est symétrique, $\eta(\widehat{V})=\eta(V), \widehat{\boldsymbol{H}}=\boldsymbol{O}(\widehat{V})$;

(ii) $\widehat{V}$ est de dimension $d-1, \widehat{Q}$ est symplectique, $\widehat{\boldsymbol{H}}=\boldsymbol{S} \boldsymbol{p}(\widehat{V})$;

(iii) $\widehat{V}$ est de dimension $d+1, \widehat{Q}$ est symétrique, $\widehat{\boldsymbol{H}}=\boldsymbol{S} \boldsymbol{O}(\widehat{V})$.

Soient $s, s^{\prime}$ deux éléments semi-simples de $\widehat{H}^{0}$. On dit qu'ils sont quadratiquement équivalents s'il existe une décomposition orthogonale $\widehat{V}=\widehat{V}^{\neq \pm} \oplus \widehat{V}^{ \pm}$et un élément $s^{\prime \prime}$ conjugué à $s^{\prime}$ par un élément de $\widehat{H}$, de sorte que :

- $\widehat{V}^{ \pm}$et $\widehat{V}^{\neq \pm}$sont stables par $s$ et $s^{\prime \prime}$;

- les restrictions de $s$ et $s^{\prime \prime}$ à $\widehat{V}^{\neq \pm}$sont égales ;

- les restrictions de $s$ et $s^{\prime \prime}$ à $\widehat{V}^{ \pm}$n'ont pour valeurs propres que 1 ou -1 .

On pose :

$$
\operatorname{Quad}(H, s)=\bigcup \operatorname{Irr}\left(H, s^{\prime}\right),
$$

où $s^{\prime}$ parcourt les éléments quadratiquement équivalents à $s$. On pose simplement :

$$
\operatorname{Quad}(H)=\operatorname{Quad}(H, 1) .
$$

On appelle représentation « quadratique-unipotente » un élément de $\operatorname{Quad}(H)$.

1.10. Les objets que nous définirons dans la suite sur différents groupes classiques ne sont pas tous invariants par l'action des groupes adjoints. Pour les préciser, on a besoin de réaliser nos groupes concrètement. Pour cela, nous fixerons, pour tout entier $n \geqslant 0$, resp. $n \geqslant 1$, un espace vectoriel $V_{+}(2 n)$, resp. $V_{-}(2 n)$, de dimension $2 n$ sur $\mathbb{F}_{q}$, muni d'une forme quadratique $Q_{+}(2 n)$, resp. $Q_{-}(2 n)$, non dégénérée, de sorte que $\eta\left(Q_{+}(2 n)\right)=1$, resp. $\eta\left(Q_{-}(2 n)\right)=-1$. On note $\boldsymbol{O}_{+}(2 n)$, resp. $\boldsymbol{O}_{-}(2 n)$, le groupe orthogonal de $V_{+}(2 n)$, resp. $V_{-}(2 n)$. On définit de même $\boldsymbol{S O}_{+}(2 n)$ etc. Désormais, pour tout espace $V$ de dimension $2 n$, muni d'une forme quadratique non dégénérée $Q$, on identifiera $\boldsymbol{O}(V)$ à $\boldsymbol{O}_{\eta(V)}(2 n)$ via le choix d'un isomorphisme de $(V, Q) \operatorname{sur}\left(V_{\eta(V)}(2 n), Q_{\eta(V)}(2 n)\right)$ (ici comme dans la suite, on identifie $\eta(V) \in\{ \pm 1\}$ à un signe \pm ).

Pour $\eta= \pm$, le groupe $\boldsymbol{O}_{\eta}(2 n)$ est isomorphe à son groupe dual, mais il peut être utile de les distinguer. Pour cela, on pose $\widehat{V}_{\eta}(2 n)=V_{\eta}(2 n), \widehat{\boldsymbol{O}}_{\eta}(2 n)=\boldsymbol{O}\left(\widehat{V}_{\eta}(2 n)\right)$ quand ce groupe intervient en tant que groupe dual.

De même, pour tout entier $n \geqslant 0$, on fixe un espace vectoriel $V(2 n)$ de dimension $2 n$ sur $\mathbb{F}_{q}$, muni d'une forme symplectique $Q(2 n)$. On pose $\boldsymbol{S} \boldsymbol{p}(2 n)=\boldsymbol{S} \boldsymbol{p}(V(2 n))$ et on adopte des conventions similaires à ci-dessus. 
Les groupes spéciaux orthogonaux impairs étant adjoints, on n'a pas vraiment besoin de telles subtilités dans leur cas. Il est néanmoins commode de fixer, pour tout entier $n \geqslant 0$, un espace vectoriel $V(2 n+1)$ de dimension $2 n+1$ sur $\mathbb{F}_{q}$, muni d'une forme quadratique $Q(2 n+1)$, non dégénérée. On pose $\boldsymbol{S} \boldsymbol{O}(2 n+1)=\boldsymbol{S} \boldsymbol{O}(V(2 n+1))$. Dans ce cas, pour tout espace $V$ de dimension $2 n+1$, muni d'une forme quadratique non dégénérée $Q$, on identifiera $\boldsymbol{S} \boldsymbol{O}(V)$ à $\boldsymbol{S} \boldsymbol{O}(2 n+1)$ via le choix d'une similitude de $(V, Q) \operatorname{sur}(V(2 n+1), Q(2 n+1))$.

Ces deux derniers cas sont duaux l'un de l'autre : on pose $\widehat{V}(2 n)=V(2 n+1)$, $\widehat{\boldsymbol{S p}}(2 n)=\boldsymbol{S O}(\widehat{V}(2 n))=\boldsymbol{S O}(2 n+1)$ etc. 



\section{CHAPITRE 2}

\section{SYMBOLES}

On introduit des ensembles de symboles. Ils sont étroitement liés aux représentations de groupes de Weyl. Dans certains espaces de symboles, on définit l'involution $\mathcal{F}$ qui intervient dans la conjecture de Lusztig. On introduit d'autres homomorphismes entre espaces de symboles qui permettront d'exprimer de façon combinatoire les foncteurs d'induction de Deligne-lusztig.

La plupart des définitions sont dues à Lusztig. On les a reprises en $[\mathbf{M W}]$ paragraphe 2. Il y a quelques modifications par rapport à $[\mathbf{M W}]$.

2.1. Pour tout ensemble fini (resp. pour toute famille finie) $X$ de nombres complexes, on note $S(X)$ la somme des éléments (resp. des termes) de $X$. Soit $\Lambda$ un sous-ensemble fini de $\mathbb{N} \times\{ \pm 1\}$. On pose :

$$
\begin{aligned}
& -\operatorname{pour} \varepsilon \in\{ \pm 1\}, \Lambda^{\varepsilon}=\{x \in \mathbb{N} ;(x, \varepsilon) \in \Lambda\} ; \\
& -\operatorname{rg}(\Lambda)=S\left(\Lambda^{+}\right)+S\left(\Lambda^{-}\right)-\left[\left(\frac{|\Lambda|-1}{2}\right)^{2}\right] ; \\
& -\widetilde{\operatorname{def}}(\Lambda)=\left|\Lambda^{+}\right|-\left|\Lambda^{-}\right| .
\end{aligned}
$$

On a l'inégalité :

$$
\operatorname{rg}(\Lambda) \geqslant\left[\left(\frac{\widetilde{\operatorname{def}}(\Lambda)}{2}\right)^{2}\right]
$$

On définit une relation d'équivalence entre sous-ensembles finis de $\mathbb{N} \times\{ \pm 1\}$, engendrée par $\Lambda \sim \Lambda^{\prime}$, où $\Lambda^{\prime}=\{(x+1, \varepsilon) ;(x, \varepsilon) \in \Lambda\} \cup\{(0,1),(0,-1)\}$. On appelle symbole ordonné une classe d'équivalence (en pratique, on choisira des représentants dans les classes d'équivalence). Remarquons que les fonctions $\mathrm{rg}$ (rang) et $\widetilde{\text { def }}$ (défaut) sont constantes sur toute classe d'équivalence. Pour $n \in \mathbb{N}$ et $D \in \mathbb{Z}$, on note $\widetilde{\mathcal{S}}_{n, D}$ l'ensemble des symboles ordonnés de rang $n$ et de défaut $D$.

Pour $\Lambda \in \widetilde{\mathcal{S}}_{n, D}$, on pose $\sigma \Lambda=\{(x,-\varepsilon) ;(x, \varepsilon) \in \Lambda\}$. On a $\sigma \Lambda \in \widetilde{\mathcal{S}}_{n,-D}$. Pour $D \in \mathbb{N}$, on note $\mathcal{S}_{n, D}$ le quotient de $\widetilde{\mathcal{S}}_{n, D} \cup \widetilde{\mathcal{S}}_{n,-D}$ par la relation d'équivalence $\Lambda \sim \sigma \Lambda$. On pose :

$$
\mathcal{S}_{n, \text { imp }}=\bigcup_{\substack{D \in \mathbb{N} \\ D \text { impair }}} \mathcal{S}_{n, D}, \quad \widetilde{\mathcal{S}}_{n, \text { pair }}=\bigcup_{\substack{D \in \mathbb{Z} \\ D \text { pair }}} \widetilde{\mathcal{S}}_{n, D} .
$$


On munit $\mathbb{C}\left[\mathcal{S}_{n, \text { imp }}\right]$, resp. $\mathbb{C}\left[\widetilde{\mathcal{S}}_{n, \text { pair }}\right]$, du produit hermitien pour lequel $\mathcal{S}_{n, \text { imp }}$, resp. $\widetilde{\mathcal{S}}_{n, \text { pair }}$, est une base orthonormée.

Soit $\Lambda \in \widetilde{\mathcal{S}}_{n \text {,pair. }}$ On dit que $\Lambda$ est spécial si $\widetilde{\operatorname{def}}(\Lambda)=0$ et si l'on peut écrire $\Lambda^{+}=\left\{x_{1}, \ldots, x_{r}\right\}, \Lambda^{-}=\left\{y_{1}, \ldots, y_{r}\right\}$, avec $x_{1} \geqslant y_{1} \geqslant x_{2} \geqslant \cdots \geqslant x_{r} \geqslant y_{r}$. Soient $\Lambda, \Lambda^{\prime} \in \widetilde{\mathcal{S}}_{n \text {,pair. }}$. On dit qu'ils appartiennent à la même famille si, quitte à les remplacer par des symboles équivalents, on a :

$$
\Lambda^{+} \cup \Lambda^{-}=\Lambda^{\prime+} \cup \Lambda^{\prime-}, \Lambda^{+} \cap \Lambda^{-}=\Lambda^{\prime+} \cap \Lambda^{\prime-} .
$$

Toute famille contient un unique symbole spécial. Soit $\widetilde{\text { Fam }}$ une famille, notons $\Lambda^{\text {sp }}$ son symbole spécial. Pour $\Lambda, \Lambda^{\prime} \in \widetilde{\text { Fam, }}$ posons :

$$
\begin{aligned}
\left\langle\Lambda, \Lambda^{\prime}\right\rangle=\left(\left|\Lambda^{+}\right|+\left|\Lambda^{\mathrm{sp},+}\right|\right)\left(\left|\Lambda^{\prime+}\right|\right. & \left.+\left|\Lambda^{\mathrm{sp},+}\right|\right) \\
& +\left|\Lambda^{+} \cap \Lambda^{\prime+} \cap \Lambda^{\mathrm{sp},-}\right|+\left|\Lambda^{-} \cap \Lambda^{\prime-} \cap \Lambda^{\mathrm{sp},+}\right| \bmod 2 \mathbb{Z} .
\end{aligned}
$$

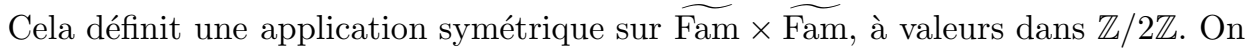
pose :

$$
\mathcal{F}(\Lambda)=|\widetilde{\mathrm{Fam}}|^{-1 / 2} \sum_{\Lambda^{\prime} \in \widetilde{\mathrm{Fam}}}(-1)^{\left\langle\Lambda, \Lambda^{\prime}\right\rangle} \Lambda^{\prime} .
$$

C'est un élément de $\mathbb{Q}\left[\widetilde{\mathcal{S}}_{n \text {,pair }}\right]$. Par linéarité, on définit ainsi un endomorphisme $\mathcal{F}$ de $\mathbb{Q}\left[\widetilde{\mathcal{S}}_{n, \text { pair }}\right]$. C'est une involution isométrique. Notons $(-1)^{\widetilde{\text { def }} / 2}$ l'involution isométrique de $\mathbb{Q}\left[\widetilde{\mathcal{S}}_{n, \text { pair }}\right]$ qui à $\Lambda$ associe $(-1)^{\widetilde{d e f}(\Lambda) / 2} \Lambda$. On vérifie les égalités :

$$
\mathcal{F} \circ \sigma=(-1)^{\widetilde{\operatorname{def}} / 2} \circ \mathcal{F}, \quad \sigma \circ \mathcal{F}=\mathcal{F} \circ(-1)^{\widetilde{\operatorname{def}} / 2} .
$$

Soit $\Lambda \in \mathcal{S}_{n, \text { imp }}$. On dit que $\Lambda$ est spécial si $|\widetilde{\operatorname{def}}(\Lambda)|=1$ et si, quitte à changer de représentant $\Lambda$, on peut écrire $\Lambda^{+}=\left\{x_{1}, \ldots, x_{r+1}\right\}, \Lambda^{-}=\left\{y_{1}, \ldots, y_{r}\right\}$, avec $x_{1} \geqslant y_{1} \geqslant x_{2} \geqslant \cdots \geqslant y_{r} \geqslant x_{r+1}$. On définit des familles dans $\mathcal{S}_{n, \text { imp }}$ comme ci-dessus et, pour une famille Fam $\subseteq \mathcal{S}_{n, \text { imp }}$, une application symétrique $\langle\bullet, \bullet\rangle$ sur Fam $\times$ Fam, à valeurs dans $\mathbb{Z} / 2 \mathbb{Z}$, par la même formule que ci-dessus. De même, on définit ensuite une involution isométrique $\mathcal{F}$ de $\mathbb{Q}\left[\mathcal{S}_{n, \text { imp }}\right]$.

2.2. Soit $n \in \mathbb{N}$. On sait que les représentations irréductibles de $W_{n}$ sont paramétrées par les couples de partitions $(\underline{\alpha}, \underline{\beta})$ tels que $S(\underline{\alpha})+S(\beta)=n$ (la représentation triviale est paramétrée par $((n), \varnothing))$. Soient $h \in \mathbb{Z}$ tel que $h^{\overline{2}} \leqslant n$ et $\rho \in \operatorname{Irr}\left(W_{n-h^{2}}\right)$. Notons $(\underline{\alpha}, \underline{\beta})$ le couple qui paramètre $\rho$. Quitte à ajouter à $\underline{\alpha}$ et $\underline{\beta}$ des termes nuls, écrivons :

$$
\underline{\alpha}=\left(\alpha_{1} \geqslant \cdots \geqslant \alpha_{a+2|h|}\right), \quad \underline{\beta}=\left(\beta_{1} \geqslant \cdots \geqslant \beta_{a}\right) .
$$

Posons :

$$
\nu(h)= \begin{cases}1, & \text { si } h \geqslant 0 \\ -1, & \text { si } h<0\end{cases}
$$


et :

$$
\begin{aligned}
\Lambda^{\nu(h)} & =\left\{\alpha_{1}+a+2|h|-1, \alpha_{2}+a+2|h|-2, \ldots, \alpha_{a+2|h|}\right\}, \\
\Lambda^{-\nu(h)} & =\left\{\beta_{1}+a-1, \ldots, \beta_{a}\right\}, \\
\Lambda & =\left(\Lambda^{+} \times\{1\}\right) \cup\left(\Lambda^{-} \times\{-1\}\right) .
\end{aligned}
$$

Alors $\Lambda \in \widetilde{\mathcal{S}}_{n, 2 h}$. L'application qui à $(h, \rho)$ associe $\Lambda$ est une bijection de l'ensemble des couples $(h, \rho)$ vérifiant les conditions ci-dessus sur $\widetilde{\mathcal{S}}_{n \text {,pair }}$.

Soient $h \in \mathbb{N}$ tel que $h^{2}+h \leqslant n$ et $\rho \in \operatorname{Irr}\left(W_{n-h^{2}-h}\right)$. Notons $(\underline{\alpha}, \underline{\beta})$ le couple qui paramètre $\rho$. Écrivons :

$$
\underline{\alpha}=\left(\alpha_{1} \geqslant \cdots \geqslant \alpha_{a+2 h+1}\right), \quad \underline{\beta}=\left(\beta_{1} \geqslant \cdots \geqslant \beta_{a}\right) .
$$

Posons :

$$
\begin{aligned}
\Lambda^{+} & =\left\{\alpha_{1}+a+2 h, \alpha_{2}+a+2 h-1, \ldots, \alpha_{a+2 h+1}\right\}, \\
\Lambda^{-} & =\left\{\beta_{1}+a-1, \ldots, \beta_{a}\right\}, \\
\Lambda & =\left(\Lambda^{+} \times\{1\}\right) \cup\left(\Lambda^{-} \times\{-1\}\right) .
\end{aligned}
$$

Alors $\Lambda \in \mathcal{S}_{n, 2 h+1}$. L'application qui à $(h, \rho)$ associe $\Lambda$ est une bijection de l'ensemble des couples $(h, \rho)$ vérifiant les conditions ci-dessus sur $\mathcal{S}_{n, \text { imp }}$.

2.3. On rappelle ici des résultats d'Asai ([A] ). Soient $n \in \mathbb{N}, a \in \mathbb{Z} \backslash\{0\}, \theta \in\{ \pm 1\}$, $\delta \in\{0,1\}$. On suppose $n+a \geqslant 0$. Notons $\widetilde{\mathcal{S}}_{n}$ l'ensemble de tous les symboles ordonnés de rang $n$. Pour $\Lambda \in \widetilde{\mathcal{S}}_{n}$, que l'on suppose représenté par un ensemble ayant un assez grand nombre d'éléments, posons :

$$
E_{a, \theta}(\Lambda)=\{(x, \varepsilon) \in \Lambda ; x+a \geqslant 0,(x+a, \theta \varepsilon) \notin \Lambda\} .
$$

Pour $(x, \varepsilon) \in E_{a, \theta}(\Lambda)$, posons :

$$
\begin{aligned}
\Lambda_{a, \theta}(x, \varepsilon) & =(\Lambda \backslash\{(x, \varepsilon)\}) \cup\{(x+a, \theta \varepsilon)\}, \\
\zeta_{a, \theta, \Lambda}(x, \varepsilon) & =\theta(-1)^{\left|\{x, \ldots, 0\} \cap \Lambda^{\varepsilon}\right|+\left|\{x+a, \ldots, 0\} \cap \Lambda_{a, \theta}(x, \varepsilon)^{\theta \varepsilon}\right|} .
\end{aligned}
$$

On définit :

$$
\mathcal{I}_{a, \theta, \delta}(\Lambda)=\sum_{(x, \varepsilon) \in E_{a, \theta}(\Lambda)} \varepsilon^{\delta} \zeta_{a, \theta, \Lambda}(x, \varepsilon) \Lambda_{a, \theta}(x, \varepsilon) .
$$

Par prolongement linéaire, on obtient un homomorphisme :

$$
\mathcal{I}_{a, \theta, \delta}: \mathbb{Z}\left[\widetilde{\mathcal{S}}_{n}\right] \longrightarrow \mathbb{Z}\left[\widetilde{\mathcal{S}}_{n+a}\right] .
$$

Remarquons que si $\Lambda$ est de défaut $D, \mathcal{I}_{a, \theta, \delta}(\Lambda)$ est combinaison linéaire de symboles ordonnés de défaut $\equiv D \bmod 2 \mathbb{Z}$. On vérifie de plus l'égalité :

$$
\mathcal{I}_{a, \theta, \delta} \circ \sigma=(-1)^{\delta} \sigma \circ \mathcal{I}_{a, \theta, \delta} .
$$

On en déduit :

- $\mathcal{I}_{a, \theta, \delta}$ se restreint en un homomorphisme de $\mathbb{Z}\left[\widetilde{\mathcal{S}}_{n, \text { pair }}\right]$ dans $\mathbb{Z}\left[\widetilde{\mathcal{S}}_{n+a, \text { pair }}\right]$; 
- l'application $\Lambda \mapsto(-1)^{\delta(\widetilde{\operatorname{def}}(\Lambda)-1) / 2} \mathcal{I}_{a, \theta, \delta}(\Lambda)$ se descend en un homomorphisme de $\mathbb{Z}\left[\mathcal{S}_{n, \text { imp }}\right]$ dans $\mathbb{Z}\left[\mathcal{S}_{n+a, \text { imp }}\right]$ (notons que $\mathbb{Z}\left[\mathcal{S}_{n, \text { imp }}\right]$ est naturellement un quotient de $\left.\mathbb{Z}\left[\widetilde{\mathcal{S}}_{n}\right]\right)$.

On définit des homomorphismes :

$$
I_{a}, I_{a}^{-}, J_{a}: \mathbb{Z}\left[\widetilde{\mathcal{S}}_{n, \text { pair }}\right] \longrightarrow \mathbb{Z}\left[\widetilde{\mathcal{S}}_{n+a, \text { pair }}\right]
$$

ainsi : $I_{a}$ est la restriction à $\mathbb{Z}\left[\widetilde{\mathcal{S}}_{n, \text { pair }}\right]$ de $\mathcal{I}_{a, 1,0}, I_{a}^{-}$, resp. $J_{a}$, est la restriction de $\mathcal{I}_{a, 1,1}$, resp. $\mathcal{I}_{a,-1,0}$. On a les égalités :

$$
I_{a} \circ \sigma=\sigma \circ I_{a}, \quad I_{a}^{-} \circ \sigma=-\sigma \circ I_{a}^{-}, \quad J_{a} \circ \sigma=\sigma \circ J_{a} .
$$

Prolongeons ces applications par linéarité en des applications linéaires de $\mathbb{Q}\left[\widetilde{\mathcal{S}}_{n, \text { pair }}\right]$ dans $\mathbb{Q}\left[\widetilde{\mathcal{S}}_{n+a, \text { pair }}\right]$. Un calcul fastidieux mais élémentaire montre que :

$$
I_{a} \circ \mathcal{F}=\mathcal{F} \circ I_{a}, \quad I_{a}^{-} \circ \mathcal{F}=\mathcal{F} \circ \sigma \circ J_{a}, \quad \sigma \circ J_{a} \circ \mathcal{F}=\mathcal{F} \circ I_{a}^{-} .
$$

Enfin, les espaces de départ et d'arrivée étant munis de produits scalaires, à chacune des applications $I_{a}, I_{a}^{-}, J_{a}$ est associée une application adjointe, qui n'est autre que, respectivement, $I_{-a}, I_{-a}^{-}, J_{-a}$, cette application étant relative à l'entier $n+a$.

On définit des homomorphismes :

$$
I_{a}, I_{a}^{-}, J_{a}: \mathbb{Z}\left[\mathcal{S}_{n, \mathrm{imp}}\right] \longrightarrow \mathbb{Z}\left[\mathcal{S}_{n+a, \mathrm{imp}}\right]
$$

ainsi : $I_{a}$ est l'application quotient de $\mathcal{I}_{a, 1,0}, J_{a}$ est celle de $\mathcal{I}_{a,-1,0}$ et $I_{a}^{-}$est celle de l'application $\Lambda \mapsto(-1)^{(\widetilde{\operatorname{def}}(\Lambda)-1) / 2} \mathcal{I}_{a, 1,1}(\Lambda)$. En prolongeant encore ces applications par linéarité, on vérifie les égalités :

$$
I_{a} \circ \mathcal{F}=\mathcal{F} \circ I_{a}, \quad I_{a}^{-} \circ \mathcal{F}=-\mathcal{F} \circ J_{a}, \quad J_{a} \circ \mathcal{F}=-\mathcal{F} \circ I_{a}^{-},
$$

ainsi que les propriétés d'adjonction ci-dessus.

On a noté de la même façon les applications dans les cas pairs et impairs. On espère que cela ne crée pas de confusion.

2.4. Pour $h \in \mathbb{Z}$, on définit le symbole ordonné :

$$
\Lambda_{\text {cusp }}(h)= \begin{cases}\{2 h-1, \ldots, 0\} \times\{1\}, & \text { si } h \geqslant 0, \\ \{2|h|-1, \ldots, 0\} \times\{-1\}, & \text { si } h<0 .\end{cases}
$$

On a les égalités :

$$
\widetilde{\operatorname{def}}\left(\Lambda_{\text {cusp }}(h)\right)=2 h, \quad \operatorname{rg}\left(\Lambda_{\text {cusp }}(h)\right)=h^{2} .
$$

On dit qu'un symbole ordonné est cuspidal s'il est de la forme $\Lambda_{\text {cusp }}(h)$ pour un $h \in \mathbb{Z}$.

Pour tout entier $n \geqslant 0$, resp. $n \geqslant 1$, on définit les symboles ordonnés de rang $n$ :

$$
\mathbf{1}_{+}(n)=\{(n, 1),(0,-1)\}, \quad \operatorname{resp} \mathbf{1}_{-}(n)=\{(n, 1),(0,1)\} .
$$

On notera souvent $\Lambda(\varnothing)=\Lambda_{\text {cusp }}(0)=\mathbf{1}_{+}(0)$. C'est l'unique symbole ordonné de rang 0 . 
2.5. Soient $n, a \in \mathbb{N}$. Définissons les éléments $w_{a}^{+}, w_{a}^{-} \in W_{a}$ par :

$$
\begin{gathered}
w_{a}^{+}(i)=w_{a}^{-}(i)=i+1, \text { pour } i \in\{1, \ldots, a-1\}, \\
w_{a}^{+}(a)=1, w_{a}^{-}(a)=-1 .
\end{gathered}
$$

À la décomposition :

$$
\{ \pm 1, \ldots, \pm(n+a)\}=\{ \pm 1, \ldots, \pm n\} \cup\{ \pm(n+1), \ldots, \pm(n+a)\},
$$

est associé un homomorphisme injectif :

$$
\begin{array}{r}
W_{n} \times W_{a} \longrightarrow W_{n+a} \\
\left(w^{\prime}, w^{\prime \prime}\right) \longmapsto w^{\prime} w^{\prime \prime} .
\end{array}
$$

Pour $\varepsilon \in\{ \pm 1\}$, on définit un homomorphisme :

$$
\operatorname{res}_{a}^{\varepsilon}: \mathbb{Q}\left[\operatorname{Irr}\left(W_{n+a}\right)\right] \longrightarrow \mathbb{Q}\left[\operatorname{Irr}\left(W_{n}\right)\right],
$$

par la formule $\operatorname{res}_{a}^{\varepsilon}(f)\left(w^{\prime}\right)=f\left(w^{\prime} w_{a}^{\varepsilon}\right)$ pour tout $f \in \mathbb{Q}\left[\operatorname{Irr}\left(W_{n+a}\right)\right]$ et tout $w^{\prime} \in W_{n}$. Grâce à 2.2, on a des isomorphismes :

$$
\begin{aligned}
\mathbb{Q}\left[\widetilde{\mathcal{S}}_{n, \text { pair }}\right] & =\oplus_{h} \mathbb{Q}\left[\operatorname{Irr}\left(W_{n-h^{2}}\right)\right], \\
\mathbb{Q}\left[\widetilde{\mathcal{S}}_{n+a, \text { pair }}\right] & =\oplus_{h} \mathbb{Q}\left[\operatorname{Irr}\left(W_{n+a-h^{2}}\right)\right],
\end{aligned}
$$

où $h$ décrit l'ensemble des entiers relatifs tels que, respectivement, $h^{2} \leqslant n, h^{2} \leqslant n+a$. On définit une application linéaire :

$$
\operatorname{Res}_{a}^{\varepsilon}: \mathbb{Q}\left[\widetilde{\mathcal{S}}_{n+a, \text { pair }}\right] \longrightarrow \mathbb{Q}\left[\widetilde{\mathcal{S}}_{n, \text { pair }}\right]
$$

de la façon suivante. Pour $h^{2}>n$, elle annule $\mathbb{Q}\left[\operatorname{Irr}\left(W_{n+a-h^{2}}\right)\right]$. Pour $h^{2} \leqslant n$, elle envoie le sommand indicé par $h$ de $\mathbb{Q}\left[\widetilde{\mathcal{S}}_{n+a}\right.$,pair $]$ dans le sommand indicé par $h$ de $\mathbb{Q}\left[\widetilde{\mathcal{S}}_{n, \text { pair }}\right]$ et y coïncide avec l'application $\operatorname{res}_{a}^{\varepsilon}$ si $h \geqslant 0, \varepsilon \operatorname{res}_{a}^{\varepsilon}$ si $h<0$.

LEMME. - On a les égalités $I_{-a}=\operatorname{Res}_{a}^{+}, I_{-a}^{-}=\operatorname{Res}_{a}^{-}$.

$C f$. [A] lemme 2.8.2. Le lemme résulte de la définition des paramétrages des représentations irréductibles des groupes $W_{m}$ et de la formule d'induction de Murnaghan ([Z], proposition 4.17).

Un lemme similaire vaut dans le cas des symboles de défaut impair.

2.6. Pour $n, a, b \in \mathbb{N}, a, b \geqslant 1$, considérons les homomorphismes :

$$
J_{a} \circ J_{b}, J_{b} \circ J_{a}, I_{a} \circ J_{b}, J_{b} \circ I_{a}: \mathbb{Q}\left[\widetilde{\mathcal{S}}_{n, \text { pair }}\right] \longrightarrow \mathbb{Q}\left[\widetilde{\mathcal{S}}_{n+a+b, \text { pair }}\right] .
$$

LEMME. - On a les égalités $J_{a} \circ J_{b}=J_{b} \circ J_{a}, I_{a} \circ J_{b}=J_{b} \circ I_{a}$.

Démonstration. - On peut le vérifier directement sur les formules de définition. Donnons une autre preuve. Par conjugaison avec l'opérateur $\mathcal{F}$, la première égalité est équivalente à $I_{a}^{-} \circ I_{b}^{-}=I_{b}^{-} \circ I_{a}^{-}$. Par adjonction, elle est encore équivalente à l'égalité $I_{-b}^{-} \circ I_{-a}^{-}=I_{-a}^{-} \circ I_{-b}^{-}$. D'après le lemme 2.5, cette égalité résulte du fait évident que pour tout entier $m \geqslant 0$, les deux applications $\operatorname{res}_{b}^{-} \circ \operatorname{res}_{a}^{-}$et $\operatorname{res}_{a}^{-} \circ \operatorname{res}_{b}^{-}$, définies sur 
$\mathbb{Q}\left[\operatorname{Irr}\left(W_{m+a+b}\right)\right]$, sont égales. La deuxième égalité de l'énoncé se démontre de la même façon.

2.7. Soit $n \in \mathbb{N}$. Notons $\mathbb{Q}\left[\widetilde{\mathcal{S}}_{n, \text { pair }}\right]_{I J}$ le sous-espace de $\mathbb{Q}\left[\widetilde{\mathcal{S}}_{n, \text { pair }}\right]$ somme des images de tous les opérateurs $I_{a}$ ou $J_{a}$ pour $a \in\{1, \ldots, n\}$.

LEMME

(i) Si n n'est pas un carré, $\mathbb{Q}\left[\widetilde{\mathcal{S}}_{n, \text { pair }}\right]_{I J}=\mathbb{Q}\left[\widetilde{\mathcal{S}}_{n, \text { pair }}\right]$.

(ii) Si $n=h^{2}$, avec $h \in \mathbb{Z}, \mathbb{Q}\left[\widetilde{\mathcal{S}}_{n, \text { pair }}\right]_{\text {IJ }}$ est l'orthogonal du sous-espace de $\mathbb{Q}\left[\widetilde{\mathcal{S}}_{n, \text { pair }}\right]$ engendré par les éléments $\mathcal{F} \Lambda_{\text {cusp }}( \pm h)$.

Démonstration. - Soit $m$ un entier $\geqslant 1$. Tout élément de $W_{m}$ est conjugué à un produit, en un sens évident, de cycles de la forme $w_{a}^{ \pm}$. Il en résulte que l'intersection dans $\mathbb{Q}\left[\operatorname{Irr}\left(W_{m}\right)\right]$ des noyaux de tous les opérateurs res $\operatorname{raur}_{a}^{ \pm}$, pour $\in\{1, \ldots, m\}$, est nulle. Notons $E$ l'orthogonal dans $\mathbb{Q}\left[\widetilde{\mathcal{S}}_{n, \text { pair }}\right]$ de $\mathbb{Q}\left[\widetilde{\mathcal{S}}_{n, \text { pair }}\right]_{I J}$. C'est le noyau de tous les opérateurs $I_{-a}$ ou $J_{-a}$ pour $a \in\{1, \ldots, n\}$. L'espace $\mathcal{F} E$ est l'intersection des noyaux de tous les opérateurs $I_{-a}^{ \pm}$pour ces mêmes $a$. Grâce au lemme 2.5 et à ce que l'on a dit ci-dessus, $\mathcal{F} E$ s'identifie par l'isomorphisme 2.5(1) à la somme des termes du type $\mathbb{Q}\left[\operatorname{Irr}\left(W_{0}\right)\right]$. Il n'y en a pas si $n$ n'est pas un carré. Si $n=h^{2}$, ces termes s'identifient aux droites portées par $\Lambda_{\text {cusp }}( \pm h)$. D'où le lemme. 


\section{CHAPITRE 3}

\section{FAISCEAUX-CARACTÈRES}

On définit des faisceaux-caractères sur les groupes classiques, en particulier sur le groupe orthogonal pair qui n'est pas connexe. On classifie certains de ces faisceauxcaractères par des symboles. On montrera au paragraphe 6 que les fonctions-traces associées à ces faisceaux engendrent le même espace que les traces de représentations irréductibles quadratiques-unipotentes.

3.1. Soient $n$ un entier $>0, \eta \in\{ \pm 1\}, r^{\prime}, r^{\prime \prime}$ deux éléments de $\mathbb{Z}$ tels que $r^{\prime 2}+r^{\prime \prime 2}=2 n$ ( $r^{\prime}$ et $r^{\prime \prime}$ sont donc de même parité). Posons $\boldsymbol{G}=\boldsymbol{O}_{\eta}(2 n)$. Fixons un élément $x \in G$, soumis aux conditions ci-dessous. Décomposons $x=s u$ en produit d'un élément semisimple $s$ et d'un unipotent $u$. On demande :

$s$ a $r^{\prime 2}$ valeurs propres 1 et $r^{\prime \prime 2}$ valeurs propres -1 .

Alors $\boldsymbol{Z}_{\boldsymbol{G}^{0}}(s)^{0}=\boldsymbol{S O}_{\eta^{\prime}}\left(r^{2}\right) \times \boldsymbol{S} \boldsymbol{O}_{\eta^{\prime \prime}}\left(r^{\prime \prime 2}\right)$, pour des $\eta^{\prime}, \eta^{\prime \prime} \in\{ \pm 1\}$ convenables. L'élément $u$ se décompose conformément en $u=u^{\prime} u^{\prime \prime}$. On demande :

$\underline{\lambda}\left(u^{\prime}\right)=\left(2\left|r^{\prime}\right|-1,2\left|r^{\prime}\right|-3, \ldots, 3,1\right), \underline{\lambda}\left(u^{\prime \prime}\right)=\left(2\left|r^{\prime \prime}\right|-1,2\left|r^{\prime \prime}\right|-3, \ldots, 3,1\right)$,

cf. 1.6. Posons $I^{\prime}=\operatorname{Jord}^{\mathrm{bp}}\left(u^{\prime}\right), I^{\prime \prime}=\operatorname{Jord}^{\mathrm{bp}}\left(u^{\prime \prime}\right)$. Il y a un isomorphisme naturel :

$$
\boldsymbol{Z}_{\boldsymbol{G}^{0}}(x) / \boldsymbol{Z}_{\boldsymbol{G}^{0}}(x)^{0} \simeq\left\{\left(\left(z_{i}^{\prime}\right)_{i \in I^{\prime}},\left(z_{i}^{\prime \prime}\right)_{i \in I^{\prime \prime}}\right) \in\{ \pm 1\}^{I^{\prime}} \times\{ \pm 1\}^{I^{\prime \prime}} ; \prod_{i \in I^{\prime}} z_{i}^{\prime}=\prod_{i \in I^{\prime \prime}} z_{i}^{\prime \prime}\right\}
$$

Le groupe des caractères de ce groupe s'identifie à $\left((\mathbb{Z} / 2 \mathbb{Z})^{I^{\prime}} \times(\mathbb{Z} / 2 \mathbb{Z})^{I^{\prime \prime}}\right) / \Delta(\mathbb{Z} / 2 \mathbb{Z})$, $\Delta$ étant le plongement diagonal. Notons $\varepsilon^{\prime}=\left(\varepsilon_{i}^{\prime}\right)_{i \in I^{\prime}}$ l'élément de $(\mathbb{Z} / 2 \mathbb{Z})^{I^{\prime}}$ tel que :

$$
\varepsilon_{i}^{\prime} \equiv\left\{\begin{array}{ll}
(i+1) / 2 \bmod 2 \mathbb{Z}, & \text { si } r^{\prime} \geqslant 0, \\
(i-1) / 2 \bmod 2 \mathbb{Z}, & \text { si } r^{\prime}<0,
\end{array} \quad \text { pour tout } i \in I^{\prime}\right.
$$

Définissons de même $\varepsilon^{\prime \prime} \in(\mathbb{Z} / 2 \mathbb{Z})^{I^{\prime \prime}}$. Le couple $\varepsilon=\left(\varepsilon^{\prime}, \varepsilon^{\prime \prime}\right)$ définit un caractère du groupe $\boldsymbol{Z}_{\boldsymbol{G}^{0}}(x) / \boldsymbol{Z}_{\boldsymbol{G}^{0}}(x)^{0}$. Notons $\boldsymbol{C}$ la classe de conjugaison de $x$ (par $\boldsymbol{G}$ ou $\boldsymbol{G}^{0}$, c'est pareil). un nombre premier $\ell \neq p$ étant fixé, on construit un système local $\ell$-adique 
$\mathcal{L}\left(r^{\prime}, r^{\prime \prime}\right)$ sur $\boldsymbol{C}$, quotient du fibré :

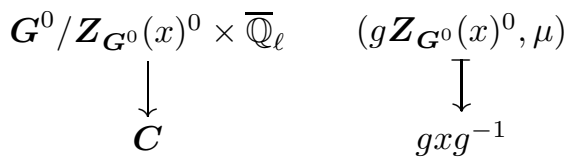

par l'action de $\boldsymbol{Z}_{\boldsymbol{G}^{0}}(x) / \boldsymbol{Z}_{\boldsymbol{G}^{0}}(x)^{0}$ agissant naturellement à droite sur $\boldsymbol{G}^{0} / \boldsymbol{Z}_{\boldsymbol{G}^{0}}(x)^{0}$ et par $\varepsilon$ sur $\overline{\mathbb{Q}}_{\ell}$. On prolonge $\mathcal{L}\left(r^{\prime}, r^{\prime \prime}\right)$ à $\boldsymbol{G}$ tout entier par 0 hors de $\boldsymbol{C}$. Le complexe $\mathcal{L}\left(r^{\prime}, r^{\prime \prime}\right)[\operatorname{dim}(\boldsymbol{C})]$ est un faisceau pervers sur $\boldsymbol{G}, \boldsymbol{G}^{0}$-équivariant, irréductible et cuspidal, au sens de $[\mathbf{L} 4]$.

Si $K$ est un complexe constructible sur $\boldsymbol{G}$ et $y \in \boldsymbol{G}$, on note $K_{y}$ la fibre de $K$ en $y$. C'est un complexe d'espaces vectoriels. Si de plus $K$ est muni d'une action de Frobenius (que l'on notera toujours $F$ ), on définit la fonction trace $(K) \operatorname{sur} G$ par :

$$
\operatorname{trace}(K)(y)=\operatorname{trace}\left(F \mid K_{y}\right)=\sum_{i \in \mathbb{Z}}(-1)^{i} \operatorname{trace}\left(F \mid K_{y}^{i}\right),
$$

pour tout $y \in G$. On munit $\mathcal{L}\left(r^{\prime}, r^{\prime \prime}\right)$ de l'action de Frobenius telle que :

$$
\begin{aligned}
& \operatorname{trace}\left(\mathcal{L}\left(r^{\prime}, r^{\prime \prime}\right)\right)(x) \\
& \quad=\eta^{r^{\prime}} q^{\delta\left(r^{\prime}, r^{\prime \prime}\right) / 2}\left(\prod_{i \in I^{\prime}}\left(\zeta(-1)^{(i+1) / 2} \eta_{u^{\prime}}(i)\right)^{\varepsilon_{i}^{\prime}}\right)\left(\prod_{i \in I^{\prime \prime}}\left(\zeta(-1)^{(i-1) / 2} \eta_{u^{\prime \prime}}(i)\right)^{\varepsilon_{i}^{\prime \prime}}\right),
\end{aligned}
$$

où $\delta\left(r^{\prime}, r^{\prime \prime}\right)=\left(\left|r^{\prime 3}-r^{\prime}\right|+\left|r^{\prime \prime 3}-r^{\prime \prime}\right|\right) / 3$. Cette normalisation ne dépend pas du choix de $x$.

Remarquons que, quand on remplace $\left(r^{\prime}, r^{\prime \prime}\right)$ par $\left(-r^{\prime},-r^{\prime \prime}\right)$, les systèmes locaux $\mathcal{L}\left(r^{\prime}, r^{\prime \prime}\right)$ et $\mathcal{L}\left(-r^{\prime},-r^{\prime \prime}\right)$ sont isomorphes, mais leurs Frobenius sont en général différents.

3.2. Soient $n, n_{c} \in \mathbb{N}, \eta, \eta_{c} \in\{ \pm 1\}$, avec $n \geqslant 1, n_{c} \leqslant n$, posons $\boldsymbol{G}=\boldsymbol{O}_{\eta}(2 n)$, $\boldsymbol{G}_{c}=\boldsymbol{O}_{\eta_{c}}\left(2 n_{c}\right)$. Supposons donné un groupe de Lévi $\boldsymbol{M}$ de $\boldsymbol{G}$, défini sur $\mathbb{F}_{q}$, de la forme $\boldsymbol{M}=\boldsymbol{T} \times \boldsymbol{G}_{c}$, où $\boldsymbol{T}$ est un tore. Soient enfin $s_{T} \in \widehat{T}$ et $r^{\prime}, r^{\prime \prime} \in \mathbb{Z}$ tels que $r^{\prime 2}+r^{\prime \prime 2}=2 n_{c}$. On a construit au paragraphe précédent un système local $\mathcal{L}\left(r^{\prime}, r^{\prime \prime}\right)$ sur une orbite, que nous noterons maintenant $\boldsymbol{C}_{c}$, de $\boldsymbol{G}_{c}$, muni d'un Frobenius.

Remarque. - Ici comme dans la suite, les données relatives à $\boldsymbol{G}_{c}$ disparaissent si $n_{c}=0$.

On construit un système local $\mathcal{L}\left(s_{T}\right)$ sur $\boldsymbol{T}$, quotient du fibré :

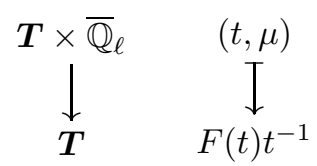

par l'action de $T$ agissant par multiplication sur $\boldsymbol{T}$ et par le caractère attaché à $s_{T}$ sur $\overline{\mathbb{Q}}_{\ell}$. On munit $\mathcal{L}\left(s_{T}\right)$ du Frobenius tel que $\operatorname{trace}\left(\mathcal{L}\left(s_{T}\right)\right)(1)=1$. Par tensorisation, on 
obtient un système local $\mathcal{L}\left(s_{T}\right) \times \mathcal{L}\left(r^{\prime}, r^{\prime \prime}\right)$ sur $\boldsymbol{T} \times \boldsymbol{C}_{c}$. Notons $\boldsymbol{M}_{\text {reg }}$ la sous-variété des $m \in \boldsymbol{M}$ tels que $\boldsymbol{Z}_{\boldsymbol{G}}(m) \subseteq \boldsymbol{M}$ et :

$$
\boldsymbol{Y}=\left\{h m h^{-1} ; m \in \boldsymbol{M}_{\mathrm{reg}} \cap\left(\boldsymbol{T} \times \boldsymbol{C}_{c}\right), h \in \boldsymbol{G}^{0}\right\} .
$$

Considérons le revêtement :

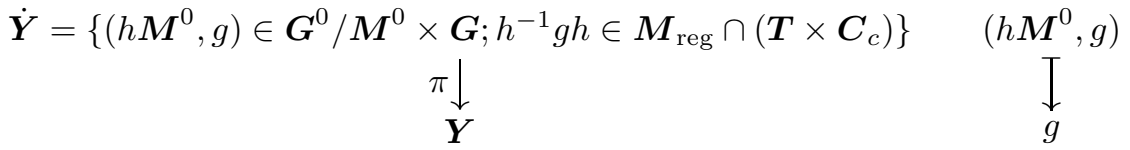

Parce que $\mathcal{L}\left(s_{T}\right) \times \mathcal{L}\left(r^{\prime}, r^{\prime \prime}\right)$ est $\boldsymbol{M}^{0}$-équivariant, de ce système local se déduit un système local sur $\dot{\boldsymbol{Y}}$, noté de la même façon. Son image $\pi_{!}\left(\mathcal{L}\left(s_{T}\right) \times \mathcal{L}\left(r^{\prime}, r^{\prime \prime}\right)\right)$ est un système local sur $\boldsymbol{Y}$, que l'on translate en un complexe $\pi_{!}\left(\mathcal{L}\left(s_{T}\right) \times \mathcal{L}\left(r^{\prime}, r^{\prime \prime}\right)\right)[\operatorname{dim}(\boldsymbol{Y})]$. Le prolongement d'intersection de ce complexe est un faisceau pervers, noté $K\left(\boldsymbol{T} ; s_{T} ; r^{\prime}, r^{\prime \prime}\right)$, porté par l'adhérence de $\boldsymbol{Y}$. Des Frobenius que l'on a fixés sur $\mathcal{L}\left(s_{T}\right)$ et $\mathcal{L}\left(r^{\prime}, r^{\prime \prime}\right)$ se déduit un Frobenius sur $K\left(\boldsymbol{T} ; s_{T} ; r^{\prime}, r^{\prime \prime}\right)$.

La fonction trace $\left(K\left(\boldsymbol{T} ; s_{T} ; r^{\prime}, r^{\prime \prime}\right)\right)$ sur $G$ est invariante par conjugaison par $G^{0}$. Elle l'est même par $G$ s'il existe $g \in G \backslash G^{0}$ tel que $g \boldsymbol{M} g^{-1}=\boldsymbol{M}, \operatorname{Ad}(g)^{*}\left(\mathcal{L}\left(s_{T}\right) \times\right.$ $\left.\mathcal{L}\left(r^{\prime}, r^{\prime \prime}\right)\right)=\mathcal{L}\left(s_{T}\right) \times \mathcal{L}\left(r^{\prime}, r^{\prime \prime}\right)$ et cet isomorphisme respecte les Frobenius. Cette condition est vérifiée si $n_{c} \neq 0$. Fixons un élément $\gamma \in G \backslash G^{0}$. On définit une fonction invariante par $G$ :

$$
k\left(\boldsymbol{T} ; s_{T} ; r^{\prime}, r^{\prime \prime}\right)= \begin{cases}\operatorname{trace}\left(K\left(\boldsymbol{T} ; s_{T} ; r^{\prime}, r^{\prime \prime}\right)\right), & \text { si } n_{c} \neq 0, \\ \operatorname{trace}\left(K\left(\boldsymbol{T} ; s_{T} ; r^{\prime}, r^{\prime \prime}\right)\right) & \\ +\operatorname{trace}\left(K\left(\boldsymbol{T} ; s_{T} ; r^{\prime}, r^{\prime \prime}\right)\right) \circ \operatorname{Ad}(\gamma), & \text { si } n_{c}=0 .\end{cases}
$$

Posons :

$$
\begin{aligned}
W\left(\boldsymbol{T} ; s_{T} ; r^{\prime}, r^{\prime \prime}\right)=\{g \in G ; & g \boldsymbol{M} g^{-1}=\boldsymbol{M}, \\
& \left.\operatorname{Ad}(g)^{*}\left(\mathcal{L}\left(s_{T}\right) \times \mathcal{L}\left(r^{\prime}, r^{\prime \prime}\right)\right)=\mathcal{L}\left(s_{T}\right) \times \mathcal{L}\left(r^{\prime}, r^{\prime \prime}\right)\right\} / M .
\end{aligned}
$$

Alors :

$$
\left(k\left(\boldsymbol{T} ; s_{T} ; r^{\prime}, r^{\prime \prime}\right), k\left(\boldsymbol{T} ; s_{T} ; r^{\prime}, r^{\prime \prime}\right)\right)= \begin{cases}\left|W\left(\boldsymbol{T} ; s_{T} ; r^{\prime}, r^{\prime \prime}\right)\right| / 2, & \text { si } n_{c} \neq 0, \\ \left|W\left(\boldsymbol{T} ; s_{T} ; r^{\prime}, r^{\prime \prime}\right)\right|, & \text { si } n_{c}=0 .\end{cases}
$$

Soient $\widetilde{\boldsymbol{M}}, \widetilde{\boldsymbol{T}}, \widetilde{s}_{\widetilde{T}}, \widetilde{r}^{\prime}, \widetilde{r}^{\prime \prime}$ des données analogues à $\boldsymbol{M}, \boldsymbol{T}, s_{T}, r^{\prime}, r^{\prime \prime}$. Supposons qu'il n'existe pas de $g \in G$ tel que

$$
\left.g \boldsymbol{M} g^{-1}=\widetilde{\boldsymbol{M}} \quad \text { et } \quad \operatorname{Ad}(g)^{*}\left(\mathcal{L}\left(\widetilde{s}_{\widetilde{T}}\right) \times \mathcal{L}\left(\widetilde{r}^{\prime}, \widetilde{r}^{\prime \prime}\right)\right)=\mathcal{L}\left(s_{T}\right) \times \mathcal{L}\left(r^{\prime}, r^{\prime \prime}\right)\right)
$$

(on ne tient pas compte ici des Frobenius). Alors :

$$
\left(k\left(\boldsymbol{T} ; s_{T} ; r^{\prime}, r^{\prime \prime}\right), k\left(\widetilde{\boldsymbol{T}} ; \widetilde{s}_{\widetilde{T}} ; \widetilde{r}^{\prime}, \widetilde{r}^{\prime \prime}\right)\right)=0 .
$$


Ces propriétés sont démontrées dans [L5] corollaire 9.9 dans le cadre des groupes connexes, mais la même démonstration s'applique ici. Le $1 / 2$ de la formule (1) vient de la normalisation du produit scalaire, qui est pour nous relatif au groupe $G$ et non pas $G^{0}$.

Prolongeons $\mathcal{L}\left(s_{T}\right) \times \mathcal{L}\left(r^{\prime}, r^{\prime \prime}\right)$ à $\boldsymbol{M}$ par 0 hors de $\boldsymbol{T} \times \boldsymbol{C}_{c}$. On a la relation :

(3) supposons $q>2 n$; alors $k\left(\boldsymbol{T} ; s_{T} ; r^{\prime}, r^{\prime \prime}\right)=(-1)^{n} R_{M}^{G}\left(\operatorname{trace}\left(\mathcal{L}\left(s_{T}\right) \times \mathcal{L}\left(r^{\prime}, r^{\prime \prime}\right)\right)\right)$.

Cette propriété est démontrée en $[\mathbf{L} 8]$, proposition 9.2, dans le cadre des groupes connexes, pour $q$ assez grand. Un examen attentif de la preuve montre que la même démonstration s'applique ici, et vaut pour $q>2 n$. Le signe intervenant dans la formule de Lusztig est $(-1)^{\operatorname{dim}\left(\boldsymbol{T} \times \boldsymbol{C}_{c}\right)}$. On calcule $\operatorname{dim}\left(\boldsymbol{T} \times \boldsymbol{C}_{c}\right) \equiv n \bmod 2 \mathbb{Z}$.

3.3. Soient $n$ un entier $>0, \eta \in\{ \pm 1\}$, posons $V=V_{\eta}(2 n), \boldsymbol{G}=\boldsymbol{O}_{\eta}(2 n)$. Donnonsnous de plus des entiers $N_{1}, N_{2} \in \mathbb{N}, h_{1}, h_{2} \in \mathbb{Z}$, tels que $n=N_{1}+N_{2}+h_{1}^{2}+h_{2}^{2}$. Posons $n_{c}=h_{1}^{2}+h_{2}^{2}$. Soient $w_{1} \in W_{N_{1}}, w_{2} \in W_{N_{2}}$. Dans le cas où $n_{c}=0$, on impose provisoirement l'égalité $\operatorname{sgn}_{C D}\left(w_{1}\right) \operatorname{sgn}_{C D}\left(w_{2}\right)=\eta$. Fixons une décomposition orthogonale :

$$
V=V_{1} \oplus V_{2} \oplus V_{c}
$$

telle que, pour $j \in\{1,2\}$, on ait $\operatorname{dim}\left(V_{j}\right)=2 N_{j}, \eta\left(V_{j}\right)=\operatorname{sgn}_{C D}\left(w_{j}\right)$. Pour un tel $j$, fixons un sous-tore maximal $\boldsymbol{T}_{w_{j}} \subseteq \boldsymbol{O}\left(V_{j}\right)$, défini sur $\mathbb{F}_{q}$ et paramétré par $w_{j}(c f .1 .7)$. Posons $\eta_{c}=\eta\left(V_{c}\right)=\operatorname{sgn}_{C D}\left(w_{1}\right) \operatorname{sgn}_{C D}\left(w_{2}\right) \eta, \boldsymbol{G}_{c}=\boldsymbol{O}\left(V_{c}\right) \simeq \boldsymbol{O}_{\eta_{c}}\left(2 n_{c}\right)$,

$$
r^{\prime}=h_{1}+h_{2}, r^{\prime \prime}=h_{1}-h_{2},
$$

$\boldsymbol{T}=\boldsymbol{T}_{w_{1}} \times \boldsymbol{T}_{w_{2}}, \boldsymbol{M}=\boldsymbol{T} \times \boldsymbol{G}_{c}$. Notons $1_{T_{w_{1}}}$ l'élément neutre de $\widehat{\boldsymbol{T}}_{w_{1}}$. Le tore dual $\widehat{\boldsymbol{T}}_{w_{2}}$ se plonge naturellement dans $\widehat{\boldsymbol{O}}\left(V_{2}\right)$. Notons $\zeta_{T_{w_{2}}}$ l'élément de $\widehat{\boldsymbol{T}}_{w_{2}}$ dont l'image dans $\widehat{\boldsymbol{O}}\left(V_{2}\right)$ est l'élément central -1 de ce groupe. Posons $s_{T}=1_{T_{w_{1}}} \times \zeta_{T_{w_{2}}} \in \widehat{\boldsymbol{T}}$. En appliquant la construction de 3.2 à $\boldsymbol{M}, \boldsymbol{T}, s_{T}, r^{\prime}, r^{\prime \prime}$, on construit un complexe $K\left(\boldsymbol{T} ; s_{T} ; r^{\prime}, r^{\prime \prime}\right)$ sur $\boldsymbol{G}$ et une fonction $k\left(\boldsymbol{T} ; s_{T} ; r^{\prime}, r^{\prime \prime}\right)$ sur $G$. Nous noterons ces objets respectivement $K\left(w_{1}, w_{2} ; r^{\prime}, r^{\prime \prime}\right)$ et $k_{\eta}\left(w_{1}, w_{2} ; r^{\prime}, r^{\prime \prime}\right)$.

Dans le cas où $n_{c}=0$, on a imposé l'égalité $\operatorname{sgn}_{C D}\left(w_{1}\right) \operatorname{sgn}_{C D}\left(w_{2}\right)=\eta$. On lève cette condition en posant $k_{\eta}\left(w_{1}, w_{2} ; 0,0\right)=0$ si elle n'est pas vérifiée.

Pour $j \in\{1,2\}$, posons :

$$
e_{j}= \begin{cases}0, & \text { si } h_{j} \geqslant 0, \\ 1, & \text { si } h_{j}<0 .\end{cases}
$$

Soient maintenant $\rho_{j} \in \operatorname{Irr}\left(W_{N_{j}}\right)$, pour $j \in\{1,2\}$. Notons $\Lambda_{j}$ l'élément de $\widetilde{\mathcal{S}}_{N_{j}+h_{j}^{2} \text {,pair }}$ correspondant au couple $\left(h_{j}, \rho_{j}\right)(c f .2 .2)$. On définit la fonction sur $G$ :

(1) $\quad k_{\eta}\left(\Lambda_{1}, \Lambda_{2}\right)=(-1)^{n}\left|W_{N_{1}}\right|^{-1}\left|W_{N_{2}}\right|^{-1}$ $\sum_{\left(w_{1}, w_{2}\right) \in W_{N_{1}} \times W_{N_{2}}} \operatorname{sgn}_{C D}\left(w_{1}\right)^{e_{1}} \operatorname{trace}\left(\rho_{1}\right)\left(w_{1}\right) \operatorname{sgn}_{C D}\left(w_{2}\right)^{e_{2}} \operatorname{trace}\left(\rho_{2}\right)\left(w_{2}\right) k_{\eta}\left(w_{1}, w_{2} ; r^{\prime}, r^{\prime \prime}\right)$. 
3.4. On conserve les notations du paragraphe précédent. Supposons d'abord $n_{c}>0$. La fonction $k_{\eta}\left(\Lambda_{1}, \Lambda_{2}\right)$ est la fonction trace d'un faisceau-caractère au sens de Lusztig. Pour voir cela, posons $N=N_{1}+N_{2}$ et fixons un sous-ensemble $\left\{v_{i} ; i \in\{ \pm 1, \ldots, \pm N\}\right\}$ de $V$ tel que, pour tous $i, j$ :

$$
Q\left(v_{i}, v_{j}\right)= \begin{cases}0, & \text { si } i \neq-j \\ -2, & \text { si } i=-j\end{cases}
$$

Prenons pour $V_{1}$, resp. $V_{2}$, le sous-espace de $V$ engendré par les $v_{i}$, pour $i \in$ $\left\{ \pm 1, \ldots, \pm N_{1}\right\}$, resp. $\left\{ \pm\left(N_{1}+1\right), \ldots, \pm N\right\}$, et pour $V_{c}$ l'orthogonal de $V_{1} \oplus V_{2}$. Pour $j \in\{1,2\}$, notons $\boldsymbol{T}_{j}$ le sous-tore maximal de $\boldsymbol{O}\left(V_{j}\right)$ qui stabilise toutes les droites $\overline{\mathbb{F}}_{q} v_{i}$, pour $v_{i} \in V_{j}$. Par la même construction qu'en 3.3 (c'est le cas $w_{1}=1, w_{2}=1$ ), on construit un complexe $K=K\left(\boldsymbol{T}_{1} \times \boldsymbol{T}_{2} ; 1_{T_{1}} \times \zeta_{T_{2}} ; r^{\prime}, r^{\prime \prime}\right)$ sur $\boldsymbol{G}$. Remarquons qu'ici, le groupe $\boldsymbol{M}=\boldsymbol{T} \times \boldsymbol{G}_{c}$ est un sous-groupe de Lévi d'un sous-groupe parabolique de $\boldsymbol{G}$ défini sur $\mathbb{F}_{q}$.

Comme en 3.1, fixons $x \in C_{c}$. Notons $\dot{\boldsymbol{T}}$ le sous-ensemble des $t \in \boldsymbol{T}$ tels que l'élément $t^{-1} F(t) x$ de $\boldsymbol{T} \times \boldsymbol{C}_{c}$ appartienne à $\boldsymbol{M}_{\text {reg }}$. Considérons le fibré :

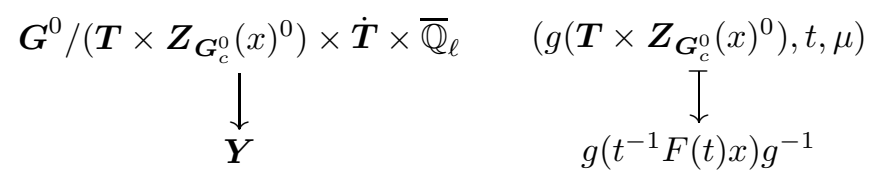

Le groupe $\left(\boldsymbol{Z}_{\boldsymbol{G}_{c}^{0}}(x) / \boldsymbol{Z}_{\boldsymbol{G}_{c}^{0}}(x)^{0}\right) \times T$ agit sur ce fibré :

- $\boldsymbol{Z}_{\boldsymbol{G}_{c}^{0}}(x) / \boldsymbol{Z}_{\boldsymbol{G}_{c}^{0}}(x)^{0}$ agit sur $\boldsymbol{G}^{0} /\left(\boldsymbol{T} \times \boldsymbol{Z}_{\boldsymbol{G}_{c}^{0}}(x)^{0}\right)$ par multiplication à droite, trivialement sur $\dot{\boldsymbol{T}}$, par le caractère $\varepsilon$ sur $\overline{\mathbb{Q}}_{\ell}$;

- $T$ agit trivialement sur $\boldsymbol{G}^{0} /\left(\boldsymbol{T} \times \boldsymbol{Z}_{\boldsymbol{G}_{c}^{0}}(x)^{0}\right)$, par multiplication sur $\dot{\boldsymbol{T}}$;

- $T_{1}$ agit trivialement sur $\overline{\mathbb{Q}}_{\ell}, T_{2}$ y agit par le caractère associé à $\zeta_{T_{2}} \in \widehat{\boldsymbol{T}}_{2}$.

Le système local $\pi_{!}\left(\mathcal{L}\left(s_{T}\right) \times \mathcal{L}\left(r^{\prime}, r^{\prime \prime}\right)\right)$ de 3.2 n'est autre que le quotient du fibré ci-dessus par l'action du groupe $\left(\boldsymbol{Z}_{\boldsymbol{G}_{c}^{0}}(x) / \boldsymbol{Z}_{\boldsymbol{G}_{c}^{0}}(x)^{0}\right) \times T$.

Fixons une décomposition orthogonale :

$$
V_{c}=\left(\oplus_{i \in I^{\prime}} V_{i}^{\prime}\right) \oplus\left(\oplus_{i \in I^{\prime \prime}} V_{i}^{\prime \prime}\right),
$$

(les notations sont celles de 3.1), telle que, pour tout $i \in I^{\prime}$, resp. $i \in I^{\prime \prime}, V_{i}^{\prime}$, resp. $V_{i}^{\prime \prime}$, soit stable par $x=s u, s$ y agisse par multiplication par 1, resp. -1 , et $u$ n'y ait qu'un seul bloc de Jordan, de longueur $i$. Fixons $i_{1}$ tel que, ou bien $i_{1} \in I^{\prime}$ et $\varepsilon_{i_{1}}^{\prime}=e_{1}$, ou bien $i_{1} \in I^{\prime \prime}$ et $\varepsilon_{i_{1}}^{\prime \prime}=e_{1}$. Un tel $i_{1}$ existe. Soit $w \in W_{N_{1}}$. On relève $w$ en un élément de $G^{0}$, encore noté $w$, de la façon suivante. Pour $i \in\left\{ \pm 1, \ldots, \pm N_{1}\right\}, w v_{i}=v_{w i}$. Pour $i \in\left\{ \pm\left(N_{1}+1\right), \ldots, \pm N\right\}, w v_{i}=v_{i} . \operatorname{Si} \operatorname{sgn}_{C D}(w)=1, w$ agit trivialement sur $V_{c}$. Si $\operatorname{sgn}_{C D}(w)=-1, w$ agit par multiplication par -1 sur $V_{i_{1}}^{\prime}$ ou $V_{i_{1}}^{\prime \prime}$, selon que $i_{1} \in I^{\prime}$ ou $i_{1} \in I^{\prime \prime}$, et agit trivialement sur les autres termes de la décomposition (2). On relève de façon similaire les éléments de $W_{N_{2}}$. On obtient ainsi un homomorphisme injectif 
$W_{N_{1}} \times W_{N_{2}} \rightarrow G$. Soit $w \in W_{N_{1}} \times W_{N_{2}}$. Il agit sur le fibré (1) par :

$$
\left(g\left(\boldsymbol{T} \times \boldsymbol{Z}_{\boldsymbol{G}_{c}^{0}}(x)^{0}\right), t, \mu\right) \longmapsto\left(g w^{-1}\left(\boldsymbol{T} \times \boldsymbol{Z}_{\boldsymbol{G}_{c}^{0}}(x)^{0}\right), w t w^{-1}, \mu\right) .
$$

Cette action se quotiente, par l'action du groupe $\left(\boldsymbol{Z}_{\boldsymbol{G}_{c}^{0}}(x) / \boldsymbol{Z}_{\boldsymbol{G}_{c}^{0}}(x)^{0}\right) \times T$, en un automorphisme du système local $\pi_{!}\left(\mathcal{L}\left(s_{T}\right) \times \mathcal{L}\left(r^{\prime}, r^{\prime \prime}\right)\right)$. On obtient une application de $W_{N_{1}} \times W_{N_{2}}$ dans le groupe d'automorphismes de ce système local. Cette application est un isomorphisme de groupes. D'après l'une des propriétés fondamentales du prolongement d'intersection, $W_{N_{1}} \times W_{N_{2}}$ s'identifie du même coup à un groupe d'automorphismes du faisceau pervers $K$, qui engendre l'algèbre des endomorphismes de ce faisceau. Notons $E$ l'espace de la représentation $\rho_{1} \times \rho_{2}$ de $W_{N_{1}} \times W_{N_{2}}$. On définit le faisceau pervers :

$$
A\left(\rho_{1}, \rho_{2}\right)=\operatorname{Hom}_{W_{N_{1}} \times W_{N_{2}}}(E, K) .
$$

Il est irréductible, $c f$. [L5] 10.4. On le munit du Frobenius déduit de celui que l'on a défini sur $K$. En utilisant [L5] 10.4.4 et en explicitant tous les termes de cette formule, on vérifie l'égalité :

$$
k_{\eta}\left(\Lambda_{1}, \Lambda_{2}\right)=(-1)^{n} \operatorname{trace}\left(A\left(\rho_{1}, \rho_{2}\right)\right) .
$$

Supposons maintenant $n_{c}=0$ et $\eta=1$. La construction se complique, le groupe d'automorphismes intervenant étant cette fois isomorphe au sous-groupe $\left(W_{N_{1}} \times W_{N_{2}}\right)^{D}$ des $\left(w_{1}, w_{2}\right) \in W_{N_{1}} \times W_{N_{2}}$ tels que $\operatorname{sgn}_{C D}\left(w_{1}\right) \operatorname{sgn}_{C D}\left(w_{2}\right)=1$. Comme ci-dessus, toute représentation irréductible $\rho$ de ce groupe donne naissance à un faisceau pervers irréductible $A(\rho)$, muni d'un Frobenius, qui est $\boldsymbol{G}^{0}$-équivariant. On a alors :

$$
k_{+}\left(\Lambda_{1}, \Lambda_{2}\right)=(-1)^{n} \sum_{\rho} \operatorname{trace}(A(\rho)),
$$

où $\rho$ parcourt les composantes irréductibles de la restriction de $\rho_{1} \times \rho_{2}$ à $\left(W_{N_{1}} \times W_{N_{2}}\right)^{D}$.

Considérons enfin le cas où $n_{c}=0$ et $\eta=-1$. On ne peut plus choisir les éléments $v_{i}$ dans $V$. On les prend dans $V \otimes_{\mathbb{F}_{q}} \overline{\mathbb{F}}_{q}$, en supposant que le Frobenius de cet espace fixe $v_{i}$ pour $i \in\{ \pm 1, \ldots, \pm(N-1)\}$ et échange $v_{N}$ et $v_{-N}$. On construit le complexe $K$. Son groupe d'automorphismes est $\left(W_{N_{1}} \times W_{N_{2}}\right)^{D}$. Mais le Frobenius $F$ de $K$ ne commute plus à l'action de ce groupe : pour $\underline{w} \in\left(W_{N_{1}} \times W_{N_{2}}\right)^{D}$, on a $F \underline{w} F^{-1}=\underline{w}_{F} \underline{w w}_{F}$, où :

$$
\underline{w}_{F}= \begin{cases}\left(1, s_{N_{2}}\right) \in W_{N_{1}} \times W_{N_{2}}, & \text { si } N_{2} \neq 0, \\ s_{N_{1}} \in W_{N_{1}}, & \text { si } N_{2}=0 .\end{cases}
$$

Si la restriction de $\rho_{1} \times \rho_{2}$ à $\left(W_{N_{1}} \times W_{N_{2}}\right)^{D}$ est réductible, on a $k_{-}\left(\Lambda_{1}, \Lambda_{2}\right)=0$ d'après la définition de cette fonction. Supposons cette restriction irréductible. On pose :

$$
A\left(\rho_{1}, \rho_{2}\right)=\operatorname{Hom}_{\left(W_{N_{1}} \times W_{N_{2}}\right)^{D}(E, K) .}
$$

C'est encore un faisceau pervers irréductible. On le munit du Frobenius tel que :

$$
F(a)=F \circ a \circ\left(\rho_{1} \times \rho_{2}\right)\left(\underline{w}_{F}\right)^{-1}
$$


pour tout $a \in A\left(\rho_{1}, \rho_{2}\right)$. On a alors l'égalité :

$$
k_{-}\left(\Lambda_{1}, \Lambda_{2}\right)=(-1)^{n} \operatorname{trace}\left(A\left(\rho_{1}, \rho_{2}\right)\right) .
$$

3.5. Dans les constructions précédentes, considérons le cas particulier où $N_{1}=n-1$, $N_{2}=0, h_{1}=1, h_{2}=0$ et $\rho_{1}$ est la représentation triviale de $W_{n-1}\left(\rho_{2}\right.$ est aussi bien sûr la représentation triviale de $\left.W_{0}\right)$. Autrement dit, $\Lambda_{2}=\Lambda(\varnothing)$ et $\Lambda_{1}=\mathbf{1}_{-}(n)$, $c f$. 2.4. Pour toute variété $\boldsymbol{X}$, notons $\overline{\mathbb{Q}}_{\ell \mid \boldsymbol{X}}$ le fibré trivial sur $\boldsymbol{X}$. Le fibré 3.4 (1) admet pour quotient $\overline{\mathbb{Q}}_{\ell \mid \boldsymbol{Y}}$ par l'application :

$$
\begin{aligned}
\boldsymbol{G}^{0} /\left(\boldsymbol{T} \times \boldsymbol{Z}_{\boldsymbol{G}_{c}^{0}}(x)^{0}\right) \times \dot{\boldsymbol{T}} \times \overline{\mathbb{Q}}_{\ell} \longrightarrow \boldsymbol{Y} \times \overline{\mathbb{Q}}_{\ell} \\
\left(g\left(\boldsymbol{T} \times \boldsymbol{Z}_{\boldsymbol{G}_{c}^{0}}(x)^{0}\right), t, \mu\right) \longmapsto\left(g\left(t^{-1} F(t) x\right) g^{-1}, \mu\right)
\end{aligned}
$$

Munissons le fibré trivial du Frobenius évident et des actions triviales de

$$
\left(\boldsymbol{Z}_{\boldsymbol{G}_{c}^{0}}(x) / \boldsymbol{Z}_{\boldsymbol{G}_{c}^{0}}(x)^{0}\right) \times T \text { et } W_{n-1} .
$$

L'application ci-dessus est équivariante pour toutes ces actions. En se reportant aux définitions, et en tenant compte des décalages qu'on y a introduits, on voit que le complexe $\overline{\mathbb{Q}}_{\ell \mid \boldsymbol{Y}}[\operatorname{dim}(\boldsymbol{Y})]$ est quotient de la restriction de $A\left(\rho_{1}, \rho_{2}\right)$ à $\boldsymbol{Y}$. Mais $\boldsymbol{Y}$ est ici un ouvert dense de $\boldsymbol{G} \backslash \boldsymbol{G}^{0}$ et $A\left(\rho_{1}, \rho_{2}\right)$ est irréductible. Donc $A\left(\rho_{1}, \rho_{2}\right)$ est isomorphe au complexe $\overline{\mathbb{Q}}_{\ell \mid \boldsymbol{G} \backslash \boldsymbol{G}^{0}}[\operatorname{dim}(\boldsymbol{G})]$. Cet isomorphisme est compatible aux actions de Frobenius. De 3.4 (3) résulte l'égalité suivante, pour tout $g \in G$ :

$$
k_{\eta}\left(\mathbf{1}_{-}(n), \Lambda(\varnothing)\right)(g)= \begin{cases}0, & \text { si } \operatorname{det}(g)=1 \\ 1, & \text { si } \operatorname{det}(g)=-1\end{cases}
$$

Considérons maintenant le cas particulier où $N_{1}=n, N_{2}=0, h_{1}=h_{2}=0$ et $\rho_{1}$ est la représentation triviale de $W_{n}$. Autrement dit, $\Lambda_{2}=\Lambda(\varnothing)$ et $\Lambda_{1}=\mathbf{1}_{+}(n)$. Un raisonnement analogue montre que, pour tout $g \in G$ :

$$
k_{\eta}\left(\mathbf{1}_{+}(n), \Lambda(\varnothing)\right)(g)= \begin{cases}1, & \text { si } \operatorname{det}(g)=1 \\ 0, & \text { si } \operatorname{det}(g)=-1\end{cases}
$$

3.6. Soit $n$ un entier $\geqslant 1$. Pour $\eta \in\{ \pm 1\}$, on a défini l'espace $\mathcal{C}\left(O_{\eta}(2 n)\right)$ des fonctions sur $O_{\eta}(2 n)$ invariantes par conjugaison. C'est une algèbre pour la multiplication point par point : $f_{1} f_{2}(g)=f_{1}(g) f_{2}(g)$. En particulier, on peut multiplier par les fonctions det ou sp. On définit de plus les automorphismes $f \mapsto f^{-}$et $f \mapsto f^{c}$ par :

$$
f^{-}(g)=f(-g), \quad f^{c}(g)=f\left(h g h^{-1}\right),
$$

pour tout $g \in O_{\eta}(2 n)$, où $h$ est un élément de $C O_{\eta}(2 n)$ tel que $\zeta \circ \lambda(h)=-1$ (la définition ne dépend pas du choix de $h$ ).

Posons :

$$
\mathcal{C}(O(2 n))=\mathcal{C}\left(O_{+}(2 n)\right) \oplus \mathcal{C}\left(O_{-}(2 n)\right)
$$


Remarquons que l'on peut considérer cet espace comme un espace de fonctions sur l'union disjointe $O_{+}(2 n) \cup O_{-}(2 n)$. En particulier, on peut évaluer un élément de cet espace en un point de $O_{+}(2 n)$ ou $O_{-}(2 n)$.

Munissons cet espace du produit hermitien somme orthogonale des produits sur chaque facteur. Des actions définies ci-dessus sur chaque facteur se déduisent des actions sur leur somme. Par exemple, pour $f=f_{+} \oplus f_{-} \in \mathcal{C}(O(2 n))$, on pose $\operatorname{det} f=$ $\left(\operatorname{det} f_{+}\right) \oplus\left(\operatorname{det} f_{-}\right)$. On note $1 \oplus-1$ l'automorphisme :

$$
f_{+} \oplus f_{-} \longmapsto f_{+} \oplus\left(-f_{-}\right) \text {. }
$$

Posons :

$$
\widetilde{\mathcal{S}} \widetilde{\mathcal{S}}_{n, \text { pair }}=\bigcup\left(\widetilde{\mathcal{S}}_{n_{1}, \text { pair }} \times \widetilde{\mathcal{S}}_{n_{2}, \text { pair }}\right),
$$

union sur les $\left(n_{1}, n_{2}\right) \in \mathbb{N} \times \mathbb{N}$ tels que $n_{1}+n_{2}=n$. Soit $\left(\Lambda_{1}, \Lambda_{2}\right) \in \widetilde{\mathcal{S}} \widetilde{\mathcal{S}}_{n \text {,pair. }}$ Pour $\eta \in\{ \pm 1\}$, on a défini en 3.3 la fonction $k_{\eta}\left(\Lambda_{1}, \Lambda_{2}\right) \in \mathcal{C}\left(O_{\eta}(2 n)\right)$. On pose :

$$
k\left(\Lambda_{1}, \Lambda_{2}\right)=k_{+}\left(\Lambda_{1}, \Lambda_{2}\right) \oplus k_{-}\left(\Lambda_{1}, \Lambda_{2}\right) \in \mathcal{C}(O(2 n)) .
$$

Proposition. - Les propriétés suivantes sont vérifiées pour tout $\left(\Lambda_{1}, \Lambda_{2}\right) \in \widetilde{\mathcal{S}} \widetilde{\mathcal{S}}_{n \text {,pair }}$.

(i) $\left(k\left(\Lambda_{1}, \Lambda_{2}\right), k\left(\Lambda_{1}, \Lambda_{2}\right)\right)=1$.

(ii) Pour tout $\left(\Lambda_{1}^{\prime}, \Lambda_{2}^{\prime}\right) \in \widetilde{\mathcal{S}} \widetilde{\mathcal{S}}_{n \text {,pair }}$ tel que $\left(\Lambda_{1}^{\prime}, \Lambda_{2}^{\prime}\right) \neq\left(\Lambda_{1}, \Lambda_{2}\right)$, on a

$$
\left(k\left(\Lambda_{1}^{\prime}, \Lambda_{2}^{\prime}\right), k\left(\Lambda_{1}, \Lambda_{2}\right)\right)=0 .
$$

(iii) $\operatorname{det} k\left(\Lambda_{1}, \Lambda_{2}\right)=(-1)^{\left(\widetilde{\operatorname{def}}\left(\Lambda_{1}\right)+\widetilde{\operatorname{def}}\left(\Lambda_{2}\right)\right) / 2} k\left(\Lambda_{1}, \Lambda_{2}\right)$.

(iv) $\operatorname{sp} k\left(\Lambda_{1}, \Lambda_{2}\right)=k\left(\Lambda_{2}, \Lambda_{1}\right)$.

(v) $k\left(\Lambda_{1}, \Lambda_{2}\right)^{c}=(-1)^{\widetilde{d e f}\left(\Lambda_{2}\right) / 2} k\left(\Lambda_{1}, \Lambda_{2}\right)$.

(vi) $k\left(\Lambda_{1}, \Lambda_{2}\right)^{-}=\zeta(-1)^{\operatorname{rg}\left(\Lambda_{2}\right)} k\left(\Lambda_{1}, \sigma \Lambda_{2}\right)$.

(vii) $(1 \oplus-1) k\left(\Lambda_{1}, \Lambda_{2}\right)=k\left(\sigma \Lambda_{1}, \sigma \Lambda_{2}\right)$.

Démonstration. - Les propriétés (iii) à (vii) résultent d'un dévissage des définitions. Démontrons seulement (iv) et (vi), et laissons les autres au lecteur. On fixe $\eta \in\{ \pm 1\}$, on pose $\boldsymbol{G}=\boldsymbol{O}_{\eta}(2 n)$

Il existe un système local $\mathcal{L}_{\text {sp }}$ sur $\boldsymbol{G}$, muni d'un Frobenius, dont sp est la fonction trace. En effet, notons $\boldsymbol{G}_{\mathrm{sc}}^{0}$ le revêtement simplement connexe de $\boldsymbol{G}^{0}$, fixons $\gamma \in$ $G \backslash G^{0}$ tel que $\gamma^{2}=1$ et $\operatorname{sp}(\gamma)=1$. L'automorphisme $\operatorname{Ad}(\gamma)$ de $\boldsymbol{G}^{0}$ se relève en un automorphisme involutif de $\boldsymbol{G}_{\mathrm{sc}}^{0}$. Formons le produit semi-direct $\boldsymbol{G}_{\mathrm{sc}} \operatorname{de}\{1, \gamma\}$ et $\boldsymbol{G}_{\mathrm{sc}}^{0}$. Alors $\mathcal{L}_{\text {sp }}$ est le quotient du fibré évident :

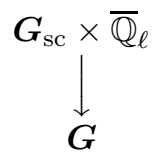

par l'action du groupe $\{ \pm 1\}$ agissant sur $\overline{\mathbb{Q}}_{\ell}$ par le caractère non trivial et sur $\boldsymbol{G}_{\text {sc }}$ par multiplication, via l'identification de $\{ \pm 1\}$ avec le noyau de la projection $\boldsymbol{G}_{\mathrm{sc}} \rightarrow \boldsymbol{G}$. 
Revenons à la définition de la fonction $k_{\eta}\left(\Lambda_{1}, \Lambda_{2}\right)$ donnée en 3.3, dont on reprend les notations. Soit $\left(w_{1}, w_{2}\right) \in W_{N_{1}} \times W_{N_{2}}$, avec $\operatorname{sgn}_{C D}\left(w_{1}\right) \operatorname{sgn}_{C D}\left(w_{2}\right)=\eta$ dans le cas où $h_{1}=h_{2}=0$. Le complexe $K\left(w_{1}, w_{2} ; r^{\prime}, r^{\prime \prime}\right)$ est induit à partir d'un système local $\mathcal{L}\left(1_{T_{w_{1}}}\right) \times \mathcal{L}\left(\zeta_{T_{w_{2}}}\right) \times \mathcal{L}\left(r^{\prime}, r^{\prime \prime}\right)$ d'un Lévi $\boldsymbol{M}=\boldsymbol{T}_{w_{1}} \times \boldsymbol{T}_{w_{2}} \times \boldsymbol{G}_{c}$. Alors $\mathcal{L}_{\mathrm{sp}} \otimes$ $K\left(w_{1}, w_{2} ; r^{\prime}, r^{\prime \prime}\right)$ est induit à partir du système local $\mathcal{L}_{\mathrm{sp} \mid \boldsymbol{M}} \otimes\left(\mathcal{L}\left(1_{T_{w_{1}}}\right) \times \mathcal{L}\left(\zeta_{T_{w_{2}}}\right) \times\right.$ $\left.\mathcal{L}\left(r^{\prime}, r^{\prime \prime}\right)\right)$, avec une notation évidente.

Soit $j \in\{1,2\}$. On vérifie que $\mathcal{L}_{\mathrm{sp} \mid \boldsymbol{T}_{w_{j}}}=\mathcal{L}\left(\zeta_{T_{w_{j}}}\right)$. Donc $\mathcal{L}_{\mathrm{sp} \mid \boldsymbol{T}_{w_{1}}} \otimes \mathcal{L}\left(1_{T_{w_{1}}}\right)=$ $\mathcal{L}\left(\zeta_{T_{w_{1}}}\right)$ et $\mathcal{L}_{\mathrm{sp} \mid \boldsymbol{T}_{w_{2}}} \otimes \mathcal{L}\left(\zeta_{T_{w_{2}}}\right)=\mathcal{L}\left(1_{T_{w_{2}}}\right)$. Autrement dit, tensoriser par $\mathcal{L}_{\mathrm{sp}}$ revient à échanger les rôles de $\boldsymbol{T}_{w_{1}}$ et $\boldsymbol{T}_{w_{2}}$ ou, ce qui revient au même, de $w_{1}$ et $w_{2}$.

Pour calculer $\mathcal{L}_{\mathrm{sp} \mid \boldsymbol{G}_{c}} \otimes \mathcal{L}\left(r^{\prime}, r^{\prime \prime}\right)$, on calcule sa fonction trace. Elle est portée par la même variété $C_{c}$ que trace $\left(\mathcal{L}\left(r^{\prime}, r^{\prime \prime}\right)\right)$. Soit $x=s u \in C_{c}$ comme en 3.1. Grâce à 1.6(1) et 1.6(2), on a les égalités :

$$
\operatorname{trace}\left(\mathcal{L}_{\mathrm{sp} \mid \boldsymbol{G}_{c}}\right)(x)=\operatorname{sp}(s)=\eta^{\prime \prime} \zeta(-1)^{\left[r^{\prime \prime 2} / 2\right]}=\zeta(-1)^{\left[\left|r^{\prime \prime}\right| / 2\right]+\left[r^{\prime \prime 2} / 2\right]} \prod_{i \in I^{\prime \prime}} \eta_{u^{\prime \prime}}(i) .
$$

Or $\left[r^{\prime \prime 2} / 2\right] \in 2 \mathbb{Z}$ et :

$$
\left[\left|r^{\prime \prime}\right| / 2\right] \equiv \sum_{i \in I^{\prime \prime}}(i-1) / 2 \bmod 2 \mathbb{Z}
$$

D'où :

$$
\operatorname{trace}\left(\mathcal{L}_{\mathrm{sp} \mid \boldsymbol{G}_{c}}\right)(x)=\prod_{i \in I^{\prime \prime}}\left(\zeta(-1)^{(i-1) / 2} \eta_{u^{\prime \prime}}(i)\right) .
$$

En examinant l'égalité $3.1(1)$, on voit que multiplier $\operatorname{trace}\left(\mathcal{L}\left(r^{\prime}, r^{\prime \prime}\right)\right)$ par $\operatorname{trace}\left(\mathcal{L}_{\mathrm{sp}} \mid \boldsymbol{G}_{c}\right)$ revient à changer l'élément $\varepsilon^{\prime \prime}=\left(\varepsilon_{i}^{\prime \prime}\right)_{i \in I^{\prime \prime}}$ en $\left(1-\varepsilon_{i}^{\prime \prime}\right)_{i \in I^{\prime \prime}}$, ou encore $r^{\prime \prime}$ en $-r^{\prime \prime}$. Autrement dit $\mathcal{L}_{\mathrm{sp} \mid \boldsymbol{G}_{c}} \otimes \mathcal{L}\left(r^{\prime}, r^{\prime \prime}\right)=\mathcal{L}\left(r^{\prime},-r^{\prime \prime}\right)$.

On obtient $\mathcal{L}_{\mathrm{sp}} \otimes K\left(w_{1}, w_{2} ; r^{\prime}, r^{\prime \prime}\right)=K\left(w_{2}, w_{1} ; r^{\prime},-r^{\prime \prime}\right)$. Grâce à ce résultat, l'égalité 3.3(1) entraîne :

$$
\begin{aligned}
\operatorname{sp} k_{\eta}\left(\Lambda_{1}, \Lambda_{2}\right)=(-1)^{n}\left|W_{N_{1}}\right|^{-1}\left|W_{N_{2}}\right|^{-1} \sum_{\left(w_{1}, w_{2}\right) \in W_{N_{1}} \times W_{N_{2}}} \operatorname{sgn}_{C D}\left(w_{1}\right)^{e_{1}} \operatorname{trace}\left(\rho_{1}\right)\left(w_{1}\right) \\
\operatorname{sgn}_{C D}\left(w_{2}\right)^{e_{2}} \operatorname{trace}\left(\rho_{2}\right)\left(w_{2}\right) k_{\eta}\left(w_{2}, w_{1} ; r^{\prime},-r^{\prime \prime}\right) .
\end{aligned}
$$

Les couples $\left(r^{\prime},-r^{\prime \prime}\right)$ et $\left(e_{2}, e_{1}\right)$ sont associés à $\left(h_{2}, h_{1}\right)$ comme $\left(r^{\prime}, r^{\prime \prime}\right)$ et $\left(e_{1}, e_{2}\right)$ ont été associés à $\left(h_{1}, h_{2}\right)$. Le membre de droite de l'égalité ci-dessus est égal à celui de l'égalité 3.3(1) relative au couple des symboles associés à $\left(h_{2}, \rho_{2}\right)$ et $\left(h_{1}, \rho_{1}\right)$. Ce couple est $\left(\Lambda_{2}, \Lambda_{1}\right)$ et le (iv) de l'énoncé s'ensuit.

Démontrons (vi). Là encore, on est ramené, pour $w_{1}, w_{2}$ fixés, à calculer $\operatorname{trace}\left(\mathcal{L}\left(1_{T_{w_{1}}}\right) \times \mathcal{L}\left(\zeta_{T_{w_{2}}}\right) \times \mathcal{L}\left(r^{\prime}, r^{\prime \prime}\right)\right)(-m)$, pour $m \in M$ (avec les notations cidessus). Pour tout $t \in T_{w_{1}}$, on a les égalités :

$$
\operatorname{trace}\left(\mathcal{L}\left(1_{T_{w_{1}}}\right)\right)(-t)=\operatorname{trace}\left(\mathcal{L}\left(1_{T_{w_{1}}}\right)\right)(t)=1
$$


Grâce à 1.6(1), pour tout $t \in T_{w_{2}}$, on a les égalités :

$$
\begin{aligned}
\operatorname{trace}\left(\mathcal{L}\left(\zeta_{T_{w_{2}}}\right)\right)(-t) & =\operatorname{sp}_{\mid T_{w_{2}}}(-1) \operatorname{trace}\left(\mathcal{L}\left(\zeta_{T_{w_{2}}}\right)\right)(t) \\
& =\operatorname{sgn}_{C D}\left(w_{2}\right) \zeta(-1)^{N_{2}} \operatorname{trace}\left(\mathcal{L}\left(\zeta_{T_{w_{2}}}\right)\right)(t) .
\end{aligned}
$$

La fonction sur $G_{c}: g \mapsto \operatorname{trace}\left(\mathcal{L}\left(r^{\prime}, r^{\prime \prime}\right)\right)(-g)$ est portée par $-C_{c}$. L'orbite $-\boldsymbol{C}_{c}$ est celle associée en 3.1 au couple $\left(\widetilde{r}^{\prime}, \widetilde{r}^{\prime \prime}\right)=\left(r^{\prime \prime}, r^{\prime}\right)$. Soit $x=s u \in-C_{c}$, notons $\widetilde{I}^{\prime}, \widetilde{I}^{\prime \prime}, \eta_{\widetilde{u}^{\prime}}, \eta_{\widetilde{u}^{\prime \prime}}$ ses données associées, et notons $I^{\prime}, I^{\prime \prime}, \eta_{u^{\prime}}, \eta_{u^{\prime \prime}}$ les données associées à $-x$. On a $\widetilde{I}^{\prime}=I^{\prime \prime}, \widetilde{I}^{\prime \prime}=I^{\prime}, \eta_{\widetilde{u}^{\prime}}=\eta_{u^{\prime \prime}}, \eta_{\widetilde{u}^{\prime \prime}}=\eta_{u^{\prime}}$. Les nombres $\operatorname{trace}\left(\mathcal{L}\left(\widetilde{r}^{\prime}, \widetilde{r}^{\prime \prime}\right)\right)(x)$ et trace $\left(\mathcal{L}\left(r^{\prime}, r^{\prime \prime}\right)\right)(-x)$ sont calculés par 3.1(1). En comparant les deux expressions, on obtient :

$$
\operatorname{trace}\left(\mathcal{L}\left(r^{\prime}, r^{\prime \prime}\right)\right)(-x)=\zeta(-1)^{a} \operatorname{trace}\left(\mathcal{L}\left(r^{\prime \prime}, r^{\prime}\right)\right)(x),
$$

où $a=\left(\sum_{i \in I^{\prime}} \varepsilon_{i}^{\prime}\right)+\left(\sum_{i \in I^{\prime \prime}} \varepsilon_{i}^{\prime \prime}\right)$. On calcule $a \equiv r^{\prime}-r^{\prime \prime} \equiv h_{2} \equiv h_{2}^{2} \bmod 2 \mathbb{Z}$.

On obtient l'égalité :

$$
\begin{aligned}
\operatorname{trace}\left(\mathcal{L}\left(1_{T_{w_{1}}}\right)\right. & \left.\times \mathcal{L}\left(\zeta_{T_{w_{2}}}\right) \times \mathcal{L}\left(r^{\prime}, r^{\prime \prime}\right)\right)(-m) \\
& =\operatorname{sgn}_{C D}\left(w_{2}\right) \zeta(-1)^{N_{2}+h_{2}^{2}} \operatorname{trace}\left(\mathcal{L}\left(1_{T_{w_{1}}}\right) \times \mathcal{L}\left(\zeta_{T_{w_{2}}}\right) \times \mathcal{L}\left(r^{\prime \prime}, r^{\prime}\right)\right)(m),
\end{aligned}
$$

pour tout $m \in M$. Remarquons que $N_{2}+h_{2}^{2}=\operatorname{rg}\left(\Lambda_{2}\right)$. On en déduit :

$$
k_{\eta}\left(w_{1}, w_{2} ; r^{\prime}, r^{\prime \prime}\right)^{-}=\operatorname{sgn}_{C D}\left(w_{2}\right) \zeta(-1)^{\mathrm{rg}\left(\Lambda_{2}\right)} k_{\eta}\left(w_{1}, w_{2} ; r^{\prime \prime}, r^{\prime}\right) .
$$

En utilisant 3.3(1), on obtient :

$$
\begin{aligned}
\zeta(-1)^{\operatorname{rg}\left(\Lambda_{2}\right)} k_{\eta}\left(\Lambda_{1}, \Lambda_{2}\right)^{-}=(-1)^{n}\left|W_{N_{1}}\right|^{-1}\left|W_{N_{2}}\right|^{-1} \sum_{\left(w_{1}, w_{2}\right) \in W_{N_{1}} \times W_{N_{2}}} \operatorname{sgn}_{C D}\left(w_{1}\right)^{e_{1}} \\
\operatorname{trace}\left(\rho_{1}\right)\left(w_{1}\right) \operatorname{sgn}_{C D}\left(w_{2}\right)^{e_{2}+1} \operatorname{trace}\left(\rho_{2}\right)\left(w_{2}\right) k_{\eta}\left(w_{2}, w_{1} ; r^{\prime \prime}, r^{\prime}\right) .
\end{aligned}
$$

Le couple $\left(r^{\prime \prime}, r^{\prime}\right)$ est associé à $\left(h_{1},-h_{2}\right)$ comme $\left(r^{\prime}, r^{\prime \prime}\right)$ a été associé à $\left(h_{1}, h_{2}\right)$. Si $h_{2} \neq 0,\left(e_{1}, e_{2}+1\right)$ est le couple associé à $\left(h_{1},-h_{2}\right)$ comme $\left(e_{1}, e_{2}\right)$ a été associé à $\left(h_{1}, h_{2}\right)$. Si $h_{2}=0$, on a $e_{2}=0$ et, bien sûr, $\left(e_{1}, e_{2}\right)$ est associé à $\left(h_{1},-h_{2}\right)$ comme à $\left(h_{1}, h_{2}\right)$. Dans les deux cas, le membre de droite de l'égalité ci-dessus est égal à $k_{\eta}\left(\Lambda_{1}^{\prime}, \Lambda_{2}^{\prime}\right)$, où $\Lambda_{1}^{\prime}$ est le symbole associé à $\left(h_{1}, \rho_{1}\right)$ et $\Lambda_{2}^{\prime}$ est celui associé à $\left(-h_{2}, \rho_{2}\right)$ si $h_{2} \neq 0$, à $\left(0, \rho_{2} \otimes \operatorname{sgn}_{C D}\right)$ si $h_{2}=0$. Or $\Lambda_{1}^{\prime}=\Lambda_{1}, \Lambda_{2}^{\prime}=\sigma \Lambda_{2}$, ce qui démontre (vi).

Supposons $\left(h_{1}, h_{2}\right) \neq(0,0)$. À partir des égalités 3.2(1), 3.2(2), 3.3(1), un calcul standard prouve que :

$$
\left(k_{\eta}\left(\Lambda_{1}, \Lambda_{2}\right), k_{\eta}\left(\Lambda_{1}, \Lambda_{2}\right)\right)=1 / 2 \text {, }
$$

et, pour $\left(\Lambda_{1}^{\prime}, \Lambda_{2}^{\prime}\right) \in \widetilde{\mathcal{S}} \widetilde{\mathcal{S}}_{n, \text { pair }}$, différent de $\left(\Lambda_{1}, \Lambda_{2}\right)$ et $\left(\sigma \Lambda_{1}, \sigma \Lambda_{2}\right)$,

$$
\left(k_{\eta}\left(\Lambda_{1}, \Lambda_{2}\right), k_{\eta}\left(\Lambda_{1}^{\prime}, \Lambda_{2}^{\prime}\right)\right)=0 .
$$

D'où le (i) de l'énoncé et aussi le (ii), pourvu que $\left(\Lambda_{1}^{\prime}, \Lambda_{2}^{\prime}\right)$ soit différent de $\left(\sigma \Lambda_{1}, \sigma \Lambda_{2}\right)$. D'après (vii), on a :

$$
k\left(\sigma \Lambda_{1}, \sigma \Lambda_{2}\right)=k_{+}\left(\Lambda_{1}, \Lambda_{2}\right) \oplus-k_{-}\left(\Lambda_{1}, \Lambda_{2}\right) .
$$


D'où encore (ii) pour $\left(\Lambda_{1}^{\prime}, \Lambda_{2}^{\prime}\right)=\left(\sigma \Lambda_{1}, \sigma \Lambda_{2}\right)$. Si $\left(h_{1}, h_{2}\right)=(0,0)$, le calcul se complique un peu. On obtient les mêmes résultats que ci-dessus, sauf si $\left(\Lambda_{1}, \Lambda_{2}\right)=\left(\sigma \Lambda_{1}, \sigma \Lambda_{2}\right)$. Dans ce cas :

$$
\left(k_{+}\left(\Lambda_{1}, \Lambda_{2}\right), k_{+}\left(\Lambda_{1}, \Lambda_{2}\right)\right)=1, k_{-}\left(\Lambda_{1}, \Lambda_{2}\right)=0 .
$$

On laisse les détails au lecteur.

Il est utile de considérer aussi le cas $n=0$. On pose par convention

$$
\begin{gathered}
O_{+}(0)=\{1\}, \quad O_{-}(0)=\varnothing, \quad \mathcal{C}(O(0))=\mathcal{C}\left(O_{+}(0)\right)=\mathbb{C}, \quad \mathcal{C}\left(O_{-}(0)\right)=\{0\}, \\
k(\Lambda(\varnothing), \Lambda(\varnothing))=k_{+}(\Lambda(\varnothing), \Lambda(\varnothing))=1, \quad k_{-}(\Lambda(\varnothing), \Lambda(\varnothing))=0 .
\end{gathered}
$$

3.7. Dans les paragraphes précédents, on a construit des fonctions sur des groupes orthogonaux pairs. Des constructions analogues valent pour les groupes spéciaux orthogonaux impairs et les groupes symplectiques. On les décrit brièvement dans les paragraphes suivants.

Soient $n, r^{\prime} \in \mathbb{N}, r^{\prime \prime} \in \mathbb{Z}$ tels que $r^{\prime}$ est impair, $r^{\prime \prime}$ est pair et $r^{\prime 2}+r^{\prime \prime 2}=2 n+1$. Posons $\boldsymbol{G}=\boldsymbol{S} \boldsymbol{O}(2 n+1), \eta=\eta(V(2 n+1))$. On fixe $x=s u \in G$ tel que $s$ ait $r^{\prime 2}$ valeurs propres 1 et $r^{\prime \prime 2}$ valeurs propres -1 et, en décomposant $u=u^{\prime} u^{\prime \prime}$ (cf. 3.1), on ait :

$$
\underline{\lambda}\left(u^{\prime}\right)=\left(2 r^{\prime}-1,2 r^{\prime}-3, \ldots, 3,1\right), \underline{\lambda}\left(u^{\prime \prime}\right)=\left(2\left|r^{\prime \prime}\right|-1,2\left|r^{\prime \prime}\right|-3, \ldots, 3,1\right) .
$$

On pose $I^{\prime}=\operatorname{Jord}^{\mathrm{bp}}\left(u^{\prime}\right), I^{\prime \prime}=\operatorname{Jord}^{\mathrm{bp}}\left(u^{\prime \prime}\right)$. On définit $\varepsilon^{\prime}=\left(\varepsilon_{i}^{\prime}\right)_{i \in I^{\prime}} \in(\mathbb{Z} / 2 \mathbb{Z})^{I^{\prime}}$ et $\varepsilon^{\prime \prime}=\left(\varepsilon_{i}^{\prime \prime}\right)_{i \in I^{\prime \prime}} \in(\mathbb{Z} / 2 \mathbb{Z})^{I^{\prime \prime}}$ par :

$$
\begin{aligned}
& \varepsilon_{i}^{\prime} \equiv(i-1) / 2 \bmod 2 \mathbb{Z} \quad \text { pour tout } i \in I^{\prime}, \\
& \varepsilon_{i}^{\prime \prime} \equiv\left\{\begin{array}{ll}
(i+1) / 2 \bmod 2 \mathbb{Z}, & \text { si } r^{\prime \prime} \geqslant 0, \\
(i-1) / 2 \bmod 2 \mathbb{Z}, & \text { si } r^{\prime \prime}<0,
\end{array} \quad \text { pour tout } i \in I^{\prime \prime} .\right.
\end{aligned}
$$

Alors $\varepsilon=\left(\varepsilon^{\prime}, \varepsilon^{\prime \prime}\right)$ définit un caractère du groupe $\boldsymbol{Z}_{\boldsymbol{G}}(x) / \boldsymbol{Z}_{\boldsymbol{G}}(x)^{0}$. On lui associe comme en 3.1 un système local $\mathcal{L}\left(r^{\prime}, r^{\prime \prime}\right)$ sur l'orbite de $x$. On munit ce système local du Frobenius tel que :

$$
\begin{aligned}
\operatorname{trace}\left(\mathcal{L}\left(r^{\prime}, r^{\prime \prime}\right)\right)(x)=\eta^{\left(r^{\prime}+r^{\prime \prime}-1\right) / 2} q^{\delta\left(r^{\prime}, r^{\prime \prime}\right) / 2}\left(\prod_{i \in I^{\prime}}\left(\zeta(-1)^{(i-1) / 2} \eta_{u^{\prime}}(i)\right)^{\varepsilon_{i}^{\prime}}\right) & \\
& \left(\prod_{i \in I^{\prime \prime}}\left(\zeta(-1)^{(i-1) / 2} \eta_{u^{\prime \prime}}(i)\right)^{\varepsilon_{i}^{\prime \prime}}\right),
\end{aligned}
$$

où $\delta\left(r^{\prime}, r^{\prime \prime}\right)=\left(r^{\prime 3}-r^{\prime}+\left|r^{\prime \prime 3}-r^{\prime \prime}\right|\right) / 3$.

3.8. Soient $n, N_{1}, N_{2}, h_{1}, h_{2} \in \mathbb{N}$ tels que $n=N_{1}+N_{2}+h_{1}^{2}+h_{1}+h_{2}^{2}+h_{2}$. On associe à $\left(h_{1}, h_{2}\right)$ un couple $\left(r^{\prime}, r^{\prime \prime}\right)$ ainsi. Le nombre $r^{\prime}$ est l'unique élément impair de $\left\{h_{1}+h_{2}+1,\left|h_{1}-h_{2}\right|\right\}$. Notons-en $R^{\prime \prime}$ l'élément pair. On pose :

$$
r^{\prime \prime}= \begin{cases}R^{\prime \prime}, & \text { si } h_{1} \geqslant h_{2}, \\ -R^{\prime \prime}, & \text { si } h_{1}<h_{2} .\end{cases}
$$


Soient $w_{1} \in W_{N_{1}}, w_{2} \in W_{N_{2}}$. Comme en 3.3, on associe à ces données un complexe $K\left(w_{1}, w_{2} ; r^{\prime}, r^{\prime \prime}\right)$ sur $\boldsymbol{S} \boldsymbol{O}(2 n+1)$ muni d'un Frobenius. On note $k\left(w_{1}, w_{2} ; r^{\prime}, r^{\prime \prime}\right)$ sa fonction trace associée.

Soient maintenant $\rho_{j} \in \operatorname{Irr}\left(W_{N_{j}}\right)$ pour $j \in\{1,2\}$. On note $\Lambda_{j}$ l'élément de $\mathcal{S}_{N_{j}+h_{j}+h_{j}^{2} \text {,imp }}$ correspondant à $\left(h_{j}, \rho_{j}\right), c f .2 .2$. On pose :

$$
\begin{aligned}
k\left(\Lambda_{1}, \Lambda_{2}\right)=(-1)^{n} \sum_{\left(w_{1}, w_{2}\right) \in W_{N_{1}} \times W_{N_{2}}} \operatorname{sgn}_{C D}\left(w_{1}\right)^{h_{1}} \operatorname{trace}\left(\rho_{1}\right)\left(w_{1}\right) \\
\operatorname{sgn}_{C D}\left(w_{2}\right)^{h_{2}} \operatorname{trace}\left(\rho_{2}\right)\left(w_{2}\right) k\left(w_{1}, w_{2} ; r^{\prime}, r^{\prime \prime}\right) .
\end{aligned}
$$

Posons :

$$
\mathcal{S S}_{n, \text { imp }}=\bigcup\left(\mathcal{S}_{n_{1}, \text { imp }} \times \mathcal{S}_{n_{2}, \text { imp }}\right),
$$

union sur les $\left(n_{1}, n_{2}\right) \in \mathbb{N} \times \mathbb{N}$ tels que $n_{1}+n_{2}=n$. On vient de définir une application :

$$
\begin{aligned}
& \mathcal{S S}_{n, \mathrm{imp}} \longrightarrow \mathcal{C}(S O(2 n+1)) \\
&\left(\Lambda_{1}, \Lambda_{2}\right) \longmapsto k\left(\Lambda_{1}, \Lambda_{2}\right) .
\end{aligned}
$$

Proposition. - Les propriétés suivantes sont vérifiées pour tout $\left(\Lambda_{1}, \Lambda_{2}\right) \in \mathcal{S S}_{n, \mathrm{imp}}$.

(i) $\left(k\left(\Lambda_{1}, \Lambda_{2}\right), k\left(\Lambda_{1}, \Lambda_{2}\right)\right)=1$.

(ii) Pour tout $\left(\Lambda_{1}^{\prime}, \Lambda_{2}^{\prime}\right) \in \mathcal{S S}_{n, \text { imp }}$, différent de $\left(\Lambda_{1}, \Lambda_{2}\right)$, on a

$$
\left(k\left(\Lambda_{1}, \Lambda_{2}\right), k\left(\Lambda_{1}^{\prime}, \Lambda_{2}^{\prime}\right)\right)=0 .
$$

(iii) $\operatorname{sp} k\left(\Lambda_{1}, \Lambda_{2}\right)=k\left(\Lambda_{2}, \Lambda_{1}\right)$.

3.9. Pour traiter le cas des groupes symplectiques, on doit fixer une fois pour toutes une racine carrée de $\zeta(-1) q$, que l'on note $(\zeta(-1) q)^{1 / 2}$.

Soient $n, r^{\prime}, r^{\prime \prime} \in \mathbb{N}$ tels que $r^{\prime 2}+r^{\prime}+r^{\prime \prime 2}+r^{\prime \prime}=2 n$. Posons $\boldsymbol{G}=\boldsymbol{S} \boldsymbol{p}(2 n)$. On fixe $x=s u \in G$ tel que $s$ ait $r^{\prime 2}+r^{\prime}$ valeurs propres 1 et $r^{\prime \prime 2}+r^{\prime \prime}$ valeurs propres -1 et, en décomposant $u=u^{\prime} u^{\prime \prime}$, on ait :

$$
\underline{\lambda}\left(u^{\prime}\right)=\left(2 r^{\prime}, 2 r^{\prime}-2, \ldots, 2\right), \quad \underline{\lambda}\left(u^{\prime \prime}\right)=\left(2 r^{\prime \prime}, 2 r^{\prime \prime}-2, \ldots, 2\right) .
$$

On pose $I^{\prime}=\operatorname{Jord}^{\mathrm{bp}}\left(u^{\prime}\right), I^{\prime \prime}=\operatorname{Jord}^{\mathrm{bp}}\left(u^{\prime \prime}\right)$. On définit $\varepsilon^{\prime}=\left(\varepsilon_{i}^{\prime}\right)_{i \in I^{\prime}} \in(\mathbb{Z} / 2 \mathbb{Z})^{I^{\prime}}$ et $\varepsilon^{\prime \prime}=\left(\varepsilon_{i}^{\prime \prime}\right)_{i \in I^{\prime \prime}} \in(\mathbb{Z} / 2 \mathbb{Z})^{I^{\prime \prime}}$ par :

$$
\begin{aligned}
\varepsilon_{i}^{\prime} \equiv i / 2 \bmod 2 \mathbb{Z} & \text { pour tout } i \in I^{\prime}, \\
\varepsilon_{i}^{\prime \prime} \equiv i / 2 \bmod 2 \mathbb{Z} & \text { pour tout } i \in I^{\prime \prime} .
\end{aligned}
$$

Alors $\varepsilon=\left(\varepsilon^{\prime}, \varepsilon^{\prime \prime}\right)$ définit un caractère du groupe $\boldsymbol{Z}_{\boldsymbol{G}}(x) / \boldsymbol{Z}_{\boldsymbol{G}}(x)^{0}$. On lui associe un système local $\mathcal{L}\left(r^{\prime}, r^{\prime \prime}\right)$ sur l'orbite de $x$, muni du Frobenius tel que :

$$
\operatorname{trace}\left(\mathcal{L}\left(r^{\prime}, r^{\prime \prime}\right)\right)(x)=(\zeta(-1) q)^{\delta\left(r^{\prime}, r^{\prime \prime}\right) / 2}\left(\prod_{i \in I^{\prime}}\left(-\zeta(-1) \eta_{u^{\prime}}(i)\right)^{\varepsilon_{i}^{\prime}}\right)\left(\prod_{i \in I^{\prime \prime}}\left(-\eta_{u^{\prime \prime}}(i)\right)^{\varepsilon_{i}^{\prime \prime}}\right)
$$

où :

$$
\delta\left(r^{\prime}, r^{\prime \prime}\right)=\left(r^{\prime}\left(2 r^{\prime}+1\right)\left(r^{\prime}+1\right)+r^{\prime \prime}\left(2 r^{\prime \prime}+1\right)\left(r^{\prime \prime}+1\right)\right) / 6
$$


Remarquons que $\delta\left(r^{\prime}, r^{\prime \prime}\right)$ est entier mais pas toujours pair.

3.10. Soient $n, N_{1}, N_{2}, h_{1} \in \mathbb{N}$ et $h_{2} \in \mathbb{Z}$ tels que $n=N_{1}+N_{2}+h_{1}^{2}+h_{1}+h_{2}^{2}$. On associe à $\left(h_{1}, h_{2}\right)$ un couple $\left(r^{\prime}, r^{\prime \prime}\right)$ en posant :

$$
\begin{aligned}
r^{\prime} & =\sup \left(h_{1}-(-1)^{h_{1}} h_{2},(-1)^{h_{1}} h_{2}-h_{1}-1\right), \\
r^{\prime \prime} & =\sup \left(h_{1}+(-1)^{h_{1}} h_{2},(-1)^{h_{1}+1} h_{2}-h_{1}-1\right) .
\end{aligned}
$$

Soient $w_{1} \in W_{N_{1}}, w_{2} \in W_{N_{2}}$. Comme en 3.3, on associe à ces données un complexe $K\left(w_{1}, w_{2} ; r^{\prime}, r^{\prime \prime}\right)$ sur $\boldsymbol{S} \boldsymbol{p}(2 n)$, muni d'un Frobenius. On note $k\left(w_{1}, w_{2} ; r^{\prime}, r^{\prime \prime}\right)$ sa fonction trace. Soient maintenant $\rho_{j} \in \operatorname{Irr}\left(W_{N_{j}}\right)$ pour $j \in\{1,2\}$. On note $\Lambda_{1}$ l'élément de $\mathcal{S}_{N_{1}+h_{1}+h_{1}^{2} \text {,imp }}$ correspondant à $\left(h_{1}, \rho_{1}\right)$ et $\Lambda_{2}$ l'élément de $\widetilde{\mathcal{S}}_{N_{2}+h_{2}^{2} \text {,pair }}$ correspondant à $\left(h_{2}, \rho_{2}\right)$. On pose :

$$
e_{2}= \begin{cases}0, & \text { si } h_{2} \geqslant 0 \\ 1, & \text { si } h_{2}<0\end{cases}
$$

et :

$$
\begin{aligned}
k\left(\Lambda_{1}, \Lambda_{2}\right)=(-1)^{n}\left|W_{N_{1}}\right|^{-1}\left|W_{N_{2}}\right|^{-1} \sum_{w_{1} \in W_{N_{1}}, w_{2} \in W_{N_{2}}} \operatorname{sgn}_{C D}\left(w_{1}\right)^{h_{1}+h_{2}} \operatorname{trace}\left(\rho_{1}\right)\left(w_{1}\right) \\
\operatorname{sgn}_{C D}\left(w_{2}\right)^{h_{2}+e_{2}+1} \operatorname{trace}\left(\rho_{2}\right)\left(w_{2}\right) k\left(w_{1}, w_{2} ; r^{\prime}, r^{\prime \prime}\right) .
\end{aligned}
$$

Posons :

$$
\mathcal{S} \widetilde{\mathcal{S}}_{n, \text { mix }}=\bigcup\left(\mathcal{S}_{n_{1}, \text { imp }} \times \widetilde{\mathcal{S}}_{n_{2}, \text { pair }}\right),
$$

union sur les $\left(n_{1}, n_{2}\right) \in \mathbb{N} \times \mathbb{N}$ tels que $n_{1}+n_{2}=n$. On vient de définir une application :

$$
\begin{aligned}
& \mathcal{S} \widetilde{\mathcal{S}}_{n, \text { mix }} \longrightarrow \mathcal{C}(S p(2 n)) \\
& \left(\Lambda_{1}, \Lambda_{2}\right) \longmapsto k\left(\Lambda_{1}, \Lambda_{2}\right) .
\end{aligned}
$$

On définit comme en 3.6 les automorphismes $f \mapsto f^{-}$et $f \mapsto f^{c}$ de $\mathcal{C}(S p(2 n))$.

Proposition. - Les propriétés suivantes sont vérifiées pour tout $\left(\Lambda_{1}, \Lambda_{2}\right) \in \mathcal{S} \widetilde{\mathcal{S}}_{n, \text { mix }}$.

(i) $\left(k\left(\Lambda_{1}, \Lambda_{2}\right), k\left(\Lambda_{1}, \Lambda_{2}\right)\right)=1$.

(ii) Pour tout $\left(\Lambda_{1}^{\prime}, \Lambda_{2}^{\prime}\right) \in \mathcal{S} \widetilde{\mathcal{S}}_{n, \text { mix }}$, différent de $\left(\Lambda_{1}, \Lambda_{2}\right)$, on a

$$
\left(\left(k\left(\Lambda_{1}, \Lambda_{2}\right), k\left(\Lambda_{1}^{\prime}, \Lambda_{2}^{\prime}\right)\right)=0 .\right.
$$

(iii) $k\left(\Lambda_{1}, \Lambda_{2}\right)^{c}=(-1)^{\widetilde{\operatorname{def}}\left(\Lambda_{2}\right) / 2} k\left(\Lambda_{1}, \Lambda_{2}\right)$.

(iv) $k\left(\Lambda_{1}, \Lambda_{2}\right)^{-}=\zeta(-1)^{\operatorname{rg}\left(\Lambda_{2}\right)} k\left(\Lambda_{1}, \sigma \Lambda_{2}\right)$. 



\section{CHAPITRE 4}

\section{REPRÉSENTATIONS QUADRATIQUES-UNIPOTENTES}

On définit et on paramètre par des symboles les représentations irréductibles quadratiques-unipotentes des groupes classiques. On commence par décrire les algèbres d'entrelacement des représentations induites de représentations cuspidales. On paramètre les représentations irréductibles quadratiques-unipotentes cuspidales, en utilisant les résultats de Lusztig. Puis, on décompose les représentations induites de ces cuspidales.

4.1. Soient $n$ un entier $>0, \eta \in\{ \pm 1\}$, posons $V=V_{\eta}(2 n), Q=Q_{\eta}(2 n)$. Supposons donnés un entier $N \in \mathbb{N}$ et un sous-ensemble $\left\{v_{i} ; i \in\{ \pm 1, \ldots, \pm N\}\right\}$ de $V$ tel que, pour tous $i, j \in\{ \pm 1, \ldots, \pm N\}$, on ait :

$$
Q\left(v_{i}, v_{j}\right)= \begin{cases}0, & \text { si } i \neq-j \\ -2, & \text { si } i=-j\end{cases}
$$

On note $V_{0}$ le sous-espace de $V$ orthogonal à tous ces vecteurs. Si $V_{0} \neq\{0\}$, on fixe un élément $x_{0} \in O\left(V_{0}\right)$ tel que $\operatorname{det}\left(x_{0}\right)=-1, \operatorname{sp}\left(x_{0}\right)=1$ et $x_{0}^{2}=1$.

Soit $w \in W_{N}$. On note encore $w$ l'élément de $O(V)$ tel que :

- $w v_{i}=v_{w i}$, pour tout $i \in\{ \pm 1, \ldots, \pm N\}$,

- si $V_{0} \neq\{0\}, w_{\mid V_{0}}= \begin{cases}1, & \text { si } \operatorname{sgn}_{C D}(w)=1, \\ x_{0}, & \text { si } \operatorname{sgn}_{C D}(w)=-1 .\end{cases}$

On a ainsi défini un homomorphisme injectif de groupes de $W_{N}$ dans $O(V)$ et même dans $S O(V)$ quand $V_{0} \neq\{0\}$. Remarquons que $\operatorname{sp}(w)=1$ pour tout $w \in W_{N}$.

Soit $\boldsymbol{G}$ l'un des groupes $\boldsymbol{O}(V)$, resp. $\boldsymbol{S O}(V), \boldsymbol{C O}(V)^{0}, \boldsymbol{C O}(V)$. Si $V_{0} \neq\{0\}$, on note $\boldsymbol{G}_{0}$ le groupe $\boldsymbol{O}\left(V_{0}\right)$, resp. $\boldsymbol{S} \boldsymbol{O}\left(V_{0}\right), \boldsymbol{C} \boldsymbol{O}\left(V_{0}\right)^{0}, \boldsymbol{C} \boldsymbol{O}\left(V_{0}\right)$. Si $V_{0}=\{0\}$, on pose $\boldsymbol{G}_{0}=\{1\}$, resp. $\{1\}, \mathbb{G}_{m}, \mathbb{G}_{m}$. Notons :

- $\boldsymbol{P}$ le sous-groupe des éléments de $\boldsymbol{G}$ qui stabilisent le drapeau de sous-espaces isotropes :

$$
\{0\} \subset \mathbb{F}_{q} v_{1} \subset \mathbb{F}_{q} v_{1} \oplus \mathbb{F}_{q} v_{2} \subset \cdots \subset \mathbb{F}_{q} v_{1} \oplus \cdots \oplus \mathbb{F}_{q} v_{N} ;
$$


- $\boldsymbol{M}$ le sous-groupe des éléments de $\boldsymbol{P}$ qui conservent la droite $\mathbb{F}_{q} v_{i}$ pour tout $i \in\{ \pm 1, \ldots, \pm N\}$.

Considérons l'application :

$$
\begin{aligned}
\boldsymbol{M} & \longrightarrow \mathbb{G}_{m}^{N} \times \boldsymbol{G}_{0} \\
m & \longmapsto\left(t_{1}, \ldots, t_{N}, g_{0}\right)
\end{aligned}
$$

définie par :

$$
\begin{aligned}
m v_{i} & =t_{i} v_{i}, \text { pour tout } i \in\{1, \ldots, N\}, \\
m_{\mid V_{0}} & =g_{0}, \text { si } V_{0} \neq\{0\}, \\
\lambda(m) & =g_{0}, \text { si } V_{0}=\{0\} .
\end{aligned}
$$

Cette application est un isomorphisme.

Soient $N_{1}, N_{2} \in \mathbb{N}$ tels que $N=N_{1}+N_{2}$ et $\left(\pi_{0}, E_{0}\right)$ une représentation irréductible et cuspidale de $G_{0}$. On note $1_{N_{1}} \times \zeta_{N_{2}} \times \pi_{0}$ la représentation de $M$ dans $E_{0}$ :

$$
\left(1_{N_{1}} \times \zeta_{N_{2}} \times \pi_{0}\right)\left(t_{1}, \ldots, t_{N}, g_{0}\right)=\left(\prod_{i=N_{1}+1, \ldots, N} \zeta\left(t_{i}\right)\right) \pi_{0}\left(g_{0}\right) .
$$

Notons $(\pi, E)$ la représentation de $G$, induite de $P$ à $G$ de la représentation $1_{N_{1}} \times$ $\zeta_{N_{2}} \times \pi_{0}$ de $M$. Notons $\operatorname{Norm}\left(M, 1_{N_{1}} \times \zeta_{N_{2}} \times \pi_{0}\right)$ le sous-groupe des $g \in G$ tels que $g M g^{-1}=M$ et $\left(1_{N_{1}} \times \zeta_{N_{2}} \times \pi_{0}\right) \circ \operatorname{Ad}(g) \simeq 1_{N_{1}} \times \zeta_{N_{2}} \times \pi_{0}$. À la décomposition $N=N_{1}+N_{2}$ est associé un plongement $W_{N_{1}} \times W_{N_{2}} \rightarrow W_{N}$, d'où un plongement $W_{N_{1}} \times W_{N_{2}} \rightarrow G$. Notons $W=\left(W_{N_{1}} \times W_{N_{2}}\right) \cap \operatorname{Norm}\left(M, 1_{N_{1}} \times \zeta_{N_{2}} \times \pi_{0}\right)$. Ce groupe dépend de $\boldsymbol{G}$ et $\pi_{0}$, mais contient toujours $W_{N_{1}}^{D} \times W_{N_{2}}^{D}$. Sa détermination exacte résulte des deux relations suivantes ( $c f .1 .7$ pour les notations) :

$$
\left(1_{N_{1}} \times \zeta_{N_{2}} \times \pi_{0}\right) \circ \operatorname{Ad}\left(s_{N_{1}}, 1\right)= \begin{cases}1_{N_{1}} \times \zeta_{N_{2}} \times\left(\pi_{0} \circ \operatorname{Ad}\left(x_{0}\right)\right), & \text { si } V_{0} \neq\{0\}, \\ 1_{N_{1}} \times \zeta_{N_{2}} \times \pi_{0}, & \text { si } V_{0}=\{0\} ;\end{cases}
$$

$$
\left(1_{N_{1}} \times \zeta_{N_{2}} \times \pi_{0}\right) \circ \operatorname{Ad}\left(1, s_{N_{2}}\right)=\left\{\begin{array}{c}
1_{N_{1}} \times \zeta_{N_{2}} \times\left((\zeta \circ \lambda) \otimes\left(\pi_{0} \circ \operatorname{Ad}\left(x_{0}\right)\right)\right), \\
\text { si } V_{0} \neq\{0\}, \\
1_{N_{1}} \times \zeta_{N_{2}} \times\left(\zeta \otimes \pi_{0}\right), \\
\text { si } V_{0}=\{0\} .
\end{array}\right.
$$

Soit $w \in W$. Fixons un automorphisme $A_{w}$ de $E_{0}$ tel que :

$$
A_{w} \circ\left(1_{N_{1}} \times \zeta_{N_{2}} \times \pi_{0}\right)(m)=\left(1_{N_{1}} \times \zeta_{N_{2}} \times \pi_{0}\right)\left(w m w^{-1}\right) \circ A_{w}
$$

pour tout $m \in M$. On définit l'opérateur d'entrelacement $T^{\prime}(w) \in \operatorname{End}_{G}(E)$ :

$$
\left(T^{\prime}(w) f\right)(g)=\sum_{u \in\left(w U w^{-1} \cap U\right) \backslash U} A_{w} f\left(w^{-1} u g\right)
$$

pour tous $f \in E, g \in G$, où $\boldsymbol{U}$ est le radical unipotent de $\boldsymbol{P}$. La droite de $\operatorname{End}_{G}(E)$ portée par $T^{\prime}(w)$ ne dépend pas des choix effectués. L'algèbre engendrée par les $T^{\prime}(w)$, pour $w \in W$, est l'algèbre d'entrelacement de la représentation $\pi$. 
4.2. On conserve les données précédentes, on suppose $\boldsymbol{G}=\boldsymbol{O}(V)$. Alors $W=W_{N_{1}} \times$ $W_{N_{2}}$. On normalise les opérateurs $T^{\prime}(w)$ en choisissant, pour tout $w \in W$ :

$$
A_{w}= \begin{cases}1, & \text { si } \operatorname{sgn}_{C D}(w)=1 \text { ou si } V_{0}=\{0\}, \\ \pi_{0}\left(x_{0}\right), & \text { si } \operatorname{sgn}_{C D}(w)=-1 \text { et } V_{0} \neq\{0\}\end{cases}
$$

On pose simplement $T_{1}^{\prime}(w)=T^{\prime}(w, 1)$ pour $w \in W_{N_{1}}, T_{2}^{\prime}(w)=T^{\prime}(1, w)$ pour $w \in$ $W_{N_{2}}$.

Lemme. - Supposons $V_{0}=\{0\}$ ou la restriction de $\pi_{0}$ à $S O\left(V_{0}\right)$ irréductible. Soit $j \in\{1,2\}$. Alors il existe un entier $c_{j} \geqslant 0$ et une fonction $C_{j}: W_{N_{j}} \rightarrow \mathbb{C}^{\times}$tels qu'en posant $T_{j}(w)=C_{j}(w) T_{j}^{\prime}(w)$ pour tout $w \in W_{N_{j}}$, les relations suivantes soient vérifiées :

(i) pour tous $w, w^{\prime} \in W_{N_{j}}$ tels que $\ell\left(w w^{\prime}\right)=\ell(w)+\ell\left(w^{\prime}\right)$, on a $T_{j}\left(w w^{\prime}\right)=$ $T_{j}(w) T_{j}\left(w^{\prime}\right)$;

(ii) pour tout $i \in\left\{1, \ldots, N_{j}-1\right\},\left(T_{j}\left(s_{i}\right)-q\right)\left(T_{j}\left(s_{i}\right)+1\right)=0$;

(iii) $\left(T_{j}\left(s_{N_{j}}\right)-p^{c_{j}}\right)\left(T_{j}\left(s_{N_{j}}\right)+1\right)=0$.

Démonstration. - Si $V_{0}=\{0\}$, on est dans le cas de la série principale, la démonstration est élémentaire. Si $V_{0} \neq\{0\}$, notons $\pi_{0}^{0}$ la restriction de $\pi_{0}$ à $S O\left(V_{0}\right)$. L'application de restriction de $G$ à $G^{0}$ identifie $E$ à l'espace de la représentation induite $\operatorname{Ind}_{P^{0}}^{G^{0}}\left(\pi_{0}^{0}\right)$. Par construction, les opérateurs $T_{j}^{\prime}(w)$ s'identifient aux opérateurs similaires de cette induite. Mais, $\pi_{0}^{0}$ étant supposée irréductible et $\boldsymbol{G}^{0}$ étant connexe, l'analogue de l'énoncé pour ces derniers opérateurs est connu, $c f$. par exemple [C] théorème 10.8.5. Notre énoncé s'en déduit.

Remarque. - En fait, le lemme reste vrai si la restriction de $\pi_{0}$ à $S O\left(V_{0}\right)$ est réductible, mais ce cas ne nous servira pas.

La détermination de $C_{j}\left(s_{i}\right)$ pour $i \in\left\{1, \ldots, N_{j}-1\right\}$ se ramène à un calcul dans $G L(2)$ : on a $C_{j}\left(s_{i}\right)=1$. Dans le cas où $c_{j}>0$, la fonction $C_{j}$ est uniquement déterminée : le système d'équations est rigide. Supposons $c_{j}=0$. Il y a deux fonctions $C_{j}$ possibles, qui se déduisent l'une de l'autre par multiplication par $\operatorname{sgn}_{C D}$. On a l'égalité $T_{j}\left(s_{N_{j}}\right)^{2}=1$, donc $T_{j}^{\prime}\left(s_{N_{j}}\right)^{2}$ est une homothétie. En appliquant $T_{j}^{\prime}\left(s_{N_{j}}\right)^{2}$ à une fonction $f \in E$ à support dans $P$, on voit que cette homothétie est de rapport $q^{2(n-N)}$ si $j=2, q^{2\left(n-N_{1}\right)}$ si $j=1$. On détermine alors $C_{j}$ par la condition $C_{j}\left(s_{N_{j}}\right)=\eta(V) q^{N-n}$ si $j=2, C_{j}\left(s_{N_{j}}\right)=\eta(V) q^{N_{1}-n}$ si $j=1$. On a ainsi identifié l'algèbre d'entrelacement de $\pi$ à un produit tensoriel de deux algèbres de Hecke. Les sous-représentations irréductibles de $\pi$ sont canoniquement en bijection avec les représentations irréductibles de ce produit tensoriel d'algèbres. Ces dernières sont canoniquement, par spécialisation de $p$ en 1, en bijection avec les représentations irréductibles de $W_{N_{1}} \times W_{N_{2}}$.

Remarque. - Comme on a défini la représentation $1_{N_{1}} \times \zeta_{N_{2}} \times \pi_{0}$ de $M$, on peut définir sa représentation $\zeta_{N_{2}} \times 1_{N_{1}} \times \pi_{0}$, puis la représentation induite $\left(\pi^{\prime}, E^{\prime}\right)$ de 
$P$ à $G$ de $\zeta_{N_{2}} \times 1_{N_{1}} \times \pi_{0}$. Comme ci-dessus, ses sous-représentations irréductibles sont canoniquement en bijection avec les représentations irréductibles de $W_{N_{2}} \times W_{N_{1}}$. Notons $w$ l'élément de $W_{N}$ défini par :

$$
w i= \begin{cases}N_{1}+i, & \text { pour } i \in\left\{1, \ldots, N_{2}\right\}, \\ i-N_{2}, & \text { pour } i \in\left\{N_{2}+1, \ldots, N\right\} .\end{cases}
$$

On définit $T^{\prime}(w) \in \operatorname{Hom}_{G}\left(E^{\prime}, E\right)$ par :

$$
\left(T^{\prime}(w) f\right)(g)=\sum_{u \in\left(w U w^{-1} \cap U\right) \backslash U} f\left(w^{-1} u g\right),
$$

pour tous $f \in E^{\prime}, g \in G$. Soit $\rho_{2} \times \rho_{1}$ une représentation irréductible de $W_{N_{2}} \times W_{N_{1}}$. On montre que $T^{\prime}(w)$ envoie tout sous-module irréductible de $E^{\prime}$ paramétré par $\rho_{2} \times \rho_{1}$ sur un sous-module irréductible de $E$ paramétré par $\rho_{1} \times \rho_{2}$.

4.3. Pour $V$ comme ci-dessus et $\pi$ une représentation irréductible de $O(V)$, l'opérateur $\pi(-1)$ est une homothétie. On note encore $\pi(-1)$ le rapport de cette homothétie. D'autre part, pour $h \in C O(V)$, la classe d'isomorphie de $\pi \circ \operatorname{Ad}(h)$ ne dépend que de $\zeta \circ \lambda(h)$. C'est la classe de $\pi$ si $\zeta \circ \lambda(h)=1$. On note $\pi^{c}$ un élément de la classe de $\pi \circ \operatorname{Ad}(h)$ pour tout $h$ tel que $\zeta \circ \lambda(h)=-1$. On vérifie la relation :

$$
(\operatorname{sp} \otimes \pi)^{c} \simeq \operatorname{det} \otimes \operatorname{sp} \otimes \pi^{c} .
$$

On note $\operatorname{Quad}_{\text {cusp }}\left(O_{\eta}(2 n)\right)$ l'ensemble des classes d'isomorphie de représentations irréductibles, cuspidales, quadratiques-unipotentes de $O_{\eta}(2 n)$. On pose :

$$
\operatorname{Quad}_{\text {cusp }}(O(2 n))=\operatorname{Quad}_{\text {cusp }}\left(O_{+}(2 n)\right) \cup \operatorname{Quad}_{\text {cusp }}\left(O_{-}(2 n)\right) .
$$

Proposition. - Il existe une bijection :

$$
\begin{aligned}
\left\{\left(h_{1}, h_{2}\right) \in \mathbb{Z} \times \mathbb{Z} ; h_{1}^{2}+h_{2}^{2}=n\right\} & \longrightarrow \operatorname{Quad}_{\text {cusp }}(O(2 n)) \\
\left(h_{1}, h_{2}\right) & \longmapsto \pi\left(h_{1}, h_{2}\right)
\end{aligned}
$$

telle que les propriétés suivantes soient vérifiées, pour tout $\left(h_{1}, h_{2}\right)$ dans l'ensemble de départ:

(i) $\pi\left(h_{1}, h_{2}\right) \in \operatorname{Quad}_{\text {cusp }}\left(O_{\eta}(2 n)\right)$, où $\eta=(-1)^{h_{1}-h_{2}}$;

(ii) $\eta$ étant comme en (i), il existe un élément semi-simple $s \in S O\left(\widehat{V}_{\eta}(2 n)\right)$, ayant $2 h_{1}^{2}$ valeurs propres 1 et $2 h_{2}^{2}$ valeurs propres -1 , tel que $\pi\left(h_{1}, h_{2}\right) \in \operatorname{Irr}\left(O_{\eta}(2 n), s\right)$;

(iii) det $\otimes \pi\left(h_{1}, h_{2}\right)=\pi\left(-h_{1},-h_{2}\right)$;

(iv) $\operatorname{sp} \otimes \pi\left(h_{1}, h_{2}\right)=\pi\left(h_{2}, h_{1}\right)$;

(v) $\pi\left(h_{1}, h_{2}\right)^{c}=\pi\left(h_{1},-h_{2}\right)$;

(vi) $\pi\left(h_{1}, h_{2}\right)(-1)=(-\zeta(-1))^{h_{2}}$. 
Démonstration. - Fixons $\eta \in\{ \pm 1\}$, posons $\boldsymbol{G}=\boldsymbol{O}_{\eta}(2 n)$ et notons $\operatorname{Quad}_{\text {cusp }}\left(G^{0}\right)$ l'analogue pour le groupe $G^{0}$ de l'ensemble $\operatorname{Quad}_{\text {cusp }}(G)$. Il s'agit d'étudier cet ensemble ainsi que la décomposition des induites de $G^{0}$ à $G$ de ses éléments. Posons $V=V_{\eta}(2 n), \widehat{V}=\widehat{V}_{\eta}(2 n)$. L'ensemble $\operatorname{Quad}_{\text {cusp }}\left(G^{0}\right)$ a été décrit par Lusztig $([\mathbf{L 2}],[\mathbf{L} 7])$. Soit $s$ un élément semi-simple de $S O(\widehat{V})$ n'ayant pour valeurs propres que \pm 1 . Pour $\varepsilon \in\{ \pm 1\}$, notons $\widehat{V}^{\varepsilon}$ l'espace propre correspondant. Notons $\operatorname{Irr}_{\text {cusp }}\left(G^{0}, s\right)$ le sous-ensemble des représentations cuspidales dans $\operatorname{Irr}\left(G^{0}, s\right)$. Alors $\operatorname{Irr}_{\text {cusp }}\left(G^{0}, s\right)$ est non vide si et seulement si le groupe $S O\left(\widehat{V}^{+}\right) \times S O\left(\widehat{V}^{-}\right)$possède une représentation unipotente cuspidale. Une telle représentation est unique, quand elle existe. Son existence équivaut à celle de $\ell_{1}, \ell_{2} \in \mathbb{N}$ tels que :

$$
\operatorname{dim}\left(\widehat{V}^{+}\right)=2 \ell_{1}^{2}, \quad \operatorname{dim}\left(\widehat{V}^{-}\right)=2 \ell_{2}^{2}, \quad(-1)^{\ell_{1}}=\eta\left(\widehat{V}^{+}\right), \quad(-1)^{\ell_{2}}=\eta\left(\widehat{V}^{-}\right) .
$$

Remarquons que, puisque $\eta\left(\widehat{V}^{+}\right) \eta\left(\widehat{V}^{-}\right)=\eta$, ces conditions entraînent $(-1)^{\ell_{1}-\ell_{2}}=\eta$. Supposons les conditions ci-dessus vérifiées. Alors $\operatorname{Irr}_{\text {cusp }}\left(G^{0}, s\right)$ a autant d'éléments que le groupe $\boldsymbol{Z}_{\boldsymbol{S O}(\widehat{V})}(s)$ a de composantes connexes, i.e. 2 si $s \neq \pm 1,1$ si $s= \pm 1$. On note $\pi_{1}^{0}, \pi_{2}^{0}$, resp. $\pi^{0}$, ces éléments si $s \neq \pm 1$, resp. $s= \pm 1$. Soit $h \in C O(V)^{0}$ tel que $\zeta \circ \lambda(h)=-1$. On a $\pi_{1}^{0} \circ \operatorname{Ad}(h)=\pi_{2}^{0}$ dans le premier cas, $\pi^{0} \circ \operatorname{Ad}(h)=\pi^{0}$ dans le second.

Supposons $s \neq \pm 1$, i.e. $\ell_{1} \ell_{2} \neq 0$. Pour $i \in\{1,2\}$, on a :

$$
\pi_{i}^{0} \circ \operatorname{Ad}\left(x_{0}\right)=\pi_{i}^{0} .
$$

Pour le démontrer, fixons, ainsi qu'il est loisible, une représentation irréductible $\Pi$ de $C O(V)^{0}$ dont la restriction à $G^{0}$ soit $\pi_{1}^{0} \oplus \pi_{2}^{0}$. D'après [L2] lemme 8.9(ii), II se prolonge en une représentation de $C O(V)$, qu'on note encore $\Pi$. Considérons un espace $V^{\prime}$ somme directe orthogonale de $V$ et d'un espace hyperbolique de dimension 2 . Appliquons les constructions de 4.1 à cet espace, à $V_{0}=V, N_{1}=N=1, N_{2}=0$, au groupe $C O\left(V^{\prime}\right)^{0}$ et à la représentation $\Pi$ de $C O(V)^{0}$. Le groupe $W$ de 4.1 se réduit à $W_{N_{1}}=W_{1}$. Pour l'unique élément non trivial $w$ de ce groupe, on choisit $A_{w}=\Pi\left(x_{0}\right)$. D'après $[\mathbf{L 2}]$ proposition 8.3 , il existe $c>0$ et $C \in \mathbb{C}^{\times}$tels qu'en posant $T(w)=C T^{\prime}(w)$, on ait l'égalité :

$$
\left(T(w)-q^{c}\right)(T(w)+1)=0 .
$$

Notons $E$ l'espace de la représentation $\operatorname{Ind}_{P}^{C O\left(V^{\prime}\right)^{0}}\left(1_{1} \times \Pi\right)$. Par restriction à $S O\left(V^{\prime}\right)$, il se décompose en $E_{1} \oplus E_{2}$ où, pour $i \in\{1,2\}, E_{i}$ est l'espace de la représentation $\operatorname{Ind}_{P \cap S O\left(V^{\prime}\right)}^{S O\left(V^{\prime}\right)}\left(1_{1} \times \pi_{i}^{0}\right)$. L'ensemble $\operatorname{Irr}_{\text {cusp }}\left(G^{0}, s\right)$ est stable par $\operatorname{Ad}\left(x_{0}\right)$. Si l'assertion $(2)$ est fausse, on a donc $\pi_{1}^{0} \circ \operatorname{Ad}\left(x_{0}\right)=\pi_{2}^{0}$. Mais alors, la construction de l'opérateur $T(w)$ entraîne que $T(w)\left(E_{1}\right) \subset E_{2}, T(w)\left(E_{2}\right) \subset E_{1}$. C'est incompatible avec l'équation (3). D'où (2).

Grâce à (2) et à la théorie de Mackey, chaque $\pi_{i}^{0}$ se prolonge en une représentation de $G$. Il y a deux prolongements possibles, qui se déduisent l'un de l'autre par tensorisation par le caractère det. Choisissons un prolongement $\pi_{1}$ de $\pi_{1}^{0}$. Alors $\pi_{1}^{c}$ 
est un prolongement de $\pi_{2}^{0}$. Les quatre représentations $\pi_{1}$, det $\otimes \pi_{1}, \pi_{1}^{c}$, det $\otimes \pi_{1}^{c}$ sont deux à deux distinctes et ce sont les quatre éléments de $\operatorname{Irr}_{\text {cusp }}(G, s)$. Notons-les respectivement $\pi\left(\ell_{1}, \ell_{2}\right), \pi\left(-\ell_{1},-\ell_{2}\right), \pi\left(\ell_{1},-\ell_{2}\right), \pi\left(-\ell_{1}, \ell_{2}\right)$. Les propriétés (iii) et (v) de l'énoncé sont vérifiées. Par un yoga standard de dualité, la tensorisation par sp envoie $\operatorname{Irr}(G, s)$ sur $\operatorname{Irr}(G,-s)$. Si $s$ n'est pas conjugué à $-s$, i.e. si $\ell_{1} \neq \ell_{2}$, on peut effectuer les choix de sorte que $\operatorname{sp} \otimes \pi\left(\ell_{1}, \ell_{2}\right)=\pi\left(\ell_{2}, \ell_{1}\right)$. Grâce à (1), on vérifie alors (iv) pour $\left(h_{1}, h_{2}\right)=\left( \pm \ell_{1}, \pm \ell_{2}\right)$. Supposons maintenant $\ell_{1}=\ell_{2}$ et soit $\pi_{1}$ comme ci-dessus. On a :

$$
\operatorname{sp} \otimes \pi_{1} \in\left\{\pi_{1}, \operatorname{det} \otimes \pi_{1}, \pi_{1}^{c}, \operatorname{det} \otimes \pi_{1}^{c}\right\} .
$$

Supposons sp $\otimes \pi_{1}=\operatorname{det}^{a} \otimes \pi_{1}^{c}$, avec $a \in\{0,1\}$. Grâce à (1), on a :

$$
\begin{aligned}
\pi_{1}=\operatorname{sp} \otimes s p \otimes \pi_{1} & =\operatorname{det}^{a} \otimes \operatorname{sp} \otimes \pi_{1}^{c}=\operatorname{det}^{a+1} \otimes\left(\operatorname{sp} \otimes \pi_{1}\right)^{c} \\
& =\operatorname{det}^{a+1} \otimes\left(\operatorname{det}^{a} \otimes \pi_{1}^{c}\right)^{c}=\operatorname{det} \otimes \pi_{1} .
\end{aligned}
$$

C'est impossible. Si maintenant $\operatorname{sp} \otimes \pi_{1}=\operatorname{det} \otimes \pi_{1}$, alors :

$$
\operatorname{sp} \otimes \pi_{1}^{c}=\operatorname{det} \otimes\left(\operatorname{sp} \otimes \pi_{1}\right)^{c}=\operatorname{det} \otimes\left(\operatorname{det} \otimes \pi_{1}^{c}\right)=\pi_{1}^{c} .
$$

Quitte à échanger $\pi_{1}$ et $\pi_{1}^{c}$, on peut donc supposer $\mathrm{sp} \otimes \pi_{1}=\pi_{1}$. Alors (iv) est vérifiée pour $\left(h_{1}, h_{2}\right)=\left( \pm \ell_{1}, \pm \ell_{2}\right)$.

Supposons maintenant $s=1$, i.e. $\ell_{2}=0$. Fixons, ainsi qu'il est loisible, une représentation irréductible $\Pi$ de $C O(V)^{0}$ dont la restriction à $G^{0}$ soit $\pi^{0}$. D'après [L2] lemme 8.9(ii), $\Pi$ se prolonge en une représentation de $C O(V)$. Notons $\pi$ la restriction à $G$ de ce prolongement. Alors $\pi$ prolonge $\pi^{0}$, donc $\operatorname{det} \otimes \pi \neq \pi$ par la théorie de Mackey. Puisque $\pi$ se prolonge à $C O(V)$, on a $\pi^{c}=\pi$. Posons $\pi\left(\ell_{1}, 0\right)=\pi$, $\pi\left(-\ell_{1}, 0\right)=\operatorname{det} \otimes \pi$. Ce sont les deux éléments de $\operatorname{Irr}_{\text {cusp }}(G, 1)$. Ils vérifient les relations (iii) et (v) de l'énoncé.

Supposons enfin $s=-1$, i.e. $\ell_{1}=0$. Comme ci-dessus, ce cas se déduit du cas $s=1$ par tensorisation par sp. Il suffit de poser $\pi\left(0, \ell_{2}\right)=\operatorname{sp} \otimes \pi\left(\ell_{2}, 0\right), \pi\left(0,-\ell_{2}\right)=$ $\mathrm{sp} \otimes \pi\left(-\ell_{2}, 0\right)$. Les propriétés (iii), (iv) et (v) de l'énoncé sont vérifiées.

Pour tout $s$ et tout $\pi^{0} \in \operatorname{Irr}\left(G^{0}, s\right)$, on a l'égalité $\pi^{0}(-1)=\operatorname{sp}(s)$ d'après un yoga standard de dualité. Or :

$$
\operatorname{sp}(s)=\eta\left(\widehat{V}^{-}\right) \zeta(-1)^{\operatorname{dim}\left(\widehat{V}^{-}\right) / 2}=(-\zeta(-1))^{\ell_{2}} .
$$

L'assertion (vi) s'en déduit.

En faisant varier $s$ parmi toutes les classes de conjugaison possibles, on obtient la proposition. QED

La démonstration montre que la bijection de l'énoncé n'est pas unique. On en fixe une, et cela pour tout $n$.

4.4. Soit $n$ un entier $\geqslant 1$. Posons :

$$
\operatorname{Quad}(O(2 n))=\operatorname{Quad}\left(O_{+}(2 n)\right) \cup \operatorname{Quad}\left(O_{-}(2 n)\right) .
$$


Soit $\left(\Lambda_{1}, \Lambda_{2}\right) \in \widetilde{\mathcal{S}} \widetilde{\mathcal{S}}_{n \text {,pair }}(c f .3 .6)$. Pour $j \in\{1,2\}$, on a associé en 2.2 un couple $\left(h_{j}, \rho_{j}\right)$ au symbole ordonné $\Lambda_{j}$. En posant $N_{j}=\operatorname{rg}\left(\Lambda_{j}\right)-h_{j}^{2}, \rho_{j}$ est une représentation irréductible de $W_{N_{j}}$. Posons $n_{0}=h_{1}^{2}+h_{2}^{2}$. La bijection de 4.3 nous fournit une représentation irréductible cuspidale $\pi\left(h_{1}, h_{2}\right)$ de $O_{\eta}(2 n)$, où $\eta=(-1)^{h_{1}-h_{2}}$ (dans le cas $n_{0}=0, \pi(0,0)$ est l'unique représentation irréductible de $\left.O_{+}(0)=\{1\}\right)$. Appliquons les constructions de 4.2 à $V_{\eta}(2 n), N_{1}, N_{2}$ et la représentation $\pi_{0}=\pi\left(h_{1}, h_{2}\right)$. On associe alors à $\rho_{1} \otimes \rho_{2}$ une sous-représentation irréductible de l'induite à $O_{\eta}(2 n)$ de la représentation $1_{N_{1}} \times \zeta_{N_{2}} \times \pi\left(h_{1}, h_{2}\right)$. On note $\pi\left(\Lambda_{1}, \Lambda_{2}\right)$ la classe de cette sousreprésentation.

\section{Proposition}

(a) Pour tout $\left(\Lambda_{1}, \Lambda_{2}\right) \in \widetilde{\mathcal{S}} \widetilde{\mathcal{S}}_{n \text {,pair }}$, on a $\pi\left(\Lambda_{1}, \Lambda_{2}\right) \in \operatorname{Quad}(O(2 n))$ et l'application :

$$
\begin{aligned}
& \widetilde{\mathcal{S}} \widetilde{\mathcal{S}}_{n, \text { pair }} \longrightarrow \operatorname{Quad}(O(2 n)) \\
& \left(\Lambda_{1}, \Lambda_{2}\right) \longmapsto \pi\left(\Lambda_{1}, \Lambda_{2}\right)
\end{aligned}
$$

est bijective.

(b) Pour tout $\left(\Lambda_{1}, \Lambda_{2}\right) \in \widetilde{\mathcal{S}} \widetilde{\mathcal{S}}_{n \text {,pair }}$, les propriétés suivantes sont vérifiées :

(i) $\pi\left(\Lambda_{1}, \Lambda_{2}\right) \in \mathrm{Quad}\left(O_{\eta}(2 n)\right)$, où $\eta=(-1)^{\left(\widetilde{\operatorname{def}}\left(\Lambda_{1}\right)-\widetilde{\operatorname{def}}\left(\Lambda_{2}\right)\right) / 2}$;

(ii) $\eta$ étant comme en (i), il existe un élément semi-simple $s \in S O\left(\widehat{V}_{\eta}(2 n)\right)$, ayant $2 \operatorname{rg}\left(\Lambda_{1}\right)$ valeurs propres 1 et $2 \operatorname{rg}\left(\Lambda_{2}\right)$ valeurs propres -1 , tel que $\pi\left(\Lambda_{1}, \Lambda_{2}\right) \in$ $\operatorname{Irr}\left(O_{\eta}(2 n), s\right)$

(iii) $\operatorname{det} \otimes \pi\left(\Lambda_{1}, \Lambda_{2}\right)=\pi\left(\sigma \Lambda_{1}, \sigma \Lambda_{2}\right)$;

(iv) $\operatorname{sp} \otimes \pi\left(\Lambda_{1}, \Lambda_{2}\right)=\pi\left(\Lambda_{2}, \Lambda_{1}\right)$;

(v) $\pi\left(\Lambda_{1}, \Lambda_{2}\right)^{c}=\pi\left(\Lambda_{1}, \sigma \Lambda_{2}\right)$;

(vi) $\pi\left(\Lambda_{1}, \Lambda_{2}\right)(-1)=(-1)^{\widetilde{\operatorname{def}}\left(\Lambda_{2}\right) / 2} \zeta(-1)^{\mathrm{rg}\left(\Lambda_{2}\right)}$.

Démonstration. - Soient $\eta \in\{ \pm 1\}$ et $\pi \in \operatorname{Irr}\left(O_{\eta}(2 n)\right)$. Grâce à Harish-Chandra, il existe un sous-groupe parabolique $\boldsymbol{P}=\boldsymbol{M U}$ de $\boldsymbol{O}_{\eta}(2 n)$, défini sur $\mathbb{F}_{q}$, et une représentation irréductible cuspidale $\pi_{M}$ de $M$ de sorte que $\pi$ soit sous-représentation de $\operatorname{Ind}_{P}^{O_{\eta}(2 n)}\left(\pi_{M}\right)$. On a un plongement naturel $\widehat{M}^{0} \rightarrow \widehat{S O}_{\eta}(2 n)$. Soit $s \in \widehat{M}^{0}$ tel que $\pi_{M} \in \operatorname{Irr}(M, s)$. D'après [L1], corollaire 6 , on a aussi $\pi \in \operatorname{Irr}\left(O_{\eta}(2 n), s\right)$. Donc $\pi$ est quadratique-unipotente si et seulement si $\pi_{M}$ l'est (cette notion étant définie pour le groupe $M$ de façon évidente). Un groupe $G L(m)$ n'a de représentation quadratiqueunipotente cuspidale que si $m=1$. Dans ce cas, il en a deux : les caractères 1 et $\zeta$. Donc $\pi$ est quadratique-unipotente si et seulement si $\boldsymbol{M}$ est de la forme :

$$
\boldsymbol{M}=\mathbb{G}_{m}^{n-n_{0}} \times \boldsymbol{O}_{\eta}\left(2 n_{0}\right),
$$

et, quitte à remplacer $\boldsymbol{P}$ par un groupe associé, $\pi_{M}$ est de la forme $1_{N_{1}} \times \zeta_{N_{2}} \times \pi_{0}$, où $\pi_{0} \in \operatorname{Quad}_{\text {cusp }}\left(O_{\eta}\left(2 n_{0}\right)\right)$. Autrement dit si et seulement si $\pi$ intervient dans l'une des induites étudiées en 4.2 , pour $\pi_{0}$ quadratique-unipotente. Le (a) de l'énoncé résulte alors de 4.2 et 4.3. Les propriétés (b)(i) et (b)(ii) résultent immédiatement de la description ci-dessus et des propriétés (i) et (ii) de la proposition 4.3. Les propriétés 
(b)(iii) à (b)(vi) résultent aussi, de façon moins immédiate, des propriétés correspondantes de cette proposition. Donnons la preuve de $(b)(v)$, en laissant les autres au lecteur.

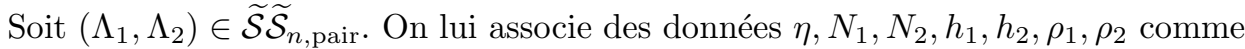
au début du paragraphe. Posons $\pi_{0}=\pi\left(h_{1}, h_{2}\right), \tilde{\pi}_{0}=\pi\left(h_{1},-h_{2}\right)$, notons $E_{0}$ et $\widetilde{E}_{0}$ leurs espaces. Comme en 4.1 et 4.2 , introduisons les représentations $(\pi, E)$, resp. $(\widetilde{\pi}, \widetilde{E})$, induites à $O_{\eta}(2 n)$ des représentations $1_{N_{1}} \times \zeta_{N_{2}} \times \pi_{0}$, resp. $1_{N_{1}} \times \zeta_{N_{2}} \times \widetilde{\pi}_{0}$, de $M$, où $\boldsymbol{M}=\mathbb{G}_{m}^{N_{1}+N_{2}} \times \boldsymbol{O}_{\eta}\left(2 n_{0}\right)$. Fixons maintenant $z \in \mathbb{F}_{q}^{\times}$tel que $\zeta(z)=-1$ et un élément $h_{0} \in C O_{\eta}\left(2 n_{0}\right)$ tel que $\lambda\left(h_{0}\right)=z$. Définissons l'élément $h \in C O_{\eta}(2 n)$ par :

$$
\begin{gathered}
h v_{i}=v_{i}, h v_{-i}=z v_{-i}, \text { pour tout } i \in\left\{1, \ldots, N_{1}+N_{2}\right\}, \\
\text { si } n_{0} \neq 0, \quad h_{\mid V_{0}}=h_{0}
\end{gathered}
$$

(les notations sont celles de 4.1). D'après la proposition 4.3(v), on peut fixer un isomorphisme $\varphi_{0}: E_{0} \rightarrow \widetilde{E}_{0}$ tel que :

$$
\varphi_{0} \circ \pi_{0}\left(h_{0} g_{0} h_{0}^{-1}\right)=\widetilde{\pi}_{0}\left(g_{0}\right) \circ \varphi_{0}
$$

pour tout $g_{0} \in O_{\eta}\left(2 n_{0}\right)$. On définit un isomorphisme $\varphi: E \rightarrow \widetilde{E}$ par :

$$
\varphi(f)(g)=\left(\varphi_{0} \circ f\right)\left(h g h^{-1}\right)
$$

pour tous $f \in E, g \in O_{\eta}(2 n)$. Il vérifie l'égalité :

$$
\varphi \circ \pi\left(h g h^{-1}\right)=\tilde{\pi}(g) \circ \varphi
$$

pour tout $g \in O_{\eta}(2 n)$. En 4.1 et 4.2 , on a introduit des opérateurs d'entrelacement dans $\operatorname{End}(E)$ et $\operatorname{End}(\widetilde{E})$. Pour les distinguer, notons $T_{j}(w), T_{j}^{\prime}(w)$ ceux de $\operatorname{End}(E)$ et $\widetilde{T}_{j}(w), \widetilde{T}_{j}^{\prime}(w)$ ceux de $\operatorname{End}(\widetilde{E})$. Soient $j \in\{1,2\}$ et $w \in W_{N_{j}}$, identifié à un élément de $O_{\eta}(2 n)$. On a $h^{-1} w^{-1} h w \in M$. Écrivons $h^{-1} w^{-1} h w=\left(t_{1}, \ldots, t_{N_{1}+N_{2}}, g_{0}\right)$, avec $t_{i} \in \mathbb{F}_{q}^{\times}$pour tout $i$ et $g_{0} \in O_{\eta}\left(2 n_{0}\right)$. Posons $\chi_{j}(w)=\prod_{i=N_{1}+1, \ldots, N_{1}+N_{2}} \zeta\left(t_{i}\right)$. Le calcul montre que :

$$
\varphi \circ T_{j}^{\prime}(w)=\chi_{j}(w) \widetilde{T}_{j}^{\prime}(w) \circ \varphi
$$

Donc $\widetilde{T}_{j}(w)$ est proportionnel à $\varphi \circ T_{j}(w) \circ \varphi^{-1}$. Soit $i \in\left\{1, \ldots, N_{j}-1\right\}$. Alors $\widetilde{T}_{j}\left(s_{i}\right)$ et $\varphi \circ T_{j}\left(s_{i}\right) \circ \varphi^{-1}$ vérifient tous deux l'équation $(T-q)(T+1)=0$, et ce ne sont pas des homothéties. Leur proportionnalité entraîne leur égalité. Le même raisonnement montre que le nombre $c_{j}$ associé en 4.2 à l'opérateur $T_{j}\left(s_{N_{j}}\right)$ est le même que celui associé à $\widetilde{T}_{j}\left(s_{N_{j}}\right)$. Si $c_{j}>0$, on a aussi l'égalité $\widetilde{T}_{j}\left(s_{N_{j}}\right)=\varphi \circ T_{j}\left(s_{N_{j}}\right) \circ \varphi^{-1}$. Si $c_{j}=0$, on obtient seulement $\widetilde{T}_{j}\left(s_{N_{j}}\right)= \pm \varphi \circ T_{j}\left(s_{N_{j}}\right) \circ \varphi^{-1}$. Mais, dans ce cas, $T_{j}\left(s_{N_{j}}\right)$, resp. $\widetilde{T}_{j}\left(s_{N_{j}}\right)$, est un multiple $>0$ de $T_{j}^{\prime}\left(s_{N_{j}}\right)$, resp. $\widetilde{T}_{j}^{\prime}\left(s_{N_{j}}\right)$. Grâce à $(2)$, le signe de l'égalité précédente est donc $\chi_{j}\left(s_{N_{j}}\right)$. Ce terme est égal à 1 si $j=1$, à -1 si $j=2$. 
D'où les relations :

$$
\begin{aligned}
& \varphi \circ T_{1}(w)=\widetilde{T}_{1}(w) \circ \varphi, \quad \text { pour tout } w \in W_{N_{1}}, \\
& \varphi \circ T_{2}(w)=\left\{\begin{array}{ll}
\widetilde{T}_{2}(w) \circ \varphi, & \text { si } c_{2}>0, \\
\operatorname{sgn}_{C D}(w) \widetilde{T}_{2}(w) \circ \varphi, & \text { si } c_{2}=0,
\end{array} \quad \text { pour tout } w \in W_{N_{2}}\right.
\end{aligned}
$$

Montrons que :

$$
c_{2}=0 \Longleftrightarrow h_{2}=0 .
$$

Si $n_{0}=0$, on a $c_{2}=h_{2}=0$. Supposons $n_{0}>0$. L'opérateur $T_{2}\left(s_{N_{2}}\right)$ vit dans le groupe spécial orthogonal. Il vit même dans la partie semi-simple d'un groupe de Lévi de ce dernier, de la forme $\mathbb{G}_{m}^{N_{1}+N_{2}-1} \times \boldsymbol{S O}_{\eta}\left(2 n_{0}+2\right)$. On peut donc supposer $n=n_{0}+1, N_{1}=0, N_{2}=1$, et travailler dans le groupe $\boldsymbol{S O}_{\eta}(2 n)$, en remplaçant $\pi_{0}$ par sa restriction à $S O_{\eta}\left(2 n_{0}\right)$. Soit $\Pi_{0}$ une représentation irréductible de $C O_{\eta}\left(2 n_{0}\right)^{0}$ dont la restriction à $S O_{\eta}\left(2 n_{0}\right)$ contienne $\pi_{0}$. Montrons que :

$$
(\zeta \circ \lambda) \otimes\left(\Pi_{0} \circ \operatorname{Ad}\left(x_{0}\right)\right)=\Pi_{0} \Longleftrightarrow h_{2} \neq 0 .
$$

Supposons $h_{1} h_{2} \neq 0$, i.e. l'élément $s$ paramétrant $\pi_{0}$ est $\neq \pm 1$. D'après [L2] lemme 8.9(ii), $\Pi_{0}$ se prolonge à $C O_{\eta}\left(2 n_{0}\right)$ et sa restriction à $S O_{\eta}\left(2 n_{0}\right)$ est réductible. Donc $\Pi_{0}=\Pi_{0} \circ \operatorname{Ad}\left(x_{0}\right)$ et $\Pi_{0}=(\zeta \circ \lambda) \otimes \Pi_{0}$. La relation (5) est vérifiée. Supposons maintenant $h_{1}=0, h_{2} \neq 0$. D'après le même lemme, on a cette fois $\Pi_{0} \neq \Pi_{0} \circ \operatorname{Ad}\left(x_{0}\right)$, $\Pi_{0} \neq(\zeta \circ \lambda) \otimes \Pi_{0}$. Il n'y a que deux représentations de $C O_{\eta}\left(2 n_{0}\right)^{0}$ dont la restriction à $S O_{\eta}\left(2 n_{0}\right)$ soit $\pi_{0}$ et dont le caractère central soit celui de $\Pi_{0}$, ce sont $\Pi_{0}$ et $(\zeta \circ \lambda) \otimes \Pi_{0}$. Or, puisque $\pi_{0} \circ \operatorname{Ad}\left(x_{0}\right)=\pi_{0}$ (on l'a vu dans la preuve de la proposition 4.3), la représentation $\Pi_{0} \circ \mathrm{Ad}\left(x_{0}\right)$ vérifie ces conditions. N'étant pas égale à $\Pi_{0}$, elle est égale à $(\zeta \circ \lambda) \otimes \Pi_{0}$, et (5) est vérifiée. Supposons enfin $h_{1} \neq 0, h_{2}=0$. Toujours d'après le même lemme, on a cette fois $\Pi_{0}=\Pi_{0} \circ \operatorname{Ad}\left(x_{0}\right), \Pi_{0} \neq(\zeta \circ \lambda) \otimes \Pi_{0}$, et (5) est encore vérifiée.

Notons $\Pi$ la représentation de $C O_{\eta}(2 n)^{0}$ induite de $\zeta_{1} \times \Pi_{0}$ comme en 4.1. Supposons $h_{2} \neq 0$. Grâce à (5) et 4.1(1), l'opérateur $T_{2}\left(s_{1}\right)$ se prolonge en un entrelacement de П. Alors, d'après [L2], proposition 8.3, on a $c_{2}>0$. Supposons maintenant $h_{2}=0$. Les mêmes arguments prouvent que $\Pi$ n'a pas d'entrelacements, donc est irréductible. Or sa restriction $\pi$ à $S O_{\eta}(2 n)$ est réductible, puisqu'elle possède l'entrelacement $T_{2}\left(s_{1}\right)$. Par la théorie de Mackey, $\pi$ se décompose en deux sous-représentations irréductibles, qui sont conjuguées par un élément de $C O_{\eta}(2 n)^{0}$, et qui ont donc même dimension. D'après $[\mathrm{C}]$ proposition $10.5 .5, p^{c_{2}}$ est égal au rapport de ces dimensions. Donc $c_{2}=0$. Cela démontre (4).

Notons $E^{\prime}$ la somme des sous- $O_{\eta}(2 n)$-modules de $E$ isomorphes à $\pi\left(\Lambda_{1}, \Lambda_{2}\right)$. Cet espace est stable par l'algèbre d'entrelacements de $E$ et isotypique pour l'action de cette algèbre, de type $\rho_{1} \times \rho_{2}$. D'après (1), $\varphi\left(E^{\prime}\right)$ est la somme des sous- $O_{\eta}(2 n)$-modules de $\widetilde{E}$ isomorphes à $\pi\left(\Lambda_{1}, \Lambda_{2}\right)^{c}$. D'après (3) et (4), $\varphi\left(E^{\prime}\right)$ est stable par l'algèbre d'entrelacements de $\widetilde{E}$ et isotypique pour l'action de cette algèbre, de type $\rho_{1} \times \rho_{2}$ si $h_{2} \neq 0$, 
de type $\rho_{1} \times\left(\operatorname{sgn}_{C D} \otimes \rho_{2}\right)$ si $h_{2}=0$. Donc $\pi\left(\Lambda_{1}, \Lambda_{2}\right)^{c}$ est paramétré par le couple de symboles ordonnés correspondant aux données $\left(h_{1}, \rho_{1}\right)$ et $\left(-h_{2}, \rho_{2}\right)$ si $h_{2} \neq 0$, aux données $\left(h_{1}, \rho_{1}\right)$ et $\left(0, \operatorname{sgn}_{C D} \otimes \rho_{2}\right)$ si $h_{2}=0$. Ce couple n'est autre que $\left(\Lambda_{1}, \sigma \Lambda_{2}\right)$, et cela démontre $(\mathrm{v})$.

Il est utile de considérer aussi le cas $n=0$. Par convention, $\pi(\Lambda(\varnothing), \Lambda(\varnothing))$ est la représentation triviale de $O_{+}(0)=\{1\}$ et $\operatorname{Quad}(O(0))$ est l'ensemble réduit à cette représentation.

4.5. Soit $n$ un entier $\geqslant 1$. On a défini en 2.4 les symboles ordonnés $\mathbf{1}_{+}(n), \mathbf{1}_{-}(n)$.

\section{LEMME}

(i) $\pi\left(\mathbf{1}_{+}(n), \Lambda(\varnothing)\right)$ est la représentation triviale de $O_{+}(2 n)$.

(ii) $\pi\left(\mathbf{1}_{-}(n), \Lambda(\varnothing)\right)$ est la représentation triviale de $O_{-}(2 n)$, pourvu qu'il en soit ainsi dans le cas où $n=1$

Démonstration. - Pour $\eta \in\{ \pm 1\}$, la droite des fonctions constantes sur $O_{\eta}(2 n)$ est sous-espace de la série principale issue du caractère trivial d'un Borel. Elle est stable par les opérateurs d'entrelacements. Le calcul de son paramétrage est immédiat, pourvu que l'on connaisse celle de la représentation cuspidale sous-jacente. Si $\eta=1$, celle-ci est une représentation du groupe $O_{+}(0)$, on n'a pas le choix pour la paramétrer. Si $\eta=-1$, il s'agit de la représentation triviale de $O_{-}(2)$. Les conditions imposées en 4.3 ne fixent pas le paramétrage de cette représentation, d'où la condition imposée dans le (ii) de l'énoncé.

4.6. Dans les paragraphes précédents, on a paramétré les représentations irréductibles quadratiques-unipotentes des groupes orthogonaux pairs. Un paramétrage analogue vaut pour les groupes spéciaux orthogonaux impairs et les groupes symplectiques. On les décrit brièvement dans les paragraphes suivants.

Soit $n \in \mathbb{N}$, posons $V=V(2 n+1), Q=Q(2 n+1), \eta=\eta(V)$. Les constructions de 4.1 et 4.2 s'adaptent à cette situation : les vecteurs $v_{i}$ vérifient les mêmes relations qu'en 4.1, on prend pour $x_{0}$ l'élément -1 de $O\left(V_{0}\right)$; le groupe $W$ est toujours égal à $W_{N_{1}} \times W_{N_{2}}$; pour $j \in\{1,2\}$ et $w \in W_{N_{j}}$, on choisit pour $A_{w}$ l'homothétie de rapport 1 si $j=1$ ou si $j=2$ et $\operatorname{sgn}_{C D}(w)=1$, de rapport $\eta \zeta(-1)^{n_{0}}$ si $j=2$ et $\operatorname{sgn}_{C D}(w)=-1$, où $n_{0}=\left(\operatorname{dim}\left(V_{0}\right)-1\right) / 2\left(\right.$ ce nombre $\eta \zeta(-1)^{n_{0}}$ est égal à $\left.\operatorname{sp}(w)\right)$. Alors les sous-représentations irréductibles de l'induite d'une représentation $1_{N_{1}} \times \zeta_{N_{2}} \times \pi_{0}$ sont paramétrées par les représentations irréductibles de $W_{N_{1}} \times W_{N_{2}}$.

\section{7.}

Proposition. - Il existe une bijection :

$$
\begin{aligned}
\left\{\left(h_{1}, h_{2}\right) \in \mathbb{N} \times \mathbb{N} ; h_{1}^{2}+h_{1}+h_{2}^{2}+h_{2}=n\right\} & \longrightarrow \operatorname{Quad}_{\text {cusp }}(S O(2 n+1)) \\
\left(h_{1}, h_{2}\right) & \longmapsto \pi\left(h_{1}, h_{2}\right)
\end{aligned}
$$


telle que les propriétés suivantes soient vérifiées pour tout $\left(h_{1}, h_{2}\right)$ dans l'ensemble de départ :

(i) soit s un élément de $S p(2 n)$ ayant $2 h_{1}^{2}+2 h_{1}$ valeurs propres 1 et $2 h_{2}^{2}+2 h_{2}$ valeurs propres -1 ; alors $\pi\left(h_{1}, h_{2}\right) \in \operatorname{Irr}(S O(2 n+1), s)$;

(ii) $\operatorname{sp} \otimes \pi\left(h_{1}, h_{2}\right)=\pi\left(h_{2}, h_{1}\right)$.

Cette bijection est unique.

4.8. On a introduit en 3.8 l'ensemble $\mathcal{S S}_{n, \text { imp. }}$ Comme en 4.4 , on associe à $\left(\Lambda_{1}, \Lambda_{2}\right) \in$ $\mathcal{S S}_{n, \text { imp }}$ une représentation $\pi\left(\Lambda_{1}, \Lambda_{2}\right)$ de $S O(2 n+1)$.

\section{Proposition}

(a) Pour tout $\left(\Lambda_{1}, \Lambda_{2}\right) \in \mathcal{S S}_{n, \text { imp }}$, on a $\pi\left(\Lambda_{1}, \Lambda_{2}\right) \in \operatorname{Quad}(S O(2 n+1))$ et l'application :

$$
\begin{aligned}
& \mathcal{S S}_{n, \text { imp }} \longrightarrow \operatorname{Quad}(S O(2 n+1)) \\
& \left(\Lambda_{1}, \Lambda_{2}\right) \longmapsto \pi\left(\Lambda_{1}, \Lambda_{2}\right)
\end{aligned}
$$

est bijective.

(b) Pour tout $\left(\Lambda_{1}, \Lambda_{2}\right) \in \mathcal{S S}_{n, \mathrm{imp}}$, les propriétés suivantes sont vérifiées :

(i) soit $s$ un élément semi-simple de $S p(2 n)$ ayant $2 \operatorname{rg}\left(\Lambda_{1}\right)$ valeurs propres 1 et $2 \operatorname{rg}\left(\Lambda_{2}\right)$ valeurs propres -1 ; alors $\pi\left(\Lambda_{1}, \Lambda_{2}\right) \in \operatorname{Irr}(S O(2 n+1), s)$;

(ii) $\operatorname{sp} \otimes \pi\left(\Lambda_{1}, \Lambda_{2}\right)=\pi\left(\Lambda_{2}, \Lambda_{1}\right)$.

4.9. Soit $n \in \mathbb{N}$, posons $V=V(2 n), Q=Q(2 n)$ (c'est une forme symplectique). Supposons donnés un entier $N \in \mathbb{N}$ et un sous-ensemble $\left\{v_{i} ; i \in\{ \pm 1, \ldots, \pm N\}\right\}$ de $V$ tel que, pour tous $i, j$,

$$
Q\left(v_{i}, v_{j}\right)= \begin{cases}0, & \text { si } i \neq-j, \\ 1, & \text { si } i=-j>0, \\ -1, & \text { si } i=-j<0 .\end{cases}
$$

On note $V_{0}$ le sous-espace de $V$ orthogonal à tous ces vecteurs. Soit $w \in W_{N}$. On note encore $w$ l'élément de $S p(V)$ tel que :

- pour tout $i \in\{1, \ldots, N\}, w v_{i}= \begin{cases}v_{w i}, & \text { si } w i>0, \\ -v_{w i}, & \text { si } w i<0\end{cases}$

- pour tout $i \in\{-1, \ldots,-N\}, w v_{i}=v_{w i}$;

$-w_{\mid V_{0}}=1$.

L'application ainsi définie de $W_{N}$ dans $S p(V)$ n'est plus un homomorphisme de groupes.

On définit comme en 4.1 les groupes $\boldsymbol{P}$ et $\boldsymbol{M}$ puis, si l'on se donne $N_{1}, N_{2}$ et $\pi_{0}$, la représentation $1_{N_{1}} \times \zeta_{N_{2}} \times \pi_{0}$ de $M$ et son induite $(\pi, E)$ à $S p(V)$. Pour tout $w \in$ $W_{N_{1}} \times W_{N_{2}}$, on définit l'opérateur d'entrelacement $T^{\prime}(w)$ de $E$ en posant $A_{w}=1$ dans la formule de 4.1. Le lemme 4.2 reste valable. Soit $j \in\{1,2\}$, supposons que l'entier 
$c_{j}$ de ce lemme soit nul. Alors $j=2$ et l'opérateur $T_{2}^{\prime}\left(s_{N_{2}}\right)^{2}$ est une homothétie. Mais, parce que l'injection de $W_{N}$ dans $S p(V)$ n'est pas un homomorphisme de groupes, son rapport n'est pas toujours réel $>0$. Il vaut $\zeta(-1) q^{2(n-N)+1}$. On a fixé en 3.9 une racine carrée $(\zeta(-1) q)^{1 / 2}$. On détermine la fonction $C_{2}$ par la condition $C_{2}\left(s_{N_{2}}\right)=$ $(\zeta(-1) q)^{-1 / 2} q^{N-n}$. On en déduit le paramétrage des sous-représentations irréductibles de $E$ par les représentations irréductibles de $W_{N_{1}} \times W_{N_{2}}$.

4.10. Soient $n$ un entier $\geqslant 1$ et $\pi \in \operatorname{Irr}(S p(2 n))$. L'opérateur $\pi(-1)$ est une homothétie, dont on note encore $\pi(-1)$ le rapport. D'autre part, on note $\pi^{c}$ la classe de la représentation $\pi \circ \operatorname{Ad}(h)$, où $h$ est un élément de $C S p(2 n)$ tel que $\zeta \circ \lambda(h)=-1$.

Proposition. - Il existe une bijection :

$$
\begin{aligned}
\left\{\left(h_{1}, h_{2}\right) \in \mathbb{N} \times \mathbb{Z} ; h_{1}^{2}+h_{1}+h_{2}^{2}=n\right\} & \longrightarrow \operatorname{Quad}_{\text {cusp }}(S p(2 n)) \\
\left(h_{1}, h_{2}\right) & \longmapsto \pi\left(h_{1}, h_{2}\right)
\end{aligned}
$$

telle que les propriétés suivantes soient vérifiées pour tout $\left(h_{1}, h_{2}\right)$ dans l'ensemble de départ :

(i) il existe un élément semi-simple $s \in S O(2 n+1)$, ayant $2 h_{1}^{2}+2 h_{1}+1$ valeurs propres 1 et $2 h_{2}^{2}$ valeurs propres -1 , tel que $\pi\left(h_{1}, h_{2}\right) \in \operatorname{Irr}(S p(2 n), s)$;

(ii) $\pi\left(h_{1}, h_{2}\right)^{c}=\pi\left(h_{1},-h_{2}\right)$;

(iii) $\pi\left(h_{1}, h_{2}\right)(-1)=(-\zeta(-1))^{h_{2}}$.

La bijection n'est pas unique. On en fixe une, et cela pour tout $n$.

4.11. On a introduit en 3.10 l'ensemble $\mathcal{S} \widetilde{\mathcal{S}}_{n, \text { mix }}$. Comme en 4.4 , on associe à $\left(\Lambda_{1}, \Lambda_{2}\right) \in \mathcal{S} \widetilde{\mathcal{S}}_{n, \text { mix }}$ une représentation $\pi\left(\Lambda_{1}, \Lambda_{2}\right)$ de $\operatorname{Sp}(2 n)$.

Proposition

(a) Pour tout $\left(\Lambda_{1}, \Lambda_{2}\right) \in \mathcal{S} \widetilde{\mathcal{S}}_{n, \text { mix }}$, on a $\pi\left(\Lambda_{1}, \Lambda_{2}\right) \in \operatorname{Quad}(S p(2 n))$ et l'application :

$$
\begin{aligned}
& \mathcal{S} \widetilde{\mathcal{S}}_{n, \text { mix }} \longrightarrow \operatorname{Quad}(S p(2 n)) \\
& \left(\Lambda_{1}, \Lambda_{2}\right) \longmapsto \pi\left(\Lambda_{1}, \Lambda_{2}\right)
\end{aligned}
$$

est bijective.

(b) Pour tout $\left(\Lambda_{1}, \Lambda_{2}\right) \in \mathcal{S} \widetilde{\mathcal{S}}_{n, \text { mix }}$, les propriétés suivantes sont vérifiées :

(i) il existe un élément semi-simple $s \in S O(2 n+1)$, ayant $2 \operatorname{rg}\left(\Lambda_{1}\right)+1$ valeurs propres 1 et $2 \operatorname{rg}\left(\Lambda_{2}\right)$ valeurs propres -1 , tel que $\pi\left(\Lambda_{1}, \Lambda_{2}\right) \in \operatorname{Irr}(\operatorname{Sp}(2 n), s)$;

(ii) $\pi\left(\Lambda_{1}, \Lambda_{2}\right)^{c}=\pi\left(\Lambda_{1}, \sigma \Lambda_{2}\right)$;

(iii) $\pi\left(\Lambda_{1}, \Lambda_{2}\right)(-1)=(-1)^{\widetilde{\operatorname{def}}\left(\Lambda_{2}\right) / 2} \zeta(-1)^{\mathrm{rg}\left(\Lambda_{2}\right)}$. 


\section{CHAPITRE 5}

\section{LES THÉORÈMES}

5.1. On considère les trois cas des groupes orthogonaux pairs, des groupes spéciaux orthogonaux impairs et des groupes symplectiques.

Dans le cas orthogonal pair, on a fixé en 4.3 une bijection :

$$
\left\{\left(h_{1}, h_{2}\right) \in \mathbb{Z} \times \mathbb{Z} ; h_{1}^{2}+h_{2}^{2}=n\right\} \longrightarrow \operatorname{Quad}_{\text {cusp }}(O(2 n)),
$$

pour tout entier $n>0$. Notons-la $\pi_{n, \text { cusp. }}$. On en a déduit en 4.4 une bijection $\widetilde{\mathcal{S}} \widetilde{\mathcal{S}}_{n, \text { pair }} \rightarrow \operatorname{Quad}(O(2 n))$, dont on déduit une application linéaire :

$$
\pi_{n}: \mathbb{Q}\left[\widetilde{\mathcal{S}}_{n, \text { pair }}\right] \longrightarrow \mathbb{Q}[\operatorname{Quad}(O(2 n))] \subset \mathcal{C}(O(2 n)) .
$$

En 3.5, on a défini une application :

$$
\begin{aligned}
& \widetilde{\mathcal{S}} \widetilde{\mathcal{S}}_{n, \text { pair }} \longrightarrow \mathcal{C}(O(2 n)) \\
& \left(\Lambda_{1}, \Lambda_{2}\right) \longmapsto k\left(\Lambda_{1}, \Lambda_{2}\right) .
\end{aligned}
$$

Par linéarité, on en déduit une application $\mathbb{Q}$-linéaire :

$$
k_{n}: \mathbb{Q}\left[\widetilde{\mathcal{S}} \widetilde{\mathcal{S}}_{n, \text { pair }}\right] \longrightarrow \mathcal{C}(O(2 n)) .
$$

D'autre part, on a l'égalité :

$$
\mathbb{Q}\left[\widetilde{\mathcal{S}} \widetilde{\mathcal{S}}_{n, \text { pair }}\right]=\oplus\left(\mathbb{Q}\left[\widetilde{\mathcal{S}}_{n_{1}, \text { pair }}\right] \otimes_{\mathbb{Q}} \mathbb{Q}\left[\widetilde{\mathcal{S}}_{n_{2}, \text { pair }}\right]\right)
$$

où l'on somme sur les $n_{1}, n_{2} \in \mathbb{N}$ tels que $n_{1}+n_{2}=n$. On a défini en 2.1 une involution $\mathcal{F}$ de chacun des termes $\mathbb{Q}\left[\widetilde{\mathcal{S}}_{n_{j} \text {,pair }}\right]$. Par tensorisation puis sommation, on en déduit une involution, que l'on note encore $\mathcal{F}$, de $\mathbb{Q}\left[\widetilde{\mathcal{S}} \widetilde{\mathcal{S}}_{n \text {,pair }}\right]$.

Dans le cas des groupes spéciaux orthogonaux impairs, on n'a plus besoin de fixer $\pi_{n, \text { cusp }}$, cette bijection étant uniquement déterminée. On définit comme ci-dessus des applications $\mathbb{Q}$-linéaires :

$$
\begin{aligned}
& \pi_{n}: \mathbb{Q}\left[\mathcal{S S}_{n, \mathrm{imp}}\right] \longrightarrow \mathbb{Q}[\operatorname{Quad}(S O(2 n+1))] \subset \mathcal{C}(S O(2 n+1)), \\
& k_{n}: \mathbb{Q}\left[\mathcal{S S}_{n, \mathrm{imp}}\right] \longrightarrow \mathcal{C}(S O(2 n+1)),
\end{aligned}
$$

et une involution $\mathcal{F}$ de l'espace $\mathbb{Q}\left[\mathcal{S} \mathcal{S}_{n, \text { imp }}\right]$. 
Dans le cas des groupes symplectiques, on fixe derechef une bijection :

$$
\pi_{n, \text { cusp }}:\left\{\left(h_{1}, h_{2}\right) \in \mathbb{N} \times \mathbb{Z} ; h_{1}^{2}+h_{1}+h_{2}^{2}=n\right\} \longrightarrow \operatorname{Quad}_{\text {cusp }}(S p(2 n)) .
$$

On définit ensuite des applications $\mathbb{Q}$-linéaires :

$$
\begin{aligned}
& \pi_{n}: \mathbb{Q}\left[\mathcal{S} \widetilde{\mathcal{S}}_{n, \text { mix }}\right] \longrightarrow \mathbb{Q}[\operatorname{Quad}(S p(2 n))] \subset \mathcal{C}(S p(2 n)), \\
& k_{n}: \mathbb{Q}\left[\mathcal{S} \widetilde{\mathcal{S}}_{n, \text { mix }}\right] \longrightarrow \mathcal{C}(S p(2 n)),
\end{aligned}
$$

ainsi qu'une involution $\mathcal{F}$ de l'espace $\mathbb{Q}\left[\mathcal{S} \widetilde{\mathcal{S}}_{n, \text { mix }}\right]$.

THÉORÈme. - Dans le cas des groupes spéciaux orthogonaux impairs, on a l'égalité $k_{n}=\pi_{n} \circ \mathcal{F}$ pour tout entier $n \in \mathbb{N}$ tel que $q>2 n$. Dans les cas des groupes orthogonaux pairs ou symplectiques, on peut choisir la famille $\left(\pi_{n, \mathrm{cusp}}\right)_{n \geqslant 1}$ de sorte que l'on ait l'égalité $k_{n}=\pi_{n} \circ \mathcal{F}$ pour tout entier $n \in \mathbb{N}$ tel que $q>2 n$.

\section{2 .}

Corollaire. - Soit $n \in \mathbb{N}$ tel que $q>2 n$. La famille formée des $k\left(\Lambda_{1}, \Lambda_{2}\right)$, pour $\left(\Lambda_{1}, \Lambda_{2}\right) \in \widetilde{\mathcal{S}} \widetilde{\mathcal{S}}_{n, \text { pair }}$, resp. $\mathcal{S} \mathcal{S}_{n, \text { imp }}, \mathcal{S} \widetilde{\mathcal{S}}_{n, \text { mix }}$, est une base sur $\mathbb{Q}$ de l'espace $\mathbb{Q}[\operatorname{Quad}(O(2 n))]$, resp. $\mathbb{Q}[\operatorname{Quad}(S O(2 n+1))], \mathbb{Q}[\operatorname{Quad}(S p(2 n))]$.

5.3. Dans les paragraphes 6 à 12, on présente la démonstration du cas orthogonal pair. On ne dira rien de celle du cas orthogonal impair, qui est similaire (il n'y a quasiment rien à faire, à partir des résultats de Lusztig). Au paragraphe 13, on donnera des indications sur les quelques modifications à apporter à la démonstration dans le cas symplectique.

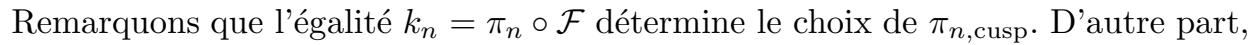
les seuls choix pouvant influer sur cette égalité sont ceux de $\pi_{n^{\prime}, \text { cusp }}$ pour $n^{\prime} \leqslant n$. On démontre le théorème par récurrence sur $n$. On fixe désormais, pour les paragraphes 6 à 12 , un entier $n \geqslant 1$ tel que $q>2 n$. On suppose effectués les choix de $\pi_{n^{\prime} \text {,cusp }}$ pour $n^{\prime}<n$, de sorte que l'assertion du théorème soit vraie pour les groupes $\boldsymbol{O}\left(2 n^{\prime}\right)$ quand $n^{\prime}<n$. On suppose également l'assertion du théorème vraie pour les groupes $\boldsymbol{S O}\left(2 n^{\prime}+1\right)$ quand $n^{\prime}<n$. 


\section{CHAPITRE 6}

\section{DÉTERMINATION DES FAISCEAUX-CARACTĖRES QUADRATIQUES-UNIPOTENTS}

On prouve que les fonctions-traces des faisceaux-caractères introduits au paragraphe 3 engendrent le même espace que les traces de représentations irréductibles quadratiques-unipotentes. Cela permet de définir l'automorphisme $i_{n}$ de l'espace $\mathbb{C}\left[\widetilde{\mathcal{S}} \widetilde{\mathcal{S}}_{n, \text { pair }}\right]$ par la formule $i_{n}=\pi_{n}^{-1} \circ k_{n} \circ \mathcal{F}$. La conjecture de Lusztig revient à dire que $i_{n}$ est l'identité, ce que l'on prouvera aux paragraphes 11 et 12 .

6.1. On aura besoin du lemme suivant, qui résulte de la théorie de Clifford.

Soient $K$ un groupe fini et $H$ un sous-groupe distingué de $K$. Le groupe $K$ agit naturellement sur l'ensemble $\operatorname{Irr}(H)$ des $H$-modules irréductibles. On note cette action $(k, M) \mapsto{ }^{k} M$ et on l'appelle conjugaison par $K$. Pour $M \in \operatorname{Irr}(H)$, on note $K_{M}=$ $\left\{k \in K ;{ }^{k} M=M\right\}$.

Soient $H_{1}, H_{2}$ deux groupes finis, posons $H=H_{1} \times H_{2}$. Pour $i=1,2$, soit $\mathcal{M}_{i}$ un sous-ensemble de $\operatorname{Irr}\left(H_{i}\right)$, et soit $R$ un $H$-module. On dit que $R$ détermine une bijection entre $\mathcal{M}_{1}$ et $\mathcal{M}_{2}$ s'il existe un sous-ensemble $\mathcal{M} \operatorname{de} \operatorname{Irr}(H)$ et, pour $i=1,2$, une bijection $\varphi_{i}: \mathcal{M} \rightarrow \mathcal{M}_{i}$ de sorte que :

$$
\left\{\begin{array}{l}
\text { pour tout } M \in \mathcal{M}, M=\varphi_{1}(M) \otimes \varphi_{2}(M) ; \\
R=\oplus_{M \in \mathcal{M}} M .
\end{array}\right.
$$

Venons-en maintenant aux hypothèses du lemme. Pour $i=1,2$, soient $K_{i}$ un groupe fini, $H_{i}$ un sous-groupe distingué, $\mathcal{M}_{i}$ un sous-ensemble de $\operatorname{Irr}\left(H_{i}\right)$. On note $\mathcal{N}_{i}$ le sous-ensemble des éléments de $\operatorname{Irr}\left(K_{i}\right)$ dont la restriction à $H_{i}$ contient un sousmodule irréductible appartenant à $\mathcal{M}_{i}$. Posons $K=K_{1} \times K_{2}, H=H_{1} \times H_{2}$. Pour $i=1,2$, on note $p r_{i}$ la projection de $K \operatorname{sur} K_{i}$ et $\overline{p r}_{i}$ celle de $K / H$ sur $K_{i} / H_{i}$. Soient $\Delta$ un sous-groupe de $K$ contenant $H$ et $R$ un $\Delta$-module. Posons $S=\operatorname{Ind}_{\Delta}^{K}(R)$.

Lemme. - On suppose vérifiées les hypothèses suivantes :

(i) pour $i=1,2, K_{i} / H_{i}$ est cyclique;

(ii) pour $i=1,2$, soient $M \in \mathcal{M}_{i}$ et $k \in K_{i} ; s i{ }^{k} M \in \mathcal{M}_{i}$, alors $k \in \operatorname{pr}_{i}(\Delta)$;

(iii) pour $i=1,2$, la restriction de $\overline{p r}_{i} \grave{a} \Delta / H$ est injective;

(iv) $R$, vu comme $H$-module, détermine une bijection entre $\mathcal{M}_{1}$ et $\mathcal{M}_{2}$.

Alors $S$ détermine une bijection entre $\mathcal{N}_{1}$ et $\mathcal{N}_{2}$. 
REmARQue. - On se convainc facilement que les hypothèses (ii) et (iii) sont nécessaires à la validité de la conclusion.

Démonstration. - Introduisons le sous-ensemble $\mathcal{M}$ de $\operatorname{Irr}(H)$ et les bijections $\varphi_{i}$ vérifiant (1). L'action de $\Delta$ sur $R$ détermine sur tout élément $M$ de $\mathcal{M}$ une action de $\Delta_{M}$. Fixons un ensemble de représentants $\mathcal{M}_{\Delta}$ des classes de conjugaison par $\Delta$ dans $\mathcal{M}$. On a alors les égalités :

$$
\begin{gathered}
\mathcal{M}=\sqcup_{M \in \mathcal{M}_{\Delta}} \sqcup_{\delta \in \Delta / \Delta_{M}}{ }^{\delta} M, \\
R=\oplus_{M \in \mathcal{M}_{\Delta}} \operatorname{Ind}_{\Delta_{M}}^{K}(M) .
\end{gathered}
$$

Fixons $M \in \mathcal{M}_{\Delta}$, pour $i=1,2$ posons $M_{i}=\varphi_{i}(M)$. On a :

$$
\overline{p r}_{i} \text { se restreint en un isomorphisme de } \Delta_{M} / H \text { sur } K_{i, M_{i}} / H_{i} \text {. }
$$

On a évidemment l'inclusion $p r_{i}\left(\Delta_{M}\right) \subseteq K_{i, M_{i}}$. Soit $k_{i} \in K_{i, M_{i}}$. D'après (ii), il existe $\delta \in \Delta$ tel que $k_{i}=p r_{i}(\delta)$. Alors $M$ et ${ }^{\delta} M$ appartiennent à $\mathcal{M}$ et on a $\varphi_{i}\left({ }^{\delta} M\right)=$ ${ }^{k_{i}} M_{i}=M_{i}=\varphi_{i}(M)$. Puisque $\varphi_{i}$ est injective, cela entraîne ${ }^{\delta} M=M$, i.e. $\delta \in \Delta_{M}$. Cela démontre l'égalité $p r_{i}\left(\Delta_{M}\right)=K_{i, M_{i}}$. Alors $\overline{p r}_{i}$ se restreint en une surjection de $\Delta_{M} / H$ sur $K_{i, M_{i}} / H_{i}$. Cette restriction est injective d'après (iii).

Posons $D=\Delta_{M} / H$, que l'on identifie par (3) à $K_{i, M_{i}} / H_{i}$. Grâce à (i), on peut utiliser la théorie de Clifford. Le $H_{i}$-module $M_{i}$ admet $|D|$ prolongements distincts à $K_{i, M_{i}}$, qui se déduisent l'un de l'autre par torsion par un caractère linéaire de $D$. Fixons-en un $\widetilde{M}_{i}$ et, pour $\chi \in \operatorname{Hom}\left(D, \mathbb{C}^{\times}\right)$, notons $\widetilde{M}_{i}(\chi)$ le module tordu. Chacun des modules $\operatorname{Ind}_{K_{i, M_{i}}}^{K_{i}}\left(\widetilde{M}_{i}(\chi)\right)$ est irréductible et ils sont tous distincts. Notons $\mathcal{N}\left(M_{i}\right)$ l'ensemble des $K_{i}$-modules irréductibles dont la restriction à $H_{i}$ contient $M_{i}$. On a l'égalité :

$$
\mathcal{N}\left(M_{i}\right)=\left\{\operatorname{Ind}_{K_{i, M_{i}}}^{K_{i}}\left(\widetilde{M}_{i}(\chi)\right) ; \chi \in \operatorname{Hom}\left(D, \mathbb{C}^{\times}\right)\right\} .
$$

Pour $\chi_{1}, \chi_{2} \in \operatorname{Hom}\left(D, \mathbb{C}^{\times}\right)$, la restriction à $\Delta_{M}$ de $\widetilde{M}_{1}\left(\chi_{1}\right) \otimes \widetilde{M}_{2}\left(\chi_{2}\right)$ ne dépend que du produit $\chi_{1} \chi_{2}$. Quand $\chi_{1}$ et $\chi_{2}$ varient, on obtient tous les $\Delta_{M}$-modules prolongeant le $H$-module $M$. Comme on l'a remarqué ci-dessus, un tel prolongement est donné par hypothèse. Quitte à changer les paramétrages, on peut supposer que c'est la restriction à $\Delta_{M}$ de $\widetilde{M}_{1} \otimes \widetilde{M}_{2}$. On a alors les égalités :

$$
\operatorname{Ind}_{\Delta_{M}}^{K_{M}}(M)=\oplus_{\chi \in \operatorname{Hom}\left(D, \mathbb{C}^{\times}\right)}\left(\widetilde{M}_{1}(\chi) \otimes \widetilde{M}_{2}\left(\chi^{-1}\right)\right),
$$

puis :

$$
\operatorname{Ind}_{\Delta_{M}}^{K}(M)=\oplus_{\chi \in \operatorname{Hom}\left(D, \mathbb{C}^{\times}\right)}\left(\operatorname{Ind}_{K_{1, M_{1}}}^{K_{1}}\left(\widetilde{M}_{1}(\chi)\right) \otimes \operatorname{Ind}_{K_{2, M_{2}}}^{K_{2}}\left(\widetilde{M}_{2}\left(\chi^{-1}\right)\right)\right) .
$$

Cela montre que le $K$-module $\operatorname{Ind}_{\Delta_{M}}^{K}(M)$ détermine une bijection entre $\mathcal{N}\left(M_{1}\right)$ et $\mathcal{N}\left(M_{2}\right)$.

Compte tenu de (2), il reste à prouver que, pour $i=1,2, \mathcal{N}_{i}$ est la réunion disjointe des $\mathcal{N}\left(\varphi_{i}(M)\right)$ quand $M$ décrit $\mathcal{M}_{\Delta}$. Soit $N_{i} \in \mathcal{N}_{i}$. Sa restriction à $H_{i}$ contient un élément, disons $M_{i}$, de $\mathcal{M}_{i}$. Soit $M$ l'élément de $\mathcal{M}_{\Delta}$ qui est conjugué par $\Delta$ à 
$\varphi_{i}^{-1}\left(M_{i}\right)$. Alors $\varphi_{i}(M)$ est conjugué par $K_{i}$ à $M_{i}$, donc $N_{i}$ contient $\varphi_{i}(M)$, i.e. $N_{i} \in$ $\mathcal{N}\left(\varphi_{i}(M)\right)$. Si maintenant $M^{\prime}$ est un autre élément de $\mathcal{M}_{\Delta}$ tel que $N_{i} \in \mathcal{N}\left(\varphi_{i}\left(M^{\prime}\right)\right)$, la restriction de $N_{i}$ à $H_{i}$ contient les deux $H_{i}$-modules $\varphi_{i}(M)$ et $\varphi_{i}\left(M^{\prime}\right)$. Il existe donc $k_{i} \in K_{i}$ tel que $\varphi_{i}\left(M^{\prime}\right)={ }^{k_{i}} \varphi_{i}(M)$. D'après (ii), il existe $\delta \in \Delta$ tel que $k_{i}=p r_{i}(\delta)$. Alors ${ }^{\delta} M \in \mathcal{M}$ et $\varphi_{i}\left({ }^{\delta} M\right)={ }^{k_{i}} \varphi_{i}(M)=\varphi_{i}\left(M^{\prime}\right)$. Puisque $\varphi_{i}$ est injective, ${ }^{\delta} M=M^{\prime}$. Mais $\mathcal{M}_{\Delta}$ est un ensemble de représentants des classes de conjugaison par $\Delta$. L'égalité ci-dessus entraîne $M=M^{\prime}$. Cela achève la démonstration.

6.2. Soit $\eta \in\{ \pm 1\}$, posons $\boldsymbol{G}=\boldsymbol{O}_{\eta}(2 n), \widehat{\boldsymbol{G}}=\widehat{\boldsymbol{O}}_{\eta}(2 n)$. Soit $s$ un élément semi-simple de $\widehat{G}^{0}$. Notons $\widehat{\boldsymbol{H}}$ son commutant dans $\widehat{\boldsymbol{G}}, \boldsymbol{Z}_{\widehat{\boldsymbol{H}}}$ le centre de $\widehat{\boldsymbol{H}}, \widehat{\boldsymbol{L}}$ le commutant de $\boldsymbol{Z}_{\widehat{\boldsymbol{H}}}^{0}$ dans $\widehat{\boldsymbol{G}}$. Le groupe $\widehat{\boldsymbol{L}}$ est un groupe de Lévi de $\widehat{\boldsymbol{G}}$ contenant $\widehat{\boldsymbol{H}}$. C'est le plus petit groupe vérifiant ces conditions. Il est défini sur $\mathbb{F}_{q}$ mais, en général, il n'existe pas de sous-groupe parabolique de $\widehat{\boldsymbol{G}}$, défini sur $\mathbb{F}_{q}$, dont $\widehat{\boldsymbol{L}}$ soit un sous-groupe de Lévi.

On peut associer à $\widehat{\boldsymbol{L}}$ un groupe de Lévi $\boldsymbol{L}$ de $\boldsymbol{G}$, défini sur $\mathbb{F}_{q}$, dont $\widehat{\boldsymbol{L}}$ soit le groupe dual. Ce groupe $\boldsymbol{L}$ est unique à conjugaison près par $G$. Cela résulte de constructions générales. Ici, il suffit d'identifier $\boldsymbol{G}$ à $\widehat{\boldsymbol{G}}$, alors $\boldsymbol{L}=\widehat{\boldsymbol{L}}$.

Concrètement, posons $\widehat{V}=\widehat{V}_{\eta}(2 n)$. Pour $\varepsilon \in\{ \pm 1\}$, notons $\widehat{V}^{\varepsilon}$ le sous-espace de $\widehat{V}$, propre pour l'action de $s$, associé à la valeur propre $\varepsilon$. Posons $\widehat{V}^{ \pm}=\widehat{V}^{+} \oplus \widehat{V}^{-}$, notons $\widehat{V}^{\neq \pm}$l'orthogonal de $\widehat{V}^{ \pm}$. Le groupe $\widehat{\boldsymbol{L}}$ se décompose en produit $\widehat{\boldsymbol{L}}=\widehat{\boldsymbol{L}}^{\neq \pm} \times \boldsymbol{O}\left(\widehat{V}^{ \pm}\right)$, où $\widehat{\boldsymbol{L}}^{\neq \pm}$est un groupe de Lévi de $\boldsymbol{S} \boldsymbol{O}\left(\widehat{V}^{\neq \pm}\right)$. Ce groupe $\widehat{\boldsymbol{L}}^{\neq \pm}$est lui-même un produit :

$$
\widehat{\boldsymbol{L}}^{\neq \pm}=\widehat{\boldsymbol{L}}_{1} \times \cdots \times \widehat{\boldsymbol{L}}_{t}
$$

où, pour tout $i \in\{1, \ldots, t\}$, il existe des entiers $n_{i}, f_{i} \geqslant 1$ tels que :

$$
\widehat{\boldsymbol{L}}_{i} \simeq \operatorname{Res}_{\mathbb{F}_{q} f_{i} / \mathbb{F}_{q}} \boldsymbol{G} \boldsymbol{L}\left(n_{i}\right), \text { ou } \widehat{\boldsymbol{L}}_{i} \simeq \operatorname{Res}_{\mathbb{F}_{q} f_{i} / \mathbb{F}_{q}} \boldsymbol{U}\left(n_{i}\right) .
$$

On a noté $\boldsymbol{U}\left(n_{i}\right)$ le groupe unitaire d'un espace de dimension $n_{i}$ sur $\mathbb{F}_{q^{2 f_{i}}}$, muni d'une forme non dégénérée, hermitienne relativement à l'extension $\mathbb{F}_{q^{2 f_{i}}} / \mathbb{F}_{q^{f_{i}}}$. L'élément $s$, qui appartient à $\widehat{L}$, se décompose conformément en $s=s_{1} \times \cdots \times s_{t} \times s^{ \pm}$. Notons que $s^{ \pm}$n'a pour valeurs propres que \pm 1 et que, pour tout $i \in\{1, \ldots, t\}, s_{i}$ est central dans $\widehat{\boldsymbol{L}}_{i}$. Le groupe $\boldsymbol{L}$ se décompose dualement en produit :

$$
\boldsymbol{L}=\boldsymbol{L}_{1} \times \cdots \times \boldsymbol{L}_{t} \times \boldsymbol{O}\left(V^{ \pm}\right) .
$$

Soit $s^{\prime}$ un autre élément semi-simple de $\widehat{G}^{0}$, supposons $s^{\prime}$ quadratiquement équivalent à $s$ (cf. 1.9). Quitte à conjuguer $s^{\prime}$ par un élément de $\widehat{G}$, on peut supposer que $s$ et $s^{\prime}$ ont même groupe $\boldsymbol{L}$ associé et que $s_{i}^{\prime}=s_{i}$ pour tout $i \in\{1, \ldots, t\}$. Quand $s^{\prime}$ décrit toutes les classes de conjugaison d'éléments quadratiquement équivalents à $s$, la composante $s^{\prime \pm}$ décrit toutes les classes de conjugaison par $O\left(\widehat{V}^{ \pm}\right)$d'éléments quadratiquement équivalents à $s^{ \pm}$(ou à 1 , puisque $s^{ \pm}$est quadratiquement équivalent à 1). 
6.3. On conserve les hypothèses précédentes. On a défini en 1.3 les ensembles $\operatorname{Irr}(L, s)$ et $\operatorname{Irr}(G, s)$. Fixons un sous-groupe parabolique $\boldsymbol{P}=\boldsymbol{L} \boldsymbol{U}$ de $\boldsymbol{G}$. En général, il n'est pas défini sur $\mathbb{F}_{q}$. Ce groupe étant choisi, on a défini en 1.4 l'application linéaire :

$$
R_{L}^{G}: \mathbb{Z}[\operatorname{Irr}(L)] \longrightarrow \mathbb{Z}[\operatorname{Irr}(G)] .
$$

Lemme. - Cette application se restreint en un isomorphisme de $\mathbb{Z}[\operatorname{Irr}(L, s)]$ sur $\mathbb{Z}[\operatorname{Irr}(G, s)]$.

L'analogue de ce lemme pour un groupe connexe est bien connu, $c f$. par exemple [DM1] théorème 13.25.

Démonstration. - Posons $D_{L}=\boldsymbol{L} / \boldsymbol{L}^{0}=\widehat{\boldsymbol{L}} / \widehat{\boldsymbol{L}}^{0}, D_{G}=\boldsymbol{G} / \boldsymbol{G}^{0}=\widehat{\boldsymbol{G}} / \widehat{\boldsymbol{G}}^{0}$. On identifie de façon naturelle $D_{L}$ à un sous-groupe de $D_{G}$. Notons $S_{L}$ le sous-groupe des éléments de $D_{L}$ qui conservent la classe de conjugaison de $s$ par $\widehat{\boldsymbol{L}}^{0}$. Définissons de façon analogue le sous-groupe $S_{G}$ de $D_{G}$. Parce que $\boldsymbol{Z}_{\widehat{\boldsymbol{G}}}(s) \subseteq \widehat{\boldsymbol{L}}$, on a l'égalité $S_{G}=S_{L}$.

Introduisons les variétés :

$$
\begin{aligned}
\boldsymbol{X} & =\left\{x(\boldsymbol{U} \cap F(\boldsymbol{U})) \in \boldsymbol{G} /(\boldsymbol{U} \cap F(\boldsymbol{U})) ; x^{-1} F(x) \in F(\boldsymbol{U})\right\}, \\
\boldsymbol{X}^{0} & =\left\{x(\boldsymbol{U} \cap F(\boldsymbol{U})) \in \boldsymbol{G}^{0} /(\boldsymbol{U} \cap F(\boldsymbol{U})) ; x^{-1} F(x) \in F(\boldsymbol{U})\right\} .
\end{aligned}
$$

La variété $\boldsymbol{X}^{0}$ est une composante connexe de $\boldsymbol{X}$. Le groupe $G \times L$, resp. $G^{0} \times L^{0}$, agit sur $\boldsymbol{X}$, resp. $\boldsymbol{X}^{0}, c f$. 1.4. En fait, posons :

$$
\Delta=\left\{(g, \ell) \in G \times L ; g \ell^{-1} \in G^{0}\right\} .
$$

Alors $\Delta$ est le stabilisateur dans $G \times L$ de la composante connexe $\boldsymbol{X}^{0}$. En particulier, ce groupe agit sur $\boldsymbol{X}^{0}$, la restriction de cette action au sous-groupe $G^{0} \times L^{0}$ de $\Delta$ étant l'action déjà introduite. La variété $\boldsymbol{X}$ étant réunion disjointe des $(g, \ell) \boldsymbol{X}^{0}$, quand $(g, \ell)$ décrit $(G \times L) / \Delta$, on en déduit que pour tout $i \in \mathbb{N}$, les $G \times L$-modules $H_{c}^{i}(\boldsymbol{X})$ et $\operatorname{Ind}_{\Delta}^{G \times L}\left(H_{c}^{i}\left(\boldsymbol{X}^{0}\right)\right)$ sont isomorphes.

Rappelons les résultats concernant le groupe connexe $G^{0}$, cf. [DM1] théorème 13.25 et $[\mathbf{B R}]$ lemme 12.6. Pour tout module $E$ sur l'un de nos groupes $G, L$ etc., on note $E^{\vee}$ le module contragrédient, et pour tout ensemble $\mathcal{E}$ de modules, on note $\breve{\mathcal{E}}=\{\check{E} ; E \in \mathcal{E}\}$. Posons $\delta=\operatorname{dim}(\boldsymbol{U})-\operatorname{dim}(\boldsymbol{U} \cap F(\boldsymbol{U}))$. Pour $i \in \mathbb{N} \backslash\{\delta\}$, le $L$-module

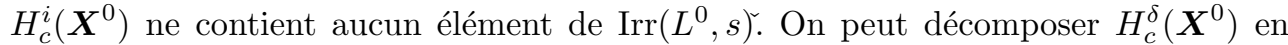
somme directe de deux $G^{0} \times L^{0}$-modules $H_{s}$ et $R_{s}$ tels que :

- le $L^{0}$-module $H_{s}$ ne contient aucun élément de $\operatorname{Irr}\left(L^{0}, s\right)^{\text {; }}$;

- $R_{s}$ détermine une bijection entre $\operatorname{Irr}\left(G^{0}, s\right)$ et $\operatorname{Irr}\left(L^{0}, s\right)^{\curlyvee}(c f .6 .1)$.

Fixons une famille de représentants $\left(s_{j}\right)_{j \in J}$ des classes de conjugaison par $\widehat{L}^{0}$ dans la classe de conjugaison par $\widehat{L}$. Dans les assertions ci-dessus, on peut remplacer $s$ par chacun des $s_{j}$. Soient $j, k$ deux éléments distincts de $J$. Par définition, les ensembles $\operatorname{Irr}\left(L^{0}, s_{j}\right)$ et $\operatorname{Irr}\left(L^{0}, s_{k}\right)$ sont disjoints. De l'égalité $S_{G}=S_{L}$ résulte que $s_{j}$ et $s_{k}$ ne sont pas conjugués par $\widehat{G}^{0}$. Les ensembles $\operatorname{Irr}\left(G^{0}, s_{j}\right)$ et $\operatorname{Irr}\left(G^{0}, s_{k}\right)$ sont donc aussi 
disjoints. On peut alors décomposer $H_{c}^{\delta}\left(\boldsymbol{X}^{0}\right)$ en somme directe de deux $G^{0} \times L^{0}$ modules $H$ et $R$ tels que :

- le $L^{0}$-module $H$ ne contient aucun élément de $\mathcal{M}_{2}$;

- $R$ détermine une bijection entre $\mathcal{M}_{1}$ et $\mathcal{M}_{2}$,

où on a posé $\mathcal{M}_{1}=\cup_{j \in J} \operatorname{Irr}\left(G^{0}, s_{j}\right), \mathcal{M}_{2}=\cup_{j \in J} \operatorname{Irr}\left(L^{0}, s_{j}\right)$.

L'action du groupe $\Delta$ sur $H_{c}^{\delta}\left(\boldsymbol{X}^{0}\right)$ conserve cette décomposition. Il est clair que ni le $L$-module $\operatorname{Ind}_{\Delta}^{G \times L}(H)$, ni le $L$-module $\operatorname{Ind}_{\Delta}^{G \times L}\left(H_{c}^{i}\left(\boldsymbol{X}^{0}\right)\right)$, pour $i \neq \delta$, ne peuvent contenir d'éléments de $\operatorname{Irr}(L, s)^{r}$. L'application $R_{L}^{G}$, restreinte au module $\mathbb{Z}[\operatorname{Irr}(L, s)]$, est donc égale, au facteur $(-1)^{\delta}$ près, à l'application qui à $M \in \operatorname{Irr}(L, s)$ associe $\left(S \otimes_{\mathbb{C}} M\right)^{L}$, où $S=\operatorname{Ind}_{\Delta}^{G \times L}(R)$. Posons $K_{1}=G, H_{1}=G^{0}, K_{2}=L, H_{2}=L^{0}$. On est exactement dans la situation du lemme 6.1. L'énoncé résulte de ce lemme, pourvu que les hypothèses de celui-ci soient vérifiées. L'unique hypothèse non évidente est la suivante :

(1) si $M \in \mathcal{M}_{1}$ et $g \in G$ vérifient ${ }^{g} M \in \mathcal{M}_{1}$, alors il existe $\ell \in L$ tel que $g \ell^{-1} \in G^{0}$. Soient $j, k \in J$ tels que $M \in \operatorname{Irr}\left(G^{0}, s_{j}\right),{ }^{g} M \in \operatorname{Irr}\left(G^{0}, s_{k}\right)$. Alors l'image $d$ de $g$ dans $D_{G}$ envoie la classe de conjugaison de $s_{j}$ par $\widehat{G}^{0}$ sur celle de $s_{k}$. Par définition de $J$, $s_{j}$ et $s_{k}$ appartiennent à la même classe de conjugaison par $\widehat{L}$. Donc $d \in D_{L} S_{G}$. On a déjà vu que $S_{G}=S_{L} \subseteq D_{L}$. Donc $d \in D_{L}$, ce qui équivaut à la conclusion de (1). Cela achève la démonstration.

6.4. On conserve les hypothèses de 6.2.

Lemme. - Supposons que s possède au moins une valeur propre différente de \pm 1 . Soit $\left(\Lambda_{1}, \Lambda_{2}\right) \in \widetilde{\mathcal{S}} \widetilde{\mathcal{S}}_{n, \text { pair }}$. Alors $k_{\eta}\left(\Lambda_{1}, \Lambda_{2}\right)$ est orthogonal à tout élément de $\mathbb{C}[\operatorname{Irr}(G, s)]$.

Démonstration. - Par construction, $k_{\eta}\left(\Lambda_{1}, \Lambda_{2}\right)$ est combinaison linéaire de fonctions $k^{G}\left(\boldsymbol{T} ; s_{T} ; r^{\prime}, r^{\prime \prime}\right), c f .3 .2$, où $s_{T}$ n'a pour valeurs propres que \pm 1 . On a ajouté un exposant $G$ à la notation pour la préciser. Reprenons les constructions de 6.2. D'après l'hypothèse sur $s$, on a $t \geqslant 1$ et $\operatorname{dim}\left(V^{ \pm}\right)<2 n$. Par récurrence, on peut appliquer le corollaire 5.2 à $O\left(V^{ \pm}\right)$. Tout élément de $\mathbb{C}\left[\operatorname{Irr}\left(O\left(V^{ \pm}\right), s^{ \pm}\right)\right]$est combinaison linéaire de fonctions $k^{O\left(V^{ \pm}\right)}\left(\boldsymbol{T}^{ \pm}, s_{T^{ \pm}} ; r^{\prime \pm}, r^{\prime \prime \pm}\right)$, où $s_{T^{ \pm}}$n'a pour valeurs propres que \pm 1 . Soit $i \in\{1, \ldots, t\}$. Le groupe $\boldsymbol{L}_{i}$ est un groupe linéaire ou unitaire. Il est bien connu que ses caractères unipotents sont uniformes, c'est-à-dire combinaisons linéaires de caractères de Deligne-Lusztig. L'élément $s_{i}$ est central dans $\widehat{\boldsymbol{L}}_{i}$ et ne modifie la situation que par torsion par un caractère. En adaptant les constructions et notations de 3.2, on peut dire que tout élément de $\mathbb{C}\left[\operatorname{Irr}\left(L_{i}, s_{i}\right)\right]$ est combinaison linéaire de fonctions $k^{L_{i}}\left(\boldsymbol{T}_{i} ; s_{i}\right)$. Donc tout élément de $\mathbb{C}[\operatorname{Irr}(L, s)]$ est combinaison linéaire de fonctions $k^{L}\left(\widetilde{\boldsymbol{T}}, s_{\widetilde{T}} ; \widetilde{r}^{\prime}, \widetilde{r}^{\prime \prime}\right)$, où $\widetilde{\boldsymbol{T}} \subseteq \boldsymbol{L}$ et $s_{\widetilde{T}}$ est un élément de $\widehat{\boldsymbol{L}}$ dont les valeurs propres différentes de \pm 1 sont les mêmes que celles de $s$. D'après le lemme 6.3 et les relations 
1.4(1) et 3.2(3), tout élément de $\mathbb{C}[\operatorname{Irr}(G, s)]$ est donc combinaison linéaire de fonctions $k^{G}\left(\widetilde{\boldsymbol{T}} ; s_{\widetilde{T}} ; \widetilde{r}^{\prime}, \widetilde{r}^{\prime \prime}\right)$. D'après $3.2(2)$, ces fonctions sont orthogonales aux fonctions $k^{G}\left(\boldsymbol{T} ; s_{T} ; r^{\prime}, r^{\prime \prime}\right)$ du début de la preuve. D'où l'énoncé.

\section{5 .}

Proposition. - La famille des $k\left(\Lambda_{1}, \Lambda_{2}\right)$, pour $\left(\Lambda_{1}, \Lambda_{2}\right) \in \widetilde{\mathcal{S}} \widetilde{\mathcal{S}}_{n \text {,pair }}$, est une base de l'espace $\mathbb{C}[\operatorname{Quad}(O(2 n))]$.

Démonstration. - Pour $\eta \in\{ \pm 1\}$, l'espace $\mathbb{C}\left[\operatorname{Quad}\left(O_{\eta}(2 n)\right)\right]$ est l'orthogonal de la somme des espaces $\mathbb{C}\left[\operatorname{Irr}\left(O_{\eta}(2 n), s\right)\right]$, quand $s$ parcourt les éléments semi-simples de $\widehat{O}_{\eta}(2 n)^{0}$ qui ne sont pas quadratiquement équivalents à 1 . Grâce au lemme 6.4 , on a donc $k_{\eta}\left(\Lambda_{1}, \Lambda_{2}\right) \in \mathbb{C}\left[\operatorname{Quad}\left(O_{\eta}(2 n)\right)\right]$ pour tout $\left(\Lambda_{1}, \Lambda_{2}\right) \in \widetilde{\mathcal{S}} \widetilde{\mathcal{S}}_{n \text {,pair }}$. La famille de l'énoncé est donc bien formée d'éléments de $\mathbb{C}[\operatorname{Quad}(O(2 n))]$. Elle est linéairement indépendante d'après la proposition 3.6(i) et (ii). Elle a le bon nombre d'éléments d'après la proposition 4.4(a).

6.6. Un élément $\left(\Lambda_{1}, \Lambda_{2}\right)$ de $\widetilde{\mathcal{S}} \widetilde{\mathcal{S}}_{n \text {,pair }}$ s'identifie à un produit tensoriel $\Lambda_{1} \otimes \Lambda_{2}$ dans un facteur $\mathbb{Q}\left[\widetilde{\mathcal{S}}_{n_{1}, \text { pair }}\right] \otimes_{\mathbb{Q}} \mathbb{Q}\left[\widetilde{\mathcal{S}}_{n_{2}, \text { pair }}\right]$ de $\mathbb{Q}\left[\widetilde{\mathcal{S}} \widetilde{\mathcal{S}}_{n, \text { pair }}\right]$. Il sera commode d'utiliser les deux notations, ce qui conduit à des égalités telles que trace $\left(\pi\left(\Lambda_{1}, \Lambda_{2}\right)\right)=\pi_{n}\left(\Lambda_{1} \otimes \Lambda_{2}\right)$, $k\left(\Lambda_{1}, \Lambda_{2}\right)=k_{n}\left(\Lambda_{1} \otimes \Lambda_{2}\right)$.

Grâce à la proposition précédente, on peut définir un endomorphisme $i_{n}$, ou simplement $i$, de l'espace $\mathbb{C}\left[\widetilde{\mathcal{S}} \widetilde{\mathcal{S}}_{n, \text { pair }}\right]$ par la formule :

$$
i=\pi_{n}^{-1} \circ k_{n} \circ \mathcal{F} \text {. }
$$

Le théorème 5.1 revient à dire que l'on peut choisir $\pi_{n, \text { cusp }}$ de sorte que $i$ soit l'identité.

Notons $\sigma \otimes 1$, resp. $1 \otimes \sigma, \tau$, les automorphismes de $\mathbb{C}\left[\widetilde{\mathcal{S}} \widetilde{\mathcal{S}}_{n \text {,pair }}\right]$ déduits des bijections $\left(\Lambda_{1}, \Lambda_{2}\right) \mapsto\left(\sigma \Lambda_{1}, \Lambda_{2}\right)$, resp. $\left(\Lambda_{1}, \sigma \Lambda_{2}\right),\left(\Lambda_{2}, \Lambda_{1}\right)$. Les propriétés suivantes résultent des propositions 3.6 et 4.4 et des propriétés de $\mathcal{F}$ :

- $i$ est une isométrie;

- $i$ commute avec $\sigma \otimes 1,1 \otimes \sigma$ et $\tau$.

On a aussi :

$$
i\left(\mathbf{1}_{+}(n) \otimes \Lambda(\varnothing)\right)=\mathbf{1}_{+}(n) \otimes \Lambda(\varnothing) .
$$

D'après le lemme 4.6 , le membre de gauche vaut 1 sur $O_{+}(2 n)$ et 0 sur $O_{-}(2 n)$. On a l'égalité :

$$
\mathcal{F} \mathbf{1}_{+}(n)=\frac{1}{2}\left(\mathbf{1}_{+}(n)+\sigma \mathbf{1}_{+}(n)+\mathbf{1}_{-}(n)+\sigma \mathbf{1}_{-}(n)\right) .
$$

On calcule $k_{n}\left(\mathcal{F} \mathbf{1}_{+}(n) \otimes \Lambda(\varnothing)\right)$ en utilisant 3.5 et la proposition 3.6 (b)(vi),(vii). On obtient le résultat requis.

Supposons $n=1$. Alors le théorème 5.1 est vrai. En effet, d'après les propriétés ci-dessus de $i$, il suffit de vérifier que cet automorphisme coïncide avec l'identité sur les deux éléments $\mathbf{1}_{+}(1) \otimes \Lambda(\varnothing)$ et $\mathbf{1}_{-}(1) \otimes \Lambda(\varnothing)$. Pour le premier, c'est l'assertion 
(1) ci-dessus. Pour le second, le même calcul montre qu'il suffit que $\pi\left(\mathbf{1}_{-}(1) \otimes \Lambda(\varnothing)\right)$ soit le caractère trivial de $O_{-}(2)$. Mais $\mathbf{1}_{-}(1)$ est cuspidal. Les conditions imposées en 4.4 laissent une liberté sur $\pi\left(\mathbf{1}_{-}(1) \otimes \Lambda(\varnothing)\right)$ : ce peut être soit le caractère trivial de $O_{-}(2)$, soit son caractère det. Il suffit de faire le bon choix.

On suppose désormais $n \geqslant 2$. On a alors :

$$
i\left(\mathbf{1}_{-}(n) \otimes \Lambda(\varnothing)\right)=\mathbf{1}_{-}(n) \otimes \Lambda(\varnothing) .
$$

La démonstration est la même que celle de (1). L'hypothèse du lemme 4.6 est vérifiée d'après ce que l'on vient de dire du cas $n=1$. 



\section{CHAPITRE 7}

\section{VALEURS DES FONCTIONS TRACES DE FAISCEAUX-CARACTĖRES}

En utilisant une formule de Lusztig (lemme 7.1), on calcule la valeur en certains points des fonctions traces de faisceaux-caractères (proposition 7.2). Il faut en 7.1 généraliser la formule de Lusztig à nos groupes orthogonaux non connexes, et calculer explicitement les normalisations des diverses fonctions qui y interviennent. Les paragraphes 7.4 à 7.11 démontrent une propriété de divisibilité de certaines valeurs de fonctions $k_{n}\left(\mathcal{F} \Lambda_{1} \otimes \mathcal{F} \Lambda_{2}\right)$ (lemme 7.11$)$. Cette propriété jouera un rôle clé au paragraphe 12 dans la dernière partie de la démonstration. Par ailleurs, on énonce en 7.3 une propriété technique qui nous permettra au paragraphe 11 de séparer des fonctions que l'on ne peut pas distinguer par des méthodes combinatoires

7.1. Soit $\eta$ un élément de $\{ \pm 1\}$, posons $\boldsymbol{G}=\boldsymbol{O}_{\eta}(2 n), V=V_{\eta}(2 n)$. Soit $g \in G$. Écrivons $g=s u$, où $s$ est semi-simple, $u$ est unipotent et $s$ et $u$ commutent. On suppose que $s$ n'a pour valeurs propres que \pm 1 . On note $V^{\prime}$, resp. $V^{\prime \prime}$, le sous-espace propre associé à la valeur propre 1 , resp. -1 . On pose $d^{\prime}=\operatorname{dim}\left(V^{\prime}\right), n^{\prime}=\left[d^{\prime} / 2\right]$, $\eta^{\prime}(g)=\eta\left(V^{\prime}\right)$. On définit de même $d^{\prime \prime}, n^{\prime \prime}$ et $\eta^{\prime \prime}(g)$. On a :

$$
\boldsymbol{Z}_{\boldsymbol{G}}(s)^{0}= \begin{cases}\boldsymbol{S O}_{\eta^{\prime}(g)}\left(2 n^{\prime}\right) \times \boldsymbol{S O}_{\eta^{\prime \prime}(g)}\left(2 n^{\prime \prime}\right), & \text { si } d^{\prime} \text { et } d^{\prime \prime} \text { sont pairs, } \\ \boldsymbol{S} \boldsymbol{O}\left(2 n^{\prime}+1\right) \times \boldsymbol{S} \boldsymbol{O}\left(2 n^{\prime \prime}+1\right), & \text { si } d^{\prime} \text { et } d^{\prime \prime} \text { sont impairs. }\end{cases}
$$

Soient $N_{1}, N_{2} \in \mathbb{N}, h_{1}, h_{2} \in \mathbb{Z}$ tels que $n=N_{1}+N_{2}+h_{1}^{2}+h_{2}^{2}$. On pose $N=N_{1}+N_{2}$, $r^{\prime}=h_{1}+h_{2}, r^{\prime \prime}=h_{1}-h_{2}$. Soient $w_{1} \in W_{N_{1}}, w_{2} \in W_{N_{2}}$. On a introduit en 3.3 une fonction $k_{\eta}\left(w_{1}, w_{2} ; r^{\prime}, r^{\prime \prime}\right)$ sur $G$. Sa construction permet de contrôler son support, ce dont on déduit :

- si $d^{\prime} \equiv d^{\prime \prime} \not \equiv r^{\prime} \equiv r^{\prime \prime} \bmod 2 \mathbb{Z}, k_{\eta}\left(w_{1}, w_{2} ; r^{\prime}, r^{\prime \prime}\right)(g)=0$;

- si $d^{\prime}<r^{\prime 2}$ ou $d^{\prime \prime}<r^{\prime \prime 2}, k_{\eta}\left(w_{1}, w_{2} ; r^{\prime}, r^{\prime \prime}\right)(g)=0$.

On suppose désormais :

$$
d^{\prime} \equiv d^{\prime \prime} \equiv r^{\prime} \equiv r^{\prime \prime} \bmod 2 \mathbb{Z}, \quad d^{\prime} \geqslant r^{\prime 2}, \quad d^{\prime \prime} \geqslant r^{\prime \prime 2} .
$$

On pose $N^{\prime}=\left(d^{\prime}-r^{\prime 2}\right) / 2, N^{\prime \prime}=\left(d^{\prime \prime}-r^{\prime \prime 2}\right) / 2$. 
Soit $w^{\prime} \in W_{N^{\prime}}$. Supposons d'abord $d^{\prime}$ pair et $r^{\prime} \neq 0$. Dans [W] II.6, on a défini (à la suite de Lusztig) une fonction $Q_{w^{\prime}}$ sur l'ensemble des éléments nilpotents de l'algèbre de Lie $s o_{\eta^{\prime}(g)}\left(2 n^{\prime}\right)$. En la remontant par la transformation de Cayley $X \mapsto$ $(1+X / 2)(1-X / 2)^{-1}$, on obtient une fonction sur l'ensemble des éléments unipotents de $S O_{\eta^{\prime}(g)}\left(2 n^{\prime}\right)$. Notons-la $Q^{\prime}\left(\left|r^{\prime}\right|, w^{\prime}\right)$. En fait, cette fonction est proportionnelle à la restriction aux unipotents de la fonction $k_{\eta^{\prime}(g)}\left(w^{\prime}, 1 ; r^{\prime}, 0\right)$ sur $S O_{\eta^{\prime}(g)}\left(2 n^{\prime}\right)$. Supposons toujours $d^{\prime}$ pair, mais $r^{\prime}=0$. La fonction $Q_{w^{\prime}}$ de $[\mathbf{W}]$ II.6 n'est définie que pour $w^{\prime} \in W_{N^{\prime}}^{D}$ et dépend de la classe de $\phi$-conjugaison de $w^{\prime}$. C'est-à-dire que, si $\eta^{\prime}(g)=1$, elle dépend de la classe de conjugaison de $w^{\prime}$ par $W_{N^{\prime}}^{D}$; et, si $\eta^{\prime}(g)=-1$, elle dépend

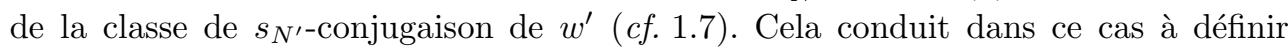
$Q^{\prime}\left(0, w^{\prime}\right)$ comme le relèvement par la transformation de Cayley de la fonction :

$$
\begin{cases}0, & \text { si } \operatorname{sgn}_{C D}\left(w^{\prime}\right) \neq \eta^{\prime}(g), \\ \frac{1}{2}\left(Q_{w^{\prime}}+Q_{s_{N^{\prime}} w^{\prime} s_{N^{\prime}}}\right), & \text { si } \operatorname{sgn}_{C D}\left(w^{\prime}\right)=\eta^{\prime}(g)=1, \\ Q_{s_{N^{\prime}} w^{\prime}}=Q_{w^{\prime} s_{N^{\prime}}}, & \text { si } \operatorname{sgn}_{C D}\left(w^{\prime}\right)=\eta^{\prime}(g)=-1 .\end{cases}
$$

Cette fonction est encore proportionnelle à la restriction de la fonction $k_{\eta^{\prime}(g)}\left(w^{\prime}, 1 ; 0,0\right)$. Supposons enfin $d^{\prime}$ impair. Dans [W] II.6, on a défini une fonction $Q_{w^{\prime}}$ sur l'ensemble des éléments nilpotents de l'algèbre de Lie $s o\left(2 n^{\prime}+1\right)$. En la remontant par la transformation de Cayley, on obtient une fonction sur l'ensemble des éléments unipotents de $S O\left(2 n^{\prime}+1\right)$. On la note encore $Q^{\prime}\left(\left|r^{\prime}\right|, w^{\prime}\right)$. Elle est proportionnelle à la restriction aux unipotents de la fonction $k\left(w^{\prime}, 1 ;\left|r^{\prime}\right|, 0\right)$ définie en 3.8.

Soit $w^{\prime \prime} \in W_{N^{\prime \prime}}$. On construit de façon similaire une fonction $Q^{\prime \prime}\left(\left|r^{\prime \prime}\right|, w^{\prime \prime}\right)$ sur l'ensemble des éléments unipotents de $S O_{\eta^{\prime \prime}(g)}\left(2 n^{\prime \prime}\right)$ ou $S O\left(2 n^{\prime \prime}+1\right)$. Pour $\left(w^{\prime}, w^{\prime \prime}\right) \in$ $W_{N^{\prime}} \times W_{N^{\prime \prime}}, Q^{\prime}\left(\left|r^{\prime}\right|, w^{\prime}\right) \times Q^{\prime \prime}\left(\left|r^{\prime \prime}\right|, w^{\prime \prime}\right)$ est une fonction sur l'ensemble des éléments unipotents de $Z_{G}(s)^{0}$.

Des décompositions :

$$
\{1, \ldots, N\}=\left\{1, \ldots, N^{\prime}\right\} \cup\left\{N^{\prime}+1, \ldots, N\right\}=\left\{1, \ldots, N_{1}\right\} \cup\left\{N_{1}+1, \ldots, N\right\}
$$

se déduisent naturellement des plongements :

$$
W_{N^{\prime}} \times W_{N^{\prime \prime}} \longrightarrow W_{N} \longleftarrow W_{N_{1}} \times W_{N_{2}} .
$$

Notons $\mathcal{N}$ l'ensemble des quadruplets $\underline{\nu}=\left(N_{1}^{\prime}, N_{2}^{\prime}, N_{1}^{\prime \prime}, N_{2}^{\prime \prime}\right)$ d'entiers $\geqslant 0$ tels que $N_{1}^{\prime}+N_{1}^{\prime \prime}=N_{1}, N_{2}^{\prime}+N_{2}^{\prime \prime}=N_{2}, N_{1}^{\prime}+N_{2}^{\prime}=N^{\prime}, N_{1}^{\prime \prime}+N_{2}^{\prime \prime}=N^{\prime \prime}$. Cet ensemble est en bijection avec celui des doubles classes :

$$
\left(W_{N^{\prime}} \times W_{N^{\prime \prime}}\right) \backslash W_{N} /\left(W_{N_{1}} \times W_{N_{2}}\right) .
$$

Au quadruplet $\underline{\nu}$, écrit comme ci-dessus, on associe la double classe de l'élément $w_{\underline{\nu}} \in W_{N}$ qui envoie de façon croissante :

- $\left\{1, \ldots, N_{1}^{\prime}\right\}$ sur lui-même;

- $\left\{N_{1}^{\prime}+1, \ldots, N_{1}\right\}$ sur $\left\{N^{\prime}+1, \ldots, N^{\prime}+N_{1}^{\prime \prime}\right\}$;

- $\left\{N_{1}+1, \ldots, N_{1}+N_{2}^{\prime}\right\}$ sur $\left\{N_{1}^{\prime}+1, \ldots, N^{\prime}\right\}$;

- $\left\{N_{1}+N_{2}^{\prime}+1, \ldots, N\right\}$ sur lui-même. 
On pose :

$$
W(\underline{\nu})=\left(W_{N^{\prime}} \times W_{N^{\prime \prime}}\right) \cap w_{\underline{\nu}}\left(W_{N_{1}} \times W_{N_{2}}\right) w_{\underline{\nu}}^{-1} .
$$

On a un isomorphisme évident :

$$
W(\underline{\nu})=W_{N_{1}^{\prime}} \times W_{N_{2}^{\prime}} \times W_{N_{1}^{\prime \prime}} \times W_{N_{2}^{\prime \prime}} .
$$

Un élément $\underline{w}$ de $W(\underline{\nu})$ s'écrit $\underline{w}=\left(w_{1}^{\prime}, w_{2}^{\prime}, w_{1}^{\prime \prime}, w_{2}^{\prime \prime}\right)$. Cette notation sera souvent implicite dans la suite. Remarquons que l'on a deux plongements naturels :

$$
\begin{aligned}
W(\underline{\nu}) & \longrightarrow W_{N^{\prime}} \times W_{N^{\prime \prime}} & W(\underline{\nu}) & \longrightarrow W_{N_{1}} \times W_{N_{2}} \\
\underline{w} & \longmapsto\left(w_{1}^{\prime} w_{2}^{\prime}, w_{1}^{\prime \prime} w_{2}^{\prime \prime}\right), & \underline{w} & \longmapsto\left(w_{1}^{\prime} w_{1}^{\prime \prime}, w_{2}^{\prime} w_{2}^{\prime \prime}\right) .
\end{aligned}
$$

Pour $\underline{w} \in W(\underline{\nu})$, on pose :

$$
\gamma\left(h_{1}, h_{2} ; g ; \underline{w}\right)= \begin{cases}1, & \text { si } r^{\prime} \geqslant 0, r^{\prime \prime} \geqslant 0, \\ \eta^{\prime \prime}(g) \operatorname{sgn}_{C D}\left(w_{1}^{\prime \prime} w_{2}^{\prime \prime}\right), & \text { si } r^{\prime} \geqslant 0, r^{\prime \prime}<0, \\ \eta^{\prime \prime}(g) \operatorname{sgn}_{C D}\left(w_{1}^{\prime} w_{2}^{\prime}\right), & \text { si } r^{\prime}<0, r^{\prime \prime} \geqslant 0, \\ \operatorname{sgn}_{C D}\left(w_{1}^{\prime} w_{2}^{\prime} w_{1}^{\prime \prime} w_{2}^{\prime \prime}\right), & \text { si } r^{\prime}<0, r^{\prime \prime}<0 .\end{cases}
$$

On pose :

$$
\begin{aligned}
& c\left(h_{1}, h_{2}\right)= \begin{cases}\zeta(-1)^{r^{\prime} / 2}, & \text { si } r^{\prime} \text { est pair, } \\
\zeta(-1)^{\left(\left|r^{\prime \prime}\right|-1\right) / 2,} \text { si } r^{\prime} \text { est impair; }\end{cases} \\
& c_{\eta}\left(h_{1}, h_{2}\right)= \begin{cases}1, & \text { si } r^{\prime} \geqslant 0, \\
\eta, & \text { si } r^{\prime}<0 ;\end{cases} \\
& \alpha\left(h_{1}, h_{2} ; g\right)=\left|\left\{\left(r^{\prime}, N^{\prime}\right)\right\} \cap(\{0\} \times(\mathbb{N} \backslash\{0\}))\right|+\left|\left\{\left(r^{\prime \prime}, N^{\prime \prime}\right)\right\} \cap(\{0\} \times(\mathbb{N} \backslash\{0\}))\right| .
\end{aligned}
$$

LEMME. - Sous l'hypothèse (1), on a l'égalité :

$$
\begin{aligned}
k_{\eta}\left(w_{1}, w_{2} ; r^{\prime}, r^{\prime \prime}\right)(g)=(-1)^{n} 2^{\alpha\left(h_{1}, h_{2} ; g\right)} c\left(h_{1}, h_{2}\right) c_{\eta}\left(h_{1}, h_{2}\right) q^{\delta\left(r^{\prime}, r^{\prime \prime}\right) / 2} \\
\cdot\left|Z_{W_{N_{1}}}\left(w_{1}\right)\right| \cdot\left|Z_{W_{N_{2}}}\left(w_{2}\right)\right| \cdot \sum_{\underline{\nu} \in \mathcal{N}}|W(\underline{\nu})|^{-1} \zeta(-1)^{N_{2}^{\prime \prime}} \\
\cdot \sum_{\underline{w}} \gamma\left(h_{1}, h_{2} ; g ; \underline{w}\right) \operatorname{sgn}_{C D}\left(w_{2}^{\prime \prime}\right) Q^{\prime}\left(\left|r^{\prime}\right|, w_{1}^{\prime} w_{2}^{\prime}\right)\left(u^{\prime}\right) Q^{\prime \prime}\left(\left|r^{\prime \prime}\right|, w_{1}^{\prime \prime} w_{2}^{\prime \prime}\right)\left(u^{\prime \prime}\right),
\end{aligned}
$$

où $\underline{\nu}$ et $\underline{w}$ sont écrits comme ci-dessus et $\underline{w}$ parcourt les éléments de $W(\underline{\nu})$ tels que $w_{1}^{\prime} w_{1}^{\prime \prime}$ soit conjugué à $w_{1}$ dans $W_{N_{1}}$ et $w_{2}^{\prime} w_{2}^{\prime \prime}$ soit conjugué à $w_{2}$ dans $W_{N_{2}}$.

Démonstration. - Lusztig a calculé le terme $k_{\eta}\left(w_{1}, w_{2} ; r^{\prime}, r^{\prime \prime}\right)(g)$ en [L5], théorème 8.5. Sa démonstration est écrite pour un groupe connexe mais s'étend à notre situation. Il faut toutefois prendre garde au fait que le nombre $\delta$ de [L5] 8.8.3, qui intervient dans les décalages effectués pour définir nos faisceaux, et qui est toujours pair dans le cas connexe, est ici de la parité de $r^{\prime}$. Cela explique le $(-1)^{r^{\prime}}$ apparaissant dans l'égalité (2) ci-dessous. Explicitons la formule de Lusztig, en reprenant les notations utilisées lors de la construction de la fonction $k_{\eta}\left(w_{1}, w_{2} ; r^{\prime}, r^{\prime \prime}\right)$, cf. 3.3. 
On suppose d'abord $\left(r^{\prime}, r^{\prime \prime}\right) \neq(0,0)$. Notre fonction est construite par induction à partir d'un Lévi $\boldsymbol{M}=\boldsymbol{T} \times \boldsymbol{G}_{c}=\boldsymbol{T}_{w_{1}} \times \boldsymbol{T}_{w_{2}} \times \boldsymbol{G}_{c}$. Dans $\boldsymbol{G}_{c}$ est donnée l'orbite $\boldsymbol{C}_{c}$ d'un élément $x_{c}=s u \in G_{c}$. Notons $\boldsymbol{C}_{c, s s}$ l'orbite dans $\boldsymbol{G}_{c}$ de $s$. La formule de Lusztig est de la forme :

(2) $k_{\eta}\left(w_{1}, w_{2} ; r^{\prime}, r^{\prime \prime}\right)(g)=(-1)^{r^{\prime}} \sum_{\substack{x \in G^{0} \\ x^{-1} s x \in T \times C_{c, s s}}}\left|x M x^{-1} \cap Z_{G}(s)^{0}\right|\left|Z_{G}(s)^{0}\right|^{-1}\left|M^{0}\right|^{-1} Q_{x}^{0}(u)$,

où $Q_{x}^{0}$ est une certaine fonction de Green sur $Z_{G}(s)^{0}$. Cette fonction est invariante par conjugaison par $Z_{G}(s)^{0}$ et ne dépend que de la classe $Z_{G}(s)^{0} x$. En fait, la définition de $Q_{x}^{0}$ s'étend aux $x \in G$ tels que $x^{-1} s x \in T \times C_{c, s s}$. Le membre de droite de (2), où l'on remplace la condition $x \in G^{0}$ par $x \in G \backslash G^{0}$, calcule $k_{\eta}\left(w_{1}, w_{2} ; r^{\prime}, r^{\prime \prime}\right)(\widetilde{g})$, où $\widetilde{g}$ est conjugué à $g$ par un élément de $G \backslash G^{0}$. Remplacer $g$ par $\widetilde{g}$ ne change rien, puisque notre fonction de départ est invariante par conjugaison par $G$. On peut donc remplacer dans (2) la condition $x \in G^{0}$ par $x \in G$, à condition de diviser par 2. À ce point, on peut lever l'hypothèse $\left(r^{\prime}, r^{\prime \prime}\right) \neq(0,0)$. Quand $\left(r^{\prime}, r^{\prime \prime}\right)=(0,0), k_{\eta}\left(w_{1}, w_{2} ; 0,0\right)(g)$ est par définition somme de deux termes. Si on exprime l'un par la formule (2), on voit que l'autre est la somme analogue où la condition $x \in G^{0}$ est remplacée par $x \in G \backslash G^{0}$. Donc $k_{\eta}\left(w_{1}, w_{2}, 0,0\right)(g)$ est donné par la somme pour $x \in G$. Cette fois, on ne doit plus diviser par 2. Maintenant, on peut remplacer chaque fonction $Q_{x}^{0}$ par :

$$
Q_{x}=\left|Z_{G}(s) / Z_{G}(s)^{0}\right|^{-1} \sum_{y \in Z_{G}(s) / Z_{G}(s)^{0}} Q_{y x}^{0},=\left|Z_{G}(s) / Z_{G}(s)^{0}\right|^{-1} \sum_{y \in Z_{G}(s) / Z_{G}(s)^{0}} Q_{x}^{0} \circ \operatorname{Ad}(y) .
$$

Cette fonction $Q_{x}$ est invariante par conjugaison par $Z_{G}(s)^{0}$ et ne dépend que de la classe $Z_{G}(s) x$. On obtient donc :

(3) $k_{\eta}\left(w_{1}, w_{2} ; r^{\prime}, r^{\prime \prime}\right)(g)=(-1)^{r^{\prime}} c_{1} \sum_{\substack{x \in G \\ x^{-1} s x \in T \times C_{c, s s}}}\left|x M x^{-1} \cap Z_{G}(s)^{0}\right|\left|Z_{G}(s)^{0}\right|^{-1}\left|M^{0}\right|^{-1} Q_{x}(u)$,

où :

$$
c_{1}= \begin{cases}1 / 2, & \text { si }\left(r^{\prime}, r^{\prime \prime}\right) \neq(0,0) \\ 1, & \text { si }\left(r^{\prime}, r^{\prime \prime}\right)=(0,0)\end{cases}
$$

Notons $\boldsymbol{Z}^{\prime}$ et $\boldsymbol{Z}^{\prime \prime}$ les deux composantes évidentes de $\boldsymbol{Z}_{\boldsymbol{G}}(s)$. Soit $x \in G$ tel que $x^{-1} s x \in T \times C_{c, s s}$. Alors il existe $\underline{\nu}=\left(N_{1}^{\prime}, N_{2}^{\prime}, N_{1}^{\prime \prime}, N_{2}^{\prime \prime}\right) \in \mathcal{N}, \underline{w}=\left(w_{1}^{\prime}, w_{2}^{\prime}, w_{1}^{\prime \prime}, w_{2}^{\prime \prime}\right) \in$ $W(\underline{\nu})$, de sorte que :

$$
\boldsymbol{Z}^{\prime} \cap x \boldsymbol{M} x^{-1}=\boldsymbol{T}_{w_{1}^{\prime}} \times \boldsymbol{T}_{w_{2}^{\prime}} \times \boldsymbol{G}_{c}^{\prime}, \quad \boldsymbol{Z}^{\prime \prime} \cap x \boldsymbol{M} x^{-1}=\boldsymbol{T}_{w_{1}^{\prime \prime}} \times \boldsymbol{T}_{w_{2}^{\prime \prime}} \times \boldsymbol{G}_{c}^{\prime \prime},
$$

où $\boldsymbol{G}_{c}^{\prime}$, resp. $\boldsymbol{G}_{c}^{\prime \prime}$ est le groupe orthogonal d'un espace $V_{c}^{\prime}$, resp. $V_{c}^{\prime \prime}$, de dimension $r^{\prime 2}$, resp. $r^{\prime \prime 2}$,

$$
\begin{aligned}
\boldsymbol{Z}^{\prime} \cap x \boldsymbol{T}_{w_{1}} x^{-1}=\boldsymbol{T}_{w_{1}^{\prime}}, & Z^{\prime} \cap x \boldsymbol{T}_{w_{2}} x^{-1}=\boldsymbol{T}_{w_{2}^{\prime}}, \\
Z^{\prime \prime} \cap x \boldsymbol{T}_{w_{1}} x^{-1}=\boldsymbol{T}_{w_{1}^{\prime \prime}}, & Z^{\prime \prime} \cap x \boldsymbol{T}_{w_{2}} x^{-1}=\boldsymbol{T}_{w_{2}^{\prime \prime}} .
\end{aligned}
$$


L'élément $\underline{w}$ n'est défini qu'à conjugaison près dans $W(\underline{\nu})$. On a ainsi associé à $x$ un élément $\underline{\nu} \in \mathcal{N}$ et une classe de conjugaison d'éléments $\underline{w}$. Remarquons que les termes indexés par $x$ dans la formule (3) ne dépendent que de ces données. En particulier, on peut noter $Q_{\underline{w}}=Q_{x}$.

Inversement, soit $\underline{\nu} \in \mathcal{N}, \underline{w} \in W(\underline{\nu})$. Calculons la somme des termes de la formule (3) à qui sont associées ces données. Pour qu'il en existe, les égalités (5), jointes au fait que $x \boldsymbol{T} x^{-1} \subseteq Z_{G}(s)$, imposent que $w_{1}^{\prime} w_{1}^{\prime \prime}$ est conjugué à $w_{1}$ dans $W_{N_{1}}$ et $w_{2}^{\prime} w_{2}^{\prime \prime}$ est conjugué à $w_{2}$ dans $W_{N_{2}}$. Supposons ces conditions satisfaites. Alors notre problème a une solution. Fixons-en une, notée $x_{\underline{w}}$. L'ensemble des solutions est égal à $Z_{G}(s) x_{\underline{w}} N_{G}\left(w_{1}, w_{2}\right)$, où on note $N_{G}\left(w_{1}, w_{2}\right)$ l'ensemble des éléments de $G$ qui normalisent à la fois $\boldsymbol{T}_{w_{1}}$ et $\boldsymbol{T}_{w_{2}}$. Avec des notations similaires, on a :

$$
Z_{G}(s) \cap x_{\underline{w}} N_{G}\left(w_{1}, w_{2}\right) x_{\underline{w}}^{-1}=N_{Z^{\prime}}\left(w_{1}^{\prime}, w_{2}^{\prime}\right) \times N_{Z^{\prime \prime}}\left(w_{1}^{\prime \prime}, w_{2}^{\prime \prime}\right) .
$$

On vérifie l'égalité :

$$
\left|N_{G}\left(w_{1}, w_{2}\right)\right|=|M| z\left(w_{1}\right) z\left(w_{2}\right)
$$

où l'on a posé par exemple $z\left(w_{1}\right)=\left|Z_{W_{N_{1}}}\left(w_{1}\right)\right|$. Des égalités analogues calculent $\left|N_{Z^{\prime}}\left(w_{1}^{\prime}, w_{2}^{\prime}\right)\right|$ et $\left|N_{Z^{\prime \prime}}\left(w_{1}^{\prime \prime}, w_{2}^{\prime \prime}\right)\right|$. Le nombre de solutions est donc égal à :

$$
\left|Z_{G}(s)\right||M|\left|x_{\underline{w}} M x_{\underline{w}}^{-1} \cap Z_{G}(s)\right|^{-1} z\left(w_{1}\right) z\left(w_{2}\right) z\left(w_{1}^{\prime}\right)^{-1} z\left(w_{2}^{\prime}\right)^{-1} z\left(w_{1}^{\prime \prime}\right)^{-1} z\left(w_{2}^{\prime \prime}\right)^{-1} .
$$

Multiplié par le coefficient intervenant dans la formule (3), on obtient :

$$
c_{2} z\left(w_{1}\right) z\left(w_{2}\right) z\left(w_{1}^{\prime}\right)^{-1} z\left(w_{2}^{\prime}\right)^{-1} z\left(w_{1}^{\prime \prime}\right)^{-1} z\left(w_{2}^{\prime \prime}\right)^{-1},
$$

où :

$$
c_{2}=c_{1}\left|Z_{G}(s) / Z_{G}(s)^{0} \| M / M^{0}\right|\left|\left(x_{\underline{w}} M x_{\underline{w}}^{-1} \cap Z_{G}(s)\right) /\left(x_{\underline{w}} M x_{\underline{w}}^{-1} \cap Z_{G}(s)^{0}\right)\right|^{-1} .
$$

En distinguant les différents cas $\left(r^{\prime}=0\right.$ ou $r^{\prime} \neq 0, d^{\prime}=0$ ou $d^{\prime} \neq 0$, etc.), on montre que $c_{2}=2^{\alpha\left(h_{1}, h_{2} ; g\right)}$. On doit sommer sur $\underline{\nu} \in \mathcal{N}$ et $\underline{w}$ dans un ensemble de représentants des classes de conjugaison dans $W(\underline{\nu})$. Il revient au même de sommer sur $\underline{w} \in W(\underline{\nu})$, en pondérant le terme à sommer par l'inverse du nombre d'éléments de la classe de conjugaison de $\underline{w}$. Ce coefficient de pondération est égal à :

$$
|W(\underline{\nu})|^{-1} z\left(w_{1}^{\prime}\right) z\left(w_{2}^{\prime}\right) z\left(w_{1}^{\prime \prime}\right) z\left(w_{2}^{\prime \prime}\right) .
$$

En le multipliant par le terme à sommer, on obtient $2^{\alpha\left(h_{1}, h_{2} ; g\right)} z\left(w_{1}\right) z\left(w_{2}\right)|W(\underline{\nu})|^{-1}$. L'égalité (3) se transforme en :

$$
k_{\eta}\left(w_{1}, w_{2} ; r^{\prime}, r^{\prime \prime}\right)(g)=(-1)^{r^{\prime}} 2^{\alpha\left(h_{1}, h_{2} ; g\right)} z\left(w_{1}\right) z\left(w_{2}\right) \sum_{\underline{\nu} \in \mathcal{N}}|W(\underline{\nu})|^{-1} \sum_{\underline{w}} Q_{\underline{w}}(u),
$$

où $\underline{w}$ parcourt l'ensemble décrit dans l'énoncé. Fixons $\underline{\nu}$ et $\underline{w}$. Il nous reste à déterminer $Q_{\underline{w}}$. Faisons-le dans le cas $r^{\prime} r^{\prime \prime} \neq 0$. D'après la définition de Lusztig, c'est la restriction aux unipotents de la fonction trace d'un complexe induit à $\boldsymbol{Z}_{\boldsymbol{G}}(s)^{0}$ à partir d'un complexe cuspidal sur $x_{\underline{w}} \boldsymbol{M} x_{\underline{w}}^{-1} \cap \boldsymbol{Z}_{\boldsymbol{G}}(s)^{0}$. Évidemment, ces complexes se décomposent en produit de complexes sur $\boldsymbol{Z}^{\prime 0}$ et $\boldsymbol{Z}^{\prime \prime 0}$. Utilisons les égalités (4) pour $x=x_{\underline{w}}$. Le complexe sur $\boldsymbol{Z}^{\prime 0} \cap x_{\underline{w}} \boldsymbol{M} x_{\underline{w}}^{-1}$ est produit des systèmes locaux triviaux sur $\boldsymbol{T}_{w_{1}^{\prime}}$ et $\boldsymbol{T}_{w_{2}^{\prime}}$ 
et d'un système local cuspidal à support unipotent sur $\boldsymbol{G}_{c}^{\prime 0}$. Il y a un seul tel système. Idem pour le complexe cuspidal sur $\boldsymbol{Z}^{\prime \prime 0} \cap x_{\underline{w}} \boldsymbol{M} x_{\underline{w}}^{-1}$. En comparant avec la définition des fonctions de Green, on voit que les fonctions $Q_{\underline{w}}$ et $Q^{\prime}\left(\left|r^{\prime}\right|, w_{1}^{\prime} w_{2}^{\prime}\right) \times Q^{\prime \prime}\left(\left|r^{\prime \prime}\right|, w_{1}^{\prime \prime} w_{2}^{\prime \prime}\right)$ sont proportionnelles. Soit $z \in \mathbb{C}^{\times}$tel que :

$$
Q_{\underline{w}}=z Q^{\prime}\left(\left|r^{\prime}\right|, w_{1}^{\prime} w_{2}^{\prime}\right) \times Q^{\prime \prime}\left(\left|r^{\prime \prime}\right|, w_{1}^{\prime \prime} w_{2}^{\prime \prime}\right) .
$$

Ce nombre $z$ est produit de deux termes $z_{1} z_{2}$. Le terme $z_{1}$ vient du fait que, en $[\mathbf{W}]$ II.6, les fonctions de Green ne sont pas définies comme fonctions traces de complexes induits, mais comme images des fonctions traces des complexes cuspidaux par induction de Lusztig. Ce terme $z_{1}$ est calculé par le théorème 1.14 de $[\mathbf{L 8}]$. On a $z_{1}=(-1)^{D}$, où $D$ est la somme des dimensions des supports des complexes cuspidaux. La dimension d'une orbite unipotente étant paire, on a :

$$
D \equiv \operatorname{dim}\left(\boldsymbol{T}_{w_{1}^{\prime}}\right)+\operatorname{dim}\left(\boldsymbol{T}_{w_{2}^{\prime}}\right)+\operatorname{dim}\left(\boldsymbol{T}_{w_{1}^{\prime \prime}}\right)+\operatorname{dim}\left(\boldsymbol{T}_{w_{2}^{\prime \prime}}\right) \equiv N \equiv n+r^{\prime} \bmod 2 \mathbb{Z} .
$$

D'où $z_{1}=(-1)^{n+r^{\prime}}$. Le terme $z_{2}$ vient de la différence de normalisation des Frobenius des complexes cuspidaux. Soit $u_{c}^{\prime} \in G_{c}^{\prime}$ un élément unipotent dans l'orbite paramétrée par la partition $\left(2\left|r^{\prime}\right|-1,2\left|r^{\prime}\right|-3, \ldots, 1\right)$ et tel que, pour tout élément $i$ de cette partition, on ait :

$$
\eta_{u_{c}^{\prime}}(i)= \begin{cases}\zeta(-1)^{r^{\prime}+(i-1) / 2}, & \text { si } i \neq 2\left|r^{\prime}\right|-1, \\ \eta_{c}^{\prime} \zeta(-1)^{r^{\prime}-1}, & \text { si } i=2\left|r^{\prime}\right|-1 .\end{cases}
$$

On a noté $\eta_{c}^{\prime}=\eta\left(V_{c}^{\prime}\right)$. On peut calculer : $\eta_{c}^{\prime}=\eta^{\prime}(g) \operatorname{sgn}_{C D}\left(w_{1}^{\prime} w_{2}^{\prime}\right)$. On définit de façon similaire $u_{c}^{\prime \prime} \in G_{c}^{\prime \prime}$. Notons $u_{c}$ l'élément de $x_{\underline{w}} M x_{\underline{w}}^{-1} \cap Z_{G}(s)^{0}$ dont les composantes sur $T_{w_{1}^{\prime}}$ etc. sont triviales et dont la composante sur $G_{c}^{\prime}$, resp. $G_{c}^{\prime \prime}$, est $u_{c}^{\prime}$, resp. $u_{c}^{\prime \prime}$. Dans [W] II.6, on a normalisé le Frobenius du complexe cuspidal de sorte que la fonction trace associée vaille 1 au point $u_{c}$. Le système cuspidal sur $\boldsymbol{M}$ est produit de systèmes locaux $\mathcal{L}\left(1_{T_{w_{1}}}\right)$ sur $\boldsymbol{T}_{w_{1}}, \mathcal{L}\left(\zeta_{T_{w_{2}}}\right)$ sur $\boldsymbol{T}_{w_{2}}$ et $\mathcal{L}\left(r^{\prime}, r^{\prime \prime}\right)$ sur $\boldsymbol{G}_{c}$, qui sont munis de Frobenius. D'après la définition de Lusztig, le Frobenius utilisé dans la construction de $Q_{\underline{w}}$ est tel que la fonction trace associée vaille :

$$
\operatorname{trace}\left(\mathcal{L}\left(1_{T_{w_{1}}}\right) \times \mathcal{L}\left(\zeta_{T_{w_{2}}}\right) \times \mathcal{L}\left(r^{\prime}, r^{\prime \prime}\right)\right)\left(x_{\underline{w}}^{-1} s u_{c} x_{\underline{w}}\right)
$$

au point $u_{c}$. Le terme $z_{2}$ est donc égal à cette expression. Notons $g_{1}, g_{2}, g_{c}$ les composantes de $x_{\underline{w}}^{-1} s u_{c} x_{\underline{w}}$ dans $T_{w_{1}}, T_{w_{2}}, G_{c}$. On a :

$$
z_{2}=\operatorname{trace}\left(\mathcal{L}\left(1_{T_{w_{1}}}\right)\right)\left(g_{1}\right) \operatorname{trace}\left(\mathcal{L}\left(\zeta_{T_{w_{2}}}\right)\right)\left(g_{2}\right) \operatorname{trace}\left(\mathcal{L}\left(r^{\prime}, r^{\prime \prime}\right)\right)\left(g_{c}\right) .
$$

Évidemment, le premier terme vaut 1 . L'élément $g_{2}$ est la composante de $x_{\underline{w}}^{-1} s x_{\underline{w}}$ dans $T_{w_{2}}$. Sa composante sur $T_{w_{2}^{\prime}}$ est triviale. Le groupe $\boldsymbol{T}_{w_{2}^{\prime \prime}}$ est naturellement un sous-tore maximal d'un groupe orthogonal, et la composante de $g_{2}$ sur $T_{w_{2}^{\prime \prime}}$ s'envoie par ce plongement sur l'élément -1 de ce groupe orthogonal. On a déjà remarqué dans la preuve de la proposition 3.6 que $\operatorname{trace}\left(\mathcal{L}\left(\zeta_{T_{w_{2}}}\right)\right)\left(g_{2}\right)=\operatorname{sp}\left(g_{2}\right)$. Grâce à 1.6(1), on calcule :

$$
\operatorname{trace}\left(\mathcal{L}\left(\zeta_{T_{w_{2}}}\right)\right)\left(g_{2}\right)=\zeta(-1)^{N_{2}^{\prime \prime}} \operatorname{sgn}_{C D}\left(w_{2}^{\prime \prime}\right) .
$$


Enfin $g_{c}$ est un élément de $C_{c}$ paramétré par les familles $\eta_{u_{c}^{\prime}}$ et $\eta_{u_{c}^{\prime \prime}}$, et $\operatorname{trace}\left(\mathcal{L}\left(r^{\prime}, r^{\prime \prime}\right)\right)\left(g_{c}\right)$ est calculé par la formule de 3.1. On trouve :

$$
\operatorname{trace}\left(\mathcal{L}\left(r^{\prime}, r^{\prime \prime}\right)\right)\left(g_{c}\right)=c\left(h_{1}, h_{2}\right) c_{\eta}\left(h_{1}, h_{2}\right) q^{\delta\left(r^{\prime}, r^{\prime \prime}\right) / 2} \gamma\left(h_{1}, h_{2} ; g ; \underline{w}\right) .
$$

Alors :

$$
z_{1} z_{2}=(-1)^{n+r^{\prime}} c\left(h_{1}, h_{2}\right) c_{\eta}\left(h_{1}, h_{2}\right) q^{\delta\left(r^{\prime}, r^{\prime \prime}\right) / 2} \gamma\left(h_{1}, h_{2} ; g ; \underline{w}\right) \zeta(-1)^{N_{2}^{\prime \prime}} \operatorname{sgn}_{C D}\left(w_{2}^{\prime \prime}\right) .
$$

La formule (6) devient celle de l'énoncé. On a supposé $r^{\prime} r^{\prime \prime} \neq 0$. Dans le cas où $r^{\prime} r^{\prime \prime}=0$, la calcul est analogue, il faut tenir compte de la définition plus embrouillée de nos fonctions qui peuvent être nulles ou sommes de plusieurs fonctions du type précédent. Le résultat est le même.

7.2. On conserve les données du paragraphe précédent, à ceci près qu'au lieu de se donner $w_{1}$ et $w_{2}$, on se donne des représentations irréductibles $\rho_{1} \in \operatorname{Irr}\left(W_{N_{1}}\right)$ et $\rho_{2} \in \operatorname{Irr}\left(W_{N_{2}}\right)$. On note $\Lambda_{1}$, resp. $\Lambda_{2}$, l'élément de $\widetilde{\mathcal{S}}_{N_{1}+h_{1}^{2} \text {,pair }}$, resp. $\widetilde{\mathcal{S}}_{N_{2}+h_{2}^{2} \text {,pair }}$, correspondant au couple $\left(h_{1}, \rho_{1}\right)$, resp. $\left(h_{2}, \rho_{2}\right)$, cf. 2.2. Du paragraphe précédent et des définitions résulte que :

- si l'hypothèse $7.1(1)$ n'est pas vérifiée, $k_{\eta}\left(\Lambda_{1}, \Lambda_{2}\right)(g)=0$.

On suppose désormais cette hypothèse 7.1(1) vérifiée et on adopte les mêmes notations qu'au paragraphe précédent.

On note $\boldsymbol{Z}^{\prime 0}$ et $\boldsymbol{Z}^{\prime \prime 0}$ les deux composantes évidentes de $\boldsymbol{Z}_{\boldsymbol{G}}(s)^{0}$. Soit $\rho^{\prime}$ une représentation irréductible de $W_{N^{\prime}}$. Supposons d'abord $r^{\prime} \neq 0$. La correspondance de Springer généralisée $(c f .[\mathbf{L} 4])$ associe au couple $\left(\left|r^{\prime}\right|, \rho^{\prime}\right)$ un couple $\iota=(\boldsymbol{C}, \mathcal{L})$ formé d'une orbite unipotente $\boldsymbol{C} \subset \boldsymbol{Z}^{\prime 0}$ et d'un système local $\mathcal{L}$ sur $\boldsymbol{C}$. Au couple $\iota$, Lusztig a associé :

- un entier $b(\iota)=\frac{1}{2}\left(\operatorname{dim}\left(\boldsymbol{Z}^{\prime 0}\right)-\operatorname{dim}(\boldsymbol{C})-N^{\prime}-\frac{\left|r^{\prime 3}-r^{\prime}\right|}{3}\right)$;

- une fonction $\chi_{\iota}$ sur $Z^{\prime 0}$, à support unipotent inclus dans $\bar{C}$, où $\bar{C}$ est l'adhérence de Zariski de $\boldsymbol{C}$.

On notera ces termes $b\left(\left|r^{\prime}\right|, \rho^{\prime}\right), \chi_{\left|r^{\prime}\right|, \rho^{\prime}}^{\prime}$. Supposons maintenant $r^{\prime}=0$. Dans la correspondance de Springer généralisée, la donnée de départ est cette fois un couple $(0, \rho)$, où $\rho$ est une représentation irréductible de $W_{N^{\prime}}^{D}$. Supposons $\eta^{\prime}(g)=1$. Si la restriction de $\rho^{\prime}$ à $W_{N^{\prime}}^{D}$ est irréductible, on associe à cette restriction un couple $\iota$ et on poursuit comme ci-dessus. Si cette restriction se décompose en deux représentations irréductibles $\rho_{a}^{\prime}$ et $\rho_{b}^{\prime}$, on a deux couples $\iota_{a}$ et $\iota_{b}$. On a alors $b\left(\iota_{a}\right)=b\left(\iota_{b}\right)$, ce qui définit $b\left(0, \rho^{\prime}\right)$ sans ambiguïté. On pose $\chi_{0, \rho^{\prime}}^{\prime}=\chi_{\iota_{a}}+\chi_{\iota_{b}}$. Supposons $\eta^{\prime}(g)=-1$. Si la restriction de $\rho^{\prime}$ à $W_{N^{\prime}}^{D}$ est réductible, on pose $\chi_{0, \rho^{\prime}}^{\prime}=0$ et la définition de $b\left(0, \rho^{\prime}\right)$ importe peu. Supposons cette restriction irréductible. On associe alors à $\left(0, \rho^{\prime}\right)$ un unique couple $\iota$. On pose $b\left(0, \rho^{\prime}\right)=b(\iota)$. La représentation $\rho^{\prime}$ est paramétrée par un couple $(\underline{\alpha}, \beta)$ de partitions tel que $S(\underline{\alpha})+S(\beta)=N^{\prime}$. On a $\alpha \neq \beta$. Ordonnons les partitions lexicographiquement, c'est-à-dire, pour $\underline{\lambda}=\left(\lambda_{1} \geqslant \lambda_{2} \geqslant \cdots\right)$ et $\underline{\mu}=\left(\mu_{1} \geqslant\right.$ 
$\left.\mu_{2} \geqslant \cdots\right)$, définissons :

$$
\underline{\lambda} \succ \underline{\mu} \Longleftrightarrow \lambda_{1}>\mu_{1} \text { ou } \lambda_{1}=\mu_{1} \text { et } \lambda_{2}>\mu_{2} \text { etc. }
$$

On pose $\chi_{0, \rho^{\prime}}^{\prime}=\chi_{\iota}$ si $\underline{\alpha} \succeq \underline{\beta}, \chi_{0, \rho^{\prime}}^{\prime}=-\chi_{\iota}$ si $\underline{\beta} \succ \underline{\alpha}$.

Soit $\rho^{\prime \prime}$ une représentation irréductible $\overline{\text { de }} W_{N^{\prime \prime}}$. On définit de même un entier $b\left(\left|r^{\prime \prime}\right|, \rho^{\prime \prime}\right)$ et une fonction $\chi_{\left|r^{\prime \prime}\right|, \rho^{\prime \prime}}^{\prime \prime} \operatorname{sur} Z^{\prime \prime 0}$, à support unipotent.

Rappelons que, pour $j \in\{1,2\}$, on a posé :

$$
e_{j}= \begin{cases}0, & \text { si } h_{j} \geqslant 0 \\ 1, & \text { si } h_{j}<0\end{cases}
$$

Soit $\underline{\nu} \in \mathcal{N}$. On définit un caractère $\chi_{h_{1}, h_{2}, \underline{\nu}}$ du groupe $W(\underline{\nu})$ par la formule suivante, où $\underline{w} \in W(\underline{\nu})$ :

$$
\chi_{h_{1}, h_{2}, \underline{\nu}}(\underline{w})= \begin{cases}\operatorname{sgn}_{C D}\left(w_{2}^{\prime \prime}\right), & \text { si }\left|h_{1}\right| \geqslant\left|h_{2}\right| \text { et } e_{1}+e_{2} \equiv 0 \bmod 2 \mathbb{Z}, \\ \operatorname{sgn}_{C D}\left(w_{1}^{\prime \prime}\right), & \text { si }\left|h_{1}\right|<\left|h_{2}\right| \text { et } e_{1}+e_{2} \equiv 0 \bmod 2 \mathbb{Z}, \\ \operatorname{sgn}_{C D}\left(w_{2}^{\prime}\right), & \text { si }\left|h_{1}\right| \geqslant\left|h_{2}\right| \text { et } e_{1}+e_{2} \equiv 1 \bmod 2 \mathbb{Z}, \\ \operatorname{sgn}_{C D}\left(w_{1}^{\prime}\right), & \text { si }\left|h_{1}\right|<\left|h_{2}\right| \text { et } e_{1}+e_{2} \equiv 1 \bmod 2 \mathbb{Z} .\end{cases}
$$

Soient maintenant $\rho^{\prime} \in \operatorname{Irr}\left(W_{N^{\prime}}\right)$ et $\rho^{\prime \prime} \in \operatorname{Irr}\left(W_{N^{\prime \prime}}\right)$. On pose :

$$
\begin{aligned}
&\left(\rho^{\prime} \times \rho^{\prime \prime}, \rho_{1} \times \rho_{2}\right)_{h_{1}, h_{2}, \underline{\nu}}=|W(\underline{\nu})|^{-1} \sum_{\underline{w} \in W(\underline{\nu})} \operatorname{trace}\left(\rho^{\prime}\right)\left(w_{1}^{\prime} w_{2}^{\prime}\right) \operatorname{trace}\left(\rho^{\prime \prime}\right)\left(w_{1}^{\prime \prime} w_{2}^{\prime \prime}\right) \\
& \cdot \operatorname{trace}\left(\rho_{1}\right)\left(w_{1}^{\prime} w_{1}^{\prime \prime}\right) \operatorname{trace}\left(\rho_{2}\right)\left(w_{2}^{\prime} w_{2}^{\prime \prime}\right) \chi_{h_{1}, h_{2}, \underline{\nu}}(\underline{w}) .
\end{aligned}
$$

Posons :

$$
c\left(h_{1}, h_{2} ; g\right)= \begin{cases}1, & \text { si }\left|h_{1}\right| \geqslant\left|h_{2}\right| \text { et } h_{1} \geqslant 0, \\ \eta, & \text { si }\left|h_{1}\right| \geqslant\left|h_{2}\right| \text { et } h_{1}<0, \\ \eta^{\prime \prime}(g), & \text { si }\left|h_{1}\right|<\left|h_{2}\right| \text { et } h_{2} \geqslant 0, \\ \eta \eta^{\prime \prime}(g), & \text { si }\left|h_{1}\right|<\left|h_{2}\right| \text { et } h_{2}<0 .\end{cases}
$$

Proposition. - Sous l'hypothèse 7.1(1), on a l'égalité :

$$
\begin{array}{r}
k_{\eta}\left(\Lambda_{1}, \Lambda_{2}\right)(g)=c\left(h_{1}, h_{2}\right) c\left(h_{1}, h_{2} ; g\right) \sum_{\underline{\nu} \in \mathcal{N}} \zeta(-1)^{N_{2}^{\prime \prime}} \sum_{\rho^{\prime}, \rho^{\prime \prime}} q^{b\left(\left|r^{\prime}\right|, \rho^{\prime}\right)+b\left(\left|r^{\prime \prime}\right|, \rho^{\prime \prime}\right)+\delta\left(r^{\prime}, r^{\prime \prime}\right) / 2} \\
\cdot\left(\rho^{\prime} \times \rho^{\prime \prime}, \rho_{1} \times \rho_{2}\right)_{h_{1}, h_{2}, \underline{\nu}} \chi_{\left|r^{\prime}\right|, \rho^{\prime}}^{\prime}\left(u^{\prime}\right) \chi_{\left|r^{\prime \prime}\right|, \rho^{\prime \prime}}^{\prime \prime}\left(u^{\prime \prime}\right),
\end{array}
$$

où $\rho^{\prime}$ et $\rho^{\prime \prime}$ décrivent respectivement $\operatorname{Irr}\left(W_{N^{\prime}}\right)$ et $\operatorname{Irr}\left(W_{N^{\prime \prime}}\right)$.

Démonstration. - Appliquons la définition 3.3(1) et le lemme 7.1. Remarquons qu'une somme triple, convenablement pondérée, sur $w_{1} \in W_{N_{1}}, w_{2} \in W_{N_{2}}$ et $\underline{w} \in$ $W(\underline{\nu})$ tel que $w_{1}^{\prime} w_{1}^{\prime \prime}$ soit conjugué à $w_{1}$ dans $W_{N_{1}}$ et $w_{2}^{\prime} w_{2}^{\prime \prime}$ soit conjugué à $w_{2}$ dans 
$W_{N_{2}}$, une telle somme, donc, se simplifie en une somme sur tout $\underline{w} \in W(\underline{\nu})$. On obtient :

$$
k_{\eta}\left(\Lambda_{1}, \Lambda_{2}\right)(g)=2^{\alpha\left(h_{1}, h_{2} ; g\right)} c\left(h_{1}, h_{2}\right) q^{\delta\left(r^{\prime}, r^{\prime \prime}\right) / 2} \sum_{\underline{\nu} \in \mathcal{N}} \zeta(-1)^{N_{2}^{\prime \prime}} X_{\underline{\nu}}
$$

où, pour $\underline{\nu} \in \mathcal{N}$, on a posé :

$$
\begin{aligned}
X_{\underline{\nu}}=c_{\eta}\left(h_{1}, h_{2}\right)|W(\underline{\nu})|^{-1} & \\
& \sum_{\underline{w} \in W(\underline{\nu})} \gamma\left(h_{1}, h_{2} ; g ; \underline{w}\right) \operatorname{sgn}_{C D}\left(w_{2}^{\prime \prime}\right) \operatorname{sgn}_{C D}\left(w_{1}^{\prime} w_{1}^{\prime \prime}\right)^{e_{1}} \operatorname{sgn}_{C D}\left(w_{2}^{\prime} w_{2}^{\prime \prime}\right)^{e_{2}} \\
& \quad \operatorname{trace}\left(\rho_{1}\right)\left(w_{1}^{\prime} w_{1}^{\prime \prime}\right) \operatorname{trace}\left(\rho_{2}\right)\left(w_{2}^{\prime} w_{2}^{\prime \prime}\right) Q^{\prime}\left(\left|r^{\prime}\right|, w_{1}^{\prime} w_{2}^{\prime}\right)\left(u^{\prime}\right) Q^{\prime \prime}\left(\left|r^{\prime \prime}\right|, w_{1}^{\prime \prime} w_{2}^{\prime \prime}\right)\left(u^{\prime \prime}\right) .
\end{aligned}
$$

Soit $\underline{w} \in W(\underline{\nu})$. Un calcul cas par cas démontre l'égalité :

$$
\begin{aligned}
c_{\eta}\left(h_{1}, h_{2}\right) \gamma\left(h_{1}, h_{2} ; g ; \underline{w}\right) \operatorname{sgn}_{C D}\left(w_{2}^{\prime \prime}\right) \operatorname{sgn}_{C D}\left(w_{1}^{\prime} w_{1}^{\prime \prime}\right)^{e_{1}} \operatorname{sgn}_{C D}\left(w_{2}^{\prime} w_{2}^{\prime \prime}\right)^{e_{2}} \\
\quad=c\left(h_{1}, h_{2} ; g\right) \chi_{h_{1}, h_{2}, \underline{\nu}}(\underline{w}),
\end{aligned}
$$

à l'exception du cas où $h_{1}<0$ et $h_{2}= \pm h_{1}$. Dans ce dernier cas, le membre de gauche vaut $\eta^{\prime \prime}(g) \operatorname{sgn}_{C D}\left(w_{1}^{\prime}\right)$ si $h_{2}=-h_{1}, \eta \eta^{\prime \prime}(g) \operatorname{sgn}_{C D}\left(w_{1}^{\prime \prime}\right)$ si $h_{2}=h_{1}$, tandis que celui de droite vaut $\eta \operatorname{sgn}_{C D}\left(w_{2}^{\prime}\right)$ si $h_{2}=-h_{1}, \eta \operatorname{sgn}_{C D}\left(w_{2}^{\prime \prime}\right)$ si $h_{2}=h_{1}$. Mais supposons par exemple $h_{2}=-h_{1}$. Alors $r^{\prime}=0$ et $Q^{\prime}\left(0, w_{1}^{\prime} w_{2}^{\prime}\right)$ est nul si $\operatorname{sgn}_{C D}\left(w_{1}^{\prime} w_{2}^{\prime}\right) \neq \eta^{\prime}(g)$. On peut donc supposer $\operatorname{sgn}_{C D}\left(w_{1}^{\prime} w_{2}^{\prime}\right)=\eta^{\prime}(g)$. Puisque $r^{\prime}=0$, a fortiori $r^{\prime}$ est pair et $\eta^{\prime}(g) \eta^{\prime \prime}(g)=\eta$. On obtient alors l'égalité (2). Un raisonnement analogue vaut si $h_{1}<0$ et $h_{2}=h_{1}$.

Grâce à (2), on obtient :

$$
\begin{aligned}
& X_{\underline{\nu}}=c_{\eta}\left(h_{1}, h_{2}\right)|W(\underline{\nu})|^{-1} \sum_{\underline{w} \in W(\underline{\nu})} \chi_{h_{1}, h_{2}, \underline{\nu}}(\underline{w}) \operatorname{trace}\left(\rho_{1}\right)\left(w_{1}^{\prime} w_{1}^{\prime \prime}\right) \\
& \operatorname{trace}\left(\rho_{2}\right)\left(w_{2}^{\prime} w_{2}^{\prime \prime}\right) Q^{\prime}\left(\left|r^{\prime}\right|, w_{1}^{\prime} w_{2}^{\prime}\right)\left(u^{\prime}\right) Q^{\prime \prime}\left(\left|r^{\prime \prime}\right|, w_{1}^{\prime \prime} w_{2}^{\prime \prime}\right)\left(u^{\prime \prime}\right) .
\end{aligned}
$$

Pour $\rho^{\prime} \in \operatorname{Irr}\left(W_{N^{\prime}}\right)$, posons :

$$
Q_{\rho^{\prime}}^{\prime}=\left|W_{N^{\prime}}\right|^{-1} \sum_{w^{\prime} \in W_{N^{\prime}}} \operatorname{trace}\left(\rho^{\prime}\right)\left(w^{\prime}\right) Q^{\prime}\left(\left|r^{\prime}\right|, w^{\prime}\right)
$$

On définit de façon similaire $Q_{\rho^{\prime \prime}}^{\prime \prime}$ pour $\rho^{\prime \prime} \in \operatorname{Irr}\left(W_{N^{\prime \prime}}\right)$. Grâce aux formules d'inversion usuelles, on transforme l'égalité (3) en :

$$
X_{\underline{\nu}}=c\left(h_{1}, h_{2} ; g\right) \sum_{\substack{\rho^{\prime} \in \operatorname{Irr}\left(W_{N^{\prime}}\right) \\ \rho^{\prime \prime} \in \operatorname{Irr}\left(W_{N^{\prime \prime}}\right)}}\left(\rho^{\prime} \times \rho^{\prime \prime}, \rho_{1} \times \rho_{2}\right)_{h_{1}, h_{2}, \underline{\nu}} Q_{\rho^{\prime}}^{\prime}\left(u^{\prime}\right) Q_{\rho^{\prime \prime}}^{\prime \prime}\left(u^{\prime \prime}\right) .
$$

Mais, grâce à [L9] 6.15(a), repris dans $[\mathbf{W}]$ proposition VIII.12, pour $\rho^{\prime} \in \operatorname{Irr}\left(W_{N^{\prime}}\right)$, on a l'égalité :

$$
Q_{\rho^{\prime}}^{\prime}=2^{-\alpha^{\prime}} q^{b\left(\left|r^{\prime}\right|, \rho^{\prime}\right)} \chi_{\left|r^{\prime}\right|, \rho^{\prime}}^{\prime}
$$


où $\alpha^{\prime}=0$ sauf si $r^{\prime}=0$ et $N^{\prime}>0$, auquel cas $\alpha^{\prime}=1$. Ce terme $2^{-\alpha^{\prime}}$ vient dans ce cas du fait que le groupe qui intervient en loc. cit. est alors $W_{N^{\prime}}^{D}$ et non $W_{N^{\prime}}$. Une formule analogue calcule $Q_{\rho^{\prime \prime}}^{\prime \prime}$ pour $\rho^{\prime \prime} \in \operatorname{Irr}\left(W_{N^{\prime \prime}}\right)$. Reportons ces égalités dans la formule $(5)$, puis dans la formule (4) et l'égalité (1). En remarquant que $\alpha^{\prime}+\alpha^{\prime \prime}=\alpha\left(h_{1}, h_{2} ; g\right)$, on obtient l'égalité de l'énoncé.

7.3. Soient $\Lambda_{1} \in \widetilde{\mathcal{S}}_{n-1, \text { pair }}$ et $\eta, \varepsilon \in\{ \pm 1\}$. On dispose des fonctions $k_{\eta}\left(\Lambda_{1}, \mathbf{1}_{-}(1)\right)$, $k_{\eta}\left(\Lambda_{1}, \sigma \mathbf{1}_{-}(1)\right)$ sur $O_{\eta}(2 n), c f .2 .4$ pour les notations. On dispose aussi de la fonction $k_{\eta \varepsilon}\left(\Lambda_{1}, \Lambda(\varnothing)\right)$ sur $O_{\eta \varepsilon}(2 n-2)$ (rappelons que $n \geqslant 2$ ). Fixons un élément semi-simple $g \in O_{\eta}(2 n)$ ayant pour valeurs propres 1 avec la multiplicité $n-1$ et -1 avec la multiplicité 1 . Pour tout entier $N \geqslant 1$, on note $N_{p^{\prime}}$ la partie première à $p$ de $N$.

LemME. - Sous ces hypothèses, on a l'égalité :

$$
\begin{aligned}
k_{\eta}\left(\Lambda_{1}, \mathbf{1}_{-}(1)\right)(g)+\varepsilon k_{\eta} & \left(\Lambda_{1}, \sigma \mathbf{1}_{-}(1)\right)(g) \\
= & 2|S O(2 n-1)|_{p^{\prime}}\left|O_{\eta \varepsilon}(2 n-2)\right|_{p^{\prime}}^{-1} \operatorname{sp}(g) k_{\eta \varepsilon}\left(\Lambda_{1}, \Lambda(\varnothing)\right)(1) .
\end{aligned}
$$

Démonstration. - On calcule $k\left(\Lambda_{1}, \mathbf{1}_{-}(1)\right)(g)$ grâce à la proposition précédente. On a $h_{2}=1, N_{2}=0, r^{\prime}=h_{1}+1, r^{\prime \prime}=h_{1}-1, d^{\prime}=2 n-1, d^{\prime \prime}=1$. La condition 7.1(1) impose $h_{1} \in\{0,2\}$. Si elle n'est pas vérifiée, $k_{\eta}\left(\Lambda_{1}, \mathbf{1}_{-}(1)\right)(g)=0$. Supposons-la vérifiée. Alors $N^{\prime}=N_{1}, N^{\prime \prime}=0$. L'ensemble $\mathcal{N}$ a pour seul élément $\underline{\nu}=\left(N_{1}, 0,0,0\right)$. La fonction $\chi_{\left|r^{\prime \prime}\right|, \rho^{\prime \prime}}^{\prime \prime}$ intervenant dans la proposition précédente est bien sûr la fonction égale à 1 sur le groupe $S O(1)$. Considérons le terme $\chi_{\left|r^{\prime}\right|, \rho^{\prime}}^{\prime}\left(u^{\prime}\right)$. Ici $u^{\prime}=1$. D'après $[\mathbf{L} \mathbf{6}]$ théorème 24.4(d), la fonction $\chi_{\left|r^{\prime}\right|, \rho^{\prime}}^{\prime}$ est supportée par des orbites unipotentes $\boldsymbol{C}$ de $\boldsymbol{S} \boldsymbol{O}(2 n-1)$ pour lesquelles il existe un système local $\boldsymbol{S} \boldsymbol{O}(2 n-1)$-équivariant $\mathcal{L}$ sur $\boldsymbol{C}$ tel que le couple $(\boldsymbol{C}, \mathcal{L})$ corresponde par la correspondance de Springer généralisée à un couple $\left(\left|r^{\prime}\right|, \widetilde{\rho}^{\prime}\right)$ où $\widetilde{\rho}^{\prime} \in \operatorname{Irr}\left(W_{N_{1}}\right)$. Or l'unique système local sur l'orbite triviale correspond au couple $(1, \operatorname{sgn})$, où sgn est le caractère signe habituel. Donc $\chi_{\left|r^{\prime}\right|, \rho^{\prime}}^{\prime}(1)=0$ si $\left|r^{\prime}\right| \neq 1$. Il en résulte que :

$$
k_{\eta}\left(\Lambda_{1}, \mathbf{1}_{-}(1)\right)(g)=0, \text { si } h_{1} \neq 0 .
$$

Supposons $h_{1}=0$. Alors la formule de la proposition précédente donne :

$$
k_{\eta}\left(\Lambda_{1}, \mathbf{1}_{-}(1)\right)(g)=\eta^{\prime \prime}(g) q^{b\left(1, \rho_{1}\right)} \chi_{1, \rho_{1}}^{\prime}(1)
$$

D'après $1.6(1)$, on a $\eta^{\prime \prime}(g)=\mathrm{sp}(g)$. D'après $7.2(4)$ et $(6)$, on a :

$$
q^{b\left(1, \rho_{1}\right)} \chi_{1, \rho_{1}}^{\prime}=\left|W_{n-1}\right|^{-1} \sum_{w \in W_{n-1}} \operatorname{trace}\left(\rho_{1}\right)(w) Q^{\prime}(1, w) .
$$

Soit $w \in W_{n-1}$. Cet élément paramètre un sous-tore $\boldsymbol{T}_{w}$ de $\boldsymbol{S} \boldsymbol{O}(2 n-1)$ et on a l'égalité $Q^{\prime}(1, w)=R_{T_{w}}^{S O(2 n-1)}(1)$. La valeur en 1 de cette fonction est calculée par [C] théorème 7.5.1. On obtient :

$$
Q^{\prime}(1, w)=\operatorname{sgn}(w)|S O(2 n-1)|_{p^{\prime}}\left|T_{w}\right|_{p^{\prime}}^{-1} .
$$


D'où l'égalité :

$$
k_{\eta}\left(\Lambda_{1}, \mathbf{1}_{-}(1)\right)(g)=\operatorname{sp}(g)|S O(2 n-1)|_{p^{\prime}} \sum_{w \in W_{n-1}} \operatorname{sgn}(w) \operatorname{trace}\left(\rho_{1}\right)(w)\left|T_{w}\right|_{p^{\prime}}^{-1} .
$$

Un raisonnement similaire permet de calculer $k_{\eta}\left(\Lambda_{1}, \sigma \mathbf{1}_{-}(1)\right)(g)$ et $k_{\eta \varepsilon}\left(\Lambda_{1}, \Lambda(\varnothing)\right)(1)$. Ces termes sont nuls si $h_{1} \neq 0$. Si $h_{1}=0$, ils valent :

$$
\begin{gathered}
k_{\eta}\left(\Lambda_{1}, \sigma \mathbf{1}_{-}(1)\right)(g)=\eta \operatorname{sp}(g)|S O(2 n-1)|_{p^{\prime}} \sum_{w \in W_{n-1}} \operatorname{sgn}(w) \operatorname{sgn}_{C D}(w) \operatorname{trace}\left(\rho_{1}\right)(w)\left|T_{w}\right|_{p^{\prime}}^{-1}, \\
k_{\eta \varepsilon}\left(\Lambda_{1}, \Lambda(\varnothing)\right)(1)=2 \eta \varepsilon\left|S O_{\eta \varepsilon}(2 n-2)\right|_{p^{\prime}} \sum_{\substack{w \in W_{n-1} \\
\operatorname{sgn}_{C D}(w)=\eta \varepsilon}} \operatorname{sgn}(w) \operatorname{trace}\left(\rho_{1}\right)(w)\left|T_{w}\right|_{p^{\prime}}^{-1} .
\end{gathered}
$$

Remarque. - Dans cette dernière formule, $\boldsymbol{T}_{w}$ est un sous-tore de $\boldsymbol{S O}_{\eta}(2 n-2)$. Mais il est isomorphe au sous-tore de $\boldsymbol{S} \boldsymbol{O}(2 n-1)$ déjà introduit.

En remarquant que $2\left|S O_{\eta \varepsilon}(2 n-2)\right|_{p^{\prime}}=\left|O_{\eta \varepsilon}(2 n-2)\right|_{p^{\prime}}$, on en déduit l'égalité de l'énoncé.

7.4. Soient $m_{1}, m_{2} \in \mathbb{N}$ tels que $n=m_{1}^{2}+m_{2}^{2}$ et $m_{1} \geqslant m_{2}$. On pose $t^{\prime}=m_{1}+m_{2}$, $t^{\prime \prime}=m_{1}-m_{2}$.

Pour $j \in\{1,2\}$, posons :

$$
\Lambda_{j}^{\mathrm{sp}}=\left\{\left(x,(-1)^{x+1}\right) ; x \in\left\{2 m_{j}-1, \ldots, 0\right\}\right\} .
$$

C'est un symbole ordonné spécial de défaut 0 et de rang $m_{j}^{2}$. On note $\widetilde{\operatorname{Fam}}_{j}$ sa famille dans $\widetilde{\mathcal{S}}_{m_{j}^{2} \text {,pair }}$. On représente tout élément de $\widetilde{\operatorname{Fam}}_{j}$ par un sous-ensemble de $\mathbb{N} \times\{ \pm 1\}$ ayant $2 m_{j}$ éléments.

Posons $M=\left\{m_{1}-m_{2}, \ldots, 1\right\}$, notons $P_{M}$ l'ensemble des sous-ensembles de $M$. On définit une application :

$$
\begin{aligned}
\widetilde{\operatorname{Fam}}_{2} \times P_{M} & \longrightarrow \widetilde{\operatorname{Fam}}_{1} \times{\widetilde{\operatorname{Fam}_{2}}}_{(\Lambda, I)} \longmapsto\left(\Lambda_{1}(\Lambda, I), \Lambda_{2}(\Lambda, I)\right)
\end{aligned}
$$

par :

$$
\Lambda_{2}(\Lambda, I)=\Lambda
$$

$$
\begin{aligned}
\Lambda_{1}(\Lambda, I)=\left\{\left(x+2\left(m_{1}-m_{2}\right) ; \varepsilon\right) ;(x, \varepsilon) \in \Lambda\right\} \cup\left(\cup_{i \in I}\{(2 i-1,1),(2 i-2,1)\}\right) \\
\cup\left(\cup_{i \in M \backslash I}\{(2 i-1,-1),(2 i-2,-1)\}\right) .
\end{aligned}
$$

Cette application est injective. Pour $\Lambda \in{\widetilde{\operatorname{Fam}_{2}}}$ et $I \in P_{M}$, on pose :

$-h(\Lambda)=\widetilde{\operatorname{def}}(\Lambda) / 2$;

- pour $j \in\{1,2\}, h_{j}(\Lambda, I)=\widetilde{\operatorname{def}}\left(\Lambda_{j}(\Lambda, I)\right) / 2$;

$-c(\Lambda, I)=h(\Lambda)^{2}+2|I|^{2}+2|I| h(\Lambda)+h(\Lambda)\left(m_{2}-m_{1}\right)+2|I|\left(m_{2}-m_{1}\right)$;

$-f(I)= \begin{cases}0, & \text { si } m_{1}-m_{2} \in I \text { ou si } m_{1}=m_{2}, \\ 1, & \text { si } m_{1}-m_{2} \notin I \text { et } m_{1} \neq m_{2} ;\end{cases}$ 
$-f(\Lambda, I)= \begin{cases}0, & \text { si }\left(2 m_{2}-1,1\right) \in \Lambda \text { et } m_{2} \geqslant 1, \\ 1, & \text { si }\left(2 m_{2}-1,-1\right) \in \Lambda \text { et } m_{2} \geqslant 1, \\ f(I), & \text { si } m_{2}=0 .\end{cases}$

Remarquons que l'on a les égalités :

$$
\left\{\begin{array}{l}
h_{2}(\Lambda, I)=h(\Lambda) \\
h_{1}(\Lambda, I)=h(\Lambda)+2|I|-m_{1}+m_{2}
\end{array}\right.
$$

Soient $\eta \in\{ \pm 1\}$ et $g \in O_{\eta}(2 n)$. Écrivons $g=s u$. On suppose que $s$ a pour valeurs propres 1 avec la multiplicité $t^{\prime 2}$ et -1 avec la multiplicité $t^{\prime \prime 2}$. Comme en 7.1, on définit $\eta^{\prime}(g)$ et $\eta^{\prime \prime}(g)$ et on décompose $u=u^{\prime} u^{\prime \prime}$. On suppose $u^{\prime}$ paramétré par les données $\underline{\lambda}^{\prime}=\underline{\lambda}\left(u^{\prime}\right), \eta^{\prime}=\eta_{u^{\prime}}$ ainsi définies :

$$
\underline{\lambda}^{\prime}=\left(2 t^{\prime}-1,2 t^{\prime}-3, \ldots, 3,1\right)
$$

pour $i \in \operatorname{Jord}^{\mathrm{bp}}\left(\underline{\lambda}^{\prime}\right)$,

$$
\eta^{\prime}(i)= \begin{cases}\zeta(-1)^{(i-1) / 2} \eta^{\prime}(g), & \text { si } t^{\prime} \text { est impair, } \\ \zeta(-1)^{(i+1) / 2}, & \text { si } t^{\prime} \text { est pair et } i \neq 2 t^{\prime}-1, \\ \eta^{\prime}(g), & \text { si } t^{\prime} \text { est pair et } i=2 t^{\prime}-1 .\end{cases}
$$

On suppose $u^{\prime \prime}$ paramétré par les données $\underline{\lambda}^{\prime \prime}, \eta^{\prime \prime}$ définies de façon similaire.

Proposition. - Soient $\eta, g$ comme ci-dessus et $\left(\Lambda_{1}, \Lambda_{2}\right) \in \widetilde{\operatorname{Fam}}_{1} \times \widetilde{\text { Fam }}_{2}$.

(a) Si $\left(\Lambda_{1}, \Lambda_{2}\right)$ n'appartient pas à l'image de l'application $(1), k_{\eta}\left(\Lambda_{1}, \Lambda_{2}\right)(g)=0$.

(b) Supposons que $\left(\Lambda_{1}, \Lambda_{2}\right)$ soit l'image de $(\Lambda, I) \in \widetilde{\mathrm{Fam}}_{2} \times P_{M}$ par l'application

(1). On a alors l'égalité :

$$
k_{\eta}\left(\Lambda_{1}, \Lambda_{2}\right)(g)=q^{a} \zeta(-1)^{b} \eta^{c} \eta^{\prime \prime}(g)^{d},
$$

où :

- $a=\delta\left(t^{\prime}, t^{\prime \prime}\right) / 2-m_{1} m_{2}+c(\Lambda, I)$,

- si $m_{1} \not \equiv m_{2} \bmod 2 \mathbb{Z}, b=0, c=h(\Lambda)+|I|-\left(m_{1}-m_{2}+1\right) / 2, d=h(\Lambda)$,

- si $m_{1} \equiv m_{2} \bmod 2 \mathbb{Z}, b=|I|+\left(m_{1}-m_{2}\right) / 2, c=f(\Lambda, I), d=f(\Lambda, I)+f(I)$.

La démonstration de cette proposition occupe les paragraphes 7.5 à 7.10. Elle est basée sur la proposition 7.2 et sur les résultats de $[\mathbf{W}]$ chapitre XI, quelque peu généralisés, que nous allons reformuler.

7.5. Pour $j \in\{1,2\}$, on fixe $\Lambda_{j} \in \widetilde{\operatorname{Fam}}_{j}$. On note $\left(h_{j}, \rho_{j}\right)$ le couple associé en 2.2 à $\Lambda_{j}$, on pose :

$$
e_{j}= \begin{cases}0, & \text { si } h_{j} \geqslant 0 \\ 1, & \text { si } h_{j}<0\end{cases}
$$


Au symbole spécial $\Lambda_{j}^{\text {sp }}$ sont associées une partition orthogonale spéciale $\underline{\lambda}_{j} \in \mathcal{P}\left(m_{j}^{2}\right)$ et une partition $\operatorname{Int}\left(\underline{\lambda}_{j}\right)$ de $\operatorname{Jord}^{\mathrm{bp}}\left(\underline{\lambda}_{j}\right), c f$. [W] VIII.20. En utilisant les définitions de [W] VIII.7 et VIII.20, on calcule :

$$
\underline{\lambda}_{j}=\left(2 m_{j}-1,2 m_{j}-1,2 m_{j}-3,2 m_{j}-3, \ldots, 3,3,1,1\right),
$$

et $\operatorname{Int}\left(\underline{\lambda}_{j}\right)$ est la partition maximale de $\operatorname{Jord}^{\mathrm{bp}}\left(\underline{\lambda}_{j}\right)$, c'est-à-dire celle formée de tous les sous-ensembles à un élément de $\operatorname{Jord}^{\mathrm{bp}}\left(\underline{\lambda}_{j}\right)$. Cela permet d'identifier $\operatorname{Int}\left(\underline{\lambda}_{j}\right)$ à $\operatorname{Jord}^{\mathrm{bp}}\left(\underline{\lambda}_{j}\right)$. On associe à $\Lambda_{j}$ l'élément $\left(\tau_{j}, \delta_{j}\right)$ de $(\mathbb{Z} / 2 \mathbb{Z})^{\operatorname{Int}\left(\underline{\lambda}_{j}\right)} \times(\mathbb{Z} / 2 \mathbb{Z})^{\operatorname{Int}\left(\underline{\lambda}_{j}\right)}$ défini par :

$$
\begin{aligned}
& \tau_{j}(i)=\left|\left\{(x, \varepsilon) ;(x, \varepsilon) \in \Lambda_{j},(x, \varepsilon) \notin \Lambda_{j}^{\mathrm{sp}}, x \geqslant i\right\}\right| \bmod 2 \mathbb{Z}, \\
& \delta_{j}(i)=\left|\left\{(x, \varepsilon) ;(x, \varepsilon) \in \Lambda_{j},(x, \varepsilon) \notin \Lambda_{j}^{\mathrm{sp}}, x \geqslant i-1\right\}\right| \bmod 2 \mathbb{Z},
\end{aligned}
$$

pour tout $i \in \operatorname{Int}\left(\underline{\lambda}_{j}\right)$.

Au couple $\left(\underline{\lambda}_{1}, \underline{\lambda}_{2}\right)$ on associe par «induction endoscopique » une partition orthogonale $\underline{\lambda} \in \mathcal{P}(n)$ et une partition $\operatorname{Int}(\underline{\lambda})$ de $\operatorname{Jord}^{\mathrm{bp}}(\underline{\lambda})$, cf. $[\mathbf{W}]$ XI.11 et XI.15. On calcule :

$$
\begin{aligned}
& \underline{\lambda}=\left(2 m_{1}+2 m_{2}-1,2 m_{1}+2 m_{2}-3, \ldots, 2\left(m_{1}-m_{2}\right)+3,2\left(m_{1}-m_{2}\right)+1,\right. \\
& \left.2\left(m_{1}-m_{2}\right)-1,2\left(m_{1}-m_{2}\right)-1,2\left(m_{1}-m_{2}\right)-3,2\left(m_{1}-m_{2}\right)-3, \ldots, 3,3,1,1\right),
\end{aligned}
$$

et $\operatorname{Int}(\underline{\lambda})$ est la partition maximale de $\operatorname{Jord}^{\mathrm{bp}}(\underline{\lambda})$. On identifie $\operatorname{Int}(\underline{\lambda})$ et $\operatorname{Jord}^{\mathrm{bp}}(\underline{\lambda})$.

On construit des fonctions :

$$
\tau^{\prime}, \delta^{\prime}, \tau^{\prime \prime}, \delta^{\prime \prime}: \operatorname{Int}(\underline{\lambda}) \longrightarrow \mathbb{Z} / 2 \mathbb{Z},
$$

de la façon suivante. Soit $i \in\left\{1, \ldots, m_{1}\right\}$; on a les égalités modulo $2 \mathbb{Z}$ :

$$
\begin{aligned}
- \text { si } i \leqslant m_{2}, & \\
\tau^{\prime}\left(2 m_{1}+2 m_{2}-4 i+3\right) & =\tau^{\prime \prime}\left(2 m_{1}+2 m_{2}-4 i+3\right) \\
& =\tau_{1}\left(2 m_{1}-2 i+1\right)+\delta_{2}\left(2 m_{2}-2 i+3\right)+h_{1}+h_{2}+e_{1}, \\
\tau^{\prime}\left(2 m_{1}+2 m_{2}-4 i+1\right) & =\tau^{\prime \prime}\left(2 m_{1}+2 m_{2}-4 i+1\right) \\
& =\tau_{1}\left(2 m_{1}-2 i+1\right)+\delta_{2}\left(2 m_{2}-2 i+1\right)+h_{1}+h_{2}+e_{1}, \\
\delta^{\prime}\left(2 m_{1}+2 m_{2}-4 i+3\right) & =\tau_{1}\left(2 m_{1}-2 i+1\right)+\tau_{2}\left(2 m_{2}-2 i+1\right)+1, \\
\delta^{\prime \prime}\left(2 m_{1}+2 m_{2}-4 i+3\right) & =\tau_{1}\left(2 m_{1}-2 i+1\right)+\tau_{2}\left(2 m_{2}-2 i+1\right), \\
\delta^{\prime}\left(2 m_{1}+2 m_{2}-4 i+1\right) & =\delta^{\prime \prime}\left(2 m_{1}+2 m_{2}-4 i+1\right) \\
& =\delta_{1}\left(2 m_{1}-2 i+1\right)+\delta_{2}\left(2 m_{2}-2 i+1\right) ;
\end{aligned}
$$

- si $i>m_{2}$,

$$
\begin{aligned}
& \tau^{\prime}\left(2 m_{1}-2 i+1\right)=\tau^{\prime \prime}\left(2 m_{1}-2 i+1\right)=\tau_{1}\left(2 m_{1}-2 i+1\right)+h_{1}+e_{1}, \\
& \delta^{\prime}\left(2 m_{1}-2 i+1\right)=\delta^{\prime \prime}\left(2 m_{1}-2 i+1\right)=\delta_{1}\left(2 m_{1}-2 i+1\right)+h_{2} .
\end{aligned}
$$


Dans la première égalité, on a $2 m_{2}-2 i+3=2 m_{2}+1$ si $i=1$, et $\delta_{2}\left(2 m_{2}+1\right)$ n'est pas défini; on pose $\delta_{2}\left(2 m_{2}+1\right)=0$.

Comme en 7.1, posons $r^{\prime}=h_{1}+h_{2}, r^{\prime \prime}=h_{1}-h_{2}$. Supposons d'abord :

$$
r^{\prime} \equiv r^{\prime \prime} \equiv t^{\prime} \equiv t^{\prime \prime} \bmod 2 \mathbb{Z},\left|r^{\prime}\right| \leqslant t^{\prime},\left|r^{\prime \prime}\right| \leqslant t^{\prime \prime}
$$

(c'est l'hypothèse $7.1(1))$. On pose $N^{\prime}=\left(t^{\prime 2}-r^{\prime 2}\right) / 2, N^{\prime \prime}=\left(t^{\prime \prime 2}-r^{\prime \prime 2}\right) / 2$. Soit $\rho^{\prime}$ une représentation irréductible de $W_{N^{\prime}}$. Supposons d'abord $r^{\prime} \neq 0$. Comme on l'a dit en 7.2, la correspondance de Springer généralisée associe à $\left(\left|r^{\prime}\right|, \rho^{\prime}\right)$ un couple $(\boldsymbol{C}, \mathcal{L})$ formé d'une orbite unipotente de $\boldsymbol{S} \boldsymbol{O}\left(V^{\prime}\right)$ ( $c f .7 .1$ pour la définition de $\left.V^{\prime}\right)$ et d'un système local $\mathcal{L}$ sur $\boldsymbol{C}$. À $\boldsymbol{C}$ est associée une partition orthogonale de $t^{\prime 2}$ que nous noterons $\underline{\lambda}_{\left|r^{\prime}\right|, \rho^{\prime}}$. Au système local $\mathcal{L}$ est associé un caractère du groupe $\boldsymbol{Z}_{\boldsymbol{S O}\left(V^{\prime}\right)}(x) / \boldsymbol{Z}_{\boldsymbol{S O}\left(V^{\prime}\right)}(x)^{0}$, où $x$ est un élément fixé de $C$. Le groupe des caractères de

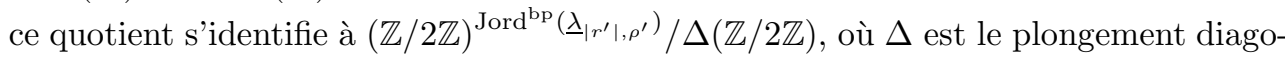
nal. À $\mathcal{L}$ est donc associé un élément de ce groupe, que l'on note $\varepsilon_{\left|r^{\prime}\right|, \rho^{\prime}}$. Supposons maintenant $r^{\prime}=0$. C'est alors à un couple $\left(0, \rho_{0}^{\prime}\right)$, où $\rho_{0}^{\prime}$ est une représentation irréductible de $W_{N^{\prime}}^{D}$, que l'on peut associer comme ci-dessus une partition $\underline{\lambda}_{0, \rho_{0}^{\prime}}$ et un

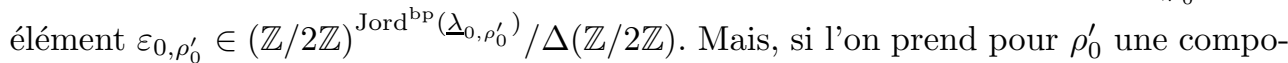
sante irréductible de la restriction de $\rho^{\prime}$ à $W_{N^{\prime}}^{D}$, ces termes ne dépendent pas du choix de $\rho_{0}^{\prime}$. Cela permet de les noter $\underline{\lambda}_{0, \rho^{\prime}}$ et $\varepsilon_{0, \rho^{\prime}}$. De façon similaire, on associe à toute $\rho^{\prime \prime} \in \operatorname{Irr}\left(W_{N^{\prime \prime}}\right)$ des termes $\underline{\lambda}_{\left|r^{\prime \prime}\right|, \rho^{\prime \prime}}$ et $\varepsilon_{\left|r^{\prime \prime}\right|, \rho^{\prime \prime}}$.

En 7.1 et 7.2, on a défini l'ensemble $\mathcal{N}$ et, pour $\underline{\nu} \in \mathcal{N}$ et $\left(\rho^{\prime}, \rho^{\prime \prime}\right) \in \operatorname{Irr}\left(W_{N^{\prime}}\right) \times$ $\operatorname{Irr}\left(W_{N^{\prime \prime}}\right)$, un entier $\left(\rho^{\prime} \times \rho^{\prime \prime}, \rho_{1} \times \rho_{2}\right)_{h_{1}, h_{2}, \underline{\nu}}$. On note $\mathcal{I}$ l'ensemble des couples $\left(\rho^{\prime}, \rho^{\prime \prime}\right) \in$ $\operatorname{Irr}\left(W_{N^{\prime}}\right) \times \operatorname{Irr}\left(W_{N^{\prime \prime}}\right)$ vérifiant les deux conditions suivantes :

$$
\begin{gathered}
\text { il existe } \underline{\nu} \in \mathcal{N} \text { tel que }\left(\rho^{\prime} \times \rho^{\prime \prime}, \rho_{1} \times \rho_{2}\right)_{h_{1}, h_{2}, \underline{\nu}} \neq 0 ; \\
\underline{\lambda}^{\prime} \leqslant \underline{\lambda}_{\left|r^{\prime}\right|, \rho^{\prime}}, \quad \underline{\lambda}^{\prime \prime} \leqslant \underline{\lambda}_{\left|r^{\prime \prime}\right|, \rho^{\prime \prime}}
\end{gathered}
$$

(il s'agit ici de l'ordre usuel des partitions de même somme : pour $m \in \mathbb{N}$, $\underline{\mu}^{\prime}=\left(\mu_{1}^{\prime} \geqslant \mu_{2}^{\prime} \geqslant \cdots\right) \in \mathcal{P}(m), \underline{\mu}^{\prime \prime}=\left(\mu_{1}^{\prime \prime} \geqslant \mu_{2}^{\prime \prime} \geqslant \cdots\right) \in \mathcal{P}(m)$, on a $\underline{\mu}^{\prime} \leqslant \underline{\mu}^{\prime \prime}$ si et seulement si $\mu_{1}^{\prime}+\cdots+\mu_{i}^{\prime} \leqslant \bar{\mu}_{1}^{\prime \prime}+\cdots+\mu_{i}^{\prime \prime}$ pour tout $\left.i \in \mathbb{N}\right)$.

Levons l'hypothèse (2). Si elle n'est pas vérifiée, on pose $\mathcal{I}=\varnothing$.

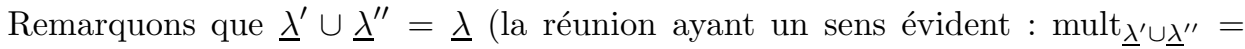
mult $_{\underline{\lambda}^{\prime}}+$ mult $_{\underline{\lambda}^{\prime \prime}}$, cf. 1.5). En particulier $\operatorname{Jord}^{\mathrm{bp}}\left(\underline{\lambda}^{\prime}\right) \cup \operatorname{Jord}^{\mathrm{bp}}\left(\underline{\lambda}^{\prime \prime}\right)=\operatorname{Jord}^{\mathrm{bp}}(\underline{\lambda})=$ $\operatorname{Int}(\underline{\lambda})$. La restriction de $\tau^{\prime}$ à $\operatorname{Jord}^{\mathrm{bp}}\left(\underline{\lambda}^{\prime}\right)$ est un élément de $(\mathbb{Z} / 2 \mathbb{Z})^{\operatorname{Jord}^{\mathrm{bp}}\left(\underline{\lambda}^{\prime}\right)}$.

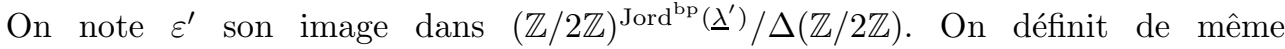

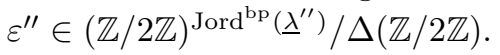

On considérera la condition :

(5) pour tout $i \in \operatorname{Int}(\underline{\lambda})$,

$$
\sum_{j \geqslant i} \operatorname{mult}_{{\underline{\lambda^{\prime}}}^{\prime}}(j) \equiv \delta^{\prime}(i) \bmod 2 \mathbb{Z}, \quad \sum_{j \geqslant i} \operatorname{mult}_{\underline{\lambda}^{\prime \prime}}(j) \equiv \delta^{\prime \prime}(i) \bmod 2 \mathbb{Z} .
$$


Enfin, soient $m \in \mathbb{N}$ et $\rho \in \operatorname{Irr}\left(W_{m}\right)$. La représentation $\rho$ est paramétrée par un couple de partitions $(\underline{\alpha}, \underline{\beta})$ tel que $S(\underline{\alpha})+S(\underline{\beta})=m$. On a introduit en 7.2 l'ordre lexicographique $\succeq$ sur l'ensemble des partitions. On note $\rho \succeq 0$ si et seulement si $\underline{\alpha} \succeq \underline{\beta}$.

\section{Proposition}

(a) Si la condition (5) n'est pas vérifiée, $\mathcal{I}=\varnothing$.

(b) Si la condition (5) est vérifiée, $\mathcal{I}$ a un unique élément, notons-le $\left(\rho^{\prime}, \rho^{\prime \prime}\right)$. On $a \underline{\lambda}_{\left|r^{\prime}\right|, \rho^{\prime}}=\underline{\lambda}^{\prime}, \underline{\lambda}_{\left|r^{\prime \prime}\right|, \rho^{\prime \prime}}=\underline{\lambda}^{\prime \prime}, \varepsilon_{\left|r^{\prime}\right|, \rho^{\prime}}=\varepsilon^{\prime}, \varepsilon_{\left|r^{\prime \prime}\right|, \rho^{\prime \prime}}=\varepsilon^{\prime \prime}$. Il existe un unique $\underline{\nu} \in \mathcal{N}$ tel que $\left(\rho^{\prime} \times \rho^{\prime \prime}, \rho_{1} \times \rho_{2}\right)_{h_{1}, h_{2}, \underline{\nu}} \neq 0$. Pour cet unique $\underline{\nu}$, on a $\left(\rho^{\prime} \times \rho^{\prime \prime}, \rho_{1} \times \rho_{2}\right)_{h_{1}, h_{2}, \underline{\nu}}=1$. Si $r^{\prime}=0$ et $t^{\prime}>0$, on $\bar{a} \rho^{\prime} \succeq 0 \Leftrightarrow \tau^{\prime}\left(2 t^{\prime}-1\right)=0$. Si $r^{\prime \prime}=0$ et $t^{\prime \prime}>0$, on a $\rho^{\prime \prime} \succeq 0 \Leftrightarrow \tau^{\prime \prime}\left(2 t^{\prime \prime}-1\right)=0$.

Démonstration. - Supposons $h_{1}, h_{2}, t^{\prime}, t^{\prime \prime}$ pairs et $h_{1} h_{2} \neq 0$. Posons $\iota_{1}=\left(\left|h_{1}\right|, \rho_{1}\right)$, $\iota_{2}=\left(\left|h_{2}\right|, \rho_{2}\right), \xi=(-1)^{e_{1}+e_{2}}, \iota_{0}=\left(\left|r^{\prime}\right|,\left|r^{\prime \prime}\right|, \xi\right)$. Ces termes $\iota_{1}, \iota_{2}, \iota_{0}$ vérifient les hypothèses de $[\mathbf{W}]$ XI.21 et XI.27, appliquées au cas d'un groupe $p$-adique $\boldsymbol{S O}(2 n)$ et de son sous-groupe endoscopique $\boldsymbol{S} \boldsymbol{O}\left(2 h_{1}^{2}+2 N_{1}\right) \times \boldsymbol{S O}\left(2 h_{2}^{2}+2 N_{2}\right)$, la forme exacte de ces groupes important peu. Appliquons les constructions de $[\mathbf{W}]$ XI.27 et XI.29 à un réseau $L$ tel que $\operatorname{dim}_{\mathbb{F}_{q}}\left(\ell^{\prime}\right)=t^{\prime 2}, \operatorname{dim}_{\mathbb{F}_{q}}\left(\ell^{\prime \prime}\right)=t^{\prime \prime 2}$. On y a construit deux ensembles $\mathcal{P}_{L}\left(\iota_{1}, \iota_{2} ; \iota_{0}\right)$ et $\mathcal{I}_{L}^{\max }\left(\iota_{1}, \iota_{2} ; \iota_{0}\right)$. L'ensemble $\mathcal{P}_{L}\left(\iota_{1}, \iota_{2} ; \iota_{0}\right)$ est vide si la condition (2) n'est pas vérifiée (car alors $\left|r^{\prime}\right| \notin \mathcal{I}_{0}\left(\ell^{\prime}\right)$ ou $\left|r^{\prime \prime}\right| \notin \mathcal{I}_{0}\left(\ell^{\prime \prime}\right)$ avec les notations de $[\mathbf{W}])$. Si elle l'est, un élément de $\mathcal{P}_{L}\left(\iota_{1}, \iota_{2} ; \iota_{0}\right)$ est une famille $\underline{p}=$ $\left(\underline{\alpha}^{\prime}, \underline{\beta}^{\prime}, \underline{\alpha}^{\prime \prime}, \underline{\beta}^{\prime \prime}, \underline{\alpha}_{1}^{\prime}, \underline{\alpha}_{2}^{\prime}, \underline{\alpha}_{1}^{\prime \prime}, \underline{\alpha}_{2}^{\prime \prime}, \underline{\beta}_{1}^{\prime}, \underline{\beta}_{2}^{\prime}, \underline{\beta}_{1}^{\prime \prime}, \underline{\beta}_{2}^{\prime \prime}\right)$ de suites finies d'entiers $\geqslant 0$, les quatre premiers termes étant des partitions telles que $S\left(\underline{\alpha}^{\prime}\right)+S\left(\underline{\beta}^{\prime}\right)=N^{\prime}, S\left(\underline{\alpha}^{\prime \prime}\right)+S\left(\underline{\beta}^{\prime \prime}\right)=$ $N^{\prime \prime}$. Associons à une telle famille $\underline{p}$ le couple $\left(\rho_{\underline{p}}^{\prime}, \rho_{\underline{p}}^{\prime \prime}\right) \in \operatorname{Irr}\left(W_{N^{\prime}}\right) \times \operatorname{Irr}\left(W_{N^{\prime \prime}}\right)$ tel que $\rho_{p}^{\prime}$ soit paramétrée par $\left(\underline{\alpha}^{\prime}, \underline{\beta}^{\prime}\right)$ et $\rho_{\underline{p}}^{\prime \prime}$ soit paramétrée par $\left(\underline{\alpha}^{\prime \prime}, \underline{\beta}^{\prime \prime}\right)$. Alors la preuve de $[\overline{\mathbf{W}}]$ XII.7(1) entraîne que l'application $\underline{p} \mapsto\left(\rho_{p}^{\prime}, \rho_{p}^{\prime \prime}\right)$ est une surjection de $\mathcal{P}_{L}\left(\iota_{1}, \iota_{2} ; \iota_{0}\right)$ sur l'ensemble des $\left(\rho^{\prime}, \rho^{\prime \prime}\right) \in \operatorname{Irr}\left(W_{N^{\prime}}\right) \times \operatorname{Irr}\left(\bar{W}_{N^{\prime \prime}}\right)$ vérifiant la condition (3). Notons $\widetilde{\mathcal{P}}_{L}\left(\iota_{1}, \iota_{2} ; \iota_{0}\right)$ l'ensemble des $\underline{p} \in \mathcal{P}_{L}\left(\iota_{1}, \iota_{2} ; \iota_{0}\right)$ tels que $\underline{\lambda}^{\prime} \leqslant \underline{\lambda}_{\left|r^{\prime}\right|, \rho_{\underline{p}}^{\prime}}, \underline{\lambda}^{\prime \prime} \leqslant \underline{\lambda}_{\left|r^{\prime \prime}\right|, \rho_{\underline{p}}^{\prime \prime}}$. Alors l'application $\underline{p} \mapsto\left(\rho_{\underline{p}}^{\prime}, \rho_{\underline{p}}^{\prime \prime}\right)$ est une surjection de $\widetilde{\mathcal{P}}_{L}\left(\iota_{1}, \iota_{2} ; \iota_{0}\right)$ sur $\mathcal{I}$. Cela reste vrai si (2) n'est pas vérifiée, les ensembles de départ et d'arrivée étant dans ce cas tous deux vides.

L'ensemble $\mathcal{I}_{L}^{\max }\left(\iota_{1}, \iota_{2} ; \iota_{0}\right)$ est formé de couples $\left(\left(\underline{\mu}^{\prime}, \nu^{\prime}\right),\left(\underline{\mu}^{\prime \prime}, \nu^{\prime \prime}\right)\right)$, où $\underline{\mu}^{\prime}$, resp. $\underline{\mu}^{\prime \prime}$, est une partition orthogonale de $t^{\prime 2}$, resp. $t^{\prime \prime 2}$, et où $\nu^{\prime} \in(\mathbb{Z} / 2 \mathbb{Z})^{\operatorname{Jord}^{\mathrm{bp}}\left(\underline{\mu}^{\prime}\right)} / \Delta(\mathbb{Z} / 2 \mathbb{Z})$,

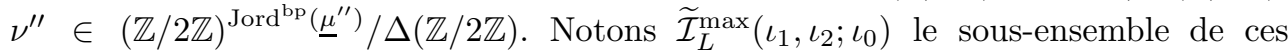
$\left(\left(\underline{\mu}^{\prime}, \nu^{\prime}\right),\left(\underline{\mu}^{\prime \prime}, \nu^{\prime \prime}\right)\right)$ tels que $\underline{\mu}^{\prime}=\underline{\lambda}^{\prime}, \underline{\mu}^{\prime \prime}=\underline{\lambda}^{\prime \prime}$. Dans la définition de $[\mathbf{W}]$ XI.29 interviennent des fonctions $\bar{\tau}^{ \pm}, \delta^{ \pm}$. On a défini ci-dessus $\tau^{\prime}, \tau^{\prime \prime}, \delta^{\prime}, \delta^{\prime \prime}$ de sorte que $\tau^{\xi}=\tau^{\prime}, \tau^{-\xi}=\tau^{\prime \prime}, \delta^{\xi}=\delta^{\prime}, \delta^{-\xi}=\delta^{\prime \prime}$. Alors la définition de [W] XI.29 nous dit que $\widetilde{\mathcal{I}}_{L}^{\max }\left(\iota_{1}, \iota_{2} ; \iota_{0}\right)=\varnothing$ si $(5)$ n'est pas vérifiée. Si elle l'est, $\widetilde{\mathcal{I}}_{L}^{\max }\left(\iota_{1}, \iota_{2} ; \iota_{0}\right)$ est réduit à l'unique élément $\left(\left(\underline{\lambda}^{\prime}, \varepsilon^{\prime}\right),\left(\underline{\lambda}^{\prime \prime}, \varepsilon^{\prime \prime}\right)\right)$. 
Soit $\underline{p} \in \widetilde{\mathcal{P}}_{L}\left(\iota_{1}, \iota_{2} ; \iota_{0}\right)$. La proposition $[\mathbf{W}]$ XI.28 nous dit que $\underline{\lambda}_{\left|r^{\prime}\right|, \rho_{\underline{p}}^{\prime}} \cup \underline{\lambda}_{\left|r^{\prime \prime}\right|, \rho_{\underline{p}}^{\prime \prime}} \leqslant$ $\underline{\lambda}$. Mais, puisque l'on a de plus $\underline{\lambda}^{\prime} \leqslant \underline{\lambda}_{\left|r^{\prime}\right|, \rho_{\underline{p}}^{\prime}}, \underline{\lambda}^{\prime \prime} \leqslant \underline{\lambda}_{\left|r^{\prime \prime}\right|, \rho_{\underline{p}}^{\prime \prime}}$ et $\underline{\lambda}^{\prime} \cup \underline{\lambda}^{\prime \prime}=\underline{\lambda}$, on a nécessairement $\underline{\lambda}^{\prime}=\underline{\lambda}_{\left|r^{\prime}\right|, \rho_{\underline{p}}^{\prime}}, \underline{\lambda}^{\prime \prime}=\underline{\lambda}_{\left|r^{\prime \prime}\right|, \rho_{\underline{p}}^{\prime \prime}}$ Alors la proposition $[\mathbf{W}]$ XI.29 (i) et (ii) nous dit que l'application :

$$
\begin{aligned}
\widetilde{\mathcal{P}}_{L}\left(\iota_{1}, \iota_{2} ; \iota_{0}\right) & \longrightarrow \widetilde{\mathcal{I}}_{L}^{\max }\left(\iota_{1}, \iota_{2} ; \iota_{0}\right) \\
\underline{p} & \longmapsto\left(\left(\underline{\lambda}_{\left|r^{\prime}\right|, \rho_{\underline{p}}^{\prime}}, \varepsilon_{\left|r^{\prime}\right|, \rho_{\underline{p}}^{\prime}}\right),\left(\underline{\lambda}_{\left|r^{\prime \prime}\right|, \rho_{\underline{p}}^{\prime \prime}}, \varepsilon_{\left|r^{\prime \prime}\right|, \rho_{\underline{p}}^{\prime \prime}}\right)\right)
\end{aligned}
$$

est bijective. Il en est donc de même de l'application :

$$
\begin{aligned}
\mathcal{I} & \longrightarrow \widetilde{\mathcal{I}}_{L}^{\max }\left(\iota_{1}, \iota_{2} ; \iota_{0}\right) \\
\left(\rho^{\prime}, \rho^{\prime \prime}\right) & \longmapsto\left(\left(\underline{\lambda}_{\left|r^{\prime}\right|, \rho^{\prime}}, \varepsilon_{\left|r^{\prime}\right|, \rho^{\prime}}\right),\left(\underline{\lambda}_{\left|r^{\prime \prime}\right|, \rho^{\prime \prime}}, \varepsilon_{\left|r^{\prime \prime}\right|, \rho^{\prime \prime}}\right)\right) .
\end{aligned}
$$

Si (5) n'est pas vérifiée, $\widetilde{\mathcal{I}}_{L}^{\max }\left(\iota_{1}, \iota_{2} ; \iota_{0}\right)$ est vide, donc $\mathcal{I}$ aussi. Si elle l'est, $\widetilde{\mathcal{I}}_{L}^{\max }\left(\iota_{1}, \iota_{2} ; \iota_{0}\right)$ a pour unique élément $\left(\left(\underline{\lambda}^{\prime}, \varepsilon^{\prime}\right),\left(\underline{\lambda}^{\prime \prime}, \varepsilon^{\prime \prime}\right)\right)$. Donc $\mathcal{I}$ a un unique élément, notons-le $\left(\rho^{\prime}, \rho^{\prime \prime}\right)$. On a $\underline{\lambda}_{\left|r^{\prime}\right|, \rho^{\prime}}=\underline{\lambda}^{\prime}, \underline{\lambda}_{\left|r^{\prime \prime}\right|, \rho^{\prime \prime}}=\underline{\lambda}^{\prime \prime}, \varepsilon_{\left|r^{\prime}\right|, \rho^{\prime}}=\varepsilon^{\prime}, \varepsilon_{\left|r^{\prime \prime}\right|, \rho^{\prime \prime}}=\varepsilon^{\prime \prime}$. La preuve de $[\mathbf{W}]$ XII.7(1) et l'unicité du $\underline{p}$ se projetant sur $\left(\rho^{\prime}, \rho^{\prime \prime}\right)$ entraînent l'unicité d'un $\nu \in \mathcal{N}$ tel que $\left(\rho^{\prime} \times \rho^{\prime \prime}, \rho_{1} \times \bar{\rho}_{2}\right)_{h_{1}, h_{2}, \nu} \neq 0$ et, pour cet unique $\nu$, on a $\left(\rho^{\prime} \times \rho^{\prime \prime}, \rho_{1} \times \rho_{2}\right)_{h_{1}, h_{2}, \nu}=1$. Enfin la dernière assertion de l'énoncé résulte de $[\mathbf{W}]$ proposition XI.29(iii).

On peut lever la condition $h_{1} h_{2} \neq 0$ tout en restant dans le cadre de $[\mathbf{W}]$. La seule difficulté est qu'en $[\mathbf{W}]$, on supposait par exemple $\rho_{1} \succeq 0$ si $h_{1}=0$. Si $h_{1}=0$ et $\rho_{1} \nsucceq 0$, on se ramène à la situation de $[\mathbf{W}]$ en remplaçant $\rho_{1} \operatorname{par} \rho_{1} \otimes \operatorname{sgn}_{C D}$. On laisse cela au lecteur car, de toutes façons, le cas où $h_{1}, h_{2}, t^{\prime}, t^{\prime \prime}$ ne sont pas tous pairs ne peut pas se ramener à la situation de $[\mathbf{W}]$. Dans ce cas, on est forcé de refaire la démonstration de $[\mathbf{W}]$. Sa longueur interdit de la reproduire ici. On ne peut que se borner à affirmer que le résultat est celui de l'énoncé.

\section{6.}

LEMME. - La condition 7.5(5) est vérifiée si et seulement si $\left(\Lambda_{1}, \Lambda_{2}\right)$ appartient à l'image de l'application $7.4(1)$.

Démonstration. - Le calcul des fonctions mult ${\underline{\underline{\lambda}^{\prime}}}$ et mult ${\underline{\underline{\lambda^{\prime \prime}}}}$ étant évident, la condition 7.5(5) s'écrit explicitement :

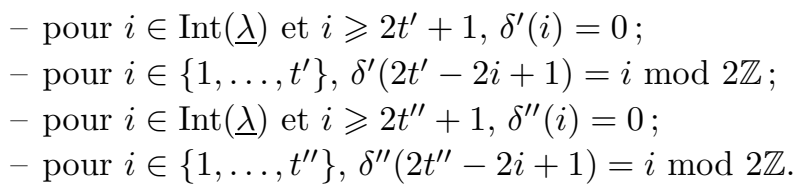


En se reportant aux définitions de $\delta^{\prime}$ et $\delta^{\prime \prime}$, ces conditions sont équivalentes aux conditions suivantes :

$$
\begin{aligned}
\text { pour } i \in\left\{1, \ldots, m_{2}\right\}, & \tau_{1}\left(2 m_{1}-2 i+1\right)+\tau_{2}\left(2 m_{2}-2 i+1\right)=0, \\
& \delta_{1}\left(2 m_{1}-2 i+1\right)+\delta_{2}\left(2 m_{2}-2 i+1\right)=0 ; \\
\text { pour } i \in\left\{m_{2}+1, \ldots, m_{1}\right\}, \quad & \delta_{1}\left(2 m_{1}-2 i+1\right)=i-m_{2}+h_{2} \bmod 2 \mathbb{Z} .
\end{aligned}
$$

Pour $j \in\{1,2\}$, introduisons la fonction $\xi_{j}:\left\{2 m_{j}-1, \ldots, 0\right\} \rightarrow\{ \pm 1\}$ telle que :

$$
\Lambda_{j}=\left\{\left(x, \xi_{j}(x)\right) ; x \in\left\{2 m_{j}-1, \ldots, 0\right\}\right\} .
$$

Les définitions 7.5(1) se traduisent par les égalités suivantes, pour $i \in\left\{1, \ldots, m_{j}\right\}$ :

$$
\begin{aligned}
& \tau_{j}\left(2 m_{j}-2 i+1\right)=\left|\left\{x \in\left\{2 m_{j}-1, \ldots, 2 m_{j}-2 i+1\right\} ; \xi_{j}(x)=(-1)^{x}\right\}\right|, \\
& \delta_{j}\left(2 m_{j}-2 i+1\right)=\left|\left\{x \in\left\{2 m_{j}-1, \ldots, 2 m_{j}-2 i\right\} ; \xi_{j}(x)=(-1)^{x}\right\}\right| .
\end{aligned}
$$

Rappelons que :

$$
h_{j}=\frac{1}{2}\left(\left|\left\{x \in\left\{2 m_{j}-1, \ldots, 0\right\} ; \xi_{j}(x)=1\right\}\right|-\left|\left\{x \in\left\{2 m_{j}-1, \ldots, 0\right\} ; \xi_{j}(x)=-1\right\}\right|\right) .
$$

D'où l'égalité $\delta_{j}(1)=h_{j} \bmod 2 \mathbb{Z}$. Si la condition (1) est vérifiée, on a

$$
\delta_{1}\left(2 m_{1}-m_{2}+1\right)=\delta_{2}(1)=h_{2} \bmod 2 \mathbb{Z}
$$

et la condition (2) est équivalente à :

(3) pour $i \in\left\{m_{2}+1, \ldots, m_{1}\right\}, \delta_{1}\left(2 m_{1}-2 i+1\right)=i-m_{2}+\delta_{1}\left(2 m_{1}-2 m_{2}+1\right) \bmod 2 \mathbb{Z}$.

À l'aide des formules ci-dessus exprimant $\tau_{j}$ et $\delta_{j}$, on voit que les conditions (1) et (3) sont respectivement équivalentes à :

$$
\begin{aligned}
\xi_{1}\left(x+2\left(m_{1}-m_{2}\right)\right) & =\xi_{2}(x) \text { pour tout } x \in\left\{2 m_{2}-1, \ldots, 0\right\} \\
\xi_{1}(2 i-1) & =\xi_{1}(2 i-2) \text { pour tout } i \in M=\left\{m_{1}-m_{2}, \ldots, 1\right\} .
\end{aligned}
$$

Mais ces dernières conditions sont équivalentes à l'appartenance de $\left(\Lambda_{1}, \Lambda_{2}\right)$ à l'image de l'application $7.4(1)$ : si elles sont vérifiées, $\left(\Lambda_{1}, \Lambda_{2}\right)$ est l'image de $(\Lambda, I)$ où $\Lambda=\Lambda_{2}$ et $I=\left\{i \in M ; \xi_{1}(2 i-1)=\xi_{1}(2 i-2)=1\right\}$.

7.7. Supposons provisoirement l'hypothèse 7.1(1) vérifiée et considérons l'égalité de la proposition 7.2. Soient $\rho^{\prime} \in \operatorname{Irr}\left(W_{N^{\prime}}\right), \rho^{\prime \prime} \in \operatorname{Irr}\left(W_{N^{\prime \prime}}\right)$. On sait que la fonction $\chi_{\left|r^{\prime}\right|, \rho^{\prime}}^{\prime}$ est supportée par les orbites unipotentes de $\boldsymbol{S} \boldsymbol{O}\left(V^{\prime}\right)$ dont la partition orthogonale associée $\underline{\mu}^{\prime}$ vérifie l'inégalité $\underline{\mu}^{\prime} \leqslant \underline{\lambda}_{\mid r^{\prime}, \rho^{\prime}}, c f$. [L6] 24.2.9 et 24.2.10. Puisqu'on calcule cette fonction au point $u^{\prime}$ dont la partition associée est $\underline{\lambda}^{\prime}$, on peut se limiter aux $\rho^{\prime}$ telles que $\underline{\lambda}^{\prime} \leqslant \underline{\lambda}_{\left|r^{\prime}\right|, \rho^{\prime}}$. De même, on peut se limiter aux $\rho^{\prime \prime}$ telles que $\underline{\lambda}^{\prime \prime} \leqslant \underline{\lambda}_{\left|r^{\prime \prime}\right|, \rho^{\prime \prime}}$. Enfin, on peut évidemment se limiter aux couples $\left(\rho^{\prime}, \rho^{\prime \prime}\right)$ tels qu'il existe $\nu \in \mathcal{N}$ pour lequel $\left(\rho^{\prime} \times \rho^{\prime \prime}, \rho_{1} \times \rho_{2}\right)_{h_{1}, h_{2}, \nu} \neq 0$. Autrement dit, on peut sommer seulement sur $\left(\rho^{\prime}, \rho^{\prime \prime}\right) \in \mathcal{I}$. Si on restreint ainsi la somme dans l'égalité de la proposition 7.2 , cette égalité devient vraie même si l'hypothèse 7.1(1) n'est pas vérifiée, puisqu'alors $\mathcal{I}=\varnothing$. 
Grâce à la proposition 7.5 et au lemme 7.6, on obtient que $k_{\eta}\left(\Lambda_{1}, \Lambda_{2}\right)(g)=0$ si $\left(\Lambda_{1}, \Lambda_{2}\right)$ n'est pas dans l'image de l'application 7.4(1).

Fixons maintenant $\Lambda \in{\widetilde{\operatorname{Fam}_{2}}}$ et $I \in P_{M}$ et supposons que $\left(\Lambda_{1}, \Lambda_{2}\right)=$ $\left(\Lambda_{1}(\Lambda, I), \Lambda_{2}(\Lambda, I)\right)$. Alors $\mathcal{I}$ a un unique élément, que l'on note $\left(\rho^{\prime}, \rho^{\prime \prime}\right)$, et il existe un unique élément de $\mathcal{N}$, que l'on note $\underline{\nu}$, pour lequel $\left(\rho^{\prime} \times \rho^{\prime \prime}, \rho_{1} \times \rho_{2}\right)_{h_{1}, h_{2}, \nu} \neq 0$. Ce dernier terme vaut 1 . La formule de la proposition 7.2 se simplifie :

$$
\begin{aligned}
& k_{\eta}\left(\Lambda_{1}, \Lambda_{2}\right)(g)=c\left(h_{1}, h_{2}\right) c\left(h_{1}, h_{2}, g\right) \zeta(-1)^{N_{2}^{\prime \prime}} \\
& \quad q^{b\left(\left|r^{\prime}\right|, \rho^{\prime}\right)+b\left(\left|r^{\prime \prime}\right|, \rho^{\prime \prime}\right)+\delta\left(r^{\prime}, r^{\prime \prime}\right) / 2} \chi_{\left|r^{\prime}\right|, \rho^{\prime}}^{\prime}\left(u^{\prime}\right) \chi_{\left|r^{\prime \prime}\right|, \rho^{\prime \prime}}^{\prime \prime}\left(u^{\prime \prime}\right) .
\end{aligned}
$$

On doit calculer tous les termes intervenant dans cette formule. On en a calculé la plupart dans $[\mathbf{W}]$ mais, malheureusement, pas le terme $N_{2}^{\prime \prime}$. On effectue ce calcul dans le paragraphe suivant.

7.8. On conserve jusqu'en 7.10 les hypothèses ci-dessus, à savoir $\left(\Lambda_{1}, \Lambda_{2}\right)=$ $\left(\Lambda_{1}(\Lambda, I), \Lambda_{2}(\Lambda, I)\right)$. Dans la preuve de la proposition 7.5 , on a introduit un ensemble $\widetilde{\mathcal{P}}_{L}\left(\iota_{1}, \iota_{2} ; \iota_{0}\right)$. On a dit qu'il était réduit à un élément :

$$
\underline{p}=\left(\underline{\alpha}^{\prime}, \underline{\beta}^{\prime}, \underline{\alpha}^{\prime \prime}, \underline{\beta}^{\prime \prime}, \underline{\alpha}_{1}^{\prime}, \underline{\alpha}_{2}^{\prime}, \underline{\alpha}_{1}^{\prime \prime}, \underline{\alpha}_{2}^{\prime \prime}, \underline{\beta}_{1}^{\prime}, \underline{\beta}_{2}^{\prime}, \underline{\beta}_{1}^{\prime \prime}, \underline{\beta}_{2}^{\prime \prime}\right)
$$

Il résulte de la preuve de [W] XII.7(1) que $N_{2}^{\prime \prime}=S\left(\underline{\alpha}_{2}^{\prime \prime}\right)+S\left(\underline{\beta}_{2}^{\prime \prime}\right)$ (on rappelle que pour toute famille finie $\underline{\alpha}$ de nombres, on note $S(\underline{\alpha})$ la somme des termes de la famille). On va écrire explicitement $\underline{p}$. Pour cela, il est commode de modifier les notations. On notera les couples de partitions $(\underline{\alpha}(0), \underline{\alpha}(1))$ au lieu de $(\underline{\alpha}, \beta), 0$ et 1 étant considérés comme les deux éléments de $\mathbb{Z} / 2 \mathbb{Z}$ (par exemple $\left(\underline{\alpha}^{\prime}(0), \underline{\alpha}^{\prime}(1)\right)=\left(\underline{\alpha}^{\prime}, \underline{\beta}^{\prime}\right)$ ). De même, pour $j \in\{1,2\}$, on pose $\Lambda_{j}(0)=\Lambda_{j}^{+}, \Lambda_{j}(1)=\Lambda_{j}^{-}$.

Pour $j \in\{1,2\}$, on définit $u_{j}^{\prime}, u_{j}^{\prime \prime} \in \mathbb{Z} / 2 \mathbb{Z}$ par :

$$
\left(u_{j}^{\prime}, u_{j}^{\prime \prime}\right)= \begin{cases}(0, j+1), & \text { si }\left|h_{1}\right| \geqslant\left|h_{2}\right| \text { et } e_{1}+e_{2} \equiv 0 \bmod 2 \mathbb{Z}, \\ (0, j), & \text { si }\left|h_{1}\right|<\left|h_{2}\right| \text { et } e_{1}+e_{2} \equiv 0 \bmod 2 \mathbb{Z}, \\ (j+1,0), & \text { si }\left|h_{1}\right| \geqslant\left|h_{2}\right| \text { et } e_{1}+e_{2} \equiv 1 \bmod 2 \mathbb{Z} \\ (j, 0), & \text { si }\left|h_{1}\right|<\left|h_{2}\right| \text { et } e_{1}+e_{2} \equiv 1 \bmod 2 \mathbb{Z} .\end{cases}
$$

On a déjà défini l'union de deux partitions. On définit la somme de deux partitions :

$$
\left(\alpha_{1} \geqslant \alpha_{2} \geqslant \cdots\right)+\left(\beta_{1} \geqslant \beta_{2} \geqslant \cdots\right)=\left(\alpha_{1}+\beta_{1} \geqslant \alpha_{2}+\beta_{2} \geqslant \cdots\right) .
$$

En particulier, on pose $2\left(\alpha_{1} \geqslant \alpha_{2} \geqslant \cdots\right)=\left(2 \alpha_{1} \geqslant 2 \alpha_{2} \geqslant \cdots\right)$. Jusque-là, on a considéré une partition comme une famille de longueur indéterminée, en s'autorisant à ajouter des 0 si besoin était. Dans la suite, les partitions auront des longueurs fixées. On peut alors additionner un entier $\geqslant 0$ et une partition :

$$
\left(\alpha_{1} \geqslant \alpha_{2} \geqslant \cdots \geqslant \alpha_{s}\right)+k=\left(\alpha_{1}+k \geqslant \alpha_{2}+k \geqslant \cdots \geqslant \alpha_{s}+k\right) .
$$

Pour $x, y \in \mathbb{N}$, avec $x \geqslant y$, on note $[x, y]$ la partition $(x \geqslant x-1 \geqslant \cdots \geqslant y+1 \geqslant y)$. Si de plus $x \equiv y \bmod 2 \mathbb{Z}$, on note $[x, y]_{2}$ la partition $(x \geqslant x-2 \geqslant \cdots \geqslant y+2 \geqslant y)$. 
Pour $j \in\{1,2\}$, notons $\left(\underline{\alpha}_{j}(0), \underline{\alpha}_{j}(1)\right)$ un couple de partitions paramétrant la représentation $\rho_{j}$. Il est facile de vérifier que, pour tout $a \in \mathbb{Z} / 2 \mathbb{Z}$, on peut supposer que $\underline{\alpha}_{j}(a)$ est de longueur $m_{j}+(-1)^{a+e_{j}} h_{j}$. Les formules de 2.2 se résument à l'égalité :

$$
\Lambda_{j}(a)=\underline{\alpha}_{j}\left(a+e_{j}\right)+\left[m_{j}+(-1)^{a} h_{j}-1,0\right],
$$

pour tous $a \in \mathbb{Z} / 2 \mathbb{Z}, j \in\{1,2\}$.

Posons $I(0)=I, I(1)=M \backslash I$. Pour $a \in \mathbb{Z} / 2 \mathbb{Z}$, notons

$$
\underline{\alpha}(I, a)=\left(\alpha(I, a)_{1} \geqslant \cdots \geqslant \alpha(I, a)_{|I(a)|}\right)
$$

la partition telle que $I(a)=\left\{\alpha(I, a)_{i}+|I(a)|-i+1 ; i \in\{1, \ldots,|I(a)|\}\right\}$. L'hypothèse $\left(\Lambda_{1}, \Lambda_{2}\right)=\left(\Lambda_{1}(\Lambda, I), \Lambda_{2}(\Lambda, I)\right)$, jointe aux égalités (1), entraîne aisément l'égalité :

$$
\underline{\alpha}_{1}(a)=\left(\underline{\alpha}_{2}\left(a+e_{1}+e_{2}\right)+2\left|I\left(a+1+e_{1}\right)\right|\right) \cup 2 \underline{\alpha}\left(I, a+e_{1}\right) \cup 2 \underline{\alpha}\left(I, a+e_{1}\right) .
$$

Pour $a \in \mathbb{Z} / 2 \mathbb{Z}$ et $j \in\{1,2\}$, on définit des partitions $\underline{\alpha}^{\prime}(a), \underline{\alpha}^{\prime \prime}(a)$ et $\underline{\alpha}_{j}^{\prime}(a), \underline{\alpha}_{j}^{\prime \prime}(a)$ par les formules suivantes :

$$
\left\{\begin{array}{l}
\underline{\alpha}^{\prime}(a)=\left(2 \underline{\alpha}_{2}\left(a+u_{2}^{\prime}\right)+2\left|I\left(a+e_{1}+1+u_{1}^{\prime}\right)\right|\right) \cup 2 \underline{\alpha}\left(I, a+e_{1}+u_{1}^{\prime}\right), \\
\underline{\alpha}^{\prime \prime}(a)=2 \underline{\alpha}\left(I, a+e_{1}+u_{1}^{\prime \prime}\right), \\
\underline{\alpha}_{1}^{\prime}(a)=\left(\underline{\alpha}_{2}\left(a+u_{2}^{\prime}\right)+2\left|I\left(a+e_{1}+1+u_{1}^{\prime}\right)\right|\right) \cup 2 \underline{\alpha}\left(I, a+e_{1}+u_{1}^{\prime}\right), \\
\underline{\alpha}_{2}^{\prime}(a)=\underline{\alpha}_{2}\left(a+u_{2}^{\prime}\right) \\
\underline{\alpha}_{1}^{\prime \prime}(a)=2 \underline{\alpha}\left(I, a+e_{1}+u_{1}^{\prime \prime}\right), \\
\underline{\alpha}_{2}^{\prime \prime}(a)=\varnothing .
\end{array}\right.
$$

On a alors l'égalité :

$\underline{p}=\left(\underline{\alpha}^{\prime}(0), \underline{\alpha}^{\prime}(1), \underline{\alpha}^{\prime \prime}(0), \underline{\alpha}^{\prime \prime}(1), \underline{\alpha}_{1}^{\prime}(0), \underline{\alpha}_{2}^{\prime}(0), \underline{\alpha}_{1}^{\prime \prime}(0), \underline{\alpha}_{2}^{\prime \prime}(0), \underline{\alpha}_{1}^{\prime}(1), \underline{\alpha}_{2}^{\prime}(1), \underline{\alpha}_{1}^{\prime \prime}(1), \underline{\alpha}_{2}^{\prime \prime}(1)\right)$.

Pour le démontrer, il suffit de vérifier les relations suivantes, qui caractérisent $\underline{p}$ :

$-S\left(\underline{\alpha}^{\prime}(0)+S\left(\underline{\alpha}^{\prime}(1)\right)=\frac{1}{2}\left(t^{\prime 2}-r^{\prime 2}\right) ;\right.$

$-S\left(\underline{\alpha}^{\prime \prime}(0)+S\left(\underline{\alpha}^{\prime \prime}(1)\right)=\frac{1}{2}\left(t^{\prime \prime 2}-r^{\prime \prime 2}\right)\right.$;

- pour tout $a \in \mathbb{Z} / 2 \mathbb{Z}, \underline{\alpha}^{\prime}(a)=\underline{\alpha}_{1}^{\prime}(a)+\underline{\alpha}_{2}^{\prime}(a)$ et $\underline{\alpha}^{\prime \prime}(a)=\underline{\alpha}_{1}^{\prime \prime}(a)+\underline{\alpha}_{2}^{\prime \prime}(a)$;

- pour tous $a \in \mathbb{Z} / 2 \mathbb{Z}$ et $j \in\{1,2\}, \underline{\alpha}_{j}(a)=\underline{\alpha}_{j}^{\prime}\left(a+u_{j}^{\prime}\right)+\underline{\alpha}_{j}^{\prime \prime}\left(a+u_{j}^{\prime \prime}\right)$;

- notons $\rho^{\prime}$ la représentation de $W_{\frac{1}{2}\left(t^{\prime 2}-r^{\prime 2}\right)}$ paramétrée par $\left(\underline{\alpha}^{\prime}(0), \underline{\alpha}^{\prime}(1)\right)$, définissons $\rho^{\prime \prime}$ de façon similaire; alors $\underline{\lambda}\left(\left|r^{\prime}\right|, \rho^{\prime}\right)=\underline{\lambda}^{\prime}, \underline{\lambda}\left(\left|r^{\prime \prime}\right|, \rho^{\prime \prime}\right)=\underline{\lambda}^{\prime \prime}$

(les quatre premières relations sont les relations $(2),(3),\left(C_{\iota_{0}}\right)$ de $[\mathbf{W}]$ XI.27; la dernière est celle que l'on a ajoutée en 7.5). Les quatre premières relations se vérifient par un calcul direct. Pour la dernière, il faut traduire les égalités à démontrer en termes de partitions. Considérons seulement la première, la seconde étant similaire. Grâce à $[\mathbf{W}]$ VIII.7, cette première égalité est équivalente à :

$$
\left(\underline{\alpha}^{\prime}(0)+\left[t^{\prime}+\left|r^{\prime}\right|-2,0\right]_{2}\right) \cup\left(\underline{\alpha}^{\prime}(1)+\left[t^{\prime}-\left|r^{\prime}\right|-2,0\right]_{2}\right)=\left[2 t^{\prime}-2,0\right]_{2},
$$

que l'on vérifie encore par un calcul direct, en utilisant (1) et l'appartenance de $\Lambda_{j}$ à $\widetilde{\operatorname{Fam}}_{j}$ pour $j \in\{1,2\}$. 
Avec nos nouvelles notations,

$$
N_{2}^{\prime \prime}=S\left(\underline{\alpha}_{2}^{\prime \prime}(0)\right)+S\left(\underline{\alpha}_{2}^{\prime \prime}(1)\right) .
$$

Les égalités (2) entraînent :

$$
N_{2}^{\prime \prime}=0
$$

7.9. On a donné la définition de $b\left(\left|r^{\prime}\right|, \rho^{\prime}\right)$ en 7.2. L'orbite unipotente $\boldsymbol{C}$ associée à $\left(\left|r^{\prime}\right|, \rho^{\prime}\right)$ est paramétrée par $\underline{\lambda}^{\prime}$. On calcule :

$$
\operatorname{dim}\left(\boldsymbol{Z}^{\prime 0}\right)-\operatorname{dim}(\boldsymbol{C})=\frac{t^{\prime 3}-t^{\prime}}{3} .
$$

Le terme $N^{\prime}$ intervenant en 7.2 est égal à $\frac{t^{\prime 2}-r^{\prime 2}}{2}$. D'où l'égalité :

$$
b\left(\left|r^{\prime}\right|, \rho^{\prime}\right)=\frac{1}{2}\left(\frac{t^{\prime 3}-t^{\prime}}{3}-\frac{t^{\prime 2}-r^{\prime 2}}{2}-\frac{\left|r^{\prime 3}-r^{\prime}\right|}{3}\right) .
$$

Une formule analogue calcule $b\left(\left|r^{\prime \prime}\right|, \rho^{\prime \prime}\right)$ et on obtient :

$$
b\left(\left|r^{\prime}\right|, \rho^{\prime}\right)+b\left(\left|r^{\prime \prime}\right|, \rho^{\prime \prime}\right)+\frac{\delta\left(r^{\prime}, r^{\prime \prime}\right)}{2}=\frac{\delta\left(t^{\prime}, t^{\prime \prime}\right)}{2}-\frac{t^{\prime 2}+t^{\prime \prime 2}}{4}+\frac{r^{\prime 2}+r^{\prime \prime 2}}{4} .
$$

On a l'égalité $\frac{r^{\prime 2}+r^{\prime \prime 2}}{4}=\frac{h_{1}^{2}+h_{2}^{2}}{2}$. Grâce à $7.4(2)$, on calcule :

$$
\frac{h_{1}^{2}+h_{2}^{2}}{2}=\frac{\left(m_{1}-m_{2}\right)^{2}}{2}+c(\Lambda, I) .
$$

On a aussi :

$$
\frac{t^{\prime 2}+t^{\prime \prime 2}}{4}=\frac{m_{1}^{2}+m_{2}^{2}}{2}
$$

et finalement :

$$
b\left(\left|r^{\prime}\right|, \rho^{\prime}\right)+b\left(\left|r^{\prime \prime}\right|, \rho^{\prime \prime}\right)+\frac{\delta\left(r^{\prime}, r^{\prime \prime}\right)}{2}=\frac{\delta\left(t^{\prime}, t^{\prime \prime}\right)}{2}-m_{1} m_{2}+c(\Lambda, I) .
$$

7.10. Les termes $\chi_{\left|r^{\prime}\right|, \rho^{\prime}}^{\prime}\left(u^{\prime}\right)$ et $\chi_{\left|r^{\prime \prime}\right|, \rho^{\prime \prime}}^{\prime \prime}\left(u^{\prime \prime}\right)$ sont calculés en [W] VIII.13(2) et VIII.11. Supposons $m_{1} \not \equiv m_{2} \bmod 2 \mathbb{Z}$. Alors $r^{\prime}$ et $r^{\prime \prime}$ sont impairs et l'on a :

$$
\chi_{\left|r^{\prime}\right|, \rho^{\prime}}^{\prime}\left(u^{\prime}\right)=\left(\zeta(-1) \eta^{\prime}(g)\right)^{\left(\left|r^{\prime}\right|-1\right) / 2}, \quad \chi_{\left|r^{\prime \prime}\right|, \rho^{\prime \prime}}^{\prime \prime}\left(u^{\prime \prime}\right)=\left(\zeta(-1) \eta^{\prime \prime}(g)\right)^{\left(\left|r^{\prime \prime}\right|-1\right) / 2} .
$$

Grâce à l'égalité $\zeta(-1) \eta^{\prime}(g) \eta^{\prime \prime}(g)=\eta$, on obtient :

$$
\chi_{\left|r^{\prime}\right|, \rho^{\prime}}^{\prime}\left(u^{\prime}\right) \chi_{\left|r^{\prime \prime}\right|, \rho^{\prime \prime}}^{\prime \prime}\left(u^{\prime \prime}\right)=\zeta(-1)^{\left(\left|r^{\prime \prime}\right|-1\right) / 2} \eta^{\left(\left|r^{\prime}\right|-1\right) / 2} \eta^{\prime \prime}(g)^{\left(\left|r^{\prime}\right|+\left|r^{\prime \prime}\right|\right) / 2-1} .
$$

En traitant tous les cas possibles, on vérifie que cette expression est égale à :

$$
c\left(h_{1}, h_{2}\right) c\left(h_{1}, h_{2}, g\right) \eta^{\left(h_{1}+h_{2}-1\right) / 2} \eta^{\prime \prime}(g)^{h_{2}} .
$$

Remarquons que $h_{2}=h(\Lambda)$, et, grâce à $7.4(2)$ :

$$
\left(h_{1}+h_{2}-1\right) / 2 \equiv h(\Lambda)+|I|-\left(m_{1}-m_{2}+1\right) / 2 \bmod 2 \mathbb{Z} .
$$


D'où :

(1) si $m_{1} \not \equiv m_{2} \bmod 2 \mathbb{Z}$,

$$
\chi_{\left|r^{\prime}\right|, \rho^{\prime}}^{\prime}\left(u^{\prime}\right) \chi_{\left|r^{\prime \prime}\right|, \rho^{\prime \prime}}^{\prime \prime}\left(u^{\prime \prime}\right)=c\left(h_{1}, h_{2}\right) c\left(h_{1}, h_{2}, g\right) \eta^{h(\Lambda)+|I|-\left(m_{1}-m_{2}+1\right) / 2} \eta^{\prime \prime}(g)^{h(\Lambda)} .
$$

Supposons maintenant $m_{1} \equiv m_{2} \bmod 2 \mathbb{Z}$. Alors $r^{\prime}$ et $r^{\prime \prime}$ sont pairs.

Si $\eta^{\prime}(g)=1$, on a $\chi_{\left|r^{\prime}\right|, \rho^{\prime}}^{\prime}\left(u^{\prime}\right)=\zeta(-1)^{r^{\prime} / 2}$.

Si $\eta^{\prime}(g)=-1$ et $r^{\prime} \neq 0$, on a introduit en $[\mathbf{W}]$ VIII.11 un élément $\varepsilon_{a_{\max }} \in \mathbb{Z} / 2 \mathbb{Z}$

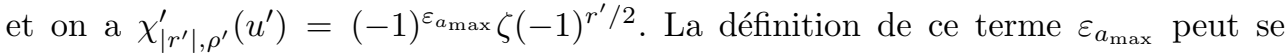
reformuler ainsi. Soit $\left(\underline{\alpha}^{\prime}, \beta^{\prime}\right)$ un couple de partitions paramétrant $\rho^{\prime}$. Comme on l'a dit en 7.8 , l'égalité $\underline{\lambda}_{\left|r^{\prime}\right|, \rho^{\prime}}=\underline{\lambda}^{\prime}$ entraîne que l'on peut supposer que $\underline{\alpha}^{\prime}$ est de longueur $\left(t^{\prime}+\left|r^{\prime}\right|\right) / 2$ et $\beta^{\prime}$ est de longueur $\left(t^{\prime}-\left|r^{\prime}\right|\right) / 2$, et on a alors l'égalité :

$$
\left(\underline{\alpha}^{\prime}+\left[t^{\prime}+\left|r^{\prime}\right|-2,0\right]_{2}\right) \cup\left(\underline{\beta}^{\prime}+\left[t^{\prime}-\left|r^{\prime}\right|-2,0\right]_{2}\right)=\left[2 t^{\prime}-2,0\right]_{2} .
$$

Le terme maximal $2 t^{\prime}-2$ de la partition de droite appartient soit à $\underline{\alpha}^{\prime}+\left[t^{\prime}+\left|r^{\prime}\right|-2,0\right]_{2}$, soit à $\beta^{\prime}+\left[t^{\prime}-\left|r^{\prime}\right|-2,0\right]_{2}$. On a $\varepsilon_{a_{\max }}=0$ dans le premier cas, $\varepsilon_{a_{\max }}=1$ dans le second. Avec les notations de 7.8, on a $\underline{\alpha}^{\prime}=\underline{\alpha}^{\prime}(0), \underline{\beta}^{\prime}=\underline{\alpha}^{\prime}(1)$. La définition ci-dessus se reformule ainsi : il y a un unique $a \in \mathbb{Z} / 2 \mathbb{Z}$ tel que le plus grand terme de $\underline{\alpha}^{\prime}(a)$ soit $t^{\prime}+(-1)^{a+1}\left|r^{\prime}\right|$. Notons-le $a^{\prime}$. Alors $\varepsilon_{a_{\max }}=a^{\prime}$ et $\chi_{\left|r^{\prime}\right|, \rho^{\prime}}^{\prime}\left(u^{\prime}\right)=(-1)^{a^{\prime}} \zeta(-1)^{r^{\prime}} / 2$.

Si $\eta^{\prime}(g)=-1$ et $r^{\prime}=0$, il n'y a plus de terme $(-1)^{\varepsilon_{a_{\max }}}$ dans les formules de [W] VIII.11. Mais il faut tenir compte du signe qu'on a introduit en 7.2 dans la définition de $\chi_{0, \rho^{\prime}}^{\prime}$. Avec les notations ci-dessus, ce signe est 1 si $\underline{\alpha}^{\prime} \succeq \underline{\beta}^{\prime},-1$ sinon. C'est encore $(-1)^{a^{\prime}}$, avec la même définition de $a^{\prime}$. On obtient en tout cas la formule :

$$
\chi_{\left|r^{\prime}\right|, \rho^{\prime}}^{\prime}\left(u^{\prime}\right)=\zeta(-1)^{r^{\prime} / 2} \eta^{\prime}(g)^{a^{\prime}} .
$$

On a de même :

$$
\chi_{\left|r^{\prime \prime}\right|, \rho^{\prime \prime}}^{\prime \prime}\left(u^{\prime \prime}\right)=\zeta(-1)^{r^{\prime \prime} / 2} \eta^{\prime \prime}(g)^{a^{\prime \prime}},
$$

où $a^{\prime \prime}$ est l'unique $a$ tel que le plus grand terme de $\underline{\alpha}^{\prime \prime}(a)$ soit $t^{\prime \prime}+(-1)^{a+1}\left|r^{\prime \prime}\right|$. En tenant compte des égalités $c\left(h_{1}, h_{2}\right)=\zeta(-1)^{r^{\prime} / 2}$ et $\eta^{\prime}(g) \eta^{\prime \prime}(g)=\eta$, on obtient :

$$
\chi_{\left|r^{\prime}\right|, \rho^{\prime}}^{\prime}\left(u^{\prime}\right) \chi_{\left|r^{\prime \prime}\right|, \rho^{\prime \prime}}^{\prime \prime}\left(u^{\prime \prime}\right)=c\left(h_{1}, h_{2}\right) \zeta(-1)^{r^{\prime \prime} / 2} \eta^{a^{\prime}} \eta^{\prime \prime}(g)^{a^{\prime}+a^{\prime \prime}} .
$$

Le calcul de $a^{\prime}$ et $a^{\prime \prime}$ est fastidieux mais élémentaire, en utilisant les relations $7.8(1)$ et 7.8(2). On obtient, avec les notations de 7.8 :

$$
a^{\prime}=f(\Lambda, I)+e_{2}+u_{2}^{\prime}, \quad a^{\prime \prime}=f(I)+e_{1}+u_{1}^{\prime \prime} .
$$

En distinguant tous les cas possibles, on calcule :

$$
\begin{aligned}
a^{\prime}+a^{\prime \prime} & =f(\Lambda, I)+f(I)+ \begin{cases}0, & \text { si }\left|h_{1}\right| \geqslant\left|h_{2}\right|, \\
1, & \text { si }\left|h_{1}\right|<\left|h_{2}\right|,\end{cases} \\
a^{\prime} & =f(\Lambda, I)+ \begin{cases}e_{1}, & \text { si }\left|h_{1}\right| \geqslant\left|h_{2}\right|, \\
e_{2}, & \text { si }\left|h_{1}\right|<\left|h_{2}\right| .\end{cases}
\end{aligned}
$$


On obtient alors l'égalité :

$$
\eta^{a^{\prime}} \eta^{\prime \prime}(g)^{a^{\prime}+a^{\prime \prime}}=c\left(h_{1}, h_{2}, g\right) \eta^{f(\Lambda, I)} \eta^{\prime \prime}(g)^{f(\Lambda, I)+f(I)} .
$$

Enfin, grâce à $7.4(2), r^{\prime \prime} / 2=\left(h_{1}-h_{2}\right) / 2 \equiv|I|+\left(m_{1}-m_{2}\right) / 2 \bmod 2 \mathbb{Z}$. D'où l'égalité :

(2) $\quad$ si $m_{1} \equiv m_{2} \bmod 2 \mathbb{Z}, \quad \chi_{\left|r^{\prime}\right|, \rho^{\prime}}^{\prime}\left(u^{\prime}\right) \chi_{\left|r^{\prime \prime}\right|, \rho^{\prime \prime}}^{\prime \prime}\left(u^{\prime \prime}\right)$

$$
=c\left(h_{1}, h_{2}\right) c\left(h_{1}, h_{2}, g\right) \zeta(-1)^{|I|+\left(m_{1}-m_{2}\right) / 2} \eta^{f(\Lambda, I)} \eta^{\prime \prime}(g)^{f(\Lambda, I)+f(I)} .
$$

En rassemblant les égalités $7.7(1), 7.8(3), 7.9(1) 7.10(1)$ et $7.10(2)$, on obtient la proposition 7.4 .

7.11. On conserve les hypothèses de 7.4 concernant $m_{1}, m_{2}, \eta$ et $g$.

Lemme. - Pour tout $\left(\Lambda_{1}, \Lambda_{2}\right) \in{\widetilde{\mathrm{Fam}_{1}}} \times{\widetilde{\mathrm{Fam}_{2}}}_{2}$, le nombre

$$
\left|\widetilde{\mathrm{Fam}}_{1}\right|^{1 / 2}\left|\widetilde{\mathrm{Fam}}_{2}\right|^{1 / 2} k_{n}\left(\mathcal{F} \Lambda_{1} \otimes \mathcal{F} \Lambda_{2}\right)(g)
$$

est entier rationnel et divisible par $2^{\text {inf }\left(3, m_{1}+m_{2}\right)}$.

Démonstration. - Le nombre en question est égal à :

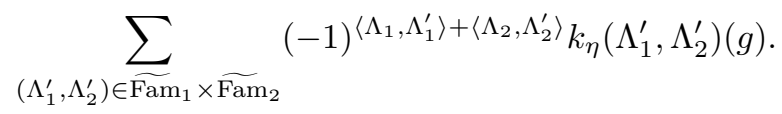

D'après la proposition 7.4, on peut restreindre la sommation à l'image de l'application 7.4(1). Les exposants de $q$ intervenant dans cette proposition sont entiers $\geqslant 0$. Pour le vérifier, il est plus simple de revenir aux calculs de 7.9. Ces exposants sont égaux à :

$$
\frac{1}{2}\left(\frac{t^{\prime 3}-t^{\prime}}{3}-\frac{t^{\prime 2}-r^{\prime 2}}{2}+\frac{t^{\prime \prime 3}-t^{\prime \prime}}{3}-\frac{t^{\prime \prime 2}-r^{\prime \prime 2}}{2}\right),
$$

et la vérification est facile, en se rappelant que $t^{\prime} \equiv r^{\prime} \bmod 2 \mathbb{Z}, t^{\prime \prime} \equiv r^{\prime \prime} \bmod 2 \mathbb{Z}$. Donc tous les termes de la somme (1) sont entiers rationnels. Pour vérifier que la somme est divisible par $2^{\text {inf }\left(3, m_{1}+m_{2}\right)}$, on peut se placer dans le complété $\mathbb{Z}_{2}$, où $q$ est inversible.

Posons $N=\left\{2 m_{2}-1, \ldots, 0\right\}$ et notons $P_{N}$ l'ensemble des sous-ensembles de $N$. Considérons l'ensemble $\Phi$ des fonctions $\varphi:{\widetilde{\mathrm{Fam}_{2}}}_{2} \times P_{M} \rightarrow \mathbb{Z}_{2}$ telles qu'il existe $c_{0}, c_{1}, c_{2} \in \mathbb{Z}_{2}^{\times}, X \in P_{M}$ et $Y \in P_{N}$ de sorte que, pour tout $(\Lambda, I) \in \widetilde{\operatorname{Fam}}_{2} \times P_{M}$, on ait la congruence :

$$
\varphi(\Lambda, I) \equiv c_{0} c_{1}^{\left|\Lambda^{+}\right|} c_{2}^{|I|}(-1)^{\left|\Lambda^{+} \cap Y\right|+|I \cap X|} \bmod 8 \mathbb{Z}_{2} .
$$

On va montrer :

(3) la fonction $(\Lambda, I) \mapsto(-1)^{\left\langle\Lambda_{1}, \Lambda_{1}(\Lambda, I)\right\rangle+\left\langle\Lambda_{2}, \Lambda_{2}(\Lambda, I)\right\rangle} k_{\eta}\left(\Lambda_{1}(\Lambda, I), \Lambda_{2}(\Lambda, I)\right)(g)$ appartient à $\Phi$.

On voit que l'ensemble $\Phi$ est stable par produit. En exprimant la fonction ci-dessus à l'aide de la proposition 7.4, il suffit de prouver que les différents facteurs appartiennent à $\Phi$. Pour les termes constants, c'est clair. Examinons les autres : 
- le terme $(-1)^{\left\langle\Lambda_{1}, \Lambda_{1}(\Lambda, I)\right\rangle+\left\langle\Lambda_{2}, \Lambda_{2}(\Lambda, I)\right\rangle}$ appartient à $\Phi$ car, en revenant à la définition du produit $\langle\bullet, \bullet\rangle$ de deux symboles ordonnés, on vérifie qu'au couple $\left(\Lambda_{1}, \Lambda_{2}\right)$ sont associés des éléments $a, b \in \mathbb{Z} / 2 \mathbb{Z}, X \in P_{M}$ et $Y \in P_{N}$ tels que :

$$
\left\langle\Lambda_{1}, \Lambda_{1}(\Lambda, I)\right\rangle+\left\langle\Lambda_{2}, \Lambda_{2}(\Lambda, I)\right\rangle=a+b\left|\Lambda^{+}\right|+\left|\Lambda^{+} \cap Y\right|+|I \cap X| \bmod 2 \mathbb{Z} ;
$$

- le terme $q^{c(\Lambda, I)}$ appartient à $\Phi$. En effet, on a $q^{2} \equiv 1 \bmod 8 \mathbb{Z}$. Si $m_{1} \not \equiv$ $m_{2} \bmod 2 \mathbb{Z}, c(\Lambda, I)$ est pair et $q^{c(\Lambda, I)} \equiv 1 \bmod 8 \mathbb{Z}$. Si $m_{1} \equiv m_{2} \bmod 2 \mathbb{Z}$, on a $c(\Lambda, I) \equiv h(\Lambda)=\left|\Lambda^{+}\right|-m_{2} \bmod 2 \mathbb{Z}$ et $q^{c(\Lambda, I)} \equiv q^{\left|\Lambda^{+}\right|-m_{2}} \bmod 8 \mathbb{Z} ;$

- pour $\varepsilon \in\{ \pm 1\}$, un terme $\varepsilon^{h(\Lambda)}$ appartient à $\Phi$ : on vient de dire que $h(\Lambda)=$ $\left|\Lambda^{+}\right|-m_{2}$

- pour $\varepsilon \in\{ \pm 1\}$, un terme $\varepsilon^{|I|}$ appartient à $\Phi$ : c'est évident ;

- pour $\varepsilon \in\{ \pm 1\}$, un terme $\varepsilon^{f(I)}$ appartient à $\Phi$ : si $m_{1}=m_{2}$, on a $f(I)=0$; si $m_{1} \neq m_{2}, f(I)=1+\left|I \cap\left\{m_{1}-m_{2}\right\}\right|$;

- pour $\varepsilon \in\{ \pm 1\}$, un terme $\varepsilon^{f(\Lambda, I)}$ appartient à $\Phi$ : si $m_{2}=0, f(\Lambda, I)=f(I)$ et on vient de traiter ce terme; si $m_{2} \neq 0, f(\Lambda, I)=1+\left|\Lambda^{+} \cap\left\{2 m_{2}-1\right\}\right|$.

Cela démontre (3). Exprimons la fonction de (3) sous la forme (2). Remarquons que l'application $\Lambda \mapsto \Lambda^{+}$est une bijection de $\widetilde{\operatorname{Fam}}_{2}$ sur $P_{N}$. Alors la somme (1) est congrue $\bmod 8 \mathbb{Z}_{2}$ à :

$$
c_{0}\left(\sum_{\Lambda^{+} \in P_{N}} c_{1}^{\left|\Lambda^{+}\right|}(-1)^{\left|\Lambda^{+} \cap Y\right|}\right)\left(\sum_{I \in P_{M}} c_{2}^{|I|}(-1)^{|I \cap X|}\right) .
$$

On a la propriété élémentaire suivante. Soient $R$ un ensemble fini, notons $P_{R}$ l'ensemble des sous-ensembles de $R$. Soient $Z \in P_{R}, a, b \in \mathbb{Z}_{2}^{\times}$. Alors :

$$
\sum_{J \in P_{R}} a^{|J|} b^{|J \cap Z|} \in 2^{|R|} \mathbb{Z}_{2}
$$

L'expression (4) appartient donc à $2^{|N|+|M|} \mathbb{Z}_{2}$. Puisque $|N|+|M|=m_{1}+m_{2}$, on obtient l'énoncé. 



\section{CHAPITRE 8}

\section{FORMULES D'INDUCTION}

On traduit en termes de symboles l'induction de Deligne-Lusztig des fonctions traces de faisceaux-caractères et l'induction de Harish-Chandra des traces de représentations irréductibles.

8.1. Soient $m, a \in \mathbb{N}$, avec $a \geqslant 1$, et $\varepsilon, \eta \in\{ \pm 1\}$, avec $\eta=1$ si $m=0$. Décomposons l'espace $V_{\eta \varepsilon}(2(m+a))$ en somme orthogonale :

$$
V_{\eta \varepsilon}(2(m+a))=V_{\varepsilon}(2 a) \oplus V_{\eta}(2 m) .
$$

Fixons un sous-tore maximal $\boldsymbol{T}$ de $\boldsymbol{O}_{\varepsilon}(2 a)$ dont la classe soit paramétrée par l'élément $w_{a}^{\varepsilon} \in W_{a}$ introduit en 2.4. Le groupe :

$$
\boldsymbol{M}=\boldsymbol{T} \times \boldsymbol{O}_{\eta}(2 m)
$$

est un groupe de Lévi de $\boldsymbol{O}_{\eta \varepsilon}(2(m+a))$. En fixant un sous-groupe parabolique de $\boldsymbol{O}_{\eta \varepsilon}(2(m+a))$ de sous-groupe de Lévi $\boldsymbol{M}$, on définit le foncteur $R_{M}^{O_{\eta \varepsilon}(2(m+a))}$. Notons $1_{T}$ le caractère trivial de $T$ et $\zeta_{T}$ son caractère quadratique paramétré par l'élément de $\widehat{T}$ noté $\zeta_{T}$ en 3.3. Pour $f \in \mathcal{C}\left(O_{\eta}(2 m)\right)$, on pose :

$$
R_{1, a}^{\varepsilon}(f)=R_{M}^{O_{\eta \varepsilon}(2(m+a))}\left(1_{T} \times f\right), R_{\zeta, a}^{\varepsilon}(f)=R_{M}^{O_{\eta \varepsilon}(2(m+a))}\left(\zeta_{T} \times f\right) .
$$

Notons que ces termes appartiennent à $\mathbb{Z}\left[\operatorname{Irr}\left(O_{\eta \varepsilon}(2(m+a))\right)\right]$ si $f$ appartient à $\mathbb{Z}\left[\operatorname{Irr}\left(O_{\eta}(2 m)\right)\right]$. D'autre part, d'après $[\mathbf{L} \mathbf{1}]$, corollaire 6 , ils sont combinaisons linéaires de représentations quadratiques-unipotentes si $f$ l'est. En faisant varier $\eta$, on obtient des homomorphismes :

$$
R_{1, a}^{\varepsilon}, R_{\zeta, a}^{\varepsilon}: \mathbb{Z}[\operatorname{Quad}(O(2 m))] \longrightarrow \mathbb{Z}[\operatorname{Quad}(O(2(m+a)))] .
$$

Dans le cas où $m=0$, ils prennent leurs valeurs dans $\mathbb{Z}\left[\operatorname{Quad}\left(O_{\varepsilon}(2 a)\right)\right]$.

Supposons maintenant $m \in \mathbb{N}, a \in \mathbb{Z}$ et $m+a \geqslant 0$. En 2.3, on a introduit diverses applications linéaires $I_{a}, I_{a}^{-}, J_{a}$ entre espaces de symboles ordonnés. On en déduit diverses applications linéaires :

$$
I_{a} \otimes 1,1 \otimes I_{a}, \text { etc. : } \mathbb{Z}\left[\widetilde{\mathcal{S}} \widetilde{\mathcal{S}}_{m, \text { pair }}\right] \longrightarrow \mathbb{Z}\left[\widetilde{\mathcal{S}} \widetilde{\mathcal{S}}_{m+a, \text { pair }}\right]
$$


Par exemple, pour $m_{1}, m_{2} \in \mathbb{N}$ tels que $m_{1}+m_{2}=m$, l'application $I_{a} \otimes 1$ envoie le facteur $\mathbb{Z}\left[\widetilde{\mathcal{S}}_{m_{1} \text {,pair }}\right] \otimes_{\mathbb{Z}} \mathbb{Z}\left[\widetilde{\mathcal{S}}_{m_{2}, \text { pair }}\right]$ de $\mathbb{Z}\left[\widetilde{\mathcal{S}} \widetilde{\mathcal{S}}_{m, \text { pair }}\right]$ sur 0 si $m_{1}+a<0$, sur le sommand $\mathbb{Z}\left[\widetilde{\mathcal{S}}_{m_{1}+a, \text { pair }}\right] \otimes_{\mathbb{Z}} \mathbb{Z}\left[\widetilde{\mathcal{S}}_{m_{2}, \text { pair }}\right]$ de $\mathbb{Z}\left[\widetilde{\mathcal{S}} \widetilde{\mathcal{S}}_{m+a, \text { pair }}\right]$ si $m_{1}+a \geqslant 0$; dans ce cas, elle coïncide avec l'application $I_{a} \otimes 1$ déjà définie.

8.2. Soit $a$ un entier tel que $1 \leqslant a \leqslant n$, posons $m=n-a$.

Proposition. - Désignons par $(I, R)$ l'un des couples $\left(I_{a} \otimes 1, R_{1, a}^{+}\right),\left(I_{a}^{-} \otimes 1, R_{1, a}^{-}\right)$, $\left(1 \otimes I_{a}, R_{\zeta, a}^{+}\right),\left(1 \otimes I_{a}^{-}, R_{\zeta, a}^{-}\right)$. Le diagramme suivant est commutatif :

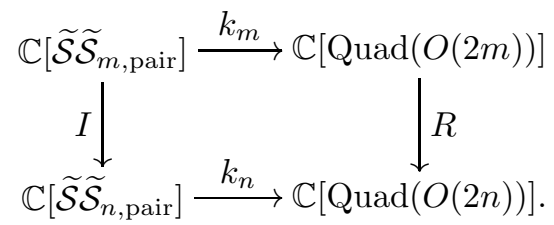

Remarque. - On renvoie à 5.1 pour la définition des applications $k_{n}$ et $k_{m}$. Elles prennent leurs valeurs dans les espaces indiqués d'après la proposition 6.4.

Démonstration. - Pour unifier les notations, on pose $I_{a}^{+}=I_{a}$. On fixe $\varepsilon \in\{ \pm 1\}$ et on traite le cas des opérateurs $\left(I_{a}^{\varepsilon} \otimes 1, R_{1, a}^{\varepsilon}\right)$, l'autre étant similaire. Soit $\left(\Lambda_{1}, \Lambda_{2}\right) \in$ $\widetilde{\mathcal{S}} \widetilde{\mathcal{S}}_{m \text {,pair. Pour }}$. $\in\{1,2\}$, notons $\left(h_{j}, \rho_{j}\right)$ le couple associé en 2.2 à $\Lambda_{j}$, posons $N_{j}=$ $\operatorname{rg}\left(\Lambda_{j}\right)-h_{j}^{2}$ et définissons $e_{j}$ comme en 3.3. Posons $r^{\prime}=h_{1}+h_{2}, r^{\prime \prime}=h_{1}-h_{2}$. Soit $\eta \in\{ \pm 1\}$, avec $\eta=1$ si $m=0$. Au paragraphe 3.3 , on a associé à tout couple $\left(w_{1}, w_{2}\right) \in W_{N_{1}} \times W_{N_{2}}$ une fonction trace $k_{\eta}\left(w_{1}, w_{2} ; r^{\prime}, r^{\prime \prime}\right)$ sur $O_{\eta}(2 m)$. Il résulte de la définition de $R_{1, a}^{\varepsilon}$, de l'égalité $3.2(3)$ et de la relation de transivité 1.4(1) que l'on a l'égalité :

$$
R_{1, a}^{\varepsilon}\left(k_{\eta}\left(w_{1}, w_{2} ; r^{\prime}, r^{\prime \prime}\right)\right)=(-1)^{n-m} k_{\eta \varepsilon}\left(w_{a}^{\varepsilon} \times w_{1}, w_{2} ; r^{\prime}, r^{\prime \prime}\right)
$$

(le terme $w_{a}^{\varepsilon} \times w_{1}$ appartient à $\left.W_{a} \times W_{N_{1}} \subseteq W_{a+N_{1}}\right)$. Pour $\sigma_{1} \in \operatorname{Irr}\left(W_{a+N_{1}}\right)$, notons $\Lambda\left(\sigma_{1}\right) \in \widetilde{\mathcal{S}}_{a+N_{1}+h_{1}^{2} \text {,pair }}$ le symbole associé au couple $\left(h_{1}, \sigma_{1}\right)$ et posons :

$$
\phi\left(\sigma_{1}, w_{2}\right)=\left|W_{a+N_{1}}\right|^{-1} \sum_{v \in W_{a+N_{1}}} \operatorname{sgn}_{C D}\left(v_{1}\right)^{e_{1}} \operatorname{trace}\left(\sigma_{1}\right)\left(v_{1}\right) k_{\eta \varepsilon}\left(v_{1}, w_{2} ; r^{\prime}, r^{\prime \prime}\right) .
$$

Par une formule d'inversion usuelle sur le groupe fini $W_{a+N_{1}}$, l'égalité (1) entraîne :

$$
\begin{aligned}
& R_{1, a}^{\varepsilon}\left(k_{\eta}\left(w_{1}, w_{2} ; r^{\prime}, r^{\prime \prime}\right)\right) \\
& =(-1)^{n-m} \operatorname{sgn}_{C D}\left(w_{a}^{\varepsilon} \times w_{1}\right)^{e_{1}} \sum_{\sigma_{1} \in \operatorname{Irr}\left(W_{a+N_{1}}\right)} \operatorname{trace}\left(\sigma_{1}\right)\left(w_{a}^{\varepsilon} \times w_{1}\right) \phi\left(\sigma_{1}, w_{2}\right) .
\end{aligned}
$$


En utilisant 3.3(1), on obtient :

(2) $\quad R_{1, a}^{\varepsilon}\left(k_{\eta}\left(\Lambda_{1}, \Lambda_{2}\right)\right)$

$$
\begin{aligned}
&=\operatorname{sgn}_{C D}\left(w_{a}^{\varepsilon}\right)^{e_{1}} \sum_{\sigma_{1} \in \operatorname{Irr}\left(W_{a+N_{1}}\right)}\left(\left|W_{N_{1}}\right|^{-1} \sum_{w_{1} \in W_{N_{1}}} \operatorname{trace}\left(\sigma_{1}\right)\left(w_{a}^{\varepsilon} \times w_{1}\right) \operatorname{trace}\left(\rho_{1}\right)\left(w_{1}\right)\right) \\
&\left((-1)^{n}\left|W_{N_{2}}\right|^{-1} \sum_{w_{2} \in W_{N_{2}}} \operatorname{sgn}_{C D}\left(w_{2}\right)^{e_{2}} \operatorname{trace}\left(\rho_{2}\right)\left(w_{2}\right) \phi\left(\sigma_{1}, w_{2}\right)\right) .
\end{aligned}
$$

En remplaçant $\phi\left(\sigma_{1}, w_{2}\right)$ par la formule qui le définit et en utilisant l'égalité 3.3(1) pour le groupe $O_{\eta \varepsilon}(2 n)$, le deuxième terme de la somme de l'expression (2) est égal à $k_{\eta \varepsilon}\left(\Lambda\left(\sigma_{1}\right), \Lambda_{2}\right)$. Le premier terme est le produit scalaire dans $\mathbb{Q}\left[\operatorname{Irr}\left(W_{N_{1}}\right)\right]$ :

$$
\left\langle\operatorname{res}_{a}^{\varepsilon}\left(\operatorname{trace}\left(\sigma_{1}\right)\right), \operatorname{trace}\left(\rho_{1}\right)\right\rangle,
$$

cf. 2.5 pour les définitions. Rappelons que $e_{1}=0$ si $h_{1} \geqslant 0, e_{1}=1$ si $h_{1}<0$, et remarquons que $\operatorname{sgn}_{C D}\left(w_{a}^{\varepsilon}\right)=\varepsilon$. Le lemme 2.5 entraîne l'égalité :

$$
\operatorname{sgn}_{C D}\left(w_{a}^{\varepsilon}\right)^{e_{1}}\left\langle\operatorname{res}_{a}^{\varepsilon}\left(\operatorname{trace}\left(\sigma_{1}\right)\right), \operatorname{trace}\left(\rho_{1}\right)\right\rangle=\left\langle\Lambda\left(\sigma_{1}\right), I_{a}^{\varepsilon}\left(\Lambda_{1}\right)\right\rangle
$$

et (2) devient :

$$
R_{1, a}^{\varepsilon}\left(k_{\eta}\left(\Lambda_{1}, \Lambda_{2}\right)\right)=\sum_{\sigma_{1} \in \operatorname{Irr}\left(W_{a+N_{1}}\right)}\left\langle\Lambda\left(\sigma_{1}\right), I_{a}^{\varepsilon}\left(\Lambda_{1}\right)\right\rangle k_{\eta \varepsilon}\left(\Lambda\left(\sigma_{1}\right), \Lambda_{2}\right)
$$

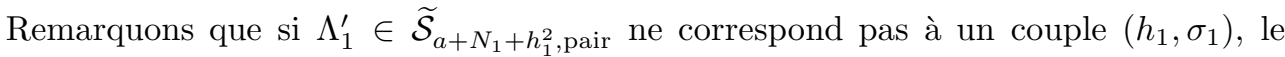
produit $\left\langle\Lambda_{1}^{\prime}, I_{a}^{\varepsilon}\left(\Lambda_{1}\right)\right\rangle$ est nul. L'égalité précédente est donc équivalente à :

$$
R_{1, a}^{\varepsilon}\left(k_{\eta}\left(\Lambda_{1}, \Lambda_{2}\right)\right)=\sum_{\Lambda_{1}^{\prime} \in \widetilde{\mathcal{S}}_{a+N_{1}+h_{1}^{2} \text {,pair }}}\left\langle\Lambda_{1}^{\prime}, I_{a}^{\varepsilon}\left(\Lambda_{1}\right)\right\rangle k_{\eta \varepsilon}\left(\Lambda_{1}^{\prime}, \Lambda_{2}\right) .
$$

Le membre de droite n'est autre que la composante dans $\mathbb{C}\left[\operatorname{Quad}\left(O_{\eta \varepsilon}(2 n)\right)\right]$ de $k_{n}$ 。 $\left(I_{a}^{\varepsilon} \otimes 1\right)\left(\Lambda_{1} \otimes \Lambda_{2}\right)$, ce qui démontre la proposition.

8.3. Soient $a, m$ comme précédemment.

Proposition. - Désignons par $(I, R)$ l'un des couples $\left(I_{a} \otimes 1, R_{1, a}^{+}\right),\left(1 \otimes I_{a}, R_{\zeta, a}^{+}\right)$. Le diagramme suivant est commutatif :

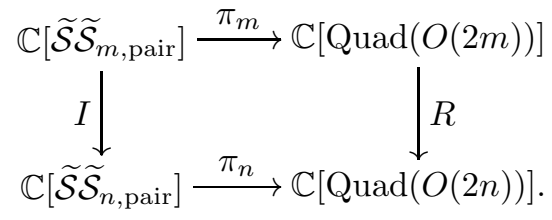


Démonstration. - On traite le cas du couple $\left(I_{a} \otimes 1, R_{1, a}^{+}\right)$. Soit $\left(\Lambda_{1}, \Lambda_{2}\right) \in \widetilde{\mathcal{S}}_{m, \text { pair }}$. Pour $j \in\{1,2\}$, notons $\left(h_{j}, \rho_{j}\right)$ le couple associé en 2.2 à $\Lambda_{j}$, et $N_{j}=\operatorname{rg}\left(\Lambda_{j}\right)-h_{j}^{2}$. Posons $N=a+N_{1}+N_{2}$, soit $\eta \in\{ \pm 1\}$, avec $\eta=1$ si $m=0$. Appliquons la construction de 4.1 à l'espace $V_{\eta}(2 n)$ et l'entier $N$. On a donc des vecteurs $v_{i}$, pour $i \in\{ \pm 1, \ldots, \pm N\}$, un sous-espace $V_{0}$ et un sous-groupe parabolique $\boldsymbol{P}=\boldsymbol{M U}$ de $\boldsymbol{O}_{\eta}(2 n)$. Notons $\left(\pi^{(n)}, E^{(n)}\right)$ la représentation induite de $P$ à $O_{\eta}(2 n)$ de la représentation $1_{a+N_{1}} \times \zeta_{N_{2}} \times \pi\left(h_{1}, h_{2}\right)$ de $M$. Dans $E^{(n)}$ agissent des opérateurs $T_{1}^{(n)}(w)$ pour $w \in W_{a+N_{1}}$ et $T_{2}^{(n)}(w)$ pour $w \in W_{N_{2}}$ (on a ajouté un exposant ${ }^{(n)}$ pour préciser le groupe ambiant). Ainsi, à tout couple $\left(\sigma_{1}, \sigma_{2}\right) \in \operatorname{Irr}\left(W_{a+N_{1}}\right) \times \operatorname{Irr}\left(W_{N_{2}}\right)$, on associe une sous-représentation irréductible $\pi^{(n)}\left(\sigma_{1}, \sigma_{2}\right)$ de $\pi^{(n)}$.

Considérons comme en 8.1 une décomposition :

$$
V_{\eta}(2 n)=V_{+}(2 a) \oplus V_{\eta}(2 m) .
$$

On peut supposer que $V_{+}(2 a)$ est engendré par $\left\{v_{i} ; i \in\{ \pm 1, \ldots, \pm a\}\right\}$ et $V_{\eta}(2 m)$ est engendré par $\left\{v_{i} ; i \in\{ \pm(a+1), \ldots, \pm N\}\right\}$ et $V_{0}$. À un décalage près, les vecteurs $\left\{v_{i} ; i \in\{ \pm(a+1), \ldots, \pm N\}\right\}$ nous permettent d'effectuer dans l'espace $V_{\eta}(2 m)$ les constructions de 4.1. Ainsi, notons $\left(\pi^{(m)}, E^{(m)}\right)$ la représentation induite de $P \cap O_{\eta}(2 m)$ à $O_{\eta}(2 m)$ de la représentation $1_{N_{1}} \times \zeta_{N_{2}} \times \pi\left(h_{1}, h_{2}\right)$ de $M \cap O_{\eta}(2 m)$. Dans $E^{(m)}$ agissent des opérateurs $T_{1}^{(m)}(w)$ pour $w \in W_{N_{1}}$ et $T_{2}^{(m)}(w)$ pour $w \in W_{N_{2}}$; à tout couple $\left(\sigma_{1}, \sigma_{2}\right) \in \operatorname{Irr}\left(W_{N_{1}}\right) \times \operatorname{Irr}\left(W_{N_{2}}\right)$, on associe une sous-représentation irréductible $\pi^{(m)}\left(\sigma_{1}, \sigma_{2}\right)$ de $\pi^{(m)}$. Notons $V^{\prime}$, resp. $V^{\prime \prime}$ le sous-espace lagrangien de $V_{+}(2 a)$ engendré par $\left\{v_{i} ; i \in\{1, \ldots, a\}\right\}$, resp. $\left\{v_{-i} ; i \in\{1, \ldots, a\}\right\}$. Notons $\boldsymbol{Q}$ le sous-groupe parabolique de $\boldsymbol{O}_{\eta}(2 n)$ formé des éléments qui stabilisent $V^{\prime}, \boldsymbol{L}$ son sous-groupe de Lévi formé des éléments qui stabilisent $V^{\prime}$ et $V^{\prime \prime}$. On a $\boldsymbol{L} \simeq \boldsymbol{G} \boldsymbol{L}\left(V^{\prime}\right) \times \boldsymbol{O}_{\eta}(2 m)$, $\boldsymbol{P} \subseteq \boldsymbol{Q}, \boldsymbol{M} \subseteq \boldsymbol{L}$. Les constructions de 4.1 s'adaptent, en se simplifiant, au groupe $G L\left(V^{\prime}\right)$. Notons $\left(\pi^{\prime}, E^{\prime}\right)$ la représentation induite de $P \cap G L\left(V^{\prime}\right)$ à $G L\left(V^{\prime}\right)$ de la représentation triviale $1_{a}$ de $M \cap G L\left(V^{\prime}\right)$. Dans $E^{\prime}$ agissent des opérateurs $T^{\prime}(w)$ pour $w \in \mathfrak{S}_{a}$. À toute représentation $\sigma \in \operatorname{Irr}\left(\mathfrak{S}_{a}\right)$, on associe une sous-représentation irréductible $\pi^{\prime}(\sigma)$ de $\pi^{\prime}$.

On a l'isomorphisme :

$$
\pi^{(n)} \simeq \operatorname{Ind}_{Q}^{O_{\eta}(2 n)}\left(\pi^{\prime} \times \pi^{(m)}\right)
$$

et un plongement naturel $\mathfrak{S}_{a} \times W_{N_{1}} \rightarrow W_{a+N_{1}}$. On vérifie que, pour $w \in \mathfrak{S}_{a}$, resp. $w_{1} \in W_{N_{1}}, w_{2} \in W_{N_{2}}$, l'opérateur $T_{1}^{(n)}(w)$, resp. $T_{1}^{(n)}\left(w_{1}\right), T_{2}^{(n)}\left(w_{2}\right)$, de l'espace $E^{(n)}$ n'est autre que l'opérateur déduit par fonctorialité de l'opérateur $T^{\prime}(w)$ de $E^{\prime}$, resp. $T_{1}^{(m)}, T_{2}^{(m)}$ de $E^{(m)}$. Il s'en déduit la propriété suivante. Soient $\sigma_{1}^{(n)} \in$ $\operatorname{Irr}\left(W_{a+N_{1}}\right), \sigma_{2}^{(n)} \in \operatorname{Irr}\left(W_{N_{2}}\right), \sigma^{\prime} \in \operatorname{Irr}\left(\mathfrak{S}_{a}\right), \sigma_{1}^{(m)} \in \operatorname{Irr}\left(W_{N_{1}}\right), \sigma_{2}^{(m)} \in \operatorname{Irr}\left(W_{N_{2}}\right)$. Alors la multiplicité de $\pi^{(n)}\left(\sigma_{1}^{(n)}, \sigma_{2}^{(n)}\right)$ dans l'induite :

$$
\operatorname{Ind}_{Q}^{O_{\eta}(2 n)}\left(\pi^{\prime}\left(\sigma^{\prime}\right) \times \pi^{(m)}\left(\sigma_{1}^{(m)}, \sigma_{2}^{(m)}\right)\right)
$$

est égale à : 
-0, si $\sigma_{2}^{(n)} \neq \sigma_{2}^{(m)}$

- la multiplicité de $\sigma^{\prime} \times \sigma_{1}^{(m)}$ dans la restriction de $\sigma_{1}^{(n)}$ à $\mathfrak{S}_{a} \times W_{N_{1}}$, dans le cas où $\sigma_{2}^{(n)}=\sigma_{2}^{(m)}$.

On peut supposer que le tore $\boldsymbol{T}$ de 8.1 est inclus dans $\boldsymbol{G} \boldsymbol{L}\left(V^{\prime}\right)$. Il en est un sous-tore maximal elliptique. Des définitions et des propriétés rappelées en 1.4 résulte l'égalité :

$$
R_{1, a}^{+}(f)=\operatorname{Ind}_{Q}^{O_{\eta}(2 n)}\left(R_{T}^{G L\left(V^{\prime}\right)}(1) \times f\right),
$$

pour tout $f \in \mathcal{C}\left(O_{\eta}(2 m)\right)$. Définissons la permutation circulaire $w_{a} \in \mathfrak{S}_{a}$ par $w_{a}(i)=$ $i+1$ pour tout $i \in\{1, \ldots, a-1\}, w_{a}(a)=1$. Cet élément s'identifie au $w_{a}^{+}$de 2.5 par le plongement $\mathfrak{S}_{a} \rightarrow W_{a}$. Il est connu que l'on a l'égalité :

$$
R_{T}^{G L\left(V^{\prime}\right)}(1)=\sum_{\sigma^{\prime} \in \operatorname{Irr}\left(\mathfrak{S}_{a}\right)} \operatorname{trace}\left(\sigma^{\prime}\right)\left(w_{a}\right) \pi^{\prime}\left(\sigma^{\prime}\right) .
$$

Soient $\sigma_{1}^{(m)} \in \operatorname{Irr}\left(W_{N_{1}}\right), \sigma_{2} \in \operatorname{Irr}\left(W_{N_{2}}\right)$. En rassemblant les propriétés ci-dessus, on obtient l'égalité :

$$
R_{1, a}^{+}\left(\pi^{(m)}\left(\sigma_{1}^{(m)}, \sigma_{2}\right)\right)=\sum_{\sigma_{1}^{(n)} \in \operatorname{Irr}\left(W_{a+N_{1}}\right)}\left\langle\operatorname{res}_{a}^{+}\left(\sigma_{1}^{(n)}\right), \sigma_{1}^{(m)}\right\rangle \pi^{(n)}\left(\sigma_{1}^{(n)}, \sigma_{2}\right) .
$$

Appliquons cette formule pour $\left(\sigma_{1}^{(m)}, \sigma_{2}\right)=\left(\rho_{1}, \rho_{2}\right)$. Le membre de gauche est égal à $R_{1, a}^{+} \circ \pi_{m}\left(\Lambda_{1} \otimes \Lambda_{2}\right)$. Grâce au lemme 2.5 , celui de droite est égal à $\pi_{n} \circ\left(I_{a} \otimes 1\right)\left(\Lambda_{1} \otimes \Lambda_{2}\right)$. D'où la conclusion. Dans le cas du couple $\left(1 \otimes I_{a}, R_{\zeta, a}^{+}\right)$un raisonnement analogue s'applique en échangeant les rôles des caractères 1 et $\zeta$, ce qui est loisible d'après la remarque de 4.2 . 



\section{CHAPITRE 9}

\section{PARAMÉTRAGE DE LUSZTIG}

Des travaux de Lusztig résulte un paramétrage des représentations irréductibles quadratiques-unipotentes des groupes spéciaux orthogonaux par des symboles qui sont des classes d'équivalence des nôtres. On montre que ce paramétrage est compatible au nôtre, c'est-à-dire que si $\pi_{0}$ est une composante irréductible de la restriction au groupe spécial orthogonal de $\pi\left(\Lambda_{1}, \Lambda_{2}\right)$, alors le paramètre de Lusztig de $\pi_{0}$ est formé des classes d'équivalence de $\Lambda_{1}$ et $\Lambda_{2}$. L'idée est que le paramétrage d'une représentation est largement déterminé par celui de ses modules de Jacquet propres. La mise en forme de cette idée nécessite deux lemmes combinatoires (9.2 et 9.3) essentiellement dus à Asai. De la comparaison des paramétrages résulte le calcul des produits scalaires (trace $\left.\left(\pi\left(\Lambda_{1}, \Lambda_{2}\right)\right), k\left(\Lambda_{1}^{\prime}, \Lambda_{2}^{\prime}\right)\right)$ quand $\Lambda_{1}^{\prime}$ et $\Lambda_{2}^{\prime}$ sont de défaut 0 . Cela sera utilisé au paragraphe 11 .

9.1. Soit $m \in \mathbb{N}$. Pour tout entier $D \in \mathbb{N}$, on a défini en 2.1 l'ensemble $\mathcal{S}_{m, D}$, quotient de $\widetilde{\mathcal{S}}_{m, D} \cup \widetilde{\mathcal{S}}_{m,-D}$ par la relation d'équivalence $\Lambda \sim \sigma \Lambda$. On pose :

$$
\begin{gathered}
\mathcal{S}_{m, \text { pair }}=\bigcup_{\substack{D \in \mathbb{N} \\
D \text { pair }}} \mathcal{S}_{m, D}, \\
\mathcal{S}_{m, \equiv 0}=\bigcup_{\substack{D \in \mathbb{N} \\
D \equiv 0 \bmod 4 \mathbb{Z}}} \mathcal{S}_{m, D}, \quad \mathcal{S}_{m, \equiv 2}=\bigcup_{\substack{D \in \mathbb{N} \\
D \equiv 2 \bmod 4 \mathbb{Z}}} \mathcal{S}_{m, D} .
\end{gathered}
$$

Remarquons que, puisque les familles de $\widetilde{\mathcal{S}}_{m \text {,pair }}$ sont stables par $\sigma$, on peut définir

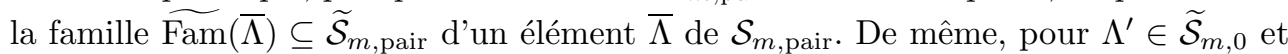
$\Lambda$ dans la famille de $\Lambda^{\prime}$, on vérifie que $\left\langle\sigma \Lambda, \Lambda^{\prime}\right\rangle=\left\langle\Lambda, \Lambda^{\prime}\right\rangle$. On peut donc définir $\left\langle\bar{\Lambda}, \Lambda^{\prime}\right\rangle$ et $\left(\bar{\Lambda}, \mathcal{F} \Lambda^{\prime}\right)$ pour $\bar{\Lambda} \in \mathcal{S}_{m, \text { pair }}$ et $\Lambda^{\prime} \in \widetilde{\operatorname{Fam}}(\bar{\Lambda}) \cap \widetilde{\mathcal{S}}_{m, 0}$. On pose :

$$
\mathcal{S S}_{m, \text { pair }}=\bigcup\left(\mathcal{S}_{m_{1}, \text { pair }} \times \mathcal{S}_{m_{2}, \text { pair }}\right)
$$

union sur les $\left(m_{1}, m_{2}\right) \in \mathbb{N} \times \mathbb{N}$ tels que $m_{1}+m_{2}=m$. On note uniformément proj les applications évidentes $\widetilde{\mathcal{S}}_{m \text {,pair }} \rightarrow \mathcal{S}_{m \text {,pair }}$ ou $\widetilde{\mathcal{S}}_{m \text {,pair }} \rightarrow \mathcal{S} \mathcal{S}_{m \text {,pair }}$, ainsi que les 
applications linéaires qui s'en déduisent :

$$
\mathbb{C}\left[\widetilde{\mathcal{S}}_{m, \text { pair }}\right] \longrightarrow \mathbb{C}\left[\mathcal{S}_{m, \text { pair }}\right], \quad \mathbb{C}\left[\widetilde{\mathcal{S}} \widetilde{\mathcal{S}}_{m, \text { pair }}\right] \longrightarrow \mathbb{C}\left[\mathcal{S} \mathcal{S}_{m, \text { pair }}\right]
$$

9.2. Soient $m, m_{1}, m_{2} \in \mathbb{N}$ tels que $m=m_{1}+m_{2}$ et $Z \in \mathbb{Z}\left[\widetilde{\mathcal{S}} \widetilde{\mathcal{S}}_{m \text {,pair }}\right]$. On considérera l'hypothèse :

(1) il existe $\Lambda_{2, Z} \in \widetilde{\mathcal{S}}_{m_{2} \text {,pair }}$ et une application $c: \widetilde{\mathcal{S}}_{m_{1} \text {,pair }} \rightarrow\{-1,0,1\}$ de sorte que les conditions suivantes soient vérifiées :

(i) $Z=\sum_{\Lambda_{1} \in \widetilde{\mathcal{S}}_{m_{1}, \mathrm{pair}}} c\left(\Lambda_{1}\right) \Lambda_{1} \otimes \Lambda_{2, Z}$;

(ii) pour toute famille $\widetilde{\text { Fam }} \subseteq \widetilde{\mathcal{S}}_{m_{1} \text {,pair }}$, l'ensemble $\left\{\Lambda_{1} \in \widetilde{\mathrm{Fam}} ; c\left(\Lambda_{1}\right) \neq 0\right\}$ a au plus 2 éléments ; quand il en a deux, notons-les $\Lambda_{1}^{a}, \Lambda_{1}^{b}$; il existe alors $\left(x^{\prime}, \varepsilon^{\prime}\right),\left(x^{\prime \prime}, \varepsilon^{\prime \prime}\right) \in \Lambda_{1}^{a}$, avec $x^{\prime}<x^{\prime \prime}$, de sorte que :

$$
\begin{gathered}
\left(x^{\prime},-\varepsilon^{\prime}\right),\left(x^{\prime \prime},-\varepsilon^{\prime \prime}\right) \notin \Lambda_{1}^{a}, \\
\Lambda_{1}^{b}=\left(\Lambda_{1}^{a} \backslash\left\{\left(x^{\prime}, \varepsilon^{\prime}\right),\left(x^{\prime \prime}, \varepsilon^{\prime \prime}\right)\right\}\right) \cup\left\{\left(x^{\prime},-\varepsilon^{\prime}\right),\left(x^{\prime \prime},-\varepsilon^{\prime \prime}\right)\right\}, \\
c\left(\Lambda_{1}^{a}\right) c\left(\Lambda_{1}^{b}\right)=(-1)^{\left|\Lambda_{1}^{a} \cap\left(\left\{x^{\prime \prime}-1, \ldots, x^{\prime}+1\right\} \times\{ \pm 1\}\right)\right|} .
\end{gathered}
$$

Remarquons que si, dans le dernier cas ci-dessus, on a $\Lambda_{1}^{b}=\sigma \Lambda_{1}^{a}$, l'ensemble $\Lambda_{1}^{a} \cap$ $\left(\left\{x^{\prime \prime}-1, \ldots, x^{\prime}+1\right\} \times\{ \pm 1\}\right)$ est symétrique donc $c\left(\Lambda_{1}^{a}\right) c\left(\Lambda_{1}^{b}\right)=1$.

On utilisera la propriété suivante, facile à prouver :

(2) soit $\widetilde{\text { Fam }} \subseteq \widetilde{\mathcal{S}}_{m \text {,pair }}$ une famille et $\Lambda, \Lambda^{\prime} \in \widetilde{\mathrm{Fam}}$; supposons $\Lambda^{\prime} \neq \Lambda, \sigma \Lambda$; alors il existe $\Lambda_{0} \in \widetilde{\mathcal{S}}_{m, 0} \cap \widetilde{\text { Fam }}$ tel que $\left\langle\Lambda, \Lambda_{0}\right\rangle=\left\langle\Lambda^{\prime}, \Lambda_{0}\right\rangle+1$.

LEMME. - Soient $m, m_{1}, m_{2}$ comme ci-dessus et $Z, \widetilde{Z} \in \mathbb{Z}\left[\widetilde{\mathcal{S}} \widetilde{\mathcal{S}}_{m \text {,pair }}\right]$. Supposons que $Z$ vérifie l'hypothèse (1) et de plus :

(a) $(Z, Z) \geqslant(\widetilde{Z}, \widetilde{Z})$;

(b) pour tous $\left(\Lambda_{1}^{\prime}, \Lambda_{2}^{\prime}\right) \in \widetilde{\mathcal{S}}_{m_{1}, 0} \times \widetilde{\mathcal{S}}_{m_{2}, 0},\left(Z, \mathcal{F} \Lambda_{1}^{\prime} \otimes \mathcal{F} \Lambda_{2}^{\prime}\right)=\left(\widetilde{Z}, \mathcal{F} \Lambda_{1}^{\prime} \otimes \mathcal{F} \Lambda_{2}^{\prime}\right)$.

Alors on a l'égalité $\operatorname{proj}(Z)=\operatorname{proj}(\widetilde{Z})$.

Évidemment, un lemme analogue vaut en échangeant les rôles des indices 1 et 2 . La preuve reprend l'idée d'Asai, $[\mathbf{A}]$ paragraphe 2.8 .

Démonstration. - On écrit $Z$ sous la forme (1)(i) et :

$$
\widetilde{Z}=\sum_{\left(\Lambda_{1}, \Lambda_{2}\right) \in \widetilde{\mathcal{S}} \widetilde{\mathcal{S}}_{m, \text { pair }}} \widetilde{c}\left(\Lambda_{1}, \Lambda_{2}\right) \Lambda_{1} \otimes \Lambda_{2},
$$

avec des coefficients $\widetilde{c}\left(\Lambda_{1}, \Lambda_{2}\right) \in \mathbb{Z}$. Notons $\widetilde{\operatorname{Fam}}_{2} \subseteq \widetilde{\mathcal{S}}_{m \text {,pair }}$ la famille de $\Lambda_{2, Z}$.

Soit $\widetilde{\mathrm{Fam}}_{1} \subseteq \widetilde{\mathcal{S}}_{m_{1} \text {,pair }}$ une famille. Montrons l'inégalité :

$$
\sum_{\left(\Lambda_{1}, \Lambda_{2}\right) \in \widetilde{\operatorname{Fam}}_{1} \times \widetilde{\operatorname{Fam}}_{2}} \widetilde{c}\left(\Lambda_{1}, \Lambda_{2}\right)^{2} \geqslant\left|\left\{\Lambda_{1} \in \widetilde{\operatorname{Fam}_{1}} ; c\left(\Lambda_{1}\right) \neq 0\right\}\right| .
$$


Soient $\Lambda_{1}^{\prime} \in \widetilde{\operatorname{Fam}}_{1} \cap \widetilde{\mathcal{S}}_{m_{1}, 0}, \Lambda_{2}^{\prime} \in \widetilde{\operatorname{Fam}}_{2} \cap \widetilde{\mathcal{S}}_{m_{2}, 0}$. L'hypothèse (b) implique :

$$
\sum_{\left(\Lambda_{1}, \Lambda_{2}\right) \in \widetilde{\operatorname{Fam}}_{1} \times \widetilde{\operatorname{Fam}}_{2}} \widetilde{c}\left(\Lambda_{1}, \Lambda_{2}\right)(-1)^{\left\langle\Lambda_{1}, \Lambda_{1}^{\prime}\right\rangle+\left\langle\Lambda_{2}, \Lambda_{2}^{\prime}\right\rangle}=\sum_{\Lambda_{1} \in \widetilde{\operatorname{Fam}}_{1}} c\left(\Lambda_{1}\right)(-1)^{\left\langle\Lambda_{1}, \Lambda_{1}^{\prime}\right\rangle+\left\langle\Lambda_{2, Z}, \Lambda_{2}^{\prime}\right\rangle} .
$$

A fortiori :

$$
\sum_{\left(\Lambda_{1}, \Lambda_{2}\right) \in \widetilde{\operatorname{Fam}_{1} \times \widetilde{\operatorname{Fam}_{2}}}} \widetilde{c}\left(\Lambda_{1}, \Lambda_{2}\right)^{2} \geqslant\left|\sum_{\Lambda_{1} \in \widetilde{\operatorname{Fam}_{1}}} c\left(\Lambda_{1}\right)(-1)^{\left\langle\Lambda_{1}, \Lambda_{1}^{\prime}\right\rangle+\left\langle\Lambda_{2, Z}, \Lambda_{2}^{\prime}\right\rangle}\right| .
$$

Posons $E=\left\{\Lambda_{1} \in \widetilde{\operatorname{Fam}_{1}} ; c\left(\Lambda_{1}\right) \neq 0\right\}$. Si $E=\varnothing$, (3) est clair. Si $E$ a un élément, (3) résulte immédiatement de l'inégalité ci-dessus. Supposons que $E$ a 2 éléments, notés $\Lambda_{1}^{a}, \Lambda_{1}^{b}$. On peut choisir $\Lambda_{1}^{\prime}$ de sorte que le membre de droite ci-dessus vaille 2 . En effet, si $c\left(\Lambda_{1}^{a}\right)=c\left(\Lambda_{1}^{b}\right)$, l'élément spécial de $\widetilde{\operatorname{Fam}}_{1}$ convient. Si $c\left(\Lambda_{1}^{a}\right)=-c\left(\Lambda_{1}^{b}\right)$, on a remarqué que $\Lambda_{1}^{b} \neq \Lambda_{1}^{a}, \sigma \Lambda_{1}^{a}$ et on applique (2). En tout cas, on obtient l'inégalité (3).

En sommant ces inégalités (3) sur toutes les familles $\widetilde{\mathrm{Fam}}_{1}$ et en utilisant l'hypothèse $(\mathrm{a})$, on voit que (3) est toujours une égalité. Cela entraîne que $\widetilde{c}$ prend ses valeurs dans $\{-1,0,1\}$ et que pour toute famille $\widetilde{\mathrm{Fam}}_{1}$, les ensembles $\widetilde{E}=\left\{\left(\Lambda_{1}, \Lambda_{2}\right) \in\right.$ $\left.\widetilde{\operatorname{Fam}_{1}} \times \widetilde{\operatorname{Fam}_{2}} ; \widetilde{c}\left(\Lambda_{1}, \Lambda_{2}\right) \neq 0\right\}$ et $E=\left\{\Lambda_{1} \in \widetilde{\operatorname{Fam}}_{1} ; c\left(\Lambda_{1}\right) \neq 0\right\}$ ont même nombre d'éléments. De plus, $\widetilde{c}\left(\Lambda_{1}, \Lambda_{2}\right)=0$ pour tout $\left(\Lambda_{1}, \Lambda_{2}\right) \in \widetilde{\mathcal{S}} \widetilde{\mathcal{S}}_{m \text {,pair }}$ tel que $\Lambda_{2} \notin \widetilde{\mathrm{Fam}}_{2}$.

On peut désormais fixer une famille $\widetilde{\mathrm{Fam}}_{1}$ et remplacer $Z$ et $\widetilde{Z}$ par leurs projections dans $\mathbb{Z}\left[\widetilde{\operatorname{Fam}}_{1} \times{\widetilde{\mathrm{Fam}_{2}}}_{2}\right.$. On définit comme ci-dessus les ensembles $\widetilde{E}$ et $E$.

Si $E$ est vide, $\widetilde{E}$ l'est aussi et $Z=\widetilde{Z}=0$.

Supposons $|E|=1$, notons $\Lambda_{1}$ l'élément de $E$ et $\left(\widetilde{\Lambda}_{1}, \widetilde{\Lambda}_{2}\right)$ celui de $\widetilde{E}$. L'égalité (4) entraîne que $\left\langle\widetilde{\Lambda}_{1}-\Lambda_{1}, \Lambda_{1}^{\prime}\right\rangle$, resp. $\left\langle\widetilde{\Lambda}_{2}-\Lambda_{2, Z}, \Lambda_{2}^{\prime}\right\rangle$, est indépendant de $\Lambda_{1}^{\prime} \in \widetilde{\operatorname{Fam}}_{1} \cap \widetilde{\mathcal{S}}_{m_{1}, 0}$, resp. $\Lambda_{2}^{\prime} \in \widetilde{\operatorname{Fam}}_{2} \cap \widetilde{\mathcal{S}}_{m_{2}, 0}$. En prenant $\Lambda_{1}^{\prime}$, resp. $\Lambda_{2}^{\prime}$, spécial, on voit que ces valeurs sont nulles. En appliquant (2), on voit que $\widetilde{\Lambda}_{1}$ est égal à $\Lambda_{1}$ ou $\sigma \Lambda_{1}$ et $\widetilde{\Lambda}_{2}$ est égal à $\Lambda_{2, Z}$ ou $\sigma \Lambda_{2, Z}$. En revenant à $(4)$, on voit que $\widetilde{c}\left(\widetilde{\Lambda}_{1}, \widetilde{\Lambda}_{2}\right)=c\left(\Lambda_{1}\right) . \operatorname{Alors} \operatorname{proj}(Z)=\operatorname{proj}(\widetilde{Z})$.

Supposons $|E|=2$, notons $\Lambda_{1}^{a}, \Lambda_{1}^{b}$ les deux éléments de $E$ et $\left(\widetilde{\Lambda}_{1}^{a}, \widetilde{\Lambda}_{2}^{a}\right),\left(\widetilde{\Lambda}_{1}^{b}, \widetilde{\Lambda}_{2}^{b}\right)$ les deux éléments de $\widetilde{E}$. Soit $\Lambda_{2}^{\prime} \in \widetilde{\mathrm{Fam}}_{2} \cap \widetilde{\mathcal{S}}_{m_{2}, 0}$. Posons :

$$
\begin{aligned}
Z\left(\Lambda_{2}^{\prime}\right)=c\left(\Lambda_{1}^{a}\right)(-1)^{\left\langle\Lambda_{2, Z}, \Lambda_{2}^{\prime}\right\rangle} \Lambda_{1}^{a} & +c\left(\Lambda_{1}^{b}\right)(-1)^{\left\langle\Lambda_{2, Z}, \Lambda_{2}^{\prime}\right\rangle} \Lambda_{1}^{b} \\
& +\widetilde{c}\left(\widetilde{\Lambda}_{1}^{a}, \widetilde{\Lambda}_{2}^{a}\right)(-1)^{\left\langle\widetilde{\Lambda}_{2}^{a}, \Lambda_{2}^{\prime}\right\rangle} \widetilde{\Lambda}_{1}^{a}+\widetilde{c}\left(\widetilde{\Lambda}_{1}^{b}, \widetilde{\Lambda}_{2}^{b}\right)(-1)^{\left\langle\widetilde{\Lambda}_{2}^{b}, \Lambda_{2}^{\prime}\right\rangle} \widetilde{\Lambda}_{1}^{b} .
\end{aligned}
$$

C'est un élément de $\widetilde{\mathcal{S}}_{m_{1}}$,pair qui, grâce à (4) et l'hypothèse (1), vérifie les hypothèses du lemme 2.8.8 de $[\mathbf{A}]$. D'après ce lemme, $\operatorname{proj}\left(Z\left(\Lambda_{2}^{\prime}\right)\right)=0$. Si $\Lambda_{1}^{b} \neq \sigma \Lambda_{1}^{a}$, cette relation entraîne que, quitte à échanger $\Lambda_{1}^{a}$ et $\Lambda_{1}^{b}$, et cela de façon indépendante de $\Lambda_{2}^{\prime}$, on a les égalités :

$$
\begin{gathered}
\operatorname{proj}\left(\Lambda_{1}^{a}\right)=\operatorname{proj}\left(\widetilde{\Lambda}_{1}^{a}\right), \quad \operatorname{proj}\left(\Lambda_{1}^{b}\right)=\operatorname{proj}\left(\widetilde{\Lambda}_{1}^{b}\right), \\
c\left(\Lambda_{1}^{a}\right)(-1)^{\left\langle\Lambda_{2, Z}, \Lambda_{2}^{\prime}\right\rangle}=\widetilde{c}\left(\widetilde{\Lambda}_{1}^{a}, \widetilde{\Lambda}_{2}^{a}\right)(-1)^{\left\langle\widetilde{\Lambda}_{2}^{a}, \Lambda_{2}^{\prime}\right\rangle}, \\
c\left(\Lambda_{1}^{b}\right)(-1)^{\left\langle\Lambda_{2, Z}, \Lambda_{2}^{\prime}\right\rangle}=\widetilde{c}\left(\widetilde{\Lambda}_{1}^{b}, \widetilde{\Lambda}_{2}^{b}\right)(-1)^{\left\langle\widetilde{\Lambda}_{2}^{b}, \Lambda_{2}^{\prime}\right\rangle} .
\end{gathered}
$$


Si $\Lambda_{1}^{b}=\sigma \Lambda_{1}^{a}$ on a remarqué que $c\left(\Lambda_{1}^{a}\right)=c\left(\Lambda_{1}^{b}\right)$ et la relation $\operatorname{proj}\left(Z\left(\Lambda_{2}^{\prime}\right)\right)=0$ entraîne encore les relations ci-dessus. Comme dans le cas $|E|=1$, ces relations entraînent les égalités $\operatorname{proj}\left(\widetilde{\Lambda}_{2}^{a}\right)=\operatorname{proj}\left(\Lambda_{2, Z}\right)=\operatorname{proj}\left(\widetilde{\Lambda}_{2}^{b}\right), c\left(\Lambda_{1}^{a}\right)=\widetilde{c}\left(\widetilde{\Lambda}_{1}^{a}, \widetilde{\Lambda}_{2}^{a}\right), c\left(\Lambda_{1}^{b}\right)=$ $\widetilde{c}\left(\widetilde{\Lambda}_{1}^{b}, \widetilde{\Lambda}_{2}^{b}\right)$. Alors $\operatorname{proj}(Z)=\operatorname{proj}(\widetilde{Z})$.

\section{3.}

LEMME. - Soient $m, m_{1}, m_{2} \in \mathbb{N}$, avec $m=m_{1}+m_{2}, Z, \widetilde{Z} \in \mathbb{Z}\left[\widetilde{\mathcal{S}} \widetilde{\mathcal{S}}_{m, \text { pair }}\right]$ et $N$ un entier $\geqslant 1$. Supposons que $Z$ et $\widetilde{Z}$ vérifient tous deux l'hypothèse (1) de 9.2 et que, pour tous $\left(\Lambda_{1}^{\prime}, \Lambda_{2}^{\prime}\right) \in \widetilde{\mathcal{S}}_{m_{1}, 0} \times \widetilde{\mathcal{S}}_{m_{2}, 0}$, on ait l'égalité

$$
\left(Z, \mathcal{F} \Lambda_{1}^{\prime} \otimes \mathcal{F} \Lambda_{2}^{\prime}\right)=N\left(\widetilde{Z}, \mathcal{F} \Lambda_{1}^{\prime} \otimes \mathcal{F} \Lambda_{2}^{\prime}\right) .
$$

Alors on a l'égalité $\operatorname{proj}(Z)=N \operatorname{proj}(\widetilde{Z})$.

Démonstration. - On peut fixer deux familles $\widetilde{\operatorname{Fam}}_{1} \subseteq \widetilde{\mathcal{S}}_{m_{1} \text {,pair }}, \widetilde{\operatorname{Fam}}_{2} \subseteq \widetilde{\mathcal{S}}_{m_{2} \text {,pair }}$,

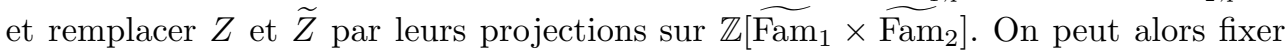
$\Lambda_{2}, \widetilde{\Lambda}_{2} \in \widetilde{\operatorname{Fam}}_{2}$ et deux fonctions $c, \widetilde{c}: \widetilde{\operatorname{Fam}}_{1} \rightarrow\{-1,0,1\}$ de sorte que :

$$
Z=\sum_{\Lambda_{1} \in \widetilde{\operatorname{Fam}}_{1}} c\left(\Lambda_{1}\right) \Lambda_{1} \otimes \Lambda_{2}, \widetilde{Z}=\sum_{\Lambda_{1} \in \widetilde{\operatorname{Fam}}_{1}} \widetilde{c}\left(\Lambda_{1}\right) \Lambda_{1} \otimes \widetilde{\Lambda}_{2}
$$

On peut de plus supposer que $Z$ ou $\widetilde{Z}$ est non nul. Posons $E=\left\{\Lambda_{1} \in \widetilde{\operatorname{Fam}}_{1} ; c\left(\Lambda_{1}\right) \neq 0\right\}$, $\widetilde{E}=\left\{\Lambda_{1} \in{\widetilde{\operatorname{Fam}_{1}}}_{\tilde{c}} \widetilde{c}\left(\Lambda_{1}\right) \neq 0\right\}$. Pour $\Lambda_{1}^{\prime} \in{\widetilde{\operatorname{Fam}_{1}}}_{\widetilde{\mathcal{S}}_{m_{1}, 0}}$ et $\Lambda_{2}^{\prime} \in{\widetilde{\operatorname{Fam}_{2}}} \cap \widetilde{\mathcal{S}}_{m_{2}, 0}$, on a l'égalité :

$$
N \sum_{\Lambda_{1} \in \widetilde{\operatorname{Fam}}_{1}} \widetilde{c}\left(\Lambda_{1}\right)(-1)^{\left\langle\Lambda_{1}, \Lambda_{1}^{\prime}\right\rangle+\left\langle\widetilde{\Lambda}_{2}, \Lambda_{2}^{\prime}\right\rangle}=\sum_{\Lambda_{1} \in \widetilde{\operatorname{Fam}}_{1}} c\left(\Lambda_{1}\right)(-1)^{\left\langle\Lambda_{1}, \Lambda_{1}^{\prime}\right\rangle+\left\langle\Lambda_{2}, \Lambda_{2}^{\prime}\right\rangle} .
$$

On a vu dans la preuve précédente que l'on pouvait choisir $\Lambda_{1}^{\prime}$ et $\Lambda_{2}^{\prime}$ de sorte que le membre de droite vaille $\pm|E|$. On en déduit $|E| \leqslant N|\widetilde{E}|$. De même, on peut choisir $\Lambda_{1}^{\prime}$ et $\Lambda_{2}^{\prime}$ de sorte que le membre de gauche vaille $\pm N|\widetilde{E}|$. D'où $N|\widetilde{E}| \leqslant|E|$ et finalement $N|\widetilde{E}|=|E|$. Puisque $E$ et $\widetilde{E}$ ont au plus 2 éléments et l'un de ces ensembles n'est pas vide, il n'y a que deux possibilités :

$-N=1,|E|=|\widetilde{E}|$,

$-N=2,|E|=2,|\widetilde{E}|=1$.

Dans le premier cas, on a $(Z, Z)=(\widetilde{Z}, \widetilde{Z})$ et on conclut grâce au lemme 9.2. Dans le second cas, notons $\widetilde{\Lambda}_{1}$ l'unique élément de $\widetilde{E}$ et $\Lambda_{1}^{a}, \Lambda_{1}^{b}$ les deux éléments de $E$. L'égalité (1) entraîne les deux égalités :

$$
\begin{aligned}
\widetilde{c}\left(\widetilde{\Lambda}_{1}\right)(-1)^{\left\langle\widetilde{\Lambda}_{1}, \Lambda_{1}^{\prime}\right\rangle+\left\langle\tilde{\Lambda}_{2}, \Lambda_{2}^{\prime}\right\rangle} & =c\left(\Lambda_{1}^{a}\right)(-1)^{\left\langle\Lambda_{1}^{a}, \Lambda_{1}^{\prime}\right\rangle+\left\langle\Lambda_{2}, \Lambda_{2}^{\prime}\right\rangle} \\
& =c\left(\Lambda_{1}^{b}\right)(-1)^{\left\langle\Lambda_{1}^{b}, \Lambda_{1}^{\prime}\right\rangle+\left\langle\Lambda_{2}, \Lambda_{2}^{\prime}\right\rangle},
\end{aligned}
$$


pour tous $\Lambda_{1}^{\prime}, \Lambda_{2}^{\prime}$ comme en (1). Comme dans la preuve du lemme précédent, on en déduit les égalités :

$$
\begin{gathered}
\operatorname{proj}\left(\Lambda_{1}^{a}\right)=\operatorname{proj}\left(\Lambda_{1}^{b}\right)=\operatorname{proj}\left(\widetilde{\Lambda}_{1}\right), \quad \operatorname{proj}\left(\Lambda_{2}\right)=\operatorname{proj}\left(\widetilde{\Lambda}_{2}\right), \\
\widetilde{c}\left(\widetilde{\Lambda}_{1}\right)=c\left(\Lambda_{1}^{a}\right)=c\left(\Lambda_{1}^{b}\right) .
\end{gathered}
$$

$\operatorname{Alors} \operatorname{proj}(Z)=2 \operatorname{proj}(\widetilde{Z})$.

9.4. Pour tout entier $x$ assez grand, posons :

$$
\begin{array}{llrl}
A(x) & =\{x-1, \ldots, 0\}, & B(x) & =\{x\} \cup\{x-2, \ldots, 0\}, \\
C(x) & =\{x+1\} \cup\{x-2, \ldots, 0\}, & D(x) & =\{x, x-1\} \cup\{x-3, \ldots, 0\} .
\end{array}
$$

Pour $h \in \mathbb{Z}$ et deux termes $a, b$ égaux à $A, B, C$ ou $D$, on définit le symbole ordonné $a b(h)$ dont un représentant vérifie :

$$
a b(h)^{+}=a(x+2 h), \quad a b(h)^{-}=b(x),
$$

$x$ étant un entier assez grand. Remarquons que $\sigma(a b(h))=b a(-h)$.

Lemme. - Soient $m \in \mathbb{N}, \Lambda, \Lambda^{\prime} \in \widetilde{\mathcal{S}}_{m \text {,pair }}$.

(a) Supposons que pour tout entier $a \in\{1, \ldots, m\}$, on ait l'égalité $I_{-a}(\Lambda)=$ $I_{-a}\left(\Lambda^{\prime}\right)$. Alors l'une des conditions suivantes est vérifiée :

$-\Lambda=\Lambda^{\prime}$;

$-\Lambda=\sigma \Lambda^{\prime}$ et $\Lambda$ et $\Lambda^{\prime}$ sont tous deux cuspidaux;

- il existe $h \in \mathbb{Z}$ tel que $\left\{\Lambda, \Lambda^{\prime}\right\}=\{A B(h), B A(h)\}$.

(b) Soit $N$ un entier $\geqslant 1$, supposons que pour tout entier $a \in\{1, \ldots, m\}$, on ait l'égalité $\operatorname{proj} \circ I_{-a}(\Lambda)=N$ proj $\circ I_{-a}\left(\Lambda^{\prime}\right)$. Alors l'une des conditions suivantes est vérifiée :

$-\operatorname{proj}(\Lambda)=\operatorname{proj}\left(\Lambda^{\prime}\right)$ et $\Lambda$ et $\Lambda^{\prime}$ sont cuspidaux;

- $N=1$ et $\operatorname{proj}(\Lambda)=\operatorname{proj}\left(\Lambda^{\prime}\right)$;

$-N=1$ et il existe $h \in \mathbb{Z}$ de sorte que $\operatorname{proj}(\Lambda)=\operatorname{proj}(A B(h)), \operatorname{proj}\left(\Lambda^{\prime}\right)=$ $\operatorname{proj}(B A(h))$.

Démonstration. - On donne seulement la démonstration de (b), celle de (a) étant similaire. On utilise les notations de 2.3 , en supprimant les indices $\theta$ qui sont égaux à 1 . On écrit ainsi :

$$
I_{-a}(\Lambda)=\sum_{(x, \varepsilon) \in E_{-a}(\Lambda)} \zeta_{-a, \Lambda}(x, \varepsilon) \Lambda_{-a}(x, \varepsilon)
$$

pour tout entier $a \in\{1, \ldots, m\}$. Remarquons que les termes de cette somme ne se compensent pas quand on applique proj. En effet, on vérifie que si $(x, \varepsilon),\left(x^{\prime}, \varepsilon^{\prime}\right)$ sont deux éléments distincts de $E_{-a}(\Lambda)$ tels que $\Lambda_{-a}(x, \varepsilon)=\sigma\left(\Lambda_{-a}\left(x^{\prime}, \varepsilon^{\prime}\right)\right)$, alors $\Lambda=\sigma \Lambda$ et $\left(x^{\prime}, \varepsilon^{\prime}\right)=(x,-\varepsilon)$. Mais dans ce cas, $\zeta_{-a, \Lambda}\left(x^{\prime}, \varepsilon^{\prime}\right)=\zeta_{-a, \Lambda}(x, \varepsilon)$ et les deux termes 
s'ajoutent au lieu de s'annuler. L'hypothèse de l'énoncé implique donc que pour tout $a \in\{1, \ldots, m\}$ et tout $(x, \varepsilon) \in E_{-a}(\Lambda)$, l'une des conditions suivantes est vérifiée :

(1) il existe $\left(x^{\prime}, \varepsilon^{\prime}\right) \in E_{-a}\left(\Lambda^{\prime}\right)$ tel que $\zeta_{-a, \Lambda}(x, \varepsilon) \Lambda_{-a}(x, \varepsilon)=\zeta_{-a, \Lambda^{\prime}}\left(x^{\prime}, \varepsilon^{\prime}\right) \Lambda_{-a}^{\prime}\left(x^{\prime}, \varepsilon^{\prime}\right)$;

(2) il existe $\left(x^{\prime}, \varepsilon^{\prime}\right) \in E_{-a}\left(\Lambda^{\prime}\right)$ tel que $\zeta_{-a, \Lambda}(x, \varepsilon) \Lambda_{-a}(x, \varepsilon)=\zeta_{-a, \Lambda^{\prime}}\left(x^{\prime}, \varepsilon^{\prime}\right) \sigma\left(\Lambda_{-a}^{\prime}\left(x^{\prime}, \varepsilon^{\prime}\right)\right)$.

On a une propriété similaire si l'on échange les rôles de $\Lambda$ et $\Lambda^{\prime}$. De ces propriétés résulte que $\Lambda$ est cuspidal si et seulement si $\Lambda^{\prime}$ l'est. Si c'est le cas, la première condition de l'énoncé est vérifiée, puisqu'il y a au plus deux éléments cuspidaux, qui se déduisent l'un de l'autre par $\sigma$. On suppose désormais que ni $\Lambda$, ni $\Lambda^{\prime}$ ne sont cuspidaux. La condition (2) est équivalente à :

(3) il existe $\left(x^{\prime}, \varepsilon^{\prime}\right) \in E_{-a}\left(\sigma \Lambda^{\prime}\right)$ tel que

$$
\zeta_{-a, \Lambda}(x, \varepsilon) \Lambda_{-a}(x, \varepsilon)=\zeta_{-a, \sigma \Lambda^{\prime}}\left(x^{\prime}, \varepsilon^{\prime}\right)\left(\sigma \Lambda^{\prime}\right)_{-a}\left(x^{\prime}, \varepsilon^{\prime}\right) .
$$

Remarquons que $\widetilde{\operatorname{def}}\left(\Lambda_{-a}(x, \varepsilon)\right)=\widetilde{\operatorname{def}}(\Lambda)$ et $\widetilde{\operatorname{def}}\left(\sigma \Lambda^{\prime}\right)=-\widetilde{\operatorname{def}}\left(\Lambda^{\prime}\right)$. Alors (1) ou (3) impliquent $\widetilde{\operatorname{def}}(\Lambda)= \pm \widetilde{\operatorname{def}}\left(\Lambda^{\prime}\right)$. Quitte à remplacer $\Lambda^{\prime}$ par $\sigma \Lambda^{\prime}$, on peut supposer $\widetilde{\operatorname{def}}(\Lambda)=\widetilde{\operatorname{def}}\left(\Lambda^{\prime}\right)$. Si ce défaut est non nul, la condition (3) devient interdite.

Supposons :

$$
\sum_{a=1, \ldots, m}\left|E_{-a}(\Lambda)\right| \geqslant \begin{cases}2, & \text { si } \widetilde{\operatorname{def}}(\Lambda) \neq 0 \\ 3, & \text { si } \widetilde{\operatorname{def}}(\Lambda)=0 .\end{cases}
$$

Alors, quitte à remplacer $\Lambda^{\prime}$ par $\sigma \Lambda^{\prime}$ dans le cas où $\widetilde{\operatorname{def}}(\Lambda)=0$, on peut fixer $a, b \in\{1, \ldots, m\},(x, \varepsilon) \in E_{-a}(\Lambda),(y, \eta) \in E_{-b}(\Lambda)$ tels que $(a, x, \varepsilon) \neq(b, y, \eta)$ et les triplets $(a, x, \varepsilon),(b, y, \eta)$ vérifient tous deux la condition (1). On note $\left(x^{\prime}, \varepsilon^{\prime}\right) \in$ $E_{-a}\left(\Lambda^{\prime}\right)$, resp. $\left(y^{\prime}, \eta^{\prime}\right) \in E_{-b}\left(\Lambda^{\prime}\right)$, le couple correspondant par cette condition à $(x, \varepsilon)$, resp. $(y, \eta)$. Notons $1_{\Lambda}$, resp. $1_{\Lambda^{\prime}}, 1_{x, \varepsilon}$ etc. les fonctions caractéristiques dans $\mathbb{N} \times\{ \pm 1\}$ de $\Lambda$, resp. $\Lambda^{\prime},\{(x, \varepsilon)\}$ etc. Les égalités (1) entraînent :

$$
\begin{aligned}
& 1_{\Lambda}-1_{x, \varepsilon}+1_{x-a, \varepsilon}=1_{\Lambda^{\prime}}-1_{x^{\prime}, \varepsilon^{\prime}}+1_{x^{\prime}-a, \varepsilon^{\prime}}, \\
& 1_{\Lambda}-1_{y, \eta}+1_{y-b, \eta}=1_{\Lambda^{\prime}}-1_{y^{\prime}, \eta^{\prime}}+1_{y^{\prime}-b, \eta^{\prime}} .
\end{aligned}
$$

D'où :

$$
1_{x, \varepsilon}+1_{x^{\prime}-a, \varepsilon^{\prime}}+1_{y^{\prime}, \eta^{\prime}}+1_{y-b, \eta}=1_{x^{\prime}, \varepsilon^{\prime}}+1_{x-a, \varepsilon}+1_{y, \eta}+1_{y^{\prime}-b, \eta^{\prime}} .
$$

Cette égalité entraîne l'une des relations suivantes :

(5) $(x, \varepsilon)=\left(x^{\prime}, \varepsilon^{\prime}\right)$ et $(y, \eta)=\left(y^{\prime}, \eta^{\prime}\right)$;

(6) $\varepsilon=\varepsilon^{\prime}=\eta=\eta^{\prime}, x=y, y^{\prime}=x-a, x^{\prime}=x-b, a \neq b$;

(7) $\varepsilon=\varepsilon^{\prime}=\eta=\eta^{\prime}, x^{\prime}=y^{\prime}, y=x^{\prime}-a, x=x^{\prime}-b, a \neq b$.

Supposons (6) vérifiée. L'égalité $\Lambda_{-a}(x, \varepsilon)=\Lambda_{-a}^{\prime}\left(x^{\prime}, \varepsilon^{\prime}\right)$ entraîne que $\Lambda^{-\varepsilon}=\Lambda^{\prime-\varepsilon}$ et qu'il existe un ensemble $X \subseteq \mathbb{N} \backslash\{x, x-a, x-b, x-a-b\}$ de sorte que :

$$
\Lambda^{\varepsilon}=X \cup\{x-a-b, x\}, \Lambda^{\varepsilon}=X \cup\{x-a, x-b\} .
$$


En supposant par exemple $a>b$, on calcule :

$$
\begin{aligned}
\zeta_{-a, \Lambda}(x, \varepsilon) \zeta_{-b, \Lambda}(y, \eta) & =(-1)^{|X \cap\{x-b, \ldots, x-a+1\}|}, \\
\zeta_{-a, \Lambda^{\prime}}\left(x^{\prime}, \varepsilon^{\prime}\right) \zeta_{-b, \Lambda^{\prime}}\left(y^{\prime}, \eta^{\prime}\right) & =(-1)^{|X \cap\{x-b, \ldots, x-a+1\}|+1} .
\end{aligned}
$$

Cela contredit (1). Cette contradiction interdit (6). On passe de (6) à (7) en échangeant les rôles de $\Lambda$ et $\Lambda^{\prime}$. Donc (7) est aussi interdite. Il reste (5), qui entraîne $\Lambda=\Lambda^{\prime}$. Cette égalité implique $N=1$ puisque $\Lambda$ n'est pas cuspidal. La deuxième condition de l'énoncé est alors vérifiée.

Il en est de même si $\Lambda^{\prime}$ vérifie l'hypothèse (4), par échange des rôles de $\Lambda$ et $\Lambda^{\prime}$. On suppose maintenant que ni $\Lambda$, ni $\Lambda^{\prime}$ ne vérifient (4). On décrit facilement tous les symboles ordonnés qui ne vérifient pas (4). Outre les cuspidaux, il y a :

- pour tout $h \in \mathbb{Z}$, les symboles $A B(h)$ et $B A(h)$;

- les symboles $B B(0), A C(0), C A(0), A D(0), D A(0)$.

Un calcul cas par cas montre que les seuls triplets $\left(\Lambda, \Lambda^{\prime}, N\right)$ vérifiant l'hypothèse de l'énoncé et tels que $\Lambda$ et $\Lambda^{\prime}$ soient chacun de l'une des formes ci-dessus, vérifient l'une des deux dernières conditions de l'énoncé.

9.5. Pour tout ensemble fini $X$ de $\mathbb{N}$, on introduit les polynômes en une variable $T$ :

$$
\Delta(X, T)=\prod_{x, x^{\prime} \in X, x>x^{\prime}}\left(T^{x}-T^{x^{\prime}}\right), \quad \Theta(X, T)=\prod_{x \in X} \prod_{y=1, \ldots, x}\left(T^{y}-1\right) .
$$

Soient $m \in \mathbb{N}$ et $\Lambda \in \widetilde{\mathcal{S}}_{m, \text { pair. }}$. Posons :

$$
\alpha=\sum_{i=1, \ldots,|\Lambda| / 2-1} \frac{(|\Lambda|-2 i)(|\Lambda|-2 i-1)}{2},
$$

et définissons la fraction rationnelle :

$$
\widetilde{D}_{\Lambda}(T)=2^{-|\Lambda| / 2} T^{-\alpha} \frac{\Delta\left(\Lambda^{+}, T\right) \Delta\left(\Lambda^{-}, T\right)}{\Theta\left(\Lambda^{+}, T^{2}\right) \Theta\left(\Lambda^{-}, T^{2}\right)} \prod_{(x, y) \in \Lambda^{+} \times \Lambda^{-}}\left(T^{x}+T^{y}\right) .
$$

\section{REMARQUES}

(1) $\widetilde{D}_{\Lambda}(T)$ diffère par un facteur 2 du terme $D_{\Lambda}(T)$ défini en $[\mathbf{L 2}]$ 2.8.1.

(2) On a l'égalité $\widetilde{D}_{\sigma \Lambda}(T)=\widetilde{D}_{\Lambda}(T)$, ce qui permet de définir $\widetilde{D}_{\bar{\Lambda}}(T)$ pour $\bar{\Lambda} \in$ $\mathcal{S}_{m, \text { pair. }}$

9.6. Soient $m \in \mathbb{N}$ et $\eta \in\{ \pm 1\}$, avec $\eta=1$ si $m=0$. Posons $\boldsymbol{G}=\boldsymbol{O}_{\eta}(2 m)$. Rappelons que pour deux entiers $N_{1}, N_{2} \geqslant 0$ tels que $m=N_{1}+N_{2}$ et pour $w_{1} \in W_{N_{1}}, w_{2} \in W_{N_{2}}$, on a construit en 3.3 une fonction $k_{\eta}\left(w_{1}, w_{2} ; 0,0\right)$ sur $G$. Son support est inclus dans $G^{0}$. Identifions-la à une fonction sur $G^{0}$. Elle est nulle $\operatorname{si~} \operatorname{sgn}_{C D}\left(w_{1}\right) \operatorname{sgn}_{C D}\left(w_{2}\right) \neq \eta$. Si $\operatorname{sgn}_{C D}\left(w_{1}\right) \operatorname{sgn}_{C D}\left(w_{2}\right)=\eta$, elle est égale à :

$$
(-1)^{m} \sum_{\gamma \in G / G^{0}} R_{T_{w_{1}} \times T_{w_{2}}}^{G^{0}}\left(1_{T_{w_{1}}} \times \zeta_{T_{w_{2}}}\right) \circ \operatorname{Ad}(\gamma) .
$$


On a fixé ici des tores $\boldsymbol{T}_{w_{1}}, \boldsymbol{T}_{w_{2}}$ comme en 3.3, on a noté $1_{T_{w_{1}}}$ le caractère trivial de $T_{w_{1}}$ et $\zeta_{T_{w_{2}}}$ le caractère correspondant à l'élément de $\widehat{T}_{w_{2}}$ noté $\zeta_{T_{w_{2}}}$ en 3.3 .

D'autre part, pour $\Lambda \in \widetilde{\mathcal{S}}_{m, 0}$, notons $\rho_{\Lambda} \in \operatorname{Irr}\left(W_{m}\right)$ la représentation telle que $\Lambda$ corresponde au couple $\left(0, \rho_{\Lambda}\right), c f .2 .2$.

Lusztig a classifié en $[\mathbf{L} 3]$ les représentations irréductibles unipotentes de $G^{0}$, c'està-dire les éléments de $\operatorname{Irr}\left(G^{0}, 1\right)$. Exprimons ses résultats dans notre terminologie. Il existe une unique application :

$$
\mathcal{L}_{1}: \operatorname{Irr}\left(G^{0}, 1\right) \longrightarrow \mathcal{S}_{m, \equiv(1-\eta)}
$$

vérifiant les propriétés suivantes :

- $\mathcal{L}_{1}$ est surjective et ses fibres sont exactement les orbites de l'action naturelle de $G / G^{0}$ sur $\operatorname{Irr}\left(G^{0}, 1\right)$;

- pour $\pi \in \operatorname{Irr}\left(G^{0}, 1\right)$, le stabilisateur de $\pi$ dans $G / G^{0}$ a autant d'éléments que la fibre au-dessus de $\mathcal{L}_{1}(\pi)$ de l'application proj : $\widetilde{\mathcal{S}}_{m, \text { pair }} \rightarrow \mathcal{S}_{m, \text { pair }}$;

- soient $\pi \in \operatorname{Irr}\left(G^{0}, 1\right)$ et $w \in W_{m} ;$ posons $\bar{\Lambda}=\mathcal{L}_{1}(\pi)$; on a les égalités :

$$
\begin{aligned}
& \operatorname{dim}(\pi)=\left|\operatorname{proj}^{-1}(\bar{\Lambda})\right|\left|G^{0}\right|_{p^{\prime}} \widetilde{D}_{\bar{\Lambda}}(q), \\
& \left(\operatorname{trace}(\pi) ; k_{\eta}(w, 1 ; 0,0)\right)_{G^{0}}=(-1)^{m}\left|\operatorname{proj}^{-1}(\bar{\Lambda})\right||\widetilde{\operatorname{Fam}}(\bar{\Lambda})|^{-1 / 2} \\
& \sum_{\Lambda \in \overline{\operatorname{Fam}}(\bar{\Lambda}) \cap \widetilde{\mathcal{S}}_{m, 0}}(-1)^{\langle\bar{\Lambda}, \Lambda\rangle} \operatorname{trace}\left(\rho_{\Lambda}\right)(w) .
\end{aligned}
$$

\section{REMARQUes}

(a) cf. 7.3 pour la définition de $\left|G^{0}\right|_{p^{\prime}}$.

(b) Le produit $(., .)_{G^{0}}$ est le produit scalaire de $\mathcal{C}\left(G^{0}\right)$.

(c) Le facteur $(-1)^{m}\left|\operatorname{proj}^{-1}(\bar{\Lambda})\right|$ ne se trouve pas dans [L3]. Il vient de notre normalisation de la fonction $k_{\eta}(w, 1 ; 0,0), c f$. (1) ci-dessus.

9.7. Soient $\eta \in\{ \pm 1\}, \boldsymbol{G}=\boldsymbol{O}_{\eta}(2 n)$ et $s$ un élément semi-simple de $\widehat{G}^{0}$ dont toutes les valeurs propres sont égales à \pm 1 . Pour $\varepsilon \in\{ \pm 1\}$, notons $\widehat{V}^{\varepsilon}$ le sous-espace de $\widehat{V}_{\eta}(2 n)$, propre pour l'action de $s$, associé à la valeur propre $\varepsilon$. Posons $\eta^{\varepsilon}=\eta\left(\widehat{V}^{\varepsilon}\right)$, $n^{\varepsilon}=\operatorname{dim}\left(\widehat{V}^{\varepsilon}\right) / 2$. Notons $\boldsymbol{H}$ le commutant de $s$ dans $\widehat{\boldsymbol{G}}$ et $\widetilde{\boldsymbol{H}}=\boldsymbol{H} \cap \widehat{\boldsymbol{G}}^{0}$. On a :

$$
\begin{aligned}
\boldsymbol{H}^{0} & =\boldsymbol{S O}_{\eta^{+}}\left(2 n^{+}\right) \times \boldsymbol{S O}_{\eta^{-}}\left(2 n^{-}\right), \\
\operatorname{Irr}\left(H^{0}, 1\right) & =\operatorname{Irr}\left(S O_{\eta^{+}}\left(2 n^{+}\right), 1\right) \times \operatorname{Irr}\left(S O_{\eta^{-}}\left(2 n^{-}\right), 1\right) .
\end{aligned}
$$

Cette décomposition nous permet d'appliquer les constructions du paragraphe précédent et de définir une application :

$$
\mathcal{L}_{1} \times \mathcal{L}_{1}: \operatorname{Irr}\left(H^{0}, 1\right) \longrightarrow \mathcal{S}_{n^{+}, \equiv\left(1-\eta^{+}\right)} \times \mathcal{S}_{n^{-}, \equiv\left(1-\eta^{-}\right)} .
$$

Les fibres de cette application sont les orbites de l'action naturelle de $H / H^{0}$ dans $\operatorname{Irr}\left(H^{0}, 1\right)$. A fortiori, l'application est constante sur les orbites de l'action de $\widetilde{H} / H^{0}$. 
Notons $\operatorname{Irr}\left(H^{0}, 1\right)^{\widetilde{H}}$ l'ensemble de ces dernières orbites. Alors $\mathcal{L}_{1} \times \mathcal{L}_{1}$ se quotiente en une application définie sur $\operatorname{Irr}\left(H^{0}, 1\right)^{\widetilde{H}}$.

En [L7], Lusztig a défini une application :

$$
\psi: \operatorname{Irr}\left(G^{0}, s\right) \longrightarrow \operatorname{Irr}\left(H^{0}, 1\right)^{\widetilde{H}} .
$$

On définit l'application :

$$
\mathcal{L}_{s}: \operatorname{Irr}\left(G^{0}, s\right) \longrightarrow \mathcal{S}_{n^{+}, \equiv\left(1-\eta^{+}\right)} \times \mathcal{S}_{n^{-}, \equiv\left(1-\eta^{-}\right)}
$$

composée de $\psi$ et de l'application $\mathcal{L}_{1} \times \mathcal{L}_{1}$ quotientée ainsi qu'on l'a expliqué ci-dessus.

Remarquons que $G / G^{0}$, qui agit naturellement sur $\operatorname{Irr}\left(G^{0}\right)$, conserve le sousensemble $\operatorname{Irr}\left(G^{0}, s\right)$. D'autre part, pour $\left(\Lambda_{1}, \Lambda_{2}\right) \in \widetilde{\mathcal{S}}_{n^{+}, \text {pair }} \times \widetilde{\mathcal{S}}_{n^{-}}$,pair , on pose :

$$
\alpha\left(\Lambda_{1}, \Lambda_{2}\right)= \begin{cases}1, & \text { si } \sigma \Lambda_{1}=\Lambda_{1} \text { et } \sigma \Lambda_{2}=\Lambda_{2}, \\ 0, & \text { sinon. }\end{cases}
$$

Cette application $\alpha$ se quotiente en une application encore notée $\alpha$ définie sur $\mathcal{S}_{n^{+}, \text {pair }} \times \mathcal{S}_{n^{-}, \text {pair }}$.

\section{LEMME}

(a) L'application $\mathcal{L}_{s}$ est surjective. Elle est constante sur les orbites de l'action de $G / G^{0}$ sur $\operatorname{Irr}\left(G^{0}, s\right)$.

(b) Soient $\pi \in \operatorname{Irr}\left(G^{0}, s\right), N_{1}, N_{2} \in \mathbb{N}$ tels que $n=N_{1}+N_{2}, w_{1} \in W_{N_{1}}$ et $w_{2} \in W_{N_{2}}$. Posons $\mathcal{L}_{s}(\pi)=\left(\bar{\Lambda}_{1}, \bar{\Lambda}_{2}\right)$. On a les égalités :

(i) $\operatorname{dim}(\pi)=2^{-\alpha\left(\bar{\Lambda}_{1}, \bar{\Lambda}_{2}\right)}|G|_{p^{\prime}} \widetilde{D}_{\bar{\Lambda}_{1}}(q) \widetilde{D}_{\bar{\Lambda}_{2}}(q) ;$

(ii) si $\left(N_{1}, N_{2}\right) \neq\left(n^{+}, n^{-}\right)$,

$$
\left(\operatorname{trace}(\pi), k_{\eta}\left(w_{1}, w_{2} ; 0,0\right)\right)_{G^{0}}=0 ;
$$

(iii) si $\left(N_{1}, N_{2}\right)=\left(n^{+}, n^{-}\right)$,

$$
\begin{aligned}
&\left(\operatorname{trace}(\pi), k_{\eta}\left(w_{1}, w_{2} ; 0,0\right)\right)_{G^{0}}=(-1)^{n} 2^{1-\alpha\left(\bar{\Lambda}_{1}, \bar{\Lambda}_{2}\right)}\left|\widetilde{\operatorname{Fam}}\left(\overline{\Lambda_{1}}\right)\right|^{-1 / 2}\left|\widetilde{\operatorname{Fam}}\left(\overline{\Lambda_{2}}\right)\right|^{-1 / 2} \\
& \sum_{\Lambda_{1}, \Lambda_{2}}(-1)^{\left\langle\bar{\Lambda}_{1}, \Lambda_{1}\right\rangle+\left\langle\bar{\Lambda}_{2}, \Lambda_{2}\right\rangle} \operatorname{trace}\left(\rho_{\Lambda_{1}}\right)\left(w_{1}\right) \operatorname{trace}\left(\rho_{\Lambda_{2}}\right)\left(w_{2}\right),
\end{aligned}
$$

où l'on somme sur $\Lambda_{j} \in \widetilde{\operatorname{Fam}}\left(\bar{\Lambda}_{j}\right) \cap \widetilde{\mathcal{S}}_{N_{j}, 0}$ pour $j \in\{1,2\}$.

Démonstration. - C'est une simple traduction de [L7] proposition 5.1. L'application $\mathcal{L}_{s}$ est surjective car $\psi$ et $\mathcal{L}_{1} \times \mathcal{L}_{1}$ le sont. Soit $\mathcal{O}$ une orbite dans $\operatorname{Irr}\left(G^{0}, s\right)$ pour l'action de $G / G^{0}$. Par transport de structure, $\psi(\mathcal{O})$ est une orbite dans $\operatorname{Irr}\left(H^{0}, 1\right)^{\widetilde{H}}$ pour l'action de $H / \widetilde{H}$. Mais $\mathcal{L}_{1} \times \mathcal{L}_{1}$ est constante sur une telle orbite. Donc $\mathcal{L}_{s}$ est constante sur $\mathcal{O}$.

Dans la situation de (b), soit $\pi_{H} \in \operatorname{Irr}\left(H^{0}, 1\right)$ tel que $\psi(\pi)$ soit la $\widetilde{H} / H^{0}$-orbite de $\pi_{H}$. Notons $\Gamma$ le stabilisateur de $\pi_{H}$ dans $\widetilde{H} / H^{0}$. Les propriétés des applications $\mathcal{L}_{1}$ permettent de calculer :

$$
|\Gamma|=\left|\operatorname{proj}^{-1}\left(\bar{\Lambda}_{1}\right)\right|\left|\operatorname{proj}^{-1}\left(\bar{\Lambda}_{2}\right)\right| 2^{\alpha\left(\bar{\Lambda}_{1}, \bar{\Lambda}_{2}\right)-1} .
$$


D'après [L7] proposition 5.1, la fibre $\psi^{-1}(\psi(\pi))$ a $|\Gamma|$ éléments. Ils ont tous même dimension. D'après la construction de $\psi$ et [L2] 7.8.1, appliqué au groupe de similitudes $C O_{\eta}(2 n)^{0}$, la somme de leurs dimensions vaut $\left|G^{0}\right|_{p^{\prime}}\left|H^{0}\right|_{p^{\prime}}^{-1} \operatorname{dim}\left(\pi_{H}\right)$. Autrement dit :

$$
\operatorname{dim}(\pi)=|\Gamma|^{-1}\left|G^{0}\right|_{p^{\prime}}\left|H^{0}\right|_{p^{\prime}}^{-1} \operatorname{dim}\left(\pi_{H}\right) .
$$

On calcule $\operatorname{dim}\left(\pi_{H}\right)$ grâce à $9.6(2)$. En utilisant (1) et l'égalité $2\left|G^{0}\right|_{p^{\prime}}=|G|_{p^{\prime}}$, on obtient le (i) de l'énoncé.

L'assertion (ii) résulte de la définition de $\operatorname{Irr}\left(G^{0}, s\right)$. Supposons $\left(N_{1}, N_{2}\right)=$ $\left(n^{+}, n^{-}\right)$. Soient $\boldsymbol{T}_{w_{1}}, \boldsymbol{T}_{w_{2}}$ comme en 9.6(1). On peut leur associer des sous-tores encore notés $\boldsymbol{T}_{w_{1}} \subseteq \boldsymbol{S O}_{\eta^{+}}\left(2 n^{+}\right), \boldsymbol{T}_{w_{2}} \subseteq \boldsymbol{S O}_{\eta^{-}}\left(2 n^{-}\right)$. La proposition 5.1 de [L7] se reformule ainsi :

$$
\begin{aligned}
& \left(\operatorname{trace}(\pi), R_{T_{w_{1}} \times T_{w_{2}}}^{G^{0}}\left(1_{T_{w_{1}}} \times \zeta_{T_{w_{2}}}\right)\right)_{G^{0}} \\
= & |\Gamma|^{-1}\left(\operatorname{trace}\left(\pi_{H}\right), \sum_{\gamma \in \widetilde{H} / H^{0}}\left(R_{T_{w_{1}}}^{S O_{\eta^{+}}\left(2 n^{+}\right)}\left(1_{T_{w_{1}}}\right) \times R_{T_{w_{2}}}^{S O_{\eta^{-}}\left(2 n^{-}\right)}\left(1_{T_{w_{2}}}\right)\right) \circ \operatorname{Ad}(\gamma)\right)_{H^{0}} .
\end{aligned}
$$

Par transport de structure, on obtient :

$$
\begin{aligned}
& \left(\operatorname{trace}(\pi), \sum_{\gamma \in G / G^{0}} R_{T_{w_{1}} \times T_{w_{2}}}^{G^{0}}\left(1_{T_{w_{1}}} \times \zeta_{T_{w_{2}}}\right) \circ \operatorname{Ad}(\gamma)\right)_{G^{0}} \\
= & |\Gamma|^{-1}\left(\operatorname{trace}\left(\pi_{H}\right), \sum_{\gamma \in H / H^{0}}\left(R_{T_{w_{1}}}^{S O_{\eta^{+}}\left(2 n^{+}\right)}\left(1_{T_{w_{1}}}\right) \times R_{T_{w_{2}}}^{S O_{\eta^{-}}\left(2 n^{-}\right)}\left(1_{T_{w_{2}}}\right)\right) \circ \operatorname{Ad}(\gamma)\right)_{H^{0}} .
\end{aligned}
$$

D'où :

$\left(\operatorname{trace}(\pi), k_{\eta}\left(w_{1}, w_{2} ; 0,0\right)\right)_{G^{0}}=|\Gamma|^{-1}\left(\operatorname{trace}\left(\pi_{H}\right), k_{\eta^{+}}\left(w_{1}, 1 ; 0,0\right) \times k_{\eta^{-}}\left(w_{2}, 1 ; 0,0\right)\right)_{H^{0}}$.

On calcule ce dernier produit scalaire grâce à 9.6(3). En utilisant (1), on obtient le (iii) de l'énoncé.

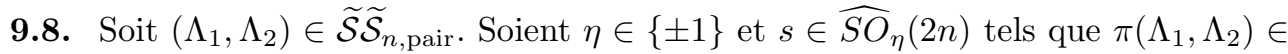
$\operatorname{Irr}\left(O_{\eta}(2 n), s\right)$. D'après le (a) du lemme précédent, toutes les composantes irréductibles de la restriction de $\pi\left(\Lambda_{1}, \Lambda_{2}\right)$ à $S O_{\eta}(2 n)$ ont même image par $\mathcal{L}_{s}$. On note $\mathcal{L}\left(\Lambda_{1}, \Lambda_{2}\right)$ cette image, ce qui définit une application :

$$
\mathcal{L}: \widetilde{\mathcal{S}} \widetilde{\mathcal{S}}_{n, \text { pair }} \longrightarrow \mathcal{S} \mathcal{S}_{n, \text { pair }}
$$

Soit toujours $\left(\Lambda_{1}, \Lambda_{2}\right) \in \widetilde{\mathcal{S}} \widetilde{\mathcal{S}}_{n \text {,pair }}$ et $\eta$ comme ci-dessus. Posons $\left(\bar{\Lambda}_{1}, \bar{\Lambda}_{2}\right)=$ $\mathcal{L}\left(\Lambda_{1}, \Lambda_{2}\right)$. Remarquons que le nombre de composantes irréductibles de la restriction de $\pi\left(\Lambda_{1}, \Lambda_{2}\right)$ à $S O_{\eta}(2 n)$ est égal à $2^{\alpha\left(\Lambda_{1}, \Lambda_{2}\right)}$. Le (b) du lemme précédent se reformule ainsi :

- on a l'égalité :

- soit $\left(\Lambda_{1}^{\prime}, \Lambda_{2}^{\prime}\right) \in \widetilde{\mathcal{S}} \widetilde{\mathcal{S}}_{n \text {,pair }}$ tel que $\widetilde{\operatorname{def}}\left(\Lambda_{1}^{\prime}\right)=\widetilde{\operatorname{def}}\left(\Lambda_{2}^{\prime}\right)=0$; on a l'égalité :

$$
\left(\operatorname{trace}\left(\pi\left(\Lambda_{1}, \Lambda_{2}\right)\right), k\left(\Lambda_{1}^{\prime}, \Lambda_{2}^{\prime}\right)\right)=2^{\alpha\left(\Lambda_{1}, \Lambda_{2}\right)-\alpha\left(\bar{\Lambda}_{1}, \bar{\Lambda}_{2}\right)}\left(\bar{\Lambda}_{1} \otimes \bar{\Lambda}_{2}, \mathcal{F} \Lambda_{1}^{\prime}, \mathcal{F} \Lambda_{2}^{\prime}\right)
$$


Le produit scalaire de gauche est celui de $\mathcal{C}(O(2 n))$. Le passage du produit dans $\mathcal{C}\left(S O_{\eta}(2 n)\right)$ à celui-là fait disparaître un facteur 2 du deuxième membre de l'égalité (b)(iii) du lemme précédent.

Proposition. - Les deux applications $\mathcal{L}$, proj : $\widetilde{\mathcal{S}} \widetilde{\mathcal{S}}_{n, \text { pair }} \rightarrow \mathcal{S S}_{n, \text { pair }}$ sont égales.

Démonstration. - Soient $n_{1}, n_{2} \in \mathbb{N}$ tels que $n_{1}+n_{2}=n, \Lambda_{1} \in \widetilde{\mathcal{S}}_{n_{1} \text {,pair }}, \Lambda_{2} \in \widetilde{\mathcal{S}}_{n_{2} \text {,pair }}$. Fixons $\left(\widetilde{\Lambda}_{1}, \widetilde{\Lambda}_{2}\right) \in \widetilde{\mathcal{S}} \widetilde{\mathcal{S}}_{n, \text { pair }}$ tel que $\operatorname{proj}\left(\widetilde{\Lambda}_{1}, \widetilde{\Lambda}_{2}\right)=\mathcal{L}\left(\Lambda_{1}, \Lambda_{2}\right)$. Soient $\eta \in\{ \pm 1\}$ et $s \in \widehat{S O}_{\eta}(2 n)$ tels que $\pi\left(\Lambda_{1}, \Lambda_{2}\right) \in \operatorname{Irr}\left(O_{\eta}(2 n), s\right)$. D'après la proposition 4.4(b)(ii), $s$ a pour valeurs propres 1 avec la multiplicité $2 n_{1}$ et -1 avec la multiplicité $2 n_{2}$. Il résulte de la construction de $\mathcal{L}$ que $\widetilde{\Lambda}_{1} \in \widetilde{\mathcal{S}}_{n_{1} \text {,pair }}, \widetilde{\Lambda}_{2} \in \widetilde{\mathcal{S}}_{n_{2} \text {,pair }}$.

Soient $a \in\left\{1, \ldots, n_{1}\right\}, \Lambda_{1}^{\prime} \in \widetilde{\mathcal{S}}_{n_{1}-a, 0}, \Lambda_{2}^{\prime} \in \widetilde{\mathcal{S}}_{n_{2}, 0}$. Montrons que l'on a l'égalité :

$$
\left(I_{-a} \Lambda_{1} \otimes \Lambda_{2}, \mathcal{F} \Lambda_{1}^{\prime} \otimes \mathcal{F} \Lambda_{2}^{\prime}\right)=c\left(I_{-a} \widetilde{\Lambda}_{1} \otimes \widetilde{\Lambda}_{2}, \mathcal{F} \Lambda_{1}^{\prime} \otimes \mathcal{F} \Lambda_{2}^{\prime}\right),
$$

où $c=2^{\alpha\left(\Lambda_{1}, \Lambda_{2}\right)-\alpha\left(\widetilde{\Lambda}_{1}, \widetilde{\Lambda}_{2}\right)}$. On a les égalités :

$$
\begin{aligned}
\left(I_{-a} \Lambda_{1} \otimes \Lambda_{2}, \mathcal{F} \Lambda_{1}^{\prime} \otimes \mathcal{F} \Lambda_{2}^{\prime}\right) & =\left(\Lambda_{1} \otimes \Lambda_{2}, I_{a} \mathcal{F} \Lambda_{1}^{\prime} \otimes \mathcal{F} \Lambda_{2}^{\prime}\right), \\
& =\left(\pi_{n}\left(\Lambda_{1} \otimes \Lambda_{2}\right), \pi_{n}\left(I_{a} \mathcal{F} \Lambda_{1}^{\prime} \otimes \mathcal{F} \Lambda_{2}^{\prime}\right)\right), \\
& =\left(\pi_{n}\left(\Lambda_{1} \otimes \Lambda_{2}\right), R_{1, a}^{+} \circ \pi_{n-a}\left(\mathcal{F} \Lambda_{1}^{\prime} \otimes \mathcal{F} \Lambda_{2}^{\prime}\right)\right), \\
& =\left(\pi_{n}\left(\Lambda_{1} \otimes \Lambda_{2}\right), R_{1, a}^{+} \circ k_{n-a}\left(\Lambda_{1}^{\prime} \otimes \Lambda_{2}^{\prime}\right)\right), \\
& =\left(\pi_{n}\left(\Lambda_{1} \otimes \Lambda_{2}\right), k_{n}\left(I_{a} \Lambda_{1}^{\prime} \otimes \Lambda_{2}^{\prime}\right)\right)
\end{aligned}
$$

(ces égalités résultent respectivement d'une formule d'adjonction, du fait que $\pi_{n}$ est une isométrie, de la proposition 8.3 , de l'hypothèse de récurrence et de la proposition 8.2). Or $I_{a} \Lambda_{1}^{\prime} \in \widetilde{\mathcal{S}}_{n_{1}, 0}$. Grâce à (2), on a :

$$
\begin{aligned}
\left(\pi_{n}\left(\Lambda_{1} \otimes \Lambda_{2}\right), k_{n}\left(I_{a} \Lambda_{1}^{\prime} \otimes \Lambda_{2}^{\prime}\right)\right) & =c\left(\widetilde{\Lambda}_{1} \otimes \widetilde{\Lambda}_{2}, \mathcal{F} I_{a} \Lambda_{1}^{\prime} \otimes \mathcal{F} \Lambda_{2}^{\prime}\right), \\
& =c\left(\widetilde{\Lambda}_{1} \otimes \widetilde{\Lambda}_{2}, I_{a} \mathcal{F} \Lambda_{1}^{\prime} \otimes \mathcal{F} \Lambda_{2}^{\prime}\right), \\
& =c\left(I_{-a} \widetilde{\Lambda}_{1} \otimes \widetilde{\Lambda}_{2}, \mathcal{F} \Lambda_{1}^{\prime} \otimes \mathcal{F} \Lambda_{2}^{\prime}\right) .
\end{aligned}
$$

D'où (3).

De la définition de l'application $I_{-a}$ résulte que les termes $I_{-a} \Lambda_{1} \otimes \Lambda_{2}$ et $I_{-a} \widetilde{\Lambda}_{1} \otimes \widetilde{\Lambda}_{2}$ vérifient tous deux l'hypothèse (1) de 9.2. De plus $c \in\left\{\frac{1}{2}, 1,2\right\}$. Quitte à échanger les deux termes, on peut, grâce à (3), leur appliquer le lemme 9.3. On en déduit :

$$
\operatorname{proj}\left(I_{-a} \Lambda_{1} \otimes \Lambda_{2}\right)=c \operatorname{proj}\left(I_{-a} \widetilde{\Lambda}_{1} \otimes \widetilde{\Lambda}_{2}\right) .
$$

Supposons $\Lambda_{1}$ cuspidal. L'égalité ci-dessus, vraie pour tout $a$, entraîne que $\widetilde{\Lambda}_{1}$ est cuspidal, donc $\widetilde{\Lambda}_{1}=\Lambda_{1}$ ou $\sigma \Lambda_{1}$ (ce sont les seuls termes cuspidaux). On peut dans ce qui précède inverser les rôles des indices 1 et 2 . Si $\Lambda_{1}$ et $\Lambda_{2}$ sont tous deux cuspidaux, on obtient alors $\operatorname{proj}\left(\Lambda_{1}, \Lambda_{2}\right)=\operatorname{proj}\left(\widetilde{\Lambda}_{1}, \widetilde{\Lambda}_{2}\right)=\mathcal{L}\left(\Lambda_{1}, \Lambda_{2}\right)$. 
Supposons $\Lambda_{1}$ non cuspidal. L'égalité (4) se décompose en deux :

$$
\begin{aligned}
\operatorname{proj}\left(\Lambda_{2}\right) & =\operatorname{proj}\left(\widetilde{\Lambda}_{2}\right), \\
\operatorname{proj}\left(I_{-a} \Lambda_{1}\right) & =c \operatorname{proj}\left(I_{-a} \widetilde{\Lambda}_{1}\right)
\end{aligned}
$$

pour tout $a \in\left\{1, \ldots, n_{1}\right\}$. En échangeant les rôles des indices 1 et 2 , on a aussi $\operatorname{proj}\left(\Lambda_{1}\right)=\operatorname{proj}\left(\widetilde{\Lambda}_{1}\right)$ si $\Lambda_{2}$ n'est pas cuspidal, donc $\operatorname{proj}\left(\Lambda_{1}, \Lambda_{2}\right)=\mathcal{L}\left(\Lambda_{1}, \Lambda_{2}\right)$ si ni $\Lambda_{1}$, ni $\Lambda_{2}$ ne sont cuspidaux.

Supposons $\Lambda_{1}$ non cuspidal et $\Lambda_{2}$ cuspidal. Grâce à (5) et au lemme 9.4, on a $c=1$ et l'une des relations suivantes est vérifiée :

(6) $\operatorname{proj}\left(\Lambda_{1}\right)=\operatorname{proj}\left(\widetilde{\Lambda}_{1}\right)$,

(7) il existe $h \in \mathbb{Z}, h \neq 0$, tel que $\operatorname{proj}\left(\Lambda_{1}\right)=\operatorname{proj}(B A(h)), \operatorname{proj}\left(\widetilde{\Lambda}_{1}\right)=\operatorname{proj}(A B(h))$

(on peut supposer $h \neq 0$, sinon la seconde relation entraîne la première). Supposons (7) vérifiée. La surjectivité des applications $\mathcal{L}_{s}$ entraîne que $\mathcal{L}$ est surjective. On peut donc fixer $\left(\Lambda_{1}^{\prime}, \Lambda_{2}^{\prime}\right) \in \widetilde{\mathcal{S}} \widetilde{\mathcal{S}}_{n \text {,pair }}$ tel que $\mathcal{L}\left(\Lambda_{1}^{\prime}, \Lambda_{2}^{\prime}\right)=\operatorname{proj}\left(\Lambda_{1}, \Lambda_{2}\right)$. En appliquant à ce couple la démonstration ci-dessus, on voit que l'une des relations :

$$
\operatorname{proj}\left(\Lambda_{1}^{\prime}, \Lambda_{2}^{\prime}\right)=\operatorname{proj}\left(\Lambda_{1}, \Lambda_{2}\right), \operatorname{ou} \operatorname{proj}\left(\Lambda_{1}^{\prime}, \Lambda_{2}^{\prime}\right)=\operatorname{proj}\left(\widetilde{\Lambda}_{1}, \widetilde{\Lambda}_{2}\right)
$$

est vérifiée. De plus la constante $c$ relative à $\left(\Lambda_{1}^{\prime}, \Lambda_{2}^{\prime}\right)$ vaut 1 . Supposons par exemple $h>0$. Montrons que l'on a :

$$
\operatorname{dim}\left(\pi\left(\Lambda_{1}^{\prime}, \Lambda_{2}^{\prime}\right)\right) \geqslant \operatorname{dim}\left(\pi\left(\Lambda_{1}, \Lambda_{2}\right)\right)
$$

Ces dimensions étant insensibles à l'application de $\sigma$ à l'une ou l'autre des variables (cf. proposition 4.4(b)(iii),(v)), on peut supposer $\Lambda_{2}^{\prime}=\Lambda_{2}, \Lambda_{1}=B A(h)$ et $\Lambda_{1}^{\prime}=\Lambda_{1}$ ou $\Lambda_{1}^{\prime}=A B(h)$. Si $\Lambda_{1}^{\prime}=\Lambda_{1}$, (8) est évident. Supposons $\Lambda_{1}^{\prime}=A B(h)$. Considérons le symbole ordonné $A A(h)=\Lambda_{\text {cusp }}(h)$. Soit $\eta \in\{ \pm 1\}$ tel que $\pi\left(\Lambda_{\text {cusp }}(h), \Lambda_{2}\right)$ soit une représentation de $O_{\eta}(2 n-2)$. Il résulte des constructions du paragraphe 4 que $\pi\left(\Lambda_{\text {cusp }}(h), \Lambda_{2}\right)$ est cuspidale, que l'induite à $O_{\eta}(2 n)$ de la représentation $1_{1} \times$ $\pi\left(\Lambda_{\text {cusp }}(h), \Lambda_{2}\right)$ d'un Lévi isomorphe à $G L(1) \times O_{\eta}(2 n-2)$ se décompose selon les représentations irréductibles du groupe $W_{1}$ et que $\pi\left(\Lambda_{1}, \Lambda_{2}\right)$, resp. $\pi\left(\Lambda_{1}^{\prime}, \Lambda_{2}^{\prime}\right)$, est la sous-représentation associée au caractère 1 , resp. sgn, de $W_{1}$. L'inégalité (8) résulte d'une formule due à Hoefsmit, $c f$. [L2 $]$ théorème 4.4.

D'autre part, on vient de voir que $\pi\left(\Lambda_{1}^{\prime}, \Lambda_{2}^{\prime}\right)$ et $\pi\left(\Lambda_{1}, \Lambda_{2}\right)$ sont des représentations d'un même groupe $O_{\eta}(2 n)$. Leurs constantes associées $c$ valent toutes deux 1. La relation (1) entraîne :

$$
\frac{\operatorname{dim}\left(\pi\left(\Lambda_{1}^{\prime}, \Lambda_{2}^{\prime}\right)\right)}{\operatorname{dim}\left(\pi\left(\Lambda_{1}, \Lambda_{2}\right)\right)}=\frac{\widetilde{D}_{\Lambda_{1}}(q)}{\widetilde{D}_{\widetilde{\Lambda}_{1}}(q)}=\frac{\widetilde{D}_{B A(h)}(q)}{\widetilde{D}_{A B(h)}(q)}
$$

On calcule ce dernier rapport. Il est $<1$, ce qui contredit (8). Cette contradiction interdit la relation (7). Il reste (6), mais alors $\operatorname{proj}\left(\Lambda_{1}, \Lambda_{2}\right)=\mathcal{L}\left(\Lambda_{1}, \Lambda_{2}\right)$. Cette égalité est donc vérifiée si $\Lambda_{1}$ n'est pas cuspidal et $\Lambda_{2}$ l'est. Par échange des indices 1 et 2 , elle l'est si $\Lambda_{1}$ est cuspidal et $\Lambda_{2}$ ne l'est pas. On a ainsi traité tous les cas. 
9.9 .

Corollaire. - Soient $\left(\Lambda_{1}, \Lambda_{2}\right),\left(\Lambda_{1}^{\prime}, \Lambda_{2}^{\prime}\right) \in \widetilde{\mathcal{S}} \widetilde{\mathcal{S}}_{n, \text { pair }}$, avec $\widetilde{\operatorname{def}}\left(\Lambda_{1}^{\prime}\right)=\widetilde{\operatorname{def}}\left(\Lambda_{2}^{\prime}\right)=0$. Notons $\eta \in\{ \pm 1\}$ l'élément tel que $\pi\left(\Lambda_{1}, \Lambda_{2}\right)$ soit une représentation de $O_{\eta}(2 n)$. On a les égalités :

(i) $\operatorname{dim}\left(\pi\left(\Lambda_{1}, \Lambda_{2}\right)\right)=\left|O_{\eta}(2 n)\right|_{p^{\prime}} \widetilde{D}_{\Lambda_{1}}(q) \widetilde{D}_{\Lambda_{2}}(q)$;

(ii) $\left(\operatorname{trace}\left(\pi\left(\Lambda_{1}, \Lambda_{2}\right)\right), k\left(\Lambda_{1}^{\prime}, \Lambda_{2}^{\prime}\right)\right)=\left(\Lambda_{1} \otimes \Lambda_{2}, \mathcal{F} \Lambda_{1}^{\prime} \otimes \mathcal{F} \Lambda_{2}^{\prime}\right)$. 



\section{CHAPITRE 10}

\section{QUELQUES CALCULS DE TRACES}

Les résultats de ce paragraphe sont techniques. Les lemmes 10.1 et 10.2 nous serviront au paragraphe suivant à séparer des représentations que l'on ne peut pas distinguer par des méthodes combinatoires. Les lemmes 10.3 à 10.6 concernent le cas $n \leqslant 4$. Ils suppléeront au paragraphe 12 à un argument de congruence qui n'est suffisant que pour $n \geqslant 5$. Le lemme d'intégralité 10.7 servira en 12.3 à prouver que la matrice $C$ est rationnelle.

10.1. Soit $\Lambda \in \widetilde{\mathcal{S}}_{n-1 \text {,pair }}$, posons $\eta=(-1)^{\widetilde{\operatorname{def}}(\Lambda) / 2}$. On dispose des trois représentations irréductibles $\pi\left(\Lambda, \mathbf{1}_{+}(1)\right), \pi\left(\Lambda, \sigma \mathbf{1}_{+}(1)\right)$ de $O_{\eta}(2 n)$ et $\pi(\Lambda, \Lambda(\varnothing))$ de $O_{\eta}(2 n-2)$, $c f .2 .4$ pour les notations. Soit $g \in O_{\eta}(2 n)$ un élément semi-simple ayant pour valeurs propres 1 , avec la multiplicité $2 n-1$, et -1 , avec la multiplicité 1 .

LEMME. - Sous ces hypothèses, on a l'égalité :

$$
\begin{aligned}
& \operatorname{trace}\left(\pi\left(\Lambda, \mathbf{1}_{+}(1)\right)\right)(g)-\operatorname{trace}\left(\pi\left(\Lambda, \sigma \mathbf{1}_{+}(1)\right)\right)(g) \\
& =2|S O(2 n-1)|_{p^{\prime}}\left|O_{\eta}(2 n-2)\right|_{p^{\prime}}^{-1} \eta \operatorname{sp}(g) \operatorname{trace}(\pi(\Lambda, \Lambda(\varnothing)))(1) .
\end{aligned}
$$

Démonstration. - Notons $\left(h_{1}, \rho_{1}\right)$ le couple associé à $\Lambda$ en 2.2. Posons $\boldsymbol{G}=\boldsymbol{O}_{\eta}(2 n)$, $V=V_{\eta}(2 n), \boldsymbol{G}_{0}=\boldsymbol{O}_{\eta}\left(2 h_{1}^{2}\right)$. Notons $\pi_{0}$ la représentation cuspidale $\pi\left(\Lambda_{\text {cusp }}\left(h_{1}\right), \Lambda(\varnothing)\right)$ de $G_{0}, E_{0}$ son espace, posons $N_{1}=n-1-h_{1}^{2}, N_{2}=1$. On effectue la construction de 4.1 pour ces données, en échangeant les rôles des indices 1 et 2 , ce qui est loisible ainsi qu'on l'a remarqué en 4.2. On introduit donc un sous-ensemble $\left\{v_{i} ; i \in\left\{ \pm 1, \ldots, \pm\left(1+N_{1}\right)\right\}\right\}$ de $V$, un sous-groupe parabolique $\boldsymbol{P}=\boldsymbol{M U}$ de $\boldsymbol{G}$, avec $\boldsymbol{M} \simeq \mathbb{G}_{m}^{1+N_{1}} \times \boldsymbol{G}_{0}$ et on note $(\pi, E)$ la représentation $\operatorname{Ind}_{P}^{G}\left(\zeta_{1} \times 1_{N_{1}} \times \pi_{0}\right)$. Son algèbre d'entrelacements est produit de deux algèbres $\mathcal{H}_{1}$ et $\mathcal{H}_{2}$. L'algèbre $\mathcal{H}_{1}$, resp. $\mathcal{H}_{2}$, est engendrée par des opérateurs $T_{1}\left(w_{1}\right)$ pour $w_{1} \in W_{N_{1}}$, resp. $T_{2}\left(w_{2}\right)$ pour $w_{2} \in W_{1}$. Pour toute représentation irréductible $\delta_{1}$ de $W_{N_{1}}$, notons $\delta_{1, \mathcal{H}_{1}}$ la représentation correspondante de l'algèbre $\mathcal{H}_{1}$ et $\Lambda_{1}\left(\delta_{1}\right)$ le symbole ordonné associé au couple $\left(h_{1}, \delta_{1}\right)$. En particulier $\Lambda=\Lambda_{1}\left(\rho_{1}\right)$. De même, pour toute représentation irréductible 
$\delta_{2}$ de $W_{1}$, notons $\delta_{2, \mathcal{H}_{2}}$ la représentation correspondante de l'algèbre $\mathcal{H}_{2}$ et $\Lambda_{2}\left(\delta_{2}\right)$ le symbole ordonné associé au couple $\left(0, \delta_{2}\right)$. En fait, $\mathbf{1}_{+}(1)=\Lambda_{2}\left(\mathbf{1}_{W_{1}}\right)$, où $\mathbf{1}_{W_{1}}$ est le caractère trivial de $W_{1}$, et $\sigma \mathbf{1}_{+}(1)=\Lambda_{2}(\operatorname{sgn})$.

Pour tous $w_{1} \in W_{N_{1}}, w_{2} \in W_{1}$, on a l'égalité :

$$
\begin{aligned}
\sum_{\substack{\delta_{1} \in \operatorname{Irr}\left(W_{N_{1}}\right) \\
\delta_{2} \in \operatorname{Irr}\left(W_{1}\right)}} \operatorname{trace}\left(\delta_{1, \mathcal{H}_{1}}\right)\left(T_{1}\left(w_{1}\right)\right) \operatorname{trace}\left(\delta_{2, \mathcal{H}_{2}}\right)\left(T_{2}\left(w_{2}\right)\right) & \operatorname{trace}\left(\pi\left(\Lambda_{1}\left(\delta_{1}\right), \Lambda_{2}\left(\delta_{2}\right)\right)\right)(g) \\
= & \operatorname{trace}\left(T_{1}\left(w_{1}\right) T_{2}\left(w_{2}\right) \pi(g) \mid E\right) .
\end{aligned}
$$

Notons $\dot{w}_{2}$ l'unique élément non trivial de $W_{1}$. D'après 4.4(4), on a l'égalité $T_{2}\left(\dot{w}_{2}\right)^{2}=1$, autrement dit $\mathcal{H}_{2} \simeq \mathbb{C}\left[W_{1}\right]$. L'égalité précédente, pour $w_{2}=\dot{w}_{2}$, devient :

$$
\begin{aligned}
& \sum_{\delta_{1} \in \operatorname{Irr}\left(W_{N_{1}}\right)} \operatorname{trace}\left(\delta_{1, \mathcal{H}_{1}}\right)\left(T_{1}\left(w_{1}\right)\right) \\
& \quad\left(\operatorname{trace}\left(\pi\left(\Lambda_{1}\left(\delta_{1}\right), \mathbf{1}_{+}(1)\right)\right)(g)-\operatorname{trace}\left(\pi\left(\Lambda_{1}\left(\delta_{1}\right), \sigma \mathbf{1}_{+}(1)\right)\right)(g)\right) \\
& =\operatorname{trace}\left(T_{1}\left(w_{1}\right) T_{2}\left(\dot{w}_{2}\right) \pi(g) \mid E\right) .
\end{aligned}
$$

Posons $\widetilde{\boldsymbol{G}}=\boldsymbol{O}_{\eta}(2 n-2)$. Appliquons la construction de 4.1 à $\widetilde{\boldsymbol{G}}, \pi_{0}, N_{1}=n-1-h_{1}^{2}$ et $N_{2}=0$. On introduit donc un sous-groupe parabolique $\widetilde{\boldsymbol{P}}=\widetilde{\boldsymbol{M}} \widetilde{\boldsymbol{U}}$ de $\widetilde{\boldsymbol{G}}$, avec $\widetilde{\boldsymbol{M}} \simeq \mathbb{G}_{m}^{N_{1}} \times \boldsymbol{G}_{0}$, et on note $(\widetilde{\pi}, \widetilde{E})$ la représentation $\operatorname{Ind}_{\widetilde{P}}^{\widetilde{G}}\left(1_{N_{1}} \times \pi_{0}\right)$. Son algèbre d'entrelacements $\widetilde{\mathcal{H}}_{1}$ est engendrée par des opérateurs $\widetilde{T}_{1}\left(w_{1}\right)$, pour $w_{1} \in W_{N_{1}}$ (on a ajouté des $\sim$ pour les distinguer des opérateurs précédents). En fait :

(2) l'application qui, pour tout $w_{1} \in W_{N_{1}}$, envoie $T_{1}\left(w_{1}\right)$ sur $\widetilde{T}_{1}\left(w_{1}\right)$ est un isomorphisme de $\mathcal{H}_{1}$ sur $\widetilde{\mathcal{H}}_{1}$.

On le démontre directement en réalisant $\widetilde{\boldsymbol{G}}$ comme sous-groupe de $\boldsymbol{G}$.

Identifions $\widetilde{\mathcal{H}}_{1}$ à $\mathcal{H}_{1}$. Pour tout $w_{1} \in W_{N_{1}}$, on a l'égalité :

$$
\sum_{\delta_{1} \in \operatorname{Irr}\left(W_{N_{1}}\right)} \operatorname{trace}\left(\delta_{1, \mathcal{H}_{1}}\right)\left(T_{1}\left(w_{1}\right)\right) \operatorname{trace}\left(\pi\left(\Lambda_{1}\left(\delta_{1}\right), \Lambda(\varnothing)\right)\right)(1)=\operatorname{trace}\left(\widetilde{T}_{1}\left(w_{1}\right) \mid \widetilde{E}\right) .
$$

Posons :

$$
c=2|S O(2 n-1)|_{p^{\prime}}\left|O_{\eta}(2 n-2)\right|_{p^{\prime}}^{-1} \eta \operatorname{sp}(g) .
$$

On va prouver l'égalité :

$$
\operatorname{trace}\left(T_{1}\left(w_{1}\right) T_{2}\left(\dot{w}_{2}\right) \pi(g) \mid E\right)=c \operatorname{trace}\left(\widetilde{T}_{1}\left(w_{1}\right) \mid \widetilde{E}\right)
$$

pour tout $w_{1} \in W_{N_{1}}$. Admettons cette égalité. Le membre de droite de (1) est égal à celui de (3) multiplié par $c$. Il en est de même des membres de gauche. Les fonctions $\operatorname{trace}\left(\delta_{1, \mathcal{H}_{1}}\right)$ étant linéairement indépendantes quand $\delta_{1}$ décrit $\operatorname{Irr}\left(W_{N_{1}}\right)$, leurs coefficients dans l'expression (1) sont égaux à ceux de l'expression (3), multipliés par $c$. Appliquée à $\delta_{1}=\rho_{1}$, cette égalité est celle de l'énoncé.

Il reste à démontrer (4). En 4.1 et 4.2 , on a introduit d'autres opérateurs $T_{1}^{\prime}\left(w_{1}\right)$ pour $w_{1} \in W_{N_{1}}, T_{2}^{\prime}\left(w_{2}\right)$ pour $w_{2} \in W_{1}$, liés aux précédents par des égalités $T_{1}\left(w_{1}\right)=$ 
$C_{1}\left(w_{1}\right) T_{1}^{\prime}\left(w_{1}\right), T_{2}\left(w_{2}\right)=C_{2}\left(w_{2}\right) T_{2}^{\prime}\left(w_{2}\right)$. On a $C_{1}(1)=C_{2}(1)=1$. On a remarqué ci-dessus que $T_{2}\left(\dot{w}_{2}\right)^{2}=1$. Comme on l'a dit en 4.2 , cela entraîne $C_{2}\left(\dot{w}_{2}\right)=\eta q^{1-n}$.

Soient $w_{1} \in W_{N_{1}}, w_{2} \in W_{1}$, notons simplement $w=w_{2} w_{1}$, identifié comme en 4.1 à un élément de $G$. Pour $f \in E$ et $h \in G$, on a l'égalité :

$$
\left(T^{\prime}(w) \pi(g) f\right)(h)=\sum_{u \in\left(w U w^{-1} \cap U\right) \backslash U} A_{w} f\left(w^{-1} u h g\right),
$$

cf. 4.1 pour les notations. Notons $X(w)$ l'ensemble des $h \in G$ tels que $h g h^{-1} \in U w P$. Pour $h \in X(w)$, notons $m(h)$ l'unique élément de $M$ tel que $h g h^{-1} \in U w m(h) U$. Remarquons que $X(w)$ est invariant à gauche par multiplication par $P$ et que la fonction $h \mapsto \operatorname{trace}\left(A_{w} \circ\left(\zeta_{1} \times 1_{N_{1}} \times \pi_{0}\right)(m(h)) \mid E_{0}\right)$ se descend à $P \backslash X(w)$. Il est standard de déduire de (5) l'égalité :

$$
\operatorname{trace}\left(T^{\prime}(w) \pi(g) \mid E\right)=\sum_{h \in P \backslash X(w)} \operatorname{trace}\left(A_{w} \circ\left(\zeta_{1} \times 1_{N_{1}} \times \pi_{0}\right)(m(h)) \mid E_{0}\right) .
$$

On poursuit la démonstration en supposant $h_{1} \neq 0$, celle-ci s'adaptant aisément au cas $h_{1}=0$. Fixons un vecteur $v \in V$ engendrant l'espace propre pour $g$ relatif à la valeur propre -1 . Posons $a=Q(v, v)$. Pour $i \in\left\{1, \ldots, 1+N_{1}\right\}$, posons $v(i)=-\frac{a}{4} v_{i}+v_{-i}$. Fixons $v(0) \in V_{0}(c f .4 .1)$, tel que $Q(v(0), v(0))=a$. Pour $i \in\left\{0, \ldots, 1+N_{1}\right\}$, notons $g(i)$ la symétrie par rapport à l'hyperplan orthogonal à $v(i)$, et fixons $h(i) \in G$ tel que $h(i) g h(i)^{-1}=g(i)$. On vérifie que l'orbite $G v$ est réunion disjointe des orbites $P v(i)$ pour $i \in\left\{0, \ldots, 1+N_{1}\right\}$. On en déduit que $G$ est réunion disjointe des sous-ensembles $\operatorname{Ph}(i) Z_{G}(g)$. Pour tout $i \in\left\{0, \ldots, 1+N_{1}\right\}$, définissons $\tau(i) \in W_{1+N_{1}}$ par $: \tau(0)=1$; si $i \geqslant 1, \tau(i)$ échange $i$ et $-i$ et fixe $j$ pour $j \in\left\{ \pm 1, \ldots, \pm\left(1+N_{1}\right)\right\} \backslash\{ \pm i\}$. Il est clair que $g(i) \in \tau(i) M$ et que, pour $h \in P h(i) Z_{G}(g)$, on a $h g h^{-1} \in U \tau(i) P$.

Si $w$ n'est pas l'un des $\tau(i)$, on a donc $X(w)=\varnothing$ et $\operatorname{trace}\left(T^{\prime}(w) \pi(g) \mid E\right)=0$.

Supposons $w=\tau(0)=1$. Alors $g(0) \in M$. Plus précisément, notons $g_{0}$ la restriction de $g(0)$ à $V_{0}$. C'est un élément analogue à $g$ : il a pour valeurs propres 1 avec la multiplicité $2 h_{1}^{2}-1$ et -1 avec la multiplicité 1 . De plus, $\operatorname{sp}\left(g_{0}\right)=\operatorname{sp}(g)$. Quand on identifie $M$ à $\left(\mathbb{F}_{q}^{\times}\right)^{1+N_{1}} \times G_{0}, g(0)$ s'identifie à $\left(1, \ldots, 1, g_{0}\right)$. Alors, pour tout $h \in X(1)=P h(0) Z_{G}(g)$, on a l'égalité :

$$
\begin{aligned}
\operatorname{trace}\left(A_{1} \circ\left(\zeta_{1} \times 1_{N_{1}} \times \pi_{0}\right)(m(h)) \mid E_{0}\right) & =\operatorname{trace}\left(\left(\zeta_{1} \times 1_{N_{1}} \times \pi_{0}\right)(g(0)) \mid E_{0}\right) \\
& =\operatorname{trace}\left(\pi_{0}\left(g_{0}\right) \mid E_{0}\right) .
\end{aligned}
$$

De plus :

$$
\begin{aligned}
|P \backslash X(1)| & =|P|^{-1}\left|P h(0) Z_{G}(g)\right| \\
& =|P|^{-1}\left|P Z_{G}(g(0)) h(0)\right|=\left|Z_{G}(g(0))\right|\left|P \cap Z_{G}(g(0))\right|^{-1} .
\end{aligned}
$$

On calcule :

$$
\left|Z_{G}(g(0))\right|=4|S O(2 n-1)|, \quad\left|P \cap Z_{G}(g(0))\right|=q^{-1-N_{1}}|P|\left|Z_{G_{0}}\left(g_{0}\right)\right|\left|G_{0}\right|^{-1} .
$$


D'où l'égalité :

(7) $\operatorname{trace}\left(T^{\prime}(1) \pi(g) \mid E\right)=4 q^{1+N_{1}}|P|^{-1}|S O(2 n-1)|\left|G_{0}\right|\left|Z_{G_{0}}\left(g_{0}\right)\right|^{-1} \operatorname{trace}\left(\pi_{0}\left(g_{0}\right) \mid E_{0}\right)$.

Supposons $w=\tau(i)$ avec $i \in\left\{1, \ldots, 1+N_{1}\right\}$. Posons :

$$
m=\left(1, \ldots, 1,4 / a, 1, \ldots, 1, x_{0}^{-1}\right) \in\left(\mathbb{F}_{q}^{\times}\right)^{1+N_{1}} \times G_{0}=M,
$$

où $4 / a$ est à la $i$-ième place. On vérifie que $g(i)=\tau(i) m$. On en déduit que pour $h \in X(w)=P h(i) Z_{G}(g)$, on a les égalités :

$$
\begin{aligned}
\operatorname{trace}\left(A_{w} \circ\left(\zeta_{1} \times 1_{N_{1}} \times \pi_{0}\right)(m(h)) \mid E_{0}\right) & =\operatorname{trace}\left(A_{w} \circ\left(\zeta_{1} \times 1_{N_{1}} \times \pi_{0}\right)(m) \mid E_{0}\right) \\
& = \begin{cases}\zeta(a) \operatorname{dim}\left(E_{0}\right)=\operatorname{sp}(g) \operatorname{dim}\left(E_{0}\right), & \text { si } i=1, \\
\operatorname{dim}\left(E_{0}\right), & \text { si } i \geqslant 2 .\end{cases}
\end{aligned}
$$

Comme précédemment,

$$
\begin{aligned}
|P \backslash X(\tau(i))| & =\left|Z_{G}(g(i))\right|\left|P \cap Z_{G}(g(i))\right|^{-1}, \\
\left|Z_{G}(g(i))\right| & =4|S O(2 n-1)|, \\
\left|P \cap Z_{G}(g(i))\right| & =2 q^{1+i-2 n}|P| .
\end{aligned}
$$

D'où les égalités :

$$
\operatorname{trace}\left(T^{\prime}(\tau(1)) \pi(g) \mid E\right)=2 q^{2 n-2}|P|^{-1}|S O(2 n-1)| \operatorname{sp}(g) \operatorname{dim}\left(E_{0}\right),
$$

et, pour $i \in\left\{2, \ldots, 1+N_{1}\right\}$,

$$
\operatorname{trace}\left(T^{\prime}(\tau(i)) \pi(g) \mid E\right)=2 q^{2 n-i-1}|P|^{-1}|S O(2 n-1)| \operatorname{dim}\left(E_{0}\right) .
$$

Supposons $w=\dot{w}_{2} w_{1}$, avec $w_{1} \in W_{N_{1}}$. On a $\dot{w}_{2}=\tau(1)$ et on voit que $w$ ne peut être de la forme $\tau(i)$ que si $w_{1}=1$. Dans ce cas $i=1$ et $T_{2}\left(\dot{w}_{2}\right) T_{1}\left(w_{1}\right)=\eta q^{1-n} T^{\prime}(\tau(1))$. En appliquant les résultats ci-dessus, on obtient :

$$
\operatorname{trace}\left(T_{2}\left(\dot{w}_{2}\right) T_{1}\left(w_{1}\right) \pi(g) \mid E\right)= \begin{cases}0, & \text { si } w_{1} \neq 1 \\ 2 q^{n-1}|P|^{-1}|S O(2 n-1)| \eta \operatorname{sp}(g) \operatorname{dim}\left(E_{0}\right), & \text { si } w_{1}=1\end{cases}
$$

Un calcul analogue (et plus simple) conduit aux égalités :

$$
\operatorname{trace}\left(\widetilde{T}_{1}\left(w_{1}\right) \mid \widetilde{E}\right)= \begin{cases}0, & \text { si } w_{1} \neq 1, \\ \left|O_{\eta}(2 n-2)\right||\widetilde{P}|^{-1} \operatorname{dim}\left(E_{0}\right), & \text { si } w_{1}=1 .\end{cases}
$$

On vérifie les égalités :

$$
\begin{aligned}
|P| & =q^{2 n-2}|\widetilde{P}| \\
|S O(2 n-1)|\left|O_{\eta}(2 n-2)\right|^{-1} & =q^{n-1}|S O(2 n-1)|_{p^{\prime}}\left|O_{\eta}(2 n-2)\right|_{p^{\prime}}^{-1} .
\end{aligned}
$$

L'égalité (4) résulte de ces calculs. 
10.2. Soit $\Lambda \in \widetilde{\mathcal{S}}_{n-1, \text { pair }}$, posons $\eta=(-1)^{1+\widetilde{\operatorname{def}}(\Lambda) / 2}$. On dispose des trois représentations irréductibles $\pi\left(\Lambda, \mathbf{1}_{-}(1)\right), \pi\left(\Lambda, \sigma \mathbf{1}_{-}(1)\right)$ de $O_{\eta}(2 n)$ et $\pi(\Lambda, \Lambda(\varnothing))$ de $O_{-\eta}(2 n-2)$. Soit $g \in O_{\eta}(2 n)$ un élément semi-simple ayant pour valeurs propres 1 , avec la multiplicité $2 n-1$, et -1 avec la multiplicité 1 .

Lemme. - On suppose que $\Lambda$ n'est pas cuspidal. Sous les hypothèses ci-dessus, on a l'égalité :

$$
\begin{aligned}
& \operatorname{trace}\left(\pi\left(\Lambda, \mathbf{1}_{-}(1)\right)\right)(g)-\operatorname{trace}\left(\pi\left(\Lambda, \sigma \mathbf{1}_{-}(1)\right)\right)(g) \\
& \quad=-2|S O(2 n-1)|_{p^{\prime}}\left|O_{-\eta}(2 n-2)\right|_{p^{\prime}}^{-1} \eta \operatorname{sp}(g) \operatorname{trace}(\pi(\Lambda, \Lambda(\varnothing)))(1) .
\end{aligned}
$$

Démonstration. - Notons $\left(h_{1}, \rho_{1}\right)$ le couple associé à $\Lambda$, posons $\boldsymbol{G}=\boldsymbol{O}_{\eta}(2 n), V=$ $V_{\eta}(2 n), \boldsymbol{G}_{0}=\boldsymbol{O}_{\eta}\left(2 h_{1}^{2}+2\right)$. Notons $\pi_{0}$ la représentation cuspidale $\pi\left(\Lambda_{\text {cusp }}\left(h_{1}\right), \mathbf{1}_{-}(1)\right)$ de $G_{0}, E_{0}$ son espace, posons $N_{1}=n-1-h_{1}^{2}, N_{2}=0$. On effectue la construction de 4.1 pour ces données. On introduit donc un sous-groupe parabolique $\boldsymbol{P}=\boldsymbol{M U}$ de $\boldsymbol{G}$, avec $\boldsymbol{M} \simeq \mathbb{G}_{m}^{N_{1}} \times \boldsymbol{G}_{0}$, on note $(\pi, E)$ la représentation $\operatorname{Ind}_{P}^{G}\left(1_{N_{1}} \times \pi_{0}\right)$. Son algèbre d'entrelacements $\mathcal{H}_{1}$ est engendrée par des opérateurs $T_{1}\left(w_{1}\right)$ pour $w_{1} \in W_{N_{1}}$. Soit $g^{c}$ un élément de $G$ conjugué à $g$ par un élément $h$ de $C O_{\eta}(2 n)$ tel que $\zeta \circ \lambda(h)=-1$. Il a aussi pour valeurs propres 1 avec la multiplicité $2 n-1$ et -1 avec la multiplicité 1. Mais $\operatorname{sp}\left(g^{c}\right)=-\operatorname{sp}(g)$. D'après la proposition $4.4(\mathrm{~b})(\mathrm{v})$, on a l'égalité :

$$
\operatorname{trace}\left(\pi\left(\Lambda, \sigma \mathbf{1}_{-}(1)\right)\right)(g)=\operatorname{trace}\left(\pi\left(\Lambda, \mathbf{1}_{-}(1)\right)\right)\left(g^{c}\right) .
$$

On a l'égalité analogue à 10.1(1) :

$$
\begin{aligned}
& \sum_{\delta_{1} \in \operatorname{Irr}\left(W_{N_{1}}\right)} \operatorname{trace}\left(\delta_{1, \mathcal{H}_{1}}\right)\left(T_{1}\left(w_{1}\right)\right) \\
& \left(\operatorname{trace}\left(\pi\left(\Lambda_{1}\left(\delta_{1}\right), \mathbf{1}_{-}(1)\right)\right)(g)-\operatorname{trace}\left(\pi\left(\Lambda_{1}\left(\delta_{1}\right), \mathbf{1}_{-}(1)\right)\right)\left(g^{c}\right)\right) \\
& =\operatorname{trace}\left(T_{1}\left(w_{1}\right)\left(\pi(g)-\pi\left(g^{c}\right)\right) \mid E\right) .
\end{aligned}
$$

Posons $\widetilde{\boldsymbol{G}}=\boldsymbol{O}_{-\eta}(2 n-2), \widetilde{\boldsymbol{G}}_{0}=\boldsymbol{O}_{-\eta}\left(2 h_{1}^{2}\right)$. Notons $\widetilde{\pi}_{0}$ la représentation cuspidale $\pi\left(\Lambda_{\text {cusp }}\left(h_{1}\right), \Lambda(\varnothing)\right)$ de $\widetilde{G}_{0}, \widetilde{E}_{0}$ son espace. Appliquons la construction de 4.1 à $\widetilde{\boldsymbol{G}}, \widetilde{\pi}_{0}$, $N_{1}=n-1-h_{1}^{2}$ et $N_{2}=0$. On introduit donc un sous groupe parabolique $\widetilde{\boldsymbol{P}}=\widetilde{\boldsymbol{M}} \widetilde{\boldsymbol{U}}$ de $\widetilde{\boldsymbol{G}}$, avec $\widetilde{\boldsymbol{M}} \simeq \mathbb{G}_{m}^{N_{1}} \times \widetilde{\boldsymbol{G}}_{0}$, on note $(\widetilde{\pi}, \widetilde{E})$ la représentation $\operatorname{Ind} \widetilde{\mathcal{G}}_{\widetilde{P}}\left(1_{N_{1}} \times \widetilde{\pi}_{0}\right)$. Son algèbre d'entrelacements $\widetilde{\mathcal{H}}_{1}$ est engendrée par des opérateurs $\widetilde{T}_{1}\left(w_{1}\right)$ pour $w_{1} \in W_{N_{1}}$. En fait :

(3) l'application qui, pour tout $w_{1} \in W_{N_{1}}$, envoie $T_{1}\left(w_{1}\right)$ sur $\widetilde{T}_{1}\left(w_{1}\right)$ est un isomorphisme de $\mathcal{H}_{1}$ sur $\widetilde{\mathcal{H}}_{1}$.

Remarquons que $N_{1}>0$ puisque $\Lambda$ n'est pas cuspidal. Le lemme 4.2 définit un entier $c_{1}$ pour l'algèbre $\mathcal{H}_{1}$ et un entier $\widetilde{c}_{1}$ pour l'algèbre $\widetilde{\mathcal{H}}_{1}$. Il s'agit de prouver l'égalité $c_{1}=\widetilde{c}_{1}$. D'après $[\mathrm{C}]$ proposition $10.5 .5, p^{c_{1}}$ est le rapport des dimensions des deux sous-quotients de la représentation $\operatorname{Ind}_{P^{\prime}}^{G^{\prime}}\left(1_{1} \times \pi_{0}\right)$. Ici $\boldsymbol{G}^{\prime}=\boldsymbol{O}_{\eta}\left(2 h_{1}^{2}+4\right), \boldsymbol{P}^{\prime}=\boldsymbol{M}^{\prime} \boldsymbol{U}^{\prime}$ est un sous-groupe parabolique de $\boldsymbol{G}^{\prime}$ tel que $\boldsymbol{M}^{\prime} \simeq \mathbb{G}_{m} \times \boldsymbol{G}_{0}$. D'après les définitions 
de 4.4 , ces deux sous-quotients sont $\pi\left(A B\left(h_{1}\right), \mathbf{1}_{-}(1)\right)$ et $\pi\left(B A\left(h_{1}\right), \mathbf{1}_{-}(1)\right)$, cf. 9.4 pour les notations. D'après le corollaire 9.9 , on a donc :

$$
p^{c_{1}}=\frac{\widetilde{D}_{A B\left(h_{1}\right)}(q)}{\widetilde{D}_{B A\left(h_{1}\right)}(q)} .
$$

On calcule $p^{\widetilde{c}_{1}}$ de façon analogue. Les deux sous-quotients sont maintenant $\pi\left(A B\left(h_{1}\right), \Lambda(\varnothing)\right)$ et $\pi\left(B A\left(h_{1}\right), \Lambda(\varnothing)\right)$. Le rapport de leurs dimensions est égal au rapport ci-dessus. D'où $c_{1}=\widetilde{c}_{1}$ et l'assertion (3).

Identifions $\widetilde{\mathcal{H}}_{1}$ à $\mathcal{H}_{1}$. Pour tout $w_{1} \in W_{N_{1}}$, on a l'égalité 10.1(3). Posons :

$$
c=-2|S O(2 n-1)|_{p^{\prime}}\left|O_{-\eta}(2 n-2)\right|_{p^{\prime}}^{-1} \eta \operatorname{sp}(g) .
$$

On va prouver l'égalité :

$$
\operatorname{trace}\left(T_{1}\left(w_{1}\right)\left(\pi(g)-\pi\left(g^{c}\right)\right) \mid E\right)=c \operatorname{trace}\left(\widetilde{T}_{1}\left(w_{1}\right) \mid \widetilde{E}\right) .
$$

Alors, comme dans la preuve du lemme précédent, l'égalité de l'énoncé résulte de la comparaison des égalités (2) ci-dessus et de 10.1(3), en tenant compte cette fois de (1) ci-dessus.

Il reste à démontrer (4). On reprend les notations de la preuve du lemme précédent, en les adaptant de façon évidente : le caractère $\zeta_{1}$ disparaît, les termes $\tau(i)$ sont définis pour $i \in\left\{0, \ldots, N_{1}\right\}$. Soit $w_{1} \in W_{N_{1}}$. Si $w_{1}$ n'est pas l'un des $\tau(i)$, on a :

$$
\operatorname{trace}\left(T_{1}^{\prime}\left(w_{1}\right) \pi(g) \mid E\right)=\operatorname{trace}\left(T_{1}^{\prime}\left(w_{1}\right) \pi\left(g^{c}\right) \mid E\right)=0 .
$$

Si $w_{1}=\tau(i)$, pour $i \in\left\{1, \ldots, N_{1}\right\}$, on a :

$$
\operatorname{trace}\left(T_{1}^{\prime}(\tau(i)) \pi(g) \mid E\right)=\operatorname{trace}\left(T_{1}^{\prime}(\tau(i)) \pi\left(g^{c}\right) \mid E\right),
$$

cf. 10.1(9). Si $w_{1}=\tau(0)=1$, les termes $\operatorname{trace}\left(T^{\prime}(1) \pi(g) \mid E\right)$ et $\operatorname{trace}\left(T^{\prime}(1) \pi\left(g^{c}\right) \mid E\right)$ sont calculés par la formule 10.1(7), où $q^{1+N_{1}}$ est remplacé par $q^{N_{1}}$. On obtient :

$\operatorname{trace}\left(T_{1}\left(w_{1}\right)\left(\pi(g)-\pi\left(g^{c}\right)\right) \mid E\right)=\left\{\begin{aligned} 0, & \text { si } w_{1} \neq 1 \\ 4 q^{N_{1}}|P|^{-1}|S O(2 n-1)|\left|G_{0}\right|\left|Z_{G_{0}}\left(g_{0}\right)\right|^{-1} & \\ \operatorname{trace}\left(\left(\pi_{0}\left(g_{0}\right)-\pi_{0}\left(g_{0}^{c}\right)\right) \mid E_{0}\right), & \text { si } w_{1}=1 .\end{aligned}\right.$

On a l'égalité analogue à 10.1(10) :

$$
\operatorname{trace}\left(\widetilde{T}_{1}\left(w_{1}\right) \mid \widetilde{E}\right)= \begin{cases}0, & \text { si } w_{1} \neq 1 \\ \left|O_{-\eta}(2 n-2)\right||\widetilde{P}|^{-1} \operatorname{dim}\left(\widetilde{E}_{0}\right), & \text { si } w_{1}=1\end{cases}
$$

On calcule toutes les constantes intervenant dans les formules ci-dessus. En posant :

$$
c^{\prime}=-2\left|S O\left(2 h_{1}^{2}+1\right)\right|_{p^{\prime}}\left|O_{-\eta}\left(2 h_{1}^{2}\right)\right|_{p^{\prime}}^{-1} \eta \operatorname{sp}\left(g_{0}\right),
$$

on obtient alors l'égalité :

$$
\begin{aligned}
\operatorname{trace}\left(T_{1}\left(w_{1}\right)(\pi(g)\right. & \left.\left.-\pi\left(g^{c}\right)\right) \mid E\right) \\
= & c c^{-1} \operatorname{trace}\left(\left(\pi_{0}\left(g_{0}\right)-\pi_{0}\left(g_{0}^{c}\right)\right) \mid E_{0}\right) \operatorname{dim}\left(\widetilde{E}_{0}\right)^{-1} \operatorname{trace}\left(\widetilde{T}_{1}\left(w_{1}\right) \mid \widetilde{E}\right)
\end{aligned}
$$


pour tout $w_{1} \in W_{N_{1}}$. L'égalité (4) résulte de l'égalité suivante :

$$
\operatorname{trace}\left(\left(\pi_{0}\left(g_{0}\right)-\pi_{0}\left(g_{0}^{c}\right)\right) \mid E_{0}\right)=c^{\prime} \operatorname{dim}\left(\widetilde{E}_{0}\right),
$$

qu'il reste à démontrer. D'après une égalité analogue à (1), le membre de gauche n'est autre que :

$$
\operatorname{trace}\left(\pi\left(\Lambda_{\text {cusp }}\left(h_{1}\right), \mathbf{1}_{-}(1)\right)\right)(g)-\operatorname{trace}\left(\pi\left(\Lambda_{\text {cusp }}\left(h_{1}\right), \sigma \mathbf{1}_{-}(1)\right)\right)(g) .
$$

Le membre de droite n'est autre que $c^{\prime} \operatorname{trace}\left(\pi\left(\Lambda_{\text {cusp }}\left(h_{1}\right), \Lambda(\varnothing)\right)\right)(1)$. On a déjà dit que, puisque $\Lambda$ n'est pas cuspidal, on a $N_{1} \geqslant 1$. Il n'intervient ci-dessus que des représentations de groupes $O_{ \pm}\left(2 n^{\prime}\right)$, avec $n^{\prime}<n$, auxquelles on peut appliquer l'hypothèse de récurrence. Le membre de gauche de (5) est égal à :

$$
\pi_{h_{1}^{2}+1}\left(\Lambda_{\text {cusp }}\left(h_{1}\right) \otimes\left(\mathbf{1}_{-}(1)-\sigma \mathbf{1}_{-}(1)\right)\right)(g) .
$$

Grâce à l'hypothèse de récurrence et à l'égalité $\mathcal{F}\left(\mathbf{1}_{-}(1)-\sigma \mathbf{1}_{-}(1)\right)=\mathbf{1}_{-}(1)-\sigma \mathbf{1}_{-}(1)$, il est aussi égal à :

$$
k_{h_{1}^{2}+1}\left(\mathcal{F} \Lambda_{\text {cusp }}\left(h_{1}\right) \otimes\left(\mathbf{1}_{-}(1)-\sigma \mathbf{1}_{-}(1)\right)\right)(g) .
$$

De même, le membre de droite de (5) est égal à :

$$
c^{\prime} k_{h_{1}^{2}}\left(\mathcal{F} \Lambda_{\text {cusp }}\left(h_{1}\right) \otimes \Lambda(\varnothing)\right)\left(1_{-\eta}\right),
$$

où on désigne par $1_{-\eta}$ l'élément neutre de $O_{-\eta}(2 n-2)$. Alors l'égalité (5) résulte du lemme 7.3 appliqué en rang $h_{1}^{2}+1$. Cela achève la démonstration.

10.3. On suppose ici $n=4$. On a fixé le corps de base $\mathbb{F}_{q}$, mais on peut le faire varier et considérer $q$ comme une variable soumise aux conditions : $q$ est une puissance d'un nombre premier impair et $q>2 n=8$. Dans l'énoncé ci-dessous, il est implicite que les polynômes intervenant sont indépendants de $q$. On définit les symboles ordonnés $\Lambda(i)$ pour $i \in\{1, \ldots, 7\}$ par :

$$
\begin{gathered}
\Lambda(1)^{+}=\{3,1\}, \Lambda(1)^{-}=\{2,0\} ; \Lambda(2)^{+}=\{3,2\}, \Lambda(2)^{-}=\{1,0\} ; \Lambda(3)^{+}=\{3,0\}, \\
\Lambda(3)^{-}=\{2,1\} ; \Lambda(4)^{+}=\{3,2,1\}, \Lambda(4)^{-}=\{0\} ; \Lambda(5)^{+}=\{3,2,0\}, \Lambda(5)^{-}=\{1\} ; \\
\Lambda(6)^{+}=\{3,1,0\}, \Lambda(6)^{-}=\{2\} ; \Lambda(7)^{+}=\{2,1,0\}, \Lambda(7)^{-}=\{3\} .
\end{gathered}
$$

LEMme. - Pour tout $\Lambda \in \widetilde{\mathcal{S}}_{4 \text {,pair }}$ tel que $\widetilde{\operatorname{def}}(\Lambda) \in\{0,2\}$, il existe un polynôme $P_{\Lambda} \in \mathbb{Q}[T]$ de sorte que :

(i) $P_{\Lambda(1)}(1)=P_{\sigma \Lambda(1)}(1)=P_{\Lambda(4)}(1)=1$;

$$
\begin{aligned}
& P_{\Lambda(2)}(1)=P_{\sigma \Lambda(2)}(1)=P_{\Lambda(7)}(1)=-1 ; \\
& P_{\Lambda(3)}(1)=P_{\sigma \Lambda(3)}(1)=P_{\Lambda(5)}(1)=P_{\Lambda(6)}(1)=0 ;
\end{aligned}
$$

(ii) en définissant, pour $d \in\{0,2\}$, l'élément $P_{d} \in \mathcal{C}(O(8))$ par :

$$
P_{d}=\sum_{\Lambda \in \widetilde{\mathcal{S}}_{4, d}} P_{\Lambda}(q) \operatorname{trace}(\pi(\Lambda, \Lambda(\varnothing))),
$$

on a les égalités :

$$
\left(k\left(\Lambda_{\text {cusp }}(2), \Lambda(\varnothing)\right), P_{0}\right)=q^{2}, \quad\left(k\left(\Lambda_{\text {cusp }}(2), \Lambda(\varnothing)\right), P_{2}\right)=-\frac{1}{2} q^{2} .
$$


Démonstration. - Commençons par traiter le cas des symboles de défaut nul. On considère la situation de 4.1 pour $n=4, \eta=1, \boldsymbol{G}=\boldsymbol{O}_{+}(8), N_{1}=4, N_{2}=0$. Autrement dit, on considère la série principale de $G$ issue de la représentation triviale d'un sous-groupe de Borel. On note $(\pi, E)$ cette série principale. Son algèbre d'entrelacements $\mathcal{H}$ est une algèbre de Hecke de type $W_{4}$. À tout symbole ordonné $\Lambda \in \widetilde{\mathcal{S}}_{4,0}$, associons la représentation irréductible $\rho_{\Lambda}$ de $W_{4}$ telle que $\Lambda$ corresponde au couple $\left(0, \rho_{\Lambda}\right)$, cf. 2.2 , et la représentation irréductible $\rho_{\Lambda, \mathcal{H}}$ de $\mathcal{H}$ correspondant à $\rho_{\Lambda}$. Ces correspondances sont bijectives. Considérons l'élément $w \in W_{4}$ défini par :

$$
w(1)=2, w(2)=3, w(3)=-1, w(4)=-4 .
$$

Il est connu que, pour tout $\Lambda \in \widetilde{\mathcal{S}}_{4,0}$, il existe un polynôme $P_{\Lambda} \in \mathbb{Q}[T]$, indépendant de $q$, tel que :

$$
\operatorname{trace}\left(\rho_{\Lambda}\right)(w)=P_{\Lambda}(1), \operatorname{trace}\left(\rho_{\Lambda, \mathcal{H}}\right)\left(T_{1}(w)\right)=P_{\Lambda}(q) .
$$

On va montrer que ce polynôme convient. Le calcul des valeurs $P_{\Lambda(i)}(1)$ et $P_{\sigma \Lambda(i)}(1)$ pour $i \in\{1,2,3\}$ se ramène au calcul de $\operatorname{trace}\left(\rho_{\Lambda(i)}\right)(w), \operatorname{trace}\left(\rho_{\sigma \Lambda(i)}(w)\right.$, qui est élémentaire. Pour tout $g \in G$, on a l'égalité :

(1) $\quad P_{0}(g)=\sum_{\Lambda \in \widetilde{\mathcal{S}}_{4,0}} \operatorname{trace}\left(\rho_{\Lambda, \mathcal{H}}\right)\left(T_{1}(w)\right) \operatorname{trace}\left(\pi(\Lambda, \Lambda(\varnothing))(g)=\operatorname{trace}\left(T_{1}(w) \pi(g) \mid E\right)\right.$.

Remarquons que la fonction $C_{1}$ du lemme 4.2 est constante égale à 1 . On a donc $T_{1}(w)=T_{1}^{\prime}(w)$. Pour calculer le produit scalaire de $P_{d}$ et $k\left(\Lambda_{\text {cusp }}(2), \Lambda(\varnothing)\right)$, on se limite à calculer $P_{d}(g)$ pour $g$ dans le support de cette dernière fonction. On suppose donc que $g$ se décompose en produit $s u$, où $s$ est semi-simple, $u$ unipotent, $s$ et $u$ commutent; $s$ a pour valeurs propres 1 et -1 , toutes deux de multiplicité 4 ; $u$ se décompose selon ces espaces propres en $u=u^{\prime} u^{\prime \prime}$, cf. 3.1 ; l'élément $u^{\prime}$, resp. $u^{\prime \prime}$, est paramétré par la partition $(3,1)$ et des données $\eta_{u^{\prime}}(3), \eta_{u^{\prime}}(1)$, resp. $\eta_{u^{\prime \prime}}(3), \eta_{u^{\prime \prime}}(1)$. Comme dans les paragraphes 10.1 et 10.2 , on calcule :

$$
\operatorname{trace}\left(T_{1}(w) \pi(g) \mid E\right)=\left|\left\{h \in P \backslash G ; h g h^{-1} \in U w P\right\}\right| .
$$

Posons $(V, Q)=\left(V_{+}(8), Q_{+}(8)\right)$, identifions $P \backslash G$ à l'ensemble des drapeaux isotropes :

$$
\{0\} \subset X_{1} \subset X_{2} \subset X_{3} \subset X_{4}
$$

de $V$ : à $h \in P \backslash G$ on associe $X_{1}=h^{-1}\left(\mathbb{F}_{q} v_{1}\right), X_{2}=h^{-1}\left(\mathbb{F}_{q} v_{1} \oplus \mathbb{F}_{q} v_{2}\right)$, etc. La condition $h g h^{-1} \in U w P$ se traduit par :

$X_{2}=X_{1}+g X_{1}, X_{3}=X_{2}+g X_{2}$, et $g X_{3}$ n'est pas orthogonal à $X_{1}$.

Considérons l'ensemble $\mathcal{D}$ des droites isotropes $D \subset V$ telles que $g D$ et $g^{2} D$ sont orthogonales à $D$ tandis que $g^{3} D$ ne l'est pas. L'application qui, au drapeau $\left(X_{i}\right)_{i=1, \ldots, 4}$, associe $D=X_{1}$ est une surjection de l'ensemble des drapeaux vérifiant les conditions ci-dessus sur $\mathcal{D}$; ses fibres ont toutes 2 éléments : on reconstruit $X_{2}$ et $X_{3}$ à partir de $D$ et il reste deux choix possibles pour $X_{4}$. Donc :

$$
\operatorname{trace}\left(T_{1}(w) \pi(g) \mid E\right)=2|\mathcal{D}| .
$$


Notons $\mathcal{V}$ l'ensemble des $v \in V$ tels que $Q(v, v)=Q(v, g v)=Q\left(v, g^{2} v\right)=0$, $Q\left(v, g^{3} v\right) \neq 0$. Décomposons $V$ en somme orthogonale :

$$
V=V_{1}^{\prime} \oplus V_{3}^{\prime} \oplus V_{1}^{\prime \prime} \oplus V_{3}^{\prime \prime}
$$

stable par $g$, de sorte que $V_{1}^{\prime}$ et $V_{1}^{\prime \prime}$ soient de dimension $1, V_{3}^{\prime}$ et $V_{3}^{\prime \prime}$ soient de dimension $3, s$ agisse trivialement dans $V_{1}^{\prime}$ et $V_{3}^{\prime}$ et par multiplication par -1 dans $V_{1}^{\prime \prime}$ et $V_{3}^{\prime \prime}$. On fixe des bases $\left\{e^{\prime}\right\}$ de $V^{\prime},\left\{f_{1}^{\prime}, f_{0}^{\prime}, f_{-1}^{\prime}\right\}$ de $V_{3}^{\prime}$, telles que $Q\left(f_{1}^{\prime}, f_{-1}^{\prime}\right)=-2, Q\left(f_{1}^{\prime}, f_{0}^{\prime}\right)=$ $Q\left(f_{0}^{\prime}, f_{-1}^{\prime}\right)=0$. On pose $a^{\prime}=Q\left(e^{\prime}, e^{\prime}\right), b^{\prime}=Q\left(f_{0}^{\prime}, f_{0}^{\prime}\right)$. On suppose que la matrice de $u$ agissant dans $V_{3}^{\prime}$ est :

$$
\left(\begin{array}{lll}
1 & b^{\prime} & b^{\prime} \\
0 & 1 & 2 \\
0 & 0 & 1
\end{array}\right)
$$

On choisit de même des bases $\left\{e^{\prime \prime}\right\}$ de $V_{1}^{\prime \prime},\left\{f_{1}^{\prime \prime}, f_{0}^{\prime \prime}, f_{-1}^{\prime \prime}\right\}$ de $V_{3}^{\prime \prime}$ et on définit $a^{\prime \prime}, b^{\prime \prime}$. Soit $v \in V$, écrivons :

$$
v=x^{\prime} e^{\prime}+y^{\prime} f_{1}^{\prime}+z^{\prime} f_{0}^{\prime}+t^{\prime} f_{-1}^{\prime}+x^{\prime \prime} e^{\prime \prime}+y^{\prime \prime} f_{1}^{\prime \prime}+z^{\prime \prime} f_{0}^{\prime \prime}+t^{\prime \prime} f_{-1}^{\prime \prime} .
$$

On a les égalités :

$$
\begin{gathered}
Q(v, v)=-4 y^{\prime} t^{\prime}-4 y^{\prime \prime} t^{\prime \prime}+a^{\prime} x^{2}+b^{\prime} z^{2}+a^{\prime \prime} x^{\prime \prime 2}+b^{\prime \prime} z^{\prime \prime 2} \\
Q(v, g v)=-4 y^{\prime} t^{\prime}+4 y^{\prime \prime} t^{\prime \prime}+a^{\prime} x^{\prime 2}+b^{\prime} z^{\prime 2}-2 b^{\prime} t^{\prime 2}-a^{\prime \prime} x^{\prime \prime 2}-b^{\prime \prime} z^{\prime \prime 2}+2 b^{\prime \prime} t^{\prime \prime 2}, \\
Q\left(v, g^{2} v\right)-Q(v, v)=-8 b^{\prime} t^{\prime 2}-8 b^{\prime \prime} t^{\prime \prime 2} \\
Q\left(v, g^{3} v\right)-Q(v, g v)=-18 b^{\prime} t^{\prime 2}+18 b^{\prime \prime} t^{\prime \prime 2}
\end{gathered}
$$

Le vecteur $v$ appartient à $\mathcal{V}$ si et seulement si les trois premières expressions sont nulles et la dernière ne l'est pas. Les solutions en $t^{\prime}, t^{\prime \prime}$ des deux dernières conditions sont faciles à calculer : il n'y en a pas si $-b^{\prime} b^{\prime \prime}$ n'est pas un carré, il y en a $2(q-1)$ si $-b^{\prime} b^{\prime \prime}$ est un carré; dans ce cas, toutes les solutions vérifient $t^{\prime} t^{\prime \prime} \neq 0$. Mais pour de tels $t^{\prime}, t^{\prime \prime}$ et pour tout choix de $x^{\prime}, z^{\prime}, x^{\prime \prime}, z^{\prime \prime}$, les deux premières équations ci-dessus forment un système linéaire inversible en $y^{\prime}, y^{\prime \prime}$, qui a donc une unique solution. On obtient :

$$
|\mathcal{V}|= \begin{cases}2 q^{4}(q-1), & \text { si } \zeta\left(-b^{\prime} b^{\prime \prime}\right)=1, \\ 0, & \text { si } \zeta\left(-b^{\prime} b^{\prime \prime}\right)=-1 .\end{cases}
$$

Remarquons que $\zeta\left(b^{\prime}\right)=\eta_{u^{\prime}}(3), \zeta\left(b^{\prime \prime}\right)=\eta_{u^{\prime \prime}}(3)$. De plus $|\mathcal{D}|=(q-1)^{-1}|\mathcal{V}|$. En rassemblant (1), (2) et les égalités ci-dessus, on obtient :

$$
P_{0}(g)= \begin{cases}4 q^{4}, & \text { si } \eta_{u^{\prime}}(3) \eta_{u^{\prime \prime}}(3)=\zeta(-1) \\ 0, & \text { sinon. }\end{cases}
$$

D'autre part, la formule de 3.1 s'explicite :

$$
k\left(\Lambda_{\text {cusp }}(2), \Lambda(\varnothing)\right)(g)=q^{2} \eta_{u^{\prime}}(1) \eta_{u^{\prime \prime}}(1) \zeta(-1) .
$$


Le stabilisateur de $g$ dans $G$ a $2^{4} q^{4}$ éléments, son orbite a $|G| 2^{-4} q^{-4}$ éléments. Les différentes orbites dans le support de $k\left(\Lambda_{\text {cusp }}(2), \Lambda(\varnothing)\right)$ correspondent aux différents choix de $\eta_{u^{\prime}}(1), \eta_{u^{\prime}}(3), \eta_{u^{\prime \prime}}(1), \eta_{u^{\prime \prime}}(3)$, soumis à la condition

$$
\eta_{u^{\prime}}(1) \eta_{u^{\prime}}(3) \eta_{u^{\prime \prime}}(1) \eta_{u^{\prime \prime}}(3)=1 .
$$

On obtient :

$$
\left(k\left(\Lambda_{\text {cusp }}(2), \Lambda(\varnothing)\right), P_{0}\right)=\frac{1}{4} q^{2} \zeta(-1) \sum \eta_{u^{\prime}}(1) \eta_{u^{\prime \prime}}(1),
$$

où l'on somme sur $\eta_{u^{\prime}}(1), \eta_{u^{\prime}}(3), \eta_{u^{\prime \prime}}(1), \eta_{u^{\prime \prime}}(3)$ soumis à la condition de produit cidessus et à la condition $\eta_{u^{\prime}}(3) \eta_{u^{\prime \prime}}(3)=\zeta(-1)$. On en déduit l'égalité de l'énoncé.

Traitons maintenant le cas des symboles de défaut 2 . On considère la situation de 4.1 pour $n=4, \eta=-1, \boldsymbol{G}=\boldsymbol{O}_{-}(8), N_{1}=3, N_{2}=0$ et $\pi_{0}=\pi\left(\Lambda_{\text {cusp }}(1), \Lambda(\varnothing)\right)$. D'après $6.5, \pi_{0}$ est la représentation triviale de $O_{-}(2)$. Autrement dit, on considère la série principale de $G$ issue de la représentation triviale d'un sous-groupe de Borel. On note $(\pi, E)$ cette série principale, $\mathcal{H}$ son algèbre d'entrelacements, qui est une algèbre de Hecke de type $W_{3}$. À tout symbole ordonné $\Lambda \in \widetilde{\mathcal{S}}_{4,2}$, on associe la représentation irréductible $\rho_{\Lambda}$ de $W_{3}$ telle que $\Lambda$ corresponde au couple $\left(1, \rho_{\Lambda}\right)$, et la représentation irréductible $\rho_{\Lambda, \mathcal{H}}$ de $\mathcal{H}$ correspondant à $\rho_{\Lambda}$. On considère l'élément $w \in W_{3}$ défini par :

$$
w(1)=2, w(2)=3, w(3)=-1 .
$$

Pour tout $\Lambda \in \widetilde{\mathcal{S}}_{4,2}$ il existe un polynôme $P_{\Lambda} \in \mathbb{Q}[T]$, indépendant de $q$, tel que :

$$
\operatorname{trace}\left(\rho_{\Lambda}\right)(w)=P_{\Lambda}(1), \operatorname{trace}\left(\rho_{\Lambda, \mathcal{H}}\right)\left(T_{1}(w)\right)=P_{\Lambda}(q) .
$$

On montre comme précédemment que ce polynôme convient.

10.4. On suppose ici $n=2$.

Lemme. - Soient $\varepsilon_{1}, \varepsilon_{2} \in\{ \pm 1\}$, avec $\left(\varepsilon_{1}, \varepsilon_{2}\right) \neq(-1,-1)$. On a l'égalité :

$$
\left(k\left(\mathbf{1}_{-}(1), \mathbf{1}_{-}(1)\right), \operatorname{trace}\left(\pi\left(\mathbf{1}_{\varepsilon_{1}}(1), \mathbf{1}_{\varepsilon_{2}}(1)\right)\right)\right)=\frac{1}{4} .
$$

Démonstration. - On considère la situation de 4.1 pour $n=2, \eta=1, \boldsymbol{G}=\boldsymbol{O}_{+}(4)$, $N_{1}=N_{2}=1$, mais en inversant les rôles des indices 1 et 2 . Autrement dit, on considère la série principale de $G$ issue de la représentation $\zeta_{1} \times 1_{1}$ d'un sous-tore maximal $M$ d'un sous-groupe de Borel $P$. Notons $(\pi, E)$ cette représentation. Son algèbre d'entrelacements est isomorphe à $\mathbb{C}\left[W_{1}\right] \otimes_{\mathbb{C}} \mathbb{C}\left[W_{1}\right]$. Les sous-quotients irréductibles de $\pi$ sont $\pi_{1}=\pi\left(\mathbf{1}_{+}(1), \mathbf{1}_{+}(1)\right), \pi_{2}=\pi\left(\sigma \mathbf{1}_{+}(1), \mathbf{1}_{+}(1)\right), \pi_{3}=\pi\left(\mathbf{1}_{+}(1), \sigma \mathbf{1}_{+}(1)\right)$, $\pi_{4}=\pi\left(\sigma \mathbf{1}_{+}(1), \sigma \mathbf{1}_{+}(1)\right)$. Soit $w$ l'élément non trivial de $W_{1}$. Notons $\varphi$ la fonction $\operatorname{sur} G$ :

$$
\varphi(g)=\operatorname{trace}\left(T_{1}(w) T_{2}(w) \pi(g) \mid E\right)
$$

pour tout $g \in G$. On a l'égalité :

$$
\varphi=\operatorname{trace}\left(\pi_{1}\right)-\operatorname{trace}\left(\pi_{2}\right)-\operatorname{trace}\left(\pi_{3}\right)+\operatorname{trace}\left(\pi_{4}\right) .
$$


Grâce aux propriétés de transformation par torsion par det et par l'automorphisme $f \mapsto f^{c}, c f$. propositions 3.5 et 4.4 , on vérifie que :

$$
\begin{aligned}
\left(k\left(\mathbf{1}_{-}(1), \mathbf{1}_{-}(1)\right), \operatorname{trace}\left(\pi_{1}\right)\right) & =\left(k\left(\mathbf{1}_{-}(1), \mathbf{1}_{-}(1)\right), \operatorname{trace}\left(\pi_{4}\right)\right) \\
& =-\left(k\left(\mathbf{1}_{-}(1), \mathbf{1}_{-}(1)\right), \operatorname{trace}\left(\pi_{2}\right)\right) \\
& =-\left(k\left(\mathbf{1}_{-}(1), \mathbf{1}_{-}(1)\right), \operatorname{trace}\left(\pi_{3}\right)\right) .
\end{aligned}
$$

Donc :

$$
\left(k\left(\mathbf{1}_{-}(1), \mathbf{1}_{-}(1)\right), \operatorname{trace}\left(\pi_{1}\right)\right)=\frac{1}{4}\left(k\left(\mathbf{1}_{-}(1), \mathbf{1}_{-}(1)\right), \varphi\right) .
$$

Comme en 10.3, on doit calculer $\varphi$ sur les éléments du support de $k\left(\mathbf{1}_{-}(1), \mathbf{1}_{-}(1)\right)$. Un tel élément $u$ est unipotent, paramétré par la partition $(3,1)$ et des données $\eta_{u}(3), \eta_{u}(1)$ vérifiant $\eta_{u}(3) \eta_{u}(1)=\zeta(-1)$. Remarquons que $T_{1}(w)=T_{1}^{\prime}(w)$, tandis que $T_{2}(w)=q^{-1} T_{2}^{\prime}(w)$, cf. 4.2. Comme en 10.1, notons $X(w)$ l'ensemble des $h \in G$ tels que $h u h^{-1} \in U w P$. Pour $h \in X(w)$, notons $m(h)$ l'unique élément de $M$ tel que $h u h^{-1} \in U w m(h) U$. Alors :

$$
\varphi(u)=q^{-1} \sum_{h \in P \backslash X(w)}\left(\zeta_{1} \times 1_{1}\right)(m(h)) .
$$

Posons $(V, Q)=\left(V_{+}(4), Q_{+}(4)\right)$ et notons $\mathcal{D}$ l'ensemble des droites isotropes $D \subset V$ telles que $u D$ ne soit pas orthogonale à $D$. Pour $D \in \mathcal{D}$, on pose $\zeta(D)=\zeta(-2 Q(v, u v))$, où $v$ est un élément non nul de $D$. Ce terme ne dépend pas du choix de $v$. L'application qui à $h \in X(w)$ associe $D=h^{-1}\left(\mathbb{F}_{q} v_{1}\right)$ se quotiente en une surjection de $P \backslash X(w)$ sur $\mathcal{D}$, dont les fibres ont 2 éléments. Pour $h, D$ ainsi reliées, on a l'égalité $\left(\zeta_{1} \times 1_{1}\right)(m(h))=\zeta(D)$. On obtient :

$$
\varphi(u)=2 q^{-1} \sum_{D \in \mathcal{D}} \zeta(D) .
$$

Le calcul de cette somme est élémentaire. On vérifie que $|\mathcal{D}|=q^{2}$ et que $\zeta(D)=\eta_{u}(3)$ pour tout $D \in \mathcal{D}$. D'où $\varphi(u)=2 q \eta_{u}(3)$. D'autre part, grâce à 3.1 , on a :

$$
k\left(\mathbf{1}_{-}(1), \mathbf{1}_{-}(1)\right)(u)=q \zeta(-1) \eta_{u}(1) .
$$

L'orbite de $u$ a $|G| 2^{-2} q^{-2}$ éléments. D'où l'égalité :

$$
\left(k\left(\mathbf{1}_{-}(1), \mathbf{1}_{-}(1)\right), \varphi\right)=\frac{1}{2} \zeta(-1) \sum \eta_{u}(1) \eta_{u}(3),
$$

où l'on somme sur les $\eta_{u}(1), \eta_{u}(3)$ vérifiant l'égalité $\eta_{u}(1) \eta_{u}(3)=\zeta(-1)$. Le terme de droite ci-dessus vaut 1 . Grâce à $(1)$, on en déduit l'énoncé pour la représentation $\pi\left(\mathbf{1}_{+}(1), \mathbf{1}_{+}(1)\right)$.

Une démonstration similaire s'applique aux deux autres représentations, en considérant des représentations principales du groupe $O_{-}(4)$. Deux signes s'introduisent dans le calcul, qui se compensent : dans la dernière somme ci-dessus, la condition est maintenant $\eta_{u}(1) \eta_{u}(3)=-\zeta(-1)$; d'après la définition de 4.2 , il y a un signe - dans les opérateurs d'entrelacements normalisés. 
10.5. Identifions $V_{+}(4)$ muni de sa forme $Q_{+}(4)$ à l'espace de matrices $g l(2)$ muni de la forme déterminant. Notons $\{1, \tau\}$ le groupe à deux éléments, considérons le groupe :

$$
\boldsymbol{R}=\left\{\left(g_{1}, g_{2}\right) \in \boldsymbol{G L}(2) \times \boldsymbol{G} \boldsymbol{L}(2) ; \operatorname{det}\left(g_{1} g_{2}\right)=1\right\} \times\{1, \tau\},
$$

où $\tau$ agit sur le premier facteur par $\tau\left(g_{1}, g_{2}\right)=\left(g_{2}, g_{1}\right) \tau$. On fait agir $R$ sur $g l(2)$ par :

$$
\left(g_{1}, g_{2}, 1\right) m=g_{1} m^{t} g_{2},(1,1, \tau) m={ }^{t} m,
$$

pour tous $\left(g_{1}, g_{2}\right) \in G L(2) \times G L(2)$ et $m \in g l(2)$. On obtient ainsi une surjection $\varphi: R \rightarrow O_{+}(4)$, de noyau :

$$
Z=\left\{\left(\left(\begin{array}{cc}
z & 0 \\
0 & z
\end{array}\right),\left(\begin{array}{cc}
z^{-1} & 0 \\
0 & z^{-1}
\end{array}\right), 1\right) ; z \in \mathbb{F}_{q}^{\times}\right\}
$$

On vérifie les propriétés suivantes :

- pour tout $a \in \mathbb{F}_{q}^{\times}$, l'élément $g_{a}=\varphi\left(\left(\begin{array}{ll}a & 0 \\ 0 & 1\end{array}\right),\left(\begin{array}{cc}a^{-1} & 0 \\ 0 & 1\end{array}\right), \tau\right)$ a pour valeurs propres 1 avec la multiplicité 3 et -1 avec la multiplicité 1 ; on a $\operatorname{sp}\left(g_{a}\right)=\zeta(a)$;

- pour tous $b, c \in \mathbb{F}_{q}^{\times}$, l'élément $u_{b, c}=\varphi\left(\left(\begin{array}{ll}1 & b \\ 0 & 1\end{array}\right),\left(\begin{array}{ll}1 & c \\ 0 & 1\end{array}\right), 1\right)$ est unipotent, classifié par la partition $(3,1)$ et les données $\eta_{u_{b, c}}(3)=\zeta(-b c), \eta_{u_{b, c}}(1)=\zeta(b c)$.

LEMME. - Le groupe $O_{+}(4)$ possède 4 représentations cuspidales quadratiquesunipotentes. Leurs caractères prennent leurs valeurs dans $\mathbb{Z}$. On peut choisir une unique indexation :

$$
\begin{aligned}
\{ \pm 1\} \times\{ \pm 1\} & \longrightarrow \operatorname{Quad}_{\text {cusp }}\left(O_{+}(4)\right) \\
\left(h_{1}, h_{2}\right) & \longmapsto \pi^{\prime}\left(h_{1}, h_{2}\right)
\end{aligned}
$$

de sorte que pour tous $a, b, c \in \mathbb{F}_{q}^{\times}$et $h_{1}, h_{2} \in\{ \pm 1\}$, on ait les égalités :

$$
\begin{aligned}
\operatorname{trace}\left(\pi^{\prime}\left(h_{1}, h_{2}\right)\right)\left(g_{a}\right) & = \begin{cases}0, & \text { si } \zeta(a) \neq h_{1} h_{2}, \\
h_{1}(1-q), & \text { si } \zeta(a)=h_{1} h_{2},\end{cases} \\
\operatorname{trace}\left(\pi^{\prime}\left(h_{1}, h_{2}\right)\right)\left(u_{b, c}\right) & =\frac{1}{2}+\frac{1}{2} q h_{1} h_{2} \zeta(-b c) .
\end{aligned}
$$

Démonstration. - La première assertion est un rappel de la proposition 4.3. Le groupe $R$ possède un sous-groupe évident $S L(2) \times S L(2)$. Le sous-groupe $R^{\prime}=$ $Z(S L(2) \times S L(2))$ de $R$ est distingué et le quotient $R / R^{\prime}$ est abélien. Les représentations irréductibles de $R$ se construisent par la théorie de Clifford à partir de celles de $R^{\prime}$. On doit considérer des représentations de $R^{\prime}$ triviales sur $Z$. Elles s'identifient à des représentations $\pi_{1} \times \pi_{2}$, où $\pi_{1}$ et $\pi_{2}$ sont des représentations irréductibles de $S L(2)$. Pour obtenir des représentations cuspidales de $R, \pi_{1}$ et $\pi_{2}$ doivent être cuspidales. Pour obtenir des représentations quadratiques-unipotentes de $R, \pi_{1}$ et $\pi_{2}$ doivent être quadratiques-unipotentes. On connaît bien les représentations de $S L(2)$ et on sait qu'il y en a exactement 2 vérifiant ces propriétés. Notons-les $\pi^{+}, \pi^{-}$. Leurs 
caractères $\chi^{+}, \chi^{-}$sont donnés par les formules suivantes. Dans celles-ci, on note $\alpha$ l'unique caractère d'ordre 2 du groupe des éléments de $\mathbb{F}_{q^{2}}$ de norme 1 et on fixe une racine carrée $(\zeta(-1) q)^{1 / 2}$ de $\zeta(-1) q$. Alors, pour $\varepsilon \in\{ \pm 1\}$ :

$$
\chi^{\varepsilon}\left(\left(\begin{array}{ll}
1 & 0 \\
0 & 1
\end{array}\right)\right)=(q-1) / 2, \quad \chi^{\varepsilon}\left(\left(\begin{array}{cc}
-1 & 0 \\
0 & -1
\end{array}\right)\right)=-\zeta(-1)(q-1) / 2,
$$

- pour $a \in \mathbb{F}_{q}^{\times} \backslash\{ \pm 1\}$,

$$
\chi^{\varepsilon}\left(\left(\begin{array}{cc}
a & 0 \\
0 & a^{-1}
\end{array}\right)\right)=0 ;
$$

- pour $g \in S L(2)$ semi-simple régulier elliptique, de valeurs propres $\mu, \mu^{-1}$,

$$
\chi^{\varepsilon}(g)=-\alpha(\mu)
$$

$-\operatorname{pour} b \in \mathbb{F}_{q}^{\times}$,

$$
\chi^{\varepsilon}\left(\left(\begin{array}{ll}
1 & b \\
0 & 1
\end{array}\right)\right)=-\frac{1}{2}+\frac{1}{2} \varepsilon \zeta(b)(\zeta(-1) q)^{1 / 2} .
$$

Pour $\varepsilon_{1}, \varepsilon_{2} \in\{ \pm 1\}$, identifions $\pi^{\varepsilon_{1}} \times \pi^{\varepsilon_{2}}$ à une représentation de $R^{\prime}$ triviale sur $Z$. À l'aide des formules ci-dessus, on vérifie que $\operatorname{Ind}_{R^{\prime}}^{R^{0}}\left(\pi^{\varepsilon_{1}} \times \pi^{\varepsilon_{2}}\right)$ est irréductible et isomorphe à $\operatorname{Ind}_{R^{\prime}}^{R^{0}}\left(\pi^{-\varepsilon_{1}} \times \pi^{-\varepsilon_{2}}\right)$. Cette représentation se prolonge de deux façons distinctes en une représentation de $R$. On obtient ainsi nos 4 représentations cherchées. Il est facile d'en calculer les caractères et de vérifier les propriétés de l'énoncé.

10.6 .

Corollaire. - On a l'égalité :

$$
\left(k\left(\mathbf{1}_{-}(1), \mathbf{1}_{-}(1)\right), \operatorname{trace}\left(\pi^{\prime}(1,1)\right)\right)=\frac{1}{4} .
$$

Le calcul est immédiat à l'aide de 3.1 et du lemme précédent.

\section{7 .}

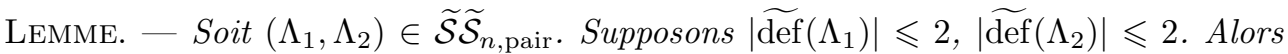
la fonction trace $\left(\pi\left(\Lambda_{1}, \Lambda_{2}\right)\right)$ prend ses valeurs dans $\mathbb{Z}$.

Démonstration. - Les valeurs des caractères de groupes finis sont des entiers algébriques. Il suffit de prouver que $\pi\left(\Lambda_{1}, \Lambda_{2}\right)$ est définie sur $\mathbb{Q}$. Soit $\eta \in\{ \pm 1\}$ tel que $\pi\left(\Lambda_{1}, \Lambda_{2}\right)$ soit une représentation de $O_{\eta}(2 n)$. Cette représentation s'obtient en décomposant une représentation induite. Utilisons pour cette induite les notations de 4.1 et 4.2. D'après l'hypothèse sur $\Lambda_{1}$ et $\Lambda_{2}$, la représentation $\pi_{0}$ est de la forme $\pi\left(\Lambda_{\text {cusp }}\left(h_{1}\right), \Lambda_{\text {cusp }}\left(h_{2}\right)\right)$, où $\left|h_{1}\right| \leqslant 1,\left|h_{2}\right| \leqslant 1$. Une telle représentation est définie $\operatorname{sur} \mathbb{Q}$ : c'est trivial si $h_{1}=h_{2}=0$; si $h_{1}=0$ et $\left|h_{2}\right|=1$, ou si $\left|h_{1}\right|=1$ et $h_{2}=0$, $\pi_{0}$ est l'un des caractères 1 , det, sp, sp det de $O_{-}(2) ;$ si $\left|h_{1}\right|=\left|h_{2}\right|=1$, l'assertion résulte du lemme 10.5. Alors la représentation $\operatorname{Ind}_{P}^{O_{\eta}(2 n)}\left(1_{N_{1}} \times \zeta_{N_{2}} \times \pi_{0}\right)$ est définie sur $\mathbb{Q}$. Pour $j \in\{1,2\}$ et $w \in W_{N_{j}}$, l'opérateur $T_{j}^{\prime}(w)$ est défini sur $\mathbb{Q}$. 
La fonction $C_{j}$ (cf. 4.2) prend ses valeurs dans $\mathbb{Q}$. En effet, il suffit de vérifier que $C_{j}\left(s_{i}\right) \in \mathbb{Q}$ pour tout $i \in\left\{1, \ldots, N_{j}\right\}$. Ce terme a été explicité en 4.2 , sauf si $i=N_{j}$ et $c_{j} \neq 0$. Dans ce cas, l'opérateur $T_{j}\left(s_{N_{j}}\right)=C_{j}\left(s_{N_{j}}\right) T_{j}^{\prime}\left(s_{N_{j}}\right)$ vérifie l'équation

$$
\left(T_{j}\left(s_{N_{j}}\right)-p^{c_{j}}\right)\left(T_{j}\left(s_{N_{j}}\right)+1\right)=0 .
$$

Si $\tau$ est un automorphisme de $\mathbb{C}$, l'opérateur $\tau\left(T_{j}\left(s_{N_{j}}\right)\right)=\tau\left(C_{j}\left(s_{N_{j}}\right)\right) T_{j}^{\prime}\left(s_{N_{j}}\right)$ vérifie la même équation. Cela impose $\tau\left(C_{j}\left(s_{N_{j}}\right)\right)=C_{j}\left(s_{N_{j}}\right)$.

Il en résulte que, pour tout $w \in W_{N_{j}}$, l'opérateur $T_{j}(w)$ est défini sur $\mathbb{Q}$. On sait bien que les représentations des algèbres de Hecke de groupes de Weyl $W_{N_{j}}$ sont définies sur $\mathbb{Q}$. Il en résulte que leurs sous-espaces isotypiques dans la représentation $\operatorname{Ind}_{P}^{O_{\eta}(2 n)}\left(1_{N_{1}} \times \zeta_{N_{2}} \times \pi_{0}\right)$ sont définis sur $\mathbb{Q}$. Donc toute sous-représentation de cette induite est définie sur $\mathbb{Q}$, en particulier $\pi\left(\Lambda_{1}, \Lambda_{2}\right)$ l'est. 


\section{CHAPITRE 11}

\section{COMMUTATION À L'INDUCTION DE DELIGNE-LUSZTIG}

On calcule en termes combinatoires l'image d'une représentation irréductible par un foncteur d'induction de Deligne-Lusztig, quand celui-ci ne provient pas d'un sousgroupe parabolique défini sur $\mathbb{F}_{q}$. Grâce aux résultats du paragraphe 9 , on connaît la restriction de cette image au groupe spécial orthogonal. On va calculer son produit scalaire contre différentes représentations induites. En général, ces deux renseignements vont bien déterminer l'image. Dans des cas particuliers, on a besoin des lemmes techniques démontrés précédemment. On doit aussi choisir convenablement le paramétrage des représentations cuspidales : c'est en 11.12 qu'est fixée l'application $\pi_{n, \text { cusp }}$

11.1. On a défini en 6.6 l'automorphisme $i_{n}$, ou simplement $i$, de l'espace $\mathbb{C}\left[\widetilde{\mathcal{S}} \widetilde{\mathcal{S}}_{n, \text { pair }}\right]$, et remarqué quelques-unes de ses propriétés. Il vérifie aussi les propriétés suivantes :

- pour tout entier $a \in\{1, \ldots, n\}, i \circ\left(I_{a} \otimes 1\right)=I_{a} \otimes 1, i \circ\left(1 \otimes I_{a}\right)=1 \otimes I_{a}$, $\left(I_{-a} \otimes 1\right) \circ i=I_{-a} \otimes 1,\left(1 \otimes I_{-a}\right) \circ i=1 \otimes I_{-a}$.

Cela résulte des propositions $8.2,8.3$ et de l'hypothèse de récurrence $\left(i_{n^{\prime}}\right.$ est l'identité pour $\left.n^{\prime}<n\right)$.

(1) Pour tout $\left(\Lambda_{1}^{\prime}, \Lambda_{2}^{\prime}\right) \in \widetilde{\mathcal{S}} \widetilde{\mathcal{S}}_{n, \text { pair }}$ tel que $\widetilde{\operatorname{def}}\left(\Lambda_{1}^{\prime}\right)=\widetilde{\operatorname{def}}\left(\Lambda_{2}^{\prime}\right)=0$, on a $i \circ \mathcal{F}\left(\Lambda_{1}^{\prime} \otimes \Lambda_{2}^{\prime}\right)=$ $\mathcal{F}\left(\Lambda_{1}^{\prime} \otimes \Lambda_{2}^{\prime}\right)$.

En effet, il suffit de prouver que pour tout $\left(\Lambda_{1}, \Lambda_{2}\right) \in \widetilde{\mathcal{S}} \widetilde{\mathcal{S}}_{n, \text { pair }}$, on a l'égalité :

$$
\left(\Lambda_{1} \otimes \Lambda_{2}, i \circ \mathcal{F}\left(\Lambda_{1}^{\prime} \otimes \Lambda_{2}^{\prime}\right)\right)=\left(\Lambda_{1} \otimes \Lambda_{2}, \mathcal{F}\left(\Lambda_{1}^{\prime} \otimes \Lambda_{2}^{\prime}\right)\right) .
$$

Parce que $\pi_{n}$ est une isométrie et $\pi_{n} \circ i \circ \mathcal{F}=k_{n}$, le membre de gauche est égal à (trace $\left.\left(\pi\left(\Lambda_{1}, \Lambda_{2}\right)\right), k\left(\Lambda_{1}^{\prime}, \Lambda_{2}^{\prime}\right)\right)$. L'égalité à démontrer résulte du corollaire 9.9.

11.2. Jusqu'en 11.11, on fixe des entiers $a, n_{1}, n_{2} \in \mathbb{N}$ tels que $n_{1}+n_{2}=n$, $a \in\left\{1, \ldots, n_{1}\right\}$ et des symboles ordonnés $\Lambda \in \widetilde{\mathcal{S}}_{n_{1}-a \text {,pair }}, \Lambda_{2} \in \widetilde{\mathcal{S}}_{n_{2}, \text { pair }}$. On simplifie les notations de 2.3 en posant $E=E_{a,-1}(\Lambda)$ et, pour $(x, \varepsilon) \in E, \Lambda(x, \varepsilon)=\Lambda_{a,-1}(x, \varepsilon)$, 
$\zeta(x, \varepsilon)=\zeta_{a,-1, \Lambda}(x, \varepsilon)$. Autrement dit, on a l'égalité :

$$
J_{a} \Lambda=\sum_{(x, \varepsilon) \in E} \zeta(x, \varepsilon) \Lambda(x, \varepsilon) .
$$

On vérifie la propriété suivante :

(1) si $(x, \varepsilon),\left(x^{\prime}, \varepsilon^{\prime}\right) \in E$ vérifient $\Lambda\left(x^{\prime}, \varepsilon^{\prime}\right)=\sigma(\Lambda(x, \varepsilon))$, alors $\left(x^{\prime}, \varepsilon^{\prime}\right)=(x, \varepsilon)$ ou $\Lambda=\sigma \Lambda$ et $\left(x^{\prime}, \varepsilon^{\prime}\right)=(x,-\varepsilon)$.

LEMME. - Le terme $i\left(J_{a} \Lambda \otimes \Lambda_{2}\right)$ appartient à $\mathbb{Z}\left[\widetilde{\mathcal{S}} \widetilde{\mathcal{S}}_{n, \text { pair }}\right]$. On a l'égalité :

$$
\operatorname{proj} \circ i\left(J_{a} \Lambda \otimes \Lambda_{2}\right)=\operatorname{proj}\left(J_{a} \Lambda \otimes \Lambda_{2}\right) .
$$

Démonstration. - On a les égalités :

$$
\begin{aligned}
i\left(J_{a} \Lambda \otimes \Lambda_{2}\right) & =\pi_{n}^{-1} \circ k_{n}\left(\mathcal{F} J_{a} \Lambda \otimes \mathcal{F} \Lambda_{2}\right)=\pi_{n}^{-1} \circ k_{n}\left(I_{a}^{-} \mathcal{F} \sigma \Lambda \otimes \mathcal{F} \Lambda_{2}\right) \\
& =\pi_{n}^{-1} \circ R_{1, a}^{-} \circ k_{n-a}\left(\mathcal{F} \sigma \Lambda \otimes \mathcal{F} \Lambda_{2}\right)=\pi_{n}^{-1} \circ R_{1, a}^{-} \circ \pi_{n-a}\left(\sigma \Lambda \otimes \Lambda_{2}\right) .
\end{aligned}
$$

L'avant-dernière égalité vient de la proposition 8.2, la dernière de l'hypothèse de récurrence. Puisque $R_{1, a}^{-}$envoie $\mathbb{Z}[\operatorname{Quad}(O(2 n-2 a))]$ dans $\mathbb{Z}[\operatorname{Quad}(O(2 n))]$, on obtient la première assertion du lemme.

Posons $Z=J_{a} \Lambda \otimes \Lambda_{2}, \widetilde{Z}=i\left(J_{a} \Lambda \otimes \Lambda_{2}\right)$. Les hypothèses du lemme 9.2 sont vérifiées pour $m=n, m_{1}=n_{1}, m_{2}=n_{2}: Z$ vérifie $9.2(1)$ d'après la définition de $J_{a}$; on vient de voir que $\widetilde{Z}$ est entier ; l'hypothèse (a) est vérifiée car $i$ est une isométrie; (b) l'est d'après 11.1(1). Ce lemme entraîne la deuxième assertion de l'énoncé.

\section{3.}

Lemme. - Pour tout $(x, \varepsilon) \in E$, il existe d'uniques symboles

$$
\widetilde{\Lambda}(x, \varepsilon) \in\{\Lambda(x, \varepsilon), \sigma(\Lambda(x, \varepsilon))\}, \quad \widetilde{\Lambda}_{2}(x, \varepsilon) \in\left\{\Lambda_{2}, \sigma \Lambda_{2}\right\}
$$

de sorte que :

(i) on a l'égalité

$$
i\left(J_{a} \Lambda \otimes \Lambda_{2}\right)=\sum_{(x, \varepsilon) \in E} \zeta(x, \varepsilon) \widetilde{\Lambda}(x, \varepsilon) \otimes \widetilde{\Lambda}_{2}(x, \varepsilon) ;
$$

(ii) si $\Lambda=\sigma \Lambda$, on a $\widetilde{\Lambda}(x, \varepsilon)=\Lambda(x, \varepsilon)$ pour tout $(x, \varepsilon) \in E$.

Démonstration. - On suppose $\Lambda_{2} \neq \sigma \Lambda_{2}$, la démonstration s'adaptant aisément au cas $\Lambda_{2}=\sigma \Lambda_{2}$. Supposons d'abord $\Lambda \neq \sigma \Lambda$. Notons $E_{\text {sym }}$, resp. $E_{\text {reg }}$, le sous-ensemble $\operatorname{des}(x, \varepsilon) \in E$ tels que $\Lambda(x, \varepsilon)=\sigma(\Lambda(x, \varepsilon))$, resp. $\Lambda(x, \varepsilon) \neq \sigma(\Lambda(x, \varepsilon))$. Notons comme il est naturel $\sigma^{0}$ l'identité et $\sigma^{1}=\sigma$. Grâce à 11.2(1), on peut écrire de façon unique :

$$
\begin{aligned}
i\left(J_{a} \Lambda \otimes \Lambda_{2}\right)=\left(\sum_{(x, \varepsilon) \in E_{\mathrm{sym}}} \sum_{j=0,1} c_{j}(x, \varepsilon) \Lambda(x, \varepsilon) \otimes \sigma^{j} \Lambda_{2}\right) & \\
& +\left(\sum_{(x, \varepsilon) \in E_{\mathrm{reg}}} \sum_{i, j=0,1} c_{i j}(x, \varepsilon) \sigma^{i}(\Lambda(x, \varepsilon)) \otimes \sigma^{j} \Lambda_{2}\right)+Z
\end{aligned}
$$


où :

- pour tous $(x, \varepsilon) \in E_{\text {sym }}$ et $j \in\{0,1\}$, resp. $(x, \varepsilon) \in E_{\text {reg }}$ et $i, j \in\{0,1\}$, on a $c_{j}(x, \varepsilon) \in \mathbb{C}$, resp. $c_{i j}(x, \varepsilon) \in \mathbb{C} ;$

- $Z$ est combinaison linéaire de termes $\Lambda_{1}^{\prime} \otimes \Lambda_{2}^{\prime}$ tels que $\left(\Lambda_{1}^{\prime}, \Lambda_{2}^{\prime}\right) \neq\left(\sigma^{i}(\Lambda(x, \varepsilon)), \sigma^{j} \Lambda_{2}\right)$ pour tous $i, j,(x, \varepsilon)$.

La première assertion du lemme 11.2 entraîne que les coefficients $c_{j}(x, \varepsilon)$ ou $c_{i j}(x, \varepsilon)$ sont des entiers relatifs. La deuxième assertion de ce lemme entraîne :

$$
\left\{\begin{array}{l}
\text { pour tout }(x, \varepsilon) \in E_{\mathrm{sym}}, \sum_{j=0,1} c_{j}(x, \varepsilon)=\zeta(x, \varepsilon), \\
\text { pour tout }(x, \varepsilon) \in E_{\mathrm{reg}}, \sum_{i, j=0,1} c_{i, j}(x, \varepsilon)=\zeta(x, \varepsilon) .
\end{array}\right.
$$

Puisque $i$ est une isométrie, $i\left(J_{a} \Lambda \otimes \Lambda_{2}\right)$ et $J_{a} \Lambda \otimes \Lambda_{2}$ ont la même norme, ce que l'on peut écrire :

$$
\left(\sum_{(x, \varepsilon) \in E_{\mathrm{sym}}}\left[\left(\sum_{j=0,1} c_{j}(x, \varepsilon)^{2}\right)-1\right]\right)+\left(\sum_{(x, \varepsilon) \in E_{\mathrm{reg}}}\left[\left(\sum_{i, j=0,1} c_{i, j}(x, \varepsilon)^{2}\right)-1\right]\right)+(Z, Z)=0
$$

Cette somme est à termes $\geqslant 0$. Ils sont donc tous nuls. Par conséquent, $Z=0$ et, dans chacune des relations (1), un seul terme du membre de gauche est non nul. Cela équivaut à l'assertion (i) de l'énoncé.

Supposons maintenant $\Lambda=\sigma \Lambda$. Alors $\sigma(\Lambda(x, \varepsilon))=\Lambda(x,-\varepsilon)$ pour tout $(x, \varepsilon) \in E$. On voit que l'on peut écrire de façon unique :

$$
i\left(J_{a} \Lambda \otimes \Lambda_{2}\right)=\left(\sum_{(x, \varepsilon) \in E} \sum_{j=0,1} c_{j}(x, \varepsilon) \Lambda(x, \varepsilon) \otimes \sigma^{j} \Lambda_{2}\right)+Z,
$$

où les $c_{j}(x, \varepsilon)$ et $Z$ sont comme précédemment. Les relations (1) deviennent, pour tout $(x, \varepsilon) \in E$ :

$$
\left(\sum_{j=0,1} c_{j}(x, \varepsilon)\right)+\left(\sum_{j=0,1} c_{j}(x,-\varepsilon)\right)=\zeta(x, \varepsilon)+\zeta(x,-\varepsilon)=2 \zeta(x, \varepsilon) .
$$

Mais $i \circ\left(\left(J_{a} \circ \sigma\right) \otimes 1\right)=(\sigma \otimes 1) \circ i \circ\left(J_{a} \otimes 1\right)$. Puisque $\Lambda=\sigma \Lambda$, on a donc $(\sigma \otimes 1) \circ i\left(J_{a} \Lambda \otimes\right.$ $\left.\Lambda_{2}\right)=i\left(J_{a} \Lambda \otimes \Lambda_{2}\right)$, d'où $c_{j}(x, \varepsilon)=c_{j}(x,-\varepsilon)$ pour tous $j,(x, \varepsilon)$. La démonstration se poursuit alors comme ci-dessus.

L'assertion d'unicité résulte de 11.2(1).

Notons les propriétés supplémentaires :

- si $\Lambda=\sigma \Lambda$, on a $\widetilde{\Lambda}_{2}(x,-\varepsilon)=\widetilde{\Lambda}_{2}(x, \varepsilon)$ pour tout $(x, \varepsilon) \in E ;$

on vient de le voir au cours de la démonstration;

- si $\Lambda=\sigma \Lambda$ et $\Lambda_{2}=\sigma \Lambda_{2}, i\left(J_{a} \Lambda \otimes \Lambda_{2}\right)=J_{a} \Lambda \otimes \Lambda_{2}$.

Dans la suite du paragraphe, on écrit $i\left(J_{a} \Lambda \otimes \Lambda_{2}\right)$ comme dans l'énoncé du lemme.

On note $\mathcal{Z}_{1}$ le sous-espace des éléments de $\mathbb{C}\left[\widetilde{\mathcal{S}} \widetilde{\mathcal{S}}_{n \text {,pair }}\right]$ qui sont combinaisons linéaires de termes $\Lambda_{1}^{\prime} \otimes \Lambda_{2}^{\prime}$ tels que $\Lambda_{1}^{\prime}$ soit cuspidal. Remarquons que $i\left(J_{a} \Lambda \otimes \Lambda_{2}\right)-$ $J_{a} \Lambda \otimes \Lambda_{2}$ appartient à $\mathcal{Z}_{1}$ si et seulement si, pour tout $(x, \varepsilon)$ tel que $\Lambda(x, \varepsilon)$ ne soit pas cuspidal, on a $\widetilde{\Lambda}(x, \varepsilon)=\Lambda(x, \varepsilon)$ et $\widetilde{\Lambda}_{2}(x, \varepsilon)=\Lambda_{2}$. 
11.4. On suppose donné un triplet $\varphi=(r, \nu, t) \in \mathbb{N} \times\{ \pm 1\} \times \mathbb{N}$ tel que $(r, \nu) \in \Lambda$, $r-t \geqslant 0,(r-t, \nu) \notin \Lambda$. On pose :

$$
\operatorname{Int}(\varphi)=\{(r, \nu),(r-t-a,-\nu)\} \cap E .
$$

Pour $(x, \varepsilon) \in E \backslash \operatorname{Int}(\varphi)$, on pose, avec les notations de 2.3 :

$$
\begin{gathered}
\Lambda_{\varphi}(x, \varepsilon)=(\Lambda \backslash\{(r, \nu),(x, \varepsilon)\}) \cup\{(r-t, \nu),(x+a,-\varepsilon)\}, \\
E_{\varphi}(x, \varepsilon)=E_{t, 1}\left(\Lambda_{\varphi}(x, \varepsilon)\right),
\end{gathered}
$$

et, pour $(y, \eta) \in E_{\varphi}(x, \varepsilon)$,

$$
\begin{aligned}
\zeta_{\varphi}(x, \varepsilon ; y, \eta) & =\zeta_{t, 1, \Lambda_{\varphi}(x, \varepsilon)}(y, \eta), \\
\Lambda_{\varphi}(x, \varepsilon ; y, \eta) & =\left(\Lambda_{\varphi}(x, \varepsilon)\right)_{t, 1}(y, \eta) .
\end{aligned}
$$

Autrement dit, on a l'égalité :

$$
I_{t} \Lambda_{\varphi}(x, \varepsilon)=\sum_{(y, \eta) \in E_{\varphi}(x, \varepsilon)} \zeta_{\varphi}(x, \varepsilon ; y, \eta) \Lambda_{\varphi}(x, \varepsilon ; y, \eta)
$$

Remarquons que $(r-t, \nu) \in E_{\varphi}(x, \varepsilon)$ et $\Lambda_{\varphi}(x, \varepsilon ; r-t, \nu)=\Lambda(x, \varepsilon)$.

LEMmE. - Soient $(x, \varepsilon) \in E \backslash \operatorname{Int}(\varphi),\left(x^{\prime}, \varepsilon^{\prime}\right) \in E$ et $(y, \eta) \in E_{\varphi}(x, \varepsilon)$. Supposons $\Lambda_{\varphi}(x, \varepsilon ; y, \eta)=\Lambda\left(x^{\prime}, \varepsilon^{\prime}\right)$. Alors $(y, \eta)=(r-t, \nu)$ et $\left(x^{\prime}, \varepsilon^{\prime}\right)=(x, \varepsilon)$.

Démonstration. - En utilisant les mêmes notations que dans la preuve du lemme 9.4, on a l'égalité :

$$
1_{\Lambda}-1_{r, \nu}-1_{x, \varepsilon}-1_{y, \eta}+1_{r-t, \nu}+1_{x+a,-\varepsilon}+1_{y+t, \eta}=1_{\Lambda}-1_{x^{\prime}, \varepsilon^{\prime}}+1_{x^{\prime}+a,-\varepsilon^{\prime}},
$$

ou encore :

$$
1_{r, \nu}+1_{x, \varepsilon}+1_{y, \eta}+1_{x^{\prime}+a,-\varepsilon^{\prime}}=1_{r-t, \nu}+1_{x+a,-\varepsilon}+1_{y+t, \eta}+1_{x^{\prime}, \varepsilon^{\prime}} .
$$

Cette égalité entraîne l'une des relations suivantes :

$$
\begin{aligned}
(x, \varepsilon)=\left(x^{\prime}, \varepsilon^{\prime}\right) \text { et }(y, \eta) & =(r-t, \nu), \\
(x, \varepsilon)=(y+t, \eta), \quad\left(x^{\prime}, \varepsilon^{\prime}\right) & =(y, \eta), \\
(x+a,-\varepsilon) & =(r, \nu), \quad\left(x^{\prime}+a,-\varepsilon^{\prime}\right)=(r-t, \nu), \\
(x, \varepsilon)=(r-t, \nu), \quad\left(x^{\prime}, \varepsilon^{\prime}\right) & =(r, \nu), \\
(x+a,-\varepsilon) & =(y, \eta), \quad\left(x^{\prime}+a,-\varepsilon^{\prime}\right)=(y+t, \eta) .
\end{aligned}
$$

Mais (2) est exclue car, par hypothèse, $(r, \nu) \in \Lambda$ et $(x+a,-\varepsilon) \notin \Lambda$. De même, (3) est exclue car $(r-t, \nu) \notin \Lambda$ et $(x, \varepsilon) \in \Lambda$. Il ne reste plus que (1), qui est la conclusion de l'énoncé.

Il résulte de ce lemme que, pour $(x, \varepsilon) \in E \backslash \operatorname{Int}(\varphi)$, on a l'égalité :

$$
\left(I_{t} \Lambda_{\varphi}(x, \varepsilon), J_{a} \Lambda\right)=\zeta_{\varphi}(x, \varepsilon ; r-t, \nu) \zeta(x, \varepsilon) .
$$


11.5. On conserve les mêmes hypothèses. On introduit les conditions (1) à (5) suivantes. Elles postulent l'existence d'un sous-ensemble fini $Z \subset \mathbb{N}$ tel que $Z \cap\{r-t, r\}=\varnothing$ et :

(1) $\Lambda^{\nu}=Z \cup\{r\}, \Lambda^{-\nu}=Z \cup\{r-t\}$;

(2) il existe $X \in \mathbb{N}$ tel que $\{X, X+a\} \cap(Z \cup\{r-t, r\})=\varnothing$ et $\Lambda^{\nu}=Z \cup\{X, X+a, r\}$, $\Lambda^{-\nu}=Z \cup\{r-t\}$;

(3) il existe $X \in \mathbb{N}$ tel que $\{X, X+a\} \cap(Z \cup\{r-t, r\})=\varnothing$ et $\Lambda^{\nu}=Z \cup\{r\}$, $\Lambda^{-\nu}=Z \cup\{X, X+a, r-t\}$;

(4) $r-t-a \notin Z, \Lambda^{\nu}=Z \cup\{r-t-a, r\}, \Lambda^{-\nu}=Z$

(5) $r+a \notin Z, \Lambda^{\nu}=Z \cup\{r\}, \Lambda^{-\nu}=Z \cup\{r-t, r, r+a\}$.

Lemme. - Soient $(x, \varepsilon) \in E \backslash \operatorname{Int}(\varphi),\left(x^{\prime}, \varepsilon^{\prime}\right) \in E$ et $(y, \eta) \in E_{\varphi}(x, \varepsilon)$. Supposons vérifiées les hypothèses suivantes :

$$
\begin{aligned}
\Lambda \neq \sigma \Lambda, \quad \Lambda\left(x^{\prime}, \varepsilon^{\prime}\right) & \neq \sigma\left(\Lambda\left(x^{\prime}, \varepsilon^{\prime}\right)\right) ; \\
\Lambda_{\varphi}(x, \varepsilon ; y, \eta) & =\sigma\left(\Lambda\left(x^{\prime}, \varepsilon^{\prime}\right)\right) ; \\
\zeta_{\varphi}(x, \varepsilon ; r-t, \nu) \zeta(x, \varepsilon) & =\zeta_{\varphi}(x, \varepsilon ; y, \eta) \zeta\left(x^{\prime}, \varepsilon^{\prime}\right) .
\end{aligned}
$$

Alors on a l'une des relations suivantes :

(i) (1) est vérifiée;

(ii) (2) est vérifiée, $\left(x^{\prime}, \varepsilon^{\prime}\right)=(x, \varepsilon)=(X, \nu)$;

(iii) (3) est vérifiée, $\left(x^{\prime}, \varepsilon^{\prime}\right)=(x, \varepsilon)=(X,-\nu)$;

(iv) (4) est vérifiée, $\left(x^{\prime}, \varepsilon^{\prime}\right)=(x, \varepsilon)=(r-t-a, \nu)$;

(v) (5) est vérifiée, $\left(x^{\prime}, \varepsilon^{\prime}\right)=(x, \varepsilon)=(r,-\nu)$.

Démonstration. — La deuxième hypothèse de l'énoncé s'écrit :

$$
\begin{array}{r}
(((\Lambda \backslash\{(r, \nu),(x, \varepsilon)\}) \cup\{(r-t, \nu),(x+a,-\varepsilon)\}) \backslash\{(y, \eta)\}) \cup\{y+t, \eta\} \\
=\left(\sigma \Lambda \backslash\left\{\left(x^{\prime},-\varepsilon^{\prime}\right\}\right) \cup\left\{\left(x^{\prime}+a, \varepsilon^{\prime}\right)\right\} .\right.
\end{array}
$$

Posons $D=\left\{r-t, r, x, x+a, y, y+t, x^{\prime}, x^{\prime}+a\right\}$. Décomposons $\Lambda$ en union disjointe $\Lambda^{\prime} \cup \Lambda^{\prime \prime}$ de sorte que, pour tout $(z, \tau) \in \Lambda^{\prime}$, resp. $(z, \tau) \in \Lambda^{\prime \prime}$, on ait $z \in D$, resp. $z \notin D$. L'égalité ci-dessus est équivalente à la conjonction de la même égalité où $\Lambda$ est remplacé par $\Lambda^{\prime}$ et de l'égalité $\Lambda^{\prime \prime}=\sigma \Lambda^{\prime \prime}$. Pour tout sous-ensemble $Z \subset \mathbb{N}$ et tout élément $z \in \mathbb{N}$, notons $1_{Z}$ la fonction caractéristique de $Z, 1_{z}$ celle de $\{z\}$ et posons $m(z, Z)=|\{z, \ldots, 0\} \cap Z|$. L'égalité (6) entraîne :

$$
1_{\Lambda^{+}}+1_{\Lambda^{-}}+1_{r-t}+1_{x+a}+1_{y+t}-1_{r}-1_{x}-1_{y}=1_{\Lambda^{+}}+1_{\Lambda^{-}}+1_{x^{\prime}+a}-1_{x^{\prime}},
$$

d'où :

$$
1_{r-t}+1_{x+a}+1_{y+t}+1_{x^{\prime}}=1_{r}+1_{x}+1_{y}+1_{x^{\prime}+a} .
$$


Exprimons la troisième hypothèse de l'énoncé en utilisant les définitions. On obtient :

(8) $m\left(r-t, \Lambda_{\varphi}(x, \varepsilon)^{\nu}\right)+m\left(r, \Lambda_{\varphi}(x, \varepsilon)^{\nu}\right)+m\left(x, \Lambda^{\varepsilon}\right)+m\left(x+a, \Lambda^{-\varepsilon}\right)$

$+m\left(y, \Lambda_{\varphi}(x, \varepsilon)^{\eta}\right)+m\left(y+t, \Lambda_{\varphi}(x, \varepsilon)^{\eta}\right)+m\left(x^{\prime}, \Lambda^{\varepsilon^{\prime}}\right)+m\left(x^{\prime}+a, \Lambda^{-\varepsilon^{\prime}}\right) \equiv 0 \bmod 2 \mathbb{Z}$.

Or la conjonction de (7) et de l'égalité $\Lambda^{\prime \prime}=\sigma \Lambda^{\prime \prime}$ montre que la contribution des éléments de $\Lambda^{\prime \prime}$ à la somme ci-dessus est paire. Autrement dit, on est ramené au cas où $\Lambda=\Lambda^{\prime}$, ce que l'on suppose désormais.

L'égalité (7) entraîne que l'une des relations suivantes est vérifiée :

$$
\begin{aligned}
& x=x^{\prime}, y=r-t ; \\
& a \neq t, r-t=x^{\prime}+a, x+a=r, y+t=x, x^{\prime}=y \\
& a \neq t, r-t=x, x+a=y, y+t=x^{\prime}+a, x^{\prime}=r \\
& a=t, r-t=x, x+a=r, y+t=x^{\prime}+a, x^{\prime}=y .
\end{aligned}
$$

La relation (12) est impossible : on a par hypothèse $(r-t, \nu) \notin \Lambda,(x, \varepsilon) \in \Lambda$, donc $\varepsilon=-\nu$ puisque $r-t=x$; on a par hypothèse $(r, \nu) \in \Lambda,(x+a,-\varepsilon) \notin \Lambda$, donc $\varepsilon=\nu$ puisque $x+a=r$.

Supposons (11) vérifiée. Comme ci-dessus, $\varepsilon=-\nu$. Montrons que $\Lambda$ est déterminé par l'égalité $(6)$. On a déjà $(r-t, \nu) \notin \Lambda,(r-t,-\nu) \in \Lambda$. On a $(r, \nu) \in \Lambda$. Si $\varepsilon^{\prime}=-\nu$, on a aussi $(r,-\nu)=\left(x^{\prime}, \varepsilon^{\prime}\right) \in \Lambda$. Si $\varepsilon^{\prime}=\nu,(r,-\nu)$ n'appartient pas au membre de droite de $(6)$, donc n'appartient pas au membre de gauche et donc n'appartient pas à $\Lambda$. On a $(r-t+a, \nu)=(x+a,-\varepsilon) \notin \Lambda$. Si $\eta=-\nu$, l'hypothèse $(y, \eta) \in E_{\varphi}(x, \varepsilon)$ entraîne $(y, \eta) \in \Lambda$, i.e. $(r-t+a,-\nu) \in \Lambda$. Si $\eta=\nu$, le terme $(r-t+a, \nu)$ n'appartient pas au membre de gauche de (6), donc pas au membre de droite, donc pas à $\sigma \Lambda$. Alors $(r-t+a,-\nu) \notin \Lambda$. Enfin, on a $\left(r+a,-\varepsilon^{\prime}\right)=\left(x^{\prime}+a,-\varepsilon^{\prime}\right) \notin \Lambda$. Si $\eta=\varepsilon^{\prime}$, l'hypothèse $(y, \eta) \in E_{\varphi}(x, \varepsilon)$ entraîne $(y+t, \eta) \notin \Lambda$, i.e. $\left(r+a, \varepsilon^{\prime}\right) \notin \Lambda$. Si $\eta=-\varepsilon^{\prime}$, $\left(r+a,-\varepsilon^{\prime}\right)=(y+t, \eta)$ appartient au membre de gauche de (6), donc au membre de droite, donc à $\sigma \Lambda$ et $\left(r+a, \varepsilon^{\prime}\right) \in \Lambda$. On obtient donc les possibilités suivantes :

$$
\begin{aligned}
& \varepsilon=-\nu, \eta=\varepsilon^{\prime}=\nu, \Lambda^{\nu}=\{r\}, \Lambda^{-\nu}=\{r-t\} \\
& \varepsilon=\varepsilon^{\prime}=-\nu, \eta=\nu, \Lambda^{\nu}=\{r\}, \Lambda^{-\nu}=\{r-t, r, r+a\}, \\
& \varepsilon=\eta=-\nu, \varepsilon^{\prime}=\nu, \Lambda^{\nu}=\{r, r+a\}, \Lambda^{-\nu}=\{r-t, r-t+a\} \\
& \varepsilon=\eta=\varepsilon^{\prime}=-\nu, \Lambda^{\nu}=\{r\}, \Lambda^{-\nu}=\{r-t, r, r-t+a\} .
\end{aligned}
$$

Dans le premier cas, la relation (i) de l'énoncé est vérifiée. Dans les trois autres cas, un calcul fastidieux mais élémentaire montre que la relation (8) n'est pas vérifiée. Ces cas sont donc exclus et cela démontre le lemme dans le cas où (11) est vérifiée.

Un raisonnement similaire s'applique quand la relation (10) est vérifiée et conduit à la même conclusion. 
Supposons (9) vérifiée. Si $\eta=\nu$, l'équation (6) devient $\Lambda(x, \varepsilon)=\sigma\left(\Lambda\left(x^{\prime}, \varepsilon^{\prime}\right)\right)$. D'après 11.2(1), $\Lambda$ ou $\Lambda\left(x^{\prime}, \varepsilon^{\prime}\right)$ est symétrique (c'est-à-dire invariant par $\sigma$ ), contrairement à la première hypothèse du lemme. Donc $\eta=-\nu$. Parce que $(x, \varepsilon) \in \Lambda$ et $(r-t, \nu) \notin \Lambda$, on a $(x, \varepsilon) \neq(r-t, \nu)$. Parce que $(x, \varepsilon) \notin \Lambda_{\varphi}(x, \varepsilon)$ et $(y, \eta)=$ $(r-t,-\nu) \in \Lambda_{\varphi}(x, \varepsilon)$, on a $(x, \varepsilon) \neq(r-t,-\nu)$. Donc $x \neq r-t$. De même, parce que $(x+a,-\varepsilon) \notin \Lambda$ et $(r, \nu) \in \Lambda$, on a $(x+a,-\varepsilon) \neq(r, \nu)$. Parce que $(x+a,-\varepsilon) \in \Lambda_{\varphi}(x, \varepsilon)$ et $(y+t, \eta)=(r,-\nu) \notin \Lambda_{\varphi}(x, \varepsilon)$, on a $(x+a,-\varepsilon) \neq(r,-\nu)$. Donc $x+a \neq r$.

Supposons $x \neq r$. On a $(r, \nu) \in \Lambda$. Parce que $(y+t, \eta)=(r,-\nu) \notin \Lambda_{\varphi}(x, \varepsilon)$, on a aussi $(r,-\nu) \notin \Lambda$. Si $\varepsilon=-\varepsilon^{\prime}$, on a $(x, \varepsilon) \in \Lambda$ et $(x,-\varepsilon)=\left(x^{\prime}, \varepsilon^{\prime}\right) \in \Lambda$. Si $\varepsilon=\varepsilon^{\prime}$, on a encore $(x, \varepsilon) \in \Lambda$. Parce que $(x,-\varepsilon)=\left(x^{\prime},-\varepsilon^{\prime}\right)$ n'appartient pas au membre de droite de (6), il n'appartient pas au membre de gauche, donc pas à $\Lambda$.

Supposons $x+a \neq r-t$. On a $(r-t, \nu) \notin \Lambda$. Parce que $(y, \eta)=(r-t,-\nu) \in \Lambda_{\varphi}(x, \varepsilon)$, on a aussi $(r-t,-\nu) \in \Lambda$. Si $\varepsilon=-\varepsilon^{\prime}$, on a $(x+a,-\varepsilon) \notin \Lambda$ et $(x+a, \varepsilon)=\left(x^{\prime}+a,-\varepsilon^{\prime}\right) \notin$ $\Lambda$. Si $\varepsilon=\varepsilon^{\prime}$, on a encore $(x+a,-\varepsilon) \notin \Lambda$. Parce que $(x+a, \varepsilon)=\left(x^{\prime}+a, \varepsilon^{\prime}\right)$ appartient au membre de droite de (6), il appartient au membre de gauche, donc à $\Lambda$.

Supposons les deux conditions ci-dessus vérifiées, i.e. $x \neq r, x+a \neq r-t$. On obtient alors les possibilités suivantes :

$$
\begin{aligned}
& \varepsilon=-\varepsilon^{\prime}, \Lambda^{\nu}=\{x, r\}, \Lambda^{-\nu}=\{x, r-t\} \\
& \varepsilon=\varepsilon^{\prime}=\nu, \Lambda^{\nu}=\{x, x+a, r\}, \Lambda^{-\nu}=\{r-t\} ; \\
& \varepsilon=\varepsilon^{\prime}=-\nu, \Lambda^{\nu}=\{r\}, \Lambda^{-\nu}=\{x, x+a, r-t\} .
\end{aligned}
$$

Dans le premier, resp. deuxième, troisième cas, la relation (i), resp. (ii), (iii) de l'énoncé est vérifiée.

Supposons maintenant $x=r$, donc $x+a \neq r-t$. Parce que $(x, \varepsilon) \notin \operatorname{Int}(\varphi)$, on a $(x, \varepsilon) \neq(r, \nu)$, d'où $\varepsilon=-\nu$. Alors $(r, \nu)$ et $(r,-\nu)=(x, \varepsilon)$ appartiennent à $\Lambda$. Puisque $(r,-\nu)=(y+t, \eta)$ appartient au membre de gauche de (6), il appartient au membre de droite, donc $\left(x^{\prime},-\varepsilon^{\prime}\right) \neq(r,-\nu)$ et $\varepsilon^{\prime}=-\nu=\varepsilon$. On obtient la seule possibilité :

$$
(x, \varepsilon)=\left(x^{\prime}, \varepsilon^{\prime}\right)=(r,-\nu), \Lambda^{\nu}=\{r\}, \Lambda^{-\nu}=\{r-t, r, r+a\},
$$

et la condition (v) de l'énoncé est vérifiée.

Supposons enfin $x+a=r-t$, donc $x \neq r$. Parce que $(x, \varepsilon) \notin \operatorname{Int}(\varphi)$, on a $(x+a,-\varepsilon) \neq(r-t, \nu)$ et $\varepsilon=\nu$. Alors $(r-t, \nu)$ et $(r-t,-\nu)=(x+a,-\varepsilon)$ n'appartiennent pas à $\Lambda$. Puisque $(r-t,-\nu)=(y, \eta)$ n'appartient pas au membre de gauche de (6), il n'appartient pas au membre de droite, donc $\left(x^{\prime}+a, \varepsilon^{\prime}\right) \neq(r-t,-\nu)$ et $\varepsilon^{\prime}=\nu=\varepsilon$. On obtient la seule possibilité :

$$
(x, \varepsilon)=\left(x^{\prime}, \varepsilon^{\prime}\right)=(r-t-a, \nu), \Lambda^{\nu}=\{r-t-a, r\}, \Lambda^{-\nu}=\varnothing,
$$

et la condition (iv) de l'énoncé est vérifiée. Cela achève la démonstration. 
11.6. Disons que $\Lambda$ est particulier s'il existe $\tau \in\{ \pm 1\}$ et trois sous-ensembles finis $Z, U, V$ de $\mathbb{N}$, deux à deux disjoints, tels que l'on ait les égalités :

$$
\Lambda^{\tau}=Z \cup U, \Lambda^{-\tau}=Z \cup V,
$$

et tels que l'une des relations suivantes soit vérifiée :

(1) $|U|=|V|=1$;

(2) $|U|=3,|V|=1$ et il existe $u \in U$ tel que $u+a \in U$;

(3) $|U|=2, V=\varnothing$ et, en notant $u_{1}>u_{2}$ les deux éléments de $U$, on a d'une part $u_{1}>u_{2}+a$, d'autre part $u_{1}-a \in Z$ ou $u_{2}+a \notin Z$.

LEMme. - Supposons que $\Lambda$ ne soit ni cuspidal, ni particulier. Alors $i\left(J_{a} \Lambda \otimes \Lambda_{2}\right)-$ $J_{a} \Lambda \otimes \Lambda_{2}$ appartient $\grave{a} \mathcal{Z}_{1}$.

Démonstration. - On va montrer que pour tout $(x, \varepsilon) \in E$ tel que $\Lambda(x, \varepsilon)$ ne soit pas cuspidal, on a les égalités $\widetilde{\Lambda}(x, \varepsilon)=\Lambda(x, \varepsilon)$ et $\widetilde{\Lambda}_{2}(x, \varepsilon)=\Lambda_{2}$.

Parce que $\Lambda$ n'est pas cuspidal, il existe un triplet $\varphi=(r, \nu, t)$ vérifiant les conditions de 11.4. Fixons-en un. Soit $(x, \varepsilon) \in E \backslash \operatorname{Int}(\varphi)$. Puisque $i$ est une isométrie et $i \circ\left(I_{t} \otimes 1\right)=I_{t} \otimes 1$, l'égalité 11.4(4) entraîne :

$$
\left(I_{t} \Lambda_{\varphi}(x, \varepsilon) \otimes \Lambda_{2}, i\left(J_{a} \Lambda \otimes \Lambda_{2}\right)\right)=\zeta_{\varphi}(x, \varepsilon ; r-t, \nu) \zeta(x, \varepsilon) .
$$

En écrivant explicitement le membre de gauche, on voit qu'il existe $(y, \eta) \in E_{\varphi}(x, \varepsilon)$ et $\left(x^{\prime}, \varepsilon^{\prime}\right) \in E$ de sorte que les deux relations suivantes soient vérifiées :

$$
\begin{aligned}
\Lambda_{\varphi}(x, \varepsilon ; y, \eta) \otimes \Lambda_{2} & =\widetilde{\Lambda}\left(x^{\prime}, \varepsilon^{\prime}\right) \otimes \widetilde{\Lambda}_{2}\left(x^{\prime}, \varepsilon^{\prime}\right), \\
\zeta_{\varphi}(x, \varepsilon ; y, \eta) \zeta\left(x^{\prime}, \varepsilon^{\prime}\right) & =\zeta_{\varphi}(x, \varepsilon ; r-t, \nu) \zeta(x, \varepsilon) .
\end{aligned}
$$

Fixons de telles données. La première relation entraîne $\widetilde{\Lambda}_{2}\left(x^{\prime}, \varepsilon^{\prime}\right)=\Lambda_{2}$. Supposons $\widetilde{\Lambda}\left(x^{\prime}, \varepsilon^{\prime}\right)=\sigma\left(\Lambda\left(x^{\prime}, \varepsilon^{\prime}\right)\right) \neq \Lambda\left(x^{\prime}, \varepsilon^{\prime}\right)$. D'après le (ii) du lemme 11.3, on a $\Lambda \neq \sigma \Lambda$. Les hypothèses du lemme 11.5 sont satisfaites. Mais sa conclusion ne l'est pas, car $\Lambda$ n'est pas particulier. Contradiction, donc $\widetilde{\Lambda}\left(x^{\prime}, \varepsilon^{\prime}\right)=\Lambda\left(x^{\prime}, \varepsilon^{\prime}\right)$. Le lemme 11.4 entraîne alors l'égalité $\left(x^{\prime}, \varepsilon^{\prime}\right)=(x, \varepsilon)$, d'où les égalités cherchées pour $(x, \varepsilon)$.

Supposons qu'il existe deux triplets $\varphi, \varphi^{\prime}$ distincts vérifiant les conditions de 11.4. D'après ce qui précède, les égalités cherchées sont vérifiées pour tout $(x, \varepsilon) \in E \backslash$ $\left(\operatorname{Int}(\varphi) \cap \operatorname{Int}\left(\varphi^{\prime}\right)\right)$. Si $\operatorname{Int}(\varphi) \cap \operatorname{Int}\left(\varphi^{\prime}\right)=\varnothing$, on a fini. Sinon, cet ensemble contient un unique élément, notons-le $(x, \varepsilon)$. Ce que l'on a démontré pour les autres éléments de $E$ entraîne l'égalité :

$$
i\left(J_{a} \Lambda \otimes \Lambda_{2}\right)-J_{a} \Lambda \otimes \Lambda_{2}=\zeta(x, \varepsilon)\left(\widetilde{\Lambda}(x, \varepsilon) \otimes \widetilde{\Lambda}_{2}(x, \varepsilon)-\Lambda(x, \varepsilon) \otimes \Lambda_{2}\right) .
$$

Si $\Lambda(x, \varepsilon)$ est cuspidal, on n'a plus rien à démontrer. Supposons que $\Lambda(x, \varepsilon)$ n'est pas cuspidal. Soit $b \in\left\{1, \ldots, n_{1}\right\}$. Les propriétés de $i$ entraînent que l'opérateur $I_{-b} \otimes 1$ annule le membre gauche ci-dessus, donc aussi le membre de droite. D'où :

$$
I_{-b} \widetilde{\Lambda}(x, \varepsilon) \otimes \widetilde{\Lambda}_{2}(x, \varepsilon)=I_{-b} \Lambda(x, \varepsilon) \otimes \Lambda_{2} .
$$


Puisque $\Lambda(x, \varepsilon)$ n'est pas cuspidal, cette égalité se décompose en deux :

$$
I_{-b} \widetilde{\Lambda}(x, \varepsilon)=I_{-b} \Lambda(x, \varepsilon), \widetilde{\Lambda}_{2}(x, \varepsilon)=\Lambda_{2} .
$$

Grâce au lemme 9.4 , ou bien $\widetilde{\Lambda}(x, \varepsilon)=\Lambda(x, \varepsilon)$, ou bien il existe $h \in \mathbb{Z}$ tel que $\{\widetilde{\Lambda}(x, \varepsilon), \Lambda(x, \varepsilon)\}=\{A B(h), B A(h)\}$. Il reste à exclure cette dernière possibilité. Si elle est vérifiée, on a aussi $\widetilde{\Lambda}(x, \varepsilon)=\sigma(\Lambda(x, \varepsilon))$, donc $h=0$. Rappelons que $(x, \varepsilon) \notin$ $\Lambda(x, \varepsilon)$ tandis que $(x+a,-\varepsilon) \in \Lambda(x, \varepsilon)$. Cela interdit à $\Lambda(x, \varepsilon)$ d'être de la forme $A B(0)$ ou $B A(0)$. Cela démontre le lemme sous l'hypothèse de l'existence de deux triplets $\varphi, \varphi^{\prime}$.

Supposons maintenant qu'il n'existe qu'un seul triplet $\varphi$. Alors $\Lambda$ est nécessairement de la forme suivante : il existe $\nu \in\{ \pm 1\}$ et deux entiers $r, y \geqslant 1$ de sorte que $\Lambda^{\nu}=$ $\{r\} \cup\{r-2, \ldots, 0\}, \Lambda^{-\nu}=\{y-1, \ldots, 0\}$. Le triplet $\varphi$ est $(r, \nu, 1)$. Si $|\operatorname{Int}(\varphi)| \leqslant 1$, le raisonnement précédent s'applique. On suppose donc $|\operatorname{Int}(\varphi)|=2$, i.e. $\operatorname{Int}(\varphi)=$ $\{(r, \nu),(r-1-a,-\nu)\}$. Posons pour simplifier :

$$
\begin{gathered}
\Lambda^{\prime}=\Lambda(r, \nu), \zeta^{\prime}=\zeta(r, \nu), \widetilde{\Lambda}^{\prime}=\widetilde{\Lambda}(r, \nu), \widetilde{\Lambda}_{2}^{\prime}=\widetilde{\Lambda}_{2}(r, \nu), \Lambda^{\prime \prime}=\Lambda(r-1-a,-\nu), \\
\zeta^{\prime \prime}=\zeta(r-1-a,-\nu), \widetilde{\Lambda}^{\prime \prime}=\widetilde{\Lambda}(r-1-a,-\nu), \widetilde{\Lambda}_{2}^{\prime \prime}=\widetilde{\Lambda}_{2}(r-1-a,-\nu) .
\end{gathered}
$$

On a maintenant :

$$
i\left(J_{a} \Lambda \otimes \Lambda_{2}\right)-J_{a} \Lambda \otimes \Lambda_{2}=\zeta^{\prime}\left(\widetilde{\Lambda}^{\prime} \otimes \widetilde{\Lambda}_{2}^{\prime}-\Lambda^{\prime} \otimes \Lambda_{2}\right)+\zeta^{\prime \prime}\left(\widetilde{\Lambda}^{\prime \prime} \otimes \widetilde{\Lambda}_{2}^{\prime \prime}-\Lambda^{\prime \prime} \otimes \Lambda_{2}\right) .
$$

Si $\Lambda^{\prime}$ est cuspidal, il n'y a rien à démontrer concernant $\widetilde{\Lambda}^{\prime}$ et $\widetilde{\Lambda}_{2}^{\prime}$. Le même raisonnement que ci-dessus détermine $\widetilde{\Lambda}^{\prime \prime}$ et $\widetilde{\Lambda}_{2}^{\prime \prime}$ car les opérateurs $I_{-b} \otimes 1$ annulent le premier terme du membre de droite ci-dessus. On conclut de même si $\Lambda^{\prime \prime}$ est cuspidal. On suppose désormais que ni $\Lambda^{\prime}$, ni $\Lambda^{\prime \prime}$ ne sont cuspidaux. On a alors :

$$
\begin{gathered}
r+a>y>r-a, \\
\Lambda^{\prime \nu}=\{r-2, \ldots, 0\}, \Lambda^{\prime-\nu}=\{r+a\} \cup\{y-1, \ldots, 0\}, \\
\Lambda^{\prime \prime \nu}=\{r, \ldots, 0\}, \Lambda^{\prime \prime-\nu}=\{y-1, \ldots, r-a\} \cup\{r-a-2, \ldots, 0\} .
\end{gathered}
$$

On a les égalités $I_{-1} \Lambda^{\prime}=\bar{\Lambda}^{\prime}, I_{-1} \Lambda^{\prime \prime}=\bar{\Lambda}^{\prime \prime}$, où :

$$
\begin{gathered}
\bar{\Lambda}^{\prime \nu}=\{r-2, \ldots, 0\}, \bar{\Lambda}^{\prime-\nu}=\{r+a-1\} \cup\{y-1, \ldots, 0\}, \\
\bar{\Lambda}^{\prime \prime \nu}=\{r, \ldots, 0\}, \bar{\Lambda}^{\prime \prime-\nu}=\{y-1, \ldots, r-a+1\} \cup\{r-a-1, \ldots, 0\} .
\end{gathered}
$$

Le terme $\bar{\Lambda}^{\prime}$ n'intervient ni dans $I_{-1} \sigma \Lambda^{\prime}$, ni dans $I_{-1} \Lambda^{\prime \prime}$. Il ne peut intervenir dans $I_{-1} \sigma \Lambda^{\prime \prime}$ que si $\bar{\Lambda}^{\prime \prime}=\sigma \bar{\Lambda}^{\prime}$. Dans ce cas $a=1$ et $y=r$. Mais alors la relation (1) est vérifiée et $\Lambda$ est particulier, contrairement à l'hypothèse. Appliquons l'opérateur $I_{-1} \otimes 1$ à l'égalité (4). Il annule le membre de gauche donc aussi celui de droite. Le terme $\bar{\Lambda}^{\prime} \otimes \Lambda_{2}$, qui intervient dans $I_{-1} \Lambda^{\prime} \otimes \Lambda_{2}$, doit être compensé par un terme de $I_{-1} \widetilde{\Lambda}^{\prime} \otimes \widetilde{\Lambda}_{2}^{\prime}$ ou $I_{-1} \widetilde{\Lambda}^{\prime \prime} \otimes \widetilde{\Lambda}_{2}^{\prime \prime}$ ou $I_{-1} \Lambda^{\prime \prime} \otimes \Lambda_{2}$. On vient de voir que ce ne peut être qu'un terme de $I_{-1} \widetilde{\Lambda}^{\prime} \otimes \widetilde{\Lambda}_{2}^{\prime}$ et alors $\widetilde{\Lambda}^{\prime}=\Lambda^{\prime}, \widetilde{\Lambda}_{2}^{\prime}=\Lambda_{2}$. Cela détermine $\widetilde{\Lambda}^{\prime}$ et $\widetilde{\Lambda}_{2}^{\prime}$. La relation 
(4) se simplifie et on peut reprendre un raisonnement déjà fait pour déterminer $\widetilde{\Lambda}^{\prime \prime}$ et $\widetilde{\Lambda}_{2}^{\prime \prime}$. Cela achève la démonstration.

11.7.

LEMme. - Supposons $n_{1}-a \geqslant 2$ et $\Lambda$ particulier. Alors $i\left(J_{a} \Lambda \otimes \Lambda_{2}\right)-J_{a} \Lambda \otimes \Lambda_{2}$ appartient à $\mathcal{Z}_{1}$.

Démonstration. - Supposons vérifiée la relation 11.6(1). Quitte à changer $\tau$ en $-\tau$, on peut supposer :

$$
\Lambda^{\tau}=Z \cup\{u\}, \Lambda^{-\tau}=Z \cup\{v\}, u>v .
$$

Le triplet $\varphi_{0}=(u, \tau, u-v)$ vérifie les conditions de 11.4. Supposons qu'il existe deux autres triplets. La démonstration du lemme précédent s'applique en utilisant ces autres triplets, le point étant que la conclusion du lemme 11.5 n'est pas vérifiée pour un triplet différent de $\varphi_{0}$. S'il n'existe aucun autre triplet, on a nécessairement $u-v=1, \Lambda^{\tau}=\{u\} \cup\{u-2, \ldots, 0\}, \Lambda^{-\tau}=\{u-1, \ldots, 0\}$. Alors $n_{1}-a=1$ contrairement à l'hypothèse. On suppose maintenant qu'il existe un unique autre triplet. Alors $u-v=2$ et l'une des relations suivantes est vérifiée :

$$
\begin{gathered}
\Lambda^{\tau}=\{u\} \cup\{u-3, \ldots, 0\}, \Lambda^{-\tau}=\{u-2, \ldots, 0\}, \\
\Lambda^{\tau}=\{u, u-1\} \cup\{u-3, \ldots, 0\}, \Lambda^{-\tau}=\{u-1, \ldots, 0\} .
\end{gathered}
$$

On suppose la première vérifiée, une démonstration similaire s'appliquant si la seconde l'est. Posons $\varphi=(u, \tau, 1)$. La démonstration du lemme précédent s'applique pour ce triplet et détermine $\widetilde{\Lambda}(x, \varepsilon)$ et $\widetilde{\Lambda}_{2}(x, \varepsilon)$ pour $(x, \varepsilon) \in E \backslash\{(u, \tau),(u-1-a,-\tau)\}$. D'où l'égalité 11.6(4) où les termes affectés d'un ', resp. d'un ", sont relatifs au couple $(u, \tau)$, resp. $(u-1-a,-\tau)$. On calcule :

$$
\begin{aligned}
\zeta^{\prime}=1, \Lambda^{\prime \tau} & =\{u-3, \ldots, 0\}, \Lambda^{\prime-\tau}=\{u+a\} \cup\{u-2, \ldots, 0\}, \\
\zeta^{\prime \prime}=(-1)^{a}, \Lambda^{\prime \prime \tau} & =\{u, u-1\} \cup\{u-3, \ldots, 0\}, \\
\Lambda^{\prime \prime-\tau} & =\{u-2, \ldots, u-a\} \cup\{u-a-2, \ldots, 0\} .
\end{aligned}
$$

On a l'égalité $I_{-1} \Lambda^{\prime}=\bar{\Lambda}^{\prime}$, où :

$$
\bar{\Lambda}^{\prime \tau}=\{u-3, \ldots, 0\}, \bar{\Lambda}^{\prime-\tau}=\{u+a-1\} \cup\{u-2, \ldots, 0\} .
$$

Appliquons l'opérateur $I_{-1} \otimes 1$ à l'égalité 11.6(4). Le terme $-\zeta^{\prime} I_{-1} \Lambda^{\prime} \otimes \Lambda_{2}$ est égal à $-\bar{\Lambda}^{\prime} \otimes \Lambda_{2}$. Ce terme doit être compensé par un terme de $\zeta^{\prime} I_{-1} \widetilde{\Lambda}^{\prime} \otimes \widetilde{\Lambda}_{2}^{\prime}$ ou $\zeta^{\prime \prime} I_{-1} \widetilde{\Lambda}^{\prime \prime} \otimes \widetilde{\Lambda}_{2}^{\prime \prime}$ ou $-\zeta^{\prime \prime} I_{-1} \Lambda^{\prime \prime} \otimes \Lambda_{2}$. On vérifie que $\bar{\Lambda}^{\prime} \otimes \Lambda_{2}$ n'intervient pas dans $I_{-1} \Lambda^{\prime \prime} \otimes \Lambda_{2}$. Il ne peut intervenir dans $I_{-1} \widetilde{\Lambda}^{\prime \prime} \otimes \widetilde{\Lambda}_{2}^{\prime \prime}$ que si $\widetilde{\Lambda}^{\prime \prime}=\sigma \Lambda^{\prime \prime}, \widetilde{\Lambda}_{2}^{\prime \prime}=\Lambda_{2}$ et $a=1$. Mais alors $\zeta^{\prime \prime}=-1$, les termes s'ajouteraient au lieu de se compenser. Donc $\bar{\Lambda}^{\prime} \otimes \Lambda_{2}$ intervient dans $I_{-1} \widetilde{\Lambda}^{\prime} \otimes \widetilde{\Lambda}_{2}^{\prime}$, ce qui force $\widetilde{\Lambda}^{\prime}=\Lambda^{\prime}, \widetilde{\Lambda}_{2}^{\prime}=\Lambda_{2}$. On détermine ensuite $\widetilde{\Lambda}^{\prime \prime}$ et $\widetilde{\Lambda}_{2}^{\prime \prime}$ comme dans la preuve précédente. 
Supposons vérifiée la relation 11.6(3). Il y a au plus deux triplets vérifiant les conditions de 11.4 pour lesquels la conclusion du lemme 11.5 peut être vérifiée. En posant $t=u_{1}-u_{2}-a$, ce sont $\varphi^{\tau}=\left(u_{1}, \tau, t\right)$, qui existe si et seulement si $u_{2}+a \notin$ $Z$, et $\varphi^{-\tau}=\left(u_{1}-a,-\tau, t\right)$, qui existe si et seulement si $u_{1}-a \in Z$. Supposons $u_{2}+a \notin Z$. En utilisant le triplet $\varphi^{\tau}$, on détermine $\widetilde{\Lambda}(x, \varepsilon)$ et $\widetilde{\Lambda}_{2}(x, \varepsilon)$ pour les $(x, \varepsilon) \in E \backslash \operatorname{Int}\left(\varphi^{\tau}\right)$ tels que la conclusion du lemme 11.5 ne soit pas vérifiée. Cette conclusion impose $(x, \varepsilon)=\left(u_{2}, \tau\right)$. Remarquons que $\operatorname{Int}\left(\varphi^{\tau}\right)$ a au plus un élément, à savoir $\left(u_{1}, \tau\right)$. En effet l'autre élément possible $\left(u_{1}-t-a,-\tau\right)=\left(u_{2},-\tau\right)$ n'appartient pas à $\Lambda$. On a donc $\widetilde{\Lambda}(x, \varepsilon)=\Lambda(x, \varepsilon)$ et $\widetilde{\Lambda}_{2}(x, \varepsilon)=\Lambda_{2}$ pour $(x, \varepsilon) \in E \backslash\left(X^{\tau} \cap E\right)$, où $X^{\tau}=\left\{\left(u_{1}, \tau\right),\left(u_{2}, \tau\right)\right\}$. Si $u_{1}-a \in Z$, en utilisant le triplet $\varphi^{-\tau}$, on obtient la même conclusion pour $(x, \varepsilon) \in E \backslash\left(X^{-\tau} \cap E\right)$, où $X^{-\tau}=\left\{\left(u_{1}-a, \tau\right),\left(u_{2}-a, \tau\right)\right\}$. Par hypothèse, l'une des relations $u_{2}+a \notin Z$ ou $u_{1}-a \in Z$ est vérifiée. Supposons par exemple $u_{2}+a \notin Z$, une démonstration similaire s'appliquant à l'autre cas. Supposons d'abord qu'il existe un triplet $\varphi \neq \varphi^{\tau}$ vérifiant les conditions de 11.4. Si $\varphi=\varphi^{-\tau}$, la conjonction des résultats ci-dessus et le fait que $X^{\tau} \cap X^{-\tau}=\varnothing$ déterminent $\widetilde{\Lambda}(x, \varepsilon)$ et $\widetilde{\Lambda}_{2}(x, \varepsilon)$ pour tout $(x, \varepsilon) \in E$. Si $\varphi \neq \varphi^{-\tau}$, le raisonnement habituel détermine ces termes pour $(x, \varepsilon) \in E \backslash \operatorname{Int}(\varphi)$. Mais $X^{\tau} \cap \operatorname{Int}(\varphi)$ a au plus un élément. De nouveau, un raisonnement déjà fait permet de conclure. Supposons maintenant qu'il n'existe pas d'autre triplet que $\varphi^{\tau}$. Alors nécessairement $u_{2}=u_{1}-2, a=1$,

$$
\Lambda^{\tau}=\left\{u_{1}\right\} \cup\left\{u_{1}-2, \ldots, 0\right\}, \Lambda^{-\tau}=\left\{u_{1}-3, \ldots, 0\right\} .
$$

Puisqu'on a déterminé les termes pour $(x, \varepsilon) \in E \backslash\left(X^{\tau} \cap E\right)$, on a l'égalité 11.6(4), les termes affectés d'un ', resp. ", étant attachés au couple $\left(u_{1}, \tau\right)$, resp. $\left(u_{1}-2, \tau\right)$. Comme dans la première partie de la démonstration, l'application de l'opérateur $I_{-1} \otimes 1$ à l'égalité 11.6(4) conduit à la conclusion.

Supposons enfin vérifiée la relation 11.6(2). Notons $u_{1}>u_{2}>u_{3}$ les éléments de $U$ et $v$ l'unique élément de $V$. Supposons d'abord $u_{2}>v$. Les deux triplets $\varphi_{2}=$ $\left(u_{2}, \tau, u_{2}-v\right), \varphi_{1}=\left(u_{1}, \tau, u_{1}-v\right)$ vérifient les conditions de 11.4. Pour $i \in\{1,2\}$, le raisonnement de 11.6 appliqué à $\varphi_{i}$ détermine $\widetilde{\Lambda}(x, \varepsilon)$ et $\widetilde{\Lambda}_{2}(x, \varepsilon)$ pour les $(x, \varepsilon) \in$ $E \backslash \operatorname{Int}\left(\varphi_{i}\right)$ tels que la conclusion du lemme 11.5 ne soit pas vérifiée. Or cette conclusion impose :

- si $i=1,(x, \varepsilon)=\left(u_{3}, \tau\right)$ et $u_{3}+a=u_{2}$;

- si $i=2,(x, \varepsilon)=\left(u_{3}, \tau\right)$ et $u_{3}+a=u_{1}$.

On a donc déterminé nos termes pour $(x, \varepsilon) \in E \backslash\left(X_{i} \cap E\right)$, où :

$$
\begin{aligned}
& X_{1}= \begin{cases}\left\{\left(u_{1}, \tau\right),(v-a,-\tau)\right\}, & \text { si } u_{3}+a \neq u_{2}, \\
\left\{\left(u_{1}, \tau\right),(v-a,-\tau),\left(u_{3}, \tau\right)\right\}, & \text { si } u_{3}+a=u_{2},\end{cases} \\
& X_{2}= \begin{cases}\left\{\left(u_{2}, \tau\right),(v-a,-\tau)\right\}, & \text { si } u_{3}+a \neq u_{1}, \\
\left\{\left(u_{2}, \tau\right),(v-a,-\tau),\left(u_{3}, \tau\right)\right\}, & \text { si } u_{3}+a=u_{1} .\end{cases}
\end{aligned}
$$


Or $X_{1} \cap X_{2}=\{(v-a,-\tau)\}$. Il reste donc au plus un élément de $E$ à traiter, ce que l'on fait comme en 11.6. Un raisonnement similaire s'applique quand $v>u_{2}$, en considérant les triplets $\left(v,-\tau, v-u_{3}\right),\left(v,-\tau, v-u_{2}\right)$.

\section{8 .}

Lemme. - Supposons $n_{1}-a \geqslant 2$ et $\Lambda$ cuspidal. Alors $i\left(J_{a} \Lambda \otimes \Lambda_{2}\right)-J_{a} \Lambda \otimes \Lambda_{2}$ appartient à $\mathcal{Z}_{1}$.

Démonstration. - Représentons $\Lambda$ de sorte que $\Lambda^{+}=A(y), \Lambda^{-}=A(z)$, où $y, z$ sont des entiers $\geqslant 1$. Supposons par exemple $y \geqslant z$. On a $y-z=\widetilde{\operatorname{def}}(\Lambda)$, qui est pair, et $n_{1}-a=(y-z)^{2} / 4$. Puisque $n_{1}-a \geqslant 2$, on a $y-z \geqslant 4, n_{1}-a \geqslant 4$. Posons :

$$
b= \begin{cases}1, & \text { si } a>1, \\ 2, & \text { si } a=1 .\end{cases}
$$

Introduisons $\Lambda^{\prime} \in \widetilde{\mathcal{S}}_{n_{1}-a-b \text {,pair }}$ tel que $\Lambda^{\prime+}=A(y-1), \Lambda^{\prime-}=\{y-1-b\} \cup A(z)$. On vérifie que $J_{b} \Lambda^{\prime}-\Lambda$, resp. $J_{a} \Lambda^{\prime}$, est combinaison linéaire d'éléments non cuspidaux de $\widetilde{\mathcal{S}}_{n_{1}-a, \text { pair }}$, resp. $\widetilde{\mathcal{S}}_{n_{1}-b, \text { pair. }}$. D'après les lemmes 11.6 et 11.7 , on a donc :

$$
\begin{gathered}
i\left(J_{a}\left(J_{b} \Lambda^{\prime}-\Lambda\right) \otimes \Lambda_{2}\right)-J_{a}\left(J_{b} \Lambda^{\prime}-\Lambda\right) \otimes \Lambda_{2} \in \mathcal{Z}_{1}, \\
i\left(J_{b} J_{a} \Lambda^{\prime} \otimes \Lambda_{2}\right)-J_{b} J_{a} \Lambda^{\prime} \otimes \Lambda_{2} \in \mathcal{Z}_{1} .
\end{gathered}
$$

Puisque $J_{a} J_{b}=J_{b} J_{a}$ d'après le lemme 2.6, on en déduit l'assertion de l'énoncé.

11.9.

LEMME

(a) Supposons $n_{1}-a=0$. Alors $i\left(J_{a} \Lambda \otimes \Lambda_{2}\right)=J_{a} \Lambda \otimes \Lambda_{2}$ ou $J_{a} \Lambda \otimes \sigma \Lambda_{2}$.

(b) Supposons $n_{1}-a=1$ et $\Lambda$ non cuspidal ou $n_{1}-a=1, n_{1} \geqslant 3$ et $a \neq 3$. Alors $i\left(J_{a} \Lambda \otimes \Lambda_{2}\right)=J_{a} \Lambda \otimes \Lambda_{2}$ ou $\sigma J_{a} \Lambda \otimes \Lambda_{2}$.

(c) Supposons $n_{1}=4, a=3$ et $\Lambda$ cuspidal. Alors $i\left(J_{a} \Lambda \otimes \Lambda_{2}\right)-J_{a} \Lambda \otimes \Lambda_{2}$ ou $i\left(J_{a} \Lambda \otimes \Lambda_{2}\right)-\sigma J_{a} \Lambda \otimes \Lambda_{2}$ appartient à $\mathcal{Z}_{1}$.

(d) Supposons $n_{1}=2$, $a=1$ et $\Lambda$ cuspidal. Alors $i\left(J_{a} \Lambda \otimes \Lambda_{2}\right)=J_{a} \Lambda \otimes \Lambda_{2}$ ou $\sigma J_{a} \Lambda \otimes \Lambda_{2}$ ou $J_{a} \Lambda \otimes \sigma \Lambda_{2}$ ou $\sigma J_{a} \Lambda \otimes \sigma \Lambda_{2}$.

Démonstration. - Supposons d'abord $n_{1}-a=0$, donc $\Lambda=\Lambda(\varnothing)$. Si $\Lambda_{2}=\sigma \Lambda_{2}$ on a vu en 11.3 que $i\left(J_{a} \Lambda \otimes \Lambda_{2}\right)=J_{a} \Lambda \otimes \Lambda_{2}$. Supposons $\Lambda_{2} \neq \sigma \Lambda_{2}$. Puisque $\Lambda=\sigma \Lambda$, on a $\widetilde{\Lambda}(x, \varepsilon)=\Lambda(x, \varepsilon)$ et $\widetilde{\Lambda}_{2}(x,-\varepsilon)=\widetilde{\Lambda}_{2}(x, \varepsilon)$ pour tout $(x, \varepsilon) \in E$. Notons $F$ le sousensemble des $(x, \varepsilon) \in E$ tels que $\widetilde{\Lambda}_{2}(x, \varepsilon)=\sigma \Lambda_{2}$. Tout revient à montrer que $F=E$ ou $\varnothing$. Ce qui précède montre que $F$ est stable par l'application $(x, \varepsilon) \mapsto(x,-\varepsilon)$. Posons :

$$
\Lambda_{F}=\sum_{(x, \varepsilon) \in F} \zeta(x, \varepsilon) \Lambda(x, \varepsilon) \text {. }
$$

On a l'égalité :

$$
i\left(J_{a} \Lambda \otimes \Lambda_{2}\right)-J_{a} \Lambda \otimes \Lambda_{2}=\Lambda_{F} \otimes\left(\sigma \Lambda_{2}-\Lambda_{2}\right) .
$$


Le membre de gauche est annulé par l'opérateur $I_{-t} \otimes 1$, pour tout $t \in\left\{1, \ldots, n_{1}\right\}$. Il en résulte que $\Lambda_{F}$ est annulé par l'opérateur $I_{-t}$ pour tout tel $t$. Représentons $\Lambda$ sous la forme $\Lambda=\{m-1, \ldots, 0\} \times\{ \pm 1\}$, pour un entier $m$ assez grand. On a l'égalité $E=\{m-1, \ldots, m-a\} \times\{ \pm 1\}$. Pour $(x, \varepsilon) \in E$, on calcule :

$$
\begin{aligned}
\Lambda(x, \varepsilon)^{\varepsilon} & =\{m-1, \ldots, x+1\} \cup\{x-1, \ldots, 0\}, \\
\Lambda(x, \varepsilon)^{-\varepsilon} & =\{x+a\} \cup\{m-1, \ldots, 0\}, \zeta(x, \varepsilon)=(-1)^{m+x-1} .
\end{aligned}
$$

Soit $y \in\{m-2, \ldots, m-a\}$, définissons $\Lambda_{y} \in \widetilde{\mathcal{S}}_{n_{1}-1 \text {,pair par }}$

$$
\Lambda_{y}^{+}=\{m-1, \ldots, y+2\} \cup\{y, \ldots, 0\}, \quad \Lambda_{y}^{-}=\{y+a\} \cup\{m-1, \ldots, 0\} .
$$

On vérifie les égalités :

$$
\begin{aligned}
& \left(I_{1} \Lambda_{y}, \Lambda(y, 1)\right)=\left(I_{1} \Lambda_{y}, \Lambda(y+1,1)\right)=1, \\
& \left(I_{1} \Lambda_{y}, \Lambda(x, \varepsilon)\right)=0 \text { pour tout }(x, \varepsilon) \in E \backslash\{(y, 1),(y+1,1)\} .
\end{aligned}
$$

En notant $1_{F}$ la fonction caractéristique de $F$ dans $E$, on en déduit :

$$
\left(I_{-1} \Lambda_{F}, \Lambda_{y}\right)=(-1)^{m+y-1}\left(1_{F}(y, 1)-1_{F}(y+1,1)\right) .
$$

La nullité de cette expression signifie que $(y, 1) \in F \Leftrightarrow(y+1,1) \in F$. Cela étant vrai pour tout $y \in\{m-2, \ldots, m-a\}$, on en déduit que $F=E$ ou $\varnothing$.

Supposons maintenant $n_{1}-a=1$. Par un argument de symétrie, on peut supposer $\Lambda=\mathbf{1}_{+}(1)$ ou $\Lambda=\mathbf{1}_{-}$(1). Supposons d'abord $\Lambda=\mathbf{1}_{+}$(1). Remarquons que $\Lambda+\sigma \Lambda=$ $I_{1} \Lambda(\varnothing)$. En utilisant le lemme 2.6 et les propriétés de $i$, on a les égalités :

$$
\begin{aligned}
i\left(J_{a}(\Lambda+\sigma \Lambda) \otimes \Lambda_{2}\right) & =i\left(J_{a} I_{1} \Lambda(\varnothing) \otimes \Lambda_{2}\right)=i\left(I_{1} J_{a} \Lambda(\varnothing) \otimes \Lambda_{2}\right) \\
& =I_{1} J_{a} \Lambda(\varnothing) \otimes \Lambda_{2}=J_{a} I_{1} \Lambda(\varnothing) \otimes \Lambda_{2}=J_{a}(\Lambda+\sigma \Lambda) \otimes \Lambda_{2} .
\end{aligned}
$$

En utilisant les propriétés de commutation à $\sigma$ de nos différents opérateurs, l'égalité des termes extrêmes ci-dessus s'écrit :

$\sum_{(x, \varepsilon) \in E} \zeta(x, \varepsilon)(\Lambda(x, \varepsilon)+\sigma(\Lambda(x, \varepsilon))) \otimes \widetilde{\Lambda}_{2}(x, \varepsilon)=\sum_{(x, \varepsilon) \in E} \zeta(x, \varepsilon)(\Lambda(x, \varepsilon)+\sigma(\Lambda(x, \varepsilon))) \otimes \Lambda_{2}$.

Grâce à $11.2(1)$, cela entraîne $\widetilde{\Lambda}_{2}(x, \varepsilon)=\Lambda_{2}$ pour tout $(x, \varepsilon) \in E$. Notons $F$ le sousensemble des $(x, \varepsilon) \in E$ tels que $\widetilde{\Lambda}(x, \varepsilon)=\sigma(\Lambda(x, \varepsilon)) \neq \Lambda(x, \varepsilon)$. De nouveau, il suffit de prouver que $F=E$ ou $\varnothing$. Posons :

$$
\Lambda_{F}=\sum_{(x, \varepsilon) \in F} \zeta(x, \varepsilon)(\sigma(\Lambda(x, \varepsilon))-\Lambda(x, \varepsilon)) .
$$

On a l'égalité :

$$
i\left(J_{a} \Lambda \otimes \Lambda_{2}\right)-J_{a} \Lambda \otimes \Lambda_{2}=\Lambda_{F} \otimes \Lambda_{2} .
$$

On en déduit encore que $\Lambda_{F}$ est annulé par l'opérateur $I_{-t}$ pour tout $t \in\left\{1, \ldots, n_{1}\right\}$. Représentons $\Lambda$ sous la forme $\Lambda^{+}=\{m\} \cup\{m-2, \ldots, 0\}, \Lambda^{-}=\{m-1, \ldots, 0\}$, avec 
$m$ assez grand. Les éléments de $E$ sont les $(x, 1)$ pour $x \in\{m\} \cup\{m-2, \ldots, m-a\}$ et les $(x,-1)$ pour $x \in\{m-1, \ldots, m+1-a\} \cup\{m-1-a\}$. On a les égalités :

$$
\Lambda(m, 1)^{+}=\{m-2, \ldots, 0\}, \Lambda(m, 1)^{-}=\{m+a\} \cup\{m-1, \ldots, 0\}, \quad \zeta(m, 1)=1 ;
$$

pour $x \in\{m-2, \ldots, m-a\}$,

$$
\begin{aligned}
& \Lambda(x, 1)^{+}=\{m\} \cup\{m-2, \ldots, x+1\} \cup\{x-1, \ldots, 0\}, \\
& \Lambda(x, 1)^{-}=\{x+a\} \cup\{m-1, \ldots, 0\}, \quad \zeta(x, 1)=(-1)^{m+x+1} ;
\end{aligned}
$$

pour $x \in\{m-1, \ldots, m+1-a\}$,

$$
\begin{aligned}
& \Lambda(x,-1)^{+}=\{x+a\} \cup\{m\} \cup\{m-2, \ldots, 0\}, \\
& \Lambda(x,-1)^{-}=\{m-1, \ldots, x+1\} \cup\{x-1, \ldots, 0\}, \quad \zeta(x,-1)=(-1)^{m+x+1} ;
\end{aligned}
$$$$
\Lambda(m-1-a,-1)^{+}=\{m, \ldots, 0\},
$$$$
\Lambda(m-1-a,-1)^{-}=\{m-1, \ldots, m-a\} \cup\{m-a-2, \ldots, 0\},
$$$$
\zeta(m-1-a,-1)=(-1)^{a+1} .
$$

Soit $y \in\{m-1, \ldots, m-a\}$, posons :

$$
y^{+}=\left\{\begin{array}{ll}
y, & \text { si } y \neq m-1 \\
m, & \text { si } y=m-1
\end{array} \quad y^{-}= \begin{cases}y, & \text { si } y \neq m-a \\
m-1-a, & \text { si } y=m-a\end{cases}\right.
$$

et définissons $\Lambda_{y}^{\prime} \in \widetilde{\mathcal{S}}_{n_{1}-1, \text { pair }}$ par

$$
\Lambda_{y}^{\prime+}=\{m-1, \ldots, y+1\} \cup\{y-1, \ldots, 0\}, \quad \Lambda_{y}^{\prime-}=\{y+a\} \cup\{m-1, \ldots, 0\} .
$$

On vérifie les égalités :

$$
\begin{aligned}
\left(I_{1} \Lambda_{y}^{\prime}, \sigma\left(\Lambda\left(y^{+}, 1\right)\right)-\Lambda\left(y^{+}, 1\right)\right) & =-1,\left(I_{1} \Lambda_{y}^{\prime}, \sigma\left(\Lambda\left(y^{-},-1\right)\right)-\Lambda\left(y^{-},-1\right)\right)=1, \\
\left(I_{1} \Lambda_{y}^{\prime}, \sigma(\Lambda(x, \varepsilon))-\Lambda(x, \varepsilon)\right) & =0 \text { pour tout }(x, \varepsilon) \in E \backslash\left\{\left(y^{+}, 1\right),\left(y^{-},-1\right)\right\} .
\end{aligned}
$$

On en déduit :

$$
\left(I_{-1} \Lambda_{F}, \Lambda_{y}^{\prime}\right)=(-1)^{m+y}\left(1_{F}\left(y^{+}, 1\right)-1_{F}\left(y^{-},-1\right)\right),
$$

où $1_{F}$ est défini comme précédemment. La nullité de cette expression signifie que :

$$
\left(y^{+}, 1\right) \in F \Longleftrightarrow\left(y^{-},-1\right) \in F .
$$

Si $a=1, E$ est réduit à $\left\{\left((m-1)^{+}, 1\right),\left((m-1)^{-},-1\right)\right\}$ et on conclut $F=E$ ou $\varnothing$. Supposons $a \geqslant 2$, soit $z \in\{m-2, \ldots, m-a\}$, définissons $\Lambda_{z}^{\prime \prime} \in \widetilde{\mathcal{S}}_{n_{1}-m+z \text {,pair par }}$ $\Lambda_{z}^{\prime \prime+}=\{m-2, \ldots, 0\}, \Lambda_{z}^{\prime \prime-}=\{z+a\} \cup\{m-1, \ldots, 0\}$. On calcule :

$$
\begin{aligned}
\left(I_{m-z} \Lambda_{z}^{\prime \prime}, \sigma(\Lambda(m, 1))-\Lambda(m, 1)\right) & =-1, \\
\left(I_{m-z} \Lambda_{z}^{\prime \prime}, \sigma(\Lambda(z, 1))-\Lambda(z, 1)\right) & =(-1)^{m+z+1} ;
\end{aligned}
$$

- si $z \neq m-a$,

$$
\left(I_{m-z} \Lambda_{z}^{\prime \prime}, \sigma(\Lambda(x, \varepsilon))-\Lambda(x, \varepsilon)\right)=0
$$

pour tout $(x, \varepsilon) \in E \backslash\{(m, 1),(z, 1)\}$; 
- si $z=m-a$,

$$
\begin{aligned}
\left(I_{-a} \Lambda_{z}^{\prime \prime}, \sigma(\Lambda(m-1,-1))-\Lambda(m-1,-1)\right) & =-1, \\
\left(I_{-a} \Lambda_{z}^{\prime \prime}, \sigma(\Lambda(m-1-a,-1))-\Lambda(m-1-a,-1)\right) & =(-1)^{a+1}, \\
\left(I_{-a} \Lambda_{z}^{\prime \prime}, \sigma(\Lambda(x, \varepsilon))-\Lambda(x, \varepsilon)\right) & =0
\end{aligned}
$$

pour tout $(x, \varepsilon) \in E \backslash\{(m, 1),(m-a, 1),(m-1,-1),(m-1-a,-1)\}$.

On en déduit :

$$
\begin{aligned}
& \text { - si } z \neq m-a,\left(I_{z-m} \Lambda_{F}, \Lambda_{z}^{\prime \prime}\right)=-1_{F}(m, 1)+1_{F}(z, 1), \\
& \text { - si } z=m-a, \\
& \left(I_{-a} \Lambda_{F}, \Lambda_{z}^{\prime \prime}\right)=-1_{F}(m, 1)+1_{F}(m-a, 1)-1_{F}(m-1,-1)+1_{F}(m-1-a,-1) .
\end{aligned}
$$

Remarquons que, grâce à (2),

$$
1_{F}(m, 1)=1_{F}(m-1,-1) \quad \text { et } \quad 1_{F}(m-a, 1)=1_{F}(m-1-a,-1) .
$$

La nullité des expressions ci-dessus signifie que $(m, 1) \in F \Leftrightarrow(z, 1) \in F$. De cette relation et de (2) se déduit que $F=E$ ou $\varnothing$.

Supposons enfin $n_{1}-a=1$ et $\Lambda=\mathbf{1}_{-}(1)$. Représentons $\Lambda$ sous la forme :

$$
\Lambda^{+}=\{m, \ldots, 0\}, \Lambda^{-}=\{m-2, \ldots, 0\} .
$$

On a les égalités :

$$
\begin{aligned}
E= & (\{m, \ldots, m-1-a\} \times\{1\}) \cup(\{m-2, \ldots, m+1-a\} \times\{-1\}) ; \\
- \text { pour } x \in\{m, \ldots, m-1-a\}, & \\
& \Lambda(x, 1)^{+}=\{m, \ldots, x+1\} \cup\{x-1, \ldots, 0\}, \\
& \Lambda(x, 1)^{-}=\{x+a\} \cup\{m-2, \ldots, 0\}, \quad \zeta(x, 1)=(-1)^{x+m} ; \\
- & \text { pour } x \in\{m-2, \ldots, m+1-a\}, \\
& \Lambda(x,-1)^{+}=\{x+a\} \cup\{m, \ldots, 0\}, \\
& \Lambda(x,-1)^{-}=\{m-2, \ldots, x+1\} \cup\{x-1, \ldots, 0\}, \quad \zeta(x,-1)=(-1)^{x+m} .
\end{aligned}
$$

Supposons d'abord $a=1$. Alors $E$ n'a que 3 éléments, il est facile d'étudier toutes les possibilités. On voit que le fait que $i\left(J_{1} \Lambda \otimes \Lambda_{2}\right)-J_{1} \Lambda \otimes \Lambda_{2}$ est annulé par l'opérateur $I_{-1} \otimes 1$ entraîne les assertions de l'énoncé.

Supposons maintenant $a \geqslant 2$. Remarquons que $\Lambda(x, \varepsilon) \neq \sigma(\Lambda(x, \varepsilon))$ pour tout $(x, \varepsilon) \in E$. Notons $E_{c}$ le sous-ensemble des $(x, \varepsilon) \in E$ tels que $\Lambda(x, \varepsilon)$ est cuspidal. On a $E_{c}=\varnothing$, sauf si $a=3$, auquel cas $E_{c}=\{(m-2,-1)\}$. On a l'égalité $\Lambda+\sigma \Lambda=$ $J_{1} \Lambda(\varnothing)$, donc :

$$
J_{a}(\Lambda+\sigma \Lambda) \otimes \Lambda_{2}=J_{1} J_{a} \Lambda(\varnothing) \otimes \Lambda_{2} .
$$

Parce que $n_{1} \geqslant 3$, on peut appliquer les lemmes 11.6, 11.7 et 11.8 à cette dernière expression. On en déduit que $i\left(J_{a}(\Lambda+\sigma \Lambda) \otimes \Lambda_{2}\right)-J_{a}(\Lambda+\sigma \Lambda) \otimes \Lambda_{2}$ appartient à $\mathcal{Z}_{1}$. Comme dans le cas $\Lambda=\mathbf{1}_{+}(1)$, cela entraîne $\widetilde{\Lambda}_{2}(x, \varepsilon)=\Lambda_{2}$ pour tout $(x, \varepsilon) \in E \backslash E_{c}$. 
Notons $F$ le sous-ensemble des $(x, \varepsilon) \in E \backslash E_{c}$ tels que $\widetilde{\Lambda}(x, \varepsilon)=\sigma(\Lambda(x, \varepsilon))$. Il suffit de prouver que $F=E \backslash E_{c}$ ou $\varnothing$. Définissons $\Lambda_{F}$ par l'égalité (1). On a l'égalité :

(3) $i\left(J_{a} \Lambda \otimes \Lambda_{2}\right)-J_{a} \Lambda \otimes \Lambda_{2}=\Lambda_{F} \otimes \Lambda_{2}+\sum_{(x, \varepsilon) \in E_{c}} \zeta(x, \varepsilon)\left(\widetilde{\Lambda}(x, \varepsilon) \otimes \widetilde{\Lambda}_{2}(x, \varepsilon)-\Lambda(x, \varepsilon) \otimes \Lambda_{2}\right)$.

Soit $z \in\{m-1, \ldots, m-a\}$, posons :

$$
\left(z^{\prime \prime}, \varepsilon^{\prime \prime}\right)= \begin{cases}(m, 1), & \text { si } z=m-1 \\ (z,-1), & \text { si } z \neq m-1, m-a \\ (m-1-a, 1), & \text { si } z=m-a\end{cases}
$$

et définissons $\Lambda_{z}^{\prime \prime} \in \widetilde{\mathcal{S}}_{n_{1}-1, \text { pair }}$ par

$$
\Lambda_{z}^{\prime \prime+}=\{m-1, \ldots, z+1\} \cup\{z-1, \ldots, 0\}, \quad \Lambda_{z}^{\prime \prime-}=\{z+a\} \cup\{m-1, \ldots, 0\} .
$$

Puisque $n_{1} \geqslant 3$, on peut appliquer à $J_{1} \Lambda_{z}^{\prime \prime} \otimes \Lambda_{2}$ l'un des lemmes 11.6 ou 11.7. Donc $i\left(J_{1} \Lambda_{z}^{\prime \prime} \otimes \Lambda_{2}\right)-J_{1} \Lambda_{z}^{\prime \prime} \otimes \Lambda_{2}$ appartient à $\mathcal{Z}_{1}$. Supposons $a \neq 3$. Alors $E_{c}=\varnothing$ et la propriété précédente entraîne que $i\left(J_{1} \Lambda_{z}^{\prime \prime} \otimes \Lambda_{2}\right)-J_{1} \Lambda_{z}^{\prime \prime} \otimes \Lambda_{2}$ est orthogonal à $i\left(J_{a} \Lambda \otimes\right.$ $\left.\Lambda_{2}\right)$. Il revient au même de dire que $J_{1} \Lambda_{z}^{\prime \prime} \otimes \Lambda_{2}$ est orthogonal au membre de gauche de (3). Toujours parce que $E_{c}=\varnothing$, cela entraîne que $J_{1} \Lambda_{z}^{\prime \prime}$ est orthogonal à $\Lambda_{F}$. On calcule :

$$
\begin{gathered}
\left(J_{1} \Lambda_{z}^{\prime \prime}, \sigma(\Lambda(z, 1)) \Lambda(z, 1)\right)=1,\left(J_{1} \Lambda_{z}^{\prime \prime}, \sigma\left(\Lambda\left(z^{\prime \prime}, \varepsilon^{\prime \prime}\right)\right)-\Lambda\left(z^{\prime \prime}, \varepsilon^{\prime \prime}\right)\right)=\varepsilon^{\prime \prime}, \\
\left(J_{1} \Lambda_{z}^{\prime \prime}, \sigma(\Lambda(x, \varepsilon))-\Lambda(x, \varepsilon)\right)=0, \text { pour tout }(x, \varepsilon) \in E \backslash\left\{(z, 1),\left(z^{\prime \prime}, \varepsilon^{\prime \prime}\right)\right\} .
\end{gathered}
$$

D'où :

$$
\left(J_{1} \Lambda_{z}^{\prime \prime}, \Lambda_{F}\right)=(-1)^{m+z}\left(1_{F}(z, 1)-1_{F}\left(z^{\prime \prime}, \varepsilon^{\prime \prime}\right)\right) .
$$

La nullité de cette expression entraîne :

$$
\text { si } a \neq 3, \quad(z, 1) \in F \Longleftrightarrow\left(z^{\prime \prime}, \varepsilon^{\prime \prime}\right) \in F \text {. }
$$

Considérons l'égalité (3). Le membre de gauche est annulé par les opérateurs $I_{-t} \otimes 1$ pour tout $t \in\left\{1, \ldots, n_{1}\right\}$. Il en est de même de la somme du membre de droite, par définition de $E_{c}$. Donc $\Lambda_{F}$ est annulé par l'opérateur $I_{-t}$ pour tout tel $t$.

Supposons $a=2$. Définissons $\Lambda^{\prime} \in \widetilde{\mathcal{S}}_{n_{1}-2 \text {,pair par }}$

$$
\Lambda^{\prime+}=\{m-1, \ldots, 0\}, \quad \Lambda^{\prime-}=\{m\} \cup\{m-2, \ldots, 0\} .
$$

On vérifie les égalités :

$$
\left(I_{2} \Lambda^{\prime}, \sigma(\Lambda(x, \varepsilon))-\Lambda(x, \varepsilon)\right)= \begin{cases}-1, & \text { si }(x, \varepsilon)=(m, 1) \text { ou }(x, \varepsilon)=(m-3,1) \\ 1, & \text { si }(x, \varepsilon)=(m-1,1) \text { ou }(x, \varepsilon)=(m-2,1)\end{cases}
$$

Alors :

$$
\left(\Lambda^{\prime}, I_{-2} \Lambda_{F}\right)=-1_{F}(m, 1)-1_{F}(m-1,1)+1_{F}(m-2,1)+1_{F}(m-3,1) .
$$


Grâce à $(4), 1_{F}(m, 1)=1_{F}(m-1,1), 1_{F}(m-2,1)=1_{F}(m-3,1)$. La nullité de l'expression ci-dessus entraine que $1_{F}$ est constante sur $E$, autrement dit $F=E$ ou $\varnothing$.

Supposons enfin $a \geqslant 3$. Soit $y \in\{m-1, \ldots, m-1-a\}$, définissons $\Lambda_{y}^{\prime} \in \widetilde{\mathcal{S}}_{n_{1}-1 \text {,pair }}$ par

$$
\Lambda_{y}^{\prime+}=\{m, \ldots, y+2\} \cup\{y, \ldots, 0\}, \quad \Lambda_{y}^{\prime-}=\{y+a\} \cup\{m-2, \ldots, 0\} .
$$

On vérifie les égalités :

$$
\begin{aligned}
& \left(I_{1} \Lambda_{y}^{\prime}, \sigma(\Lambda(y, 1))-\Lambda(y, 1)\right)=\left(I_{1} \Lambda_{y}^{\prime}, \sigma(\Lambda(y+1,1))-\Lambda(y+1,1)\right)=-1, \\
& \left(I_{1} \Lambda_{y}^{\prime}, \sigma(\Lambda(x, \varepsilon))-\Lambda(x, \varepsilon)\right)=0, \text { pour tout }(x, \varepsilon) \in E \backslash\{(y, 1),(y+1,1)\} .
\end{aligned}
$$

On en déduit :

$$
\left(\Lambda_{y}^{\prime}, I_{-1} \Lambda_{F}\right)=(-1)^{m+y+1}\left(1_{F}(y, 1)-1_{F}(y+1,1)\right) .
$$

La nullité de cette expression entraîne que $(y, 1) \in F \Leftrightarrow(y+1,1) \in F$. Si $a=3$, $E \backslash E_{c}$ est justement l'ensemble des $(x, \varepsilon) \in E$ tels que $\varepsilon=1$. L'équivalence ci-dessus entraîne que $F=E \backslash E_{c}$ ou $\varnothing$. Si $a \geqslant 4$, cette équivalence et (4) entraînent que $F=E$ ou $\varnothing$.

\subsection{0 .}

\section{LEMME}

(a) Supposons que $\Lambda_{2}$ n'est pas cuspidal et $n_{2} \geqslant 2$. Alors $i\left(J_{a} \Lambda \otimes \Lambda_{2}\right)=J_{a} \Lambda \otimes \Lambda_{2}$.

(b) Supposons que $\Lambda_{2}$ n'est pas cuspidal et $n_{2}=1$. Alors $\widetilde{\Lambda}(x, \varepsilon)=\Lambda(x, \varepsilon)$ pour tout $(x, \varepsilon) \in E$.

Démonstration. - Soit $t \in\left\{1, \ldots, n_{2}\right\}$. L'opérateur $1 \otimes I_{-t}$ annule $i\left(J_{a} \Lambda \otimes \Lambda_{2}\right)-$ $J_{a} \Lambda \otimes \Lambda_{2}$. Grâce à 11.2(1), cela entraîne que pour tout $(x, \varepsilon) \in E$, on a l'égalité :

$$
\widetilde{\Lambda}(x, \varepsilon) \otimes I_{-t} \widetilde{\Lambda}_{2}(x, \varepsilon)=\Lambda(x, \varepsilon) \otimes I_{-t} \Lambda_{2} .
$$

Puisque $\Lambda_{2}$ n'est pas cuspidal, cette égalité se scinde en les deux égalités :

$$
\widetilde{\Lambda}(x, \varepsilon)=\Lambda(x, \varepsilon), I_{-t} \widetilde{\Lambda}_{2}(x, \varepsilon)=I_{-t} \Lambda_{2} .
$$

Si $n_{2}=1$, on a terminé. Sinon, la dernière égalité, vraie pour tout $t$, jointe au fait que $\widetilde{\Lambda}_{2}(x, \varepsilon)=\Lambda_{2}$ ou $\sigma \Lambda_{2}$, entraîne que $\widetilde{\Lambda}_{2}(x, \varepsilon)=\Lambda_{2}$ grâce au lemme 9.4.

11.11. On note $\mathcal{Z}$ le sous-espace des éléments de $\mathbb{C}\left[\widetilde{\mathcal{S}} \widetilde{\mathcal{S}}_{n, \text { pair }}\right]$ qui sont combinaisons linéaires de termes $\Lambda_{1}^{\prime} \otimes \Lambda_{2}^{\prime}$ tels que $\Lambda_{1}^{\prime}$ et $\Lambda_{2}^{\prime}$ soient cuspidaux.

Proposition. - Si $\Lambda_{2}$ n'est pas cuspidal, on a l'égalité $i\left(J_{a} \Lambda \otimes \Lambda_{2}\right)=J_{a} \Lambda \otimes \Lambda_{2}$. Si $\Lambda_{2}$ est cuspidal, $i\left(J_{a} \Lambda \otimes \Lambda_{2}\right)-J_{a} \Lambda \otimes \Lambda_{2}$ appartient à $\mathcal{Z}$. 
Démonstration. - Si $\Lambda_{2}$ n'est pas cuspidal et $n_{2} \geqslant 2$, cela résulte du lemme précédent.

Supposons $\Lambda_{2}$ non cuspidal et $n_{2}=1$. Par un argument de symétrie, on peut supposer $\Lambda_{2}=\mathbf{1}_{+}(1)$. D'après le lemme précédent, on a déjà $\widetilde{\Lambda}(x, \varepsilon)=\Lambda(x, \varepsilon)$ pour tout $(x, \varepsilon) \in E$. Supposons $n_{1}-a \geqslant 2$. Les lemmes $11.6,11.7$ et 11.8 entraînent l'égalité $\widetilde{\Lambda}_{2}(x, \varepsilon)=\mathbf{1}_{+}(1)$ pour tout $(x, \varepsilon) \in E$ tel que $\Lambda(x, \varepsilon)$ ne soit pas cuspidal. Or il y a au plus un élément $(x, \varepsilon)$ tel que $\Lambda(x, \varepsilon)$ soit cuspidal. Si l'énoncé n'est pas vérifié, cet élément existe et, en le notant $\left(x_{c}, \varepsilon_{c}\right)$, on a $\widetilde{\Lambda}_{2}\left(x_{c}, \varepsilon_{c}\right)=\sigma \mathbf{1}_{+}(1)$. En posant $Z=\Lambda\left(x_{c}, \varepsilon_{c}\right)$ et $Z_{2}=\sigma \mathbf{1}_{+}(1)-\mathbf{1}_{+}(1)$, on a l'égalité :

$$
i\left(J_{a} \Lambda \otimes \mathbf{1}_{+}(1)\right)-J_{a} \Lambda \otimes \mathbf{1}_{+}(1)=Z \otimes Z_{2},
$$

que l'on peut symétriser en :

$$
J_{a} \Lambda \otimes Z_{2}-i\left(J_{a} \Lambda \otimes Z_{2}\right)=2 Z \otimes Z_{2} .
$$

En revenant à la définition de l'application $i$, c'est équivalent à :

$$
\pi_{n}\left(J_{a} \Lambda \otimes Z_{2}\right)-k_{n}\left(\mathcal{F} J_{a} \Lambda \otimes \mathcal{F} Z_{2}\right)=\pi_{n}\left(2 Z \otimes Z_{2}\right) .
$$

Posons $\eta=(-1)^{1+\widetilde{\operatorname{def}}(\Lambda) / 2}=(-1)^{\widetilde{\operatorname{def}}(Z) / 2}$. Soit $g$ un élément semi-simple de $O_{\eta}(2 n)$ ayant pour valeurs propres 1 , avec la multiplicité $2 n-1$, et -1 , avec la multiplicité 1 . Posons :

$$
c=2|S O(2 n-1)|_{p^{\prime}}\left|O_{\eta}(2 n-2)\right|_{p^{\prime}}^{-1} \operatorname{sp}(g)
$$

et notons $1_{\eta}$ l'élément neutre de $O_{\eta}(2 n-2)$. On calcule $\mathcal{F} Z_{2}=\mathbf{1}_{-}(1)+\sigma \mathbf{1}_{-}(1)$. Grâce aux lemmes 7.3 et 10.1, on a les égalités :

$$
\begin{aligned}
k_{n}\left(\mathcal{F} J_{a} \Lambda \otimes \mathcal{F} Z_{2}\right)(g) & =c k_{n-1}\left(\mathcal{F} J_{a} \Lambda \otimes \Lambda(\varnothing)\right)\left(1_{\eta}\right), \\
\pi_{n}\left(J_{a} \Lambda \otimes Z_{2}\right)(g) & =c \pi_{n-1}\left(J_{a} \Lambda \otimes \Lambda(\varnothing)\right)\left(1_{\eta}\right) .
\end{aligned}
$$

D'après l'hypothèse de récurrence, , $k_{n-1}\left(\mathcal{F} J_{a} \Lambda \otimes \Lambda(\varnothing)\right)=\pi_{n-1}\left(J_{a} \Lambda \otimes \Lambda(\varnothing)\right)$. Les deux expressions ci-dessus sont égales. L'égalité (2) entraîne $\pi_{n}\left(2 Z \otimes Z_{2}\right)(g)=$ 0 . Or, toujours d'après le lemme 10.1, le membre de gauche de cette égalité vaut $2 c \operatorname{dim}(\pi(Z, \Lambda(\varnothing))$, qui n'est pas nul. Cette contradiction démontre l'énoncé, sous nos hypothèses.

Supposons toujours $\Lambda_{2}=\mathbf{1}_{+}$(1) et maintenant $n_{1}-a=1$ et $\Lambda$ non cuspidal ou $n_{1}-a=1, n_{1} \geqslant 3$ et $\Lambda$ cuspidal. De nouveau, d'après le lemme 11.9 , il y a au plus un élément $(x, \varepsilon) \in E$ tel que $\widetilde{\Lambda}(x, \varepsilon)=\sigma \mathbf{1}_{+}(1)$ et le raisonnement ci-dessus s'applique.

Supposons maintenant $\Lambda_{2}=\mathbf{1}_{+}$(1) et $n_{1}-a=0$, ou $n_{1}=2, a=1$ et $\Lambda$ cuspidal. Le lemme 11.9 entraîne que la fonction $(x, \varepsilon) \mapsto \widetilde{\Lambda}_{2}(x, \varepsilon)$ est constante sur $E$. Si elle vaut $\Lambda_{2}$, c'est terminé. Sinon, on a l'égalité $i\left(J_{a} \Lambda \otimes \mathbf{1}_{+}(1)\right)=J_{a} \Lambda \otimes \sigma \mathbf{1}_{+}(1)$, et, en posant $Z=2 J_{a} \Lambda$, on a encore l'égalité (1). Le même raisonnement que ci-dessus conduit à une contradiction, pourvu que $\pi_{n-1}(Z \otimes \Lambda(\varnothing))\left(1_{\eta}\right) \neq 0$. Vérifions cette relation. 
Grâce au corollaire 9.9, on a l'égalité :

$$
\pi_{n-1}(Z \otimes \Lambda(\varnothing))\left(1_{\eta}\right)=\left|O_{\eta}(2 n-2)\right|_{p^{\prime}} \sum_{(x, \varepsilon) \in E} \zeta(x, \varepsilon) \widetilde{D}_{\Lambda(x, \varepsilon)}(q) .
$$

Pour tout entier $m \in \mathbb{N}$, définissons le polynôme $c_{m}(T)=\prod_{h=1, \ldots, m}\left(T^{h}-1\right)$. Supposons $n_{1}-a=0$, donc $\Lambda=\Lambda(\varnothing)$. On a décrit les termes $\Lambda(x, \varepsilon)$ dans la preuve du lemme 11.9. Avec les notations de ce lemme et grâce aux formules de 9.5 , on calcule, pour $y \in\{0, \ldots, a-1\}$ et $\varepsilon \in\{ \pm 1\}$ :

$$
\zeta(m-1-y, \varepsilon)=(-1)^{y}, \widetilde{D}_{\Lambda(m-1-y, \varepsilon)}=\frac{1}{2} q^{y(y+1)} c_{y}\left(q^{2}\right)^{-1} c_{a-1-y}\left(q^{2}\right)^{-1} .
$$

Le membre de droite de (3) est égal à :

$$
\left|O_{-}(2 n-2)\right|_{p^{\prime}} \sum_{y=0, \ldots, a-1}(-1)^{y} q^{y(y+1)} c_{y}\left(q^{2}\right)^{-1} c_{a-1-y}\left(q^{2}\right)^{-1} .
$$

Grâce à $[\mathbf{Z}]$, p. 119 , ceci vaut $(-1)^{a-1}\left|O_{-}(2 n-2)\right|_{p^{\prime}}$, qui est non nul comme on le voulait. Un calcul similaire vaut dans le cas $n_{1}=2, a=1$ et $\Lambda$ cuspidal, où l'on obtient $\left|O_{+}(4)\right|_{p^{\prime}}(q-1)^{-2}$. Cela achève la preuve dans le cas où $\Lambda_{2}$ n'est pas cuspidal.

Supposons $\Lambda_{2}$ cuspidal. Si $n_{1}=1$, il n'y a rien à démontrer : on a $a=1, \Lambda=\Lambda(\varnothing)$, $J_{1} \Lambda=\mathbf{1}_{-}(1)+\sigma \mathbf{1}_{-}(1)$ qui est une somme de termes cuspidaux. Si $n_{1}-a \geqslant 2$, les lemmes 11.6, 11.7 et 11.8 entraînent l'énoncé. Supposons $n_{1} \geqslant 2$ et $n_{2} \geqslant 2$. Posons $h=\widetilde{\operatorname{def}}\left(\Lambda_{2}\right) / 2$, supposons par exemple $h \geqslant 0$. Puisque $n_{2}=h^{2}$, on a $h \geqslant 2$.

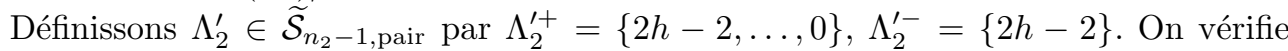
que $\left(\Lambda_{2}, J_{1} \Lambda_{2}^{\prime}\right)=1,\left(\sigma \Lambda_{2}, J_{1} \Lambda_{2}^{\prime}\right)=0$. Soit $(x, \varepsilon) \in E$, supposons $\Lambda(x, \varepsilon)$ non cuspidal. Appliquons le lemme 11.10 en échangeant les rôles des indices 1 et 2 . On obtient :

$$
i\left(\Lambda(x, \varepsilon) \otimes J_{1} \Lambda_{2}^{\prime}\right)=\Lambda(x, \varepsilon) \otimes J_{1} \Lambda_{2}^{\prime} .
$$

Parce que $i$ est une isométrie, cela entraîne :

$$
\left(i\left(J_{a} \Lambda \otimes \Lambda_{2}\right), \Lambda(x, \varepsilon) \otimes J_{1} \Lambda_{2}^{\prime}\right)=\left(J_{a} \Lambda \otimes \Lambda_{2}, \Lambda(x, \varepsilon) \otimes J_{1} \Lambda_{2}^{\prime}\right) .
$$

Le membre de droite vaut $\zeta(x, \varepsilon)$. Grâce à $11.2(1)$, celui de gauche vaut $\zeta(x, \varepsilon)(\widetilde{\Lambda}(x, \varepsilon) \otimes$ $\left.\widetilde{\Lambda}_{2}(x, \varepsilon), \Lambda(x, \varepsilon) \otimes \Lambda_{2}\right)$. D'où les égalités $\widetilde{\Lambda}(x, \varepsilon)=\Lambda(x, \varepsilon), \widetilde{\Lambda}_{2}(x, \varepsilon)=\Lambda_{2}$.

Supposons $n_{1} \geqslant 2, n_{1}-a \leqslant 1$ et $n_{2}=1$. Par un argument de symétrie, on peut supposer $\Lambda_{2}=\mathbf{1}_{-}(1)$. Soit $(x, \varepsilon) \in E$ tel que $\Lambda(x, \varepsilon)$ ne soit pas cuspidal. Remarquons que $J_{1} \Lambda(\varnothing)=\mathbf{1}_{-}(1)+\sigma \mathbf{1}_{-}(1)$. Le même calcul que ci-dessus conduit à l'égalité $\zeta(x, \varepsilon)=\zeta(x, \varepsilon)(\widetilde{\Lambda}(x, \varepsilon), \Lambda(x, \varepsilon))$, donc $\widetilde{\Lambda}(x, \varepsilon)=\Lambda(x, \varepsilon)$. Si $n_{1}-a=1$ et $\Lambda$ n'est pas cuspidal, ou si $n_{1}-a=1$ et $n_{1} \geqslant 3$, on a aussi $\Lambda_{2}(x, \varepsilon)=\mathbf{1}_{-}$(1) d'après le lemme 11.9. Supposons $n_{1}-a=0$ ou $n_{1}=2, a=1$ et $\Lambda$ est cuspidal. D'après ce lemme 11.9 et ce que l'on vient de démontrer, on a $i\left(J_{a} \Lambda \otimes \mathbf{1}_{-}(1)\right)=J_{a} \Lambda \otimes \mathbf{1}_{-}(1)$ ou $J_{a} \Lambda \otimes \sigma \mathbf{1}_{-}(1)$. Il faut exclure cette deuxième possibilité. Mais celle-ci conduit à l'égalité (1), où $Z=J_{a} \Lambda$ et $Z_{2}=\sigma \mathbf{1}_{-}(1)-\mathbf{1}_{-}(1)$. On peut reprendre le raisonnement du cas $\Lambda_{2}=\mathbf{1}_{+}(1)$. On choisit cette fois $\eta=(-1)^{\widetilde{\operatorname{def}}(\Lambda) / 2}$, on remarque que $\mathcal{F} Z_{2}=Z_{2}$, 
on utilise le lemme 10.2 au lieu de 10.1. Le raisonnement conduit à une contradiction, qui démontre l'énoncé sous nos hypothèses.

Supposons enfin $n_{1} \geqslant 2, n_{1}-a \leqslant 1$ et $n_{2}=0$. On a $\Lambda_{2}=\Lambda(\varnothing)$ qui est symétrique, donc $\widetilde{\Lambda}_{2}(x, \varepsilon)=\Lambda(\varnothing)$ pour tout $(x, \varepsilon) \in E$. Si $n_{1}-a=0, \Lambda=\Lambda(\varnothing)$ est aussi symétrique et $\widetilde{\Lambda}(x, \varepsilon)=\Lambda(x, \varepsilon)$ pour tout $(x, \varepsilon) \in E$. Reste le cas $n_{1}-a=1$. Par un argument de symétrie, on peut supposer $\Lambda=\mathbf{1}_{\nu}(1)$, avec $\nu \in\{ \pm 1\}$. D'après le lemme 11.9, $i\left(J_{a} \Lambda \otimes \Lambda(\varnothing)\right)-J_{a} \Lambda \otimes \Lambda(\varnothing)$ ou $i\left(J_{a} \Lambda \otimes \Lambda(\varnothing)\right)-\sigma J_{a} \Lambda \otimes \Lambda(\varnothing)$ est orthogonal à $\Lambda_{1} \otimes \Lambda(\varnothing)$ pour tout $\Lambda_{1}$ non cuspidal. Il faut exclure la deuxième possibilité. Supposons-la vérifiée et prenons $\Lambda_{1}=\sigma \mathbf{1}_{\nu}(1)$. Grâce à $6.5(1)$ ou $6.5(2)$, on a l'égalité $i\left(\sigma \mathbf{1}_{\nu}(n) \otimes \Lambda(\varnothing)\right)=\sigma \mathbf{1}_{\nu}(n) \otimes \Lambda(\varnothing)$. Or on vérifie que :

$$
\left(J_{a} \mathbf{1}_{\nu}(1), \sigma \mathbf{1}_{\nu}(n)\right)=1,\left(\sigma J_{a} \mathbf{1}_{\nu}(1), \sigma \mathbf{1}_{\nu}(n)\right)=0 .
$$

Ces égalités et le fait que $i$ est une isométrie entraînent que $i\left(J_{a} \Lambda \otimes \Lambda(\varnothing)\right)-\sigma J_{a} \Lambda \otimes$ $\Lambda(\varnothing)$ n'est pas orthogonal à $\sigma \mathbf{1}_{\nu}(1) \otimes \Lambda(\varnothing)$. Contradiction qui achève la démonstration.

11.12. Notons $\mathbb{C}\left[\widetilde{\mathcal{S}} \widetilde{\mathcal{S}}_{n, \text { pair }}\right]_{I J}$ le sous-espace de $\mathbb{C}\left[\widetilde{\mathcal{S}} \widetilde{\mathcal{S}}_{n, \text { pair }}\right]$ somme des images de tous les opérateurs $I_{a} \otimes 1,1 \otimes I_{a}, J_{a} \otimes 1,1 \otimes J_{a}$, pour $a \in\{1, \ldots, n\}$.

Proposition. - On peut choisir l'application $\pi_{n, \text { cusp }}$ de sorte que la restriction de $i$ au sous-espace $\mathbb{C}\left[\widetilde{\mathcal{S}} \widetilde{\mathcal{S}}_{n, \text { pair }}\right]_{I J}$ soit l'identité.

Démonstration. - Quel que soit le choix de $\pi_{n, \text { cusp }}, i$ est l'identité sur l'image d'un opérateur $I_{a} \otimes 1$ ou $1 \otimes I_{a}$. Posons $H=\left\{\left(h_{1}, h_{2}\right) \in \mathbb{Z} \times \mathbb{Z} ; h_{1}^{2}+h_{2}^{2}=n\right\}$. L'application :

$$
\begin{aligned}
H & \longrightarrow \widetilde{\mathcal{S}} \widetilde{\mathcal{S}}_{n, \text { pair }} \\
\left(h_{1}, h_{2}\right) & \longmapsto\left(\Lambda_{\text {cusp }}\left(h_{1}\right), \Lambda_{\text {cusp }}\left(h_{2}\right)\right)
\end{aligned}
$$

est une bijection de $H$ sur l'ensemble des éléments de $\widetilde{\mathcal{S}} \widetilde{\mathcal{S}}_{n \text {,pair }}$ dont les deux termes sont cuspidaux. Rappelons que l'on a noté $\mathcal{Z}$ le sous-espace de $\mathbb{C}\left[\widetilde{\mathcal{S}} \widetilde{\mathcal{S}}_{n, \text { pair }}\right]$ engendré par ces éléments. Notons $p_{\mathcal{Z}}$ la projection orthogonale sur $\mathcal{Z}$. Notons $\mathcal{L}$ l'ensemble des quadruplets $L=\left(\Lambda_{1}, \Lambda_{2}, a, j\right)$ où $j \in\{1,2\}, a \in \mathbb{N}$ et $\Lambda_{1}, \Lambda_{2}$ sont deux symboles ordonnés tels que $\operatorname{rg}\left(\Lambda_{1}\right)+\operatorname{rg}\left(\Lambda_{2}\right)+a=n$. Pour un tel quadruplet, on pose :

$$
J_{L}= \begin{cases}J_{a} \Lambda_{1} \otimes \Lambda_{2}, & \text { si } j=1, \\ \Lambda_{1} \otimes J_{a} \Lambda_{2}, & \text { si } j=2 .\end{cases}
$$

La proposition 11.11, et celle obtenue en échangeant les rôles des indices 1 et 2 signifient que $i\left(J_{L}\right)-p_{\mathcal{Z}} \circ i\left(J_{L}\right)=J_{L}-p_{\mathcal{Z}}\left(J_{L}\right)$ pour tout $L \in \mathcal{L}$. Puisque $i$ est une isométrie, on en déduit l'égalité :

$$
\left(p_{\mathcal{Z}} \circ i\left(J_{L}\right), p_{\mathcal{Z}} \circ i\left(J_{L^{\prime}}\right)\right)=\left(p_{\mathcal{Z}}\left(J_{L}\right), p_{\mathcal{Z}}\left(J_{L^{\prime}}\right)\right)
$$

pour tous $L, L^{\prime} \in \mathcal{L}$. 
Montrons qu'il existe une unique application $\gamma: H \rightarrow H$ telle que, pour tout $\left(h_{1}, h_{2}\right) \in H$ et tout $L \in \mathcal{L}$, on ait l'égalité :

$$
\left(\Lambda_{\text {cusp }}\left(h_{1}^{\prime}\right) \otimes \Lambda_{\text {cusp }}\left(h_{2}^{\prime}\right), i\left(J_{L}\right)\right)=\left(\Lambda_{\text {cusp }}\left(h_{1}\right) \otimes \Lambda_{\text {cusp }}\left(h_{2}\right), J_{L}\right),
$$

où $\left(h_{1}^{\prime}, h_{2}^{\prime}\right)=\gamma\left(h_{1}, h_{2}\right)$. Soit $\left(h_{1}, h_{2}\right) \in H$. Supposons d'abord $h_{1} \geqslant 2$. Comme dans la démonstration précédente, définissons $\Lambda_{1} \in \widetilde{\mathcal{S}}_{h_{1}^{2}-1 \text {,pair }}$ par $\Lambda_{1}^{+}=\left\{2 h_{1}-2, \ldots, 0\right\}$, $\Lambda_{1}^{-}=\left\{2 h_{1}-2\right\}$. Posons $L_{0}=\left(\Lambda_{1}, \Lambda_{\text {cusp }}\left(h_{2}\right), 1,1\right)$. On vérifie que $p_{\mathcal{Z}}\left(J_{L_{0}}\right)=$ $\Lambda_{\text {cusp }}\left(h_{1}\right) \otimes \Lambda_{\text {cusp }}\left(h_{2}\right)$. D'après le lemme 11.3 , il existe $\varepsilon_{1}, \varepsilon_{2} \in\{ \pm 1\}$ tels que $p_{\mathcal{Z}} \circ i\left(J_{L_{0}}\right)=\Lambda_{\text {cusp }}\left(\varepsilon_{1} h_{1}\right) \otimes \Lambda_{\text {cusp }}\left(\varepsilon_{2} h_{2}\right)$. En prenant $L=L_{0}$, l'égalité (2) impose $\gamma\left(h_{1}, h_{2}\right)=\left(\varepsilon_{1} h_{1}, \varepsilon_{2} h_{2}\right)$. Inversement, pour ce choix de $\gamma\left(h_{1}, h_{2}\right)$, l'égalité (2) pour tout $L$ résulte de (1) appliqué au couple $\left(L, L_{0}\right)$. On traite de façon similaire les cas $h_{1} \leqslant-2, h_{2} \geqslant 2, h_{2} \leqslant-2$. Puisque $n \geqslant 2$, il ne reste à considérer que le cas $n=2$, $\left|h_{1}\right|=\left|h_{2}\right|=1$. Posons $L_{1}=\left(\Lambda(\varnothing), \Lambda_{\text {cusp }}\left(h_{2}\right), 1,1\right), L_{2}=\left(\Lambda_{\text {cusp }}\left(h_{1}\right), \Lambda(\varnothing), 1,2\right)$. On a les égalités :

$$
\begin{aligned}
& J_{L_{1}}=\left(\Lambda_{\text {cusp }}\left(h_{1}\right)+\Lambda_{\text {cusp }}\left(-h_{1}\right)\right) \otimes \Lambda_{\text {cusp }}\left(h_{2}\right), \\
& J_{L_{2}}=\Lambda_{\text {cusp }}\left(h_{1}\right) \otimes\left(\Lambda_{\text {cusp }}\left(h_{2}\right)+\Lambda_{\text {cusp }}\left(-h_{2}\right)\right) .
\end{aligned}
$$

D'après le lemme 11.3 , il existe $\varepsilon_{1}, \varepsilon_{2} \in\{ \pm 1\}$ de sorte que :

$$
\begin{aligned}
& i\left(J_{L_{1}}\right)=\left(\Lambda_{\text {cusp }}\left(h_{1}\right)+\Lambda_{\text {cusp }}\left(-h_{1}\right)\right) \otimes \Lambda_{\text {cusp }}\left(\varepsilon_{2} h_{2}\right), \\
& i\left(J_{L_{2}}\right)=\Lambda_{\text {cusp }}\left(\varepsilon_{1} h_{1}\right) \otimes\left(\Lambda_{\text {cusp }}\left(h_{2}\right)+\Lambda_{\text {cusp }}\left(-h_{2}\right)\right) .
\end{aligned}
$$

En prenant $L=L_{1}$ puis $L=L_{2}$, l'égalité (2) impose $\gamma\left(h_{1}, h_{2}\right)=\left(\varepsilon_{1} h_{1}, \varepsilon_{2} h_{2}\right)$. Inversement, pour ce choix de $\gamma\left(h_{1}, h_{2}\right)$, les seuls $L$ pour lesquels l'égalité (2) n'est pas évidente vérifient $J_{L^{\prime}}=J_{L_{1}}$ ou $J_{L_{2}}$ ou $(1 \otimes \sigma) J_{L_{1}}$ ou $(\sigma \otimes 1) J_{L_{2}}$. La commutation de $i$ à $\sigma \otimes 1$ et $1 \otimes \sigma$ permet de vérifier (2) pour ces termes.

Il résulte de la preuve que $\gamma\left(h_{1}, h_{2}\right)=\left( \pm h_{1}, \pm h_{2}\right)$ pour tout $\left(h_{1}, h_{2}\right) \in H$. En utilisant l'unicité de $\gamma$ et la commutation de $i$ à $\sigma \otimes 1,1 \otimes \sigma$ et $\tau$ (cf. 6.5), on voit que $\gamma$ commute aux applications $\left(h_{1}, h_{2}\right) \mapsto\left(-h_{1}, h_{2}\right)$ ou $\left(h_{1},-h_{2}\right)$ ou $\left(h_{2}, h_{1}\right)$. Rappelons que $\pi_{n, \text { cusp }}$ est une application de $H$ dans $\operatorname{Quad}(O(2 n))$. Posons $\pi_{n, \text { cusp }}^{\prime}=\pi_{n, \text { cusp }} \circ \gamma$. Cette nouvelle application vérifie les mêmes propriétés que $\pi_{n, \text { cusp }}$. On en déduit de nouvelles applications $\pi_{n}^{\prime}, i^{\prime}$. La relation (2) devient:

$$
\left(\Lambda_{\text {cusp }}\left(h_{1}\right) \otimes \Lambda_{\text {cusp }}\left(h_{2}\right), i^{\prime}\left(J_{L}\right)\right)=\left(\Lambda_{\text {cusp }}\left(h_{1}\right) \otimes \Lambda_{\text {cusp }}\left(h_{2}\right), J_{L}\right)
$$

pour tous $\left(h_{1}, h_{2}\right) \in H$ et $L \in \mathcal{L}$. Autrement dit, $p_{\mathcal{Z}} \circ i^{\prime}\left(J_{L}\right)=p_{\mathcal{Z}}\left(J_{L}\right)$, ce qui entraîne $i^{\prime}\left(J_{L}\right)=J_{L}$. Cela vérifie l'énoncé.

Remarque. - De la démonstration résulte l'unicité du choix de $\pi_{n, \text { cusp }}$. 



\section{CHAPITRE 12}

\section{FIN DE LA PREUVE}

On achève la preuve en reprenant la méthode de Shoji, rendue possible par le lemme 7.11. Comme dans [S2], on a des difficultés quand $n$ est trop petit, ici $n \leqslant 4$. On traite ces cas séparément.

12.1. On choisit $\pi_{n \text {,cusp }}$ de sorte que la conclusion de la proposition 11.12 soit vérifiée. On simplifie les notations en posant $k\left(h_{1}, h_{2}\right)=k\left(\Lambda_{\text {cusp }}\left(h_{1}\right), \Lambda_{\text {cusp }}\left(h_{2}\right)\right)$ pour tout $\left(h_{1}, h_{2}\right) \in H, c f .11 .12$ pour la définition de $H$. D'après le lemme 2.7, l'orthogonal dans $\mathbb{C}\left[\widetilde{\mathcal{S}} \widetilde{\mathcal{S}}_{n, \text { pair }}\right]$ du sous-espace $\mathbb{C}\left[\widetilde{\mathcal{S}} \widetilde{\mathcal{S}}_{n, \text { pair }}\right]_{I J}$ a pour base la famille $\left(\mathcal{F} \Lambda_{\text {cusp }}\left(h_{1}\right) \otimes \mathcal{F} \Lambda_{\text {cusp }}\left(h_{2}\right)\right)_{\left(h_{1}, h_{2}\right) \in H}$. Cette base est orthonormée. On définit la matrice carrée $C$, d'ordre $|H| \times|H|$ par :

$$
C_{h_{1}, h_{2} ; h_{1}^{\prime}, h_{2}^{\prime}}=\left(i\left(\mathcal{F} \Lambda_{\text {cusp }}\left(h_{1}\right) \otimes \mathcal{F} \Lambda_{\text {cusp }}\left(h_{2}\right)\right), \mathcal{F} \Lambda_{\text {cusp }}\left(h_{1}^{\prime}\right) \otimes \mathcal{F} \Lambda_{\text {cusp }}\left(h_{2}^{\prime}\right)\right)
$$

pour tous $\left(h_{1}, h_{2}\right),\left(h_{1}^{\prime}, h_{2}^{\prime}\right) \in H$. Remarquons que cette égalité équivaut à :

$$
C_{h_{1}, h_{2} ; h_{1}^{\prime}, h_{2}^{\prime}}=\left(k\left(h_{1}, h_{2}\right), \pi_{n}\left(\mathcal{F} \Lambda_{\text {cusp }}\left(h_{1}^{\prime}\right) \otimes \mathcal{F} \Lambda_{\text {cusp }}\left(h_{2}^{\prime}\right)\right)\right) .
$$

La proposition 11.12 entraîne que la matrice $C$ est unitaire.

LEMmE. - Soient $\left(h_{1}, h_{2}\right),\left(h_{1}^{\prime}, h_{2}^{\prime}\right) \in H$. On a les égalités :

$$
\begin{aligned}
C_{h_{2}, h_{1} ; h_{2}^{\prime}, h_{1}^{\prime}} & =C_{h_{1}, h_{2} ; h_{1}^{\prime}, h_{2}^{\prime}} ; \\
C_{-h_{1}, h_{2} ;-h_{1}^{\prime}, h_{2}^{\prime}} & =C_{h_{1},-h_{2} ; h_{1}^{\prime},-h_{2}^{\prime}}=C_{h_{1}, h_{2} ; h_{1}^{\prime}, h_{2}^{\prime}} ; \\
C_{h_{1}, h_{2} ; h_{1}^{\prime}, h_{2}^{\prime}} & =0, \text { si } h_{2} \not \equiv h_{2}^{\prime} \bmod 2 \mathbb{Z} .
\end{aligned}
$$

Démonstration. - Cela résulte des propositions 3.5, 4.4 et des propriétés de $\mathcal{F}$ notées en 2.1. Par exemple, on a introduit en 3.5 l'automorphisme $f \mapsto f^{c} \operatorname{de} \mathcal{C}(O(2 n))$. Dans le membre de droite de l'égalité (1), on peut remplacer chacun des termes dont on prend le produit scalaire par son image par cet automorphisme. Grâce à la proposition $4.4(\mathrm{~b})(\mathrm{v})$, on a :

$$
\begin{aligned}
\pi_{n}\left(\mathcal{F} \Lambda_{\text {cusp }}\left(h_{1}^{\prime}\right) \otimes \mathcal{F} \Lambda_{\text {cusp }}\left(h_{2}^{\prime}\right)\right)^{c} & =\pi_{n}\left(\mathcal{F} \Lambda_{\text {cusp }}\left(h_{1}^{\prime}\right) \otimes \sigma \circ \mathcal{F}\left(\Lambda_{\text {cusp }}\left(h_{2}^{\prime}\right)\right)\right) \\
& =\pi_{n}\left(\mathcal{F} \Lambda_{\text {cusp }}\left(h_{1}^{\prime}\right) \otimes \mathcal{F} \circ(-1)^{\widetilde{\operatorname{def}} / 2}\left(\Lambda_{\text {cusp }}\left(h_{2}^{\prime}\right)\right)\right) \\
& =(-1)^{h_{2}^{\prime}} \pi_{n}\left(\mathcal{F} \Lambda_{\text {cusp }}\left(h_{1}^{\prime}\right) \otimes \mathcal{F} \Lambda_{\text {cusp }}\left(h_{2}^{\prime}\right)\right) .
\end{aligned}
$$


Grâce à la proposition $3.5(\mathrm{v})$, on a :

$$
k\left(h_{1}, h_{2}\right)^{c}=(-1)^{h_{2}} k\left(h_{1}, h_{2}\right) .
$$

D’où l'égalité :

$$
C_{h_{1}, h_{2} ; h_{1}^{\prime}, h_{2}^{\prime}}=(-1)^{h_{2}+h_{2}^{\prime}} C_{h_{1}, h_{2} ; h_{1}^{\prime}, h_{2}^{\prime}},
$$

dont on déduit la dernière assertion de l'énoncé. Les autres se démontrent de façon similaire.

12.2. Soient $m_{1}, m_{2} \in \mathbb{N}$ tels que $m_{1}^{2}+m_{2}^{2}=n$. Pour $j \in\{1,2\}$, introduisons le symbole spécial :

$$
\Lambda_{j}^{0}=\left\{\left(x,(-1)^{x+1}\right) ; x \in\left\{2 m_{j}-1, \ldots, 0\right\}\right\},
$$

notons $\widetilde{\operatorname{Fam}}_{j}$ sa famille dans $\widetilde{\mathcal{S}}_{m_{j}^{2}}$,pair. Notons $\widetilde{\operatorname{Fam}}_{n c}$ l'ensemble des $\left(\Lambda_{1}, \Lambda_{2}\right) \in \widetilde{\operatorname{Fam}}_{1} \times$ $\widetilde{\text { Fam }}_{2}$ tels que $\Lambda_{1}$ ou $\Lambda_{2}$ ne soit pas cuspidal. Notons $H\left(m_{1}, m_{2}\right)$ l'ensemble des $\left(h_{1}, h_{2}\right) \in H$ tels que $\left|h_{1}\right|=m_{1},\left|h_{2}\right|=m_{2}$. Les éléments $\left(\Lambda_{1}, \Lambda_{2}\right)$ de $\widetilde{\operatorname{Fam}}_{1} \times \widetilde{\operatorname{Fam}}_{2}$ tels que $\Lambda_{1}$ et $\Lambda_{2}$ sont tous deux cuspidaux sont précisément les $\left(\Lambda_{\text {cusp }}\left(h_{1}\right), \Lambda_{\text {cusp }}\left(h_{2}\right)\right)$ pour $\left(h_{1}, h_{2}\right) \in H\left(m_{1}, m_{2}\right)$. Soit $\left(\Lambda_{1}, \Lambda_{2}\right) \in \widetilde{\operatorname{Fam}}_{n c}$. Alors $\mathcal{F} \Lambda_{1} \otimes \mathcal{F} \Lambda_{2}$ appartient à $\mathbb{C}\left[\widetilde{\mathcal{S}} \widetilde{\mathcal{S}}_{n, \text { pair }}\right]_{I J}$ d'après le lemme 2.7. Cet élément est donc fixé par $i$, autrement dit :

$$
\pi_{n}\left(\mathcal{F} \Lambda_{1} \otimes \mathcal{F} \Lambda_{2}\right)=k\left(\Lambda_{1}, \Lambda_{2}\right) .
$$

Soit $\left(h_{1}^{\prime}, h_{2}^{\prime}\right) \in H\left(m_{1}, m_{2}\right)$. Alors $\mathcal{F} \Lambda_{\text {cusp }}\left(h_{1}^{\prime}\right) \otimes \mathcal{F} \Lambda_{\text {cusp }}\left(h_{2}^{\prime}\right)$ appartient à l'orthogonal de $\mathbb{C}\left[\widetilde{\mathcal{S}} \widetilde{\mathcal{S}}_{n, \text { pair }}\right]_{I J}$ et on a l'égalité :

$$
\mathcal{F} \Lambda_{\text {cusp }}\left(h_{1}^{\prime}\right) \otimes \mathcal{F} \Lambda_{\text {cusp }}\left(h_{2}^{\prime}\right)=\sum_{\left(h_{1}, h_{2}\right) \in H} C_{h_{1}, h_{2} ; h_{1}^{\prime}, h_{2}^{\prime}} i\left(\mathcal{F} \Lambda_{\text {cusp }}\left(h_{1}\right) \otimes \mathcal{F} \Lambda_{\text {cusp }}\left(h_{2}\right)\right) .
$$

Autrement dit :

$$
\pi_{n}\left(\mathcal{F} \Lambda_{\text {cusp }}\left(h_{1}^{\prime}\right) \otimes \mathcal{F} \Lambda_{\text {cusp }}\left(h_{2}^{\prime}\right)\right)=\sum_{\left(h_{1}, h_{2}\right) \in H} C_{h_{1}, h_{2} ; h_{1}^{\prime}, h_{2}^{\prime}} k\left(h_{1}, h_{2}\right) .
$$

Soit maintenant $\left(\Lambda_{1}, \Lambda_{2}\right) \in{\widetilde{\operatorname{Fam}_{1}}} \times \widetilde{\text { Fam }}_{2}$. On a l'égalité :

$$
\begin{aligned}
& \operatorname{trace}\left(\pi\left(\Lambda_{1}, \Lambda_{2}\right)\right)=\pi_{n}\left(\mathcal{F F} \Lambda_{1} \otimes \mathcal{F F} \Lambda_{2}\right) \\
& =\left|\widetilde{\operatorname{Fam}_{1}}\right|^{-1 / 2} \mid{\widetilde{\operatorname{Fam}_{2}}}_{\mid}^{-1 / 2} \sum_{\left(\Lambda_{1}^{\prime}, \Lambda_{2}^{\prime}\right) \in \widetilde{\operatorname{Fam}}_{1} \times \widetilde{\operatorname{Fam}}_{2}}^{(-1)^{\left\langle\Lambda_{1}, \Lambda_{1}^{\prime}\right\rangle+\left\langle\Lambda_{2}, \Lambda_{2}^{\prime}\right\rangle} \pi_{n}\left(\mathcal{F} \Lambda_{1}^{\prime} \otimes \mathcal{F} \Lambda_{2}^{\prime}\right) .}
\end{aligned}
$$

En rassemblant les égalités précédentes, on obtient l'égalité :

(1) $\operatorname{trace}\left(\pi\left(\Lambda_{1}, \Lambda_{2}\right)\right)$

$$
\begin{gathered}
=\left|\widetilde{\operatorname{Fam}}_{1}\right|^{-1 / 2} \mid{\widetilde{\operatorname{Fam}_{2}}}^{-1 / 2}\left(\left[\sum_{\left(\Lambda_{1}^{\prime}, \Lambda_{2}^{\prime}\right) \in \widetilde{\operatorname{Fam}}_{n c}}(-1)^{\left\langle\Lambda_{1}, \Lambda_{1}^{\prime}\right\rangle+\left\langle\Lambda_{2}, \Lambda_{2}^{\prime}\right\rangle} k\left(\Lambda_{1}^{\prime}, \Lambda_{2}^{\prime}\right)\right]\right. \\
\left.+\sum_{\left(h_{1}, h_{2}\right) \in H}\left[\sum_{\left(h_{1}^{\prime}, h_{2}^{\prime}\right) \in H\left(m_{1}, m_{2}\right)}(-1)^{\left\langle\Lambda_{1}, \Lambda_{\text {cusp }}\left(h_{1}^{\prime}\right)\right\rangle+\left\langle\Lambda_{2}, \Lambda_{\text {cusp }}\left(h_{2}^{\prime}\right)\right\rangle} C_{h_{1}, h_{2} ; h_{1}^{\prime}, h_{2}^{\prime}}\right] k\left(h_{1}, h_{2}\right)\right) .
\end{gathered}
$$


C'est la décomposition de la fonction trace $\left(\pi\left(\Lambda_{1}, \Lambda_{2}\right)\right)$ dans la base orthonormée de $\mathbb{C}[$ Quad $(O(2 n))]$ formée des fonctions $k\left(\Lambda_{1}^{\prime}, \Lambda_{2}^{\prime}\right)$ pour $\left(\Lambda_{1}^{\prime}, \Lambda_{2}^{\prime}\right) \in \widetilde{\mathcal{S}} \widetilde{\mathcal{S}}_{n \text {,pair }}$.

Introduisons des objets qui nous seront utiles. Soit $j \in\{1,2\}$, supposons $m_{j}>0$. Pour $e \in \mathbb{Z} / 2 \mathbb{Z}$, on définit un symbole ordonné $\Lambda_{j}^{e} \in \widetilde{\operatorname{Fam}}_{j}$. Si $e=0$, c'est le symbole spécial déjà introduit. Si $e=1$,

$\Lambda_{j}^{1+}=\left\{2 m_{j}-1\right\} \cup\left\{2 m_{j}-2,2 m_{j}-4, \ldots, 2,0\right\}, \Lambda_{j}^{1-}=\left\{2 m_{j}-3,2 m_{j}-5, \ldots, 3,1\right\}$.

On a $\widetilde{\operatorname{def}}\left(\Lambda_{j}^{0}\right)=0, \widetilde{\operatorname{def}}\left(\Lambda_{j}^{1}\right)=2$. Pour $e \in \mathbb{Z} / 2 \mathbb{Z}$ et $\varepsilon \in\{ \pm 1\}$, on vérifie l'égalité :

$$
\left\langle\Lambda_{j}^{e}, \Lambda_{\text {cusp }}\left(\varepsilon m_{j}\right)\right\rangle=\varepsilon^{e} .
$$

\section{3.}

LEMME. - Les coefficients de la matrice $C$ sont rationnels.

Démonstration. - On peut fixer $\left(h_{1}, h_{2}\right) \in H, m_{1}, m_{2} \in \mathbb{N}$ tels que $m_{1}^{2}+m_{2}^{2}=n$ et prouver que $C_{h_{1}, h_{2} ; h_{1}^{\prime}, h_{2}^{\prime}} \in \mathbb{Q}$ pour tout $\left(h_{1}^{\prime}, h_{2}^{\prime}\right) \in H\left(m_{1}, m_{2}\right)$. On reprend les notations du paragraphe précédent. On suppose $m_{1}, m_{2}>0$, le raisonnement s'adaptant aisément au cas où l'un de ces termes s'annule. Soient $\varepsilon_{1}, \varepsilon_{2} \in\{ \pm 1\}$. On déduit de 12.2(1) et 12.2(2) l'égalité :

(1) $\quad\left(k\left(h_{1}, h_{2}\right), \sum_{e_{1}, e_{2} \in \mathbb{Z} / 2 \mathbb{Z}} \varepsilon_{1}^{e_{1}} \varepsilon_{2}^{e_{2}} \operatorname{trace}\left(\pi\left(\Lambda_{1}^{e_{1}}, \Lambda_{2}^{e_{2}}\right)\right)\right)$

$$
=4\left|{\widetilde{\operatorname{Fam}_{1}}}_{1}\right|^{-1 / 2}\left|\widetilde{\operatorname{Fam}}_{2}\right|^{-1 / 2} C_{h_{1}, h_{2} ; \varepsilon_{1} m_{1}, \varepsilon_{2} m_{2}} .
$$

La fonction $k\left(h_{1}, h_{2}\right)$ prend ses valeurs dans $\mathbb{Z}, c f$. 3.1. Grâce au lemme 10.4, les fonctions trace $\left(\pi\left(\Lambda_{1}^{e_{1}}, \Lambda_{2}^{e_{2}}\right)\right.$ intervenant ci-dessus prennent leurs valeurs dans $\mathbb{Z}$. De plus, pour $j \in\{1,2\},\left|\widetilde{\operatorname{Fam}}_{j}\right|^{1 / 2}$ est entier, égal à $2^{m_{j}}$. Le lemme résulte de (1).

12.4.

Lemme. - Soit $\left(h_{1}, h_{2}\right) \in H$. Supposons $h_{1} \neq \pm h_{2}$. Alors il existe un entier $N \in \mathbb{N}$ tel que $C_{h_{1}, h_{2} ; h_{1}^{\prime}, h_{2}^{\prime}} \in 2^{-N} \mathbb{Z}$ pour tout $\left(h_{1}^{\prime}, h_{2}^{\prime}\right) \in H$.

Démonstration. - On reprend la preuve du lemme précédent. Il suffit de fixer un couple intervenant dans l'égalité $12.3(1)$, notons-le $\left(\Lambda_{1}, \Lambda_{2}\right)$, et de prouver qu'il existe $N \in \mathbb{N}$ tel que :

$$
\left(k\left(h_{1}, h_{2}\right), \operatorname{trace}\left(\pi\left(\Lambda_{1}, \Lambda_{2}\right)\right)\right) \in 2^{-N} \mathbb{Z} .
$$

Soit $\eta \in\{ \pm 1\}$ tel que $\pi\left(\Lambda_{1}, \Lambda_{2}\right)$ soit une représentation de $O_{\eta}(2 n)$. Posons $r^{\prime}=h_{1}+h_{2}$, $r^{\prime \prime}=h_{1}-h_{2}$. Par définition, la partie semi-simple de tout élément du support de $k\left(h_{1}, h_{2}\right)$ a pour valeurs propres 1 avec la multiplicité $r^{\prime 2}$ et -1 avec la multiplicité $r^{\prime \prime 2}$. Fixons un ensemble de représentants $\left(s_{i}\right)_{i \in I}$ des classes de conjugaison d'éléments semi-simples de $O_{\eta}(2 n)$ vérifiant ces propriétés. Un calcul simple montre que :

$$
\left(k\left(h_{1}, h_{2}\right), \operatorname{trace}\left(\pi\left(\Lambda_{1}, \Lambda_{2}\right)\right)\right)=\sum_{i \in I} X_{i},
$$


où, pour tout $i \in I$ :

$$
X_{i}=\left|Z_{O_{\eta}(2 n)}\left(s_{i}\right)\right|^{-1} \sum_{u} \bar{k}_{\eta}\left(h_{1}, h_{2}\right)\left(s_{i} u\right) \operatorname{trace}\left(\pi\left(\Lambda_{1}, \Lambda_{2}\right)\right)\left(s_{i} u\right),
$$

la somme portant sur les éléments unipotents $u \in Z_{O_{\eta}(2 n)}\left(s_{i}\right)$. Fixons $i \in I$ et supposons d'abord $r^{\prime}, r^{\prime \prime}$ pairs. Le groupe $\boldsymbol{Z}_{\boldsymbol{O}_{\eta}(2 n)}\left(s_{i}\right)$ se décompose en produit :

$$
\boldsymbol{Z}_{\boldsymbol{O}_{\eta}(2 n)}\left(s_{i}\right) \simeq \boldsymbol{O}_{\eta^{\prime}}\left(r^{2}\right) \times \boldsymbol{O}_{\eta^{\prime \prime}}\left(r^{\prime \prime 2}\right) .
$$

Introduisons les fonctions $k\left(r^{\prime} / 2, r^{\prime} / 2\right) \in \mathcal{C}\left(O\left(r^{2}\right)\right), k\left(r^{\prime \prime} / 2, r^{\prime \prime} / 2\right) \in \mathcal{C}\left(O\left(r^{\prime \prime 2}\right)\right)$. Elles sont à support unipotent. En comparant les formules de définition, on voit qu'il existe $\varepsilon \in\{ \pm 1\}$ tel que, pour tous les éléments unipotents $u^{\prime} \in O_{\eta^{\prime}}\left(r^{2}\right), u^{\prime \prime} \in O_{\eta^{\prime \prime}}\left(r^{\prime \prime 2}\right)$, on ait l'égalité :

$$
k_{\eta}\left(h_{1}, h_{2}\right)\left(s_{i} u^{\prime} u^{\prime \prime}\right)=\varepsilon k_{\eta^{\prime}}\left(r^{\prime} / 2, r^{\prime} / 2\right)\left(u^{\prime}\right) k_{\eta^{\prime \prime}}\left(r^{\prime \prime} / 2, r^{\prime \prime} / 2\right)\left(u^{\prime \prime}\right) .
$$

Notons $f$ l'élément de $\mathcal{C}\left(Z_{O_{\eta}(2 n)}\left(s_{i}\right)\right)$ défini par :

$$
f(g)=\operatorname{trace}\left(\pi\left(\Lambda_{1}, \Lambda_{2}\right)\right)\left(s_{i} g\right) .
$$

Identifions $f$ à un élément de $\mathcal{C}\left(O\left(r^{\prime 2}\right)\right) \otimes_{\mathbb{C}} \mathcal{C}\left(O\left(r^{\prime \prime 2}\right)\right)$. On obtient l'égalité :

$$
X_{i}=\varepsilon\left(k\left(r^{\prime} / 2, r^{\prime} / 2\right) \otimes k\left(r^{\prime \prime} / 2, r^{\prime \prime} / 2\right), f\right) .
$$

D'après l'hypothèse $h_{1} \neq \pm h_{2}$, on a $r^{\prime 2}<2 n, r^{\prime \prime 2}<2 n$. On peut appliquer l'hypothèse de récurrence. On a remarqué (corollaire 5.2) qu'elle entraînait que, par exemple, $k\left(r^{\prime} / 2, r^{\prime} / 2\right)$ appartenait à $\mathbb{Q}\left[\operatorname{Quad}\left(O\left(r^{\prime 2}\right)\right)\right]$. En fait, en remarquant que les familles ont toutes pour cardinal une puissance de 4, on voit qu'il existe un entier $N$ tel que $k\left(r^{\prime} / 2, r^{\prime} / 2\right) \in 2^{-N} \mathbb{Z}\left[\operatorname{Quad}\left(O\left(r^{\prime 2}\right)\right)\right]$. Il existe donc $N \in \mathbb{N}$ tel que :

(4) $k\left(r^{\prime} / 2, r^{\prime} / 2\right) \otimes k\left(r^{\prime \prime} / 2, r^{\prime \prime} / 2\right) \in 2^{-N} \mathbb{Z}\left[\operatorname{Quad}\left(O\left(r^{\prime 2}\right)\right)\right] \otimes_{\mathbb{Z}} \mathbb{Z}\left[\operatorname{Quad}\left(O\left(r^{\prime \prime 2}\right)\right)\right]$.

Notons $E$ l'espace de la représentation $\pi\left(\Lambda_{1}, \Lambda_{2}\right)$. Puisque $s_{i}^{2}=1$, l'opérateur $\pi\left(\Lambda_{1}, \Lambda_{2}\right)\left(s_{i}\right)$ est semi-simple et n'a pour valeurs propres que \pm 1 . Notons $E^{+}$et $E^{-}$les sous-espaces propres correspondants. Ces sous-espaces sont stables par $\pi\left(\Lambda_{1}, \Lambda_{2}\right)(g)$ pour tout $g \in Z_{O_{\eta}(2 n)}\left(s_{i}\right)$. On obtient ainsi deux représentations $\pi^{+}$ et $\pi^{-}$de ce groupe. On a l'égalité $f=\operatorname{trace}\left(\pi^{+}\right)-\operatorname{trace}\left(\pi^{-}\right)$. Donc $f$ appartient à $\mathbb{Z}\left[\operatorname{Irr}\left(O\left(r^{\prime 2}\right)\right)\right] \otimes \mathbb{Z}\left[\operatorname{Irr}\left(O\left(r^{\prime \prime 2}\right)\right)\right]$. De (3) et (4) résulte alors que $X_{i} \in 2^{-N} \mathbb{Z}$. Grâce à (2), cela entraîne (1).

Le raisonnement s'adapte si $r^{\prime}$ et $r^{\prime \prime}$ sont impairs. Le groupe $\boldsymbol{Z}_{\boldsymbol{O}_{\eta}(2 n)}\left(s_{i}\right)^{0}$ se décompose en produit :

$$
\boldsymbol{Z}_{\boldsymbol{O}_{\eta}(2 n)}\left(s_{i}\right)^{0}=\boldsymbol{S O}\left(r^{2}\right) \times \boldsymbol{S O}\left(r^{\prime \prime 2}\right) .
$$

On introduit cette fois les fonctions

$$
\begin{aligned}
k^{\prime} & =k\left(\Lambda_{\text {cusp }}\left(\frac{\left|r^{\prime}\right|-1}{2}\right), \Lambda_{\text {cusp }}\left(\frac{\left|r^{\prime}\right|-1}{2}\right)\right) \in \mathcal{C}\left(S O\left(r^{2}\right)\right), \\
k^{\prime \prime} & =k\left(\Lambda_{\text {cusp }}\left(\frac{\left|r^{\prime \prime}\right|-1}{2}\right), \Lambda_{\text {cusp }}\left(\frac{\left|r^{\prime \prime}\right|-1}{2}\right)\right) \in \mathcal{C}\left(S O\left(r^{\prime \prime 2}\right)\right),
\end{aligned}
$$


cf. 3.7. On définit $f \in \mathcal{C}\left(Z_{O_{\eta}(2 n)}\left(s_{i}\right)^{0}\right)$ par la même formule que ci-dessus et on obtient une égalité :

$$
X_{i}=\frac{\varepsilon}{4}\left(k^{\prime} \otimes k^{\prime \prime}, f\right) .
$$

On conclut comme précédemment. Remarquons que l'on doit utiliser par récurrence le théorème 5.1 pour des groupes spéciaux orthogonaux impairs.

\section{5 .}

Lemme. - Il existe un entier $N \in \mathbb{N}$ tel que les coefficients de la matrice $C$ appartiennent $\grave{a} 2^{-N} \mathbb{Z}$.

Démonstration. - On peut fixer $m_{1}, m_{2}, m_{1}^{\prime}, m_{2}^{\prime} \in \mathbb{N}$ tels que $m_{1}^{2}+m_{2}^{2}=m_{1}^{\prime 2}+$ $m_{2}^{\prime 2}=n$ et prouver qu'il existe un entier $N$ tel que $C_{h_{1}, h_{2} ; h_{1}^{\prime}, h_{2}^{\prime}} \in 2^{-N} \mathbb{Z}$ pour tous $\left(h_{1}, h_{2}\right) \in H\left(m_{1}, m_{2}\right),\left(h_{1}^{\prime}, h_{2}^{\prime}\right) \in H\left(m_{1}^{\prime}, m_{2}^{\prime}\right)$. D'après le lemme précédent, c'est vrai si $m_{1} \neq m_{2}$. Supposons $m_{1}=m_{2}$ et d'abord $m_{1}^{\prime}, m_{2}^{\prime} \neq 0$. Rangeons les éléments de $H\left(m_{1}^{\prime}, m_{2}^{\prime}\right)$ dans cet ordre : $\left(m_{1}^{\prime}, m_{2}^{\prime}\right),\left(m_{1}^{\prime},-m_{2}^{\prime}\right),\left(-m_{1}^{\prime}, m_{2}^{\prime}\right),\left(-m_{1}^{\prime},-m_{2}^{\prime}\right)$. Rangeons de même ceux de $H\left(m_{1}, m_{2}\right)$. Grâce aux propriétés relevées en 12.1, la matrice extraite $\left(C_{h_{1}, h_{2} ; h_{1}^{\prime}, h_{2}^{\prime}}\right)_{\left(h_{1}, h_{2}\right) \in H\left(m_{1}, m_{2}\right),\left(h_{1}^{\prime}, h_{2}^{\prime}\right) \in H\left(m_{1}^{\prime}, m_{2}^{\prime}\right)}$ est de la forme :

$$
\left(\begin{array}{llll}
a & b & c & d \\
b & a & d & c \\
c & d & a & b \\
d & c & b & a
\end{array}\right)
$$

Les coefficients $a, b, c, d$ sont rationnels, $c f$. lemme 12.3. La matrice $C$ étant unitaire, chaque colonne est de norme 1 et le produit de deux colonnes distinctes est nul. Appliquons cela aux colonnes $\left(h_{1}^{\prime}, h_{2}^{\prime}\right) \in H\left(m_{1}^{\prime}, m_{2}^{\prime}\right)$. Les coefficients de ces colonnes autres que ceux de la matrice ci-dessus appartiennent à $2^{-N} \mathbb{Z}$ pour un certain entier $N$. Il existe donc un tel entier tel que :

$$
a b+c d, a c+b d, a d+b c, a^{2}+b^{2}+c^{2}+d^{2} \in 2^{-N} \mathbb{Z} .
$$

On en déduit aisément l'existence de $N$ tel que $a, b, c, d \in 2^{-N} \mathbb{Z}$.

Dans le cas où, par exemple, $m_{2}^{\prime}=0$, la matrice ci-dessus se simplifie et devient de la forme :

$$
\left(\begin{array}{ll}
a & b \\
a & b \\
b & a \\
b & a
\end{array}\right)
$$

On conclut de même.

12.6.

Lemme. - Soient $m_{1}, m_{2} \in \mathbb{N}$ tels que $m_{1} \geqslant m_{2}, m_{1}^{2}+m_{2}^{2}=n$ et $m_{1}+m_{2} \geqslant 3$. Alors $C_{m_{1}, m_{2} ; m_{1}, m_{2}}=1$. 
Démonstration. - On utilise les constructions de 12.2 pour le couple $\left(m_{1}, m_{2}\right)$. Pour $\left(\Lambda_{1}, \Lambda_{2}\right) \in{\widetilde{\operatorname{Fam}_{1}}} \times \widetilde{\operatorname{Fam}}_{2}$ et $\left(h_{1}, h_{2}\right) \in H$, posons :

$$
y_{h_{1}, h_{2}}\left(\Lambda_{1}, \Lambda_{2}\right)=\sum_{\left(h_{1}^{\prime}, h_{2}^{\prime}\right) \in H\left(m_{1}, m_{2}\right)}(-1)^{\left\langle\Lambda_{1}, \Lambda_{\text {cusp }}\left(h_{1}^{\prime}\right)\right\rangle+\left\langle\Lambda_{2}, \Lambda_{\text {cusp }}\left(h_{2}^{\prime}\right)\right\rangle}\left(C_{h_{1}, h_{2} ; h_{1}^{\prime}, h_{2}^{\prime}}-\delta_{\left.h_{1}, h_{2} ; h_{1}^{\prime}, h_{2}^{\prime}\right),}\right.
$$

où $\delta_{h_{1}, h_{2} ; h_{1}^{\prime}, h_{2}^{\prime}}$ est le symbole de Kronecker, valant 1 si $\left(h_{1}, h_{2}\right)=\left(h_{1}^{\prime}, h_{2}^{\prime}\right), 0$ sinon. On pose :

$$
Y\left(\Lambda_{1}, \Lambda_{2}\right)=\sum_{\left(h_{1}, h_{2}\right) \in H} y_{h_{1}, h_{2}}\left(\Lambda_{1}, \Lambda_{2}\right) k\left(h_{1}, h_{2}\right) .
$$

Posons $t^{\prime}=m_{1}+m_{2}, t^{\prime \prime}=m_{1}-m_{2}$. Soient $\eta \in\{ \pm 1\}$ et $e_{0} \in \mathbb{Z} / 2 \mathbb{Z}$, avec $e_{0}=0$ si $t^{\prime \prime}=0$. On fixe un élément $g\left(\eta, e_{0}\right) \in O_{\eta}(2 n)$ vérifiant les conditions de 7.4 et tel que $\eta^{\prime \prime}\left(g\left(\eta, e_{0}\right)\right)=(-1)^{e_{0}}$. C'est-à-dire, décomposons $g\left(\eta, e_{0}\right)$ en produit su d'un élément semi-simple $s$ et d'un unipotent $u$ qui commutent entre eux. L'élément $s$ a pour valeurs propres 1 avec la multiplicité $t^{\prime 2}$ et -1 avec la multiplicité $t^{\prime \prime 2}$. Décomposons $u$ en $u^{\prime} u^{\prime \prime}$ selon les espaces propres de $s$. La partition associée à $u^{\prime}$, resp. $u^{\prime \prime}$, est $\left(2 t^{\prime}-1,2 t^{\prime}-3, \ldots, 3,1\right)$, resp. $\left(2 t^{\prime \prime}-1,2 t^{\prime \prime}-3, \ldots, 3,1\right)$. On renvoie à 7.4 pour la description des données $\eta_{u^{\prime}}$ et $\eta_{u^{\prime \prime}}$.

Les formules de 3.1 montrent que, pour $\left(h_{1}, h_{2}\right) \in H$, on a les égalités :

$$
\begin{aligned}
& k\left(h_{1}, h_{2}\right)\left(g\left(\eta, e_{0}\right)\right)=0, \\
& \text { si }\left(h_{1}, h_{2}\right) \notin\left\{\left(m_{1}, m_{2}\right),\left(-m_{1},-m_{2}\right),\left(m_{2}, m_{1}\right),\left(-m_{2},-m_{1}\right)\right\} ; \\
& \text { (2) } k\left(m_{1}, m_{2}\right)\left(g\left(\eta, e_{0}\right)\right)=\eta k\left(-m_{1},-m_{2}\right)\left(g\left(\eta, e_{0}\right)\right) \\
& =(-1)^{e_{0}} k\left(m_{2}, m_{1}\right)\left(g\left(\eta, e_{0}\right)\right)=\eta(-1)^{e_{0}} k\left(-m_{2},-m_{1}\right)\left(g\left(\eta, e_{0}\right)\right) .
\end{aligned}
$$

Supposons $m_{1} \neq m_{2}$ et $m_{2} \neq 0$. Pour $\varepsilon_{1}, \varepsilon_{2} \in\{ \pm 1\}$, posons :

$$
\begin{gathered}
c\left(1, \varepsilon_{1}, \varepsilon_{2}\right)=C_{m_{1}, m_{2} ; \varepsilon_{1} m_{1}, \varepsilon_{2} m_{2}}-\delta_{m_{1}, m_{2} ; \varepsilon_{1} m_{1}, \varepsilon_{2} m_{2}}, \\
c\left(-1, \varepsilon_{1}, \varepsilon_{2}\right)=C_{m_{2}, m_{1} ; \varepsilon_{1} m_{1}, \varepsilon_{2} m_{2}}=C_{m_{1}, m_{2} ; \varepsilon_{2} m_{2}, \varepsilon_{1} m_{1}} .
\end{gathered}
$$

Soient $e_{1}, e_{2} \in \mathbb{Z} / 2 \mathbb{Z}$. Les propriétés de symétrie de la matrice $C$ et la formule 12.2(2) entraînent les égalités :

$$
\begin{aligned}
& y_{m_{1}, m_{2}}\left(\Lambda_{1}^{e_{1}}, \Lambda_{2}^{e_{2}}\right)=(-1)^{e_{1}+e_{2}} y_{-m_{1},-m_{2}}\left(\Lambda_{1}^{e_{1}}, \Lambda_{2}^{e_{2}}\right)=\sum_{\varepsilon_{1}, \varepsilon_{2} \in\{ \pm 1\}} \varepsilon_{1}^{e_{1}} \varepsilon_{2}^{e_{2}} c\left(1, \varepsilon_{1}, \varepsilon_{2}\right), \\
& y_{m_{2}, m_{1}}\left(\Lambda_{1}^{e_{1}}, \Lambda_{2}^{e_{2}}\right)=(-1)^{e_{1}+e_{2}} y_{-m_{2},-m_{1}}\left(\Lambda_{1}^{e_{1}}, \Lambda_{2}^{e_{2}}\right)=\sum_{\varepsilon_{1}, \varepsilon_{2} \in\{ \pm 1\}} \varepsilon_{1}^{e_{1}} \varepsilon_{2}^{e_{2}} c\left(-1, \varepsilon_{1}, \varepsilon_{2}\right) .
\end{aligned}
$$

Grâce à (1) et (2) ci-dessus, on obtient l'égalité :

$$
\begin{aligned}
& Y\left(\Lambda_{1}^{e_{1}}, \Lambda_{2}^{e_{2}}\right)\left(g\left(\eta, e_{0}\right)\right) \\
& =\left(1+\eta(-1)^{e_{1}+e_{2}}\right) \sum_{\varepsilon_{0}, \varepsilon_{1}, \varepsilon_{2} \in\{ \pm 1\}} \varepsilon_{0}^{e_{0}} \varepsilon_{1}^{e_{1}} \varepsilon_{2}^{e_{2}} c\left(\varepsilon_{0}, \varepsilon_{1}, \varepsilon_{2}\right) k\left(m_{1}, m_{2}\right)\left(g\left(\eta, e_{0}\right)\right) .
\end{aligned}
$$


Spécialisée au cas $\eta=(-1)^{e_{1}+e_{2}}$, elle devient :

(3) $Y\left(\Lambda_{1}^{e_{1}}, \Lambda_{2}^{e_{2}}\right)\left(g\left((-1)^{e_{1}+e_{2}}, e_{0}\right)\right)$

$$
=2 \sum_{\varepsilon_{0}, \varepsilon_{1}, \varepsilon_{2} \in\{ \pm 1\}} \varepsilon_{0}^{e_{0}} \varepsilon_{1}^{e_{1}} \varepsilon_{2}^{e_{2}} c\left(\varepsilon_{0}, \varepsilon_{1}, \varepsilon_{2}\right) k\left(m_{1}, m_{2}\right)\left(g\left((-1)^{e_{1}+e_{2}}, e_{0}\right)\right) .
$$

On peut inverser cette formule; pour $\varepsilon_{0}, \varepsilon_{1}, \varepsilon_{2} \in\{ \pm 1\}$, on a l'égalité :

$$
\begin{aligned}
c\left(\varepsilon_{0}, \varepsilon_{1}, \varepsilon_{2}\right)=2^{-4} \sum_{e_{0}, e_{1}, e_{2} \in \mathbb{Z} / 2 \mathbb{Z}} \varepsilon_{0}^{e_{0}} \varepsilon_{1}^{e_{1}} \varepsilon_{2}^{e_{2}} & \\
& k\left(m_{1}, m_{2}\right)\left(g\left((-1)^{e_{1}+e_{2}}, e_{0}\right)\right)^{-1} Y\left(\Lambda_{1}^{e_{1}}, \Lambda_{2}^{e_{2}}\right)\left(g\left((-1)^{e_{1}+e_{2}}, e_{0}\right)\right) .
\end{aligned}
$$

Pour $\left(\Lambda_{1}, \Lambda_{2}\right) \in \widetilde{\operatorname{Fam}}_{1} \times \widetilde{\operatorname{Fam}}_{2}$, posons :

$$
X\left(\Lambda_{1}, \Lambda_{2}\right)=\left|\widetilde{\operatorname{Fam}}_{1}\right|^{1 / 2}\left|\widetilde{\operatorname{Fam}}_{2}\right|^{1 / 2}\left(\operatorname{trace}\left(\pi\left(\Lambda_{1}, \Lambda_{2}\right)\right)-k_{n}\left(\mathcal{F} \Lambda_{1} \otimes \mathcal{F} \Lambda_{2}\right)\right) .
$$

L'égalité 12.2(1) équivaut à $X\left(\Lambda_{1}, \Lambda_{2}\right)=Y\left(\Lambda_{1}, \Lambda_{2}\right)$. Remarquons que :

$$
\left|\widetilde{\operatorname{Fam}}_{1}\right|^{1 / 2}\left|\widetilde{\operatorname{Fam}}_{2}\right|^{1 / 2}=2^{m_{1}+m_{2}} \text {. }
$$

Grâce à l'hypothèse $m_{1}+m_{2} \geqslant 3$, les lemmes 10.7 et 7.11 entraînent que, pour tous $e_{0}, e_{1}, e_{2} \in \mathbb{Z} / 2 \mathbb{Z}, X\left(\Lambda_{1}^{e_{1}}, \Lambda_{2}^{e_{2}}\right)\left(g\left((-1)^{e_{1}+e_{2}}, e_{0}\right)\right)$ est un entier rationnel divisible par 8. Il en est donc de même de $Y\left(\Lambda_{1}^{e_{1}}, \Lambda_{2}^{e_{2}}\right)\left(g\left((-1)^{e_{1}+e_{2}}, e_{0}\right)\right)$. Remarquons que $k\left(m_{1}, m_{2}\right)\left(g\left((-1)^{e_{1}+e_{2}}, e_{0}\right)\right)$ est un entier impair. En se plaçant un instant dans le corps 2-adique $\mathbb{Q}_{2}$, l'égalité (4) jointe à la propriété ci-dessus entraîne :

- pour tous $\varepsilon_{0}, \varepsilon_{1}, \varepsilon_{2} \in\{ \pm 1\}, c\left(\varepsilon_{0}, \varepsilon_{1}, \varepsilon_{2}\right) \in 2^{-1} \mathbb{Z}_{2}$;

- pour tous $\varepsilon_{0}, \varepsilon_{1}, \varepsilon_{2}, \varepsilon_{0}^{\prime}, \varepsilon_{1}^{\prime}, \varepsilon_{2}^{\prime}, c\left(\varepsilon_{0}, \varepsilon_{1}, \varepsilon_{2}\right)-c\left(\varepsilon_{0}^{\prime}, \varepsilon_{1}^{\prime}, \varepsilon_{2}^{\prime}\right) \in \mathbb{Z}_{2}$.

Rappelons que les valeurs $c\left(\varepsilon_{0}, \varepsilon_{1}, \varepsilon_{2}\right)$ sont rationnelles, de dénominateurs des puissances de 2. D'autre part, l'unitarité de la matrice $C$ entraîne :

$$
(c(1,1,1)+1)^{2}+\sum_{\left(\varepsilon_{0}, \varepsilon_{1}, \varepsilon_{2}\right) \neq(1,1,1)} c\left(\varepsilon_{0}, \varepsilon_{1}, \varepsilon_{2}\right)^{2} \leqslant 1 .
$$

Supposons que l'une des valeurs $c\left(\varepsilon_{0}, \varepsilon_{1}, \varepsilon_{2}\right)$ ne soit pas entière. Des relations cidessus résulte qu'aucune ne l'est et qu'elles ont toutes pour dénominateur 2. Donc $c\left(\varepsilon_{0}, \varepsilon_{1}, \varepsilon_{2}\right)^{2} \geqslant 1 / 4$ pour tout $\left(\varepsilon_{0}, \varepsilon_{1}, \varepsilon_{2}\right)$ et cela contredit (5). Donc $c$ est à valeurs entières. Supposons qu'il existe $\left(\varepsilon_{0}, \varepsilon_{1}, \varepsilon_{2}\right) \neq(1,1,1)$ tel que $c\left(\varepsilon_{0}, \varepsilon_{1}, \varepsilon_{2}\right) \neq 0$. Fixons un tel $\left(\varepsilon_{0}, \varepsilon_{1}, \varepsilon_{2}\right)$. Grâce à (5), on a nécessairement $c\left(\varepsilon_{0}, \varepsilon_{1}, \varepsilon_{2}\right)= \pm 1, c\left(\varepsilon_{0}^{\prime}, \varepsilon_{1}^{\prime}, \varepsilon_{2}^{\prime}\right)=0$ pour $\left(\varepsilon_{0}^{\prime}, \varepsilon_{1}^{\prime}, \varepsilon_{2}^{\prime}\right) \neq(1,1,1)$ et $\neq\left(\varepsilon_{0}, \varepsilon_{1}, \varepsilon_{2}\right)$, et $c(1,1,1)=-1$. On peut alors choisir $e_{0}, e_{1}, e_{2}$ tel que le membre de droite de (3) ne soit pas divisible par 8 , contradiction. Donc $c\left(\varepsilon_{0}, \varepsilon_{1}, \varepsilon_{2}\right)=0$ pour tout $\left(\varepsilon_{0}, \varepsilon_{1}, \varepsilon_{2}\right) \neq(1,1,1)$. Maintenant (3) entraîne que $c(1,1,1)$ est divisible par 4 . Grâce à $(5)$, il ne peut être que nul. Cette nullité signifie que $C_{m_{1}, m_{2} ; m_{1}, m_{2}}=1$.

Dans le cas où $m_{1}=m_{2}$, resp. $m_{2}=0$, un raisonnement similaire s'applique. Les termes $\varepsilon_{0}, e_{0}$, resp. $\varepsilon_{2}, e_{2}$, disparaissent. Dans la formule (4), le coefficient n'est 
plus $2^{-4}$ mais $2^{-3}$ et on obtient tout de suite que $c$ est à valeurs entières. La fin du raisonnement est sans changement.

\section{7 .}

Lemme. - Supposons $n=4$. Alors $C_{2,0 ; 2,0}=1$.

Démonstration. - Le même raisonnement que dans la démonstration précédente montre que $C_{2,0 ; 2,0}$ et $C_{2,0 ; 0,2}$ sont demi-entiers. La matrice $C$ étant unitaire, il n'y a qu'un nombre fini de valeurs possibles pour ces coefficients. Notons $\widetilde{\text { Fam }}_{1}$ la famille de $\Lambda_{\text {cusp }}(2)$. On a introduit en 10.3 des symboles $\Lambda(i)$ pour $i \in\{1, \ldots, 7\}$ et des éléments $P_{0}, P_{2} \in \mathcal{C}(0(8))$. Soit $\Lambda \in \widetilde{\mathcal{S}}_{4,0}$. Si $\Lambda \notin \widetilde{\operatorname{Fam}}_{1}$, alors $\Lambda \otimes \Lambda(\varnothing)$ est orthogonal à $\mathcal{F} \Lambda_{\text {cusp }}\left(h_{1}\right) \otimes \mathcal{F} \Lambda_{\text {cusp }}\left(h_{2}\right)$ pour tout $\left(h_{1}, h_{2}\right) \in H$, donc $\Lambda \otimes \Lambda(\varnothing)$ est fixe par $i$ et cela entraîne que trace $(\pi(\Lambda, \Lambda(\varnothing)))$ est orthogonal à $k(2,0)$. Les seuls éléments de $\widetilde{\mathcal{S}}_{4,0} \cap \widetilde{\operatorname{Fam}}_{1}$ sont $\Lambda(i)$ et $\sigma(\Lambda(i))$ pour $i \in\{1,2,3\}$. Pour ceux-là, on calcule grâce à $12.2(1)$ :

$$
\begin{aligned}
(k(2,0), \operatorname{trace}(\pi(\Lambda(i), \Lambda(\varnothing)))) & =(k(2,0), \operatorname{trace}(\pi(\sigma(\Lambda(i)), \Lambda(\varnothing)))) \\
& =\frac{1}{4} \varepsilon_{i}\left(C_{2,0 ; 2,0}+C_{2,0 ; 0,2}\right),
\end{aligned}
$$

où $\varepsilon_{1}=1, \varepsilon_{2}=\varepsilon_{3}=-1$. On a donc l'égalité :

$$
\left(k(2,0), P_{0}\right)=\frac{1}{4}\left(C_{2,0 ; 2,0}+C_{2,0 ; 0,2}\right)\left(\sum_{i=1,2,3} \varepsilon_{i}\left(P_{\Lambda(i)}(q)+P_{\sigma(\Lambda(i))}(q)\right)\right) .
$$

D'après le lemme 10.3, le membre de gauche vaut $q^{2}$. Alors $C_{2,0 ; 2,0}+C_{2,0 ; 0,2}$ apparaît comme la valeur en $q$ d'une fraction rationnelle. On ne sait pas a priori que $C_{2,0 ; 2,0}$ et $C_{2,0 ; 0,2}$ sont indépendants de $q$ mais on a vu ci-dessus qu'ils ne pouvaient varier que dans un ensemble fini. Cela entraîne que cette fraction rationnelle est constante. On peut la remplacer par sa valeur au point 1 . Cela revient à remplacer le membre de gauche de (1) par 1 et les $P_{\Lambda}(q)$ apparaissant dans le membre de droite par $P_{\Lambda}(1)$. En utilisant le lemme 10.3, on obtient l'égalité :

$$
C_{2,0 ; 2,0}+C_{2,0 ; 0,2}=1 .
$$

Un calcul similaire utilisant l'élément $P_{2}$ conduit à l'égalité :

$$
C_{2,0 ; 2,0}-C_{2,0 ; 0,2}=1 \text {. }
$$

D'où l'assertion de l'énoncé.

12.8. Supposons $n=2$. Puisqu'on a fixé $\pi_{2, \text { cusp }}$, on dispose d'un paramétrage des représentations cuspidales quadratiques-unipotentes de $O_{+}(4)$ : ce sont les représentations $\pi_{2, \text { cusp }}\left(h_{1}, h_{2}\right)=\pi\left(\Lambda_{\text {cusp }}\left(h_{1}\right), \Lambda_{\text {cusp }}\left(h_{2}\right)\right)$ pour $h_{1}, h_{2} \in\{ \pm 1\}$. En 10.4, on a défini un autre paramétrage de cet ensemble de représentations.

Lemme. - Pour tous $h_{1}, h_{2} \in\{ \pm 1\}$, on a l'égalité $\pi^{\prime}\left(h_{1}, h_{2}\right)=\pi_{2, \text { cusp }}\left(h_{1}, h_{2}\right)$. 
Démonstration. - Rappelons que $\pi_{2, \text { cusp }}(1,1)$ et $\pi_{2, \text { cusp }}(-1,-1)$ sont invariantes par torsion par sp, tandis que $\pi_{2, \text { cusp }}(1,-1)$ et $\pi_{2 \text {,cusp }}(-1,1)$ sont échangées par cette torsion. D'après les formules du lemme 10.5 , ni $\pi^{\prime}(1,-1)$ ni $\pi^{\prime}(-1,1)$ ne sont invariantes par cette torsion. On en déduit les égalités :

$$
\begin{aligned}
& \left\{\pi_{2, \text { cusp }}(1,1), \pi_{2, \text { cusp }}(-1,-1)\right\}=\left\{\pi^{\prime}(1,1), \pi^{\prime}(-1,-1)\right\}, \\
& \left\{\pi_{2, \text { cusp }}(1,-1), \pi_{2, \text { cusp }}(-1,1)\right\}=\left\{\pi^{\prime}(1,-1), \pi^{\prime}(-1,1)\right\} .
\end{aligned}
$$

Soit $g$ un élément semi-simple de $O_{+}(4)$ ayant pour valeurs propres 1 avec la multiplicité 3 , et -1 avec la multiplicité 1 . Cet élément n'appartient pas au support de $k\left(h_{1}, h_{2}\right)$, quels que soient $h_{1}, h_{2} \in\{ \pm 1\}$. La formule $12.2(1)$ se simplifie en :

$$
\operatorname{trace}\left(\pi\left(\Lambda_{1}, \Lambda_{2}\right)\right)(g)=k_{2}\left(\mathcal{F} \Lambda_{1} \otimes \mathcal{F} \Lambda_{2}\right)(g) .
$$

En particulier, pour $h_{1}, h_{2} \in\{ \pm 1\}$ :

$$
\operatorname{trace}\left(\pi_{2, \text { cusp }}\left(h_{1}, h_{2}\right)\right)(g)=k_{2}\left(\mathcal{F} \Lambda_{\text {cusp }}\left(h_{1}\right) \otimes \mathcal{F} \Lambda_{\text {cusp }}\left(h_{2}\right)\right)(g) \text {. }
$$

En utilisant le lemme 7.3 et l'égalité $\mathcal{F}\left(\Lambda_{\text {cusp }}(1)-\Lambda_{\text {cusp }}(-1)\right)=\Lambda_{\text {cusp }}(1)-\Lambda_{\text {cusp }}(-1)$, on en déduit, pour tout $h \in\{ \pm 1\}$ :

$$
\begin{aligned}
\operatorname{trace}\left(\pi_{2, \text { cusp }}(h, 1)\right)(g) & -\operatorname{trace}\left(\pi_{2, \text { cusp }}(h,-1)\right)(g) \\
& =-2|S O(3)|_{p^{\prime}}\left|O_{-}(2)\right|_{p^{\prime}}^{-1} \operatorname{sp}(g) k_{-}\left(\mathcal{F} \Lambda_{\text {cusp }}(h) \otimes \Lambda(\varnothing)\right) \\
& =-(q-1) \operatorname{sp}(g) \operatorname{trace}\left(\pi\left(\Lambda_{\text {cusp }}(h), \Lambda(\varnothing)\right)(1)=-(q-1) \operatorname{sp}(g) .\right.
\end{aligned}
$$

En comparant avec les formules du lemme 10.5, on voit que le couple

$$
\left(\pi_{2, \mathrm{cusp}}(h, 1), \pi_{2, \operatorname{cusp}}(h,-1)\right)
$$

est nécessairement l'un des couples $\left(\pi^{\prime}(1,1), \pi^{\prime}(1,-1)\right)$ ou $\left(\pi^{\prime}(-1,1), \pi^{\prime}(-1,-1)\right)$. Pour $h=1$, le deuxième couple est exclu par (1). Pour $h=-1$, c'est le premier couple qui est exclu. On en déduit l'énoncé.

12.9 .

Lemme. - Supposons $n=2$. Alors $C_{1,1 ; 1,1}=1$.

Démonstration. - On considère la situation de 12.2 pour $m_{1}=m_{2}=1$. Les lemmes 10.4, 10.6 et 12.8 entraînent l'égalité :

$$
\left(k(1,1), \operatorname{trace}\left(\pi\left(\Lambda_{1}^{e_{1}}, \Lambda_{2}^{e_{2}}\right)\right)\right)=\frac{1}{4}
$$

pour tous $e_{1}, e_{2} \in \mathbb{Z} / 2 \mathbb{Z}$. L'énoncé résulte de l'égalité $12.3(1)$ appliquée à $\left(h_{1}, h_{2}\right)=$ $(1,1), \varepsilon_{1}=\varepsilon_{2}=1$. 
12.10. Achevons la preuve du théorème 5.1. Puisque $n \geqslant 2$, les lemmes 12.6, 12.7 et 12.9 entraînent que $C_{m_{1}, m_{2} ; m_{1}, m_{2}}=1$ pour tous $m_{1}, m_{2} \in \mathbb{N}$ tels que $m_{1}^{2}+m_{2}^{2}=$ $n$ et $m_{1} \geqslant m_{2}$. Les propriétés de symétrie de la matrice $C$ entraînent alors que $C_{h_{1}, h_{2} ; h_{1}, h_{2}}=1$ pour tout $\left(h_{1}, h_{2}\right) \in H$. Puisque $C$ est unitaire, cela force l'égalité $C=1$. Donc $i$ est l'identité sur l'orthogonal dans $\mathbb{C}\left[\widetilde{\mathcal{S}} \widetilde{\mathcal{S}}_{n, \text { pair }}\right]$ du sous-espace $\mathbb{C}\left[\widetilde{\mathcal{S}} \widetilde{\mathcal{S}}_{n, \text { pair }}\right]_{I J}$. On sait déjà que c'est aussi l'identité sur ce sous-espace. Donc $i$ est l'identité, ce qui équivaut à l'assertion du théorème. 


\section{CHAPITRE 13}

\section{LE CAS DU GROUPE SYMPLECTIQUE}

On indique rapidement comment adapter la preuve dans le cas du groupe symplectique.

13.1. Nous allons expliquer comment s'adapte la démonstration dans le cas du groupe symplectique $S p(2 n)$. On ne donnera de preuves que quand elles diffèrent substantiellement du cas du groupe orthogonal pair.

On note désormais $\Lambda_{2}(\varnothing)$ le symbole ordonné noté $\Lambda(\varnothing)$ dans les paragraphes précédents, c'est-à-dire l'unique élément de $\widetilde{\mathcal{S}}_{0, \text { pair }}$. On note $\Lambda_{1}(\varnothing)$ l'unique élément de $\mathcal{S}_{0, \text { imp }}$, que l'on peut représenter de sorte que $\Lambda_{1}(\varnothing)^{+}=\{0\}, \Lambda_{1}(\varnothing)^{-}=\varnothing$. Pour $h \in \mathbb{Z}$, on note désormais $\Lambda_{2, \text { cusp }}(h)$ le symbole ordonné noté $\Lambda_{\text {cusp }}(h)$ dans les paragraphes précédents. Pour $h \in \mathbb{N}$, on note $\Lambda_{1, \text { cusp }}(h)$ l'unique élément cuspidal de $\mathcal{S}_{h^{2}+h, \mathrm{imp}}$, que l'on peut représenter de sorte que

$$
\Lambda_{1, \text { cusp }}(h)^{+}=\{2 h, 2 h-1, \ldots, 1,0\}, \quad \Lambda_{1, \text { cusp }}(h)^{-}=\varnothing .
$$

Tout d'abord, le théorème 5.1 est vrai pour $n=1$ : on le vérifie explicitement grâce à la table des caractères de $S p(2)=S L(2)$. La représentation cuspidale $\pi\left(\Lambda_{1}(\varnothing), \mathbf{1}_{-}(1)\right)$ doit être choisie telle que :

$$
\operatorname{trace}\left(\pi\left(\Lambda_{1}(\varnothing), \mathbf{1}_{-}(1)\right)\right)(u)=-\frac{1}{2}-\frac{1}{2} \eta_{u}(2)(\zeta(-1) q)^{-1 / 2} q
$$

pour tout unipotent régulier $u$ paramétré par la partition (2) et la donnée $\eta_{u}(2)$.

On suppose dans la suite $n \geqslant 2$ et le théorème vrai pour les groupes $S p(2 m)$ si $m<n$.

Comme en 6.4, on démontre que la famille $\left(k\left(\Lambda_{1}, \Lambda_{2}\right)\right)_{\left(\Lambda_{1}, \Lambda_{2}\right) \in \mathcal{S} \widetilde{\mathcal{S}}_{n, \text { mix }}}$ est une base orthonormée de $\mathbb{C}[\operatorname{Quad}(S p(2 n))]$.

13.2. Soit $\left(\Lambda_{1}, \Lambda_{2}\right) \in \mathcal{S} \widetilde{\mathcal{S}}_{n, \text { mix }}$. On note $\left(h_{1}, \rho_{1}\right),\left(h_{2}, \rho_{2}\right)$ les couples associés en 2.2 à $\Lambda_{1}, \Lambda_{2}$. $\grave{A}\left(h_{1}, h_{2}\right)$ on associe $r^{\prime}, r^{\prime \prime} \in \mathbb{N}$ comme en 3.10. On pose $N_{1}=\operatorname{rg}\left(\Lambda_{1}\right)-h_{1}^{2}-h_{1}$, 
$N_{2}=\operatorname{rg}\left(\Lambda_{2}\right)-h_{2}^{2}$. Soit $g \in S p(2 n)$, que l'on écrit sous la forme $g=s u$, où $s$ est semisimple, $u$ unipotent et $s$ et $u$ commutent. On suppose que $s$ n'a pour valeurs propres que \pm 1 . On note $2 n^{\prime}$, resp. $2 n^{\prime \prime}$, la multiplicité de la valeur propre 1 , resp. -1 . On a :

$$
\boldsymbol{Z}_{\boldsymbol{S p}(2 n)}(s) \simeq \boldsymbol{S} \boldsymbol{p}\left(2 n^{\prime}\right) \times \boldsymbol{S} \boldsymbol{p}\left(2 n^{\prime \prime}\right) .
$$

On décompose conformément l'élément $u$ en produit $u=u^{\prime} u^{\prime \prime}$.

On vérifie :

- si $2 n^{\prime}<r^{\prime}\left(r^{\prime}+1\right)$ ou $2 n^{\prime \prime}<r^{\prime \prime}\left(r^{\prime \prime}+1\right), k\left(\Lambda_{1}, \Lambda_{2}\right)(g)=0$.

Supposons :

$$
2 n^{\prime} \geqslant r^{\prime}\left(r^{\prime}+1\right), 2 n^{\prime \prime} \geqslant r^{\prime \prime}\left(r^{\prime \prime}+1\right) .
$$

On pose $N^{\prime}=n^{\prime}-r^{\prime}\left(r^{\prime}+1\right) / 2, N^{\prime \prime}=n^{\prime \prime}-r^{\prime \prime}\left(r^{\prime \prime}+1\right) / 2$. À toute représentation irréductible $\rho^{\prime}$ de $W_{N^{\prime}}$, on associe comme en 7.2 un entier $b\left(r^{\prime}, \rho^{\prime}\right)$ et une fonction $\chi_{r^{\prime}, \rho^{\prime}}$ sur $S p\left(2 n^{\prime}\right)$, à support unipotent. De même, à toute représentation irréductible $\rho^{\prime \prime}$ de $W_{N^{\prime \prime}}$, on associe $b\left(r^{\prime \prime}, \rho^{\prime \prime}\right)$ et $\chi_{r^{\prime \prime}, \rho^{\prime \prime}}$. Remarquons qu'un signe se glisse dans les formules (4) et (6) de 7.2. Avec des notations similaires à celles de ce paragraphe, on a maintenant :

$$
\chi_{r^{\prime}, \rho^{\prime}}=q^{-b\left(r^{\prime}, \rho^{\prime}\right)}\left|W_{N^{\prime}}\right|^{-1} \sum_{w \in W_{N^{\prime}}} \operatorname{sgn}_{C D}^{r^{\prime}}(w) \operatorname{trace}\left(\rho^{\prime}\right)(w) Q\left(r^{\prime}, w\right)
$$

cf. $[\mathbf{W}]$ lemme VIII.9. On définit comme en 7.1 l'ensemble $\mathcal{N}$ et, pour $\underline{\nu} \in \mathcal{N}$, l'ensemble $W(\underline{\nu})$. Rappelons que l'on a posé :

$$
e_{2}= \begin{cases}0, & \text { si } h_{2} \geqslant 0 \\ 1, & \text { si } h_{2}<0\end{cases}
$$

Pour $\underline{\nu} \in \mathcal{N}$, on définit un caractère $\chi_{h_{1}, h_{2}, \underline{\nu}}$ du groupe $W(\underline{\nu})$ par la formule :

$$
\chi_{h_{1}, h_{2}, \underline{\nu}}(\underline{w})= \begin{cases}\operatorname{sgn}_{C D}\left(w_{2}^{\prime \prime}\right), & \text { si } h_{1} \geqslant\left|h_{2}\right| \text { et } h_{1}+e_{2} \equiv 1 \bmod 2 \mathbb{Z}, \\ \operatorname{sgn}_{C D}\left(w_{1}^{\prime \prime}\right), & \text { si } h_{1}<\left|h_{2}\right| \text { et } h_{1}+e_{2} \equiv 1 \bmod 2 \mathbb{Z}, \\ \operatorname{sgn}_{C D}\left(w_{2}^{\prime}\right), & \text { si } h_{1} \geqslant\left|h_{2}\right| \text { et } h_{1}+e_{2} \equiv 0 \bmod 2 \mathbb{Z}, \\ \operatorname{sgn}_{C D}\left(w_{1}^{\prime}\right), & \text { si } h_{1}<\left|h_{2}\right| \text { et } h_{1}+e_{2} \equiv 0 \bmod 2 \mathbb{Z} .\end{cases}
$$

Pour $\rho^{\prime} \in \operatorname{Irr}\left(W_{N^{\prime}}\right), \rho^{\prime \prime} \in \operatorname{Irr}\left(W_{N^{\prime \prime}}\right)$, on définit $\left(\rho^{\prime} \times \rho^{\prime \prime}, \rho_{1} \times \rho_{2}\right)_{h_{1}, h_{2}, \underline{\nu}}$ comme en 7.2 .

Proposition. - Sous l'hypothèse (1), on a l'égalité :

$$
\begin{aligned}
& k\left(\Lambda_{1}, \Lambda_{2}\right)(g)=\zeta(-1)^{r^{\prime}\left(r^{\prime}+1\right) / 2}(\zeta(-1) q)^{\delta\left(r^{\prime}, r^{\prime \prime}\right) / 2} \\
& \sum_{\underline{\nu} \in \mathcal{N}} \zeta(-1)^{N_{2}^{\prime \prime}} \sum_{\substack{\rho^{\prime} \in \operatorname{Irr}\left(W_{N^{\prime}}\right) \\
\rho^{\prime \prime} \in \operatorname{Irr}\left(W_{N^{\prime \prime}}\right)}} q^{b\left(r^{\prime}, \rho^{\prime}\right)+b\left(r^{\prime \prime}, \rho^{\prime \prime}\right)}\left(\rho^{\prime} \times \rho^{\prime \prime}, \rho_{1} \times \rho_{2}\right)_{h_{1}, h_{2}, \underline{\nu}} \chi_{r^{\prime}, \rho^{\prime}}\left(u^{\prime}\right) \chi_{r^{\prime \prime}, \rho^{\prime \prime}}\left(u^{\prime \prime}\right) .
\end{aligned}
$$


13.3. Pour $\eta \in\{ \pm 1\}$, on fixe un élément unipotent $u_{\eta} \in S p(2 n)$ paramétré par la partition $(2,1, . ., 1)$, où 1 est répété $2 n-2$ fois, et par la donnée $\eta_{u_{\eta}}(2)=\eta$. On note $\ell$ la forme linéaire sur $\mathcal{C}(S p(2 n))$ définie par $\ell(f)=f\left(u_{+}\right)-f\left(u_{-}\right)$pour $f \in \mathcal{C}(S p(2 n))$. Comme en 7.3 , le lemme suivant se déduit de la proposition précédente.

LEMME. - Soit $\Lambda_{1} \in \mathcal{S}_{n-1, \mathrm{imp}}$

(a) Pour $\Lambda_{2}=\mathbf{1}_{+}(1), \sigma \mathbf{1}_{+}(1)$ ou $\mathbf{1}_{-}(1), \ell\left(k\left(\Lambda_{1}, \Lambda_{2}\right)\right)=0$.

(b) $\ell\left(k\left(\Lambda_{1}, \sigma \mathbf{1}_{-}(1)\right)\right)=2(\zeta(-1) q)^{-1 / 2} q^{n} k\left(\Lambda_{1}, \Lambda_{2}(\varnothing)\right)(1)$.

13.4. Pour tout entier $m \in \mathbb{N}$, notons $E_{m}$ l'espace $\mathbb{C}^{m}$ muni de la représentation naturelle de $W_{m}$. Pour tout $\rho \in \operatorname{Irr}\left(W_{m}\right)$, définissons la fraction rationnelle :

$$
\delta_{\rho}(T)=(-1)^{m}\left|W_{m}\right|^{-1} \sum_{w \in W_{m}} \operatorname{trace}(\rho)(w) \operatorname{det}\left(1-T w \mid E_{m}\right)^{-1} .
$$

Soit $\Lambda_{2} \in \widetilde{\mathcal{S}}_{n \text {,pair }}$, notons $\left(h_{2}, \rho_{2}\right)$ le couple associé à $\Lambda_{2}$.

\section{LEMME}

(a) Si $h_{2} \neq-1, \quad \ell\left(k\left(\Lambda_{1}(\varnothing), \Lambda_{2}\right)\right)=0$.

(b) Si $h_{2}=-1, \quad \ell\left(k\left(\Lambda_{1}(\varnothing), \Lambda_{2}\right)\right)=2(\zeta(-1) q)^{-1 / 2} q^{n}|S p(2 n-2)|_{p^{\prime}} \delta_{\rho_{2}}(q)$.

Démonstration. - Pour $\eta \in\{ \pm 1\}$, on calcule $k\left(\Lambda_{1}(\varnothing), \Lambda_{2}\right)\left(u_{\eta}\right)$ grâce à la proposition 13.2. L'hypothèse 13.2(1) impose $r^{\prime \prime}=0$, i.e. $h_{2} \in\{0,-1\}$. Si $h_{2}=0$, on a $r^{\prime}=0$ et on vérifie que $\chi_{0, \rho^{\prime}}\left(u_{\eta}\right)$ est indépendant de $\eta$ pour tout $\rho^{\prime} \in \operatorname{Irr}\left(W_{n}\right)$, d'où le (a) de l'énoncé. Supposons donc $h_{2}=-1$. La formule de la proposition 13.2 devient :

$$
k\left(\Lambda_{1}(\varnothing), \Lambda_{2}\right)\left(u_{\eta}\right)=\zeta(-1)(\zeta(-1) q)^{1 / 2} q^{b\left(1, \rho_{2}\right)} \chi_{1, \rho_{2}}\left(u_{\eta}\right) .
$$

Pour toute représentation irréductible $\rho$ de $W_{n-1}$, Lusztig associe au couple $(1, \rho)$ :

- une orbite unipotente $\boldsymbol{C}_{\rho} \subseteq \boldsymbol{S} \boldsymbol{p}(2 n)$ et un système local $\mathcal{L}$ sur $\boldsymbol{C}_{\rho}$;

- une fonction $\mathcal{Y}_{\rho}$ sur $S p(2 n)$, nulle hors de $C_{\rho}$.

On définit un préordre sur l'ensemble $\operatorname{Irr}\left(W_{n-1}\right)$ par : $\rho \leqslant \rho^{\prime} \Leftrightarrow \boldsymbol{C}_{\rho} \subseteq \overline{\boldsymbol{C}}_{\rho^{\prime}}$, où $\overline{\boldsymbol{C}}_{\rho^{\prime}}$ est l'adhérence de Zariski de $\boldsymbol{C}_{\rho^{\prime}}$. On note $\sim$ la relation d'équivalence associée : $\rho \sim \rho^{\prime} \Leftrightarrow \rho \leqslant \rho^{\prime}$ et $\rho^{\prime} \leqslant \rho \Leftrightarrow \boldsymbol{C}_{\rho}=\boldsymbol{C}_{\rho^{\prime}}$. L'unique plus petit élément $\operatorname{de} \operatorname{Irr}\left(W_{n-1}\right)$ est le caractère sgn, pour lequel $\boldsymbol{C}_{\text {sgn }}$ est l'orbite de $u_{\eta}$. On a :

$$
\mathcal{Y}_{\mathrm{sgn}}\left(u_{\eta}\right)=\eta
$$

cf. [W] VIII.13. Définissons la matrice carrée $\omega$, d'ordre $\left|\operatorname{Irr}\left(W_{n-1}\right)\right| \times\left|\operatorname{Irr}\left(W_{n-1}\right)\right|$, par :

$$
\begin{aligned}
\omega_{\rho, \rho^{\prime}}=(-1)^{n-1}|S p(2 n)| q^{-\operatorname{dim}(\boldsymbol{S} \boldsymbol{p}(2 n))+\operatorname{dim}\left(\boldsymbol{C}_{\rho}\right) / 2+\operatorname{dim}\left(\boldsymbol{C}_{\rho^{\prime}}\right) / 2+n-1}\left|W_{n-1}\right|^{-1} \\
\sum_{w \in W_{n-1}} \operatorname{trace}(\rho)(w) \operatorname{trace}\left(\rho^{\prime}\right)(w) \operatorname{sgn}(w) \operatorname{det}\left(1-q w \mid E_{n-1}\right)^{-1}
\end{aligned}
$$


pour $\rho, \rho^{\prime} \in \operatorname{Irr}\left(W_{n-1}\right)$. D'après [L6] paragraphe 24, il existe deux matrices carrées $\left(P_{\rho, \rho^{\prime}}\right)$ et $\left(\lambda_{\rho, \rho^{\prime}}\right)$ telles que, pour tous $\rho, \rho^{\prime} \in \operatorname{Irr}\left(W_{n-1}\right)$, on ait :

$$
\begin{aligned}
P_{\rho, \rho^{\prime}} & \neq 0 \Longrightarrow \rho<\rho^{\prime} \text { ou } \rho=\rho^{\prime} ; \\
P_{\rho, \rho} & =1 ; \\
\lambda_{\rho, \rho^{\prime}} & \neq 0 \Longrightarrow \rho \sim \rho^{\prime} ; \\
{ }^{t} P \lambda P & =\omega ; \\
\chi_{1, \rho^{\prime}} & =\sum_{\rho \leqslant \rho^{\prime}} P_{\rho, \rho^{\prime}} \mathcal{Y}_{\rho} .
\end{aligned}
$$

Parce que sgn est l'unique plus petit élément de $\operatorname{Irr}\left(W_{n-1}\right)$, on calcule grâce à (3) :

$$
P_{\mathrm{sgn}, \rho^{\prime}}=\frac{\omega_{\mathrm{sgn}, \rho^{\prime}}}{\omega_{\mathrm{sgn}, \mathrm{sgn}}} .
$$

En utilisant (1),(2) et (4), on obtient :

$$
k\left(\Lambda_{1}(\varnothing), \Lambda_{2}\right)\left(u_{\eta}\right)=\zeta(-1)(\zeta(-1) q)^{1 / 2} q^{b\left(1, \rho_{2}\right)} \frac{\omega_{\text {sgn }, \rho_{2}}}{\omega_{\text {sgn }, \text { sgn }}} \eta
$$

Il reste à expliciter :

$$
\begin{aligned}
b\left(1, \rho_{2}\right) & =\left(\operatorname{dim}(\boldsymbol{S p}(2 n))-\operatorname{dim}\left(\boldsymbol{C}_{\rho_{2}}\right)-n\right) / 2, \\
\frac{\omega_{\mathrm{sgn}, \rho_{2}}}{\omega_{\mathrm{sgn}, \mathrm{sgn}}} & =q^{\operatorname{dim}\left(\boldsymbol{C}_{\rho_{2}}\right) / 2-n} \delta_{\rho_{2}}(q) \delta_{\mathrm{sgn}}(q)^{-1} \\
\delta_{\mathrm{sgn}}(q) & =q^{(\operatorname{dim}(\boldsymbol{S p}(2 n-2))-n+1) / 2}|S p(2 n-2)|_{p^{\prime}}^{-1},
\end{aligned}
$$

cf. [L2] lemme 2.4 pour cette dernière égalité. De (5) se déduit le (b) de l'énoncé.

13.5.

Corollaire. - Soit $\varepsilon \in\{ \pm 1\}$. Alors :

$$
\begin{gathered}
\ell \circ k_{n}\left(\Lambda_{1}(\varnothing) \otimes \mathcal{F} \mathbf{1}_{\varepsilon}(n)\right)=\varepsilon(\zeta(-1) q)^{-1 / 2} q^{n} ; \\
\ell \circ k_{n}\left(\Lambda_{1}(\varnothing) \otimes \mathcal{F} J_{n-1} \mathbf{1}_{\varepsilon}(1)\right) \neq 0 .
\end{gathered}
$$

Démonstration. - On a :

$$
\mathcal{F} \mathbf{1}_{\varepsilon}(n)=\frac{1}{2}\left(\mathbf{1}_{+}(n)+\varepsilon \sigma \mathbf{1}_{+}(n)+\mathbf{1}_{-}(n)+\varepsilon \sigma \mathbf{1}_{-}(n)\right)
$$

Le seule terme de cette expression correspondant à un couple $\left(h_{2}, \rho_{2}\right)$ pour lequel $h_{2}=-1$ est $\sigma \mathbf{1}_{-}(n)$. Son couple correspondant est $\left(-1, \mathbf{1}_{W_{n-1}}\right)$, où $\mathbf{1}_{W_{n-1}}$ est la représentation triviale de $W_{n-1}$. Le (a) de l'énoncé résulte alors du lemme précédent, en calculant grâce à $[\mathbf{L 2}]$ lemme 2.4 :

$$
\delta_{\mathbf{1}_{W_{n-1}}}(q)=\left(q^{2(n-1)}-1\right)^{-1} \cdots\left(q^{4}-1\right)^{-1}\left(q^{2}-1\right)^{-1}=|S p(2 n-2)|_{p^{\prime}}^{-1} .
$$


On a :

$$
\begin{aligned}
\mathcal{F} \circ J_{n-1}\left(\mathbf{1}_{\varepsilon}(1)\right) & =I_{n-1}^{-} \circ \mathcal{F} \circ \sigma\left(\mathbf{1}_{\varepsilon}(1)\right) \\
& =\frac{1}{2} I_{n-1}^{-}\left(\mathbf{1}_{+}(1)+\varepsilon \sigma \mathbf{1}_{+}(1)-\mathbf{1}_{-}(1)-\varepsilon \sigma \mathbf{1}_{-}(1)\right) .
\end{aligned}
$$

De nouveau, le seul terme contribuant à l'expression (b) est le terme $I_{n-1}^{-} \sigma \mathbf{1}_{-}(1)$. Pour $\rho \in \operatorname{Irr}\left(W_{n-1}\right)$, soit $\Lambda(\rho)$ l'élément de $\widetilde{\mathcal{S}}_{n \text {,pair }}$ correspondant au couple $(-1, \rho)$. Grâce au lemme 2.4, on a :

$$
\left\langle\Lambda(\rho), I_{n-1}^{-} \sigma \mathbf{1}_{-}(1)\right\rangle=-\operatorname{trace}(\rho)\left(w_{n-1}^{-}\right) .
$$

Le membre de gauche du (b) de l'énoncé est donc égal à :

$$
\frac{\varepsilon}{2} \sum_{\rho \in \operatorname{Irr}\left(W_{n-1}\right)} \operatorname{trace}(\rho)\left(w_{n-1}^{-}\right) \ell\left(k\left(\Lambda_{1}(\varnothing), \Lambda(\rho)\right)\right) .
$$

Grâce au lemme précédent et à la définition de $\delta_{\rho}$, ceci s'explicite en :

$$
\varepsilon(-1)^{n-1}(\zeta(-1) q)^{-1 / 2} q^{n}|S p(2 n-2)|_{p^{\prime}} \operatorname{det}\left(1-q w_{n-1}^{-} \mid E_{n-1}\right)^{-1}
$$

qui n'est pas nul.

13.6. Soient $m_{1}, m_{2} \in \mathbb{N}$ tels que $m_{1}^{2}+m_{1}+m_{2}^{2}=n$. Posons $t^{\prime}=m_{1}+m_{2}$, $t^{\prime \prime}=\sup \left(m_{1}-m_{2}, m_{2}-m_{1}-1\right)$,

$$
\Lambda_{1}^{\mathrm{sp}}=\left\{\left(x,(-1)^{x}\right) ; x \in\left\{2 m_{1}, \ldots, 0\right\}\right\}, \Lambda_{2}^{\mathrm{sp}}=\left\{\left(x,(-1)^{x+1}\right) ; x \in\left\{2 m_{2}-1, \ldots, 0\right\}\right\} .
$$

On considère $\Lambda_{1}$ comme un élément de $\mathcal{S}_{m_{1}^{2}+m_{1} \text {,imp }}$. Il est spécial, de défaut 1 . Le symbole ordonné $\Lambda_{2}^{\mathrm{sp}}$ appartient à $\widetilde{\mathcal{S}}_{m_{2}^{2}}$,pair , il est spécial de défaut 0 . On note $\mathrm{Fam}_{1}$, resp. ${\widetilde{\operatorname{Fam}_{2}}}_{2}$, la famille de $\Lambda_{1}^{\mathrm{sp}}$, resp. $\Lambda_{2}^{\mathrm{sp}}$, dans $\mathcal{S}_{m_{1}^{2}+m_{1} \text {,imp }}$, resp. $\widetilde{\mathcal{S}}_{m_{2}^{2}}$,pair . On représente tout élément de $\widetilde{\mathrm{Fam}}_{2}$ comme un sous-ensemble de $\mathbb{N} \times\{ \pm 1\}$ à $2 m_{2}$ éléments. On représente tout élément de $\mathrm{Fam}_{1}$ comme un sous-ensemble de $\mathbb{N} \times\{ \pm 1\}$ à $2 m_{1}+1$ éléments, en convenant d'identifier un tel sous-ensemble avec son image par $\sigma$.

Posons $M=\left\{\left|m_{1}-m_{2}\right|, \ldots, 1\right\}$, notons $P_{M}$ l'ensemble des sous-ensembles de $M$. Si $m_{1} \geqslant m_{2}$, on définit une application :

$$
\begin{aligned}
\widetilde{\operatorname{Fam}}_{2} \times P_{M} & \longrightarrow \operatorname{Fam}_{1} \times{\widetilde{\operatorname{Fam}_{2}}}_{(\Lambda, I)} \longmapsto\left(\Lambda_{1}(\Lambda, I), \Lambda_{2}(\Lambda, I)\right)
\end{aligned}
$$

par :

$$
\begin{aligned}
\Lambda_{2}(\Lambda, I) & =\Lambda, \\
\Lambda_{1}(\Lambda, I) & =\left\{\left(x+2 m_{1}-2 m_{2}+1,(-1)^{h(\Lambda)+m_{1}-m_{2}+1} \varepsilon\right) ;(x, \varepsilon) \in \Lambda\right\} \\
& \cup\left(\cup_{i \in I}\{2 i, 2 i-1\} \times\{1\}\right) \cup\left(\cup_{i \in M \backslash I}\{2 i, 2 i-1\} \times\{-1\}\right) \cup\{(0,1)\},
\end{aligned}
$$


où $h(\Lambda)=\widetilde{\operatorname{def}}(\Lambda) / 2$. On pose :

$$
\begin{array}{r}
c(\Lambda, I)=h(\Lambda)^{2}+2|I|^{2}+2(-1)^{h(\Lambda)+m_{1}-m_{2}+1}|I| h(\Lambda)+|I|\left(1-2 m_{1}+2 m_{2}\right) \\
+(-1)^{h(\Lambda)+m_{1}-m_{2}+1}\left(1-2 m_{1}+2 m_{2}\right) \frac{h(\Lambda)}{2} .
\end{array}
$$

Si $m_{1}<m_{2}$, on définit une application :

$$
\begin{aligned}
\operatorname{Fam}_{1} \times P_{M} & \longrightarrow \operatorname{Fam}_{1} \times{\widetilde{\operatorname{Fam}_{2}}}_{(\Lambda, I)} \longmapsto\left(\Lambda_{1}(\Lambda, I), \Lambda_{2}(\Lambda, I)\right)
\end{aligned}
$$

par :

$$
\begin{aligned}
\Lambda_{1}(\Lambda, I) & =\Lambda, \\
\Lambda_{2}(\Lambda, I) & =\left\{\left(x+2 m_{2}-2 m_{1}-1,(-1)^{h(\Lambda)+1} \varepsilon\right) ;(x, \varepsilon) \in \Lambda\right\} \\
\cup & \left(\cup_{i \in I}(\{2 i-2,2 i-3\} \cap \mathbb{N}) \times\{1\}\right) \cup\left(\cup_{i \in M \backslash I}(\{2 i-2,2 i-3\} \cap \mathbb{N}) \times\{-1\}\right),
\end{aligned}
$$

où $h(\Lambda)=(\widetilde{\operatorname{def}}(\Lambda)-1) / 2$. On pose :

$$
f(\Lambda, I)= \begin{cases}m_{1}-m_{2}-1, & \text { si } 1 \in I \text { et } h(\Lambda) \equiv 0 \bmod 2 \mathbb{Z}, \\ m_{1}-m_{2}, & \text { si } 1 \notin I \text { et } h(\Lambda) \equiv 0 \bmod 2 \mathbb{Z} \\ & \text { ou si } 1 \in I \text { et } h(\Lambda) \equiv 1 \bmod 2 \mathbb{Z}, \\ m_{1}-m_{2}+1, & \text { si } 1 \notin I \text { et } h(\Lambda) \equiv 1 \bmod 2 \mathbb{Z}\end{cases}
$$

$$
\begin{aligned}
c(\Lambda, I)=h(\Lambda)^{2}+2|I|^{2}+2(-1)^{h(\Lambda)+1}|I| h(\Lambda)+2|I| f(\Lambda, I) & \\
& +(-1)^{h(\Lambda)+1} h(\Lambda) f(\Lambda, I)+\frac{h(\Lambda)}{2}+\frac{f(\Lambda, I)^{2}}{2} .
\end{aligned}
$$

Remarquons que les termes $h(\Lambda)$ et $f(\Lambda, I)$ dépendent du choix d'un relèvement de $\Lambda$ en un symbole ordonné, mais ni $\Lambda_{2}(\Lambda, I)$, ni $c(\Lambda, I)$ n'en dépendent.

Soit $g \in S p(2 n)$, écrivons $g=s u$ comme en 13.2. On suppose que $s$ a pour valeurs propres 1 avec la multiplicité $t^{\prime}\left(t^{\prime}+1\right)$ et -1 avec la multiplicité $t^{\prime \prime}\left(t^{\prime \prime}+1\right)$. Décomposons $u$ en $u^{\prime} u^{\prime \prime}$. On suppose $u^{\prime}$ paramétré par les données $\underline{\lambda}\left(u^{\prime}\right)$ et $\eta_{u^{\prime}}$ ainsi définies :

$$
\underline{\lambda}\left(u^{\prime}\right)=\left(2 t^{\prime}, 2 t^{\prime}-2, \ldots, 4,2\right), \quad \text { pour } i \in \operatorname{Jord}^{\mathrm{bP}}\left(\underline{\lambda}\left(u^{\prime}\right)\right), \eta_{u^{\prime}}(i)=\zeta(-1)^{t^{\prime}+1+i / 2} .
$$

On suppose $u^{\prime \prime}$ paramétré de façon analogue.

Proposition. - Soient $g$ comme ci-dessus et $\left(\Lambda_{1}, \Lambda_{2}\right) \in \mathrm{Fam}_{1} \times \widetilde{\mathrm{Fam}}_{2}$.

(a) Si $\left(\Lambda_{1}, \Lambda_{2}\right)$ n'appartient pas à l'image de l'application (1) dans le cas où $m_{1} \geqslant m_{2}$, de l'application (2) dans le cas où $m_{1}<m_{2}$, on a $k\left(\Lambda_{1}, \Lambda_{2}\right)(g)=0$. 
(b) Supposons que $\left(\Lambda_{1}, \Lambda_{2}\right)$ soit l'image de $(\Lambda, I)$ par l'application (1), resp. (2). Alors on a l'égalité :

$$
\begin{aligned}
& \text { si } m_{1} \geqslant m_{2}, \quad k\left(\Lambda_{1}, \Lambda_{2}\right)(g)=q^{\frac{\delta\left(t^{\prime}, t^{\prime \prime}\right)}{2}-\frac{t^{\prime}\left(t^{\prime}+1\right)}{4}-\frac{t^{\prime \prime}\left(t^{\prime \prime}-3\right)}{4}} \zeta(-1)^{|I|}(\zeta(-1) q)^{c(\Lambda, I)} \text {; }
\end{aligned}
$$

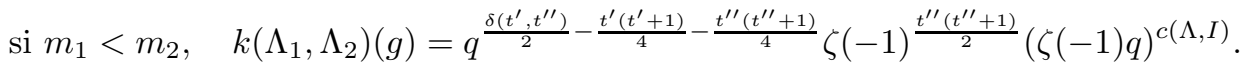

La démonstration est similaire à celle de la proposition 7.4 .

13.7. On conserve les mêmes hypothèses concernant $m_{1}, m_{2}$ et $g$. On note o l'anneau des entiers du corps $\mathbb{Q}\left((\zeta(-1) q)^{1 / 2}\right)$ (ce corps est soit une extension quadratique de $\mathbb{Q}$, soit $\mathbb{Q}$ lui-même).

Lemme. - Pour tout $\left(\Lambda_{1}, \Lambda_{2}\right) \in \mathrm{Fam}_{1} \times{\widetilde{\mathrm{Fam}_{2}}}_{2}$, le nombre

$$
\left|\operatorname{Fam}_{1}\right|^{1 / 2}\left|\widetilde{\operatorname{Fam}}_{2}\right|^{1 / 2} k_{n}\left(\mathcal{F} \Lambda_{1} \otimes \mathcal{F} \Lambda_{2}\right)(g)
$$

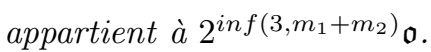

13.8. Pour $a \in\{1, \ldots, n\}$ et $\varepsilon \in\{ \pm 1\}$, on définit des foncteurs $R_{1, a}^{\varepsilon}$ et $R_{\zeta, a}^{\varepsilon}$ comme en 8.1. On pose $m=n-a$.

Proposition

(a) Désignons par $(I, R)$ l'un des couples

$$
\left(I_{a} \otimes 1, R_{1, a}^{+}\right),\left(1 \otimes I_{a}, R_{\zeta, a}^{+}\right),\left(I_{a}^{-} \otimes(-1)^{\widetilde{\operatorname{def}} / 2}, R_{1, a}^{-}\right),\left(1 \otimes I_{a}^{-} \circ(-1)^{\widetilde{\operatorname{def}} / 2},-R_{\zeta, a}^{-}\right) .
$$

Le diagramme suivant est commutatif :

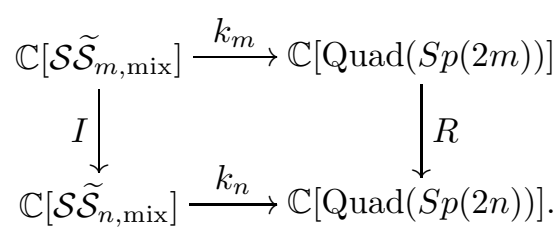

(b) Désignons par $(I, R)$ l'un des couples $\left(I_{a} \otimes 1, R_{1, a}^{+}\right),\left(1 \otimes I_{a}, R_{\zeta, a}^{+}\right)$. Le diagramme suivant est commutatif :

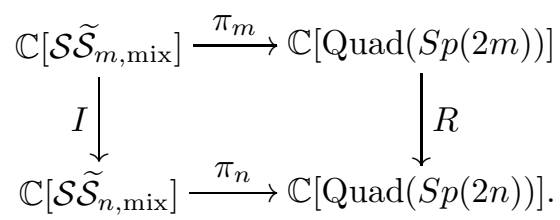




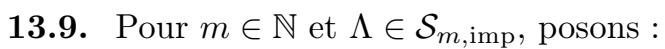

$$
\begin{aligned}
\alpha & =\sum_{i=1, \ldots,(|\Lambda|-1) / 2} \frac{(|\Lambda|-2 i)(|\Lambda|-2 i-1)}{2}, \\
D_{\Lambda}(T) & =2^{-(|\Lambda|-1) / 2} T^{-\alpha} \frac{\Delta\left(\Lambda^{+}, T\right) \Delta\left(\Lambda^{-}, T\right)}{\Theta\left(\Lambda^{+}, T^{2}\right) \Theta\left(\Lambda^{-}, T^{2}\right)} \prod_{(x, y) \in \Lambda^{+} \times \Lambda^{-}}\left(T^{x}+T^{y}\right),
\end{aligned}
$$

cf. 9.5 pour les notations.

Proposition. - Soient $\left(\Lambda_{1}, \Lambda_{2}\right),\left(\Lambda_{1}^{\prime}, \Lambda_{2}^{\prime}\right) \in \mathcal{S} \widetilde{\mathcal{S}}_{n, \text { mix }}$, supposons $\left|\widetilde{\operatorname{def}}\left(\Lambda_{1}^{\prime}\right)\right|=1$, $\widetilde{\operatorname{def}}\left(\Lambda_{2}^{\prime}\right)=0$. On a les égalités :

(i) $\operatorname{dim}\left(\pi\left(\Lambda_{1}, \Lambda_{2}\right)\right)=|S p(2 n)|_{p^{\prime}} D_{\Lambda_{1}}(q) \widetilde{D}_{\Lambda_{2}}(q)$;

(ii) $\left(\operatorname{trace}\left(\pi\left(\Lambda_{1}, \Lambda_{2}\right)\right), k\left(\Lambda_{1}^{\prime}, \Lambda_{2}^{\prime}\right)\right)=\left(\Lambda_{1} \otimes \Lambda_{2}, \mathcal{F} \Lambda_{1}^{\prime} \otimes \mathcal{F} \Lambda_{2}^{\prime}\right)$.

13.10. On considère la situation de 4.10, dont on reprend les notations. On pose $n_{0}=n-N, \pi_{0}$ étant donc une représentation de $S p\left(2 n_{0}\right)$. Pour $i \in\{1, \ldots, N\}$, on note $\tau(i)$ l'élément de $W_{N}$ qui envoie $i$ sur $-i$ et fixe $j$ pour $j \in\{ \pm 1, \ldots, \pm N\} \backslash\{ \pm i\}$. On a fixé en 13.3 un élément unipotent $u_{\eta} \in S p(2 n)$ pour $\eta \in\{ \pm 1\}$. Dans le cas où $n_{0}>0$, on fixe de même un élément unipotent $u_{0, \eta} \in S p\left(2 n_{0}\right)$.

Lemme. - Soit $w \in W_{N_{1}} \times W_{N_{2}}$. On a les égalités :

$$
\begin{aligned}
\operatorname{trace}\left(T^{\prime}(w)\right. & \left.\left(\pi\left(u_{+}\right)-\pi\left(u_{-}\right)\right) \mid E\right) \\
= & \left\{\begin{array}{l}
q^{n-n_{0}}(q-1)^{n_{0}-n}|S p(2 n-2)|_{p^{\prime}}\left|S p\left(2 n_{0}-2\right)\right|_{p^{\prime}}^{-1} \\
\quad\left(\operatorname{trace}\left(\pi_{0}\right)\left(u_{0,+}\right)-\operatorname{trace}\left(\pi_{0}\right)\left(u_{0,-}\right)\right) \text { si } w=1 \text { et } n_{0}>0, \\
2 q^{2 n-i}(q-1)^{n_{0}-n+1}|S p(2 n-2)|_{p^{\prime}}\left|S p\left(2 n_{0}\right)\right|_{p^{\prime}}^{-1} \operatorname{dim}\left(\pi_{0}\right) \\
\text { si } w=w_{i} \text { pour } i \in\left\{N_{1}+1, \ldots, N\right\}, \\
0, \text { dans les autres cas. }
\end{array}\right.
\end{aligned}
$$

Démonstration. - On suppose $n_{0}>0$, la démonstration s'adaptant aisément au cas $n_{0}=0$. Fixons $\eta \in\{ \pm 1\}$ et $b \in \mathbb{F}_{q}^{\times}$tel que $\zeta(b)=\eta$. On note $G=S p(2 n)$, $G_{0}=S p\left(2 n_{0}\right), X(w)$ l'ensemble des $h \in G$ tels que $h g h^{-1} \in U w P$. Pour $h \in X(w)$, on note $m(h)$ l'unique élément de $M$ tel que $h u_{\eta} h^{-1} \in U w m(h) U$. Comme en 10.1(6), on a l'égalité :

$$
\operatorname{trace}\left(T^{\prime}(w) \pi\left(u_{\eta}\right) \mid E\right)=\sum_{P \backslash X(w)} \operatorname{trace}\left(1_{N_{1}} \times \zeta_{N_{2}} \times \pi_{0}\right)(m(h)) .
$$

Pour tout $i \in\{ \pm 1, \ldots, \pm N\}$, notons $u_{i, \eta}$ l'élément de $G$ qui envoie $v_{i}$ sur $-b v_{-i}$ si $i>0$, sur $b v_{-i}$ si $i<0$, et fixe $v_{j}$ pour tout $j \in\{ \pm 1, \ldots, \pm N\} \backslash\{i\}$ ainsi que tout élément de $V_{0}$. Pour tout $i \in\{0\} \cup\{ \pm 1, \ldots, \pm N\}$, on fixe $h(i) \in G$ tel que $h(i) u_{\eta} h(i)^{-1}=u_{i, \eta}$. On vérifie que la classe de conjugaison de $u_{\eta}$ par $G$ est réunion disjointe des classes de conjugaison par $P$ des $u_{i, \eta}$ pour $i \in\{0\} \cup\{ \pm 1, \ldots, \pm N\}$. Autrement dit, $G$ est réunion disjointe des ensembles $P h(i) Z_{G}\left(u_{\eta}\right)$. 
On a $h(0) u_{\eta} h(0)^{-1}=u_{0, \eta} \in G_{0} \subseteq M$. On en déduit que pour $h \in P h(0) Z_{G}\left(u_{\eta}\right)$, on a $h \in X(w) \Leftrightarrow w=1$ et, dans le cas où $w=1, \operatorname{trace}\left(1_{N_{1}} \times \zeta_{N_{2}} \times \pi_{0}\right)(m(h))=$ $\operatorname{trace}\left(\pi_{0}\right)\left(u_{0, \eta}\right)$.

Soit $i \in\{-1, \ldots,-N\}$. On a $h(i) u_{\eta} h(i)^{-1}=u_{i, \eta} \in U$. On en déduit que pour $h \in P h(i) Z_{G}\left(u_{\eta}\right)$, on a $h \in X(w) \Leftrightarrow w=1$ et, dans le cas où $w=1, \operatorname{trace}\left(1_{N_{1}} \times\right.$ $\left.\zeta_{N_{2}} \times \pi_{0}\right)(m(h))=\operatorname{dim}\left(\pi_{0}\right)$.

Soit $i \in\{1, \ldots, N\}$. On a $h(i) u_{\eta} h(i)^{-1}=u_{i, \eta} \in U \tau(i) m_{i} U$, où $m_{i}$ est l'élément de $M$ qui envoie $v_{i}$ sur $b v_{i}, v_{-i}$ sur $b^{-1} v_{-i}$ et fixe $v_{j}$ pour $j \in\{ \pm 1, \ldots, \pm N\} \backslash\{ \pm i\}$, ainsi que tout élément de $V_{0}$. On en déduit que pour $h \in P h(i) Z_{G}\left(u_{\eta}\right)$, on a $h \in X(w) \Leftrightarrow$ $w=\tau(i)$ et, dans le cas où $w=\tau(i)$,

$$
\operatorname{trace}\left(1_{N_{1}} \times \zeta_{N_{2}} \times \pi_{0}\right)(m(h))= \begin{cases}\operatorname{dim}\left(\pi_{0}\right), & \text { si } i \in\left\{1, \ldots, N_{1}\right\}, \\ \eta \operatorname{dim}\left(\pi_{0}\right), & \text { si } i \in\left\{N_{1}+1, \ldots, N\right\} .\end{cases}
$$

On obtient donc :

- si $w=1$

$$
\begin{aligned}
\operatorname{trace}\left(T^{\prime}(w) \pi\left(u_{\eta}\right) \mid E\right)=\left|P \backslash P h(0) Z_{G}\left(u_{\eta}\right)\right| & \operatorname{trace}\left(\pi_{0}\right)\left(u_{0, \eta}\right) \\
& +\sum_{i=1, \ldots, N}\left|P \backslash P h(-i) Z_{G}\left(u_{\eta}\right)\right| \operatorname{dim}\left(\pi_{0}\right) ;
\end{aligned}
$$

- si $w=\tau(i)$, pour $i \in\left\{1, \ldots, N_{1}\right\}$,

$$
\operatorname{trace}\left(T^{\prime}(w) \pi\left(u_{\eta}\right) \mid E\right)=\left|P \backslash P h(i) Z_{G}\left(u_{\eta}\right)\right| \operatorname{dim}\left(\pi_{0}\right) ;
$$

- si $w=\tau(i)$, pour $i \in\left\{N_{1}+1, \ldots, N\right\}$,

$$
\operatorname{trace}\left(T^{\prime}(w) \pi\left(u_{\eta}\right) \mid E\right)=\eta\left|P \backslash P h(i) Z_{G}\left(u_{\eta}\right)\right| \operatorname{dim}\left(\pi_{0}\right) ;
$$

- dans les autres cas, trace $\left(T^{\prime}(w) \pi\left(u_{\eta}\right) \mid E\right)=0$.

L'énoncé se déduit de ces formules, après avoir calculé les nombres d'éléments des ensembles $P \backslash P h(i) Z_{G}\left(u_{\eta}\right)$, ce qui est élémentaire.

\subsection{1.}

LEMme. - Soient $\varepsilon \in\{ \pm 1\}$ et $\Lambda_{1} \in \mathcal{S}_{n-1, \mathrm{imp}}$. Si $\varepsilon=-1$, on suppose $\Lambda_{1}$ non cuspidal. On a l'égalité :

$$
\ell\left(\operatorname{trace}\left(\pi\left(\Lambda_{1}, \mathbf{1}_{\varepsilon}(1)\right)\right)\right)=\varepsilon q^{n}(\zeta(-1) q)^{-1 / 2} \operatorname{dim}\left(\pi\left(\Lambda_{1}, \Lambda_{2}(\varnothing)\right)\right) .
$$

Cela se déduit du lemme précédent comme en 10.1 et 10.2.

13.12.

Lemme. - On a l'égalité :

$$
\ell\left(\operatorname{trace}\left(\pi\left(\Lambda_{1}(\varnothing), \mathbf{1}_{+}(n)\right)\right)\right)=(\zeta(-1) q)^{-1 / 2} q^{n} .
$$


Démonstration. - On considère la situation de 13.10 pour $N_{1}=0, N_{2}=n$. Notons $\mathcal{H}$ l'algèbre d'entrelacements de la représentation induite $\pi$. Elle a pour base les opérateurs $T(w)=T_{2}(w)=C_{2}(w) T_{2}^{\prime}(w)$ pour $w \in W_{n}$. Pour toute représentation irréductible $\rho$ de $W_{n}$, notons $\rho_{\mathcal{H}}$ la représentation correspondante de $\mathcal{H}$ et $\Lambda_{2}(\rho)$ le symbole ordonné associé en 2.2 au couple $(0, \rho)$. Pour $w \in W_{n}$, posons :

$$
Y(w)=\sum_{\rho \in \operatorname{Irr}\left(W_{n}\right)} \operatorname{trace}\left(\rho_{\mathcal{H}}\right)(T(w)) \ell\left(\operatorname{trace}\left(\pi\left(\Lambda_{1}(\varnothing), \Lambda_{2}(\rho)\right)\right)\right) .
$$

On a l'égalité :

$Y(w)=\operatorname{trace}\left(T(w)\left(\pi\left(u_{+}\right)-\pi\left(u_{-}\right)\right) \mid E\right)=C_{2}(w) \operatorname{trace}\left(T^{\prime}(w)\left(\pi\left(u_{+}\right)-\pi\left(u_{-}\right)\right) \mid E\right)$.

En 4.10, on a dit que $C_{2}\left(s_{i}\right)=1$ pour $i \in\{1, \ldots, n-1\}$ et $C_{2}\left(s_{n}\right)=(\zeta(-1) q)^{-1 / 2}$. Il en résulte que $C_{2}(\tau(i))=(\zeta(-1) q)^{-1 / 2}$ pour tout $i \in\{1, \ldots, n\}$. Grâce au lemme 13.10, on obtient :

$$
Y(w)=\left\{\begin{array}{l}
\left.2(\zeta(-1) q)^{-1 / 2} q^{2 n-i}(q-1)^{1-n}|S p(2 n-2)|\right|_{p^{\prime}} \\
\quad \text { si } w=\tau(i) \text { pour } i \in\{1, \ldots, n\} \\
0, \text { sinon. }
\end{array}\right.
$$

On a les relations :

- pour $i \in\{1, \ldots, n-1\},\left(T_{2}\left(s_{i}\right)+1\right)\left(T_{2}\left(s_{i}\right)-q\right)=0$;

$-\left(T_{2}\left(s_{n}\right)+1\right)\left(T_{2}\left(s_{n}\right)-1\right)=0$.

Plaçons-nous pour un instant dans une situation plus générale où la dernière égalité est remplacée par :

$-\left(T_{2}\left(s_{n}\right)+1\right)\left(T_{2}\left(s_{n}\right)-q^{c}\right)=0$,

où $c \in \mathbb{N}$. On définit une fonction $l_{\mathcal{H}}$ sur $W_{n}$ par :

- pour $i \in\{1, \ldots, n-1\}, l_{\mathcal{H}}\left(s_{i}\right)=1$;

$-l_{\mathcal{H}}\left(s_{n}\right)=c$;

- pour $w \in W_{n}$, écrit sous forme réduite $w=s_{i_{1}} \cdots s_{i_{t}}, l_{\mathcal{H}}(w)=l_{\mathcal{H}}\left(s_{i_{1}}\right)+\cdots+$ $l_{\mathcal{H}}\left(s_{i_{t}}\right)$.

Pour $\rho, \rho^{\prime} \in \operatorname{Irr}\left(W_{n}\right)$, posons :

$$
\left\langle\rho, \rho^{\prime}\right\rangle_{\mathcal{H}}=\sum_{w \in W_{n}} \operatorname{trace}\left(\rho_{\mathcal{H}}\right)(T(w)) \operatorname{trace}\left(\rho_{\mathcal{H}}^{\prime}\right)\left(T\left(w^{-1}\right)\right) q^{-l_{\mathcal{H}}(w)} .
$$

Il est connu que :

$$
\left\langle\rho, \rho^{\prime}\right\rangle_{\mathcal{H}}=0, \text { si } \rho \neq \rho^{\prime},
$$

cf. [CR] 9.17. Notons $\mathbf{1}_{W_{n}}$ la représentation triviale de $W_{n}$. On a

$$
\operatorname{trace}\left(\mathbf{1}_{W_{n}, \mathcal{H}}\right)(T(w))=q^{l_{\mathcal{H}}(w)}
$$

pour tout $w \in W_{n}$ et on calcule aisément :

$$
\left\langle\mathbf{1}_{W_{n}}, \mathbf{1}_{W_{n}}\right\rangle_{\mathcal{H}}=(q-1)^{-n} \prod_{i=1, \ldots, n}\left(q^{i}-1\right)\left(q^{i+c-1}+1\right) .
$$


Revenons à notre situation où $c=0$. Posons :

$$
Y=\sum_{w \in W_{n}} Y(w) \operatorname{trace}\left(\mathbf{1}_{W_{n}, \mathcal{H}}\right)(T(w)) q^{-l_{\mathcal{H}}(w)} .
$$

Remarquons que $\Lambda_{2}\left(\mathbf{1}_{W_{n}}\right)=\mathbf{1}_{+}(n)$. De (1) et (3) se déduit l'égalité :

$$
Y=\left\langle\mathbf{1}_{W_{n}}, \mathbf{1}_{W_{n}}\right\rangle_{\mathcal{H}} \ell\left(\operatorname{trace}\left(\pi\left(\Lambda_{1}(\varnothing), \mathbf{1}_{+}(n)\right)\right)\right)
$$

Puisque trace $\left(\mathbf{1}_{W_{n}, \mathcal{H}}\right)(T(w))=q^{l_{\mathcal{H}}(w)}$ pour tout $w \in W_{n}$, on a $Y=\sum_{w \in W_{n}} Y(w)$ et on calcule grâce à $(2)$ :

$$
Y=2\left(\zeta(-1) q^{-1 / 2} q^{n}(q-1)^{-n}\left(q^{n}-1\right)|S p(2 n-2)|_{p^{\prime}} .\right.
$$

On obtient l'énoncé en comparant les deux formules ci-dessus et en calculant grâce à (4) :

$$
\begin{aligned}
\left\langle\mathbf{1}_{W_{n}}, \mathbf{1}_{W_{n}}\right\rangle_{\mathcal{H}} & =2(q-1)^{-n}\left(q^{n}-1\right) \prod_{i=1, \ldots, n-1}\left(q^{2 i}-1\right) \\
& =2(q-1)^{-n}\left(q^{n}-1\right)|S p(2 n-2)|_{p^{\prime}}
\end{aligned}
$$

13.13.

Lemme. - On a l'égalité :

$$
\ell\left(\operatorname{trace}\left(\pi\left(\Lambda_{1}(\varnothing), \mathbf{1}_{-}(n)\right)\right)\right)=-(\zeta(-1) q)^{-1 / 2} q^{n} .
$$

Démonstration. - On considère la situation de 13.10 pour $N_{1}=0, N_{2}=n-1$, $\pi_{0}=\pi\left(\Lambda_{1}(\varnothing), \mathbf{1}_{-}(1)\right)$. La démonstration est similaire à celle du lemme précédent, une fois calculées les constantes de structure de l'algèbre $\mathcal{H}$, c'est-à-dire le nombre $C_{2}\left(s_{n-1}\right)$ et l'entier $c$ tel que :

$$
\left(T_{2}\left(s_{n-1}\right)+1\right)\left(T_{2}\left(s_{n-1}\right)-q^{c}\right)=0 .
$$

Pour faire ce calcul, on peut supposer $n=2$. L'équation précédente s'écrit :

$$
C_{2}\left(s_{1}\right)^{2} T_{2}^{\prime}\left(s_{1}\right)^{2}+C_{2}\left(s_{1}\right)\left(1-q^{c}\right) T_{2}^{\prime}\left(s_{1}\right)-q^{c}=0 .
$$

Soit $f \in E$ un élément non nul à support dans $P$. Pour tout $g \in S p(4)$, on a l'égalité :

$$
C_{2}\left(s_{1}\right)^{2}\left(T_{2}^{\prime}\left(s_{1}\right)^{2} f\right)(g)+C_{2}\left(s_{1}\right)\left(1-q^{c}\right)\left(T_{2}^{\prime}\left(s_{1}\right) f\right)(g)-q^{c} f(g)=0 .
$$

Connaissant le caractère de $\pi_{0}$, un calcul matriciel permet de calculer tous les termes ci-dessus pour $g=1$ et pour $g=s_{1}$. On obtient deux équations qui déterminent les inconnues. On obtient $c=2, C_{2}\left(s_{1}\right)=-(\zeta(-1) q)^{-1 / 2}$. Le lemme s'en déduit. 
13.14. On suppose ici $n=2$. On définit les symboles $\Lambda(i)$ pour $i \in\{0,1,2\}$ par :

$$
\begin{gathered}
\Lambda(0)^{+}=\{2,1\}, \Lambda(0)^{-}=\{0\} ; \Lambda(1)^{+}=\{2,0\}, \Lambda(1)^{-}=\{1\} ; \\
\Lambda(2)^{+}=\{1,0\}, \Lambda(2)^{-}=\{2\} .
\end{gathered}
$$

Lemme. - Pour tout $\Lambda \in \mathcal{S}_{2,1}$ (cf. 2.1), il existe un polynôme $P_{\Lambda} \in \mathbb{Q}[T]$ de sorte que :

$$
P_{\Lambda(0)}(1)=-1, \quad P_{\Lambda(1)}(1)=0, \quad P_{\Lambda(2)}(1)=-1 ;
$$

en posant $P=\sum_{\Lambda \in \mathcal{S}_{2,1}} P_{\Lambda}(q) \operatorname{trace}\left(\pi\left(\Lambda, \Lambda_{2}(\varnothing)\right)\right)$, on a l'égalité :

$$
\left(k\left(\Lambda_{1, \operatorname{cusp}}(1), \Lambda_{2}(\varnothing)\right), P\right)=q^{2} .
$$

13.15. Comme en 13.7 , on note o l'anneau des entiers du corps $\mathbb{Q}\left((\zeta(-1) q)^{1 / 2}\right)$. Dans le cas où ce corps est une extension quadratique de $\mathbb{Q}$, on note $\theta$ son automorphisme galoisien non trivial.

LEMME. - Soit $\left(\Lambda_{1}, \Lambda_{2}\right) \in \mathcal{S} \widetilde{\mathcal{S}}_{n, \text { mix }}$. Supposons $\left|\widetilde{\operatorname{def}}\left(\Lambda_{1}\right)\right|=1,\left|\widetilde{\operatorname{def}}\left(\Lambda_{2}\right)\right| \leqslant 2$. Alors la fonction trace $\left(\pi\left(\Lambda_{1}, \Lambda_{2}\right)\right)$ prend ses valeurs dans o. Quand $\mathbb{Q}\left((\zeta(-1) q)^{1 / 2}\right)$ est une extension quadratique de $\mathbb{Q}$, l'image de cette fonction par $\theta$ est la fonction $\operatorname{trace}\left(\pi\left(\Lambda_{1}, \sigma \Lambda_{2}\right)\right)$.

Démonstration. - Ces assertions sont vraies pour $\Lambda_{1}=\Lambda_{1}(\varnothing), \Lambda_{2}=\mathbf{1}_{-}(1)$ : cela se voit sur la table des caractères de $S L(2)$. Le lemme s'en déduit comme en 10.7.

13.16. Soit $m \in \mathbb{N}$. Pour $\varepsilon \in\{ \pm 1\}$, on a introduit en 2.4 l'élément $w_{m}^{\varepsilon} \in W_{m}$. Notons $\mathcal{P}_{2}(m)$ l'ensemble des couples $(\underline{\alpha}, \underline{\beta})$ de partitions tels que $S(\underline{\alpha})+S(\underline{\beta})=m$. Pour un tel couple, notons $\underline{\alpha}=\left(\alpha_{1} \geqslant \cdots \geqslant \alpha_{r}\right), \underline{\beta}=\left(\beta_{1} \geqslant \cdots \geqslant \beta_{s}\right)$, avec $\alpha_{r}>0, \beta_{s}>0$. On note $w_{\underline{\alpha}, \underline{\beta}}$ l'image dans $W_{m}$ de $\left(w_{\alpha_{1}}^{+}, \ldots, w_{\alpha_{r}}^{+}, w_{\beta_{1}}^{-}, \ldots, w_{\beta_{s}}^{-}\right)$par le plongement naturel :

$$
W_{\alpha_{1}} \times \cdots \times W_{\alpha_{r}} \times W_{\beta_{1}} \times \cdots \times W_{\beta_{s}} \longrightarrow W_{m} .
$$

On note $C(\underline{\alpha}, \underline{\beta})$ la classe de conjugaison de $w_{\underline{\alpha}, \underline{\beta}}$ dans $W_{m}$. On pose $\varepsilon(\underline{\beta})=(-1)^{s}$, $I_{\underline{\alpha}}=I_{\alpha_{1}} \circ \cdots \circ I_{\alpha_{r}}, J_{\underline{\beta}}=J_{\beta_{1}} \circ \cdots \circ J_{\beta_{s}}$.

LEMME. - Soit $\varepsilon \in\{ \pm 1\}$. On a l'égalité :

$$
\mathbf{1}_{\varepsilon}(n)-\sigma \mathbf{1}_{\varepsilon}(n)=\sum_{(\underline{\alpha}, \underline{\beta}) \in \mathcal{P}_{2}(n-1)}|C(\underline{\alpha}, \underline{\beta})|\left|W_{n-1}\right|^{-1} \varepsilon(\underline{\beta}) I_{\underline{\alpha}} \circ J_{\underline{\beta}}\left(\mathbf{1}_{\varepsilon \varepsilon(\underline{\beta})}(1)-\sigma \mathbf{1}_{\varepsilon \varepsilon(\underline{\beta})}(1)\right) .
$$

Deux termes d'indices distincts de cette somme sont orthogonaux. 
Démonstration. - Soit $m \in \mathbb{N}$. Pour $a \in\{1, \ldots, m\}$, on a défini en 2.4 les homomorphismes :

$$
\operatorname{res}_{a}^{+}, \operatorname{res}_{a}^{-}: \mathcal{C}\left(W_{m}\right) \longrightarrow \mathcal{C}\left(W_{m-a}\right) .
$$

Pour $(\underline{\alpha}, \underline{\beta}) \in \mathcal{P}_{2}(m)$, que l'on écrit comme ci-dessus, on définit l'homomorphisme :

$$
\operatorname{res}_{\underline{\alpha}, \underline{\beta}}: \mathcal{C}\left(W_{m}\right) \longrightarrow \mathcal{C}\left(W_{0}\right)=\mathbb{C}
$$

par :

$$
\operatorname{res}_{\underline{\alpha}, \underline{\beta}}=\operatorname{res}_{\beta_{s}}^{-} \circ \cdots \circ \operatorname{res}_{\beta_{1}}^{-} \circ \operatorname{res}_{\alpha_{r}}^{+} \circ \cdots \circ \operatorname{res}_{\alpha_{1}}^{+} .
$$

On note ind $\underline{\alpha}, \underline{\beta}_{1}$ l'homomorphisme adjoint. Notons $\mathbf{1}_{W_{m}}$, resp. $\mathbf{1}_{W_{0}}$, la représentation triviale de $W_{m}$, resp. $W_{0}$. Pour $f \in \mathcal{C}\left(W_{m}\right)$, on a par définition :

$$
\left(f, \operatorname{ind}_{\underline{\alpha}, \underline{\beta}} \mathbf{1}_{W_{0}}\right)=f\left(w_{\underline{\alpha}}, \underline{\beta}\right) .
$$

On en déduit que $\operatorname{ind}_{\underline{\alpha}, \underline{\beta}} \mathbf{1}_{W_{0}}$ est le produit de $\left|W_{m}\right||C(\underline{\alpha}, \underline{\beta})|^{-1}$ par la fonction caractéristique de $C(\underline{\alpha}, \underline{\beta})$. D'où l'égalité :

$$
\mathbf{1}_{W_{m}}=\sum_{(\underline{\alpha}, \underline{\beta}) \in \mathcal{P}_{2}(m)}|C(\underline{\alpha}, \underline{\beta})|\left|W_{m}\right|^{-1} \operatorname{ind}_{\underline{\alpha}, \underline{\beta}} \mathbf{1}_{W_{0}} .
$$

Deux termes d'indices distincts de cette somme sont orthogonaux.

Appliquons ceci pour $m=n-1$, transférons cette égalité au terme indicé par $h=1$ de la décomposition 2.4(1). Grâce au lemme 2.4, on obtient :

$$
\mathbf{1}_{-}(n)=\sum_{(\underline{\alpha}, \underline{\beta}) \in \mathcal{P}_{2}(n-1)}|C(\underline{\alpha}, \underline{\beta})|\left|W_{n-1}\right|^{-1} I_{\underline{\alpha}} \circ I_{\underline{\beta}}^{-} \mathbf{1}_{-}(1),
$$

avec une définition évidente de $I_{\underline{\beta}}^{-}$. En utilisant les formules de commutation de 2.3, on a aussi :

$$
\sigma \mathbf{1}_{-}(n)=\sum_{(\underline{\alpha}, \underline{\beta}) \in \mathcal{P}_{2}(n-1)}|C(\underline{\alpha}, \underline{\beta})|\left|W_{n-1}\right|^{-1} \varepsilon(\underline{\beta}) I_{\underline{\alpha}} \circ I_{\underline{\beta}}^{-} \sigma \mathbf{1}_{-}(1) .
$$

Dans ces deux formules, les termes d'indices distincts sont orthogonaux. Ceux de la première sont orthogonaux à ceux de la seconde : ils ont des défauts distincts. Alors :

$$
\mathbf{1}_{-}(n)+\varepsilon \sigma \mathbf{1}_{-}(n)=\sum_{(\underline{\alpha}, \underline{\beta}) \in \mathcal{P}_{2}(n-1)}|C(\underline{\alpha}, \underline{\beta})|\left|W_{n-1}\right|^{-1} I_{\underline{\alpha}} \circ I_{\underline{\beta}}^{-}\left(\mathbf{1}_{-}(1)+\varepsilon \varepsilon(\underline{\beta}) \sigma \mathbf{1}_{-}(1)\right),
$$

et deux termes d'indices distincts de cette somme sont orthogonaux. Appliquons $\mathcal{F}$. Grâce aux formules de commutation de 2.2 et 2.3 , on obtient :

$$
\mathcal{F}\left(\mathbf{1}_{-}(n)+\varepsilon \sigma \mathbf{1}_{-}(n)\right)=\sum_{(\underline{\alpha}, \underline{\beta}) \in \mathcal{P}_{2}(n-1)}|C(\underline{\alpha}, \underline{\beta})|\left|W_{n-1}\right|^{-1} \varepsilon(\underline{\beta}) I_{\underline{\alpha}} \circ J_{\underline{\beta}} \circ \mathcal{F}\left(\mathbf{1}_{-}(1)+\varepsilon \varepsilon(\underline{\beta}) \sigma \mathbf{1}_{-}(1)\right) .
$$

Deux termes d'indices distincts de cette somme restent orthogonaux. On remarque maintenant que :

$$
\mathcal{F}\left(\mathbf{1}_{-}(n)+\varepsilon \sigma \mathbf{1}_{-}(n)\right)=\mathbf{1}_{\varepsilon}(n)-\sigma \mathbf{1}_{\varepsilon}(n),
$$


et, pour $(\underline{\alpha}, \underline{\beta}) \in \mathcal{P}_{2}(n-1)$,

$$
\mathcal{F}\left(\mathbf{1}_{-}(1)+\varepsilon \varepsilon(\underline{\beta}) \sigma \mathbf{1}_{-}(1)\right)=\mathbf{1}_{\varepsilon \varepsilon(\underline{\beta})}(1)-\sigma \mathbf{1}_{\varepsilon \varepsilon(\underline{\beta})}(1) .
$$

13.17. On définit l'endomorphisme $i=\pi_{n}^{-1} \circ k_{n} \circ \mathcal{F}$ de l'espace $\mathbb{C}\left[\mathcal{S} \widetilde{\mathcal{S}}_{n \text {,mix }}\right]$. C'est une isométrie qui possède des propriétés similaires à celles relevées en 6.5 et 11.1. On note $\mathcal{Z}$ le sous-espace de $\mathbb{C}\left[\mathcal{S} \widetilde{\mathcal{S}}_{n, \text { mix }}\right]$ engendré par les $\Lambda_{1} \otimes \Lambda_{2}$ où $\Lambda_{1}$ et $\Lambda_{2}$ sont cuspidaux.

\section{Proposition}

(a) Soient $n_{1}, n_{2}, a \in \mathbb{N}$ tels que $n_{1}+n_{2}=n, 1 \leqslant a \leqslant n_{1}$, soient $\Lambda \in \mathcal{S}_{n_{1}-a, \mathrm{imp}}$, $\Lambda_{2} \in \widetilde{\mathcal{S}}_{n_{2}, \text { pair }}$. Alors $i\left(J_{a} \Lambda \otimes \Lambda_{2}\right)-J_{a} \Lambda \otimes \Lambda_{2}$ appartient à $\mathcal{Z}$.

(b) Soient $n_{1}, n_{2}, a \in \mathbb{N}$ tels que $n_{1}+n_{2}=n, 1 \leqslant a \leqslant n_{2}$, soient $\Lambda_{1} \in \mathcal{S}_{n_{1}, \mathrm{imp}}$, $\Lambda \in \widetilde{\mathcal{S}}_{n_{2}-a, \text { pair }}$. Alors $i\left(\Lambda_{1} \otimes J_{a} \Lambda\right)-\Lambda_{1} \otimes J_{a} \Lambda$ appartient à $\mathcal{Z}$.

Démonstration. - Dans la situation de (a), écrivons comme en 11.2 :

$$
J_{a} \Lambda=\sum_{(x, \varepsilon) \in E} \zeta(x, \varepsilon) \Lambda(x, \varepsilon)
$$

notons $E_{c}$ le sous-ensemble des $(x, \varepsilon) \in E$ tels que $\Lambda(x, \varepsilon)$ est cuspidal. Remarquons que $E_{c}$ a au plus 1 élément. On pose :

$$
\left(J_{a} \Lambda\right)_{n c}=\sum_{(x, \varepsilon) \in E \backslash E_{c}} \zeta(x, \varepsilon) \Lambda(x, \varepsilon),\left(J_{a} \Lambda\right)_{c}=\sum_{(x, \varepsilon) \in E_{c}} \zeta(x, \varepsilon) \Lambda(x, \varepsilon) .
$$

Comme au paragraphe 11, des raisonnements purement combinatoires permettent de démontrer l'assertion de la proposition sauf dans les cas (1) et (2) suivants :

(1) $n_{1}-a>0, \Lambda_{2}=\mathbf{1}_{+}(1)$ ou $\sigma \mathbf{1}_{+}(1)$;

(2) $n_{1}-a=0$, il existe $\varepsilon \in\{ \pm 1\}$ tel que $\Lambda_{2}=\mathbf{1}_{\varepsilon}(1)$ ou $\sigma \mathbf{1}_{\varepsilon}(1)$.

Dans le cas (1), on peut seulement prouver :

$$
i\left(J_{a} \Lambda \otimes \Lambda_{2}\right)=J_{a} \Lambda \otimes \Lambda_{2} \text { ou }\left(J_{a} \Lambda\right)_{n c} \otimes \Lambda_{2}+\left(J_{a} \Lambda\right)_{c} \otimes \sigma \Lambda_{2} .
$$

Si $E_{c}=\varnothing$, c'est terminé. Supposons $E_{c} \neq \varnothing$, notons $\left(x_{c}, \varepsilon_{c}\right)$ l'unique élément de $E_{c}$. Il faut exclure la deuxième possibilité ci-dessus. Supposons-la vérifiée. Elle équivaut à :

$$
k_{n}\left(\mathcal{F} J_{a} \Lambda \otimes \mathcal{F} \Lambda_{2}\right)-\pi_{n}\left(J_{a} \Lambda \otimes \Lambda_{2}\right)=\pi_{n}\left(\zeta\left(x_{c}, \varepsilon_{c}\right) \Lambda\left(x_{c}, \varepsilon_{c}\right) \otimes\left(\sigma \Lambda_{2}-\Lambda_{2}\right)\right) .
$$

Par symétrie, on peut supposer $\Lambda_{2}=\mathbf{1}_{+}(1)$. On a l'égalité :

$$
\ell \circ k_{n}\left(\mathcal{F} J_{a} \Lambda \otimes \mathcal{F} \mathbf{1}_{+}(1)\right)=\ell \circ \pi_{n}\left(J_{a} \Lambda \otimes \mathbf{1}_{+}(1)\right) .
$$

En effet, cela résulte des lemmes 13.3 et 13.11 et de l'hypothèse de récurrence qui nous dit que $k_{n-1}\left(\mathcal{F} J_{a} \Lambda \otimes \Lambda_{2}(\varnothing)\right)=\pi_{n-1}\left(J_{a} \Lambda \otimes \Lambda_{2}(\varnothing)\right)$. On en déduit :

$$
\ell \circ \pi_{n}\left(\Lambda\left(x_{c}, \varepsilon_{c}\right) \otimes\left(\sigma \Lambda_{2}-\Lambda_{2}\right)\right)=0 .
$$

Grâce à la proposition 4.12 b(ii), on a l'égalité :

$$
\ell \circ \pi_{n}\left(\Lambda\left(x_{c}, \varepsilon_{c}\right) \otimes \sigma \Lambda_{2}\right)=-\ell \circ \pi_{n}\left(\Lambda\left(x_{c}, \varepsilon_{c}\right) \otimes \Lambda_{2}\right),
$$


et l'égalité précédente devient :

$$
\ell \circ \pi_{n}\left(\Lambda\left(x_{c}, \varepsilon_{c}\right) \otimes \mathbf{1}_{+}(1)\right)=0 .
$$

Grâce au lemme 13.11, cela entraîne $\operatorname{dim}\left(\pi\left(\Lambda\left(x_{c}, \varepsilon_{c}\right), \Lambda_{2}(\varnothing)\right)\right)=0$, ce qui est évidemment faux. Cette contradiction entraîne l'énoncé sous l'hypothèse (1).

Dans le cas (2), on peut seulement prouver l'une des égalités :

$$
\begin{gathered}
i\left(J_{a} \Lambda \otimes \Lambda_{2}\right)=J_{a} \Lambda \otimes \Lambda_{2} \text { ou } J_{a} \Lambda \otimes \sigma \Lambda_{2} \\
\text { ou }\left(J_{a} \Lambda\right)_{n c} \otimes \Lambda_{2}+\left(J_{a} \Lambda\right)_{c} \otimes \sigma \Lambda_{2} \text { ou }\left(J_{a} \Lambda\right)_{n c} \otimes \sigma \Lambda_{2}+\left(J_{a} \Lambda\right)_{c} \otimes \Lambda_{2} .
\end{gathered}
$$

Remarquons que $\Lambda=\Lambda_{1}(\varnothing)$ et que, par symétrie, on peut supposer $\Lambda_{2}=\mathbf{1}_{\varepsilon}(1)$. Supposons d'abord $E_{c}=\varnothing$. Les quatre possibilités ci-dessus se ramènent aux deux premières. On doit exclure la deuxième. Un raisonnement analogue à celui du cas précédent y pourvoit, pourvu que $\pi_{n}\left(J_{a} \Lambda \otimes \Lambda_{2}(\varnothing)\right)(1) \neq 0$, i.e.

$$
\sum_{(x, \varepsilon) \in E} \zeta(x, \varepsilon) \operatorname{dim}\left(\pi\left(\Lambda(x, \varepsilon) \otimes \Lambda_{2}(\varnothing)\right)\right) \neq 0
$$

Un calcul analogue à celui effectué dans la preuve de la proposition 11.11 montre que la somme ci-dessus vaut $(-1)^{a+1}\left(q^{a}+1\right)^{-1}|S p(2 n-2)|_{p^{\prime}}$. Cela démontre l'assertion dans ce cas. Supposons maintenant $E_{c} \neq \varnothing$. Notons $\sigma_{c}$ et $\sigma_{n c}$ les automorphismes, égaux à 1 ou $\sigma$, tels que :

$$
i\left(J_{a} \Lambda \otimes \Lambda_{2}\right)=\left(J_{a} \Lambda\right)_{n c} \otimes \sigma_{n c} \Lambda_{2}+\left(J_{a} \Lambda\right)_{c} \otimes \sigma_{c} \Lambda_{2} .
$$

On doit prouver que $\sigma_{n c}=1$ et, si $\varepsilon=1, \sigma_{c}=1$. Puisque $E_{c} \neq \varnothing$, on a $a=2$, $n=3$ et, en notant $\left(x_{c}, \varepsilon_{c}\right)$ l'unique élément de $E_{c}$, on a $\Lambda\left(x_{c}, \varepsilon_{c}\right)=\Lambda_{1, \text { cusp }}(1)$ et $\left(J_{2} \Lambda\right)_{c}=-\Lambda\left(x_{c}, \varepsilon_{c}\right)$. Définissons $\Lambda^{\prime} \in \mathcal{S}_{1, \text { imp }}$ par $\Lambda^{\prime+}=\{1,0\}, \Lambda^{\prime-}=\{1\}$. On vérifie que, avec une notation évidente, $\left(J_{1} \Lambda^{\prime}\right)_{c}=-\Lambda\left(x_{c}, \varepsilon_{c}\right)$. D'après ce que l'on a déjà démontré pour $\Lambda^{\prime}$, il existe un automorphisme $\sigma^{\prime}$, égal à 1 ou $\sigma$, de sorte que :

$$
i\left(J_{1} \Lambda^{\prime} \otimes \Lambda_{2}\right)=\left(J_{1} \Lambda^{\prime}\right)_{n c} \otimes \Lambda_{2}+\left(J_{1} \Lambda^{\prime}\right)_{c} \otimes \sigma^{\prime} \Lambda_{2} .
$$

De plus $\sigma^{\prime}=1$ si $\varepsilon=1$. On vérifie que $\left(J_{1} \Lambda^{\prime} \otimes \Lambda_{2}, J_{2} \Lambda \otimes \Lambda_{2}\right)=0$. Parce que $i$ est une isométrie, on en déduit l'égalité $\sigma_{n c} \sigma^{\prime}=\sigma_{c}$. D'où :

$$
i\left(J_{1} \Lambda^{\prime} \otimes \Lambda_{2}-J_{2} \Lambda \otimes \sigma_{n c} \Lambda_{2}\right)=\left(J_{1} \Lambda^{\prime}\right)_{n c} \otimes \Lambda_{2}-\left(J_{2} \Lambda\right)_{n c} \otimes \Lambda_{2} .
$$

Ou encore :

$$
k_{3}\left(\mathcal{F} J_{1} \Lambda^{\prime} \otimes \mathcal{F} \Lambda_{2}-\mathcal{F} J_{2} \Lambda \otimes \mathcal{F} \sigma_{n c} \Lambda_{2}\right)=\pi_{3}\left(\left(J_{1} \Lambda^{\prime}\right)_{n c} \otimes \Lambda_{2}-\left(J_{2} \Lambda\right)_{n c} \otimes \Lambda_{2}\right) .
$$

Appliquons la forme linéaire $\ell$ aux deux membres de cette égalité. Grâce au lemme 13.3 et à l'hypothèse de récurrence, on trouve, pour le membre de gauche :

$$
(\zeta(-1) q)^{-1 / 2} q^{3} \varepsilon\left(\pi_{2}\left(J_{1} \Lambda^{\prime} \otimes \Lambda_{2}(\varnothing)\right)(1)-\nu \pi_{2}\left(J_{2} \Lambda \otimes \Lambda_{2}(\varnothing)\right)(1)\right),
$$

où :

$$
\nu= \begin{cases}1, & \text { si } \sigma_{n c}=1 \\ -1, & \text { si } \sigma_{n c}=\sigma\end{cases}
$$


Remarquons que $\left(J_{1} \Lambda^{\prime}\right)_{n c}$ et $\left(J_{2} \Lambda\right)_{n c}$ n'ont pas de termes cuspidaux. Grâce au lemme 13.11, l'image par $\ell$ du membre de droite de (3) vaut

$$
(\zeta(-1) q)^{-1 / 2} q^{3} \varepsilon\left(\pi_{2}\left(\left(J_{1} \Lambda^{\prime}\right)_{n c} \otimes \Lambda_{2}(\varnothing)\right)(1)-\pi_{2}\left(\left(J_{2} \Lambda\right)_{n c} \otimes \Lambda_{2}(\varnothing)\right)(1),\right.
$$

qui n'est autre que :

$$
(\zeta(-1) q)^{-1 / 2} q^{3} \varepsilon\left(\pi_{2}\left(J_{1} \Lambda^{\prime} \otimes \Lambda_{2}(\varnothing)\right)(1)-\pi_{2}\left(J_{2} \Lambda \otimes \Lambda_{2}(\varnothing)\right)(1)\right) .
$$

L'égalité de (4) et (5) entraîne que :

$$
(1-\nu) \pi_{2}\left(J_{2} \Lambda \otimes \Lambda_{2}(\varnothing)\right)(1)=0 .
$$

On a vu ci-dessus que le deuxième facteur était non nul. Donc $\nu=1$ et $\sigma_{n c}=1$. Si $\varepsilon=1$, les égalités $\sigma_{n c} \sigma^{\prime}=\sigma_{c}$ et $\sigma^{\prime}=1$ entraînent aussi $\sigma_{c}=1$. Cela achève de démontrer le (a) de l'énoncé.

Dans la situation de (b), des raisonnements purement combinatoires analogues à ceux du paragraphe 11 permettent de démontrer l'assertion, sauf dans le cas où $n_{1}=0$ et $n_{2}-a=1$. Dans ce cas, on peut seulement prouver que :

$$
i\left(\Lambda_{1} \otimes J_{a} \Lambda\right)-\Lambda_{1} \otimes J_{a} \Lambda \in \mathcal{Z} \text { ou } i\left(\Lambda_{1} \otimes J_{a} \Lambda\right)-\Lambda_{1} \otimes \sigma J_{a} \Lambda \in \mathcal{Z} .
$$

On doit exclure la deuxième possibilité. Supposons-la vérifiée. Remarquons que $\Lambda_{1}=$ $\Lambda_{1}(\varnothing)$ et que l'on peut supposer $\Lambda=\mathbf{1}_{\varepsilon}(1)$, avec $\varepsilon \in\{ \pm 1\}$. Par symétrie, on a :

$$
i\left(\Lambda_{1} \otimes J_{a}\left(\mathbf{1}_{\varepsilon}(1)-\sigma \mathbf{1}_{\varepsilon}(1)\right)\right)+\Lambda_{1} \otimes J_{a}\left(\mathbf{1}_{\varepsilon}(1)-\sigma \mathbf{1}_{\varepsilon}(1)\right) \in \mathcal{Z} .
$$

Notons $\left(\underline{\alpha}_{0}, \underline{\beta}_{0}\right)$ l'élément de $\mathcal{P}_{2}(n-1)$ défini par $\underline{\alpha}_{0}=\varnothing, \underline{\beta}_{0}=(n-1)$. D'après le lemme 13.16 , on a une égalité :

$$
\mathbf{1}_{-\varepsilon}(n)-\sigma \mathbf{1}_{-\varepsilon}(n)=\sum_{(\underline{\alpha}, \underline{\beta}) \in \mathcal{P}_{2}(n-1)} c_{\underline{\alpha}, \underline{\beta}} \Lambda_{\underline{\alpha}, \underline{\beta}},
$$

où :

$$
\Lambda_{\underline{\alpha}, \underline{\beta}}=I_{\underline{\alpha}} \circ J_{\underline{\beta}}\left(\mathbf{1}_{-\varepsilon \varepsilon(\underline{\beta})}(1)-\sigma \mathbf{1}_{-\varepsilon \varepsilon(\underline{\beta})}(1)\right)
$$

et les $c_{\underline{\alpha}, \underline{\beta}}$ sont des coefficients non nuls. Deux termes d'indices distincts de cette somme sont orthogonaux. Posons :

$$
\begin{aligned}
\Lambda^{\prime} & =\mathbf{1}_{-\varepsilon}(n)-\sigma \mathbf{1}_{-\varepsilon}(n)-2 c_{\underline{\alpha}_{0}, \underline{\beta}_{0}} J_{a}\left(\mathbf{1}_{\varepsilon}(1)-\sigma \mathbf{1}_{\varepsilon}(1)\right) \\
& =\left(\sum_{(\underline{\alpha}, \underline{\beta}) \in \mathcal{P}_{2}(n-1) \backslash\left\{\left(\underline{\alpha}_{0}, \underline{\beta}_{0}\right)\right\}} c_{\underline{\alpha}, \underline{\beta}} \Lambda_{\underline{\alpha}, \underline{\beta}}\right)-c_{\underline{\alpha}_{0}, \underline{\beta}_{0}} \Lambda_{\underline{\alpha}_{0}, \underline{\beta}_{0}} .
\end{aligned}
$$

Ce terme $\Lambda^{\prime}$ a même norme que $\mathbf{1}_{-\varepsilon}(n)-\sigma \mathbf{1}_{-\varepsilon}(n)$. Pour $(\underline{\alpha}, \underline{\beta}) \in \mathcal{P}_{2}(n-1) \backslash$ $\left\{\left(\underline{\alpha}_{0}, \underline{\beta}_{0}\right)\right\}$, on a déjà montré que $i\left(\Lambda_{1} \otimes \Lambda_{\underline{\alpha}, \underline{\beta}}\right)-\Lambda_{1} \otimes \Lambda_{\underline{\alpha}, \underline{\beta}}$ appartenait à $\mathcal{Z}$. La relation (6) nous dit que $i\left(\Lambda_{1} \otimes \Lambda_{\underline{\alpha}_{0}, \underline{\beta}_{0}}\right)+\Lambda_{1} \otimes \Lambda_{\underline{\alpha}_{0}, \underline{\beta}_{0}}$ appartient à $\mathcal{Z}$. On en déduit que $i\left(\Lambda_{1} \otimes \Lambda^{\prime}\right)-\Lambda_{1} \otimes\left(\mathbf{1}_{-\varepsilon}(n)-\sigma \mathbf{1}_{-\varepsilon}(n)\right)$ appartient à $\mathcal{Z}$, ou encore :

$$
i\left(\Lambda_{1} \otimes \Lambda^{\prime}\right)=\Lambda_{1} \otimes\left(\mathbf{1}_{-\varepsilon}(n)-\sigma \mathbf{1}_{-\varepsilon}(n)\right)+Z,
$$


avec $Z \in \mathcal{Z}$. Parce que $i$ est une isométrie et que les deux termes du membre de droite ci-dessus sont orthogonaux, on a en fait $Z=0$ et l'égalité précédente s'écrit :

$$
\begin{aligned}
k_{n}\left(\Lambda_{1}(\varnothing) \otimes \mathcal{F}\left(\mathbf{1}_{-\varepsilon}(n)-\sigma \mathbf{1}_{-\varepsilon}(n)\right)\right) & -\pi_{n}\left(\Lambda_{1}(\varnothing) \otimes\left(\mathbf{1}_{-\varepsilon}(n)-\sigma \mathbf{1}_{-\varepsilon}(n)\right)\right) \\
= & 2 c_{\underline{\alpha}_{0}, \underline{\beta}_{0}} k_{n}\left(\Lambda_{1}(\varnothing) \otimes \mathcal{F} \circ J_{a}\left(\mathbf{1}_{\varepsilon}(1)-\sigma \mathbf{1}_{\varepsilon}(1)\right)\right) .
\end{aligned}
$$

Appliquons la forme linéaire $\ell$ aux deux membres. Grâce aux lemmes 13.5(a), 13.12 et 13.13 , et aux propositions 3.10 (iii) et 4.12 (b)(ii), le membre de gauche s'annule. Grâce au lemme 13.5(b) et à la proposition 3.10(iii), le membre de droite ne s'annule pas. Cette contradiction achève la démonstration.

13.18. Comme en 11.13 , on définit un espace $\mathbb{C}\left[\mathcal{S} \widetilde{\mathcal{S}}_{n, \operatorname{mix}}\right]_{I J}$.

Corollaire. - On peut choisir l'application $\pi_{n, \text { cusp }}$ de sorte que la restriction de $i$ au sous-espace $\mathbb{C}\left[\mathcal{S} \widetilde{\mathcal{S}}_{n, \text { mix }}\right]_{I J}$ soit l'identité.

On fixe ainsi $\pi_{n, \text { cusp }}$.

13.19. On pose :

$$
H=\left\{\left(h_{1}, h_{2}\right) \in \mathbb{N} \times \mathbb{Z} ; h_{1}^{2}+h_{1}+h_{2}^{2}=n\right\} .
$$

On définit une matrice carrée $C$ d'ordre $|H| \times|H|$ comme en 12.1. Elle est unitaire. Pour $\left(h_{1}, h_{2}\right),\left(h_{1}^{\prime}, h_{2}^{\prime}\right) \in H$, on a les égalités :

$$
\begin{aligned}
C_{h_{1},-h_{2} ; h_{1}^{\prime},-h_{2}^{\prime}} & =C_{h_{1}, h_{2} ; h_{1}^{\prime}, h_{2}^{\prime}} \\
C_{h_{1}, h_{2} ; h_{1}^{\prime}, h_{2}^{\prime}} & =0 \quad \text { si } h_{2} \not \equiv h_{2}^{\prime} \bmod 2 \mathbb{Z} .
\end{aligned}
$$

Définissons o et $\theta$ comme en 13.15. Il est visible sur les définitions, que, pour $\left(h_{1}, h_{2}\right) \in H$, la fonction $k\left(h_{1}, h_{2}\right)=k\left(\Lambda_{1, \text { cusp }}\left(h_{1}\right), \Lambda_{2, \text { cusp }}\left(h_{2}\right)\right)$ prend ses valeurs dans o et, quand $\mathbb{Q}\left((\zeta(-1) q)^{1 / 2}\right)$ est une extension quadratique de $\mathbb{Q}$, on a l'égalité $\theta\left(k\left(h_{1}, h_{2}\right)\right)=(-1)^{h_{2}} k\left(h_{1}, h_{2}\right)$. En utilisant cela et le lemme 13.15 , on démontre comme en 12.3 que les coefficients de $C$ sont rationnels. On montre ensuite comme en 12.4 et 12.5 qu'ils appartiennent à $2^{-N} \mathbb{Z}$ pour un entier $N$ assez grand.

LEMme. - Soient $m_{1}, m_{2} \in \mathbb{N}$ tels que $m_{1}^{2}+m_{1}+m_{2}^{2}=n$ et $m_{1}+m_{2} \geqslant 2$. Alors $C_{m_{1},(-1)^{m_{1}+1} m_{2} ; m_{1},(-1)^{m_{1}+1} m_{2}}=1$.

Démonstration. - On définit $\widetilde{\operatorname{Fam}}_{2}$ comme en 12.2 , ainsi que $\Lambda_{2}^{e}$ pour $e \in \mathbb{Z} / 2 \mathbb{Z}$. On introduit le symbole spécial :

$$
\Lambda_{1}^{\mathrm{sp}}=\left\{\left(x,(-1)^{x}\right) ; x \in\left\{2 m_{1}, \ldots, 0\right\}\right\},
$$


on note Fam $_{1}$ sa famille dans $\mathcal{S}_{m_{1}^{2}+m_{1} \text {,imp }}$. Comme en 12.6, pour $\left(\Lambda_{1}, \Lambda_{2}\right) \in \operatorname{Fam}_{1} \times$ $\widetilde{\mathrm{Fam}_{2}}$, on pose :

$$
\begin{aligned}
& Y\left(\Lambda_{1}, \Lambda_{2}\right) \\
= & \sum_{\left(h_{1}, h_{2}\right) \in H} k\left(h_{1}, h_{2}\right) \sum_{h_{2}^{\prime} \in\left\{ \pm m_{2}\right\}}(-1)^{\left\langle\Lambda_{1}, \Lambda_{1, \text { cusp }}\left(m_{1}\right)\right\rangle+\left\langle\Lambda_{2}, \Lambda_{2, \text { cusp }}\left(h_{2}^{\prime}\right)\right\rangle}\left(C_{h_{1}, h_{2} ; m_{1}, h_{2}^{\prime}}-\delta_{h_{1}, h_{2} ; m_{1}, h_{2}^{\prime}}\right) .
\end{aligned}
$$

Soit $g \in S p(2 n)$ vérifiant les conditions de 13.6. Les formules de 3.9 montrent que, pour $\left(h_{1}, h_{2}\right) \in H \backslash\left\{\left(m_{1},(-1)^{m_{1}+1} m_{2}\right)\right\}$, on a l'égalité $k\left(h_{1}, h_{2}\right)(g)=0$. Posons $K=k\left(m_{1},(-1)^{m_{1}+1} m_{2}\right)(g)$, qui est non nul. Supposons $m_{2} \neq 0$ (le raisonnement s'adapte aisément au cas $\left.m_{2}=0\right)$. Pour $\varepsilon \in\{ \pm 1\}$, posons :

$$
c(\varepsilon)=C_{m_{1},(-1)^{m_{1}+1} m_{2} ; m_{1}, \varepsilon m_{2}}-\delta_{m_{1},(-1)^{m_{1}+1} m_{2} ; m_{1}, \varepsilon m_{2}} .
$$

Alors, pour $e \in \mathbb{Z} / 2 \mathbb{Z}$, on a l'égalité :

$$
Y\left(\Lambda_{1}^{\mathrm{sp}}, \Lambda_{2}^{e}\right)(g)=K \sum_{\varepsilon \in\{ \pm 1\}} \varepsilon^{e} c(\varepsilon) .
$$

Cette égalité s'inverse en :

$$
c(\varepsilon)=\frac{1}{2 K} \sum_{e \in \mathbb{Z} / 2 \mathbb{Z}} \varepsilon^{e} Y\left(\Lambda_{1}^{\mathrm{sp}}, \Lambda_{2}^{e}\right)(g) .
$$

Mais on a l'égalité :

$$
Y\left(\Lambda_{1}, \Lambda_{2}\right)=\left|\operatorname{Fam}_{1}\right|^{1 / 2}\left|\widetilde{\operatorname{Fam}}_{2}\right|^{1 / 2}\left(\operatorname{trace}\left(\pi\left(\Lambda_{1}, \Lambda_{2}\right)\right)-k_{n}\left(\mathcal{F} \Lambda_{1} \otimes \mathcal{F} \Lambda_{2}\right)\right)
$$

pour tous $\left(\Lambda_{1}, \Lambda_{2}\right) \in \operatorname{Fam}_{1} \times \widetilde{\operatorname{Fam}}_{2}$. Grâce à l'hypothèse $m_{1}+m_{2} \geqslant 2$, les lemmes 13.7 et 13.15 entraînent que, pour tout $e \in \mathbb{Z} / 2 \mathbb{Z}, Y\left(\Lambda_{1}^{\mathrm{sp}}, \Lambda_{2}^{e}\right)(g) \in 4 \mathfrak{o}$. Parce que $K$ est premier à 2 et $c(\varepsilon)$ est rationnel pour tout $\varepsilon \in\{ \pm 1\}$, on déduit de (1) les relations :

$$
c(1), c(-1) \in 2 \mathbb{Z}, c(1)-c(-1) \in 4 \mathbb{Z} .
$$

D'autre part, l'unitarité de la matrice $C$ entraîne :

$$
\left(c\left((-1)^{m_{1}+1}\right)+1\right)^{2}+c\left((-1)^{m_{1}}\right)^{2} \leqslant 1 .
$$

Prises ensemble, ces relations entraînent les égalités $c\left((-1)^{m_{1}}\right)=c\left((-1)^{m_{1}+1}\right)=0$. La dernière entraîne l'énoncé.

13.20. Si $m_{1}, m_{2} \in \mathbb{N}$ vérifient $m_{1}^{2}+m_{1}+m_{2}^{2}=n$ et $m_{1}+m_{2} \leqslant 1$, on a soit $m_{1}=0$, $m_{2}=n=1$, cas que l'on a déjà traité, soit $m_{1}=1, m_{2}=0, n=2$. Dans ce cas, en utilisant le lemme 13.14, on démontre comme en 12.7 que $C_{1,0 ; 1,0}=1$. On a ainsi supprimé la restriction $m_{1}+m_{2} \geqslant 1$ de l'énoncé du lemme précédent. On démontre ensuite comme en 12.10 que $C$ est la matrice identité, ce qui achève la preuve du théorème. 


\section{BIBLIOGRAPHIE}

[A] T. AsAI - « Unipotent class functions of split special orthogonal groups $S \mathrm{O}_{2 n}^{+}$ over finite fields », Comm. Algebra 12 (1984), p. 517-615.

[BR] C. Bonnafé \& R. Rouquier - « Catégories dérivées et vériétés de DeligneLusztig», Publ. Math. Inst. Hautes Études Sci. 97 (2003), p. 1-59.

[C] R. CARTER - Finite groups of Lie type; conjugacy class and complex characters, Wiley Interscience Publ., 1993.

[CR] C. CuRTis \& I. ReINER - Methods of representation theory, with applications to finite groups and orders, vol. 1, Wiley Interscience, 1981.

[DM1] F. Digne \& J. MicheL - Representations of finite groups of Lie type, London Math. Soc. student texts, vol. 21, Cambridge Univ. Press, 1991.

[DM2] - « Groupes réductifs non connexes», Ann. scient. Éc. Norm. Sup. $4^{\mathrm{e}}$ série 27 (1994), p. 345-406.

[E] M. EfTeKharI - «Faisceaux caractères sur les groupes non connexes », J. Algebra 184 (1996), p. 516-537.

[L1] G. LuszTig - « On the finiteness of the number of unipotent classes », Invent. Math. 34 (1976), p. 201-213.

[L2] , «Irreductible representations of finite classical groups», Invent. Math. 43 (1977), p. 125-175.

[L3] - « Unipotent characters of the even orthogonal groups over a finite field», Trans. Amer. Math. Soc. 272 (1982), p. 733-751.

[L4] , «Intersection cohomology complexes on a reductive group », Invent. Math. 75 (1984), p. 205-272.

[L5] — « Character sheaves II », Adv. in Math. 57 (1985), p. 226-265. 
[L6] _ « Character sheaves V», Adv. in Math. 61 (1986), p. 103-155.

[L7] « «On the representations of reductive groups with disconnected center », in Orbites unipotentes et représentations I, Astérisque, vol. 168, Société Mathématique de France, Paris, 1988, p. 157-166.

[L8] _ « Green functions and character sheaves », Ann. of Math. 131 (1990), p. $355-408$.

[L9] , «A unipotent support for irreducible representations », Adv. in Math. 94 (1992), p. 139-179.

[L10] _ « Remarks on computing irreductible characters », J. Amer. Math. Soc. 5 (1992), p. 971-986.

[M] C. Moeglin - «Représentations quadratiques unipotentes des groupes classiques p-adiques », Duke Math. J. 84 (1996), p. 267-332.

[MW] C. Moeglin \& J.-L. Waldspurger - «Paquets stables de représentations tempérées et de réduction unipotente pour $S O(2 n+1) »$, Invent. Math 152 (2003), p. 461-623.

[S1] T. ShoJI - « Character sheaves and almost characters of reductive groups I et II », Adv. in Math. 111 (1995), p. 244-313 et 314-354.

[S2] _ _ Unipotent characters of finite classical groups », in Finite reductive groups (Luminy 1994), Progress in Math., vol. 141, Birkhäuser, 1997.

[W] J.-L. WALDSPURGER - Intégrales orbitales nilpotentes et endoscopie pour les groupes classiques non ramifiés, Astérisque, vol. 269, Société Mathématique de France, Paris, 2001.

[Z] A. ZeLEVINSKY - Representations of finite classical groups, Lect. Notes in Math., vol. 869, Springer, 1981. 